\title{
A Radical-Initiated Fragmentary Rearrangement Cascade of Ene- Ynamides to [1,2]-Annulated Indoles via Site-Selective Cyclization
}

Sifan Li, ${ }^{\dagger}+$ Yu Wang, ${ }^{\dagger}$ Zibo Wu ${ }^{\S}$ Weiliang Shi, ${ }^{\S}$ Yibo Lei, ${ }^{\S}$ Paul W. Davies, ${ }^{*}{ }^{\star}$ and Wei Shu*,†

† Shenzhen Grubbs Institute, Department of Chemistry, and Guangdong Provincial Key Laboratory of Catalysis, Southern University of Science and Technology, Shenzhen, Guangdong (P. R. China).

* School of Chemistry, University of Birmingham, Edgbaston, Birmingham, B15 2TT, U.K.

$\S$ Key Laboratory of Synthetic and Natural Functional Molecule of the Ministry of Education, College of Chemistry and Materials Science, Northwest University, Xi'an, Shaanxi (P. R. China)

\section{*E-mail:shuw@sustech.edu.cn; p.w.davies@bham.ac.uk}

Contents

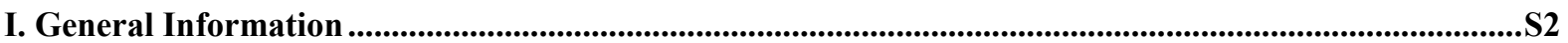

II. Preparation of Alkene Tethered Ynamides.......................................................................................................S3

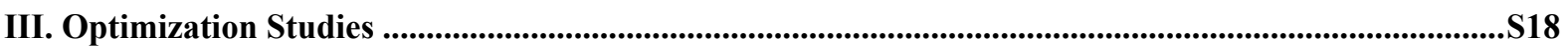

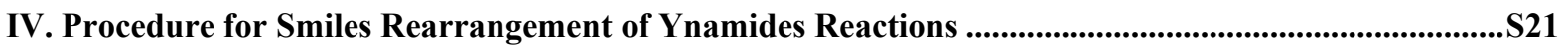

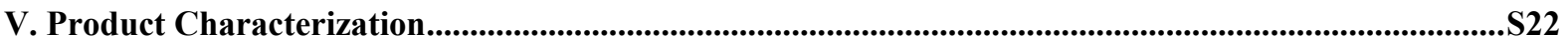

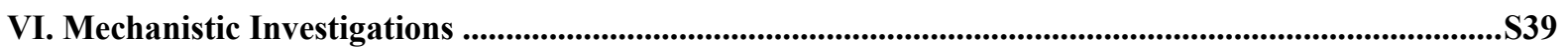

VII. Crystal Data and Structure Refinement for 3e, 3m, 3t, 10a, 10b......................................................S83

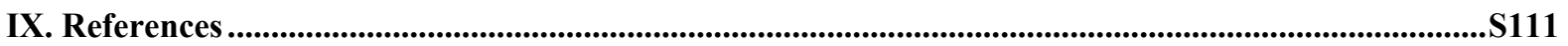

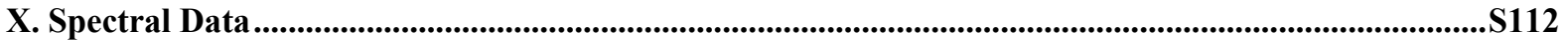




\section{General Information}

NMR spectra were recorded on $400 \mathrm{MHz}$ or $600 \mathrm{MHz}$ Bruker spectrometers. Chemical shifts are given in ppm. The spectra are calibrated to the residual $1 \mathrm{H}$ and $13 \mathrm{C}$ signals of the solvents. Multiplicities are abbreviated as follows: singlet (s), doublet (d), triplet ( $\mathrm{t}$ ), quartet (q), doublet-doublet (dd), quintet (quint), septet (sept), multiplet (m), and broad (br). High-resolution electrospray ionization and electronic impact mass spectrometry was performed on a Thermo Scientific Q Exactive mass spectrometer (mass analyzer type: Orbitrap). A mass accuracy $\leq 2 \mathrm{ppm}$ was obtained in the peak matching acquisition mode by using a solution containing $2<1$ PEG200, $2<1$ PPG450, and $1.5 \mathrm{mg}$ $\mathrm{NaOAc}$ (all obtained from Sigma-Aldrich, CH-Buchs) dissolved in $100 \mathrm{~mL} \mathrm{MeOH}$ (HPLC Supra grade, Scharlau, E-Barcelona) as internal standard.

Materials and Methods: Unless otherwise stated, starting materials were used upon purchased. Solvents were purchased in HPLC quality, degassed by purging with nitrogen and dried over activated molecular sieves of appropriate size. Alternatively, they were purged with argon and passed through alumina columns in a solvent purification system (Innovative Technology). Conversion was monitored by thin layer chromatography (TLC) using Merck TLC silica gel 60 F254. Compounds were visualized by UV light at $254 \mathrm{~nm}$ and by dipping the plates in an ethanolic vanillin/sulfuric acid solution or an aqueous potassium permanganate solution followed by heating. Flash column chromatography was performed over silica gel (230-400 mesh). 


\section{Preparation of Alkene Tethered Ynamides}

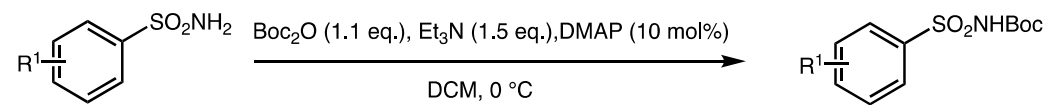

Scheme S1. Boc-protection of sulfonamide

To a flame-dried round-bottomed flask charged with a stir bar was added the corresponding sulfonamide (1.0 eq.) and DMAP (10 mol\%). Dichloromethane was added followed by addition of triethylamine (1.5 eq.), and dropwise addition of a solution of $\mathrm{Boc}_{2} \mathrm{O}$ (1.1 eq.) in dichloromethane at $0{ }^{\circ} \mathrm{C}$. The resulting reaction mixture was stirred at room temperature overnight. After completion the solvent was removed on a rotary evaporator, the residue was diluted with ethyl acetate and $1.0 \mathrm{M} \mathrm{HCl}$ was added. The organic layer was washed with water, brine, then dried over $\mathrm{Na}_{2} \mathrm{SO}_{4}$ and concentrated on a rotary evaporator to give Boc-protected sulfonamide, which was used for the next step without further purification.

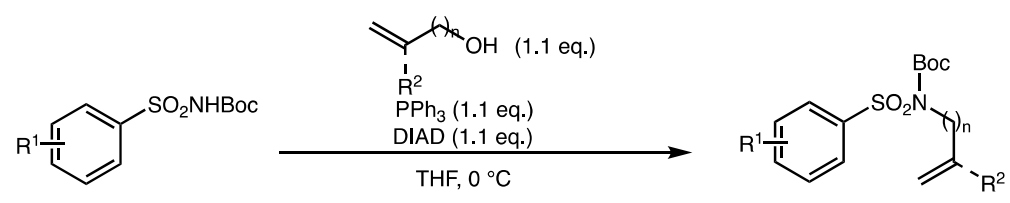

Scheme S2. Synthesis of alkene tethered sulfonamide ${ }^{1}$

To a flame-dried round-bottomed flask charged with a stir bar was added the Boc-protected sulfonamide (1.0 eq.), triphenylphosphine (1.1 eq.) under $\mathrm{N}_{2}$ atmosphere. Dry THF was then added followed by slow addition of corresponding alcohol (1.1 eq.), and the reaction mixture was stirred for 15 minutes. Azodicarbonic acid diisopropyl ester (DIAD, 1.1 eq.) was then added dropwise at $0{ }^{\circ} \mathrm{C}$. The reaction was stirred at room temperature until full consumption of the sulfonamide (typically 2-12 h). The solvent was removed on a rotary evaporator and the residue was purified by silica gel flash chromatography to afford the pure product $(70-90 \%)$.
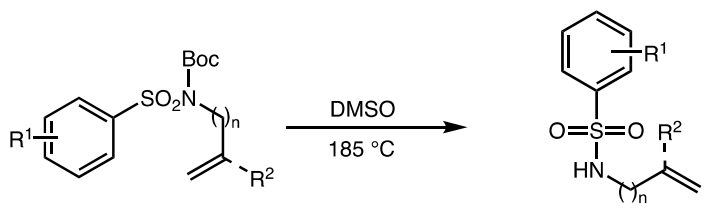

Scheme S3. Boc-deprotection

A solution of alkene-tethered sulfonamide in DMSO was vigorously stirred while being heated at $185^{\circ} \mathrm{C}$ using a metal heating block. After full consumption of starting material, the reaction mixture was allowed to cool to room temperature. Ethyl acetate was then added, and the organic layer was washed with water, brine and then dried over $\mathrm{Na}_{2} \mathrm{SO}_{4}$ and concentrated on a rotary evaporator. The residue was purified by flash chromatography to afford the pure product (85-95\%). 


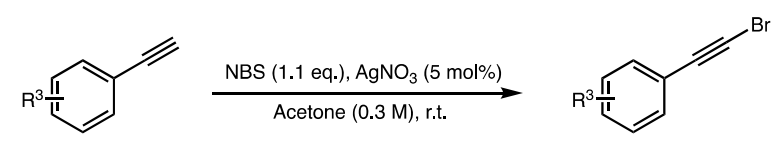

Scheme S4. Synthesis of bromoacetylenes

A solution of terminal acetylene (1.0 eq.), NBS (1.1 eq.) and $\mathrm{AgNO}_{3}(5 \mathrm{~mol} \%)$ in acetone (0.3 M) was stirred at room temperature for $3 \mathrm{~h}$. Silica gel was added to the reaction mixture which was concentrated under reduced pressure. The silica-absorbed crude reaction mixture was added to a $3 \mathrm{~cm}$ pad of silica in a sintered funnel and the product eluted with hexane, concentrated on a rotary evaporator. Bromoacetylene was used for the next step without further purification.

\section{Synthesis of alkene tethered ynamides $(\operatorname{Method} \mathbf{A})^{2}$}

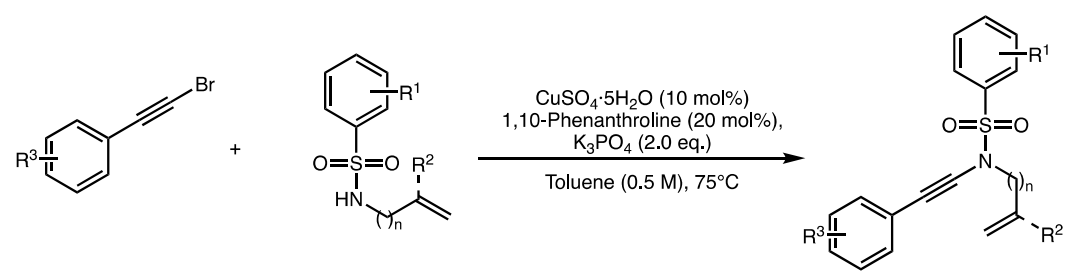

To a mixture of alkene tethered sulfonamide (if solid, 1.0 eq.), $\mathrm{K}_{3} \mathrm{PO}_{4}\left(2.0\right.$ eq.), $\mathrm{CuSO}_{4} \cdot 5 \mathrm{H}_{2} \mathrm{O}(10 \mathrm{~mol} \%$ ), 1,10-phenanthroline (20 mol\%) in a Schlenk flask was added dry toluene, sulfonamide (if oil, 1.0 eq.) and corresponding bromoalkyne (1.2 eq.) under $\mathrm{N}_{2}$ atmosphere. The reaction mixture was heated at $75{ }^{\circ} \mathrm{C}$ in an oil-bath until full consumption of the starting material (typically 8-32 h). The reaction mixture was cooled to room temperature and diluted with ethyl acetate and filtered through a pad of Celite. The filtrate was concentrated on a rotary evaporator. The crude products were then purified by silica gel flash chromatography to afford the pure product (38-85\%).

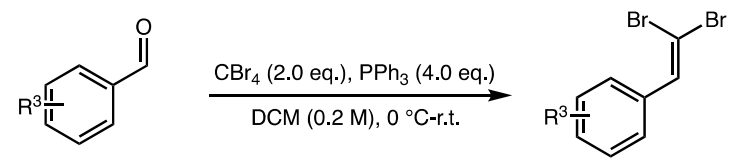

Scheme S5. Synthesis of dibromoalkenes

$\mathrm{CBr}_{4}$ (2.0 eq.) and $\mathrm{PPh}_{3}$ (4.0 eq.) were stirred at $0{ }^{\circ} \mathrm{C}$ in $\mathrm{DCM}(0.2 \mathrm{M})$. Benzaldehyde (1.0 eq.) was added dropwise to the reaction mixture at $0{ }^{\circ} \mathrm{C}$. The ice bath was removed, and the reaction mixture was stirred at room temperature overnight. The reaction mixture was quenched with pentane and filtered through a silica plug $\left(\mathrm{Et}_{2} \mathrm{O}\right)$. The solvent was removed on a rotary evaporator and dibromoalkenes was isolated by flash chromatography (60-95\%). 


\section{Synthesis of alkene tethered ynamides $\left(\operatorname{Method~B)^{3}}\right.$}

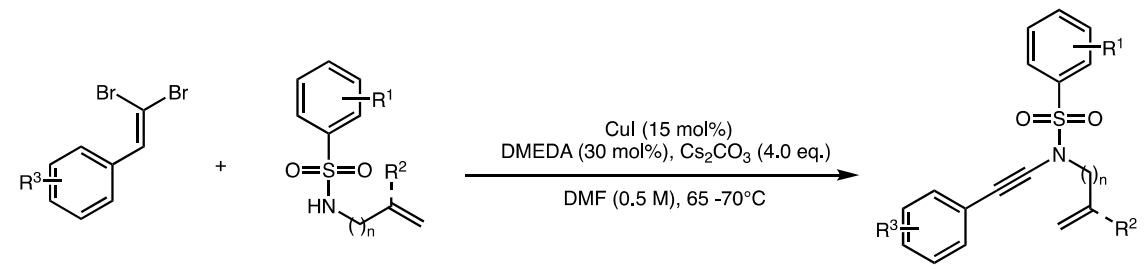

To a mixture of alkene tethered sulfonamide (if solid, 1.0 eq.), $\mathrm{Cs}_{2} \mathrm{CO}_{3}$ (4.0 eq.), $\mathrm{CuI}$ (15 mol\%) in a Schlenk flask was added DMF, sulfonamide (if oil, 1.0 eq.) and corresponding dibromoalkene (1.5 eq.), $N, N^{\prime}$-dimethylethylenediamine (DMEDA $30 \mathrm{~mol} \%$ ) under $\mathrm{N}_{2}$ atmosphere. The reaction mixture was heated at $65^{\circ} \mathrm{C}$ in an oil-bath until full consumption of the starting material. The reaction mixture was cooled to room temperature and diluted with ethyl acetate and filtered through a pad of Celite. The filtrate was washed with water, brine and then dried over $\mathrm{Na}_{2} \mathrm{SO}_{4}$ and concentrated on a rotary evaporator. The crude products were purified by silica gel flash chromatography to afford the pure product (45-89\%) 


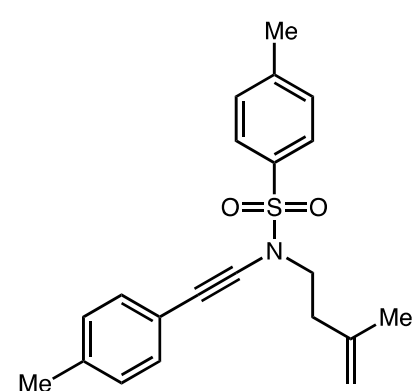

Following the method $\mathbf{A}$, purification by flash column chromatography (5$10 \%$ EtOAc in hexane), Colourless oil. ${ }^{1} \mathrm{H}$ NMR $\left(600 \mathrm{MHz}, \mathrm{CDCl}_{3}\right) \delta 7.87$ (d, $J=8.1 \mathrm{~Hz}, 2 \mathrm{H}), 7.36$ (d, $J=8.1 \mathrm{~Hz}, 2 \mathrm{H}), 7.0$ (d, $J=8.1 \mathrm{~Hz}, 2 \mathrm{H}), 7.12$ $(\mathrm{d}, J=8.1 \mathrm{~Hz}, 2 \mathrm{H}), 4.85-4.82(\mathrm{~m}, 1 \mathrm{H}), 4.80-4.77(\mathrm{~m}, 1 \mathrm{H}), 3.55(\mathrm{t}, J=$ $7.5 \mathrm{~Hz}, 2 \mathrm{H}), 2.44(\mathrm{~s}, 3 \mathrm{H}), 2.43(\mathrm{t}, J=7.4 \mathrm{~Hz}, 2 \mathrm{H}), 2.34(\mathrm{~s}, 3 \mathrm{H}), 1.77$ (s, $3 \mathrm{H}) ;{ }^{13} \mathrm{C} \mathrm{NMR}\left(151 \mathrm{MHz}, \mathrm{CDCl}_{3}\right) \delta 144.6,141.2,137.9,134.5,131.4(2 \mathrm{C})$, 129.7 (2C), 129.0 (2C), $127.6(2 \mathrm{C}), 119.6,112.8,81.4,70.8,49.9,35.8$,

\section{$\mathrm{N}$-(3-Methylbut-3-en-1-yl)- $\mathrm{N}$-(phenylethynyl)benzenesulfonamide (1b)}

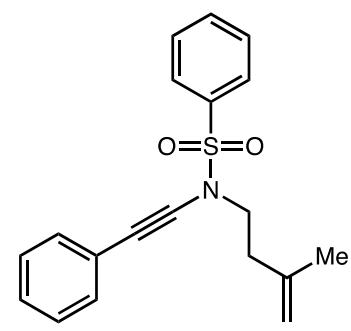

Following the method A, purification by flash column chromatography (5-10\% EtOAc in hexane), Colourless oil. ${ }^{1} \mathrm{H}$ NMR $\left(400 \mathrm{MHz}, \mathrm{CDCl}_{3}\right) \delta 8.01-7.93$ (m, 2H), $7.70-7.62(\mathrm{~m}, 1 \mathrm{H}), 7.61-7.53(\mathrm{~m}, 2 \mathrm{H}), 7.41-7.34(\mathrm{~m}, 2 \mathrm{H}), 7.32$ $-7.27(\mathrm{~m}, 3 \mathrm{H}), 4.82-4.79(\mathrm{~m}, 1 \mathrm{H}), 4.76-4.73(\mathrm{~m}, 1 \mathrm{H}), 3.57-3.51(\mathrm{~m}, 2 \mathrm{H})$, $2.41(\mathrm{~m}, 2 \mathrm{H}), 1.75(\mathrm{~s}, 3 \mathrm{H}) ;{ }^{13} \mathrm{C} \mathrm{NMR}\left(101 \mathrm{MHz}, \mathrm{CDCl}_{3}\right) \delta 141.2,137.7,133.7$, 131.5 (2C), 129.2 (2C), 128.4 (2C), 128.0, 127.8 (2C), 122.9, 113.1, 82.1, 71.1, 50.2, 36.1, 22.4. HRMS ESI $[\mathrm{M}+\mathrm{H}]^{+}$calculated for $\mathrm{C}_{19} \mathrm{H}_{20} \mathrm{NO}_{2} \mathrm{~S} 326.1215$, found 326.1208 .

\section{$N$-([1,1'-Biphenyl]-4-ylethynyl)- $N$-(3-methylbut-3-en-1-yl)-[1,1'-biphenyl]-4-sulfonamide (1c)}

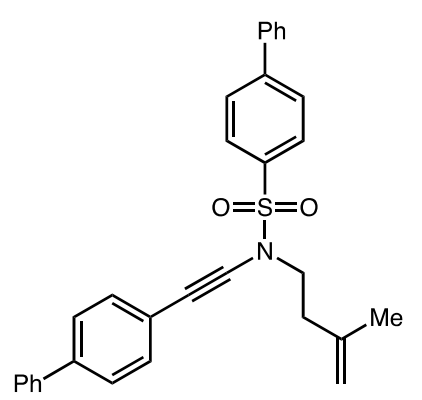

Following the method $\mathbf{B}$, purification by flash column chromatography (5$10 \%$ EtOAc in hexane), Colourless oil. ${ }^{1} \mathrm{H}$ NMR (400 MHz, $\left.\mathrm{CDCl}_{3}\right) \delta 7.92$ (d, $J=8.2 \mathrm{~Hz}, 2 \mathrm{H}), 7.64$ (d, $J=8.2 \mathrm{~Hz}, 2 \mathrm{H}), 7.53-7.38$ (m, 6H), 7.32 (m, $7 \mathrm{H}), 7.23$ (d, $J=7.3 \mathrm{~Hz}, 1 \mathrm{H}), 4.71(\mathrm{~s}, 1 \mathrm{H}), 4.67$ (s, 1H), 3.49 (t, $J=7.5 \mathrm{~Hz}$, 2H), $2.34(\mathrm{t}, J=7.5 \mathrm{~Hz}, 2 \mathrm{H}), 1.66(\mathrm{~s}, 3 \mathrm{H}) ;{ }^{13} \mathrm{C} \mathrm{NMR}\left(101 \mathrm{MHz}, \mathrm{CDCl}_{3}\right) \delta$ 146.6, 141.3, 140.7, 140.4, 139.1, 136.1, 131.9 (2C), 129.2 (2C), 128.9 (2C), $128.8,128.3(2 \mathrm{C}), 127.8(2 \mathrm{C}), 127.7,127.4(2 \mathrm{C}), 127.1(2 \mathrm{C}), 127.0(2 \mathrm{C})$, 121.8, 113.1, 82.8, 71.1, 50.2, 36.1, 22.4. HRMS ESI $[\mathrm{M}+\mathrm{H}]^{+}$calculated for $\mathrm{C}_{31} \mathrm{H}_{28} \mathrm{NO}_{2} \mathrm{~S}$ 478.1841, found 478.1834 .

4-Methoxy- $N$-((4-methoxyphenyl)ethynyl)- $N$-(3-methylbut-3-en-1-yl) benzenesulfonamide (1d)

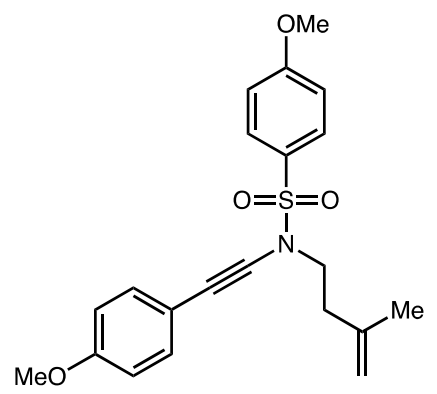
Following the method A, purification by flash column chromatography $\left(10 \%\right.$ EtOAc in hexane) white solid. ${ }^{1} \mathrm{H}$ NMR $\left(400 \mathrm{MHz}, \mathrm{CDCl}_{3}\right) \delta 7.88$ (d, $J=8.9 \mathrm{~Hz}, 2 \mathrm{H}), 7.32$ (d, $J=8.9 \mathrm{~Hz}, 2 \mathrm{H}), 7.01(\mathrm{~d}, J=9.0 \mathrm{~Hz}, 2 \mathrm{H})$, $6.82(\mathrm{~d}, J=8.9 \mathrm{~Hz}, 2 \mathrm{H}), 4.82-4.78(\mathrm{~m}, 1 \mathrm{H}), 4.75-4.73(\mathrm{~m}, 1 \mathrm{H}), 3.88$ $(\mathrm{s}, 3 \mathrm{H}), 3.79(\mathrm{~s}, 3 \mathrm{H}), 3.54-3.45(\mathrm{~m}, 2 \mathrm{H}), 2.44-2.34(\mathrm{~m}, 2 \mathrm{H}), 1.75(\mathrm{~s}$, $3 \mathrm{H}) .{ }^{13} \mathrm{C}$ NMR (101 MHz, $\left.\mathrm{CDCl}_{3}\right) \delta 163.7,159.6,141.5,133.4(2 \mathrm{C})$, 129.9 (2C), 129.2, 114.8, 114.3 (2C), 114.0 (2C), 112.8, 80.9, 70.6, 55.7, 55.4, 50.0, 36.0, 22.4. HRMS ESI $[\mathrm{M}+\mathrm{H}]^{+}$calculated for $\mathrm{C}_{21} \mathrm{H}_{24} \mathrm{NO}_{4} \mathrm{~S} 386.1426$, found 386.1417 . 


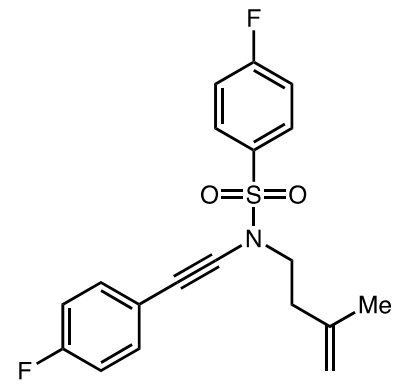

Following the method $\mathbf{B}$, purification by flash column chromatography (5$10 \%$ EtOAc in hexane), colourless oil. ${ }^{1} \mathrm{H}$ NMR $\left(400 \mathrm{MHz}, \mathrm{CDCl}_{3}\right) \delta 7.93$ $-7.85(\mathrm{~m}, 2 \mathrm{H}), 7.30-7.22(\mathrm{~m}, 2 \mathrm{H}), 7.22-7.11(\mathrm{~m}, 2 \mathrm{H}), 6.98-6.85(\mathrm{~m}$, $2 \mathrm{H}), 4.74-4.71(\mathrm{~m}, 1 \mathrm{H}), 4.67-4.64(\mathrm{~m}, 1 \mathrm{H}), 3.48-3.42(\mathrm{~m}, 2 \mathrm{H}), 2.38-$ $2.25(\mathrm{~m}, 2 \mathrm{H}), 1.66(\mathrm{~s}, 3 \mathrm{H}) .{ }^{13} \mathrm{C} \mathrm{NMR}\left(101 \mathrm{MHz}, \mathrm{CDCl}_{3}\right) \delta 165.8\left(\mathrm{~d},{ }^{1} J_{\mathrm{C}-\mathrm{F}}\right.$ $=257.6 \mathrm{~Hz}), 162.5\left(\mathrm{~d},{ }^{1} J_{\mathrm{C}-\mathrm{F}}=250.5 \mathrm{~Hz}\right), 141.1,133.7,133.7\left(\mathrm{~d},{ }^{3} J_{\mathrm{C}-\mathrm{F}}=8.1\right.$

$\mathrm{Hz}, 2 \mathrm{C}), 130.5\left(\mathrm{~d},{ }^{3} J_{\mathrm{C}-\mathrm{F}}=10.1 \mathrm{~Hz}, 2 \mathrm{C}\right), 118.7\left(\mathrm{~d},{ }^{4} J_{\mathrm{C}-\mathrm{F}}=4.0 \mathrm{~Hz}\right), 116.6\left(\mathrm{~d},{ }^{2} J_{\mathrm{C}-\mathrm{F}}=23.2 \mathrm{~Hz}, 2 \mathrm{C}\right), 115.7$ $\left(\mathrm{d},{ }^{2} \mathrm{~J}_{\mathrm{C}-\mathrm{F}}=22.2 \mathrm{~Hz}, 2 \mathrm{C}\right), 113.2,81.4,70.2,50.1,36.0,22.4 .{ }^{19} \mathrm{~F}$ NMR $\left(376 \mathrm{MHz}, \mathrm{CDCl}_{3}\right) \delta-103.20-$ $103.30(\mathrm{~m}, 1 \mathrm{~F}),-111.01--111.10(\mathrm{~m}, 1 \mathrm{~F})$. HRMS ESI $[\mathrm{M}+\mathrm{H}]^{+}$calculated for $\mathrm{C}_{19} \mathrm{H}_{18} \mathrm{NO}_{2} \mathrm{~S} 362.1026$, found 362.1018 .

\section{$N$-((4-(tert-Butyl)phenyl)ethynyl)-4-methyl- $N$-(3-methylbut-3-en-1-yl)benzenesulfonamide (1f)}

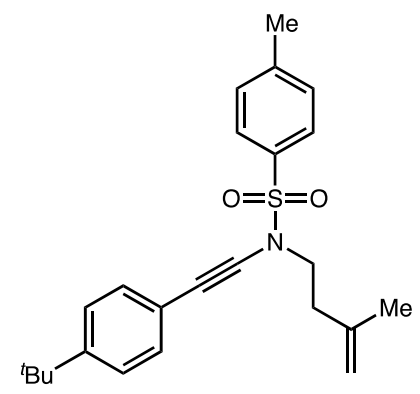

Following the method $\mathbf{B}$, purification by flash column chromatography ( $5 \%$ EtOAc in hexane), colourless oil. ${ }^{1} \mathrm{H}$ NMR $\left(400 \mathrm{MHz}, \mathrm{CDCl}_{3}\right) \delta 7.86(\mathrm{~d}$, $J=8.3 \mathrm{~Hz}, 2 \mathrm{H}), 7.36(\mathrm{~d}, J=8.3 \mathrm{~Hz}, 2 \mathrm{H}), \mathrm{j} 7.34(\mathrm{~s}, 4 \mathrm{H}), 4.82-4.80(\mathrm{~m}$, $1 \mathrm{H}), 4.76-4.74(\mathrm{~m}, 1 \mathrm{H}), 3.55-3.50(\mathrm{~m}, 2 \mathrm{H}), 2.46(\mathrm{~s}, 3 \mathrm{H}), 2.44-2.37$ $(\mathrm{m}, 2 \mathrm{H}), 1.75(\mathrm{~s}, 3 \mathrm{H}), 1.32(\mathrm{~s}, 9 \mathrm{H}) .{ }^{13} \mathrm{C}$ NMR $\left(101 \mathrm{MHz}, \mathrm{CDCl}_{3}\right) \delta$ 151.3, 144.7, 141.4, 134.7, $131.4(2 \mathrm{C}), 129.8(2 \mathrm{C}), 127.7$ (2C), 125.3 (2C), 119.8, $112.9,81.5,70.9,50.1,36.0,34.8,31.2(3 \mathrm{C}), 22.4,21.7$. HRMS ESI $[\mathrm{M}+\mathrm{H}]^{+}$calculated for $\mathrm{C}_{24} \mathrm{H}_{30} \mathrm{NO}_{2} \mathrm{~S}$ 396.1997, found 396.1989.

\section{$N$-((3-Methoxyphenyl)ethynyl)-4-methyl- $N$-(3-methylbut-3-en-1-yl)benzenesulfonamide (1g)}

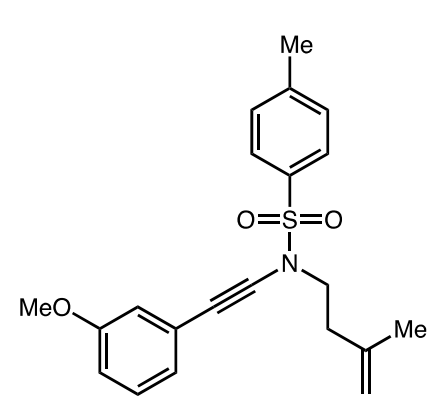

Following the method $\mathbf{A}$, purification by flash column chromatography $\left(10 \%\right.$ EtOAc in hexane), colourless oil. ${ }^{1} \mathrm{H}$ NMR $\left(600 \mathrm{MHz}, \mathrm{CDCl}_{3}\right) \delta$ $7.85(\mathrm{~d}, J=8.3 \mathrm{~Hz}, 2 \mathrm{H}), 7.36(\mathrm{~d}, J=8.3 \mathrm{~Hz}, 2 \mathrm{H}), 7.20(\mathrm{t}, J=8.0 \mathrm{~Hz}, 1 \mathrm{H})$, $6.97(\mathrm{dt}, J=7.6,1.2 \mathrm{~Hz}, 1 \mathrm{H}), 6.91(\mathrm{dd}, J=2.6,1.4 \mathrm{~Hz}, 1 \mathrm{H}), 6.86-6.83$ $(\mathrm{m}, 1 \mathrm{H}), 4.82-4.80(\mathrm{~m}, 1 \mathrm{H}), 4.78-4.73(\mathrm{~m}, 1 \mathrm{H}), 3.79(\mathrm{~s}, 3 \mathrm{H}), 3.55-$ $3.51(\mathrm{~m}, 2 \mathrm{H}), 2.45(\mathrm{~s}, 3 \mathrm{H}), 2.43-2.39(\mathrm{~m}, 2 \mathrm{H}), 1.76(\mathrm{~s}, 3 \mathrm{H}) .{ }^{13} \mathrm{C} \mathrm{NMR}$ $\left(151 \mathrm{MHz}, \mathrm{CDCl}_{3}\right) \delta 159.3,144.7,141.2,134.6,129.8(2 \mathrm{C}), 129.3,127.7$ (2C), 123.9, 123.8, 116.3, 114.1, 112.9, 82.1, 70.9, 55.2, 50.0, 35.9, 22.3, 21.6. HRMS ESI $[\mathrm{M}+\mathrm{H}]^{+}$ calculated for $\mathrm{C}_{21} \mathrm{H}_{24} \mathrm{NO}_{3} \mathrm{~S} 370.1477$, found 370.1467 .

$N$-((4-Fluorophenyl)ethynyl)-4-methyl- $N$-(3-methylbut-3-en-1-yl)benzenesulfonamide (1h)<smiles>C=C(C)CCN(C#Cc1ccc(F)cc1)S(=O)(=O)c1ccc([N+](=O)[O-])cc1</smiles>

Following the method $\mathbf{B}$, purification by flash column chromatography (5$10 \%$ EtOAc in hexane), colourless oil. ${ }^{1} \mathrm{H}$ NMR (600 MHz, $\left.\mathrm{CDCl}_{3}\right) \delta 7.83$ $(\mathrm{d}, J=8.3 \mathrm{~Hz}, 2 \mathrm{H}), 7.38-7.32(\mathrm{~m}, 4 \mathrm{H}), 7.01-6.96(\mathrm{~m}, 2 \mathrm{H}), 4.82-4.79$ (m, 1H), $4.75-4.73(\mathrm{~m}, 1 \mathrm{H}), 3.54-3.50(\mathrm{~m}, 2 \mathrm{H}), 2.45$ (s, 3H), $2.41-2.37$ $(\mathrm{m}, 2 \mathrm{H}), 1.74(\mathrm{~s}, 3 \mathrm{H}) .{ }^{13} \mathrm{C}$ NMR $\left(151 \mathrm{MHz}, \mathrm{CDCl}_{3}\right) \delta 162.3\left(\mathrm{~d},{ }^{1} J_{\mathrm{C}-\mathrm{F}}=249.2\right.$ $\mathrm{Hz}), 144.8,141.2,134.6,133.5\left(\mathrm{~d},{ }^{3} \mathrm{~J}_{\mathrm{C}-\mathrm{F}}=8.4 \mathrm{~Hz}, 2 \mathrm{C}\right), 129.8(2 \mathrm{C}), 127.7$ 
(2C), $118.9\left(\mathrm{~d},{ }^{4} J_{\mathrm{C}-\mathrm{F}}=3.4 \mathrm{~Hz}\right), 115.6\left(\mathrm{~d},{ }^{2} J_{\mathrm{C}-\mathrm{F}}=21.1 \mathrm{~Hz}, 2 \mathrm{C}\right), 112.9,81.8,69.9,50.0,36.0,22.3,21.71$. ${ }^{19} \mathrm{~F}$ NMR $\left(565 \mathrm{MHz}, \mathrm{CDCl}_{3}\right) \delta-111.41--111.47(\mathrm{~m}, 1 \mathrm{~F})$. HRMS ESI $[\mathrm{M}+\mathrm{H}]^{+}$calculated for $\mathrm{C}_{20} \mathrm{H}_{21} \mathrm{NFO}_{2} \mathrm{~S} 358.1277$, found 358.1269.

$N$-((3-Fluorophenyl)ethynyl)-4-methyl- $N$-(3-methylbut-3-en-1-yl)benzenesulfonamide (1i)

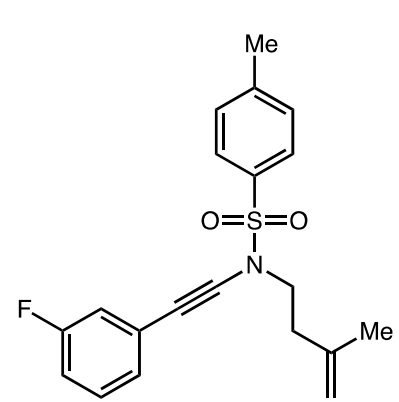

Following the method $\mathbf{A}$, purification by flash column chromatography (5$10 \%$ EtOAc in hexane), colourless oil. ${ }^{1} \mathrm{H}$ NMR $\left(400 \mathrm{MHz}, \mathrm{CDCl}_{3}\right) \delta 7.86$ $(\mathrm{d}, J=8.3 \mathrm{~Hz}, 2 \mathrm{H}), 7.39$ (d, $J=8.3 \mathrm{~Hz}, 2 \mathrm{H}), 7.31-7.24(\mathrm{~m}, 1 \mathrm{H}), 7.18-$ $7.15(\mathrm{~m}, 1 \mathrm{H}), 7.09-7.05(\mathrm{~m}, 1 \mathrm{H}), 7.02-6.98(\mathrm{~m}, 1 \mathrm{H}), 4.85-4.82(\mathrm{~m}, 1 \mathrm{H})$, $4.80-4.75(\mathrm{~m}, 1 \mathrm{H}), 3.61-3.50(\mathrm{~m}, 2 \mathrm{H}), 2.47(\mathrm{~s}, 3 \mathrm{H}), 2.45-2.38(\mathrm{~m}, 2 \mathrm{H})$, $1.78(\mathrm{~s}, 3 \mathrm{H}) .{ }^{13} \mathrm{C} \mathrm{NMR}\left(101 \mathrm{MHz}, \mathrm{CDCl}_{3}\right) \delta 162.3\left(\mathrm{~d},{ }^{1} J_{\mathrm{C}-\mathrm{F}}=247.5 \mathrm{~Hz}\right)$, $144.8,141.0,134.4,129.9,129.8(2 \mathrm{C}), 127.6(2 \mathrm{C}), 126.9\left(\mathrm{~d},{ }^{4} J_{\mathrm{C}-\mathrm{F}}=3.0\right.$ $\mathrm{Hz}), 124.7\left(\mathrm{~d},{ }^{3} J_{\mathrm{C}-\mathrm{F}}=9.6 \mathrm{~Hz}\right), 117.7\left(\mathrm{~d},{ }^{2} J_{\mathrm{C}-\mathrm{F}}=23.3 \mathrm{~Hz}\right), 114.9\left(\mathrm{~d},{ }^{2} J_{\mathrm{C}-\mathrm{F}}=21.2 \mathrm{~Hz}\right), 113.0,83.2,70.08$ $\left(\mathrm{d},{ }^{3} J_{\mathrm{C}-\mathrm{F}}=3.5 \mathrm{~Hz}\right), 49.8,35.9,22.2,21.5 .{ }^{19} \mathrm{~F} \mathrm{NMR}\left(376 \mathrm{MHz}, \mathrm{CDCl}_{3}\right) \delta-112.84--112.97(\mathrm{~m}, 1 \mathrm{~F})$. HRMS ESI $[\mathrm{M}+\mathrm{H}]^{+}$calculated for $\mathrm{C}_{20} \mathrm{H}_{21} \mathrm{NFO}_{2} \mathrm{~S} 358.1277$, found 358.1270 .

\section{$N$-((2-Fluorophenyl)ethynyl)-4-methyl- $N$-(3-methylbut-3-en-1-yl)benzenesulfonamide (1j)}

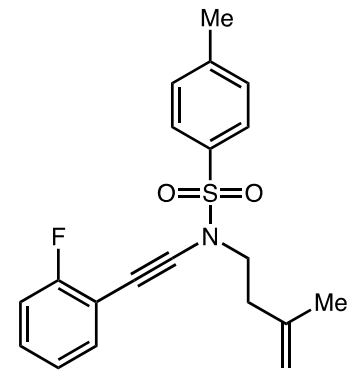

Following the method $\mathbf{B}$, purification by flash column chromatography $(5-10 \%$ EtOAc in hexane), colourless oil. ${ }^{1} \mathrm{H}$ NMR $\left(600 \mathrm{MHz}, \mathrm{CDCl}_{3}\right) \delta 7.78(\mathrm{~d}, J=$ $8.3 \mathrm{~Hz}, 2 \mathrm{H}), 7.30-7.24(\mathrm{~m}, 3 \mathrm{H}), 7.18-7.13(\mathrm{~m}, 1 \mathrm{H}), 7.01-6.92(\mathrm{~m}, 2 \mathrm{H})$, $4.72-4.70(\mathrm{~m}, 1 \mathrm{H}), 4.67-4.66(\mathrm{~s}, 1 \mathrm{H}), 3.45(\mathrm{t}, J=7.5 \mathrm{~Hz}, 2 \mathrm{H}), 2.35(\mathrm{~s}, 3 \mathrm{H})$, $2.34-2.30(\mathrm{~m}, 2 \mathrm{H}), 1.66(\mathrm{~s}, 3 \mathrm{H}) .{ }^{13} \mathrm{C} \mathrm{NMR}\left(151 \mathrm{MHz}, \mathrm{CDCl}_{3}\right) \delta 162.5\left(\mathrm{~d},{ }^{1} J\right.$ C-F $=250.7 \mathrm{~Hz}), 144.8,141.2,134.6,133.0,129.8(2 \mathrm{C}), 129.4\left(\mathrm{~d},{ }^{3} \mathrm{~J}_{\mathrm{C}-\mathrm{F}}=7.6\right.$ $\mathrm{Hz}), 127.8(2 \mathrm{C}), 123.9\left(\mathrm{~d},{ }^{4} J_{\mathrm{C}-\mathrm{F}}=3.7 \mathrm{~Hz}\right), 115.4\left(\mathrm{~d},{ }^{2} J_{\mathrm{C}-\mathrm{F}}=21.1 \mathrm{~Hz}\right), 113.0,111.6\left(\mathrm{~d},{ }^{2} J_{\mathrm{C}-\mathrm{F}}=15.1 \mathrm{~Hz}\right)$, 87.0, 64.8, 50.0, 35.9, 22.3, 21.7. ${ }^{19} \mathrm{~F}$ NMR $\left(565 \mathrm{MHz}, \mathrm{CDCl}_{3}\right) \delta-110.24--110.37(\mathrm{~m}, 1 \mathrm{~F})$. HRMS ESI $[\mathrm{M}+\mathrm{H}]^{+}$calculated for $\mathrm{C}_{20} \mathrm{H}_{21} \mathrm{NFO}_{2} \mathrm{~S} 358.1277$, found 358.1269 .

\section{4-(tert-Butyl)- $N$-((3-fluorophenyl)ethynyl)- $N$-(3-methylbut-3-en-1-yl)benzenesulfonamide (1k)}

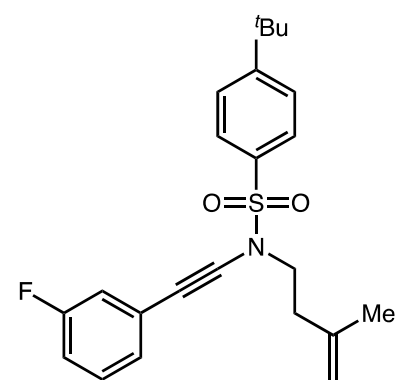

Following the method A, purification by flash column chromatography (5$10 \%$ EtOAc in hexane), colourless oil. ${ }^{1} \mathrm{H}$ NMR $\left(600 \mathrm{MHz}, \mathrm{CDCl}_{3}\right) \delta 7.86$ (d, $J=8.6 \mathrm{~Hz}, 2 \mathrm{H}), 7.56(\mathrm{~d}, J=8.6 \mathrm{~Hz}, 2 \mathrm{H}), 7.26-7.20(\mathrm{~m}, 1 \mathrm{H}), 7.15-$ $7.10(\mathrm{~m}, 1 \mathrm{H}), 7.04-7.01(\mathrm{~m}, 1 \mathrm{H}), 6.98-6.93(\mathrm{~m}, 1 \mathrm{H}), 4.79-4.78(\mathrm{~m}$, $1 \mathrm{H}), 4.72-4.71(\mathrm{~m}, 1 \mathrm{H}), 3.55-3.51(\mathrm{~m}, 2 \mathrm{H}), 2.42-2.35(\mathrm{~m}, 2 \mathrm{H}), 1.73$ $(\mathrm{s}, 3 \mathrm{H}), 1.34(\mathrm{~s}, 9 \mathrm{H}) \cdot{ }^{13} \mathrm{C} \mathrm{NMR}\left(151 \mathrm{MHz}, \mathrm{CDCl}_{3}\right) \delta 162.4\left(\mathrm{~d},{ }^{1} J_{\mathrm{C}-\mathrm{F}}=\right.$ $246.13 \mathrm{~Hz}), 157.8,141.2,134.6,129.9\left(\mathrm{~d},{ }^{3} \mathrm{~J}_{\mathrm{C}-\mathrm{F}}=9.1 \mathrm{~Hz}\right), 127.6(2 \mathrm{C}), 127.0\left(\mathrm{~d},{ }^{4} \mathrm{~J}_{\mathrm{C}-\mathrm{F}}=3.0 \mathrm{~Hz}\right), 126.33$ (2C), $124.9\left(\mathrm{~d},{ }^{3} J_{\mathrm{C}-\mathrm{F}}=9.7 \mathrm{~Hz}\right), 117.9\left(\mathrm{~d},{ }^{2} J_{\mathrm{C}-\mathrm{F}}=22.7 \mathrm{~Hz}\right), 115.0\left(\mathrm{~d},{ }^{2} J_{\mathrm{C}-\mathrm{F}}=21.2 \mathrm{~Hz}\right), 113.0,83.4,70.25$, 49.9, 36.1, 35.4, $31.1(3 \mathrm{C}), 22.4 .{ }^{19} \mathrm{~F}$ NMR $\left(565 \mathrm{MHz}, \mathrm{CDCl}_{3}\right) \delta-113.01--113.07$ (m, 1F). HRMS ESI $[\mathrm{M}+\mathrm{H}]^{+}$calculated for $\mathrm{C}_{23} \mathrm{H}_{27} \mathrm{NFO}_{2} \mathrm{~S} 400.1747$, found 400.1740 . 
4-(tert-Butyl)- $N$-((4-chlorophenyl)ethynyl)- $N$-(3-methylbut-3-en-1-yl)benzenesulfonamide (11)

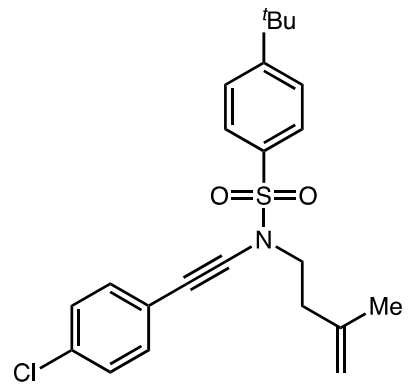

Following the method $\mathbf{B}$, purification by flash column chromatography (5$10 \%$ EtOAc in hexane), yellow oil. ${ }^{1} \mathrm{H}$ NMR $\left(600 \mathrm{MHz}, \mathrm{CDCl}_{3}\right) \delta 7.79(\mathrm{~d}$, $J=8.6 \mathrm{~Hz}, 2 \mathrm{H}), 7.49$ (d, $J=8.6 \mathrm{~Hz}, 2 \mathrm{H}), 7.23-7.17$ (m, 4H), $4.73-4.71$ (m, 1H), $4.67-4.62(\mathrm{~m}, 1 \mathrm{H}), 3.49-3.40(\mathrm{~m}, 2 \mathrm{H}), 2.36-2.27(\mathrm{~m}, 2 \mathrm{H})$, $2.33(\mathrm{~s}, 3 \mathrm{H}), 1.28(\mathrm{~s}, 9 \mathrm{H}) .{ }^{13} \mathrm{C} \mathrm{NMR}\left(151 \mathrm{MHz}, \mathrm{CDCl}_{3}\right) \delta 157.7,141.2$, 134.7, 133.8, 132.5 (2C), 128.7 (2C), 127.6 (2C), 126.3 (2C), 121.5, 113.0, 83.3, 70.1, 50.0, 36.1, 35.4, 31.1 (3C), 22.4. HRMS ESI [M+H] $]^{+}$calculated for $\mathrm{C}_{23} \mathrm{H}_{27} \mathrm{NClO}_{2} \mathrm{~S} 416.1451$, found 416.1443 .

$N$-((3-Chlorophenyl)ethynyl)-4-methyl- $N$-(3-methylbut-3-en-1-yl)benzenesulfonamide (1m)

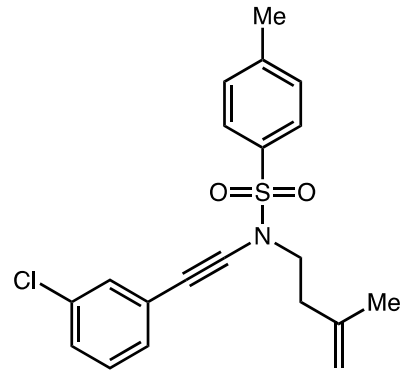

Following the method $\mathbf{B}$, purification by flash column chromatography (5$10 \%$ EtOAc in hexane), pale yellow oil. ${ }^{1} \mathrm{H}$ NMR $\left(600 \mathrm{MHz}, \mathrm{CDCl}_{3}\right) \delta 7.74$ (d, $J=8.3 \mathrm{~Hz}, 2 \mathrm{H}), 7.27$ (d, $J=8.3 \mathrm{~Hz}, 2 \mathrm{H}), 7.24-7.22(\mathrm{~m}, 1 \mathrm{H}), 7.17-$ $7.09(\mathrm{~m}, 3 \mathrm{H}), 4.73-4.69(\mathrm{~m}, 1 \mathrm{H}), 4.67-4.62(\mathrm{~m}, 1 \mathrm{H}), 3.46-3.41(\mathrm{~m}$, $2 \mathrm{H}), 2.35(\mathrm{~s}, 3 \mathrm{H}), 2.30(\mathrm{t}, J=7.7 \mathrm{~Hz}, 2 \mathrm{H}), 1.65(\mathrm{~s}, 3 \mathrm{H}) .{ }^{13} \mathrm{C}$ NMR $(151$ $\mathrm{MHz}, \mathrm{CDCl} 3) \delta 144.9,141.1,134.5,134.1,130.9,129.9$ (2C), 129.5, 129.3, 127.9, $127.7(2 \mathrm{C}), 124.7,113.0,83.5,70.0,49.9,36.0,22.3,21.7$. HRMS ESI $[\mathrm{M}+\mathrm{H}]^{+}$calculated for $\mathrm{C}_{20} \mathrm{H}_{21} \mathrm{NClO}_{2} \mathrm{~S} 374.0982$, found 374.0973.

\section{$N$-((4-Bromophenyl)ethynyl)-4-methoxy- $N$-(3-methylbut-3-en-1-yl)benzenesulfonamide (1n)}

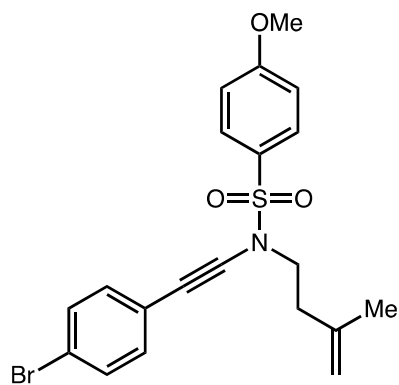

Following the method $\mathbf{B}$, purification by flash column chromatography (10\% EtOAc in hexane), pale yellow oil. ${ }^{1} \mathrm{H}$ NMR (600 $\left.\mathrm{MHz}, \mathrm{CDCl}_{3}\right) \delta$ $7.87(\mathrm{~d}, J=8.9 \mathrm{~Hz}, 2 \mathrm{H}), 7.41(\mathrm{~d}, J=8.5 \mathrm{~Hz}, 2 \mathrm{H}), 7.21(\mathrm{~d}, J=8.5 \mathrm{~Hz}, 2 \mathrm{H})$, $7.01(\mathrm{~d}, J=8.9 \mathrm{~Hz}, 2 \mathrm{H}), 4.82-4.79(\mathrm{~m}, 1 \mathrm{H}), 4.75-4.72(\mathrm{~m}, 1 \mathrm{H}), 3.88$ $(\mathrm{s}, 3 \mathrm{H}), 3.51(\mathrm{t}, J=7.5 \mathrm{~Hz}, 2 \mathrm{H}), 2.39(\mathrm{t}, J=7.5 \mathrm{~Hz}, 2 \mathrm{H}), 1.74(\mathrm{~s}, 3 \mathrm{H}) .{ }^{13} \mathrm{C}$ NMR (151 MHz, $\left.\mathrm{CDCl}_{3}\right) \delta 163.8,141.3,132.7$ (2C), 131.6 (2C), 129.9 (2C), 129.2, 122.0, 121.9, 114.4 (2C), 113.0, 83.6, 70.2, 55.8, 49.9, 36.1, 22.4. HRMS ESI [M+H] calculated for $\mathrm{C}_{20} \mathrm{H}_{21} \mathrm{NO}_{3} \mathrm{SBr} 434.0426$, found 434.0419.

Methyl 4-(((4-(tert-butyl)- $N$-(3-methylbut-3-en-1-yl)phenyl) sulfonamido)ethynyl)benzoate (10)

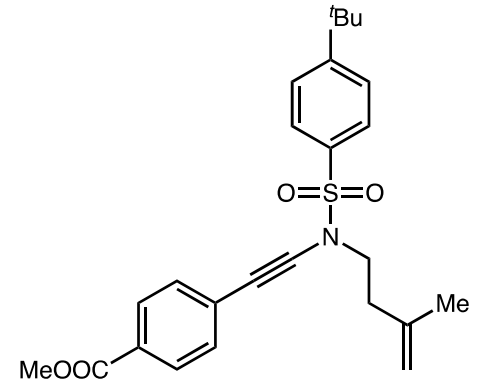
Following the method $\mathbf{B}$, purification by flash column chromatography (10\% EtOAc in hexane), pale yellow oil. ${ }^{1} \mathrm{H}$ NMR $\left(600 \mathrm{MHz}, \mathrm{CDCl}_{3}\right)$ $\delta 7.96(\mathrm{~d}, J=8.1 \mathrm{~Hz}, 2 \mathrm{H}), 7.87(\mathrm{~d}, J=8.3 \mathrm{~Hz}, 2 \mathrm{H}), 7.57(\mathrm{~d}, J=8.3 \mathrm{~Hz}$, $2 \mathrm{H}), 7.39(\mathrm{~d}, J=8.1 \mathrm{~Hz}, 2 \mathrm{H}), 4.80-4.78(\mathrm{~m}, 1 \mathrm{H}), 4.74-4.72(\mathrm{~m}, 1 \mathrm{H})$, $3.90(\mathrm{~s}, 3 \mathrm{H}), 3.55(\mathrm{t}, J=7.5 \mathrm{~Hz}, 2 \mathrm{H}), 2.41(\mathrm{t}, J=7.5 \mathrm{~Hz}, 2 \mathrm{H}), 1.74(\mathrm{~s}$, $3 \mathrm{H}), 1.34(\mathrm{~s}, 9 \mathrm{H}) .{ }^{13} \mathrm{C} \mathrm{NMR}\left(151 \mathrm{MHz}, \mathrm{CDCl}_{3}\right) \delta 166.6,157.8,141.14$, 134.6, 130.6 (2C), 129.5 (2C), 128.8, 128.0, 127.6 (2C), 126.3 (2C), 113.1, 85.7, 71.1, 52.2, 49.9, 36.1, 35.4, 31.1 (3C), 22.4. HRMS ESI [M+H] $]^{+}$calculated for $\mathrm{C}_{25} \mathrm{H}_{30} \mathrm{NO}_{4} \mathrm{~S} 440.1896$, found 440.1887. 


\section{$N$-((4-Cyanophenyl)ethynyl)-4-methyl- $N$-(3-methylbut-3-en-1-yl)benzenesulfonamide (1p)}

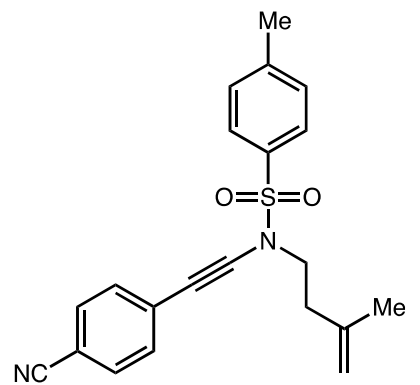

Following the method $\mathbf{B}$, purification by flash column chromatography (20$30 \%$ EtOAc in hexane), white solid. ${ }^{1} \mathrm{H}$ NMR $\left(600 \mathrm{MHz}, \mathrm{CDCl}_{3}\right) \delta 7.82(\mathrm{~d}$, $J=8.3 \mathrm{~Hz}, 2 \mathrm{H}), 7.56(\mathrm{~d}, J=8.4 \mathrm{~Hz}, 2 \mathrm{H}), 7.40$ (d, $J=8.4 \mathrm{~Hz}, 2 \mathrm{H}), 7.36$ (d, $J=8.3 \mathrm{~Hz}, 2 \mathrm{H}), 4.81-4.79(\mathrm{~m}, 1 \mathrm{H}), 4.73-4.71(\mathrm{~m}, 1 \mathrm{H}), 3.56-3.52(\mathrm{~m}$, 2H), $2.44(\mathrm{~s}, 3 \mathrm{H}), 2.40-2.36(\mathrm{~m}, 2 \mathrm{H}), 1.73(\mathrm{~s}, 3 \mathrm{H}) .{ }^{13} \mathrm{C} \mathrm{NMR}(151 \mathrm{MHz}$, $\left.\mathrm{CDCl}_{3}\right) \delta 145.1,140.9,134.5,132.0$ (2C), 131.0 (2C), 130.0 (2C), 128.2, $127.6(2 \mathrm{C}), 118.6,113.2,110.5,87.1,70.6,49.9,36.1,22.3,21.7$. HRMS ESI $[\mathrm{M}+\mathrm{H}]^{+}$calculated for $\mathrm{C}_{21} \mathrm{H}_{21} \mathrm{~N}_{2} \mathrm{O}_{2} \mathrm{~S} 365.1324$, found 365.1314 .

\section{$N$-((4-Cyanophenyl)ethynyl)-4-methoxy- $N$-(3-methylbut-3-en-1-yl)benzenesulfonamide (1q)}

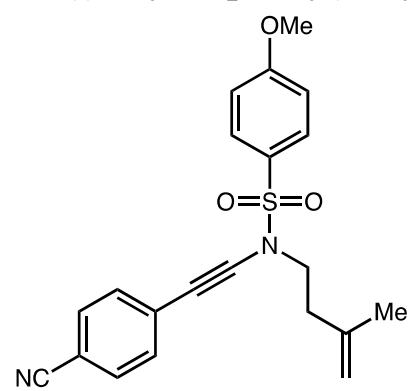

Following the method $\mathbf{B}$, purification by flash column chromatography (20$30 \%$ EtOAc in hexane), white solid. ${ }^{1} \mathrm{H}$ NMR $\left(600 \mathrm{MHz}, \mathrm{CDCl}_{3}\right) \delta 7.87(\mathrm{~d}$, $J=8.9 \mathrm{~Hz}, 2 \mathrm{H}), 7.57$ (d, $J=8.4 \mathrm{~Hz}, 2 \mathrm{H}), 7.40$ (d, $J=8.4 \mathrm{~Hz}, 2 \mathrm{H}), 7.02$ (d, $J=8.9 \mathrm{~Hz}, 2 \mathrm{H}), 4.84-4.79(\mathrm{~m}, 1 \mathrm{H}), 4.77-4.25(\mathrm{~m}, 1 \mathrm{H}), 3.89$ (s, 3H), 3.57 - $3.53(\mathrm{~m}, 2 \mathrm{H}), 2.41-2.37(\mathrm{~m}, 2 \mathrm{H}), 1.75(\mathrm{~s}, 3 \mathrm{H}) .{ }^{13} \mathrm{C} \mathrm{NMR}(151 \mathrm{MHz}$, $\left.\mathrm{CDCl}_{3}\right) \delta 164.1,141.1,132.1(2 \mathrm{C}), 131.0(2 \mathrm{C}), 130.0(2 \mathrm{C}), 129.1,128.4$, 118.7, $114.6(2 \mathrm{C}), 113.2,110.6,87.4,70.8,55.8,49.9,36.2,22.4$. HRMS ESI $[\mathrm{M}+\mathrm{H}]^{+}$calculated for $\mathrm{C}_{21} \mathrm{H}_{21} \mathrm{~N}_{2} \mathrm{O}_{3} \mathrm{~S} 381.1273$, found 381.1265 .

\section{$(1 S, 3 S, 5 S)-3-I s o p r o p y l-5-m e t h y l c y c l o h e x y l 4-(((4-m e t h o x y-N$-(3-methylbut-3-en-1-}

\section{yl)phenyl)sulfonamido) ethynyl)benzoate (1r)}

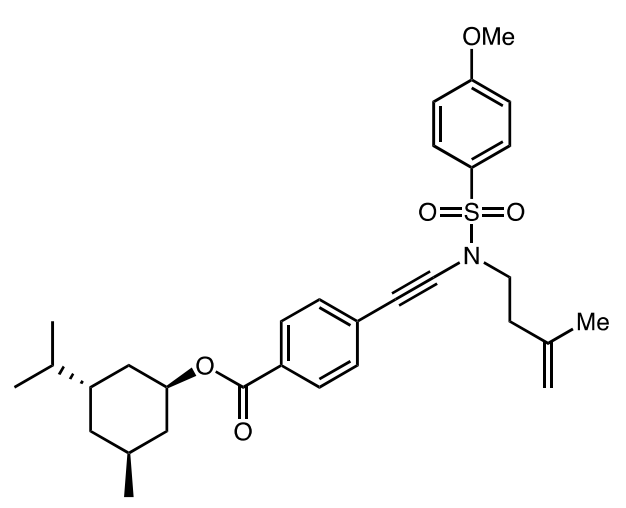

Following the method B, purification by flash column chromatography $\left(10 \%\right.$ EtOAc in hexane), colourless oil. ${ }^{1} \mathrm{H}$ NMR (600 MHz, $\left.\mathrm{CDCl}_{3}\right) \delta 7.99-7.92(\mathrm{~m}, 2 \mathrm{H}), 7.91-7.85$ (m, 2H), $7.41-7.35(\mathrm{~m}, 2 \mathrm{H}), 7.04-6.99(\mathrm{~m}, 2 \mathrm{H}), 4.91(\mathrm{dt}, J$ $=10.9,4.4 \mathrm{~Hz}, 1 \mathrm{H}), 4.80(\mathrm{~s}, 1 \mathrm{H}), 4.74(\mathrm{~s}, 1 \mathrm{H}), 3.87(\mathrm{~s}, 3 \mathrm{H})$, $3.54(\mathrm{t}, J=7.5 \mathrm{~Hz}, 2 \mathrm{H}), 2.40(\mathrm{t}, J=7.5 \mathrm{~Hz}, 2 \mathrm{H}), 2.13-2.08$ (m, 1H), $1.96-1.90(\mathrm{~m}, 1 \mathrm{H}), 1.75(\mathrm{~s}, 3 \mathrm{H}), 1.74-1.68(\mathrm{~m}$, $2 \mathrm{H}), 1.58-1.50(\mathrm{~m}, 2 \mathrm{H}), 1.17-1.05(\mathrm{~m}, 2 \mathrm{H}), 0.91(\mathrm{t}, J=6.9$ $\mathrm{Hz}, 6 \mathrm{H}), 0.78(\mathrm{~d}, J=7.0 \mathrm{~Hz}, 3 \mathrm{H}) .{ }^{13} \mathrm{C} \mathrm{NMR}\left(151 \mathrm{MHz}, \mathrm{CDCl}_{3}\right) \delta 165.6,163.9,141.1,130.5(2 \mathrm{C}), 129.9$ (2C), 129.5 (2C), 129.5, 129.0, 127.7, 114.4 (2C), 113.0, 85.6, 75.0, 71.1, 55.7, 49.9, 47.3, 41.0, 36.1, 34.3, 31.5, 26.6, 23.7, 22.3, 22.1, 20.8, 16.6. HRMS ESI [M+H] ${ }^{+}$calculated for $\mathrm{C}_{31} \mathrm{H}_{40} \mathrm{NO}_{5} \mathrm{~S} 538.2627$, found 538.2631 . 


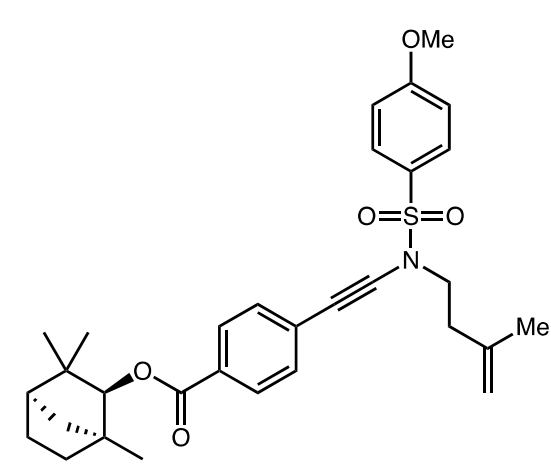

Following the method B, purification by flash column chromatography (10\% EtOAc in hexane), colourless oil. ${ }^{1} \mathrm{H}$ NMR (600 MHz, CDCl3) $\delta 1 \mathrm{H}$ NMR $\left(600 \mathrm{MHz}, \mathrm{CDCl}_{3}\right) \delta 8.01-$ $7.94(\mathrm{~m}, 2 \mathrm{H}), 7.92-7.87(\mathrm{~m}, 2 \mathrm{H}), 7.43-7.37(\mathrm{~m}, 2 \mathrm{H}), 7.05-7.00$ $(\mathrm{m}, 2 \mathrm{H}), 4.83-4.80(\mathrm{~m}, 1 \mathrm{H}), 4.74(\mathrm{~s}, 1 \mathrm{H}), 4.61(\mathrm{~d}, J=2.0 \mathrm{~Hz}, 1 \mathrm{H})$, $3.88(\mathrm{~s}, 3 \mathrm{H}), 3.55(\mathrm{t}, J=7.5 \mathrm{~Hz}, 2 \mathrm{H}), 2.41(\mathrm{t}, J=7.5 \mathrm{~Hz}, 2 \mathrm{H}), 1.95$ $-1.88(\mathrm{~m}, 1 \mathrm{H}), 1.81-1.77(\mathrm{~m}, 2 \mathrm{H}), 1.76(\mathrm{~s}, 3 \mathrm{H}), 1.66(\mathrm{~d}, J=10.6$ $\mathrm{Hz}, 1 \mathrm{H}), 1.55-1.47(\mathrm{~m}, 1 \mathrm{H}), 1.25(\mathrm{~d}, J=10.4 \mathrm{~Hz}, 1 \mathrm{H}), 1.23-1.19(\mathrm{~m}, 1 \mathrm{H}), 1.18(\mathrm{~s}, 3 \mathrm{H}), 1.10(\mathrm{~s}, 3 \mathrm{H})$, $0.83(\mathrm{~s}, 3 \mathrm{H}) .{ }^{13} \mathrm{C}$ NMR $\left(151 \mathrm{MHz}, \mathrm{CDCl}_{3}\right) \delta 166.5,163.9,141.2,130.7$ (2C), $130.0(2 \mathrm{C}), 129.5(2 \mathrm{C})$, 129.4, 129.1, 127.9, 114.5 (2C), 113.1, 86.9, 85.7, 71.2, 55.8, 49.9, 48.7, 48.5, 41.5, 39.9, 36.1, 29.8, 27.0, 26.0, 22.4, 20.4, 19.6. HRMS ESI $[\mathrm{M}+\mathrm{H}]^{+}$calculated for $\mathrm{C}_{31} \mathrm{H}_{38} \mathrm{NO}_{5} \mathrm{~S} 536.2471$, found 536.2477 .

\section{4-Fluoro- $N$-((4-methoxyphenyl)ethynyl)- $N$-(3-methylbut-3-en-1-yl)benzenesulfonamide (1t)}

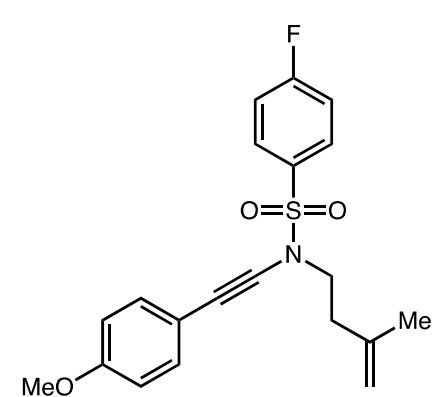

Following the method A, purification by flash column chromatography $\left(10 \%\right.$ EtOAc in hexane), colourless oil. ${ }^{1} \mathrm{H}$ NMR $\left(600 \mathrm{MHz}, \mathrm{CDCl}_{3}\right) \delta$ $7.92-7.86(\mathrm{~m}, 2 \mathrm{H}), 7.23(\mathrm{~d}, J=8.8 \mathrm{~Hz}, 2 \mathrm{H}), 7.19-7.13(\mathrm{~m}, 2 \mathrm{H}), 6.75$ $(\mathrm{d}, J=8.8 \mathrm{~Hz}, 2 \mathrm{H}), 4.73-4.71(\mathrm{~m}, 1 \mathrm{H}), 4.68-4.63(\mathrm{~m}, 1 \mathrm{H}), 3.72(\mathrm{~s}$, $3 \mathrm{H}), 3.47-3.42(\mathrm{~m}, 2 \mathrm{H}), 2.34-2.30(\mathrm{~m}, 2 \mathrm{H}), 1.66(\mathrm{~s}, 3 \mathrm{H}) .{ }^{13} \mathrm{C} \mathrm{NMR}$ $\left(151 \mathrm{MHz}, \mathrm{CDCl}_{3}\right) \delta 165.7\left(\mathrm{~d},{ }^{1} J_{\mathrm{C}-\mathrm{F}}=255.19 \mathrm{~Hz}\right), 159.7,141.2,133.7$ $\left(\mathrm{d},{ }^{4} J_{\mathrm{C}-\mathrm{F}}=3.1 \mathrm{~Hz}\right), 133.6(2 \mathrm{C}), 130.5\left(\mathrm{~d},{ }^{3} \mathrm{~J}_{\mathrm{C}-\mathrm{F}}=9.5 \mathrm{~Hz}, 2 \mathrm{C}\right), 116.5\left(\mathrm{~d},{ }^{2} J_{\mathrm{C}-\mathrm{F}}=22.7 \mathrm{~Hz}, 2 \mathrm{C}\right), 114.5$, 114.0 (2C), 113.0, 80.3, 70.9, 55.4, 50.2, 36.0, 22.4. $\left.{ }^{19} \mathrm{~F} \mathrm{NMR} \mathrm{(565} \mathrm{MHz,} \mathrm{CDCl}_{3}\right) \delta-103.47--103.34$ (m, 1F). HRMS ESI $[\mathrm{M}+\mathrm{H}]^{+}$calculated for $\mathrm{C}_{20} \mathrm{H}_{21} \mathrm{NFO}_{3} \mathrm{~S} 374.1226$, found 374.1220.

\section{2-Fluoro- $N$-((4-methoxyphenyl)ethynyl)- $N$-(3-methylbut-3-en-1-yl)benzenesulfonamide (1u)}

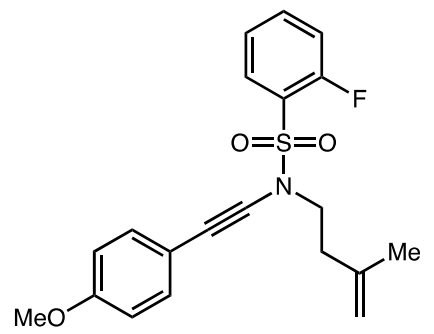

Following the method $\mathbf{A}$, purification by flash column chromatography $(10 \%$ EtOAc in hexane), colourless oil. ${ }^{1} \mathrm{H}$ NMR $\left(400 \mathrm{MHz}, \mathrm{CDCl}_{3}\right) \delta 7.97-$ $7.87(\mathrm{~m}, 1 \mathrm{H}), 7.65-7.53(\mathrm{~m}, 1 \mathrm{H}), 7.29-7.23(\mathrm{~m}, 1 \mathrm{H}), 7.23-7.18(\mathrm{~m}$, $3 \mathrm{H}), 6.75(\mathrm{~d}, J=8.8 \mathrm{~Hz}, 2 \mathrm{H}), 4.80-4.77(\mathrm{~m}, 1 \mathrm{H}), 4.76-4.74(\mathrm{~m}, 1 \mathrm{H})$, $3.74(\mathrm{~s}, 3 \mathrm{H}), 3.71-3.64(\mathrm{~m}, 2 \mathrm{H}), 2.48-2.35(\mathrm{~m}, 2 \mathrm{H}), 1.73(\mathrm{~s}, 3 \mathrm{H}) .{ }^{13} \mathrm{C}$ $\operatorname{NMR}\left(101 \mathrm{MHz}, \mathrm{CDCl}_{3}\right) \delta 159.6,159.1\left(\mathrm{~d},{ }^{1} J_{\mathrm{C}-\mathrm{F}}=259.6 \mathrm{~Hz}\right), 141.3,135.96\left(\mathrm{~d},{ }^{3} J_{\mathrm{C}-\mathrm{F}}=8.5 \mathrm{~Hz}\right), 133.5$ (2C), 131.6, $125.9\left(\mathrm{~d},{ }^{3} J_{\mathrm{C}-\mathrm{F}}=14.2 \mathrm{~Hz}\right), 124.4\left(\mathrm{~d},{ }^{4} J_{\mathrm{C}-\mathrm{F}}=3.9 \mathrm{~Hz}\right), 117.5\left(\mathrm{~d},{ }^{2} J_{\mathrm{C}-\mathrm{F}}=21.2 \mathrm{~Hz}\right), 114.6$, 113.9 (2C), 113.0, 79.6, 71.2, 55.4, 50.5, 36.4, 22.4. $\left.{ }^{19} \mathrm{~F} \mathrm{NMR} \mathrm{(376} \mathrm{MHz,} \mathrm{CDCl}_{3}\right) \delta-106.78--106.87$ (m, 1F). HRMS ESI $[\mathrm{M}+\mathrm{H}]^{+}$calculated for $\mathrm{C}_{20} \mathrm{H}_{21} \mathrm{NFO}_{3} \mathrm{~S} 374.1226$, found 374.1216. 


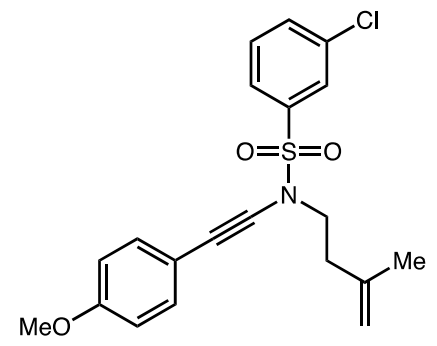

Following the method $\mathbf{A}$, purification by flash column chromatography $\left(10 \%\right.$ EtOAc in hexane), colourless oil. ${ }^{1} \mathrm{H}$ NMR $\left(600 \mathrm{MHz}, \mathrm{CDCl}_{3}\right) \delta$ $7.97-7.95(\mathrm{~m}, 1 \mathrm{H}), 7.86-7.82(\mathrm{~m}, 1 \mathrm{H}), 7.64-7.60(\mathrm{~m}, 1 \mathrm{H}), 7.53-$ $7.48(\mathrm{~m}, 1 \mathrm{H}), 7.34(\mathrm{~d}, J=8.8 \mathrm{~Hz}, 2 \mathrm{H}), 6.84(\mathrm{~d}, J=8.8 \mathrm{~Hz}, 2 \mathrm{H}), 4.82-$ $4.79(\mathrm{~m}, 1 \mathrm{H}), 4.78-4.74(\mathrm{~m}, 1 \mathrm{H}), 3.79(\mathrm{~s}, 3 \mathrm{H}), 3.58-3.53(\mathrm{~m}, 2 \mathrm{H}), 2.42$ -2.39 (m, 2H), 1.75 (s, 3H). ${ }^{13} \mathrm{C} \mathrm{NMR}\left(151 \mathrm{MHz}, \mathrm{CDCl}_{3}\right) \delta$ 159.7, 141.0, 139.0, 135.2, 133.6, 133.5 (2C), 130.4, 127.6, 125.6, $114.2,113.9$ (2C), 113.1, 79.9, 71.1, 55.2, 50.2, 35.8, 22.2. HRMS ESI [M+H] $]^{+}$calculated for $\mathrm{C}_{20} \mathrm{H}_{21} \mathrm{NClO}_{3} \mathrm{~S} 390.0931$, found 390.0928 .

\section{$N$-((4-Methoxyphenyl)ethynyl)- $N$-(3-methylbut-3-en-1-yl)-4-(trifluoromethyl)}

benzenesulfonamide (1w)

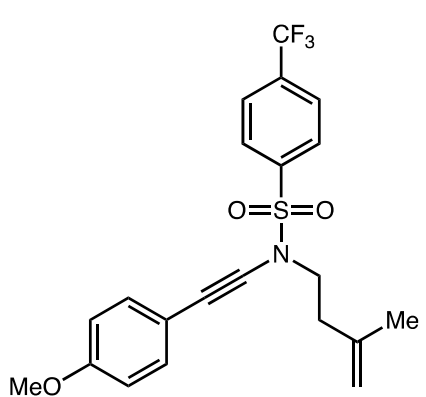

Following the method A, purification by flash column chromatography (10\% EtOAc in hexane), white solid. ${ }^{1} \mathrm{H}$ NMR $\left(600 \mathrm{MHz}, \mathrm{CDCl}_{3}\right) \delta 8.09$ $(\mathrm{d}, J=8.2 \mathrm{~Hz}, 2 \mathrm{H}), 7.84(\mathrm{~d}, J=8.2 \mathrm{~Hz}, 2 \mathrm{H}), 7.33(\mathrm{~d}, J=8.8 \mathrm{~Hz}, 2 \mathrm{H}), 6.84$ $(\mathrm{d}, J=8.8 \mathrm{~Hz}, 2 \mathrm{H}), 4.83-4.77(\mathrm{~m}, 1 \mathrm{H}), 4.75-4.73(\mathrm{~m}, 1 \mathrm{H}), 3.81(\mathrm{~s}$, $3 \mathrm{H}), 3.56(\mathrm{t}, J=7.4 \mathrm{~Hz}, 2 \mathrm{H}), 2.41(\mathrm{t}, J=7.4 \mathrm{~Hz}, 2 \mathrm{H}), 1.75(\mathrm{~s}, 3 \mathrm{H}) .{ }^{13} \mathrm{C}$ $\operatorname{NMR}\left(151 \mathrm{MHz}, \mathrm{CDCl}_{3}\right) \delta 159.9,141.1,135.2\left(\mathrm{q},{ }^{2} J_{\mathrm{C}-\mathrm{F}}=33.2 \mathrm{~Hz}, 1 \mathrm{C}\right)$, 133.7 (2C), $128.2(2 \mathrm{C}), 126.3\left(\mathrm{q},{ }^{3} J_{\mathrm{C}-\mathrm{F}}=3.7 \mathrm{~Hz}, 2 \mathrm{C}\right), 123.3\left(\mathrm{q},{ }^{1} J_{\mathrm{C}-\mathrm{F}}=\right.$ $273.2 \mathrm{~Hz}, 1 \mathrm{C}), 114.3,114.1(2 \mathrm{C}), 113.2,79.9,71.2,55.4,50.4,36.0,22.4 .{ }^{19} \mathrm{~F}$ NMR (565 MHz, $\left.\mathrm{CDCl}_{3}\right)$ $\delta$-63.13 (s, 3F). HRMS ESI $[\mathrm{M}+\mathrm{H}]^{+}$calculated for $\mathrm{C}_{21} \mathrm{H}_{21} \mathrm{NF}_{3} \mathrm{O}_{3} \mathrm{~S} 424.1194$, found 424.1187.

\section{$N$-((4-(tert-Butyl)phenyl)ethynyl)- $N$-(3-methylbut-3-en-1-yl)-4-}

\section{(trifluoromethyl)benzenesulfonamide (1x)}

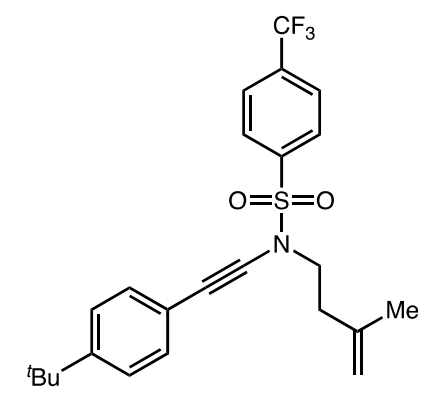

Following the method A, purification by flash column chromatography (5-10\% EtOAc in hexane), colourless oil. ${ }^{1} \mathrm{H} \mathrm{NMR}\left(400 \mathrm{MHz}, \mathrm{CDCl}_{3}\right) \delta$ $8.10(\mathrm{~d}, J=8.2 \mathrm{~Hz}, 2 \mathrm{H}), 7.84(\mathrm{~d}, J=8.2 \mathrm{~Hz}, 2 \mathrm{H}), 7.38-7.28(\mathrm{~m}, 4 \mathrm{H})$, $4.82-4.79(\mathrm{~m}, 1 \mathrm{H}), 4.78-4.72(\mathrm{~m}, 1 \mathrm{H}), 3.62-3.50(\mathrm{~m}, 2 \mathrm{H}), 2.47-2.38$ $(\mathrm{m}, 2 \mathrm{H}), 1.76$ (s 3H), 1.32 (s, 9H). ${ }^{13} \mathrm{C}$ NMR $\left(101 \mathrm{MHz}, \mathrm{CDCl}_{3}\right) \delta 151.8$ $141.1\left(\mathrm{~d},{ }^{4} J_{\mathrm{C}-\mathrm{F}}=0.9 \mathrm{~Hz}\right), 141.1,135.30\left(\mathrm{q},{ }^{2} J_{\mathrm{C}-\mathrm{F}}=33.2 \mathrm{~Hz}\right), 131.6(2 \mathrm{C})$, $128.3(2 \mathrm{C}), 126.4\left(\mathrm{q},{ }^{3} J_{\mathrm{C}-\mathrm{F}}=3.7 \mathrm{~Hz}, 2 \mathrm{C}\right), 125.5(2 \mathrm{C}), 123.3\left(\mathrm{q},{ }^{1} J_{\mathrm{C}-\mathrm{F}}=274.1 \mathrm{~Hz}\right), 119.3,113.3,80.5$, 71.5, 50.4, 36.0, 34.9, $31.3(2 \mathrm{C}), 22.4 .{ }^{19} \mathrm{~F}$ NMR (376 MHz, $\left.\mathrm{CDCl}_{3}\right) \delta-63.14(\mathrm{~s}, 3 \mathrm{~F})$. HRMS ESI $[\mathrm{M}+\mathrm{H}]^{+}$calculated for $\mathrm{C}_{24} \mathrm{H}_{27} \mathrm{NF}_{3} \mathrm{O}_{2} \mathrm{~S} 450.1715$, found 450.1707 . 


\section{$N$-(But-3-en-1-yl)- $N$-((4-methoxyphenyl)ethynyl)-4-nitrobenzenesulfonamide (1y)}

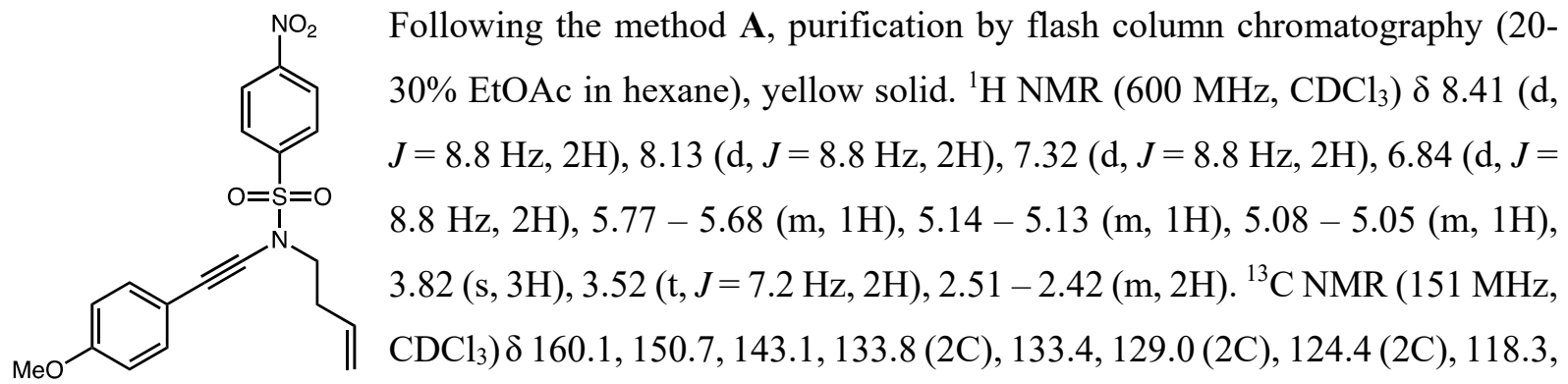

114.2 (2C), 113.9, 79.4, 71.5, 55.4, 51.6, 32.4. HRMS ESI $[\mathrm{M}+\mathrm{H}]^{+}$calculated for $\mathrm{C}_{19} \mathrm{H}_{19} \mathrm{~N}_{2} \mathrm{O}_{5} \mathrm{~S}$ 387.1015 , found 387.1020 .

3,5-Difluoro- $N$-((4-methoxyphenyl)ethynyl)- $N$-(3-methylbut-3-en-1-yl)benzenesulfonamide (1z)

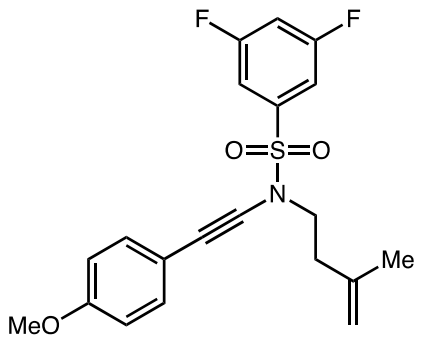

Following the method A, purification by flash column chromatography (10\% EtOAc in hexane), white solid. ${ }^{1} \mathrm{H}$ NMR (600 $\left.\mathrm{MHz}, \mathrm{CDCl}_{3}\right) \delta 7.53$ $-7.47(\mathrm{~m}, 2 \mathrm{H}), 7.34(\mathrm{~d}, J=8.8 \mathrm{~Hz}, 2 \mathrm{H}), 7.14-7.09(\mathrm{~m}, 1 \mathrm{H}), 6.85(\mathrm{~d}, J=$ $8.8 \mathrm{~Hz}, 2 \mathrm{H}), 4.83-4.81(\mathrm{~m}, 1 \mathrm{H}), 4.79-4.74(\mathrm{~m}, 1 \mathrm{H}), 3.81(\mathrm{~s}, 3 \mathrm{H}), 3.59$ $-3.53(\mathrm{~m}, 2 \mathrm{H}), 2.45-2.40(\mathrm{~m}, 2 \mathrm{H}), 1.76(\mathrm{~s}, 3 \mathrm{H}) .{ }^{13} \mathrm{C} \mathrm{NMR}(151 \mathrm{MHz}$, $\left.\mathrm{CDCl}_{3}\right) \delta 162.8(\mathrm{~d}, J=255.3 \mathrm{~Hz}), 162.8(\mathrm{~d}, J=255.3 \mathrm{~Hz}), 159.9,141.0$, $140.6(\mathrm{t}, J=8.5 \mathrm{~Hz}), 133.7$ (2C), $114.1(2 \mathrm{C}), 113.3,111.3$ (d, $J=28.7 \mathrm{~Hz}), 111.3$ (d, $J=15.5 \mathrm{~Hz})$, $109.3(\mathrm{~d}, J=25.0 \mathrm{~Hz}), 109.2(\mathrm{~d}, J=25.0 \mathrm{~Hz}), 79.5,71.4,55.4,50.5,36.0,22.3 .{ }^{19} \mathrm{~F}$ NMR $(565 \mathrm{MHz}$, $\left.\mathrm{CDCl}_{3}\right) \delta-105.26(\mathrm{dd}, J=8.5,5.6 \mathrm{~Hz}, 2 \mathrm{~F})$. HRMS ESI $[\mathrm{M}+\mathrm{H}]^{+}$calculated for $\mathrm{C}_{20} \mathrm{H}_{20} \mathrm{NF}_{3} \mathrm{O}_{3} \mathrm{~S} 392.1132$, found 392.1123 .

\section{$N$-((3,5-Dimethylphenyl)ethynyl)-3-fluoro- $N$-(3-methylbut-3-en-1-yl)benzenesulfonamide (1aa)}

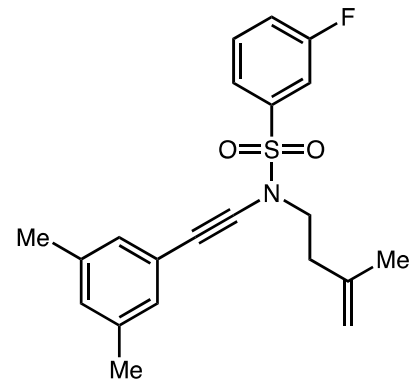

Following the method $\mathbf{B}$, purification by flash column chromatography (5$10 \%$ EtOAc in hexane), colourless solid. ${ }^{1} \mathrm{H}$ NMR $\left(600 \mathrm{MHz}, \mathrm{CDCl}_{3}\right) \delta$ $7.79-7.76(\mathrm{~m}, 1 \mathrm{H}), 7.71-7.67(\mathrm{~m}, 1 \mathrm{H}), 7.60-7.54(\mathrm{~m}, 1 \mathrm{H}), 7.40-7.34$ $(\mathrm{m}, 1 \mathrm{H}), 7.04-7.02(\mathrm{~m}, 2 \mathrm{H}), 6.95(\mathrm{~s}, 1 \mathrm{H}), 4.84-4.81(\mathrm{~m}, 1 \mathrm{H}), 4.79-$ $4.74(\mathrm{~m}, 1 \mathrm{H}), 3.56(\mathrm{t}, J=7.4 \mathrm{~Hz}, 2 \mathrm{H}), 2.43(\mathrm{t}, J=7.5 \mathrm{~Hz}, 2 \mathrm{H}), 2.30(\mathrm{~s}$, $6 \mathrm{H}), 1.77(\mathrm{~s}, 3 \mathrm{H}) \cdot{ }^{13} \mathrm{C} \mathrm{NMR}\left(151 \mathrm{MHz}, \mathrm{CDCl}_{3}\right) \delta 162.44\left(\mathrm{~d},{ }^{1} J_{\mathrm{C}-\mathrm{F}}=252.17\right.$ $\mathrm{Hz}), 141.12,139.52\left(\mathrm{~d},{ }^{3} J_{\mathrm{C}-\mathrm{F}}=7.6 \mathrm{~Hz}\right), 138.03,131.07\left(\mathrm{~d},{ }^{3} J_{\mathrm{C}-\mathrm{F}}=9.1 \mathrm{~Hz}\right), 130.1,129.2(2 \mathrm{C}), 123.5(\mathrm{~d}$, $\left.{ }^{4} J_{\mathrm{C}-\mathrm{F}}=3.4 \mathrm{~Hz}\right), 122.1,120.9\left(\mathrm{~d},{ }^{2} J_{\mathrm{C}-\mathrm{F}}=21.1 \mathrm{~Hz}\right), 115.1\left(\mathrm{~d},{ }^{2} J_{\mathrm{C}-\mathrm{F}}=25.7 \mathrm{~Hz}\right), 113.2,80.7,71.6,50.3$, 36.0, 22.3, $21.1(2 \mathrm{C}) .{ }^{19} \mathrm{~F}$ NMR (565 MHz, $\left.\mathrm{CDCl}_{3}\right) \delta-109.17--109.30(\mathrm{~m}, 1 \mathrm{~F})$. HRMS ESI $[\mathrm{M}+\mathrm{H}]^{+}$ calculated for $\mathrm{C}_{21} \mathrm{H}_{23} \mathrm{NFO}_{3} \mathrm{~S} 372.1434$, found 372.1426 . 
4-Chloro- $N$-(3-methylbut-3-en-1-yl)- $N$-((4-(methylthio)phenyl)ethynyl)benzenesulfonamide (1ab)

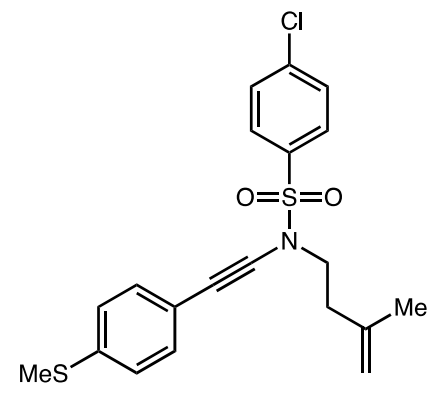

Following the method $\mathbf{B}$, purification by flash column chromatography (5-10\% EtOAc in hexane), yellow oil. ${ }^{1} \mathrm{H}$ NMR $\left(400 \mathrm{MHz}, \mathrm{CDCl}_{3}\right) \delta 7.88$ $(\mathrm{d}, J=8.6 \mathrm{~Hz}, 2 \mathrm{H}), 7.53(\mathrm{~d}, J=8.6 \mathrm{~Hz}, 2 \mathrm{H}), 7.28(\mathrm{~d}, J=8.5 \mathrm{~Hz}, 2 \mathrm{H})$, $7.16(\mathrm{~d}, J=8.5 \mathrm{~Hz}, 2 \mathrm{H}), 4.82-4.80(\mathrm{~m}, 1 \mathrm{H}), 4.75-4.73(\mathrm{~m}, 1 \mathrm{H}), 3.56$ $-3.52(\mathrm{~m}, 2 \mathrm{H}), 2.471(\mathrm{~s}, 3 \mathrm{H}), 2.42-2.38(\mathrm{~m}, 2 \mathrm{H}), 1.74(\mathrm{~s}, 3 \mathrm{H}) .{ }^{13} \mathrm{C} \mathrm{NMR}$ $\left(101 \mathrm{MHz}, \mathrm{CDCl}_{3}\right) \delta 141.0,140.2,139.2,135.9,131.9$ (2C), 129.4 (2C), $129.0(2 \mathrm{C}), 125.8(2 \mathrm{C}), 118.6,113.0,81.5,70.9,50.0,35.8,22.2,15.3$. HRMS ESI $[\mathrm{M}+\mathrm{H}]^{+}$calculated for $\mathrm{C}_{20} \mathrm{H}_{21} \mathrm{NFO}_{3} \mathrm{~S}$ 406.0702, found 406.0692 .

\section{3-Fluoro- $N$-((4-isopropylphenyl)ethynyl)- $N$-(3-methylbut-3-en-1-yl)benzenesulfonamide (1ac)}

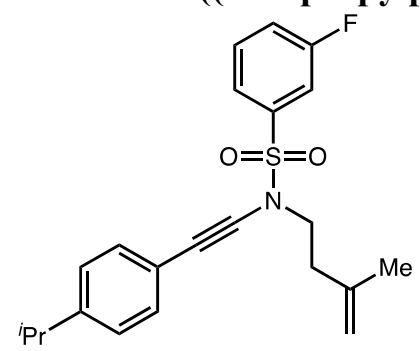

Following the method B, purification by flash column chromatography (5$10 \%$ EtOAc in hexane), colourless oil. ${ }^{1} \mathrm{H}$ NMR (600 MHz, $\left.\mathrm{CDCl}_{3}\right) \delta 7.79$ $-7.75(\mathrm{~m}, 1 \mathrm{H}), 7.70-7.67(\mathrm{~m}, 1 \mathrm{H}), 7.59-7.54(\mathrm{~m}, 1 \mathrm{H}), 7.39-7.35(\mathrm{~m}$, $1 \mathrm{H}), 7.34-7.31(\mathrm{~m}, 2 \mathrm{H}), 7.18(\mathrm{~d}, J=8.1 \mathrm{~Hz}, 2 \mathrm{H}), 4.83-4.80(\mathrm{~m}, 1 \mathrm{H})$, $4.76(\mathrm{~s}, 1 \mathrm{H}), 3.56(\mathrm{t}, J=7.4 \mathrm{~Hz}, 2 \mathrm{H}), 2.95-2.84(\mathrm{~m}, 1 \mathrm{H}), 2.42(\mathrm{t}, J=7.4$ $\mathrm{Hz}, 2 \mathrm{H}), 1.76(\mathrm{~s}, 3 \mathrm{H}), 1.25(\mathrm{~s}, 3 \mathrm{H}), 1.24(\mathrm{~s}, 3 \mathrm{H}) .{ }^{13} \mathrm{C} \mathrm{NMR}\left(151 \mathrm{MHz}, \mathrm{CDCl}_{3}\right) \delta 162.4\left(\mathrm{~d},{ }^{1} J_{\mathrm{C}-\mathrm{F}}=252.17\right.$ $\mathrm{Hz}), 149.4,141.1,139.5\left(\mathrm{~d},{ }^{3} J_{\mathrm{C}-\mathrm{F}}=6.8 \mathrm{~Hz}\right), 131.8(2 \mathrm{C}), 131.0\left(\mathrm{~d},{ }^{3} \mathrm{~J}_{\mathrm{C}-\mathrm{F}}=7.7 \mathrm{~Hz}\right), 126.6(2 \mathrm{C}), 123.5$ $\left(\mathrm{d},{ }^{4} J_{\mathrm{C}-\mathrm{F}}=3.4 \mathrm{~Hz}\right), 120.9\left(\mathrm{~d},{ }^{2} J_{\mathrm{C}-\mathrm{F}}=21.2 \mathrm{~Hz}\right), 119.7,115.1\left(\mathrm{~d},{ }^{2} J_{\mathrm{C}-\mathrm{F}}=24.7 \mathrm{~Hz}\right), 113.2,80.7,71.4,50.3$, 36.0, 34.1, $23.9(2 \mathrm{C}), 22.4 .{ }^{19} \mathrm{~F}$ NMR (565 MHz, $\left.\mathrm{CDCl}_{3}\right) \delta-109.16--109.28(\mathrm{~m}, 1 \mathrm{~F})$. HRMS ESI $[\mathrm{M}+\mathrm{H}]^{+}$calculated for $\mathrm{C}_{22} \mathrm{H}_{25} \mathrm{NFO}_{2} \mathrm{~S} 386.1590$, found 386.1581 .

\section{$N$-((4-Methoxyphenyl)ethynyl)- $N$-(3-methylbut-3-en-1-yl)pyridine-3-sulfonamide (1ad)}

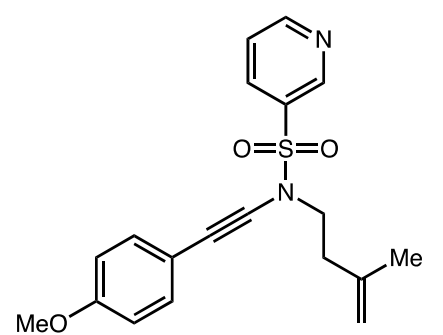

Following the method A, purification by flash column chromatography (10-15\% EtOAc in hexane), yellow solid. ${ }^{1} \mathrm{H}$ NMR (400 MHz, $\left.\mathrm{CDCl}_{3}\right)$ $\delta 9.17-9.13(\mathrm{~m}, 1 \mathrm{H}), 8.88-8.84(\mathrm{~m}, 1 \mathrm{H}), 8.23-8.18(\mathrm{~m}, 1 \mathrm{H}), 7.53-$ $7.47(\mathrm{~m}, 1 \mathrm{H}), 7.31(\mathrm{~d}, J=8.8 \mathrm{~Hz}, 2 \mathrm{H}), 6.82(\mathrm{~d}, J=8.8 \mathrm{~Hz}, 2 \mathrm{H}), 4.79-$ $4.76(\mathrm{~m}, 1 \mathrm{H}), 4.75-4.69(\mathrm{~m}, 1 \mathrm{H}), 3.79(\mathrm{~s}, 3 \mathrm{H}), 3.62-3.52(\mathrm{~m}, 2 \mathrm{H})$, $2.46-2.32(\mathrm{~m}, 2 \mathrm{H}), 1.73(\mathrm{~s}, 3 \mathrm{H}) .{ }^{13} \mathrm{C} \mathrm{NMR}\left(101 \mathrm{MHz}, \mathrm{CDCl}_{3}\right) \delta 159.8,154.0,148.3,141.0,135.2$, 134.3, 133.6 (2C), 123.7, 114.1, 114.0 (2C), 113.2, 79.6, 71.4, 55.3, 50.3, 35.9, 22.3. HRMS ESI $[\mathrm{M}+\mathrm{H}]^{+}$calculated for $\mathrm{C}_{19} \mathrm{H}_{21} \mathrm{~N}_{2} \mathrm{O}_{3} \mathrm{~S} 357.1273$, found 357.1264 .

\section{$N$-((3-Fluorophenyl)ethynyl)-4-methoxy- $N$-(3-methylbut-3-en-1-yl)benzenesulfonamide (4a)}

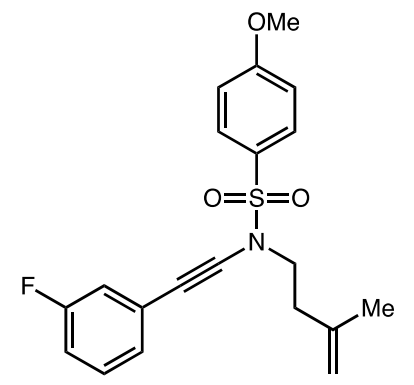

Following the method $\mathbf{A}$, purification by flash column chromatography $(10 \%$ EtOAc in hexane), colourless oil. ${ }^{1} \mathrm{H}$ NMR $\left(600 \mathrm{MHz}, \mathrm{CDCl}_{3}\right) \delta 7.78(\mathrm{~d}, J$ $=9.0 \mathrm{~Hz}, 2 \mathrm{H}), 7.16-7.11(\mathrm{~m}, 1 \mathrm{H}), 7.04-7.01(\mathrm{~m}, 1 \mathrm{H}), 6.95-6.90(\mathrm{~m}$, $3 \mathrm{H}), 6.88-6.84(\mathrm{~m}, 1 \mathrm{H}), 4.72-4.70(\mathrm{~m}, 1 \mathrm{H}), 4.65-4.63(\mathrm{~m}, 1 \mathrm{H}), 3.77$ (s, 3H), $3.44-3.41(\mathrm{~m}, 2 \mathrm{H}), 2.33-2.27(\mathrm{~m}, 2 \mathrm{H}), 1.66-1.64(\mathrm{~m}, 3 \mathrm{H}) .{ }^{13} \mathrm{C}$ $\operatorname{NMR}\left(151 \mathrm{MHz}, \mathrm{CDCl}_{3}\right) \delta 163.8,162.3\left(\mathrm{~d},{ }^{1} J_{\mathrm{C}-\mathrm{F}}=247.6 \mathrm{~Hz}\right), 141.1,129.9$, $129.8(2 \mathrm{C}), 129.0,127.0\left(\mathrm{~d},{ }^{4} J_{\mathrm{C}-\mathrm{F}}=3.0 \mathrm{~Hz}\right), 124.9\left(\mathrm{~d},{ }^{3} J_{\mathrm{C}-\mathrm{F}}=9.7 \mathrm{~Hz}\right), 117.8\left(\mathrm{~d},{ }^{2} J_{\mathrm{C}-\mathrm{F}}=22.8 \mathrm{~Hz}\right), 114.9$ 
$\left(\mathrm{d},{ }^{2} \mathrm{~J}_{\mathrm{C}-\mathrm{F}}=21.2 \mathrm{~Hz}\right), 114.4(2 \mathrm{C}), 113.0,83.4,70.1,55.7,49.8,36.0,22.3 .{ }^{19} \mathrm{~F}$ NMR $\left(565 \mathrm{MHz}, \mathrm{CDCl}_{3}\right)$ $\delta-112.98--113.04(\mathrm{~m}, 1 \mathrm{~F})$. HRMS ESI $[\mathrm{M}+\mathrm{H}]^{+}$calculated for $\mathrm{C}_{20} \mathrm{H}_{21} \mathrm{NFO}_{3} \mathrm{~S}$ 374.1226, found 374.1219 .

4-Methoxy- $N$-(3-methylbut-3-en-1-yl)- $N$-((4-nitrophenyl)ethynyl) benzenesulfonamide (4b)

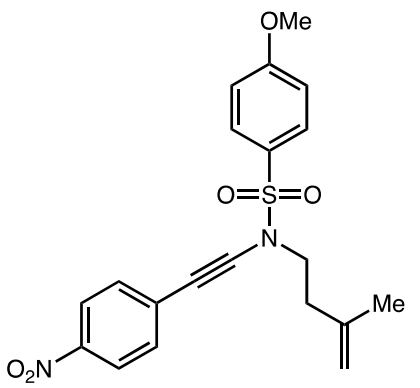

Following the method $\mathbf{B}$, purification by flash column chromatography (20-30\% EtOAc in hexane), yellow solid. ${ }^{1} \mathrm{H}$ NMR $\left(600 \mathrm{MHz}, \mathrm{CDCl}_{3}\right) \delta$ $8.16(\mathrm{~d}, J=8.8 \mathrm{~Hz}, 2 \mathrm{H}), 7.88(\mathrm{~d}, J=8.9 \mathrm{~Hz}, 2 \mathrm{H}), 7.45(\mathrm{~d}, J=8.8 \mathrm{~Hz}, 2 \mathrm{H})$, $7.03(\mathrm{~d}, J=8.9 \mathrm{~Hz}, 2 \mathrm{H}), 4.84-4.80(\mathrm{~m}, 1 \mathrm{H}), 4.75-4.73(\mathrm{~m}, 1 \mathrm{H}), 3.89$ (s, $3 \mathrm{H}), 3.59-3.54(\mathrm{~m}, 2 \mathrm{H}), 2.43-2.39(\mathrm{~m}, 2 \mathrm{H}), 1.76(\mathrm{~s}, 3 \mathrm{H}) .{ }^{13} \mathrm{C} \mathrm{NMR}$ $\left(151 \mathrm{MHz}, \mathrm{CDCl}_{3}\right) \delta 164.2,146.4,141.1,131.0(2 \mathrm{C}), 130.6,130.0(2 \mathrm{C})$, 129.1, 123.8 (2C), $114.6(2 \mathrm{C}), 113.3,88.6,71.0,55.9,49.9,36.2,22.4$. HRMS ESI [M+H] ${ }^{+}$calculated for $\mathrm{C}_{20} \mathrm{H}_{21} \mathrm{~N}_{2} \mathrm{O}_{5} \mathrm{~S} 401.1171$, found 401.1162 .

\section{3-Fluoro- $N$-((4-methoxyphenyl)ethynyl)- $N$-(3-methylbut-3-en-1-yl)benzenesulfonamide (4a')}

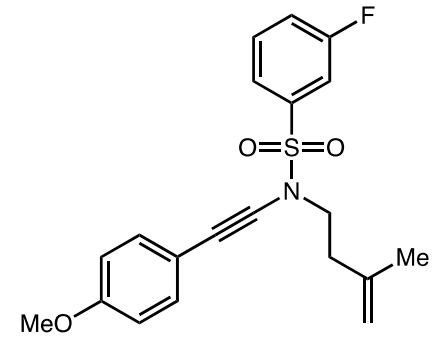

Following the method $\mathbf{A}$, purification by flash column chromatography $\left(10 \%\right.$ EtOAc in hexane), colourless oil. ${ }^{1} \mathrm{H}$ NMR (400 MHz, $\left.\mathrm{CDCl}_{3}\right) \delta$ $7.77-7.74(\mathrm{~m}, 1 \mathrm{H}), 7.69-7.65(\mathrm{~m}, 1 \mathrm{H}), 7.59-7.53(\mathrm{~m}, 1 \mathrm{H}), 7.40-$ $7.34(\mathrm{~m}, 1 \mathrm{H}), 7.34-7.30(\mathrm{~m}, 2 \mathrm{H}), 6.4(\mathrm{~d}, J=8.8 \mathrm{~Hz}, 2 \mathrm{H}), 4.82-4.79$ (m, 1H), $4.78-4.73(\mathrm{~m}, 1 \mathrm{H}), 3.81(\mathrm{~s}, 3 \mathrm{H}), 3.57-3.51(\mathrm{~m}, 2 \mathrm{H}), 2.45-$ $2.36(\mathrm{~m}, 2 \mathrm{H}), 1.75(\mathrm{~s}, 3 \mathrm{H}) \cdot{ }^{13} \mathrm{C} \mathrm{NMR}\left(101 \mathrm{MHz}, \mathrm{CDCl}_{3}\right) \delta 162.4\left(\mathrm{~d},{ }^{1} J_{\mathrm{C}-\mathrm{F}}=252.9 \mathrm{~Hz}\right), 159.8,141.2$, $139.5\left(\mathrm{~d},{ }^{3} J_{\mathrm{C}-\mathrm{F}}=6.9 \mathrm{~Hz}\right), 133.6(2 \mathrm{C}), 131.0\left(\mathrm{~d},{ }^{3} J_{\mathrm{C}-\mathrm{F}}=7.8 \mathrm{~Hz}\right), 123.5\left(\mathrm{~d},{ }^{4} J_{\mathrm{C}-\mathrm{F}}=3.4 \mathrm{~Hz}\right), 120.9\left(\mathrm{~d},{ }^{2} J\right.$ $\mathrm{C}-\mathrm{F}=21.3 \mathrm{~Hz}), 115.1\left(\mathrm{~d},{ }^{2} J_{\mathrm{C}-\mathrm{F}}=24.7 \mathrm{~Hz}\right), 114.4,114.1(2 \mathrm{C}), 113.1,80.0,71.0,55.4,50.3,36.0,22.4$. ${ }^{19} \mathrm{~F}$ NMR $\left(376 \mathrm{MHz}, \mathrm{CDCl}_{3}\right) \delta-109.24--109.33(\mathrm{~m}, 1 \mathrm{~F})$. HRMS ESI $[\mathrm{M}+\mathrm{H}]^{+}$calculated for $\mathrm{C}_{20} \mathrm{H}_{21} \mathrm{NFO}_{3} \mathrm{~S} 374.1226$, found 374.1219 .

\section{$N$-((4-Methoxyphenyl)ethynyl)- $N$-(3-methylbut-3-en-1-yl)-4-nitrobenzenesulfonamide (4b')}

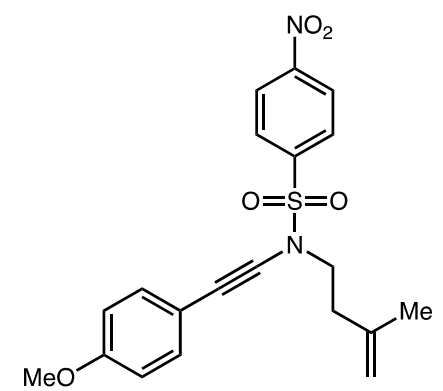

Following the method A, purification by flash column chromatography (20-30\% EtOAc in hexane), yellow solid. ${ }^{1} \mathrm{H}$ NMR (600 MHz, $\left.\mathrm{CDCl}_{3}\right) \delta$ $8.40(\mathrm{~d}, J=8.8 \mathrm{~Hz}, 2 \mathrm{H}), 8.14(\mathrm{~d}, J=8.8 \mathrm{~Hz}, 2 \mathrm{H}), 7.32(\mathrm{~d}, J=8.7 \mathrm{~Hz}$, $2 \mathrm{H}), 6.84(\mathrm{~d}, J=8.7 \mathrm{~Hz}, 2 \mathrm{H}), 4.82-4.79(\mathrm{~m}, 1 \mathrm{H}), 4.75-4.73(\mathrm{~m}, 1 \mathrm{H})$, $3.81(\mathrm{~s}, 3 \mathrm{H}), 3.58(\mathrm{t}, J=7.4 \mathrm{~Hz}, 2 \mathrm{H}), 2.41(\mathrm{t}, J=7.4 \mathrm{~Hz}, 2 \mathrm{H}), 1.75$ (s, $3 \mathrm{H}) .{ }^{13} \mathrm{C}$ NMR $\left(151 \mathrm{MHz}, \mathrm{CDCl}_{3}\right) \delta 160.1,150.7,143.1,141.0,133.8$ (2C), 129.0 (2C), 124.5 (2C), 114.2 (2C), 114.0, 113.4, 79.4, 71.5, 55.4, 50.6, 36.0, 22.3. HRMS ESI $[\mathrm{M}+\mathrm{H}]^{+}$calculated for $\mathrm{C}_{20} \mathrm{H}_{21} \mathrm{~N}_{2} \mathrm{O}_{5} \mathrm{~S} 401.1171$, found 401.1175 . 


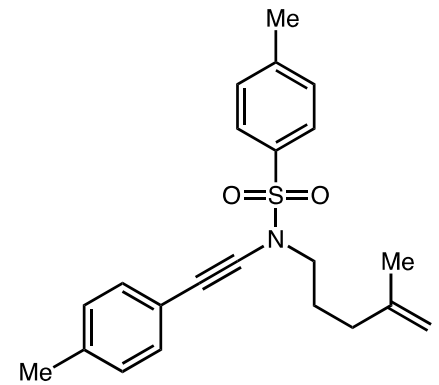

Following the method A, purification by flash column chromatography (5-10\% EtOAc in hexane), colourless oil. ${ }^{1} \mathrm{H}$ NMR $\left(600 \mathrm{MHz}, \mathrm{CDCl}_{3}\right) \delta$ 7.84 (d, $J=7.9 \mathrm{~Hz}, 2 \mathrm{H}), 7.35$ (d, $J=7.9 \mathrm{~Hz}, 2 \mathrm{H}), 7.26$ (d, $J=7.7 \mathrm{~Hz}$, 2H), $7.10(\mathrm{~d}, J=7.7 \mathrm{~Hz}, 2 \mathrm{H}), 4.75-4.73(\mathrm{~m}, 1 \mathrm{H}), 4.70-4.68(\mathrm{~m}, 1 \mathrm{H})$, $3.41-3.37(\mathrm{~m}, 1 \mathrm{H}), 2.45(\mathrm{~s}, 3 \mathrm{H}), 2.34(\mathrm{~s}, 3 \mathrm{H}), 2.09-2.04(\mathrm{~m}, 2 \mathrm{H}), 1.86$

$-1.82(\mathrm{~m}, 2 \mathrm{H}), 1.71(\mathrm{~s}, 3 \mathrm{H}) .{ }^{13} \mathrm{C} \mathrm{NMR}\left(151 \mathrm{MHz}, \mathrm{CDCl}_{3}\right) \delta 144.6,144.4$, 138.0, 134.7, 131.5 (2C), 129.8 (2C), 129.2 (2C), 127.8 (2C), 119.8, 110.8, 81.7, 70.7, 51.4, 34.4, 25.94, 22.4, 21.7, 21.5. HRMS ESI $[\mathrm{M}+\mathrm{H}]^{+}$calculated for $\mathrm{C}_{22} \mathrm{H}_{26} \mathrm{NO}_{2} \mathrm{~S} 368.1684$, found 368.1676.

\section{$N$-((3-Fluorophenyl)ethynyl)-4-methyl- $N$-(pent-4-en-1-yl)benzenesulfonamide (6b)}

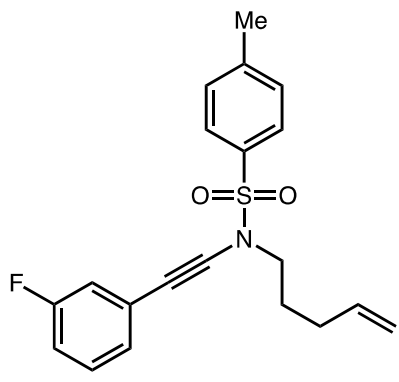

Following the method A, purification by flash column chromatography (5$10 \%$ EtOAc in hexane), colourless oil. ${ }^{1} \mathrm{H}$ NMR $\left(600 \mathrm{MHz}, \mathrm{CDCl}_{3}\right) \delta 7.85$ (d, $J=8.2 \mathrm{~Hz}, 2 \mathrm{H}), 7.39$ (d, $J=8.2 \mathrm{~Hz}, 2 \mathrm{H}), 7.30-7.24$ (m, 1H), $7.18-$ $7.14(\mathrm{~m}, 1 \mathrm{H}), 7.08-7.04(\mathrm{~m}, 1 \mathrm{H}), 7.03-6.94(\mathrm{~m}, 1 \mathrm{H}), 5.85-5.76(\mathrm{~m}, 1 \mathrm{H})$, $5.10-5.05(\mathrm{~m}, 1 \mathrm{H}), 5.05-5.01(\mathrm{~m}, 1 \mathrm{H}), 3.44(\mathrm{t}, J=7.2 \mathrm{~Hz}, 2 \mathrm{H}), 2.48(\mathrm{~s}$, $3 \mathrm{H}), 2.19-2.12(\mathrm{~m}, 2 \mathrm{H}), 1.83$ (p, $J=7.3 \mathrm{~Hz}, 2 \mathrm{H}) .{ }^{13} \mathrm{C}$ NMR $(151 \mathrm{MHz}$, $\left.\mathrm{CDCl}_{3}\right) \delta 162.4\left(\mathrm{~d},{ }^{1} J_{\mathrm{C}-\mathrm{F}}=246.13 \mathrm{~Hz}\right), 144.9,137.1,134.6,129.9\left(\mathrm{~d},{ }^{3} J_{\mathrm{C}-\mathrm{F}}=7.6 \mathrm{~Hz}\right), 129.9(2 \mathrm{C}), 127.7$ (2C), $127.0\left(\mathrm{~d},{ }^{4} J_{\mathrm{C}-\mathrm{F}}=2.9 \mathrm{~Hz}\right), 124.9\left(\mathrm{~d},{ }^{3} J_{\mathrm{C}-\mathrm{F}}=9.1 \mathrm{~Hz}\right), 117.9\left(\mathrm{~d},{ }^{2} J_{\mathrm{C}-\mathrm{F}}=22.7 \mathrm{~Hz}\right), 115.8,115.0(\mathrm{~d}$, $\left.{ }^{2} J_{\mathrm{C}-\mathrm{F}}=21.1 \mathrm{~Hz}\right), 83.4,69.9,51.0,30.3,27.2,21.7 .{ }^{19} \mathrm{~F} \mathrm{NMR}\left(565 \mathrm{MHz}, \mathrm{CDCl}_{3}\right) \delta-113.02--113.10$ (m, 1F). HRMS ESI $[\mathrm{M}+\mathrm{H}]^{+}$calculated for $\mathrm{C}_{20} \mathrm{H}_{21} \mathrm{NFO}_{2} \mathrm{~S} 358.1277$, found 358.1269.

\section{3,5-Difluoro- $N$-((4-methoxyphenyl)ethynyl)- $N$-(5-methylenenonyl)benzenesulfonamide (6c)}

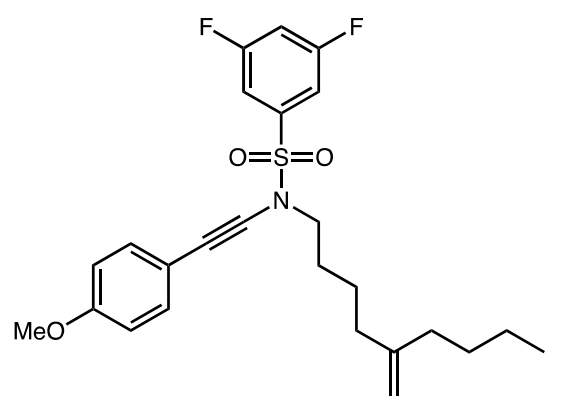

Following the method A, purification by flash column chromatography (5-10\% EtOAc in hexane), colourless oil. ${ }^{1} \mathrm{H}$ NMR (400 MHz, $\left.\mathrm{CDCl}_{3}\right) \delta 7.54-7.43(\mathrm{~m}, 2 \mathrm{H}), 7.33(\mathrm{~d}, J=8.4$ $\mathrm{Hz}, 2 \mathrm{H}), 7.14-7.08(\mathrm{~m}, 1 \mathrm{H}), 6.89-6.80 \mathrm{j}(\mathrm{m}, 2 \mathrm{H}), 4.75-4.70$ (m, 1H), $4.68(\mathrm{~s}, 1 \mathrm{H}), 3.81(\mathrm{~s}, 3 \mathrm{H}), 3.44$ (t, $J=7.1 \mathrm{~Hz}, 2 \mathrm{H}), 2.04$ $(\mathrm{t}, J=7.6 \mathrm{~Hz}, 2 \mathrm{H}), 1.98(\mathrm{t}, J=7.6 \mathrm{~Hz}, 2 \mathrm{H}), 1.71(\mathrm{p}, J=7.2 \mathrm{~Hz}$, $2 \mathrm{H}), 1.54-1.45(\mathrm{~m}, 2 \mathrm{H}), 1.43-1.34(\mathrm{~m}, 2 \mathrm{H}), 1.33-1.24(\mathrm{~m}, 2 \mathrm{H}), 0.89(\mathrm{t}, J=7.2 \mathrm{~Hz}, 3 \mathrm{H}) .{ }^{13} \mathrm{C} \mathrm{NMR}$ $\left(101 \mathrm{MHz}, \mathrm{CDCl}_{3}\right) \delta 162.9\left(\mathrm{~d},{ }^{1} J_{\mathrm{C}-\mathrm{F}}=256.1 \mathrm{~Hz}, 1 \mathrm{C}\right), 162.8\left(\mathrm{~d},{ }^{1} \mathrm{~J}_{\mathrm{C}-\mathrm{F}}=256.1 \mathrm{~Hz}, 1 \mathrm{C}\right), 159.9,149.2$, $140.6\left(\mathrm{t},{ }^{3} \mathrm{~J}_{\mathrm{C}-\mathrm{F}}=8.8 \mathrm{~Hz}, 1 \mathrm{C}\right), 133.7(2 \mathrm{C}), 114.2,114.2(2 \mathrm{C}), 111.4\left(\mathrm{~d},{ }^{2} J_{\mathrm{C}-\mathrm{F}}=28.5 \mathrm{~Hz}, 1 \mathrm{C}\right), 111.3(\mathrm{~d}$, $\left.{ }^{3} J_{\mathrm{C}-\mathrm{F}}=11.4 \mathrm{~Hz}, 1 \mathrm{C}\right), 109.3,109.3\left(\mathrm{t},{ }^{2} J_{\mathrm{C}-\mathrm{F}}=25.0 \mathrm{~Hz}, 1 \mathrm{C}\right), 79.8,71.1,55.5,52.2,35.7,35.5,30.1,27.6$, 24.4, 22.6, 14.1. ${ }^{19} \mathrm{~F}$ NMR (565 MHz, $\left.\mathrm{CDCl}_{3}\right) \delta-106.19--106.27(\mathrm{~m}, 2 \mathrm{~F})$. HRMS ESI $[\mathrm{M}+\mathrm{H}]^{+}$ calculated for $\mathrm{C}_{25} \mathrm{H}_{30} \mathrm{NF}_{2} \mathrm{O}_{3} \mathrm{~S} 462.1914$, found 462.1919 . 


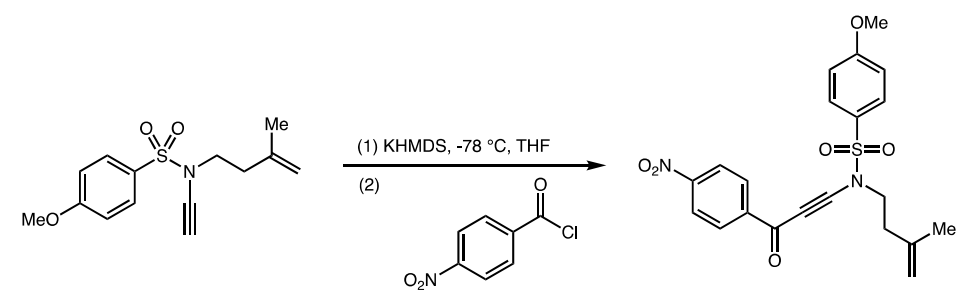

Scheme 6 Synthesis of $9^{4}$

To a solution of terminal ynamide (1 eq.) in THF was added KHMDS (1.0 M in THF, 1.5 eq.) at -78

${ }^{\circ} \mathrm{C}$. The reaction mixture was stirred at $-78^{\circ} \mathrm{C}$ for $1 \mathrm{~h}$, and corresponding benzoyl chloride (1 eq.) was added dropwise. The reaction mixture was stirred for another $30 \mathrm{~min}$ at $-78{ }^{\circ} \mathrm{C}$, and then quenched with sat. aqueous $\mathrm{NH}_{4} \mathrm{Cl}$. This mixture was diluted with EtOAc and partitioned. The organic layer was washed with sat. aqueous $\mathrm{NaHCO}_{3}$, then brine, dried over $\mathrm{Na}_{2} \mathrm{SO}_{4}$, filtered, and concentrated on a rotary evaporator. The crude residue was purified using silica gel flash chromatography to afford ynamide 9 .

\section{4-Methoxy- $N$-(3-methylbut-3-en-1-yl)- $N$-(3-(4-nitrophenyl)-3-oxoprop-1-yn-1-yl)}

benzenesulfonamide (9)

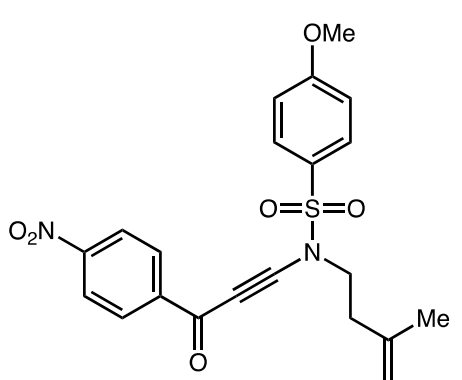

Purification by flash column chromatography $(10-20 \%$ EtOAc in hexane), yellow solid. ${ }^{1} \mathrm{H}$ NMR $\left(600 \mathrm{MHz}, \mathrm{CDCl}_{3}\right) \delta 8.36-8.29(\mathrm{~m}$, 4H), 7.85 (d, $J=8.9 \mathrm{~Hz}, 2 \mathrm{H}), 7.03$ (d, $J=8.9 \mathrm{~Hz}, 2 \mathrm{H}), 4.80(\mathrm{~s}, 1 \mathrm{H})$, $4.71(\mathrm{~s}, 1 \mathrm{H}), 3.88(\mathrm{~s}, 3 \mathrm{H}), 3.60(\mathrm{t}, J=7.5 \mathrm{~Hz}, 2 \mathrm{H}), 2.41(\mathrm{t}, J=7.5 \mathrm{~Hz}$, $2 \mathrm{H}), 1.72(\mathrm{~s}, 3 \mathrm{H}) .{ }^{13} \mathrm{C} \mathrm{NMR}\left(151 \mathrm{MHz}, \mathrm{CDCl}_{3}\right) \delta 174.6,164.7,150.7$, $141.4,140.4,130.1$ (2C), 130.1 (2C), 128.4, 123.9 (2C), 115.0 (2C), 113.7, 93.4, 77.8, 55.9, 49.6, 36.1, 22.3. HRMS ESI $[\mathrm{M}+\mathrm{H}]^{+}$calculated for $\mathrm{C}_{21} \mathrm{H}_{21} \mathrm{~N}_{2} \mathrm{O}_{6} \mathrm{~S} 429.1120$, found 429.1125 . 


\section{Optimization Studies}

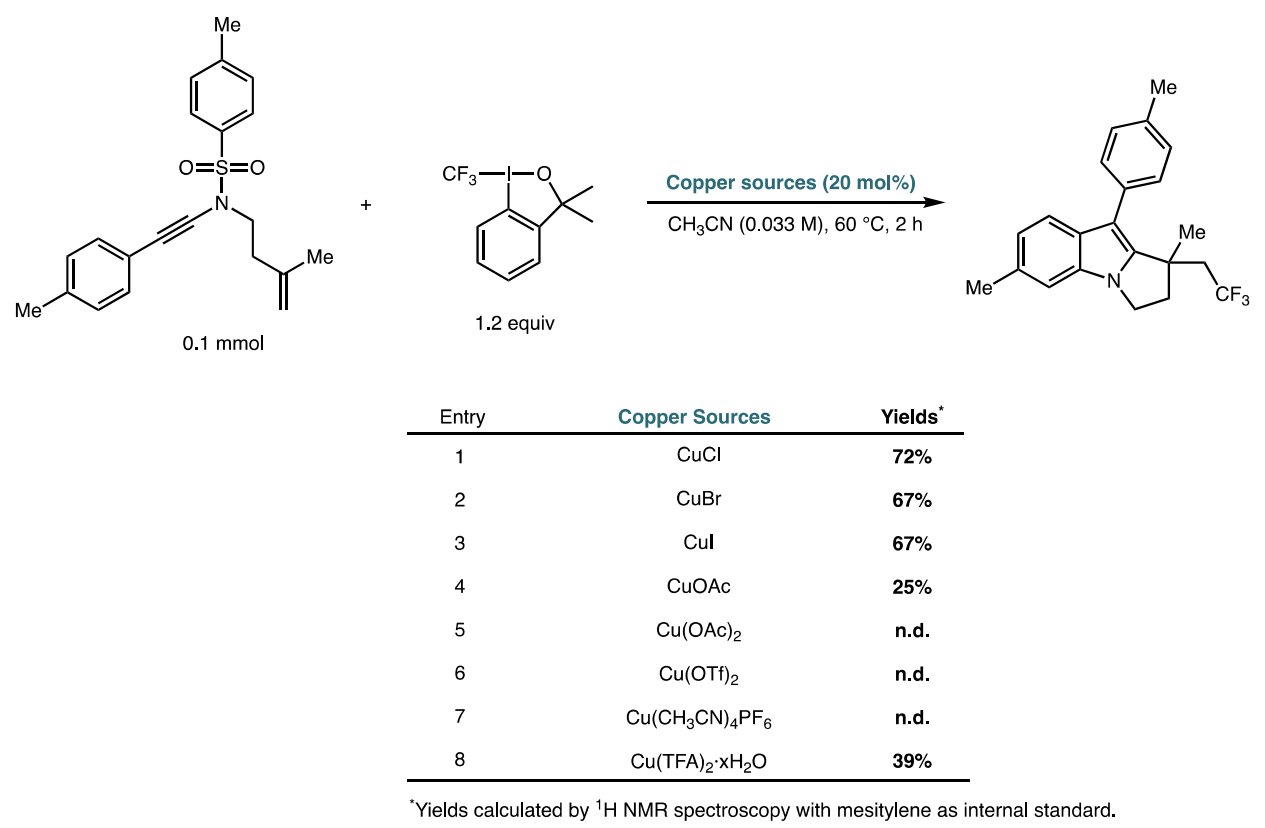

Table S1. Evaluation of copper sources
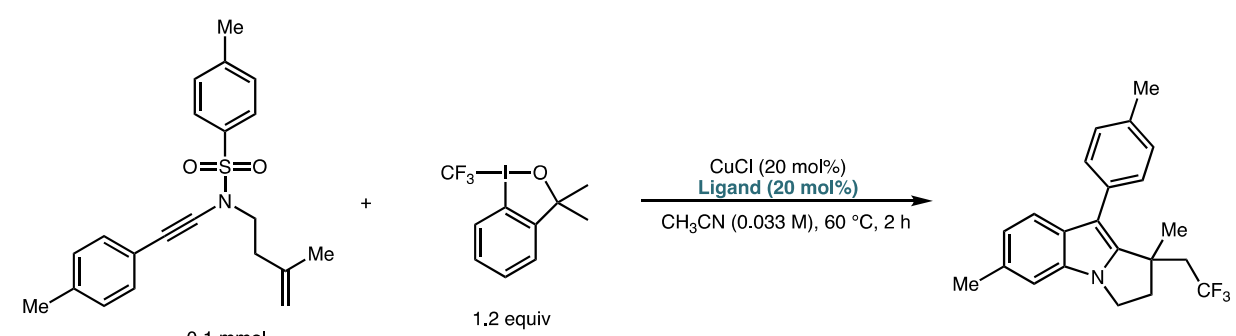

$0.1 \mathrm{mmol}$

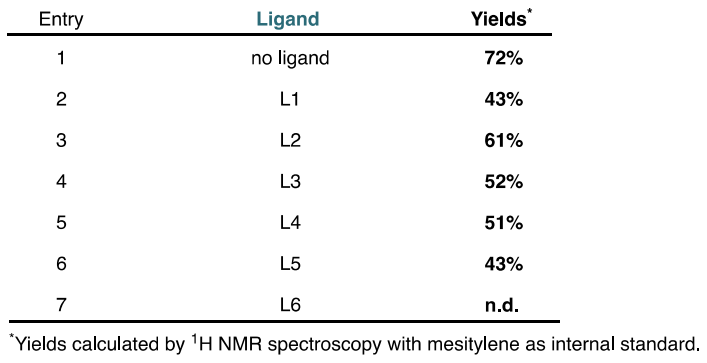

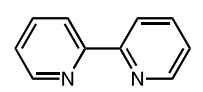

L1

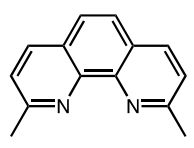

L4<smiles>FC(F)(F)c1ccc(-c2ccc(C(F)(F)F)cn2)nc1</smiles>

L2

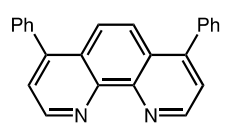

L5

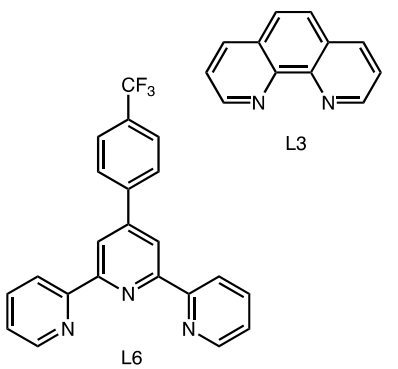

L6

Table S2. Evaluation of ligands 
<smiles>C=C(C)CCN(C#Cc1ccc(C)cc1)S(=O)(=O)c1ccc(C)cc1</smiles>

$0.1 \mathrm{mmol}$

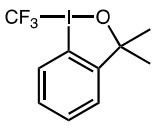

1.2 equiv
$\frac{\mathrm{CuCl}(20 \mathrm{~mol} \%)}{\text { Solvents }(0.033 \mathrm{M}), 60^{\circ} \mathrm{C}, 2 \mathrm{~h}}$

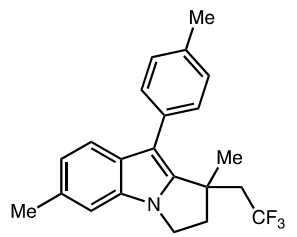

\begin{tabular}{cll} 
Entry & Solvents & Yields* \\
\hline 1 & $\mathrm{CH}_{3} \mathrm{CN}$ & $72 \%$ \\
2 & $\mathrm{DCM}$ & $47 \%$ \\
3 & $\mathrm{DCE}$ & $\mathbf{4 4 \%}$ \\
4 & $\mathrm{THF}$ & n.d. \\
5 & $\mathrm{PhCF}_{3}$ & $\mathbf{3 8} \%$ \\
6 & $\mathrm{Et}_{2} \mathrm{O}$ & n.d. \\
7 & $\mathrm{MeOH}$ & n.d. \\
8 & $\mathrm{DMA}$ & trace \\
\hline Yields calculated by ${ }^{1} \mathrm{H}$ NMR spectroscopy with mesitylene as internal standard.
\end{tabular}

Table S3. Evaluation of solvents
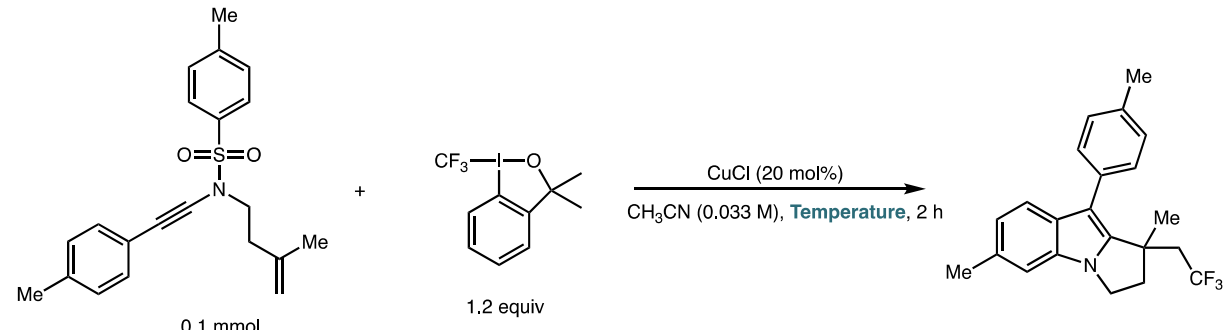

\begin{tabular}{ccc} 
Entry & Temperature & Yields \\
\hline 1 & room temperature & $46 \%$ \\
2 & $40^{\circ} \mathrm{C}$ & $62 \%$ \\
3 & $60^{\circ} \mathrm{C}$ & $72 \%$ \\
4 & $80^{\circ} \mathrm{C}$ & $59 \%$ \\
\hline
\end{tabular}

Table S4. Evaluation of temperature 

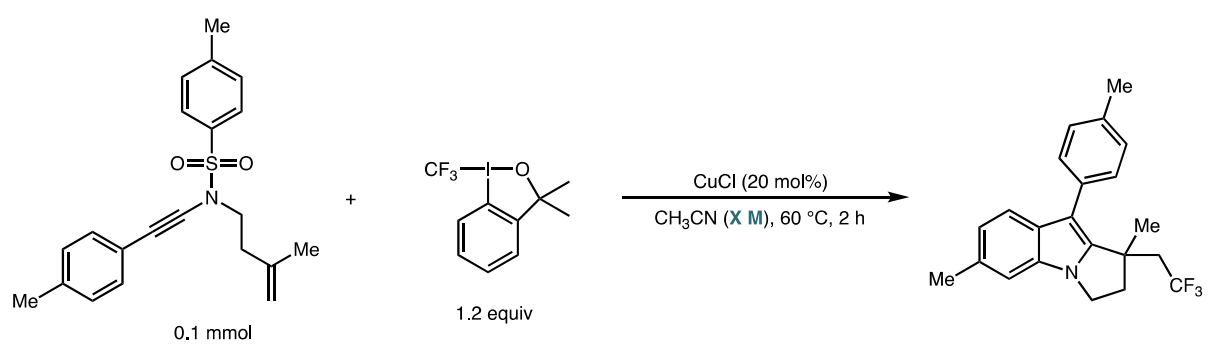

\begin{tabular}{ccc} 
Entry & Concentration (X M) & Yields* $^{*}$ \\
\hline 1 & $0.100 \mathrm{M}$ & $\mathbf{5 3} \%$ \\
2 & $0.050 \mathrm{M}$ & $\mathbf{6 2 \%}$ \\
3 & $0.033 \mathrm{M}$ & $\mathbf{7 2} \%$ \\
4 & $0.020 \mathrm{M}$ & $\mathbf{4 7 \%}$ \\
\hline
\end{tabular}

Yields calculated by ${ }^{1} \mathrm{H}$ NMR spectroscopy with mesitylene as internal standard.

Table S5. Evaluation of solvent concentration
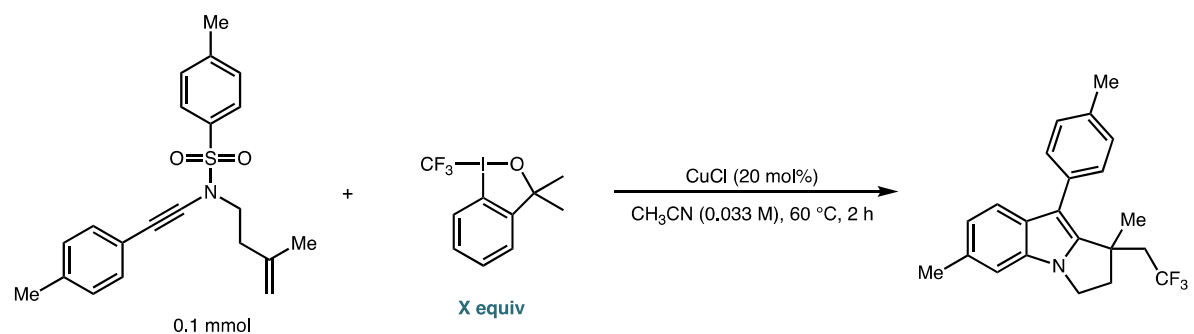

\begin{tabular}{ccc} 
Entry & Equiv of Togni's reagent & Yields $^{*}$ \\
\hline 1 & 1.0 equiv & $\mathbf{6 6 \%}$ \\
2 & 1.2 equiv & $\mathbf{7 2 \%}$ \\
3 & 1.5 equiv & $\mathbf{5 5 \%}$ \\
\hline "Yields calculated by ${ }^{1} \mathrm{H}$ NMR spectroscopy with mesitylene as internal standard.
\end{tabular}

Table S6. Evaluation of the equivalents of Togni I reagent
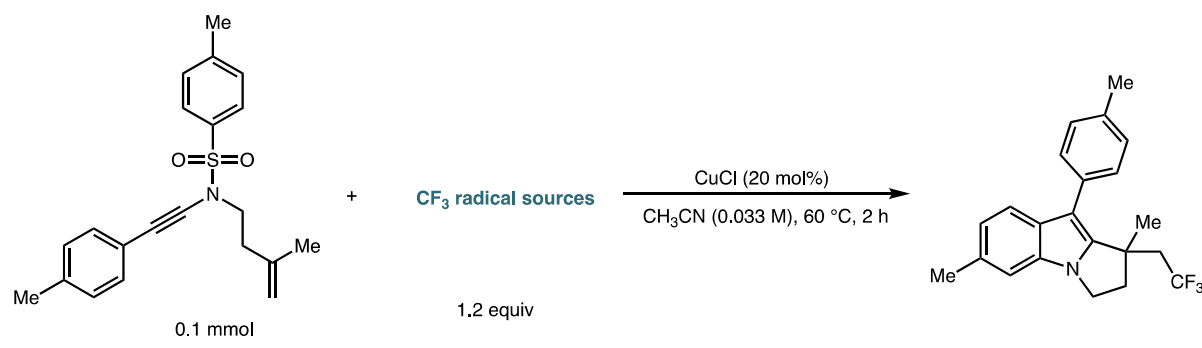

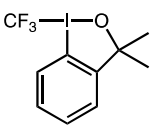

Yields

$72 \%$

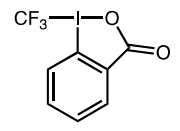

$55 \%$

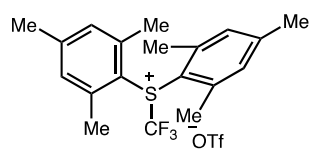

n.d.

'Yields calculated by ${ }^{1} \mathrm{H}$ NMR spectroscopy with mesitylene as internal standard.

Table S7. Evaluation of trifluoromethyl radical sources 


\section{Procedure for Smiles Rearrangement of Ynamides Reactions}

General Procedure A (Copper catalyzed condition): A 10-mL Schlenk tube equipped with a magnetic stir bar was charged with ynamide ( $0.2 \mathrm{mmol}$, if solid), $\mathrm{CuCl}$ (4 mg, $0.04 \mathrm{mmol}, 20 \mathrm{~mol} \%$ ), Togni I reagent (79.2 $\mathrm{mg}, 0.24 \mathrm{mmol}, 1.2$ equiv). The flask was evacuated and backfilled with $\mathrm{N}_{2}$ for 3 times. $\mathrm{CH}_{3} \mathrm{CN}(6 \mathrm{~mL})$ or a solution of ynamide $(0.2 \mathrm{mmol})$ in $\mathrm{CH}_{3} \mathrm{CN}(6 \mathrm{~mL})$ was then added via syringe under $\mathrm{N}_{2}$. The reaction mixture was then vigorously stirred at $60^{\circ} \mathrm{C}$ in an oil-bath. After the reaction was completed (as judged by TLC analysis), the mixture was diluted with ethyl acetate and poured into a separatory funnel, washed with brine for three times. The organic layer was dried over $\mathrm{Na}_{2} \mathrm{SO}_{4}$ and concentrated under reduced pressure after filtration. The crude product was purified by flash chromatography on silica gel to afford the desired product.

General Procedure B (Photoredox catalyzed condition): A 10-mL Schlenk tube equipped with a magnetic stir bar was charged with $f a c-\operatorname{Ir}(\mathrm{ppy})_{3}(1.3 \mathrm{mg}, 2 \mathrm{~mol} \%$ ) (for the reaction with $\mathbf{1 2}, 2 \mathrm{~mol} \%$ of $\operatorname{Ir}(\text { ppy })_{2}$ (dtbbpy)PF 6 was used), ynamide (0.2 mmol, if solid), $\mathrm{NaHCO}_{3}(16.8 \mathrm{mg}, 0.4 \mathrm{mmol}, 2.0$ equiv, for the reaction with $\mathbf{1 2}, \mathrm{NaHCO}_{3}$ was not added), corresponding radical precursor $(0.24 \mathrm{mmol}, 1.2$ equiv., if solid). The flask was evacuated and backfilled with $\mathrm{N}_{2}$ for 3 times. $\mathrm{CH}_{3} \mathrm{CN}(6 \mathrm{~mL})$ or a solution of ynamide $(0.2 \mathrm{mmol})$ in $\mathrm{CH}_{3} \mathrm{CN}(6 \mathrm{~mL})$, corresponding radical precursor $(0.24 \mathrm{mmol}, 1.2$ equiv) were then added via syringe under $\mathrm{N}_{2}$. The reaction mixture was then vigorously stirred under blue LED light $(30 \mathrm{~W})$ at room temperature (two fans were used to cool down the reaction mixture) overnight. After the reaction was completed, the mixture was diluted with ethyl acetate and poured into a separatory funnel, washed with brine for three times. The organic layer was dried over $\mathrm{Na}_{2} \mathrm{SO}_{4}$ and concentrated under reduced pressure after filtration. The crude product was purified by flash chromatography on silica gel to afford the desired product.

Gram-Scale Synthesis of 3e (6 mmol scale): To a flame-dried Schlenk flask charged with a stir bar was added Togni I reagent (2.37 g, $7.2 \mathrm{mmol}, 1.2$ equiv) and $\mathrm{CuCl}$ (119 mg, $1.2 \mathrm{mmol}, 20 \mathrm{~mol} \%$ ) under $\mathrm{N}_{2}$ atmosphere. The flask was evacuated and backfilled with $\mathrm{N}_{2}$ for 3 times. A solution of ynamide $\mathbf{1 f}$ (2.16 g, 6 mmol, 1.0 equiv) in $\mathrm{CH}_{3} \mathrm{CN}(180 \mathrm{~mL})$ was then added. The resulting reaction mixture was stirred at $60{ }^{\circ} \mathrm{C}$ in an oil-bath for about 2 hours (as judged by TLC analysis), the mixture was cooled down to room temperature, diluted with ethyl acetate and poured into a separatory funnel, washed with brine for three times. The organic layer was dried over $\mathrm{Na}_{2} \mathrm{SO}_{4}$ and concentrated under reduced pressure after filtration. The crude product was purified by flash chromatography on silica gel to afford the desired product $3 \mathbf{e}(1.12 \mathrm{~g}, 51 \%)$. 


\section{Product Characterization}

\section{1,6-Dimethyl-9-(p-tolyl)-1-(2,2,2-trifluoroethyl)-2,3-dihydro-1H-pyrrolo[1,2-a]indole (3a)}

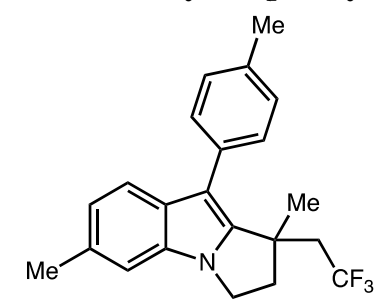

Following the general procedure A, purification by flash column chromatography (3\% EtOAc in hexane), the product $(45.1 \mathrm{mg}$ ) was obtained in $63 \%$ yield as white solid. ${ }^{1} \mathrm{H}$ NMR $\left(600 \mathrm{MHz}, \mathrm{CDCl}_{3}\right) \delta 7.42$ (d, $J=8.2$ $\mathrm{Hz}, 1 \mathrm{H}), 7.35$ (d, $J=8.0 \mathrm{~Hz}, 2 \mathrm{H}), 7.27$ (d, $J=8.0 \mathrm{~Hz}, 2 \mathrm{H}), 7.10(\mathrm{~s}, 1 \mathrm{H})$, $6.95(\mathrm{dd}, J=8.2,1.5 \mathrm{~Hz}, 1 \mathrm{H}), 4.19-4.15(\mathrm{~m}, 1 \mathrm{H}), 4.14-4.09(\mathrm{~m}, 1 \mathrm{H}), 2.79$ $-2.71(\mathrm{~m}, 1 \mathrm{H}), 2.71-2.65(\mathrm{~m}, 1 \mathrm{H}), 2.56-2.52(\mathrm{~m}, 1 \mathrm{H}), 2.51(\mathrm{~s}, 3 \mathrm{H}), 2.45(\mathrm{~s}, 3 \mathrm{H}), 2.39-2.27(\mathrm{~m}$, 1H), $1.58(\mathrm{~s}, 3 \mathrm{H}) .{ }^{13} \mathrm{C} \mathrm{NMR}\left(151 \mathrm{MHz}, \mathrm{CDCl}_{3}\right) \delta 144.7,136.1,132.3,131.7,131.4,130.1,130.1(2 \mathrm{C})$, $129.3(2 \mathrm{C}), 126.7$ (q, $\left.{ }^{1} J_{\mathrm{C}-\mathrm{F}}=278.82 \mathrm{~Hz}\right), 121.3,119.4,109.5,108.7,42.5,42.2\left(\mathrm{q},{ }^{2} J_{\mathrm{C}-\mathrm{F}}=27.2 \mathrm{~Hz}\right)$, 42.1, 39.8, 26.1, 21.8, 21.4. ${ }^{19} \mathrm{~F}$ NMR $\left(565 \mathrm{MHz}, \mathrm{CDCl}_{3}\right) \delta-60.31(\mathrm{t}, J=11.8 \mathrm{~Hz}, 3 \mathrm{~F})$. HRMS ESI $[\mathrm{M}+\mathrm{H}]^{+}$calculated for $\mathrm{C}_{22} \mathrm{H}_{23} \mathrm{NF}_{3} 358.1783$, found 358.1773 .

\section{1-Methyl-9-phenyl-1-(2,2,2-trifluoroethyl)-2,3-dihydro-1H-pyrrolo[1,2-a]indole (3b)}

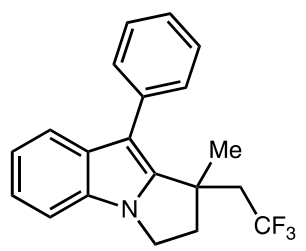

Following the general procedure $\mathbf{A}$, purification by flash column chromatography ( $3 \%$ EtOAc in hexane), the product $(32.3 \mathrm{mg})$ was obtained in $49 \%$ yield as white solid. ${ }^{1} \mathrm{H}$ NMR $\left(400 \mathrm{MHz}, \mathrm{CDCl}_{3}\right) \delta 7.53-7.49(\mathrm{~m}, 1 \mathrm{H})$,

$7.44(\mathrm{~s}, 2 \mathrm{H}), 7.42(\mathrm{~s}, 2 \mathrm{H}), 7.35-7.30(\mathrm{~m}, 1 \mathrm{H}), 7.27-7.24(\mathrm{~m}, 1 \mathrm{H}), 7.24-7.16$ (m, 1H), $7.10-7.05(\mathrm{~m}, 1 \mathrm{H}), 4.23-4.09$ (m, 2H), $2.80-2.72(\mathrm{~m}, 1 \mathrm{H}), 2.67-2.57(\mathrm{~m}, 1 \mathrm{H}), 2.57-$ $2.46(\mathrm{~m}, 1 \mathrm{H}), 2.37-2.25(\mathrm{~m}, 1 \mathrm{H}), 1.56(\mathrm{~s}, 3 \mathrm{H}) .{ }^{13} \mathrm{C} \mathrm{NMR}\left(101 \mathrm{MHz}, \mathrm{CDCl}_{3}\right) \delta 145.5,134.6,132.2$, 131.9, 130.4 (2C), 128.6 (2C), 126.7 (q, $\left.{ }^{1} J_{\text {C-F }}=279.7 \mathrm{~Hz}\right), 126.6,121.6,119.8,119.7,109.5,109.0$, $42.5,42.3\left(\mathrm{q},{ }^{2} J_{\mathrm{C}-\mathrm{F}}=26.9 \mathrm{~Hz}\right), 42.3,39.9,26.1 .{ }^{19} \mathrm{~F} \mathrm{NMR}\left(376 \mathrm{MHz}, \mathrm{CDCl}_{3}\right) \delta-60.35(\mathrm{t}, J=11.4 \mathrm{~Hz}$, 3F). HRMS ESI $[\mathrm{M}+\mathrm{H}]^{+}$calculated for $\mathrm{C}_{20} \mathrm{H}_{19} \mathrm{NF}_{3} 330.1470$, found 330.1462 .

9-([1,1'-Biphenyl]-4-yl)-1-methyl-6-phenyl-1-(2,2,2-trifluoroethyl)-2,3-dihydro-1H-pyrrolo[1,2a]indole (3c)

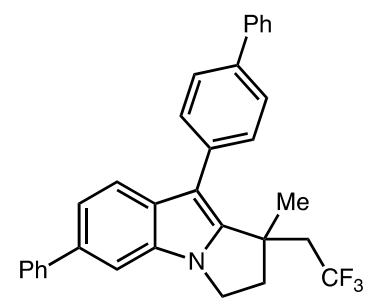

Following the general procedure $\mathbf{A}$, purification by flash column chromatography ( $3 \%$ EtOAc in hexane), the product $(62.5 \mathrm{mg})$ was obtained in $65 \%$ yield as white solid. ${ }^{1} \mathrm{H}$ NMR $\left(400 \mathrm{MHz}, \mathrm{CDCl}_{3}\right) \delta 7.78-7.70(\mathrm{~m}$, $6 \mathrm{H}), 7.68(\mathrm{~d}, J=8.3 \mathrm{~Hz}, 1 \mathrm{H}), 7.62-7.56(\mathrm{~m}, 2 \mathrm{H}), 7.56-7.46(\mathrm{~m}, 5 \mathrm{H}), 7.46$ $-7.34(\mathrm{~m}, 3 \mathrm{H}), 4.31-4.18(\mathrm{~m}, 2 \mathrm{H}), 2.89-2.69(\mathrm{~m}, 2 \mathrm{H}), 2.66-2.54(\mathrm{~m}$, $1 \mathrm{H}), 2.50-2.34(\mathrm{~m}, 1 \mathrm{H}), 1.66(\mathrm{~s}, 3 \mathrm{H}) .{ }^{13} \mathrm{C} \mathrm{NMR}\left(101 \mathrm{MHz}, \mathrm{CDCl}_{3}\right) \delta 146.3,142.5,140.9,139.3,135.5$, 133.6, 132.5, 131.5, 130.6 (2C), 128.9 (2C), 128.8 (2C), 127.5 (2C), 127.4, 127.3 (2C), 127.1 (2C), $126.7,126.6\left(\mathrm{q},{ }^{1} J_{\mathrm{C}-\mathrm{F}}=279.9 \mathrm{~Hz}\right), 120.0,119.8,108.6,108.2,42.5,42.3,42.2\left(\mathrm{q},{ }^{2} J_{\mathrm{C}-\mathrm{F}}=26.9 \mathrm{~Hz}\right)$, 40.1, 26.2. ${ }^{19} \mathrm{~F}$ NMR $\left(376 \mathrm{MHz}, \mathrm{CDCl}_{3}\right) \delta-60.24(\mathrm{t}, J=11.4 \mathrm{~Hz}, 3 \mathrm{~F})$. HRMS ESI $[\mathrm{M}+\mathrm{H}]^{+}$calculated for $\mathrm{C}_{32} \mathrm{H}_{27} \mathrm{NF}_{3} 482.2096$, found 482.2089 .

6-Methoxy-9-(4-methoxyphenyl)-1-methyl-1-(2,2,2-trifluoroethyl)-2,3-dihydro-1H-pyrrolo[1,2a]indole (3d) 


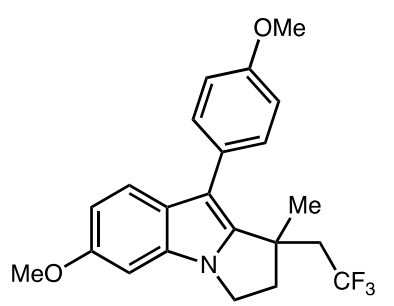

Following the general procedure $\mathbf{A}$, reaction was conducted at room temperature for 2 hours. Purification by flash column chromatography (5-10\% EtOAc in hexane), the product ( $35.0 \mathrm{mg}$ ) was obtained in $45 \%$ yield as white solid. ${ }^{1} \mathrm{H}$ NMR $\left(600 \mathrm{MHz}, \mathrm{CDCl}_{3}\right) \delta 7.38-7.35(\mathrm{~m}, 3 \mathrm{H}), 7.00(\mathrm{~d}, J=8.6$ $\mathrm{Hz}, 2 \mathrm{H}), 6.79-6.75(\mathrm{~m}, 2 \mathrm{H}), 4.16-4.06(\mathrm{~m}, 2 \mathrm{H}), 3.89(\mathrm{~s}, 6 \mathrm{H}), 2.77-2.72$ $(\mathrm{m}, 1 \mathrm{H}), 2.66-2.58(\mathrm{~m}, 1 \mathrm{H}), 2.57-2.47(\mathrm{~m}, 1 \mathrm{H}), 2.36-2.26(\mathrm{~m}, 1 \mathrm{H}), 1.56(\mathrm{~s}, 3 \mathrm{H}) .{ }^{13} \mathrm{C}$ NMR $(151$ $\left.\mathrm{MHz}, \mathrm{CDCl}_{3}\right) \delta 158.4,156.3,144.1,132.3,131.2(2 \mathrm{C}), 126.9,126.8,126.7\left(\mathrm{q},{ }^{1} J_{\mathrm{C}-\mathrm{F}}=278.9 \mathrm{~Hz}\right), 120.37$, 114.0 (2C), 109.4, 108.3, 93.2, 55.9, 55.4, 42.5, 42.3 (q, $\left.{ }^{2} J_{\mathrm{C}-\mathrm{F}}=26.6 \mathrm{~Hz}\right), 42.1,39.7,26.2 .{ }^{19} \mathrm{~F}$ NMR $\left(565 \mathrm{MHz}, \mathrm{CDCl}_{3}\right) \delta-60.35(\mathrm{t}, J=11.5 \mathrm{~Hz}, 3 \mathrm{~F})$. HRMS ESI $[\mathrm{M}+\mathrm{H}]^{+}$calculated for $\mathrm{C}_{22} \mathrm{H}_{23} \mathrm{NO}_{2} \mathrm{~F}_{3}$ 390.1681 , found 390.1672 .

\section{6-Fluoro-9-(4-fluorophenyl)-1-methyl-1-(2,2,2-trifluoroethyl)-2,3-dihydro-1H-pyrrolo[1,2- a]indole (3e)}

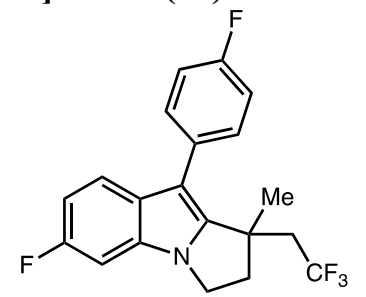

Following the general procedure A, purification by flash column chromatography ( $3 \%$ EtOAc in hexane), the product $(51.8 \mathrm{mg}$ ) was obtained in $71 \%$ yield as white solid. ${ }^{1} \mathrm{H}$ NMR $\left(600 \mathrm{MHz}, \mathrm{CDCl}_{3}\right) \delta 7.38-7.35(\mathrm{~m}, 2 \mathrm{H})$, $7.35-7.32(\mathrm{~m}, 1 \mathrm{H}), 7.17-7.12(\mathrm{~m}, 2 \mathrm{H}), 6.95(\mathrm{dd}, J=9.4,2.3 \mathrm{~Hz}, 1 \mathrm{H}), 6.87-$ $6.82(\mathrm{~m}, 1 \mathrm{H}), 4.18-4.05(\mathrm{~m}, 2 \mathrm{H}), 2.81-2.76(\mathrm{~m}, 1 \mathrm{H}), 2.62-2.46(\mathrm{~m}, 2 \mathrm{H})$, $2.41-2.24(\mathrm{~m}, 1 \mathrm{H}), 1.55(\mathrm{~s}, 3 \mathrm{H}) \cdot{ }^{13} \mathrm{C} \mathrm{NMR}\left(151 \mathrm{MHz}, \mathrm{CDCl}_{3}\right) \delta 162.0\left(\mathrm{~d},{ }^{1} J_{\mathrm{C}-\mathrm{F}}=246.0 \mathrm{~Hz}\right), 159.8(\mathrm{~d}$, $\left.{ }^{1} J_{\mathrm{C}-\mathrm{F}}=238.2 \mathrm{~Hz}\right), 145.7\left(\mathrm{~d},{ }^{4} J_{\mathrm{C}-\mathrm{F}}=3.4 \mathrm{~Hz}\right), 131.8\left(\mathrm{~d},{ }^{3} J_{\mathrm{C}-\mathrm{F}}=7.6 \mathrm{~Hz}, 2 \mathrm{C}\right), 131.7\left(\mathrm{~d},{ }^{3} J_{\mathrm{C}-\mathrm{F}}=13.6 \mathrm{~Hz}\right)$, $130.2\left(\mathrm{~d},{ }^{4} J_{\mathrm{C}-\mathrm{F}}=3.0 \mathrm{~Hz}\right), 128.9,126.5\left(\mathrm{q},{ }^{1} J_{\mathrm{C}-\mathrm{F}}=278.8 \mathrm{~Hz}\right), 120.2\left(\mathrm{~d},{ }^{3} J_{\mathrm{C}-\mathrm{F}}=10.6 \mathrm{~Hz}\right), 115.6\left(\mathrm{~d},{ }^{2} J_{\mathrm{C}-}\right.$ $\mathrm{F}=21.1 \mathrm{~Hz}, 2 \mathrm{C}), 108.4\left(\mathrm{~d},{ }^{2} J_{\mathrm{C}-\mathrm{F}}=25.7 \mathrm{~Hz}\right), 108.0,96.0\left(\mathrm{~d},{ }^{2} J_{\mathrm{C}-\mathrm{F}}=27.2 \mathrm{~Hz}\right), 42.4\left(\mathrm{q},{ }^{2} J_{\mathrm{C}-\mathrm{F}}=26.4 \mathrm{~Hz}\right)$, 42.3, 42.3, 39.9, 26.3. ${ }^{19} \mathrm{~F}$ NMR $\left(565 \mathrm{MHz}, \mathrm{CDCl}_{3}\right) \delta-60.50(\mathrm{t}, J=11.3 \mathrm{~Hz}, 3 \mathrm{~F}),-115.72--115.78(\mathrm{~m}$, 1F), $-120.98--121.02(\mathrm{~m}, 1 \mathrm{~F})$. HRMS ESI $[\mathrm{M}+\mathrm{H}]^{+}$calculated for $\mathrm{C}_{20} \mathrm{H}_{17} \mathrm{NF}_{5}$ 366.1281, found 366.1277.

\section{9-(4-(Tert-butyl)phenyl)-1,6-dimethyl-1-(2,2,2-trifluoroethyl)-2,3-dihydro-1H-pyrrolo[1,2-} a]indole (3f) \& 6-(Tert-butyl)-1-methyl-9-(p-tolyl)-1-(2,2,2-trifluoroethyl)-2,3-dihydro-1 Hpyrrolo[1,2-a]indole (3f')
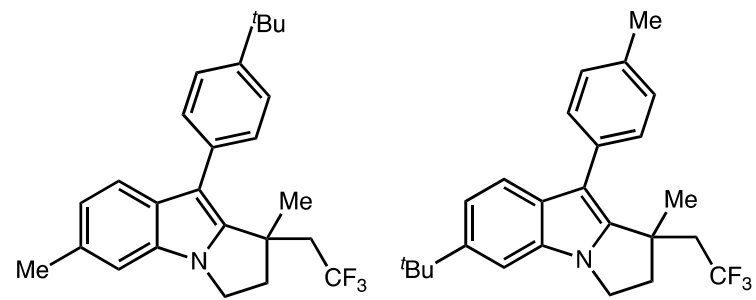

Following the general procedure A, purification by flash column chromatography $(2-3 \%$ EtOAc in hexane), two regioisomers were obtained $(47.9 \mathrm{mg})$ in $60 \%$ yield as colourless oil. The ratio (1.0:1.0) of two regioisomers was calculated by ${ }^{19} \mathrm{~F}$ NMR spectroscopy of the crude reaction mixture. ${ }^{1} \mathrm{H}$ NMR $\left(600 \mathrm{MHz}, \mathrm{CDCl}_{3}\right) \delta 7.38-7.33(\mathrm{~m}, 4 \mathrm{H}), 7.30-7.26(\mathrm{~m}, 2 \mathrm{H})$, $7.25-7.22(\mathrm{~m}, 2 \mathrm{H}), 7.18-7.13(\mathrm{~m}, 3 \mathrm{H}), 7.09$ (dd, $J=8.5,1.8 \mathrm{~Hz}, 1 \mathrm{H}), 6.98(\mathrm{~s}, 1 \mathrm{H}), 6.83(\mathrm{dd}, J=8.2$, $1.5 \mathrm{~Hz}, 1 \mathrm{H}), 4.13-3.97$ (m, 4H), $2.67-2.51$ (m, 4H), $2.45-2.38$ (m, 5H), 2.33 (s, 3H), $2.26-2.17$ $(\mathrm{m}, 2 \mathrm{H}), 1.48(\mathrm{~s}, 3 \mathrm{H}), 1.46(\mathrm{~s}, 3 \mathrm{H}), 1.32(\mathrm{~s}, 9 \mathrm{H}), 1.30(\mathrm{~s}, 9 \mathrm{H}) .{ }^{13} \mathrm{C} \mathrm{NMR}\left(151 \mathrm{MHz}, \mathrm{CDCl}_{3}\right) \delta 149.2$, 145.2 , 145.2, 144.7, 136.0, 132.3, 131.8, 131.8, 131.7, 131.5, 130.2, 130.2 (2C), 130.0, $129.8(2 \mathrm{C})$, 
$129.3(2 \mathrm{C}), 126.8\left(\mathrm{q},{ }^{1} J_{\mathrm{C}-\mathrm{F}}=278.9 \mathrm{~Hz}\right), 126.7\left(\mathrm{q},{ }^{1} J_{\mathrm{C}-\mathrm{F}}=279.0 \mathrm{~Hz}\right), 125.4(2 \mathrm{C}), 121.3,119.6,119.3$, $118.0,109.5,108.7,108.6,105.7,42.6(\mathrm{~d}, J=6.9 \mathrm{~Hz}), 42.6,42.3\left(\mathrm{q},{ }^{2} J_{\mathrm{C}-\mathrm{F}}=26.7 \mathrm{~Hz}\right), 42.1\left(\mathrm{q},{ }^{3} J_{\mathrm{C}-\mathrm{F}}=\right.$ $6.2 \mathrm{~Hz}), 39.8,34.9,34.6,32.0$ (3C), 31.5 (3C), 26.0, 26.0, 21.8, 21.4. ${ }^{19} \mathrm{~F}$ NMR (565 MHz, $\left.\mathrm{CDCl}_{3}\right) \delta$ $60.22--60.32(\mathrm{~m}, 6 \mathrm{~F})$. HRMS ESI $[\mathrm{M}+\mathrm{H}]^{+}$calculated for $\mathrm{C}_{25} \mathrm{H}_{29} \mathrm{NF}_{3} 400.2252$, found 400.2256 .

\section{9-(3-Methoxyphenyl)-1,6-dimethyl-1-(2,2,2-trifluoroethyl)-2,3-dihydro-1H-pyrrolo[1,2-a]indole}

(3g)

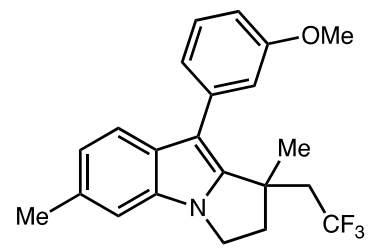

Following the general procedure A, purification by flash column chromatography (5-10\% EtOAc in hexane), two regioisomers were obtained $(40.3 \mathrm{mg})$ in $54 \%$ yield as white solid. The ratio (2.8:1.0) of two regioisomers was calculated by ${ }^{19} \mathrm{~F}$ NMR spectroscopy of the crude reaction mixture. Preparative TLC plates were used for further purification (5\% EtOAc in hexane). Major regioisomer: ${ }^{1} \mathrm{H}$ NMR $\left(600 \mathrm{MHz}, \mathrm{CDCl}_{3}\right) \delta 7.47(\mathrm{~d}, J=8.2 \mathrm{~Hz}, 1 \mathrm{H}), 7.38(\mathrm{t}, J=7.9 \mathrm{~Hz}, 1 \mathrm{H}), 7.12-7.08(\mathrm{~m}, 1 \mathrm{H})$, $7.06-7.04(\mathrm{~m}, 1 \mathrm{H}), 7.03-7.02(\mathrm{~m}, 1 \mathrm{H}), 6.96(\mathrm{dd}, J=8.2,1.5 \mathrm{~Hz}, 1 \mathrm{H}), 6.91$ (ddd, $J=8.3,2.7,1.0 \mathrm{~Hz}$, $1 \mathrm{H}), 4.20-4.09(\mathrm{~m}, 2 \mathrm{H}), 3.87(\mathrm{~s}, 3 \mathrm{H}), 2.80-2.66(\mathrm{~m}, 2 \mathrm{H}), 2.56-2.47(\mathrm{~m}, 4 \mathrm{H}), 2.40-2.30(\mathrm{~m}, 1 \mathrm{H})$, $1.60(\mathrm{~s}, 3 \mathrm{H}) .{ }^{13} \mathrm{C} \mathrm{NMR}\left(151 \mathrm{MHz}, \mathrm{CDCl}_{3}\right) \delta 159.7,144.8,136.3,132.3,131.6,129.9,129.5,126.7$ (q, $\left.{ }^{1} J_{\mathrm{C}-\mathrm{F}}=278.8 \mathrm{~Hz}\right), 122.7,121.5,119.5,115.7,112.1,109.5,108.7,55.3,42.6,42.2\left(\mathrm{q},{ }^{2} J_{\mathrm{C}-\mathrm{F}}=26.9 \mathrm{~Hz}\right)$, 42.1, 39.8, 26.0, 21.8. ${ }^{19} \mathrm{~F}$ NMR (565 MHz, $\left.\mathrm{CDCl}_{3}\right) \delta-60.32(\mathrm{t}, J=11.4 \mathrm{~Hz}, 3 \mathrm{~F})$. HRMS ESI $[\mathrm{M}+\mathrm{H}]^{+}$ calculated for $\mathrm{C}_{22} \mathrm{H}_{23} \mathrm{NOF}_{3} 374.1732$, found 374.1740.

\section{7-Methoxy-1-methyl-9-(p-tolyl)-1-(2,2,2-trifluoroethyl)-2,3-dihydro-1 $\mathrm{H}$-pyrrolo[1,2-a]indole (3g')}

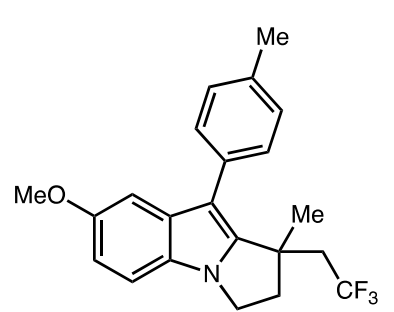

Following the general procedure $\mathbf{A}$, purification by flash column chromatography (5-10\% EtOAc in hexane), two regioisomers were obtained $(40.3 \mathrm{mg})$ in $54 \%$ yield as white solid. The ratio (2.8:1.0) of two regioisomers was calculated by ${ }^{19} \mathrm{~F}$ NMR spectroscopy of the crude reaction mixture. Preparative TLC plates were used for further purification $(5 \%$ EtOAc in hexane). Minor regioisomer: ${ }^{1} \mathrm{H}$ NMR (600 MHz, $\left.\mathrm{CDCl}_{3}\right) \delta 7.33-7.30(\mathrm{~m}, 2 \mathrm{H}), 7.27-7.24$ (m, 2H), $7.15(\mathrm{~d}, J=8.7 \mathrm{~Hz}, 1 \mathrm{H}), 6.95(\mathrm{~d}, J=2.4 \mathrm{~Hz}, 1 \mathrm{H}), 6.85(\mathrm{dd}, J=8.8,2.4 \mathrm{~Hz}, 1 \mathrm{H}), 4.17-4.06$ (m, 2H), $3.77(\mathrm{~s}, 3 \mathrm{H}), 2.77-2.70(\mathrm{~m}, 1 \mathrm{H}), 2.65-2.57(\mathrm{~m}, 1 \mathrm{H}), 2.52-2.46(\mathrm{~m}, 1 \mathrm{H}), 2.42(\mathrm{~s}, 3 \mathrm{H}), 2.37$ $-2.23(\mathrm{~m}, 1 \mathrm{H}), 1.54(\mathrm{~s}, 3 \mathrm{H}) .{ }^{13} \mathrm{C} \mathrm{NMR}\left(151 \mathrm{MHz}, \mathrm{CDCl}_{3}\right) \delta 154.5,146.1,136.2,132.6,131.7,130.2$ (2C), $129.4(2 \mathrm{C}), 127.2,126.7\left(\mathrm{q},{ }^{1} J_{\mathrm{C}-\mathrm{F}}=278.8 \mathrm{~Hz}\right), 111.8,110.2,108.6,101.7,56.2,42.5,42.4,42.3$ $\left(\mathrm{q},{ }^{2} J_{\mathrm{C}-\mathrm{F}}=26.7 \mathrm{~Hz}\right), 40.1,26.1,21.4 .{ }^{19} \mathrm{~F} \mathrm{NMR}\left(565 \mathrm{MHz}, \mathrm{CDCl}_{3}\right) \delta-60.36(\mathrm{t}, J=11.4 \mathrm{~Hz}, 3 \mathrm{~F}) . \mathrm{HRMS}$ ESI $[\mathrm{M}+\mathrm{H}]^{+}$calculated for $\mathrm{C}_{22} \mathrm{H}_{23} \mathrm{NOF}_{3} 374.1732$, found 374.1739 . 


\section{9-(4-Fluorophenyl)-1,6-dimethyl-1-(2,2,2-trifluoroethyl)-2,3-dihydro-1H-pyrrolo[1,2-a]indole}

(3h)

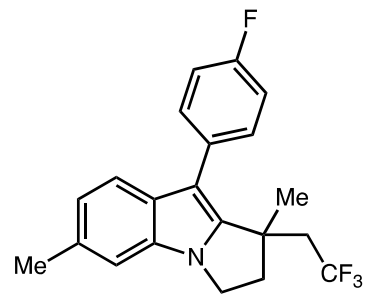

Following the general procedure A, purification by flash column chromatography (3\% EtOAc in hexane), two regioisomers were obtained (38.3 $\mathrm{mg})$ in 53\% yield as white solid. The ratio (4.0:1.0) of two regioisomers was calculated by ${ }^{19} \mathrm{~F}$ NMR spectroscopy of the crude reaction mixture. The major product 3ah was obtained by recrystallization (DCM/Hexane). ${ }^{1} \mathrm{H}$ $\operatorname{NMR}\left(600 \mathrm{MHz}, \mathrm{CDCl}_{3}\right) \delta 7.41-7.35(\mathrm{~m}, 2 \mathrm{H}), 7.33(\mathrm{~d}, J=8.1 \mathrm{~Hz}, 1 \mathrm{H}), 7.17-7.10(\mathrm{~m}, 2 \mathrm{H}), 7.08(\mathrm{~s}$, $1 \mathrm{H}), 6.93(\mathrm{dd}, J=8.2,1.5 \mathrm{~Hz}, 1 \mathrm{H}), 4.18-4.08(\mathrm{~m}, 2 \mathrm{H}), 2.77-2.73(\mathrm{~m}, 1 \mathrm{H}), 2.61-2.50(\mathrm{~m}, 2 \mathrm{H}), 2.49$ (s, 3H), $2.35-2.26(\mathrm{~m}, 1 \mathrm{H}), 1.54(\mathrm{~s}, 3 \mathrm{H}) .{ }^{13} \mathrm{C} \mathrm{NMR}\left(151 \mathrm{MHz}, \mathrm{CDCl}_{3}\right) \delta 161.8\left(\mathrm{q},{ }^{1} J_{\mathrm{C}-\mathrm{F}}=246.1 \mathrm{~Hz}\right)$, 144.8, 132.2, $131.8\left(\mathrm{~d},{ }^{3} J_{\mathrm{C}-\mathrm{F}}=7.9 \mathrm{~Hz}, 2 \mathrm{C}\right), 131.6,130.7$ (d, $\left.{ }^{4} J_{\mathrm{C}-\mathrm{F}}=3.3 \mathrm{~Hz}\right), 130.2,126.5\left(\mathrm{q},{ }^{1} J_{\mathrm{C}-\mathrm{F}}=\right.$ $278.9 \mathrm{~Hz}), 121.6,119.2,115.5\left(\mathrm{~d},{ }^{2} J_{\mathrm{C}-\mathrm{F}}=21.23 \mathrm{~Hz}, 2 \mathrm{C}\right), 109.6,107.7,42.4,42.4\left(\mathrm{q},{ }^{2} J_{\mathrm{C}-\mathrm{F}}=26.8 \mathrm{~Hz}\right)$, 42.1, 39.8, 26.3, 21.8. ${ }^{19} \mathrm{~F}$ NMR $\left(565 \mathrm{MHz}, \mathrm{CDCl}_{3}\right) \delta-60.45(\mathrm{t}, J=11.4 \mathrm{~Hz}),-116.28--116.34(\mathrm{~m}, J$ $=14.2,8.8,5.6 \mathrm{~Hz}$ ). HRMS ESI $[\mathrm{M}+\mathrm{H}]^{+}$calculated for $\mathrm{C}_{21} \mathrm{H}_{20} \mathrm{NF}_{4} 362.1532$, found 362.1523 .

9-(3-Fluorophenyl)-1,6-dimethyl-1-(2,2,2-trifluoroethyl)-2,3-dihydro-1 H-pyrrolo[1,2-a]indole (3i) Following the general procedure $\mathbf{A}$, purification by flash column chromatography (3\% EtOAc in

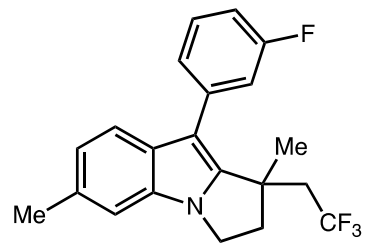
hexane), the product $\left(46.2 \mathrm{mg}\right.$ ) was obtained in $64 \%$ yield as white solid. ${ }^{1} \mathrm{H}$ NMR (600 MHz, $\left.\mathrm{CDCl}_{3}\right) \delta 7.46-7.38(\mathrm{~m}, 2 \mathrm{H}), 7.25$ - $7.22(\mathrm{~m}, 1 \mathrm{H}), 7.19-$ $7.16(\mathrm{~m}, 1 \mathrm{H}), 7.11(\mathrm{~s}, 1 \mathrm{H}), 7.07-7.03(\mathrm{~m}, 1 \mathrm{H}), 6.98(\mathrm{dd}, J=8.2,1.5 \mathrm{~Hz}, 1 \mathrm{H})$, $1 \mathrm{H}), 2.52(\mathrm{~s}, 3 \mathrm{H}), 2.42-2.28(\mathrm{~m}, 1 \mathrm{H}), 1.60(\mathrm{~s}, 3 \mathrm{H}) .{ }^{13} \mathrm{C} \mathrm{NMR}\left(151 \mathrm{MHz}, \mathrm{CDCl}_{3}\right) \delta 163.0\left(\mathrm{~d},{ }^{1} J_{\mathrm{C}-\mathrm{F}}=\right.$ $246.1 \mathrm{~Hz}), 144.9,137.2\left(\mathrm{~d},{ }^{3} J_{\mathrm{C}-\mathrm{F}}=9.1 \mathrm{~Hz}\right), 132.3,131.8,129.9\left(\mathrm{~d},{ }^{3} \mathrm{~J}_{\mathrm{C}-\mathrm{F}}=9.1 \mathrm{~Hz}\right), 129.7,126.6\left(\mathrm{q},{ }^{1} \mathrm{~J}\right.$ C-F $=277.8 \mathrm{~Hz}), 125.9\left(\mathrm{~d},{ }^{4} J_{\mathrm{C}-\mathrm{F}}=3.0 \mathrm{~Hz}\right), 121.8,119.2,116.9\left(\mathrm{~d},{ }^{2} J_{\mathrm{C}-\mathrm{F}}=21.1 \mathrm{~Hz}\right), 113.4\left(\mathrm{~d},{ }^{2} J_{\mathrm{C}-\mathrm{F}}=\right.$ $21.1 \mathrm{~Hz}), 109.7,107.7\left(\mathrm{~d},{ }^{4} J_{\mathrm{C}-\mathrm{F}}=3.0 \mathrm{~Hz}\right), 42.5,42.3\left(\mathrm{q},{ }^{2} J_{\mathrm{C}-\mathrm{F}}=27.2 \mathrm{~Hz}\right), 42.1,39.9,26.2,21.8 .{ }^{19} \mathrm{~F}$ NMR $\left(376 \mathrm{MHz}, \mathrm{CDCl}_{3}\right) \delta-60.39(\mathrm{t}, J=11.3 \mathrm{~Hz}, 3 \mathrm{~F}),-113.23--113.32(\mathrm{~m}, 1 \mathrm{~F})$. HRMS ESI $[\mathrm{M}+\mathrm{H}]^{+}$ calculated for $\mathrm{C}_{21} \mathrm{H}_{20} \mathrm{NF}_{4} 362.1532$, found 362.1525 .

\section{9-(2-Fluorophenyl)-1,6-dimethyl-1-(2,2,2-trifluoroethyl)-2,3-dihydro-1H-pyrrolo[1,2-a]indole (3j)}

Following the general procedure $\mathbf{A}$, purification by flash column chromatography (3\% EtOAc in

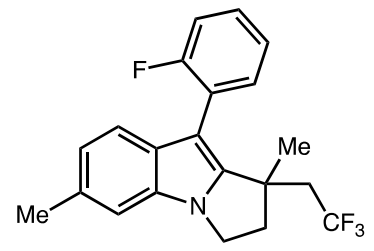
hexane), the product ( $47.7 \mathrm{mg}$ ) was obtained in $66 \%$ yield as white solid. ${ }^{1} \mathrm{H}$ NMR $\left(600 \mathrm{MHz}, \mathrm{CDCl}_{3}\right) \delta 7.38-7.35(\mathrm{~m}, 1 \mathrm{H}), 7.33-7.30(\mathrm{~m}, 1 \mathrm{H})$, $7.22(\mathrm{~d}, J=8.1 \mathrm{~Hz}, 1 \mathrm{H}), 7.20-7.14(\mathrm{~m}, 2 \mathrm{H}), 7.07$ (s, 1H), $6.91(\mathrm{dd}, J=8.1$, $1.5 \mathrm{~Hz}, 1 \mathrm{H}), 4.16-4.04(\mathrm{~m}, 2 \mathrm{H}), 2.76-2.70(\mathrm{~m}, 1 \mathrm{H}), 2.60-2.49(\mathrm{~m}, 2 \mathrm{H})$, $2.46(\mathrm{~s}, 3 \mathrm{H}), 2.41-2.29(\mathrm{~m}, 1 \mathrm{H}), 1.46(\mathrm{~s}, 3 \mathrm{H}) .{ }^{13} \mathrm{C} \mathrm{NMR}\left(151 \mathrm{MHz}, \mathrm{CDCl}_{3}\right) \delta 160.6\left(\mathrm{~d},{ }^{1} J_{\mathrm{C}-\mathrm{F}}=244.6\right.$ $\mathrm{Hz}), 145.9,133.2\left(\mathrm{~d},{ }^{4} J_{\mathrm{C}-\mathrm{F}}=1.5 \mathrm{~Hz}\right), 132.4,131.6,130.3,128.9\left(\mathrm{~d},{ }^{3} J_{\mathrm{C}-\mathrm{F}}=7.6 \mathrm{~Hz}\right), 126.6\left(\mathrm{q},{ }^{1} J_{\mathrm{C}-\mathrm{F}}=\right.$ $279.4 \mathrm{~Hz}), 124.0\left(\mathrm{~d},{ }^{4} J_{\mathrm{C}-\mathrm{F}}=3.0 \mathrm{~Hz}\right), 122.1\left(\mathrm{~d},{ }^{2} J_{\mathrm{C}-\mathrm{F}}=16.6 \mathrm{~Hz}\right), 121.5,119.4,115.9\left(\mathrm{~d},{ }^{2} J_{\mathrm{C}-\mathrm{F}}=24.2\right.$ $\mathrm{Hz}), 109.6,101.4,42.2,41.8\left(\mathrm{q},{ }^{2} J_{\mathrm{C}-\mathrm{F}}=25.7 \mathrm{~Hz}\right), 39.8,29.8,24.9,21.8 .{ }^{19} \mathrm{~F}$ NMR $\left(565 \mathrm{MHz}, \mathrm{CDCl}_{3}\right)$ 
$\delta-60.40(\mathrm{t}, J=11.9 \mathrm{~Hz}, 3 \mathrm{~F}),-112.18--112.25(\mathrm{~m}, 1 \mathrm{~F})$. HRMS ESI $[\mathrm{M}+\mathrm{H}]^{+}$calculated for $\mathrm{C}_{21} \mathrm{H}_{20} \mathrm{NF}_{4}$ 362.1532 , found 362.1527 .

6-(tert-Butyl)-9-(3-fluorophenyl)-1-methyl-1-(2,2,2-trifluoroethyl)-2,3-dihydro-1H-pyrrolo[1,2a]indole (31)

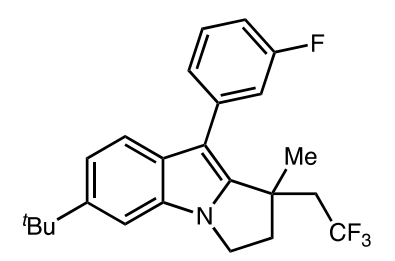

Following the general procedure A, purification by flash column chromatography (1-3\% EtOAc in hexane), the product $(47.7 \mathrm{mg})$ was obtained in 59\% yield as colourless oil. ${ }^{1} \mathrm{H}$ NMR $\left(400 \mathrm{MHz}, \mathrm{CDCl}_{3}\right) \delta 7.58$ 1H), $7.28-7.26(\mathrm{~m}, 1 \mathrm{H}), 7.25-7.21(\mathrm{~m}, 1 \mathrm{H}), 7.11-7.06(\mathrm{~m}, 1 \mathrm{H}), 4.30-4.16(\mathrm{~m}, 2 \mathrm{H}), 2.86-2.76$ $(\mathrm{m}, 1 \mathrm{H}), 2.75-2.65(\mathrm{~m}, 1 \mathrm{H}), 2.64-2.52(\mathrm{~m}, 1 \mathrm{H}), 2.48-2.30(\mathrm{~m}, 1 \mathrm{H}), 1.63(\mathrm{~s}, 3 \mathrm{H}), 1.48(\mathrm{~s}, 9 \mathrm{H}) .{ }^{13} \mathrm{C}$ $\operatorname{NMR}\left(101 \mathrm{MHz}, \mathrm{CDCl}_{3}\right) \delta 163.0\left(\mathrm{~d},{ }^{1} J_{\mathrm{C}-\mathrm{F}}=246.4 \mathrm{~Hz}\right), 145.6,145.4,137.3\left(\mathrm{~d},{ }^{3} J_{\mathrm{C}-\mathrm{F}}=8.1 \mathrm{~Hz}\right), 131.9$, $130.0\left(\mathrm{~d},{ }^{3} J_{\mathrm{C}-\mathrm{F}}=9.1 \mathrm{~Hz}\right), 129.6,126.61\left(\mathrm{q},{ }^{1} J_{\mathrm{C}-\mathrm{F}}=279.8 \mathrm{~Hz}\right), 125.9\left(\mathrm{~d},{ }^{4} J_{\mathrm{C}-\mathrm{F}}=2.0 \mathrm{~Hz}\right), 119.0,118.4$, $116.9\left(\mathrm{~d},{ }^{2} J_{\mathrm{C}-\mathrm{F}}=20.2 \mathrm{~Hz}\right), 113.3\left(\mathrm{~d},{ }^{2} J_{\mathrm{C}-\mathrm{F}}=21.2 \mathrm{~Hz}\right), 107.6,105.8,42.5\left(\mathrm{q},{ }^{4} J_{\mathrm{C}-\mathrm{F}}=1.0 \mathrm{~Hz}\right), 42.2\left(\mathrm{q},{ }^{2} J\right.$ C-F $=26.8 \mathrm{~Hz}), 42.1,39.9,34.9,31.9(3 \mathrm{C}), 26.1 .{ }^{19} \mathrm{~F} \mathrm{NMR}\left(376 \mathrm{MHz}, \mathrm{CDCl}_{3}\right) \delta-60.29(\mathrm{t}, J=11.4 \mathrm{~Hz}$, $3 \mathrm{~F}),-113.16--113.24(\mathrm{~m}, 1 \mathrm{~F})$. HRMS ESI $[\mathrm{M}+\mathrm{H}]^{+}$calculated for $\mathrm{C}_{24} \mathrm{H}_{26} \mathrm{NF}_{4}$ 404.2001, found 404.1993.

\section{6-(tert-Butyl)-9-(4-chlorophenyl)-1-methyl-1-(2,2,2-trifluoroethyl)-2,3-dihydro-1H-pyrrolo[1,2-} a]indole (3m)

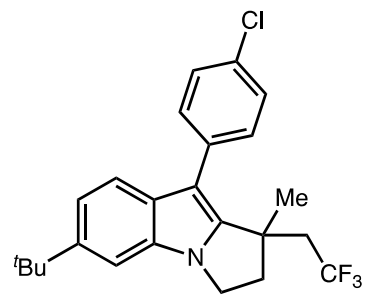

Following the general procedure $\mathbf{A}$, purification by flash column chromatography (1-3\% EtOAc in hexane), the product $(52.9 \mathrm{mg})$ was obtained in $63 \%$ yield as colourless oil. ${ }^{1} \mathrm{H}$ NMR $(600 \mathrm{MHz}, \mathrm{CDCl} 3) \delta 7.41$ $-7.35(\mathrm{~m}, 3 \mathrm{H}), 7.36-7.31(\mathrm{~m}, 2 \mathrm{H}), 7.24(\mathrm{~d}, J=1.7 \mathrm{~Hz}, 1 \mathrm{H}), 7.19-7.16(\mathrm{~m}$,

$1 \mathrm{H}), 4.19-4.14(\mathrm{~m}, 1 \mathrm{H}), 4.13-4.08(\mathrm{~m}, 1 \mathrm{H}), 2.75-2.67(\mathrm{~m}, 1 \mathrm{H}), 2.60-$ $2.53(\mathrm{~m}, 1 \mathrm{H}), 2.50-2.45(\mathrm{~m}, 1 \mathrm{H}), 2.30-2.22(\mathrm{~m}, 1 \mathrm{H}), 1.51(\mathrm{~s}, 3 \mathrm{H}), 1.38(\mathrm{~s}, 9 \mathrm{H}) .{ }^{13} \mathrm{C}$ NMR $(151 \mathrm{MHz}$, $\left.\mathrm{CDCl}_{3}\right) \delta 145.5,145.3,133.4,132.3,131.9,131.4(2 \mathrm{C}), 129.7,128.8(2 \mathrm{C}), 126.5\left(\mathrm{q},{ }^{1} J_{\mathrm{C}-\mathrm{F}}=279.1 \mathrm{~Hz}\right)$, $118.9,118.3,107.4,105.8,42.5,42.3\left(\mathrm{q},{ }^{2} J_{\mathrm{C}-\mathrm{F}}=26.8 \mathrm{~Hz}\right), 42.1,39.8,34.9,31.9(3 \mathrm{C}), 26.1 .{ }^{19} \mathrm{~F}$ NMR $\left(565 \mathrm{MHz}, \mathrm{CDCl}_{3}\right) \delta-60.27(\mathrm{t}, J=11.4 \mathrm{~Hz}, 3 \mathrm{~F})$. HRMS ESI $[\mathrm{M}+\mathrm{H}]^{+}$calculated for $\mathrm{C}_{24} \mathrm{H}_{26} \mathrm{NClF}_{3}$ 420.1706 , found 420.1699 .

\section{9-(3-Chlorophenyl)-1,6-dimethyl-1-(2,2,2-trifluoroethyl)-2,3-dihydro-1H-pyrrolo[1,2-a]indole} $(3 \mathbf{k})$

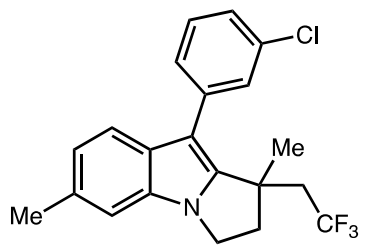

Following the general procedure A, purification by flash column chromatography ( $3 \%$ EtOAc in hexane), the product $(48.3 \mathrm{mg}$ ) was obtained in $64 \%$ yield as white solid. ${ }^{1} \mathrm{H}$ NMR $\left(600 \mathrm{MHz}, \mathrm{CDCl}_{3}\right) \delta 7.45(\mathrm{t}, J=1.8 \mathrm{~Hz}$,

$1 \mathrm{H}), 7.39(\mathrm{t}, J=7.7 \mathrm{~Hz}, 1 \mathrm{H}), 7.38-7.36(\mathrm{~m}, 1 \mathrm{H}), 7.33-7.30(\mathrm{~m}, 2 \mathrm{H}), 7.09$ (s, 1H), $6.96(\mathrm{dd}, J=8.2,1.5 \mathrm{~Hz}, 1 \mathrm{H}), 4.19-4.09(\mathrm{~m}, 2 \mathrm{H}), 2.80-2.73(\mathrm{~m}, 1 \mathrm{H}), 2.66-2.56(\mathrm{~m}, 1 \mathrm{H})$, $2.56-2.50(\mathrm{~m}, 1 \mathrm{H}), 2.50(\mathrm{~s}, 3 \mathrm{H}), 2.37-2.28(\mathrm{~m}, 1 \mathrm{H}), 1.57(\mathrm{~s}, 3 \mathrm{H}) .{ }^{13} \mathrm{C} \mathrm{NMR}\left(151 \mathrm{MHz}, \mathrm{CDCl}_{3}\right) \delta$ $145.1,136.9,134.3,132.2,131.8,130.1,129.7,129.7,128.3,126.2,126.5\left(\mathrm{q},{ }^{1} J_{\text {C-F }}=278.8 \mathrm{~Hz}\right), 121.80$, 
$119.1,109.6,107.5,42.4,42.3\left(\mathrm{q},{ }^{2} \mathrm{~J}_{\mathrm{C}-\mathrm{F}}=25.7 \mathrm{~Hz}\right), 42.1,39.9,26.3,21.8 .{ }^{19} \mathrm{~F}$ NMR $\left(565 \mathrm{MHz}, \mathrm{CDCl}_{3}\right)$ $\delta-60.40(\mathrm{t}, J=11.3 \mathrm{~Hz}, 3 \mathrm{~F})$. HRMS ESI $[\mathrm{M}+\mathrm{H}]^{+}$calculated for $\mathrm{C}_{21} \mathrm{H}_{20} \mathrm{NOClF}_{3} 378.1236$, found 378.1230 .

\section{9-(4-Bromophenyl)-6-methoxy-1-methyl-1-(2,2,2-trifluoroethyl)-2,3-dihydro-1H-pyrrolo[1,2-} a]indole (3n)

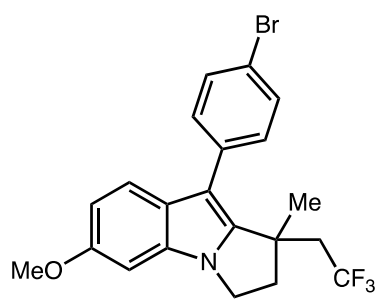

Following the general procedure A, purification by flash column chromatography (3-10\% EtOAc in hexane), the product $(49.8 \mathrm{mg}$ ) was obtained in $57 \%$ yield as pale yellow solid. ${ }^{1} \mathrm{H}$ NMR $\left(600 \mathrm{MHz}, \mathrm{CDCl}_{3}\right) \delta$ $7.57(\mathrm{~d}, J=8.3 \mathrm{~Hz}, 2 \mathrm{H}), 7.35(\mathrm{~d}, J=8.6 \mathrm{~Hz}, 1 \mathrm{H}), 7.31(\mathrm{~d}, J=8.3 \mathrm{~Hz}, 2 \mathrm{H})$, $6.78(\mathrm{dd}, J=8.7,2.3 \mathrm{~Hz}, 1 \mathrm{H}), 6.76(\mathrm{~d}, J=2.2 \mathrm{~Hz}, 1 \mathrm{H}), 4.17-4.06(\mathrm{~m}, 2 \mathrm{H})$, $3.88(\mathrm{~s}, 1 \mathrm{H}), 2.77-2.73(\mathrm{~m}, 1 \mathrm{H}), 2.65-2.56(\mathrm{~m}, 1 \mathrm{H}), 2.55-2.50(\mathrm{~m}, 1 \mathrm{H}), 2.36-2.27(\mathrm{~m}, 1 \mathrm{H}), 1.56$ (s, 3H). ${ }^{13} \mathrm{C}$ NMR $\left(151 \mathrm{MHz}, \mathrm{CDCl}_{3}\right) \delta 156.5,144.3,133.8,132.5,131.8(2 \mathrm{C}), 131.7$ (2C), $126.6\left(\mathrm{q},{ }^{1} \mathrm{~J}\right.$ C-F $=277.8 \mathrm{~Hz}), 126.2,120.4,120.1,109.8,107.6,93.2,55.9,42.4,42.4\left(\mathrm{q},{ }^{2} J_{\mathrm{C}-\mathrm{F}}=26.9 \mathrm{~Hz}\right), 42.2,39.8$, 26.3. ${ }^{19} \mathrm{~F}$ NMR $\left(565 \mathrm{MHz}, \mathrm{CDCl}_{3}\right) \delta-60.39(\mathrm{t}, J=11.4 \mathrm{~Hz}, 3 \mathrm{~F})$. HRMS ESI $[\mathrm{M}+\mathrm{H}]^{+}$calculated for $\mathrm{C}_{21} \mathrm{H}_{20} \mathrm{NOBrF}_{3} 438.0680$, found 438.0675 .

Methyl 4-(6-(tert-butyl)-1-methyl-1-(2,2,2-trifluoroethyl)-2,3-dihydro-1H-pyrrolo[1,2-a]indol-9yl)benzoate (3o)

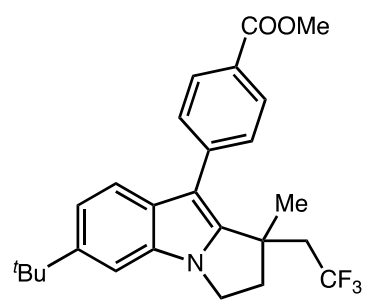

Following the general procedure A, purification by flash column chromatography (3-5\% EtOAc in hexane), the product $(46.1 \mathrm{mg})$ was obtained in $52 \%$ yield as white solid. ${ }^{1} \mathrm{H}$ NMR $\left(400 \mathrm{MHz} \mathrm{CDCl}_{3}\right) \delta 8.03(\mathrm{~d}, J=8.4$ $\mathrm{Hz}, 2 \mathrm{H}), 7.44$ (d, $J=8.4 \mathrm{~Hz}, 2 \mathrm{H}), 7.40$ (d, $J=8.5 \mathrm{~Hz}, 1 \mathrm{H}), 7.19$ (d, $J=1.6$ $\mathrm{Hz}, 1 \mathrm{H}), 7.13$ (dd, $J=8.4,1.7 \mathrm{~Hz}, 1 \mathrm{H}), 4.17-4.02(\mathrm{~m}, 2 \mathrm{H}), 3.87$ (s, 3H), 2.72 $-2.60(\mathrm{~m}, 1 \mathrm{H}), 2.59-2.49(\mathrm{~m}, 1 \mathrm{H}), 2.49-2.40(\mathrm{~m}, 1 \mathrm{H}), 2.27-2.15(\mathrm{~m}, 1 \mathrm{H}), 1.50(\mathrm{~s}, 3 \mathrm{H}), 1.32(\mathrm{~s}$, 9H). ${ }^{13} \mathrm{C}$ NMR (101 MHz, $\left.\mathrm{CDCl}_{3}\right) \delta 167.2,145.7,145.7,140.2,132.1,129.9$ (2C), 129.9 (2C), 126.5 $\left(\mathrm{q},{ }^{1} J_{\mathrm{C}-\mathrm{F}}=279.8 \mathrm{~Hz}\right), 129.3,128.0,119.0,118.5,107.9,105.9,52.2,42.6,42.2,42.2\left(\mathrm{q},{ }^{2} J_{\mathrm{C}-\mathrm{F}}=27.3\right.$ $\mathrm{Hz}), 40.0\left(\mathrm{~d},{ }^{4} J_{\mathrm{C}-\mathrm{F}}=2.0 \mathrm{~Hz}\right), 34.9,31.9(3 \mathrm{C}), 26.1 .{ }^{19} \mathrm{~F} \mathrm{NMR}\left(376 \mathrm{MHz}, \mathrm{CDCl}_{3}\right) \delta-60.32(\mathrm{t}, J=11.3$ $\mathrm{Hz}, 3 \mathrm{~F})$. HRMS ESI [M+H] $]^{+}$calculated for $\mathrm{C}_{26} \mathrm{H}_{29} \mathrm{NO}_{2} \mathrm{~F}_{3} 444.2150$, found 444.2144.

4-(1,6-Dimethyl-1-(2,2,2-trifluoroethyl)-2,3-dihydro-1 H-pyrrolo[1,2-a]indol-9-yl)benzonitrile (3p)

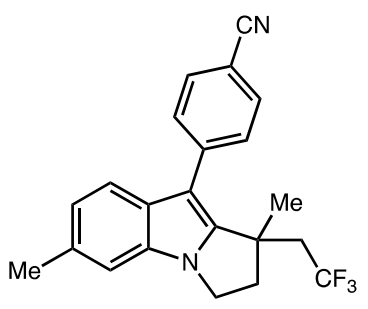

Following the general procedure $\mathbf{A}$, purification by flash column chromatography (5-10\% EtOAc in hexane), the product $(25.6 \mathrm{mg})$ was obtained in 35\% yield as white solid. ${ }^{1} \mathrm{H}$ NMR $(600 \mathrm{MHz}, \mathrm{CDCl} 3) \delta 7.73$ (d, $J=8.0 \mathrm{~Hz}, 2 \mathrm{H}), 7.57$ (d, $J=8.0 \mathrm{~Hz}, 2 \mathrm{H}), 7.41(\mathrm{~d}, J=8.2 \mathrm{~Hz}, 1 \mathrm{H}), 7.12$ (s, $1 \mathrm{H}), 6.99(\mathrm{dd}, J=8.3,1.5 \mathrm{~Hz}, 1 \mathrm{H}), 4.22-4.11(\mathrm{~m}, 2 \mathrm{H}), 2.82-2.76(\mathrm{~m}, 1 \mathrm{H})$, $2.63-2.53(\mathrm{~m}, 1 \mathrm{H}), 2.51(\mathrm{~s}, 3 \mathrm{H}), 2.37-2.30(\mathrm{~m}, 1 \mathrm{H}), 1.60(\mathrm{~s}, 3 \mathrm{H}) .{ }^{13} \mathrm{C} \mathrm{NMR}\left(151 \mathrm{MHz}, \mathrm{CDCl}_{3}\right) \delta$ 145.4, 140.4, 132.5, 132.4 (2C), 132.2, 130.5 (2C), 129.2, 126.4 (q, $\left.{ }^{1} J_{\mathrm{C}-\mathrm{F}}=279.4 \mathrm{~Hz}\right), 122.2,119.2$, 
118.8, 109.8, 109.7, 107.3, 42.5, $42.3\left(\mathrm{q},{ }^{2} \mathrm{~J}_{\mathrm{C}-\mathrm{F}}=27.3 \mathrm{~Hz}\right), 42.2,40.0,26.3,21.7 .{ }^{19} \mathrm{~F}$ NMR $(565 \mathrm{MHz}$, $\left.\mathrm{CDCl}_{3}\right) \delta-60.40(\mathrm{t}, J=11.3 \mathrm{~Hz}, 3 \mathrm{~F})$. HRMS ESI $[\mathrm{M}+\mathrm{H}]^{+}$calculated for $\mathrm{C}_{22} \mathrm{H}_{20} \mathrm{~N}_{2} \mathrm{~F}_{3} 369.1579$, found 369.1570 .

4-(6-Methoxy-1-methyl-1-(2,2,2-trifluoroethyl)-2,3-dihydro-1H-pyrrolo[1,2-a]indol-9yl)benzonitrile (3q)

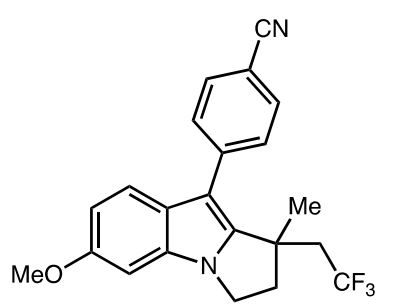

Following the general procedure A, purification by flash column chromatography (8-15\% EtOAc in hexane), the product $(48.2 \mathrm{mg}$ ) was obtained in $63 \%$ yield as white solid. ${ }^{1} \mathrm{H}$ NMR $\left(600 \mathrm{MHz}, \mathrm{CDCl}_{3}\right) \delta 7.73$ (d, $J=7.9 \mathrm{~Hz}, 2 \mathrm{H}), 7.55$ (d, $J=7.9 \mathrm{~Hz}, 2 \mathrm{H}), 7.38$ (d, $J=8.7 \mathrm{~Hz}, 1 \mathrm{H}), 6.80$ (dd, $J=8.6,2.3 \mathrm{~Hz}, 1 \mathrm{H}), 6.77(\mathrm{~d}, J=2.3 \mathrm{~Hz}, 1 \mathrm{H}), 4.19-4.11(\mathrm{~m}, 2 \mathrm{H}), 3.89$ $(\mathrm{s}, 3 \mathrm{H}), 2.82-2.75(\mathrm{~m}, 1 \mathrm{H}), 2.64-2.50(\mathrm{~m}, 2 \mathrm{H}), 2.42-2.25(\mathrm{~m}, 1 \mathrm{H}), 1.59(\mathrm{~s}, 3 \mathrm{H}) .{ }^{13} \mathrm{C} \mathrm{NMR}(151$ $\left.\mathrm{MHz}, \mathrm{CDCl}_{3}\right) \delta 156.7,144.8,140.2,132.8,132.4(2 \mathrm{C}), 130.4(2 \mathrm{C}), 126.3\left(\mathrm{q},{ }^{1} J_{\mathrm{C}-\mathrm{F}}=279.4 \mathrm{~Hz}\right), 125.5$, $119.9,119.2,110.3,109.8,107.5,93.3,55.9,42.5,42.3\left(\mathrm{q},{ }^{2} J_{\mathrm{C}-\mathrm{F}}=25.7 \mathrm{~Hz}\right), 42.2,40.0,26.3 .{ }^{19} \mathrm{~F}$ NMR $\left(565 \mathrm{MHz}, \mathrm{CDCl}_{3}\right) \delta-60.42(\mathrm{t}, J=11.3 \mathrm{~Hz}, 3 \mathrm{~F})$. HRMS ESI $[\mathrm{M}+\mathrm{H}]^{+}$calculated for $\mathrm{C}_{22} \mathrm{H}_{20} \mathrm{~N}_{2} \mathrm{OF}_{3}$ 385.1528 , found 385.1520 .

(1S,3S,5S)-3-Isopropyl-5-methylcyclohexyl 4-(6-methoxy-1-methyl-1-(2,2,2-trifluoroethyl)-2,3dihydro-1H-pyrrolo[1,2-a]indol-9-yl)benzoate (3r)

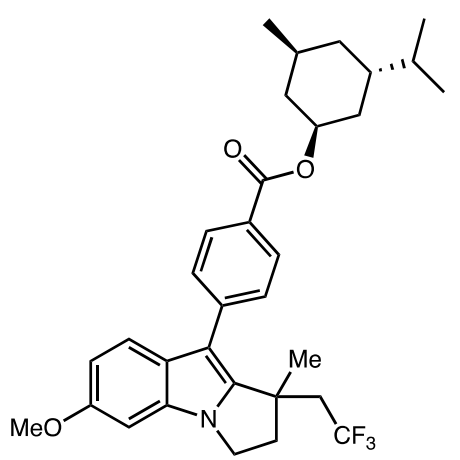

Following the general procedure A, purification by flash column chromatography (3-10\% EtOAc in hexane), the product $(60.6 \mathrm{mg})$ was obtained in $56 \%$ as inseparable mixture of 2 diastereomers (1:1, estimated by HPLC), colourless oil. ${ }^{1} \mathrm{H}$ NMR $\left(600 \mathrm{MHz}, \mathrm{CDCl}_{3}\right) \delta 8.13$ $(\mathrm{d}, J=7.9 \mathrm{~Hz}, 2 \mathrm{H}), 7.52(\mathrm{~d}, J=7.8 \mathrm{~Hz}, 2 \mathrm{H}), 7.42(\mathrm{~d}, J=8.7 \mathrm{~Hz}, 1 \mathrm{H})$, $6.79(\mathrm{~d}, J=8.7 \mathrm{~Hz}, 1 \mathrm{H}), 6.77-6.76(\mathrm{~m}, 1 \mathrm{H}), 4.99(\mathrm{dt}, J=10.9,4.4 \mathrm{~Hz}$, $1 \mathrm{H}), 4.18-4.09(\mathrm{~m}, 1 \mathrm{H}), 3.89(\mathrm{~s}, 3 \mathrm{H}), 2.81-2.74(\mathrm{~m}, 1 \mathrm{H}), 2.70-2.60$ (m, 1H), $2.59-2.50(\mathrm{~m}, 1 \mathrm{H}), 2.37-2.27(\mathrm{~m}, 1 \mathrm{H}), 2.24-2.14(\mathrm{~m}, 1 \mathrm{H}), 2.08-2.03(\mathrm{~m}, 1 \mathrm{H}), 1.80-$ $1.72(\mathrm{~m}, 2 \mathrm{H}), 1.66-1.55(\mathrm{~m}, 5 \mathrm{H}), 1.21-1.11(\mathrm{~m}, 2 \mathrm{H}), 1.00-0.92(\mathrm{~m}, 8 \mathrm{H}), 0.85(\mathrm{dd}, J=7.1,2.4 \mathrm{~Hz}$, $3 \mathrm{H}) .{ }^{13} \mathrm{C} \mathrm{NMR}\left(151 \mathrm{MHz}, \mathrm{CDCl}_{3}\right) \delta 13 \mathrm{C} \mathrm{NMR}\left(151 \mathrm{MHz}, \mathrm{CDCl}_{3}\right) \delta 166.3,156.6,144.7,139.8,132.71$, $129.9(2 \mathrm{C}), 129.9(2 \mathrm{C}), 128.8,126.6\left(\mathrm{q},{ }^{1} J_{\mathrm{C}-\mathrm{F}}=278.8 \mathrm{~Hz}\right), 126.0,120.3,110.0,108.2,93.3,74.9,55.9$, $47.5,42.6,42.2$ (q, $\left.{ }^{2} J_{\mathrm{C}-\mathrm{F}}=27.2 \mathrm{~Hz}, 1 \mathrm{C}\right), 42.2,42.1,39.9,34.5,31.6,26.6,26.2,23.7,22.1,20.9$, 16.6. ${ }^{19} \mathrm{~F}$ NMR $\left(565 \mathrm{MHz}, \mathrm{CDCl}_{3}\right) \delta-60.30--60.40(\mathrm{~m}, 3 \mathrm{~F})$. HRMS ESI $[\mathrm{M}+\mathrm{H}]^{+}$calculated for $\mathrm{C}_{32} \mathrm{H}_{39} \mathrm{~F}_{3} \mathrm{NO}_{3}$ 542.2882, found 542.2889. 


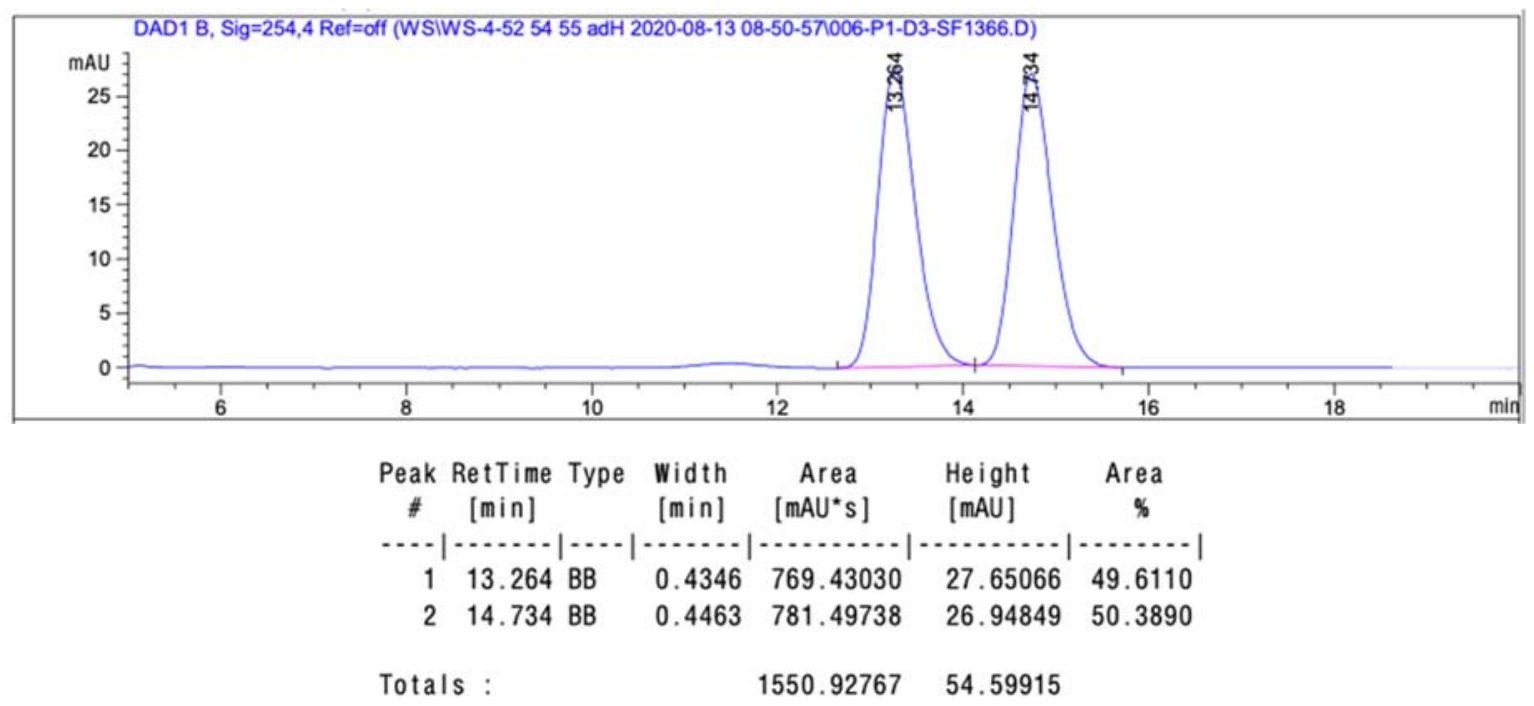

$(1 S, 2 S, 4 R)-1,3,3-T r i m e t h y l b i c y c l o[2.2 .1]$ heptan-2-yl 4-(6-methoxy-1-methyl-1-(2,2,2-

trifluoroethyl)-2,3-dihydro-1H-pyrrolo[1,2-a]indol-9-yl)benzoate (3s)

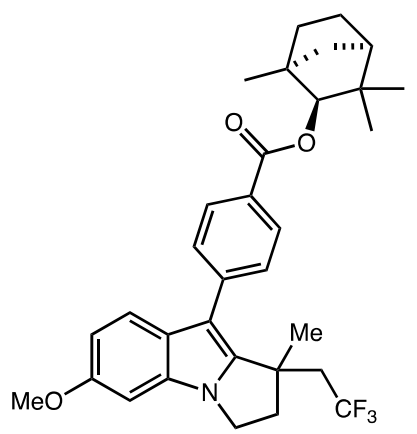

Following the general procedure A, purification by flash column chromatography (3-10\% EtOAc in hexane), the product $(58.3 \mathrm{mg}$ ) was obtained in $54 \%$ as inseparable mixture of 2 diastereomers $(1: 1$, estimated by HPLC), colourless oil. ${ }^{1} \mathrm{H}$ NMR $\left(600 \mathrm{MHz}, \mathrm{CDCl}_{3}\right) \delta 8.14$ (d, $J=7.9$ $\mathrm{Hz}, 2 \mathrm{H}), 7.53$ (d, $J=7.9 \mathrm{~Hz}, 2 \mathrm{H}), 7.43$ (d, $J=8.7 \mathrm{~Hz}, 1 \mathrm{H}), 6.79$ (dd, $J=$ 8.6, 2.2 Hz, 1H), $6.76(\mathrm{~d}, J=2.3 \mathrm{~Hz}, 1 \mathrm{H}), 4.67(\mathrm{~d}, J=2.2 \mathrm{~Hz}, 1 \mathrm{H}), 4.18-$ $4.09(\mathrm{~m}, 2 \mathrm{H}), 3.89(\mathrm{~s}, 3 \mathrm{H}), 2.82-2.75(\mathrm{~m}, 1 \mathrm{H}), 2.70-2.62(\mathrm{~m}, 1 \mathrm{H}), 2.57$ $-2.53(\mathrm{~m}, 1 \mathrm{H}), 2.40-2.30(\mathrm{~m}, 1 \mathrm{H}), 2.06-1.97(\mathrm{~m}, 1 \mathrm{H}), 1.88-1.77(\mathrm{~m}, 2 \mathrm{H}), 1.70(\mathrm{~d}, J=10.4 \mathrm{~Hz}$, $1 \mathrm{H}), 1.61(\mathrm{~s}, 3 \mathrm{H}), 1.57-1.52(\mathrm{~m}, 1 \mathrm{H}), 1.28(\mathrm{~d}, J=10.4 \mathrm{~Hz}, 1 \mathrm{H}), 1.25-1.20(\mathrm{~m}, 4 \mathrm{H}), 1.16(\mathrm{~d}, J=4.9$ $\mathrm{Hz}, 3 \mathrm{H}), 0.92(\mathrm{~d}, J=3.6 \mathrm{~Hz}, 3 \mathrm{H}) .{ }^{13} \mathrm{C} \mathrm{NMR}\left(151 \mathrm{MHz}, \mathrm{CDCl}_{3}\right) \delta 167.0,156.6,144.6,139.9,132.7$, $129.9(2 \mathrm{C}), 129.9(2 \mathrm{C}), 128.6,126.6\left(\mathrm{q},{ }^{1} J_{\mathrm{C}-\mathrm{F}}=278.87 \mathrm{~Hz}\right), 126.0,120.3,110.0,108.2,93.3,86.8,55.9$, $48.8,48.6,42.6,42.3\left(\mathrm{q},{ }^{2} J_{\mathrm{C}-\mathrm{F}}=27.6 \mathrm{~Hz}, 1 \mathrm{H}\right), 42.2,41.6,40.0,40.0,29.9,27.1,26.2,26.1,20.5,19.70$. ${ }^{19} \mathrm{~F}$ NMR $\left(565 \mathrm{MHz}, \mathrm{CDCl}_{3}\right) \delta-60.32(\mathrm{t}, J=11.3 \mathrm{~Hz}, 3 \mathrm{~F})$. HRMS ESI $[\mathrm{M}+\mathrm{H}]^{+}$calculated for $\mathrm{C}_{32} \mathrm{H}_{37} \mathrm{~F}_{3} \mathrm{NO}_{3}$ 540.2726, found 540.2731.

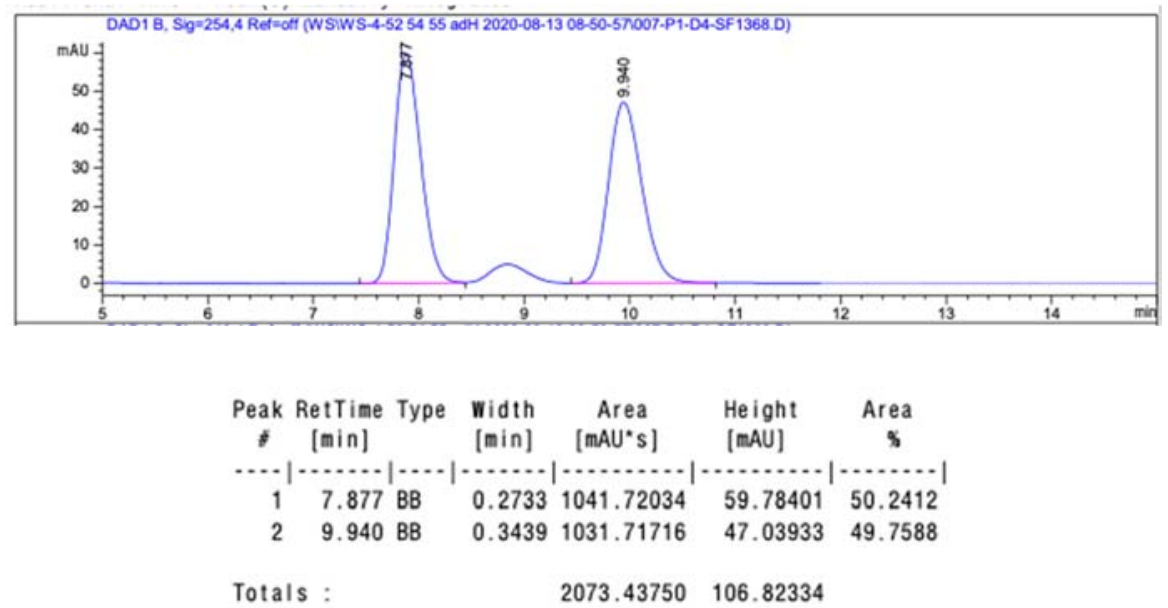




\section{9-(4-Fluorophenyl)-6-methoxy-1-methyl-1-(2,2,2-trifluoroethyl)-2,3-dihydro-1H-pyrrolo[1,2-}

a]indole (3t)

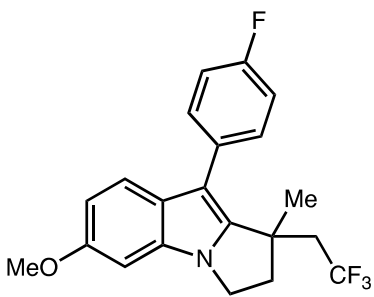

Following the general procedure A, purification by flash column chromatography (3-10\% EtOAc in hexane), the product (42.2 $\mathrm{mg}$ ) was obtained in $56 \%$ yield as white solid. ${ }^{1} \mathrm{H}$ NMR $\left(600 \mathrm{MHz}, \mathrm{CDCl}_{3}\right) \delta 7.42-$ $7.37(\mathrm{~m}, 2 \mathrm{H}), 7.34$ (dd, $J=8.5,0.9 \mathrm{~Hz}, 1 \mathrm{H}), 7.18-7.12(\mathrm{~m}, 2 \mathrm{H}), 6.80-6.76$ $(\mathrm{m}, 2 \mathrm{H}), 4.18-4.07(\mathrm{~m}, 2 \mathrm{H}), 3.89(\mathrm{~s}, 3 \mathrm{H}), 2.78-2.73(\mathrm{~m}, 1 \mathrm{H}), 2.63-2.56$ $(\mathrm{m}, 1 \mathrm{H}), 2.56-2.50(\mathrm{~m}, 1 \mathrm{H}), 2.39-2.27(\mathrm{~m}, 1 \mathrm{H}), 1.56(\mathrm{~s}, 3 \mathrm{H}) .{ }^{13} \mathrm{C} \mathrm{NMR}\left(151 \mathrm{MHz}, \mathrm{CDCl}_{3}\right) \delta 161.8$ $\left(\mathrm{d},{ }^{1} J_{\mathrm{C}-\mathrm{F}}=244.6 \mathrm{~Hz}\right), 156.4,144.2,132.4,131.7\left(\mathrm{~d},{ }^{3} J_{\mathrm{C}-\mathrm{F}}=7.6 \mathrm{~Hz}, 2 \mathrm{C}\right), 130.6\left(\mathrm{~d},{ }^{4} J_{\mathrm{C}-\mathrm{F}}=3.0 \mathrm{~Hz}\right)$, 126.6, $126.6\left(\mathrm{q},{ }^{1} J_{\mathrm{C}-\mathrm{F}}=278.9 \mathrm{~Hz}\right), 120.2,115.5\left(\mathrm{~d},{ }^{2} J_{\mathrm{C}-\mathrm{F}}=21.1 \mathrm{~Hz}, 2 \mathrm{C}\right), 109.7,107.8,93.2,55.9,42.43$, $42.4\left(\mathrm{q},{ }^{2} J_{\mathrm{C}-\mathrm{F}}=26.8 \mathrm{~Hz}\right), 42.1,39.7,26.2 .{ }^{19} \mathrm{~F}$ NMR $\left(565 \mathrm{MHz}, \mathrm{CDCl}_{3}\right) \delta-60.45(\mathrm{t}, J=17.1 \mathrm{~Hz}, 3 \mathrm{~F})$, $116.19--116.28(\mathrm{~m}, 1 \mathrm{~F})$. HRMS ESI $[\mathrm{M}+\mathrm{H}]^{+}$calculated for $\mathrm{C}_{21} \mathrm{H}_{20} \mathrm{NOF}_{4} 378.1481$, found 378.1473.

9-(2-Fluorophenyl)-6-methoxy-1-methyl-1-(2,2,2-trifluoroethyl)-2,3-dihydro-1H-pyrrolo[1,2a]indole (3u)

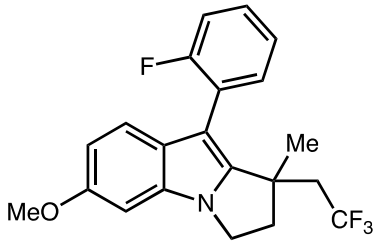

Following the general procedure $\mathbf{A}$, purification by flash column chromatography (3-8\% EtOAc in hexane), the product $(43.7 \mathrm{mg}$ ) was obtained in $58 \%$ yield as white solid. ${ }^{1} \mathrm{H}$ NMR $\left(400 \mathrm{MHz}, \mathrm{CDCl}_{3}\right) \delta 7.44-$ $7.33(\mathrm{~m}, 2 \mathrm{H}), 7.29-7.16(\mathrm{~m}, 3 \mathrm{H}), 6.80-6.76(\mathrm{~m}, 2 \mathrm{H}), 4.21-4.08(\mathrm{~m}, 2 \mathrm{H})$, $3.89(\mathrm{~s}, 3 \mathrm{H}), 2.82-2.74(\mathrm{~m}, 1 \mathrm{H}), 2.63-2.44(\mathrm{~m}, 2 \mathrm{H}), 2.44-2.31(\mathrm{~m}, 1 \mathrm{H}), 1.51(\mathrm{~s}, 3 \mathrm{H}) .{ }^{13} \mathrm{C}$ NMR $(101 \mathrm{MHz}, \mathrm{CDCl} 3) \delta 160.6\left(\mathrm{~d},{ }^{1} J_{\mathrm{C}-\mathrm{F}}=245.4 \mathrm{~Hz}\right), 156.4,145.3,133.1\left(\mathrm{~d},{ }^{4} J_{\mathrm{C}-\mathrm{F}}=4.0 \mathrm{~Hz}\right), 132.5,128.91$ $\left(\mathrm{d},{ }^{3} J_{\mathrm{C}-\mathrm{F}}=8.1 \mathrm{~Hz}\right), 126.7,126.6\left(\mathrm{q},{ }^{1} J_{\mathrm{C}-\mathrm{F}}=279.8 \mathrm{~Hz}\right), 124.1\left(\mathrm{~d},{ }^{4} J_{\mathrm{C}-\mathrm{F}}=4.0 \mathrm{~Hz}\right), 122.0\left(\mathrm{~d},{ }^{2} J_{\mathrm{C}-\mathrm{F}}=16.7\right.$ $\mathrm{Hz}), 120.4,115.9\left(\mathrm{~d},{ }^{2} J_{\mathrm{C}-\mathrm{F}}=22.2 \mathrm{~Hz}\right), 109.7,101.5,93.3,55.9,42.2(2 \mathrm{C}), 41.8\left(\mathrm{q},{ }^{2} J_{\mathrm{C}-\mathrm{F}}=28.3 \mathrm{~Hz}\right)$, 39.7, 25.0. ${ }^{19} \mathrm{~F}$ NMR $\left(376 \mathrm{MHz}, \mathrm{CDCl}_{3}\right) \delta-60.41(\mathrm{t}, J=11.4 \mathrm{~Hz}, 3 \mathrm{~F}),-112.27--112.35(\mathrm{~m}, 1 \mathrm{~F})$. HRMS ESI $[\mathrm{M}+\mathrm{H}]^{+}$calculated for $\mathrm{C}_{21} \mathrm{H}_{20} \mathrm{NOF}_{4} 378.1481$, found 378.1476 .

9-(3-Chlorophenyl)-6-methoxy-1-methyl-1-(2,2,2-trifluoroethyl)-2,3-dihydro-1 H-pyrrolo[1,2a]indole (3v)

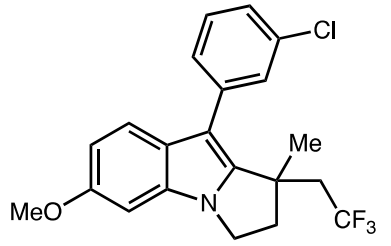

Following the general procedure $\mathbf{A}$, purification by flash column chromatography (3-8\% EtOAc in hexane), the product $(44.0 \mathrm{mg}$ ) was obtained in $56 \%$ yield as white solid. ${ }^{1} \mathrm{H}$ NMR $\left(600 \mathrm{MHz}, \mathrm{CDCl}_{3}\right) \delta 7.45(\mathrm{t}$, $J=1.8 \mathrm{~Hz}, 1 \mathrm{H}), 7.40-7.36(\mathrm{~m}, 2 \mathrm{H}), 7.35-7.30$ (m, 2H), 6.79 (dd, $J=8.7$, $2.3 \mathrm{~Hz}, 1 \mathrm{H}), 6.76(\mathrm{~d}, J=2.3 \mathrm{~Hz}, 1 \mathrm{H}), 4.18-4.08(\mathrm{~m}, 2 \mathrm{H}), 3.89$ (s, 3H), $2.80-2.74(\mathrm{~m}, 1 \mathrm{H}), 2.67-$ $2.57(\mathrm{~m}, 1 \mathrm{H}), 2.57-2.50(\mathrm{~m}, 1 \mathrm{H}), 2.39-2.30(\mathrm{~m}, 1 \mathrm{H}), 1.58(\mathrm{~s}, 3 \mathrm{H}) .{ }^{13} \mathrm{C} \mathrm{NMR}\left(151 \mathrm{MHz}, \mathrm{CDCl}_{3}\right) \delta$ $156.5,144.4,136.8,134.4,132.5,130.1,129.8,128.3,126.6,126.5$ (q, $\left.{ }^{1} J_{\mathrm{C}-\mathrm{F}}=278.9 \mathrm{~Hz}\right), 126.2,120.2$, $109.9,107.6,93.3,55.9,42.4,42.4\left(\mathrm{q},{ }^{2} \mathrm{~J}_{\mathrm{C}-\mathrm{F}}=26.7 \mathrm{~Hz}\right), 42.2,39.9,26.3 .{ }^{19} \mathrm{~F}$ NMR $\left(565 \mathrm{MHz}, \mathrm{CDCl}_{3}\right)$ $\delta-60.40(\mathrm{t}, J=11.4 \mathrm{~Hz}, 3 \mathrm{~F})$. HRMS ESI $[\mathrm{M}+\mathrm{H}]^{+}$calculated for $\mathrm{C}_{21} \mathrm{H}_{20} \mathrm{NClOF}_{3} 394.1186$, found 394.1176. 
6-Methoxy-1-methyl-1-(2,2,2-trifluoroethyl)-9-(4-(trifluoromethyl)phenyl)-2,3-dihydro-1Hpyrrolo[1,2-a]indole (3w)

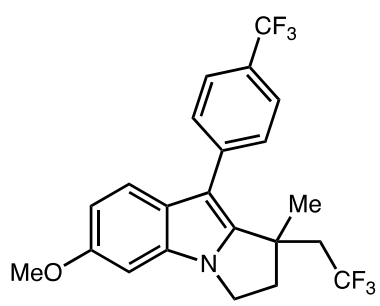

Following the general procedure A, purification by flash column chromatography (3-10\% EtOAc in hexane), the product $(43.6 \mathrm{mg}$ ) was obtained in $51 \%$ yield as white solid. ${ }^{1} \mathrm{H}$ NMR $\left(600 \mathrm{MHz}, \mathrm{CDCl}_{3}\right) \delta 7.72$ (d, $J=7.9 \mathrm{~Hz}, 2 \mathrm{H}), 7.58$ (d, $J=7.9 \mathrm{~Hz}, 2 \mathrm{H}), 7.40$ (d, $J=8.7 \mathrm{~Hz}, 1 \mathrm{H}), 6.82$ (dd, $J=8.7,2.3 \mathrm{~Hz}, 1 \mathrm{H}), 6.79(\mathrm{~d}, J=2.3 \mathrm{~Hz}, 1 \mathrm{H}), 4.20-4.12(\mathrm{~m}, 2 \mathrm{H})$, $3.91(\mathrm{~s}, 3 \mathrm{H}), 2.83-2.76(\mathrm{~m}, 1 \mathrm{H}), 2.68-2.59(\mathrm{~m}, 1 \mathrm{H}), 2.58-2.53(\mathrm{~m}, 1 \mathrm{H}), 2.42-2.29(\mathrm{~m}, 1 \mathrm{H}), 1.61$ $(\mathrm{s}, 3 \mathrm{H}) .{ }^{13} \mathrm{C}$ NMR $\left(151 \mathrm{MHz}, \mathrm{CDCl}_{3}\right) \delta$ 156.6, 144.7, 138.9, 132.7, $130.3(2 \mathrm{C}), 128.5\left(\mathrm{q},{ }^{2} J_{\mathrm{C}-\mathrm{F}}=32.4\right.$ $\mathrm{Hz}), 128.4\left(\mathrm{q},{ }^{1} J_{\mathrm{C}-\mathrm{F}}=278.8 \mathrm{~Hz}\right), 126.1,125.6\left(\mathrm{q},{ }^{4} J_{\mathrm{C}-\mathrm{F}}=3.7 \mathrm{~Hz}\right), 124.5\left(\mathrm{q},{ }^{1} J_{\mathrm{C}-\mathrm{F}}=272.0 \mathrm{~Hz}\right), 123.8$, $120.1,110.1,107.7,93.3,55.9,42.5,42.4\left(\mathrm{q},{ }^{2} J_{\mathrm{C}-\mathrm{F}}=26.8 \mathrm{~Hz}\right), 42.2,39.9,26.3$. HRMS ESI $[\mathrm{M}+\mathrm{H}]^{+}$ calculated for $\mathrm{C}_{22} \mathrm{H}_{20} \mathrm{NOF}_{6} 428.1449$, found 428.1442 .

6-(tert-Butyl)-1-methyl-1-(2,2,2-trifluoroethyl)-9-(4-(trifluoromethyl)phenyl)-2,3-dihydro-1Hpyrrolo[1,2-a]indole $(3 \mathbf{x})$

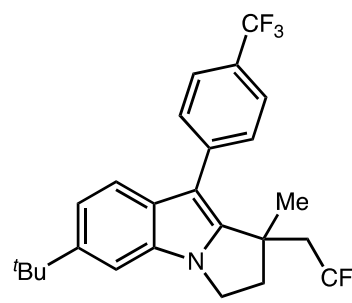

Following the general procedure A, purification by flash column chromatography (3-8\% EtOAc in hexane), the product ( $63.4 \mathrm{mg}$ ) was obtained in $70 \%$ yield as white solid. ${ }^{1} \mathrm{H}$ NMR $\left(400 \mathrm{MHz}, \mathrm{CDCl}_{3}\right) \delta 7.72(\mathrm{~d}, J=8.0$ $\mathrm{Hz}, 2 \mathrm{H}), 7.59$ (d, $J=8.0 \mathrm{~Hz}, 2 \mathrm{H}), 7.48(\mathrm{dd}, J=8.5,0.7 \mathrm{~Hz}, 1 \mathrm{H}), 7.31$ (dd, $J$ $2.76(\mathrm{~m}, 1 \mathrm{H}), 2.70-2.61(\mathrm{~m}, 1 \mathrm{H}), 2.60-2.50(\mathrm{~m}, 1 \mathrm{H}), 2.41-2.27(\mathrm{~m}, 1 \mathrm{H}), 1.63-1.56(\mathrm{~m}, 3 \mathrm{H}), 1.44$ (s, 9H). ${ }^{13} \mathrm{C} \mathrm{NMR}\left(101 \mathrm{MHz}, \mathrm{CDCl}_{3}\right) \delta 145.8,145.7,139.0\left(\mathrm{~d},{ }^{4} \mathrm{~J}_{\mathrm{C}-\mathrm{F}}=1.2 \mathrm{~Hz}\right), 132.1,130.3(2 \mathrm{C}), 129.5$, $128.5\left(\mathrm{q},{ }^{2} J_{\mathrm{C}-\mathrm{F}}=32.5 \mathrm{~Hz}\right), 126.5\left(\mathrm{q},{ }^{1} J_{\mathrm{C}-\mathrm{F}}=279.7 \mathrm{~Hz}\right), 124.6\left(\mathrm{q},{ }^{1} J_{\mathrm{C}-\mathrm{F}}=272.9 \mathrm{~Hz}\right), 118.9(2 \mathrm{C}), 118.6$ (2C), 107.5, 106.0, 42.6, 42.6, 42.4 (q, $\left.{ }^{2} J_{\mathrm{C}-\mathrm{F}}=27.0 \mathrm{~Hz}\right), 40.0\left(\mathrm{q},{ }^{4} J_{\mathrm{C}-\mathrm{F}}=1.8 \mathrm{~Hz}\right), 35.0,31.9(3 \mathrm{C}), 26.2$. ${ }^{19} \mathrm{~F} \mathrm{NMR}\left(376 \mathrm{MHz}, \mathrm{CDCl}_{3}\right) \delta-60.35(\mathrm{t}, J=11.3 \mathrm{~Hz}, 3 \mathrm{~F}),-62.23(\mathrm{~s}, 3 \mathrm{~F})$. HRMS ESI $\left.[\mathrm{M}+\mathrm{H}]\right]^{+}$calculated for $\mathrm{C}_{25} \mathrm{H}_{26} \mathrm{NF}_{6} 454.1969$, found 454.1963 .

6-Methoxy-9-(4-nitrophenyl)-1-(2,2,2-trifluoroethyl)-2,3-dihydro-1H-pyrrolo[1,2-a]indole

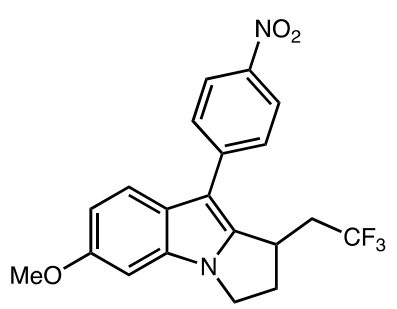

Following the general procedure $\mathbf{A}$, purification by flash column chromatography (8-15\% EtOAc in hexane), the product $(40.6 \mathrm{mg})$ was obtained in $52 \%$ yield as yellow solid. ${ }^{1} \mathrm{H}$ NMR $\left(600 \mathrm{MHz}, \mathrm{CDCl}_{3}\right) \delta 8.30$ (d, $J=8.8 \mathrm{~Hz}, 2 \mathrm{H}), 7.72-7.64(\mathrm{~m}, 3 \mathrm{H}), 6.87$ (dd, $J=8.8,2.3 \mathrm{~Hz}, 1 \mathrm{H}), 6.79$ (d, $J=2.3 \mathrm{~Hz}, 1 \mathrm{H}), 4.20-4.10(\mathrm{~m}, 2 \mathrm{H}), 4.02-3.97(\mathrm{~m}, 1 \mathrm{H}), 3.89(\mathrm{~s}, 3 \mathrm{H})$, $3.07-3.01(\mathrm{~m}, 1 \mathrm{H}), 2.63-2.47(\mathrm{~m}, 2 \mathrm{H}), 2.15-2.05(\mathrm{~m}, 1 \mathrm{H}) .{ }^{13} \mathrm{C}$ NMR $\left(151 \mathrm{MHz}, \mathrm{CDCl}_{3}\right) \delta 156.7,145.4,142.3,141.9,133.6,127.8(2 \mathrm{C}), 126.4\left(\mathrm{q},{ }^{1} J_{\mathrm{C}-\mathrm{F}}=277.9 \mathrm{~Hz}\right), 124.6$, 124.5 (2C), 120.2, 110.9, 107.2, 93.9, 55.9, 42.9, 36.9 (q, $\left.{ }^{2} J_{\mathrm{C}-\mathrm{F}}=27.7 \mathrm{~Hz}\right), 34.4,32.3 .{ }^{19} \mathrm{~F}$ NMR $(565$ $\left.\mathrm{MHz}, \mathrm{CDCl}_{3}\right) \delta-64.94(\mathrm{t}, J=10.6 \mathrm{~Hz}, 3 \mathrm{~F})$. HRMS ESI $[\mathrm{M}+\mathrm{H}]^{+}$calculated for $\mathrm{C}_{20} \mathrm{H}_{18} \mathrm{~N}_{2} \mathrm{O}_{3} \mathrm{~F}_{3} 391.1270$, found 391.1261 . 


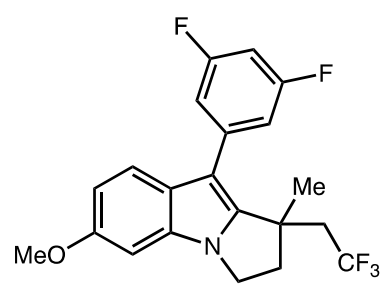

Following the general procedure $\mathbf{A}$, purification by flash column chromatography (5-10\% EtOAc in hexane), the product $(45.0 \mathrm{mg})$ was obtained in $57 \%$ yield as white solid. ${ }^{1} \mathrm{H}$ NMR $\left(600 \mathrm{MHz}, \mathrm{CDCl}_{3}\right) \delta 7.40$ $(\mathrm{d}, J=8.7 \mathrm{~Hz}, 1 \mathrm{H}), 6.98-6.94(\mathrm{~m}, 2 \mathrm{H}), 6.82-6.78(\mathrm{~m}, 2 \mathrm{H}), 6.76(\mathrm{~d}, J=$ $2.3 \mathrm{~Hz}, 1 \mathrm{H}), 4.17-4.09(\mathrm{~m}, 2 \mathrm{H}), 3.89(\mathrm{~s}, 3 \mathrm{H}), 2.81-2.75(\mathrm{~m}, 1 \mathrm{H}), 2.69-$ $2.58(\mathrm{~m}, 1 \mathrm{H}), 2.58-2.52(\mathrm{~m}, 1 \mathrm{H}), 2.41-2.31(\mathrm{~m}, 1 \mathrm{H}), 1.59(\mathrm{~s}, 3 \mathrm{H}) \cdot{ }^{13} \mathrm{C} \mathrm{NMR}\left(151 \mathrm{MHz}, \mathrm{CDCl}_{3}\right) \delta$ $163.2\left(\mathrm{~d},{ }^{1} J_{\mathrm{C}-\mathrm{F}}=248.7 \mathrm{~Hz}\right), 163.1\left(\mathrm{~d},{ }^{1} J_{\mathrm{C}-\mathrm{F}}=235.1 \mathrm{~Hz}\right), 156.6,144.5,138.4\left(\mathrm{t},{ }^{3} J_{\mathrm{C}-\mathrm{F}}=10.1 \mathrm{~Hz}\right), 132.6$, $126.5\left(\mathrm{q},{ }^{1} J_{\mathrm{C}-\mathrm{F}}=278.8 \mathrm{~Hz}\right), 125.8,120.0,112.8\left(\mathrm{~d},{ }^{2} J_{\mathrm{C}-\mathrm{F}}=24.5 \mathrm{~Hz}\right), 112.8\left(\mathrm{~d},{ }^{2} J_{\mathrm{C}-\mathrm{F}}=14.4 \mathrm{~Hz}\right), 110.2$, $107.1\left(\mathrm{t},{ }^{4} \mathrm{~J}_{\mathrm{C}-\mathrm{F}}=2.3 \mathrm{~Hz}\right), 101.9\left(\mathrm{t},{ }^{2} J_{\mathrm{C}-\mathrm{F}}=25.4 \mathrm{~Hz}\right), 93.3,55.9,42.5,42.3,42.2,39.9,26.3 .{ }^{19} \mathrm{~F}$ NMR $\left(565 \mathrm{MHz}, \mathrm{CDCl}_{3}\right) \delta-60.45(\mathrm{t}, J=11.2 \mathrm{~Hz}, 3 \mathrm{~F}),-110.03--110.10(\mathrm{~m}, 1 \mathrm{~F})$. HRMS ESI $[\mathrm{M}+\mathrm{H}]^{+}$ calculated for $\mathrm{C}_{21} \mathrm{H}_{19} \mathrm{NOF}_{5} 396.1387$, found 396.1380.

\section{9-(3-Fluorophenyl)-1,5,7-trimethyl-1-(2,2,2-trifluoroethyl)-2,3-dihydro-1H-pyrrolo[1,2-a]indole} (3aa)

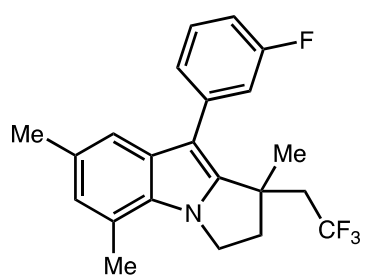

Following the general procedure $\mathbf{A}$, purification by flash column chromatography (3\% EtOAc in hexane), the product $(33.1 \mathrm{mg})$ was obtained in $44 \%$ yield as colourless oil. ${ }^{1} \mathrm{H}$ NMR $\left(600 \mathrm{MHz}, \mathrm{CDCl}_{3}\right) \delta 7.43-7.38(\mathrm{~m}$, $1 \mathrm{H}), 7.21-7.18(\mathrm{~m}, 1 \mathrm{H}), 7.16-7.13(\mathrm{~m}, 1 \mathrm{H}), 7.08(\mathrm{~s}, 1 \mathrm{H}), 7.08-7.03(\mathrm{~m}$, $1 \mathrm{H}), 6.80(\mathrm{~s}, 1 \mathrm{H}), 4.53-4.48(\mathrm{~m}, 1 \mathrm{H}), 4.44-4.40(\mathrm{~m}, 1 \mathrm{H}), 2.77-2.73(\mathrm{~m}$, $1 \mathrm{H}), 2.67(\mathrm{~s}, 3 \mathrm{H}), 2.63-2.54(\mathrm{~m}, 1 \mathrm{H}), 2.53-2.46(\mathrm{~m}, 1 \mathrm{H}), 2.37(\mathrm{~s}, 3 \mathrm{H}), 2.35-2.30(\mathrm{~m}, 1 \mathrm{H}), 1.55(\mathrm{~s}$, 3H). ). ${ }^{13} \mathrm{C}$ NMR (151 MHz, $\left.\mathrm{CDCl}_{3}\right) \delta 162.9\left(\mathrm{~d},{ }^{1} J_{\mathrm{C}-\mathrm{F}}=246.1 \mathrm{~Hz}\right), 146.1,137.4\left(\mathrm{~d},{ }^{3} J_{\mathrm{C}-\mathrm{F}}=7.6 \mathrm{~Hz}\right)$, $132.6,129.9\left(\mathrm{~d},{ }^{3} J_{\mathrm{C}-\mathrm{F}}=9.1 \mathrm{~Hz}\right), 129.7,126.6\left(\mathrm{q},{ }^{1} J_{\mathrm{C}-\mathrm{F}}=277.8 \mathrm{~Hz}\right), 126.3\left(\mathrm{~d},{ }^{4} J_{\mathrm{C}-\mathrm{F}}=2.7 \mathrm{~Hz}\right), 125.2$, 120.5, $117.3\left(\mathrm{~d},{ }^{2} J_{\mathrm{C}-\mathrm{F}}=21.1 \mathrm{~Hz}\right), 116.7(2 \mathrm{C}), 113.5\left(\mathrm{~d},{ }^{2} J_{\mathrm{C}-\mathrm{F}}=21.1 \mathrm{~Hz}\right), 107.6\left(\mathrm{~d},{ }^{4} J_{\mathrm{C}-\mathrm{F}}=2.0 \mathrm{~Hz}\right), 45.1$, $42.4,42.4\left(\mathrm{q},{ }^{2} J_{\mathrm{C}-\mathrm{F}}=26.9 \mathrm{~Hz}\right), 39.2,26.2,21.4,18.0 .{ }^{19} \mathrm{~F} \mathrm{NMR}\left(565 \mathrm{MHz}, \mathrm{CDCl}_{3}\right) \delta-60.37(\mathrm{t}, J=11.4$ $\mathrm{Hz}, 3 \mathrm{~F}),-113.31--113.37(\mathrm{~m}, 1 \mathrm{~F})$. HRMS ESI $[\mathrm{M}+\mathrm{H}]^{+}$calculated for $\mathrm{C}_{22} \mathrm{H}_{22} \mathrm{NF}_{4} 376.1688$, found 376.1681 .

\section{9-(4-Chlorophenyl)-1-methyl-6-(methylthio)-1-(2,2,2-trifluoroethyl)-2,3-dihydro-1H-} pyrrolo[1,2-a]indole (3ab)

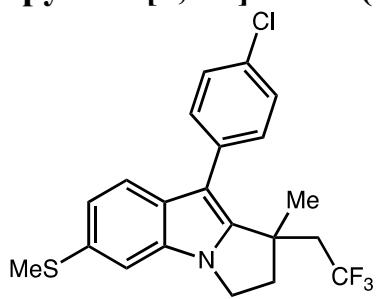

Following the general procedure A, purification by flash column chromatography (3\% EtOAc in hexane), the product ( $46.6 \mathrm{mg}$ ) was obtained in $57 \%$ yield as colourless oil. ${ }^{1} \mathrm{H}$ NMR $\left(400 \mathrm{MHz}, \mathrm{CDCl}_{3}\right) \delta 7.44-7.40(\mathrm{~m}$, $2 \mathrm{H}), 7.37(\mathrm{dd}, J=8.4,0.6 \mathrm{~Hz}, 1 \mathrm{H}), 7.36-7.32(\mathrm{~m}, 2 \mathrm{H}), 7.25(\mathrm{dd}, J=1.7,0.6$ $\mathrm{Hz}, 1 \mathrm{H}), 7.10(\mathrm{dd}, J=8.4,1.7 \mathrm{~Hz}, 1 \mathrm{H}), 4.22-4.08(\mathrm{~m}, 2 \mathrm{H}), 2.81-2.72(\mathrm{~m}$, $1 \mathrm{H}), 2.64-2.56(\mathrm{~m}, 1 \mathrm{H}), 2.54(\mathrm{~s}, 3 \mathrm{H}), 2.57-2.48(\mathrm{~m}, 1 \mathrm{H}), 2.40-2.24(\mathrm{~m}, 1 \mathrm{H}), 1.55(\mathrm{~s}, 3 \mathrm{H}) .{ }^{13} \mathrm{C} \mathrm{NMR}$ $\left(101 \mathrm{MHz}^{\mathrm{CDCl}} \mathrm{CDC}_{3}\right) \delta 145.6,132.9,132.6,132.4,131.5$ (2C), 131.0, 130.5, 128.9 (2C), 126.5 (q, ${ }^{1} \mathrm{~J}_{\mathrm{C}-\mathrm{F}}$ $=279.5 \mathrm{~Hz}), 121.3,119.8,109.7,107.9,42.4,42.3\left(\mathrm{q},{ }^{2} J_{\mathrm{C}-\mathrm{F}}=27.4 \mathrm{~Hz}\right), 42.3,39.9,26.3,18.3 .{ }^{19} \mathrm{~F}$ NMR 
$\left(376 \mathrm{MHz}, \mathrm{CDCl}_{3}\right) \delta-60.43(\mathrm{t}, J=11.3 \mathrm{~Hz}, 3 \mathrm{~F})$. HRMS ESI $[\mathrm{M}+\mathrm{H}]^{+}$calculated for $\mathrm{C}_{21} \mathrm{H}_{20} \mathrm{NClSF}_{3}$ 410.0957 , found 410.0948 .

\section{9-(3-Fluorophenyl)-6-isopropyl-1-methyl-1-(2,2,2-trifluoroethyl)-2,3-dihydro-1H-pyrrolo[1,2-} a]indole (3ac)

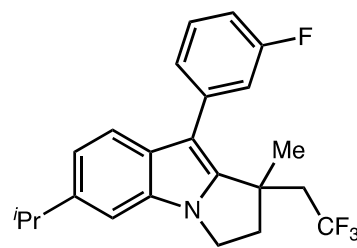

Following the general procedure A, purification by flash column chromatography (3\% EtOAc in hexane), the product $(39.0 \mathrm{mg}$ ) was obtained in $50 \%$ yield as colourless oil. ${ }^{1} \mathrm{H}$ NMR $\left(600 \mathrm{MHz}, \mathrm{CDCl}_{3}\right) \delta 7.50-7.46(\mathrm{~m}$, $1 \mathrm{H}), 7.45-7.41(\mathrm{~m}, 1 \mathrm{H}), 7.25-7.22(\mathrm{~m}, 1 \mathrm{H}), 7.21-7.18(\mathrm{~m}, 1 \mathrm{H}), 7.18-$ $7.16(\mathrm{~m}, 1 \mathrm{H}), 7.08-7.04(\mathrm{~m}, 2 \mathrm{H}), 4.24-4.20(\mathrm{~m}, 1 \mathrm{H}), 4.19-4.14(\mathrm{~m}, 1 \mathrm{H}), 3.11-3.05(\mathrm{~m}, 1 \mathrm{H}), 2.82$ $-2.76(\mathrm{~m}, 1 \mathrm{H}), 2.72-2.60(\mathrm{~m}, 1 \mathrm{H}), 2.59-2.52(\mathrm{~m}, 1 \mathrm{H}), 2.41-2.29(\mathrm{~m}, 1 \mathrm{H}), 1.62-1.59(\mathrm{~m}, 3 \mathrm{H})$, $1.37(\mathrm{~s}, 3 \mathrm{H}), 1.37(\mathrm{~s}, 3 \mathrm{H}) .{ }^{13} \mathrm{C}$ NMR $\left(151 \mathrm{MHz}, \mathrm{CDCl}_{3}\right) \delta 163.0\left(\mathrm{~d},{ }^{1} \mathrm{~J}_{\mathrm{C}-\mathrm{F}}=244.6 \mathrm{~Hz}\right), 145.2,143.4$, $137.3\left(\mathrm{~d},{ }^{3} J_{\mathrm{C}-\mathrm{F}}=7.6 \mathrm{~Hz}\right), 132.1,130.1,129.9\left(\mathrm{~d},{ }^{3} J_{\mathrm{C}-\mathrm{F}}=9.1 \mathrm{~Hz}\right), 126.6\left(\mathrm{q},{ }^{1} J_{\mathrm{C}-\mathrm{F}}=279.4 \mathrm{~Hz}\right), 125.9(\mathrm{~d}$, $\left.{ }^{4} J_{\mathrm{C}-\mathrm{F}}=2.7 \mathrm{~Hz}\right), 119.4,119.3,116.9\left(\mathrm{~d},{ }^{2} J_{\mathrm{C}-\mathrm{F}}=19.6 \mathrm{~Hz}\right), 113.4\left(\mathrm{~d},{ }^{2} J_{\mathrm{C}-\mathrm{F}}=21.1 \mathrm{~Hz}\right), 107.7\left(\mathrm{~d},{ }^{4} J_{\mathrm{C}-\mathrm{F}}=\right.$ $2.1 \mathrm{~Hz}), 106.8,42.6,42.3$ (q, $\left.{ }^{2} J_{\mathrm{C}-\mathrm{F}}=26.8 \mathrm{~Hz}\right), 42.2,39.9,34.6,26.2,24.7$ (2C). ${ }^{19} \mathrm{~F}$ NMR $(565 \mathrm{MHz}$, $\left.\mathrm{CDCl}_{3}\right) \delta-60.35(\mathrm{t}, J=11.4 \mathrm{~Hz}, 3 \mathrm{~F}),-113.24--113.29(\mathrm{~m}, 1 \mathrm{~F})$. HRMS ESI $[\mathrm{M}+\mathrm{H}]^{+}$calculated for $\mathrm{C}_{23} \mathrm{H}_{24} \mathrm{NF}_{4} 390.1845$, found 390.1836 .

\section{6-Methoxy-1-methyl-9-(pyridin-3-yl)-1-(2,2,2-trifluoroethyl)-2,3-dihydro-1H-pyrrolo[1,2-} a]indole (3ad)

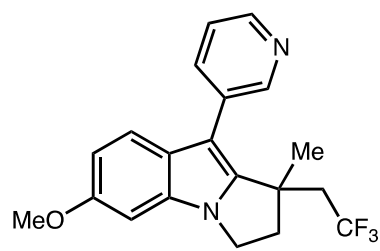

Following the general procedure A, purification by flash column chromatography (3-10\% EtOAc in hexane), the product $(30.9 \mathrm{mg}$ ) was obtained in $43 \%$ yield as yellow solid. ${ }^{1} \mathrm{H}$ NMR $\left(400 \mathrm{MHz}, \mathrm{CDCl}_{3}\right) \delta 8.73(\mathrm{~s}$, $1 \mathrm{H}), 8.60(\mathrm{~d}, J=5.0 \mathrm{~Hz}, 1 \mathrm{H}), 7.83(\mathrm{dt}, J=7.8,1.9 \mathrm{~Hz}, 1 \mathrm{H}), 7.45(\mathrm{dd}, J=7.9$, $4.9 \mathrm{~Hz}, 1 \mathrm{H}), 7.33(\mathrm{dd}, J=8.5,0.8 \mathrm{~Hz}, 1 \mathrm{H}), 6.81-6.74(\mathrm{~m}, 2 \mathrm{H}), 4.20-4.08$ (m, 2H), $3.88(\mathrm{~s}, 3 \mathrm{H}), 2.82-2.74(\mathrm{~m}, 1 \mathrm{H}), 2.61-2.46(\mathrm{~m}, 2 \mathrm{H}), 2.42-2.27(\mathrm{~m}, 1 \mathrm{H}), 1.57(\mathrm{~s}, 3 \mathrm{H}) .{ }^{13} \mathrm{C}$ $\operatorname{NMR}\left(101 \mathrm{MHz}, \mathrm{CDCl}_{3}\right) \delta 156.7,149.9,146.9,145.1,138.2,132.7,131.6,126.4\left(\mathrm{q},{ }^{1} J_{\mathrm{C}-\mathrm{F}}=279.8 \mathrm{~Hz}\right)$, $126.1,123.9,119.7,110.2,104.5,93.4,55.9,42.5\left(\mathrm{q},{ }^{2} J_{\mathrm{C}-\mathrm{F}}=27.0 \mathrm{~Hz}\right), 42.3,42.3,40.0\left(\mathrm{~d},{ }^{4} J_{\mathrm{C}-\mathrm{F}}=1.8\right.$ $\mathrm{Hz})$, 26.6. ${ }^{19} \mathrm{~F}$ NMR $\left(376 \mathrm{MHz}, \mathrm{CDCl}_{3}\right) \delta-60.47(\mathrm{t}, J=11.2 \mathrm{~Hz}, 3 \mathrm{~F})$. HRMS ESI $[\mathrm{M}+\mathrm{H}]^{+}$calculated for $\mathrm{C}_{20} \mathrm{H}_{20} \mathrm{~N}_{2} \mathrm{OF}_{3} 361.1528$, found 361.1519 .

\section{9-(3-Fluorophenyl)-6-methoxy-1-methyl-1-(2,2,2-trifluoroethyl)-2,3-dihydro-1H-pyrrolo[1,2-}

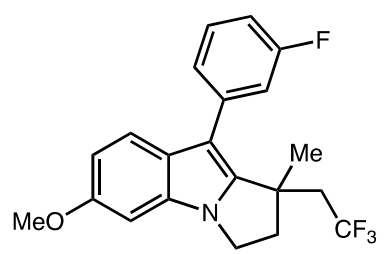
a]indole (5a)

Following the general procedure A, purification by flash column chromatography (3-5\% EtOAc in hexane), the product $(42.4 \mathrm{mg})$ was obtained in $56 \%$ yield as white solid. ${ }^{1} \mathrm{H}$ NMR $\left(400 \mathrm{MHz}, \mathrm{CDCl}_{3}\right) \delta 7.45-$ $7.36(\mathrm{~m}, 2 \mathrm{H}), 7.24-7.20(\mathrm{~m}, 1 \mathrm{H}), 7.18-7.13(\mathrm{~m}, 1 \mathrm{H}), 7.10-6.99(\mathrm{~m}, 1 \mathrm{H})$, $6.79(\mathrm{dd}, J=8.6,2.3 \mathrm{~Hz}, 1 \mathrm{H}), 6.77-6.75(\mathrm{~m}, 1 \mathrm{H}), 4.19-4.06(\mathrm{~m}, 2 \mathrm{H}), 3.89(\mathrm{~s}, 3 \mathrm{H}), 2.82-2.73(\mathrm{~m}$, $1 \mathrm{H}), 2.70-2.59(\mathrm{~m}, 1 \mathrm{H}), 2.57-2.50(\mathrm{~m}, 1 \mathrm{H}), 2.42-2.27(\mathrm{~m}, 1 \mathrm{H}), 1.62-1.53(\mathrm{~m}, 3 \mathrm{H}) .{ }^{13} \mathrm{C}$ NMR $(101$ $\left.\mathrm{MHz}, \mathrm{CDCl}_{3}\right) \delta 163.0\left(\mathrm{~d},{ }^{1} J_{\mathrm{C}-\mathrm{F}}=247.5 \mathrm{~Hz}\right), 156.5,144.4,137.2\left(\mathrm{~d},{ }^{3} J_{\mathrm{C}-\mathrm{F}}=8.1 \mathrm{~Hz}\right), 132.5,130.0\left(\mathrm{~d},{ }^{3} \mathrm{~J}\right.$ 
C-F $=8.1 \mathrm{~Hz}), 126.6\left(\mathrm{q},{ }^{1} J_{\mathrm{C}-\mathrm{F}}=279.8 \mathrm{~Hz}\right), 126.2,125.9\left(\mathrm{~d},{ }^{4} J_{\mathrm{C}-\mathrm{F}}=3.0 \mathrm{~Hz}\right), 120.2,116.9\left(\mathrm{~d},{ }^{2} J_{\mathrm{C}-\mathrm{F}}=21.1\right.$ $\mathrm{Hz}), 113.4\left(\mathrm{~d},{ }^{2} J_{\mathrm{C}-\mathrm{F}}=21.2 \mathrm{~Hz}\right), 109.9,107.9\left(\mathrm{~d},{ }^{4} J_{\mathrm{C}-\mathrm{F}}=2.0 \mathrm{~Hz}\right), 93.3,55.9,42.5\left(\mathrm{~d},{ }^{4} J_{\mathrm{C}-\mathrm{F}}=1.0 \mathrm{~Hz}\right)$, $42.3\left(\mathrm{q},{ }^{2} J_{\mathrm{C}-\mathrm{F}}=27.4 \mathrm{~Hz}\right), 42.2,39.9,26.2 .{ }^{19} \mathrm{~F}$ NMR $\left(376 \mathrm{MHz}, \mathrm{CDCl}_{3}\right) \delta-60.41$ (t, $\left.J=11.4 \mathrm{~Hz}, 3 \mathrm{~F}\right),-$ $113.19--113.27$ (m, 1F). HRMS ESI [M+H] ${ }^{+}$calculated for $\mathrm{C}_{21} \mathrm{H}_{20} \mathrm{NOF}_{4} 378.1481$, found 378.1482.

\section{6-Methoxy-1-methyl-9-(4-nitrophenyl)-1-(2,2,2-trifluoroethyl)-2,3-dihydro-1H-pyrrolo[1,2-} a]indole (5b)

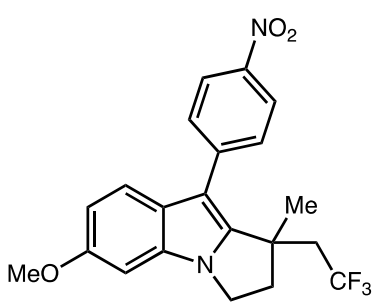

Following the general procedure A, purification by flash column chromatography $(8-15 \%$ EtOAc in hexane), the product $(50.2 \mathrm{mg})$ was obtained in $62 \%$ yield as yellow solid. ${ }^{1} \mathrm{H}$ NMR $\left(400 \mathrm{MHz}, \mathrm{CDCl}_{3}\right) \delta 8.31(\mathrm{~d}$, $J=8.8 \mathrm{~Hz}, 2 \mathrm{H}), 7.60(\mathrm{~d}, J=8.8 \mathrm{~Hz}, 2 \mathrm{H}), 7.41(\mathrm{~d}, J=8.7 \mathrm{~Hz}, 1 \mathrm{H}), 6.82(\mathrm{dd}$, $J=8.7,2.3 \mathrm{~Hz}, 1 \mathrm{H}), 6.78(\mathrm{~d}, J=2.2 \mathrm{~Hz}, 1 \mathrm{H}), 4.22-4.10(\mathrm{~m}, 2 \mathrm{H}), 3.89$ (s, $3 \mathrm{H}), 2.84-2.75(\mathrm{~m}, 1 \mathrm{H}), 2.68-2.52(\mathrm{~m}, 1 \mathrm{H}), 2.43-2.30(\mathrm{~m}, 1 \mathrm{H}), 1.62(\mathrm{~s}, 3 \mathrm{H}) .{ }^{13} \mathrm{C} \mathrm{NMR}(101 \mathrm{MHz}$, $\left.\mathrm{CDCl}_{3}\right) \delta 156.8,146.1,145.2,142.5,132.9,130.2(2 \mathrm{C}), 126.4\left(\mathrm{q},{ }^{1} J_{\mathrm{C}-\mathrm{F}}=279.8 \mathrm{~Hz}\right), 125.5,124.0(2 \mathrm{C})$, $120.0,110.5,107.3,93.5,55.9,42.6,42.3\left(\mathrm{q},{ }^{2} J_{\mathrm{C}-\mathrm{F}}=27.3 \mathrm{~Hz}\right), 42.3,40.2,26.4 .{ }^{19} \mathrm{~F}$ NMR $(376 \mathrm{MHz}$, $\left.\mathrm{CDCl}_{3}\right) \delta-60.42(\mathrm{t}, J=11.3 \mathrm{~Hz}, 3 \mathrm{~F})$. HRMS ESI $[\mathrm{M}+\mathrm{H}]^{+}$calculated for $\mathrm{C}_{21} \mathrm{H}_{20} \mathrm{~N}_{2} \mathrm{O}_{3} \mathrm{~F}_{3} 405.1425$, found 405.1420 .

\section{3,9-Dimethyl-10-(p-tolyl)-9-(2,2,2-trifluoroethyl)-6,7,8,9-tetrahydropyrido[1,2-a]indole (7a)}

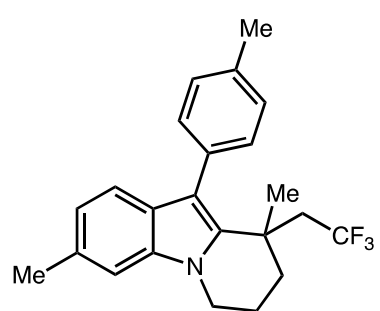

Following the general procedure A, purification by flash column chromatography (3\% EtOAc in hexane), the product $(53.4 \mathrm{mg})$ was obtained in $72 \%$ yield as colourless oil. ${ }^{1} \mathrm{H}$ NMR $\left(400 \mathrm{MHz}, \mathrm{CDCl}_{3}\right) \delta 7.21(\mathrm{~s}, 4 \mathrm{H})$, $7.07(\mathrm{~s}, 1 \mathrm{H}), 7.04(\mathrm{~d}, J=8.1 \mathrm{~Hz}, 1 \mathrm{H}), 6.86(\mathrm{~d}, J=8.0 \mathrm{~Hz}, 1 \mathrm{H}), 4.14-4.06$ (m, 1H), $4.05-3.96(\mathrm{~m}, 1 \mathrm{H}), 2.60-2.29(\mathrm{~m}, 8 \mathrm{H}), 2.17-2.02(\mathrm{~m}, 3 \mathrm{H}), 1.85$ - $1.75(\mathrm{~m}, 1 \mathrm{H}), 1.41$ (s, 3H). ${ }^{13} \mathrm{C}$ NMR $\left(151 \mathrm{MHz}, \mathrm{CDCl}_{3}\right) \delta 137.6,136.6,135.2,133.5,131.5,131.4$ (2C), $129.1(2 \mathrm{C}), 127.6,126.5\left(\mathrm{q},{ }^{1} J_{\mathrm{C}-\mathrm{F}}=279.35 \mathrm{~Hz}\right), 121.6,118.8,113.5,108.8,43.5\left(\mathrm{q},{ }^{2} J_{\mathrm{C}-\mathrm{F}}=25.9\right.$ $\mathrm{Hz}$ ), 42.6, 34.8, 33.8, 29.0, 21.9, 21.4, 19.5. ${ }^{19} \mathrm{~F} \mathrm{NMR}\left(565 \mathrm{MHz}, \mathrm{CDCl}_{3}\right) \delta-59.03$ (t, $J=11.5 \mathrm{~Hz}, 3 \mathrm{~F}$ ). HRMS ESI $[\mathrm{M}+\mathrm{H}]^{+}$calculated for $\mathrm{C}_{23} \mathrm{H}_{25} \mathrm{NF}_{3} 372.1939$, found 372.1931 .

10-(3-Fluorophenyl)-3-methyl-9-(2,2,2-trifluoroethyl)-6,7,8,9-tetrahydropyrido[1,2-a]indole (7b)

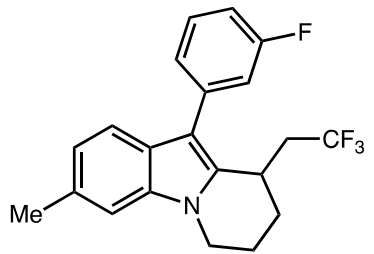

Following the general procedure $\mathbf{A}$, purification by flash column chromatography (3\% EtOAc in hexane), the product $(29.4 \mathrm{mg}$ ) was obtained in $42 \%$ yield as colourless oil. ${ }^{1} \mathrm{H}$ NMR $\left(600 \mathrm{MHz}, \mathrm{CDCl}_{3}\right) \delta 7.51(\mathrm{~d}, J=8.1$

$\mathrm{Hz}, 1 \mathrm{H}), 7.44-7.40$ (m, 1H), $7.27-7.23$ (m, 1H), $7.23-7.19(\mathrm{~m}, 1 \mathrm{H}), 7.16-$ $7.13(\mathrm{~m}, 1 \mathrm{H}), 7.05-6.99(\mathrm{~m}, 2 \mathrm{H}), 4.22-4.17(\mathrm{~m}, 1 \mathrm{H}), 4.04-3.97(\mathrm{~m}, 1 \mathrm{H}), 3.95-3.88(\mathrm{~m}, 1 \mathrm{H}), 2.52$ (s, 3H), $2.30-2.23(\mathrm{~m}, 2 \mathrm{H}), 2.21-2.14(\mathrm{~m}, 2 \mathrm{H}), 2.12-1.99(\mathrm{~m}, 2 \mathrm{H}) .{ }^{13} \mathrm{C} \mathrm{NMR}\left(151 \mathrm{MHz}, \mathrm{CDCl}_{3}\right) \delta$ $163.3\left(\mathrm{~d},{ }^{1} J_{\mathrm{C}-\mathrm{F}}=246.1 \mathrm{~Hz}\right), 137.5\left(\mathrm{~d},{ }^{3} J_{\mathrm{C}-\mathrm{F}}=9.1 \mathrm{~Hz}\right), 136.3,133.5,131.8,130.3\left(\mathrm{~d},{ }^{3} J_{\mathrm{C}-\mathrm{F}}=7.6 \mathrm{~Hz}\right)$, $126.4\left(\mathrm{q},{ }^{1} J_{\mathrm{C}-\mathrm{F}}=277.8 \mathrm{~Hz}\right), 125.1\left(\mathrm{~d},{ }^{4} J_{\mathrm{C}-\mathrm{F}}=3.0 \mathrm{~Hz}\right), 125.1,122.4,118.5,116.2\left(\mathrm{~d},{ }^{2} J_{\mathrm{C}-\mathrm{F}}=19.6 \mathrm{~Hz}\right)$, $113.3\left(\mathrm{~d},{ }^{2} J_{\mathrm{C}-\mathrm{F}}=21.1 \mathrm{~Hz}\right), 112.1\left(\mathrm{~d},{ }^{4} J_{\mathrm{C}-\mathrm{F}}=2.0 \mathrm{~Hz}\right), 109.1,42.5,37.6\left(\mathrm{q},{ }^{2} J_{\mathrm{C}-\mathrm{F}}=27.2 \mathrm{~Hz}\right), 27.4,25.5$, 
21.9, 19.6. ${ }^{19} \mathrm{~F}$ NMR (565 MHz, $\left.\mathrm{CDCl}_{3}\right) \delta-63.81(\mathrm{t}, J=11.9 \mathrm{~Hz}, 3 \mathrm{~F}),-113.15--113.24(\mathrm{~m}, 1 \mathrm{~F})$. HRMS ESI $[\mathrm{M}+\mathrm{H}]^{+}$calculated for $\mathrm{C}_{21} \mathrm{H}_{20} \mathrm{NF}_{4} 362.1532$, found 362.1525 .

10-Butyl-11-(3,5-difluorophenyl)-3-methoxy-10-(2,2,2-trifluoroethyl)-7,8,9,10-tetrahydro-6Hazepino[1,2-a]indole (7c)

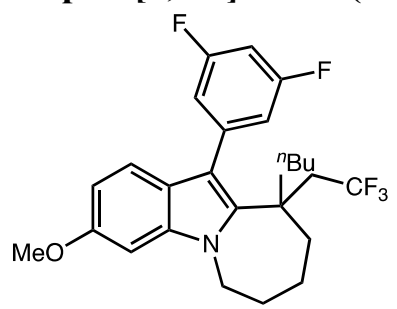

Following the general procedure A, purification by flash column chromatography ( $3 \%$ EtOAc in hexane), the product $(34.1 \mathrm{mg}$ ) was obtained in $37 \%$ yield as colourless oil. ${ }^{1} \mathrm{H}$ NMR $\left(600 \mathrm{MHz}, \mathrm{CDCl}_{3}\right) \delta 6.91(\mathrm{~d}, J=$ $8.6 \mathrm{~Hz}, 1 \mathrm{H}), 6.89-6.85(\mathrm{~m}, 2 \mathrm{H}), 6.84-6.80(\mathrm{~m}, 1 \mathrm{H}), 6.78(\mathrm{~d}, J=2.2 \mathrm{~Hz}$, $1 \mathrm{H}), 6.70(\mathrm{dd}, J=8.6,2.2 \mathrm{~Hz}, 1 \mathrm{H}), 4.38-4.33(\mathrm{~m}, 1 \mathrm{H}), 4.25-4.19(\mathrm{~m}, 1 \mathrm{H})$, $3.87(\mathrm{~s}, 3 \mathrm{H}), 2.64-2.47(\mathrm{~m}, 2 \mathrm{H}), 2.08-2.03(\mathrm{~m}, 1 \mathrm{H}), 2.01-1.96(\mathrm{~m}, 1 \mathrm{H}), 1.95-1.87(\mathrm{~m}, 2 \mathrm{H}), 1.87$ $-1.82(\mathrm{~m}, 2 \mathrm{H}), 1.78-1.70(\mathrm{~m}, 2 \mathrm{H}), 1.22-1.15(\mathrm{~m}, 2 \mathrm{H}), 1.10-1.01(\mathrm{~m}, 1 \mathrm{H}), 0.97-0.90(\mathrm{~m}, 1 \mathrm{H})$, $0.78(\mathrm{t}, J=7.3 \mathrm{~Hz}, 3 \mathrm{H}) .{ }^{13} \mathrm{C}$ NMR $\left(151 \mathrm{MHz}, \mathrm{CDCl}_{3}\right) \delta 162.8(\mathrm{~d}, J=248.6 \mathrm{~Hz}), 162.7\left(\mathrm{~d},{ }^{1} J_{\mathrm{C}-\mathrm{F}}=248.7\right.$ $\mathrm{Hz}), 156.9,141.4\left(\mathrm{t},{ }^{3} J_{\mathrm{C}-\mathrm{F}}=10.0 \mathrm{~Hz}\right), 138.3,136.6,126.6\left(\mathrm{q},{ }^{1} J_{\mathrm{C}-\mathrm{F}}=279.5 \mathrm{~Hz}\right), 123.8,119.7,114.6(\mathrm{~d}$, $\left.{ }^{2} J_{\mathrm{C}-\mathrm{F}}=21.1 \mathrm{~Hz}\right), 114.4\left(\mathrm{~d},{ }^{2} J_{\mathrm{C}-\mathrm{F}}=22.7 \mathrm{~Hz}\right), 114.2\left(\mathrm{t},{ }^{4} J_{\mathrm{C}-\mathrm{F}}=2.4 \mathrm{~Hz}\right), 109.5,102.5\left(\mathrm{t},{ }^{2} J_{\mathrm{C}-\mathrm{F}}=25.3 \mathrm{~Hz}\right)$, 92.9, 56.0, 43.9, 42.4 (q, $\left.{ }^{2} J_{\mathrm{C}-\mathrm{F}}=25.2 \mathrm{~Hz}\right), 42.2,40.3,35.3,27.7,26.7,23.0,22.8,14.0 .{ }^{19} \mathrm{~F}$ NMR (565 $\left.\mathrm{MHz}, \mathrm{CDCl}_{3}\right) \delta-58.43(\mathrm{t}, J=10.7 \mathrm{~Hz}, 3 \mathrm{~F}),-110.43--110.50(\mathrm{~m}, 1 \mathrm{~F}),-110.94--110.02(\mathrm{~m}, 1 \mathrm{~F})$. HRMS ESI $[\mathrm{M}+\mathrm{H}]^{+}$calculated for $\mathrm{C}_{26} \mathrm{H}_{29} \mathrm{~F}_{5} \mathrm{NO} 466.2169$, found 466.2172 .

\section{3-(1,6-Dimethyl-9-(p-tolyl)-2,3-dihydro-1H-pyrrolo[1,2-a]indol-1-yl)-2,2-difluoro- $N$ -}

\section{Propylpropanamide (8a)}

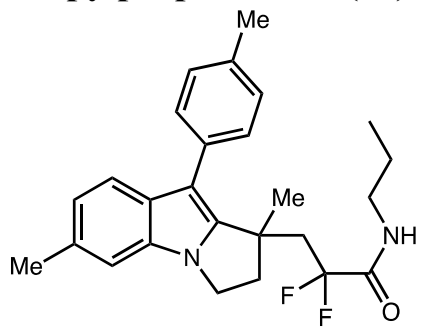

Following the general procedure $\mathbf{B}$, purification by flash column chromatography (3-10\% EtOAc in hexane), 2-bromo-2,2-difluoro- $N$ propylacetamide was used as radical precursor, the product $(41.8 \mathrm{mg})$ was obtained in $51 \%$ yield as white solid. ${ }^{1} \mathrm{H}$ NMR $\left(400 \mathrm{MHz}, \mathrm{CDCl}_{3}\right) \delta 7.43$ (d, $J=8.2 \mathrm{~Hz}, 1 \mathrm{H}), 7.38$ (dd, $J=8.0,1.5 \mathrm{~Hz}, 2 \mathrm{H}), 7.29-7.22(\mathrm{~m}, 2 \mathrm{H})$, 7.08 (s, 1H), 6.93 (dd, $J=8.1,1.5 \mathrm{~Hz}, 1 \mathrm{H}), 6.17$ (brs, 1H), $4.20-4.03(\mathrm{~m}, 2 \mathrm{H}), 3.25-3.15(\mathrm{~m}, 1 \mathrm{H})$, $3.15-3.05(\mathrm{~m}, 1 \mathrm{H}), 2.86-2.77(\mathrm{~m}, 1 \mathrm{H}), 2.74-2.58(\mathrm{~m}, 1 \mathrm{H}), 2.57-2.45(\mathrm{~m}, 5 \mathrm{H}), 2.43(\mathrm{~s}, 3 \mathrm{H}), 1.55$ (s, 3H), 1.48 (p, J=7.2 Hz, 2H), $0.90(\mathrm{t}, J=7.4 \mathrm{~Hz}, 3 \mathrm{H}) .{ }^{13} \mathrm{C} \mathrm{NMR}\left(101 \mathrm{MHz}, \mathrm{CDCl}_{3}\right) \delta 164.4\left(\mathrm{t},{ }^{2} J_{\mathrm{C}-}\right.$ $\mathrm{F}=28.4 \mathrm{~Hz}), 145.2,135.7,132.3,132.0,131.2,130.2,130.2(2 \mathrm{C}), 129.2(2 \mathrm{C}), 121.2,119.4,118.3$ (t, $\left.{ }^{1} J_{\mathrm{C}-\mathrm{F}}=255.4 \mathrm{~Hz}\right), 109.4,108.6,42.6,42.1,41.9\left(\mathrm{t},{ }^{2} J_{\mathrm{C}-\mathrm{F}}=21.9 \mathrm{~Hz}\right), 41.3,40.3,26.9,22.4,21.8,21.3$, 11.3. ${ }^{19} \mathrm{~F}$ NMR $\left(376 \mathrm{MHz}, \mathrm{CDCl}_{3}\right) \delta-101.0--105.3(\mathrm{~m}, 2 \mathrm{~F})$. HRMS ESI $[\mathrm{M}+\mathrm{H}]^{+}$calculated for $\mathrm{C}_{26} \mathrm{H}_{31} \mathrm{~F}_{2} \mathrm{~N}_{2} \mathrm{O}$ 425.2404, found 425.2404 . 


\section{3-(1,6-Dimethyl-9-(p-tolyl)-2,3-dihydro-1H-pyrrolo[1,2-a]indol-1-yl)-2,2-difluoro- $N$ -}

\section{Phenylpropanamide (8b)}

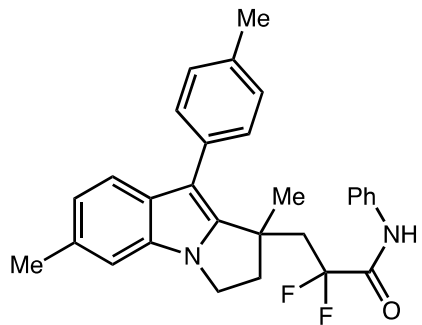

Following the general procedure B, 2-bromo-2,2-difluoro- $N$ phenylacetamide was used as radical precursor, purification by flash column chromatography (3-10\% EtOAc in hexane), the product (39.1 mg) was obtained in $44 \%$ yield as white solid. ${ }^{1} \mathrm{H}$ NMR $\left(400 \mathrm{MHz}, \mathrm{CDCl}_{3}\right) \delta$ 7.66 (brs, 1H), $7.36-7.32$ (m, 2H), $7.31-7.28$ (m, 1H), $7.28-7.20(\mathrm{~m}$, 4H), $7.15-7.12(\mathrm{~m}, 2 \mathrm{H}), 7.10-7.04(\mathrm{~m}, 1 \mathrm{H}), 6.96(\mathrm{~s}, 1 \mathrm{H}), 6.83-6.79(\mathrm{~m}, 1 \mathrm{H}), 4.09-3.92(\mathrm{~m}, 2 \mathrm{H})$, $2.77-2.71(\mathrm{~m}, 1 \mathrm{H}), 2.64(\mathrm{dt}, J=22.9,13.7 \mathrm{~Hz}, 1 \mathrm{H}), 2.56-2.42(\mathrm{~m}, 1 \mathrm{H}), 2.39(\mathrm{~s}, 3 \mathrm{H}), 2.28(\mathrm{~s}, 3 \mathrm{H})$, $1.48(\mathrm{~s}, 3 \mathrm{H}) .{ }^{13} \mathrm{C}$ NMR $\left(101 \mathrm{MHz}, \mathrm{CDCl}_{3}\right) \delta 162.1\left(\mathrm{t},{ }^{2} J_{\mathrm{C}-\mathrm{F}}=28.7 \mathrm{~Hz}\right), 144.9,135.9,135.8,132.3$, $131.9,131.3,130.2,130.2(2 \mathrm{C}), 129.3$ (2C), $129.2(2 \mathrm{C}), 125.7,121.3,120.4(2 \mathrm{C}), 119.4,118.2\left(\mathrm{t},{ }^{1} J_{\mathrm{C}}-\right.$ $\mathrm{F}=256.6 \mathrm{~Hz}), 109.5,108.7,42.7,42.1,41.7\left(\mathrm{t},{ }^{2} J_{\mathrm{C}-\mathrm{F}}=21.8 \mathrm{~Hz}\right), 40.3,27.2,21.8,21.3 .{ }^{19} \mathrm{~F}$ NMR $(376$ $\left.\mathrm{MHz}, \mathrm{CDCl}_{3}\right) \delta-100.3--104.1(\mathrm{~m}, 2 \mathrm{~F})$. HRMS ESI $[\mathrm{M}+\mathrm{H}]^{+}$calculated for $\mathrm{C}_{29} \mathrm{H}_{29} \mathrm{~F}_{2} \mathrm{~N}_{2} \mathrm{O} 459.2248$, found 459.2249 .

3-(1,6-Dimethyl-9-(p-tolyl)-2,3-dihydro-1H-pyrrolo[1,2-a]indol-1-yl)-2,2-difluoro-1-(piperidin-1yl)propan-1-one (8c)

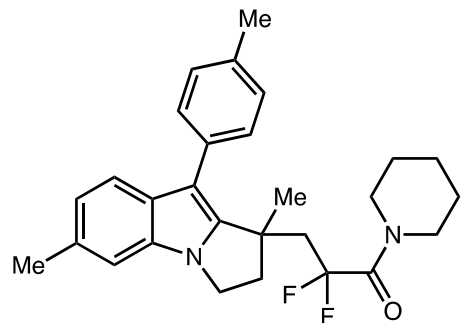

Following the general procedure B, 2-bromo-2,2-difluoro-1-(piperidin1-yl)ethan-1-one was used as radical precursor, purification by flash column chromatography (3-8\% EtOAc in hexane), the product ( 40.5 $\mathrm{mg}$ ) was obtained in $46 \%$ yield as colourless oil. ${ }^{1} \mathrm{H}$ NMR (600 MHz, $\left.\mathrm{CDCl}_{3}\right) \delta 7.43-7.42(\mathrm{~m}, 1 \mathrm{H}), 7.40-7.35(\mathrm{~m}, 2 \mathrm{H}), 7.25-7.23(\mathrm{~m}, 2 \mathrm{H})$, $7.10-7.07$ (m, 1H), $6.94-6.92(\mathrm{~m}, 1 \mathrm{H}), 4.18-4.13(\mathrm{~m}, 1 \mathrm{H}), 4.12-4.07(\mathrm{~m}, 1 \mathrm{H}), 3.58-3.53(\mathrm{~m}$, $1 \mathrm{H}), 3.52(\mathrm{t}, J=5.7 \mathrm{~Hz}, 1 \mathrm{H}), 3.49-3.44(\mathrm{~m}, 2 \mathrm{H}), 2.84-2.78(\mathrm{~m}, 1 \mathrm{H}), 2.77-2.66(\mathrm{~m}, 1 \mathrm{H}), 2.58-2.52$ (m, 1H), $2.50(\mathrm{~s}, 3 \mathrm{H}), 2.49-2.43(\mathrm{~m}, 1 \mathrm{H}), 2.42(\mathrm{~s}, 3 \mathrm{H}), 1.67-1.63(\mathrm{~m}, 2 \mathrm{H}), 1.61-1.54(\mathrm{~m}, 5 \mathrm{H}), 1.53$ $-1.44(\mathrm{~m}, 2 \mathrm{H}) .{ }^{13} \mathrm{C} \mathrm{NMR}\left(151 \mathrm{MHz}, \mathrm{CDCl}_{3}\right) \delta 162.2\left(\mathrm{t},{ }^{2} J_{\mathrm{C}-\mathrm{F}}=28.6 \mathrm{~Hz}\right), 146.0,135.7,132.2,132.1$, 131.1, 130.2 (2C), 129.1 (2C), 121.2, 121.2, 119.5 (t, $\left.{ }^{1} J_{\mathrm{C}-\mathrm{F}}=254.8 \mathrm{~Hz}\right), 119.3,109.5,108.4,47.0\left(\mathrm{t},{ }^{3} \mathrm{~J}\right.$ C-F $=6.1 \mathrm{~Hz}), 44.6,43.0,42.6\left(\mathrm{t},{ }^{2} \mathrm{~J}_{\mathrm{C}-\mathrm{F}}=21.5 \mathrm{~Hz}\right), 42.2,40.7,26.7,26.5,25.7,24.5,21.8,21.3 .{ }^{19} \mathrm{~F}$ NMR $\left(565 \mathrm{MHz}, \mathrm{CDCl}_{3}\right) \delta-96.2--98.0(\mathrm{~m}, 2 \mathrm{~F})$. HRMS ESI $[\mathrm{M}+\mathrm{H}]^{+}$calculated for $\mathrm{C}_{28} \mathrm{H}_{33} \mathrm{~F}_{2} \mathrm{~N}_{2} \mathrm{O}$ 451.2561, found 451.2561.

Ethyl 3-(9-(3,5-difluorophenyl)-6-methoxy-1-methyl-2,3-dihydro-1H-pyrrolo[1,2-a]indol-1-yl)2,2-difluoropropanoate (8d)

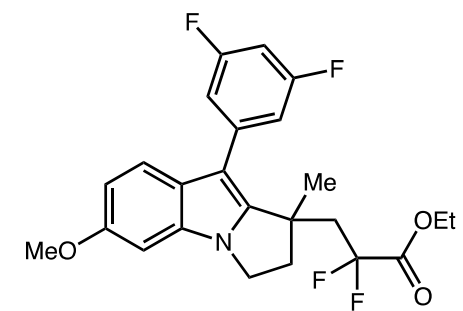

Following the general procedure B, ethyl 2-bromo-2,2-difluoroacetate was used as radical precursor, purification by flash column chromatography (3-8\% EtOAc in hexane), the product (48.4 mg) was obtained in 54\% yield as colourless oil. ${ }^{1} \mathrm{H}$ NMR $\left(400 \mathrm{MHz}, \mathrm{CDCl}_{3}\right) \delta$ $7.42-7.37(\mathrm{~m}, 1 \mathrm{H}), 7.02-6.94(\mathrm{~m}, 2 \mathrm{H}), 6.81-6.78(\mathrm{~m}, 1 \mathrm{H}), 6.77-$ $6.75(\mathrm{~m}, 1 \mathrm{H}), 6.74-6.73(\mathrm{~m}, 1 \mathrm{H}), 4.20-4.13(\mathrm{~m}, 1 \mathrm{H}), 4.11-3.99(\mathrm{~m}, 3 \mathrm{H}), 3.88(\mathrm{~s}, 3 \mathrm{H}), 2.85-2.77$ 
(m, 1H), $2.64(\mathrm{dt}, J=18.7,15.2 \mathrm{~Hz}, 1 \mathrm{H}), 2.57-2.38(\mathrm{~m}, 2 \mathrm{H}), 1.53-1.50(\mathrm{~m}, 3 \mathrm{H}), 1.21(\mathrm{t}, J=7.1 \mathrm{~Hz}$, $3 \mathrm{H}) .{ }^{13} \mathrm{C}$ NMR $\left(101 \mathrm{MHz}, \mathrm{CDCl}_{3}\right) \delta 164.0\left(\mathrm{t},{ }^{2} J_{\mathrm{C}-\mathrm{F}}=32.7 \mathrm{~Hz}\right), 163.2\left(\mathrm{~d},{ }^{1} J_{\mathrm{C}-\mathrm{F}}=248.7 \mathrm{~Hz}\right), 163.0(\mathrm{~d}$, $\left.{ }^{1} J_{\mathrm{C}-\mathrm{F}}=248.7 \mathrm{~Hz}\right), 156.6,144.6,138.5\left(\mathrm{t},{ }^{3} J_{\mathrm{C}-\mathrm{F}}=10.3 \mathrm{~Hz}\right), 132.6,125.7,120.0,116.1\left(\mathrm{t},{ }^{1} J_{\mathrm{C}-\mathrm{F}}=252.9\right.$ $\mathrm{Hz}), 112.8\left(\mathrm{~d},{ }^{3} J_{\mathrm{C}-\mathrm{F}}=11.4 \mathrm{~Hz}, 1 \mathrm{C}\right), 112.7\left(\mathrm{~d},{ }^{2} J_{\mathrm{C}-\mathrm{F}}=24.8 \mathrm{~Hz}\right), 110.2,107.1\left(\mathrm{t},{ }^{4} \mathrm{~J}_{\mathrm{C}-\mathrm{F}}=2.6 \mathrm{~Hz}\right), 101.7$ $\left(\mathrm{t},{ }^{2} J_{\mathrm{C}-\mathrm{F}}=25.5 \mathrm{~Hz}\right), 93.3,63.3,55.9,43.5\left(\mathrm{t},{ }^{2} J_{\mathrm{C}-\mathrm{F}}=22.3 \mathrm{~Hz}\right), 42.7,42.3,40.4\left(\mathrm{t},{ }^{4} J_{\mathrm{C}-\mathrm{F}}=2.4 \mathrm{~Hz}\right), 26.9$, 13.8. ${ }^{19} \mathrm{~F}$ NMR (376 MHz, $\left.\mathrm{CDCl}_{3}\right) \delta-100.2--103.6(\mathrm{~m}, 2 \mathrm{~F}),-110.3--110.5(\mathrm{~m}, 2 \mathrm{~F})$. HRMS ESI $[\mathrm{M}+\mathrm{H}]^{+}$calculated for $\mathrm{C}_{24} \mathrm{H}_{24} \mathrm{~F}_{4} \mathrm{NO}_{3} 450.1692$, found 450.1683 .

\section{Ethyl 3-(6-(tert-butyl)-9-(3-fluorophenyl)-1-methyl-2,3-dihydro-1H-pyrrolo[1,2-a]indol-1-yl)-}

\section{2,2-difluoropropanoate (8e)}

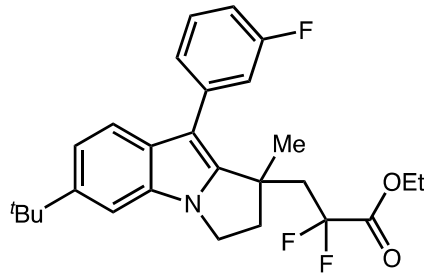

Following the general procedure B, ethyl 2-bromo-2,2-difluoroacetate was used as radical precursor, purification by flash column chromatography (3-8\% EtOAc in hexane), the product $(50.1 \mathrm{mg})$ was obtained in $55 \%$ yield as colourless oil. ${ }^{1} \mathrm{H}$ NMR $\left(400 \mathrm{MHz}, \mathrm{CDCl}_{3}\right) \delta$ $7.50-7.45(\mathrm{~m}, 1 \mathrm{H}), 7.43-7.32(\mathrm{~m}, 1 \mathrm{H}), 7.28-7.24(\mathrm{~m}, 2 \mathrm{H}), 7.24-7.17$ (m, 2H), $7.06-6.99(\mathrm{~m}, 1 \mathrm{H}), 4.25-4.18(\mathrm{~m}, 1 \mathrm{H}), 4.17-4.10(\mathrm{~m}, 2 \mathrm{H}), 4.08-4.00(\mathrm{~m}, 1 \mathrm{H}), 2.81$ (dt, $J=12.9,7.4 \mathrm{~Hz}, 1 \mathrm{H}), 2.66(\mathrm{dt}, J=19.4,15.4 \mathrm{~Hz}, 1 \mathrm{H}), 2.58-2.50(\mathrm{~m}, 1 \mathrm{H}), 2.43$ (dt, $J=20.5,15.5 \mathrm{~Hz}$, $1 \mathrm{H}), 1.53(\mathrm{~d}, J=1.3 \mathrm{~Hz}, 3 \mathrm{H}), 1.42(\mathrm{~s}, 9 \mathrm{H}), 1.22(\mathrm{t}, J=7.2 \mathrm{~Hz}, 3 \mathrm{H}) .{ }^{13} \mathrm{C} \mathrm{NMR}\left(101 \mathrm{MHz}, \mathrm{CDCl}_{3}\right) \delta$ $164.1\left(\mathrm{t},{ }^{2} J_{\mathrm{C}-\mathrm{F}}=32.7 \mathrm{~Hz}, 1 \mathrm{C}\right), 163.0\left(\mathrm{~d},{ }^{1} J_{\mathrm{C}-\mathrm{F}}=246.5 \mathrm{~Hz}\right), 145.6,145.5,137.4\left(\mathrm{~d},{ }^{3} J_{\mathrm{C}-\mathrm{F}}=8.4 \mathrm{~Hz}\right)$, $132.0,129.8\left(\mathrm{~d},{ }^{3} J_{\mathrm{C}-\mathrm{F}}=8.7 \mathrm{~Hz}\right), 129.6,126.0\left(\mathrm{~d},{ }^{4} J_{\mathrm{C}-\mathrm{F}}=2.7 \mathrm{~Hz}\right), 118.9,118.3,116.9\left(\mathrm{~d},{ }^{2} J_{\mathrm{C}-\mathrm{F}}=21.0\right.$ $\mathrm{Hz}), 116.2\left(\mathrm{t},{ }^{1} J_{\mathrm{C}-\mathrm{F}}=252.7 \mathrm{~Hz}\right), 113.1\left(\mathrm{~d},{ }^{2} J_{\mathrm{C}-\mathrm{F}}=21.1 \mathrm{~Hz}\right), 107.6\left(\mathrm{~d},{ }^{4} J_{\mathrm{C}-\mathrm{F}}=2.2 \mathrm{~Hz}\right), 105.9,63.2,43.2$ $\left(\mathrm{t},{ }^{2} J_{\mathrm{C}-\mathrm{F}}=22.1 \mathrm{~Hz}\right), 42.8,42.3,40.4,35.0,32.0(3 \mathrm{C}), 26.6,13.8 .{ }^{19} \mathrm{~F} \mathrm{NMR}\left(376 \mathrm{MHz}, \mathrm{CDCl}_{3}\right) \delta-100.4$ - -103.5 (m, 2F), -113.4 - -113.6 (m, F). HRMS ESI $[\mathrm{M}+\mathrm{H}]^{+}$calculated for $\mathrm{C}_{27} \mathrm{H}_{31} \mathrm{~F}_{3} \mathrm{NO}_{2}$ 458.2307, found 458.2310 .

(6-Methoxy-1-methyl-1-(2,2,2-trifluoroethyl)-2,3-dihydro-1H-pyrrolo[1,2-a]indol-9-yl)(4nitrophenyl)methanone (10a)

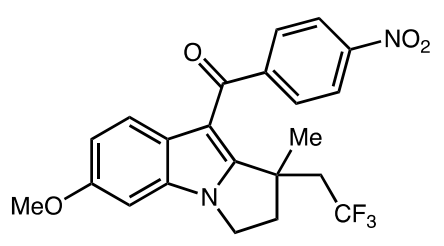

Following the general procedure B, ethyl 2-bromo-2,2-difluoroacetate was used as radical precursor, the product $(43.9 \mathrm{mg})$ was obtained in $51 \%$ yield as yellow solid. ${ }^{1} \mathrm{H}$ NMR $\left(600 \mathrm{MHz}, \mathrm{CDCl}_{3}\right) \delta 8.32(\mathrm{~d}, J=$ $8.7 \mathrm{~Hz}, 2 \mathrm{H}), 7.86(\mathrm{~d}, J=8.7 \mathrm{~Hz}, 2 \mathrm{H}), 6.77(\mathrm{~m}, 1 \mathrm{H}), 6.67(\mathrm{~d}, J=2.3 \mathrm{~Hz}$, $1 \mathrm{H}), 6.62-6.59(\mathrm{~m}, 1 \mathrm{H}), 4.28-4.16(\mathrm{~m}, 2 \mathrm{H}), 3.84(\mathrm{~s}, 3 \mathrm{H}), 3.13-3.02(\mathrm{~m}, 2 \mathrm{H}), 3.01-2.96(\mathrm{~m}, 1 \mathrm{H})$, $2.65-2.59(\mathrm{~m}, 1 \mathrm{H}), 1.68(\mathrm{~s}, 3 \mathrm{H}) .{ }^{13} \mathrm{C}$ NMR $\left(101 \mathrm{MHz}, \mathrm{CDCl}_{3}\right) \delta 189.5,157.1,156.7,149.4,147.1$, $132.9,129.7$ (2C), $126.8\left(\mathrm{q},{ }^{1} J_{\mathrm{C}-\mathrm{F}}=279.5 \mathrm{~Hz}\right) 124.5,123.8(2 \mathrm{C}), 121.8,111.7,107.9,94.3,55.8,43.6$, $41.7\left(\mathrm{q},{ }^{4} J_{\mathrm{C}-\mathrm{F}}=2.0 \mathrm{~Hz}\right), 40.5\left(\mathrm{q},{ }^{2} J_{\mathrm{C}-\mathrm{F}}=26.9 \mathrm{~Hz}\right), 40.5,25.1 .{ }^{19} \mathrm{~F} \mathrm{NMR}\left(565 \mathrm{MHz}, \mathrm{CDCl}_{3}\right) \delta-60.71(\mathrm{t}$, $J=11.1 \mathrm{~Hz}, 3 \mathrm{~F}$ ). HRMS ESI $[\mathrm{M}+\mathrm{H}]^{+}$calculated for $\mathrm{C}_{22} \mathrm{H}_{20} \mathrm{~F}_{3} \mathrm{~N}_{2} \mathrm{O}_{4} 433.1375$, found 433.1376 . 


\section{Unsuccessful substrates}

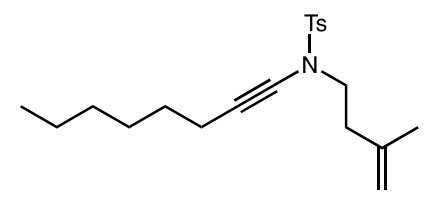

complex reaction mixture

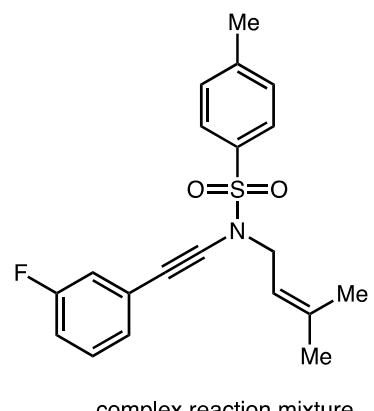

complex reaction mixture

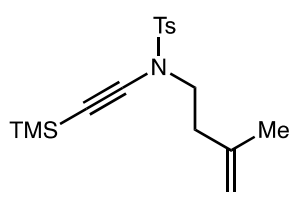

complex reaction mixture

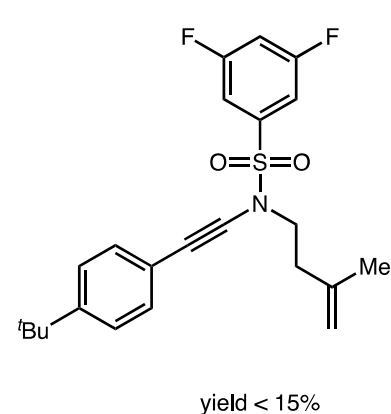

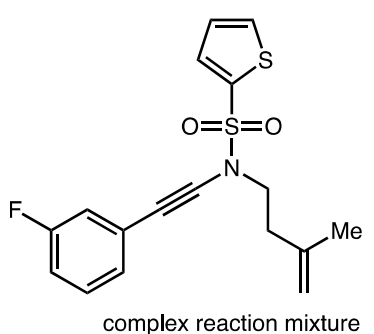

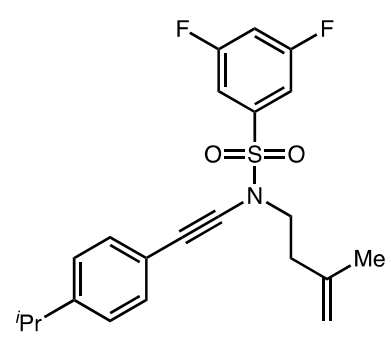

yield $<15 \%$ 


\section{Mechanistic Investigations}

\section{a. Radical Trapping experiments}

(a) Reaction with 1.0 equiv of TEMPO

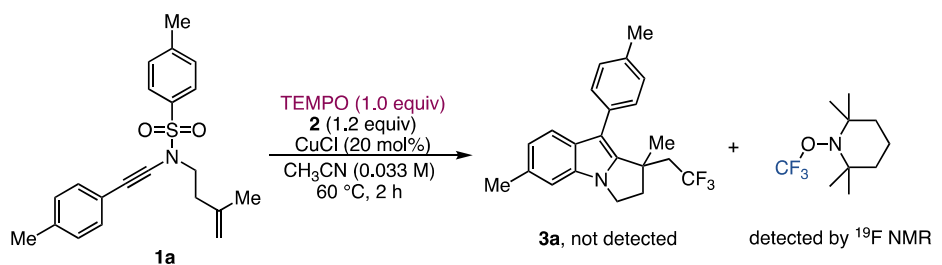

SF1754-2.10.fid
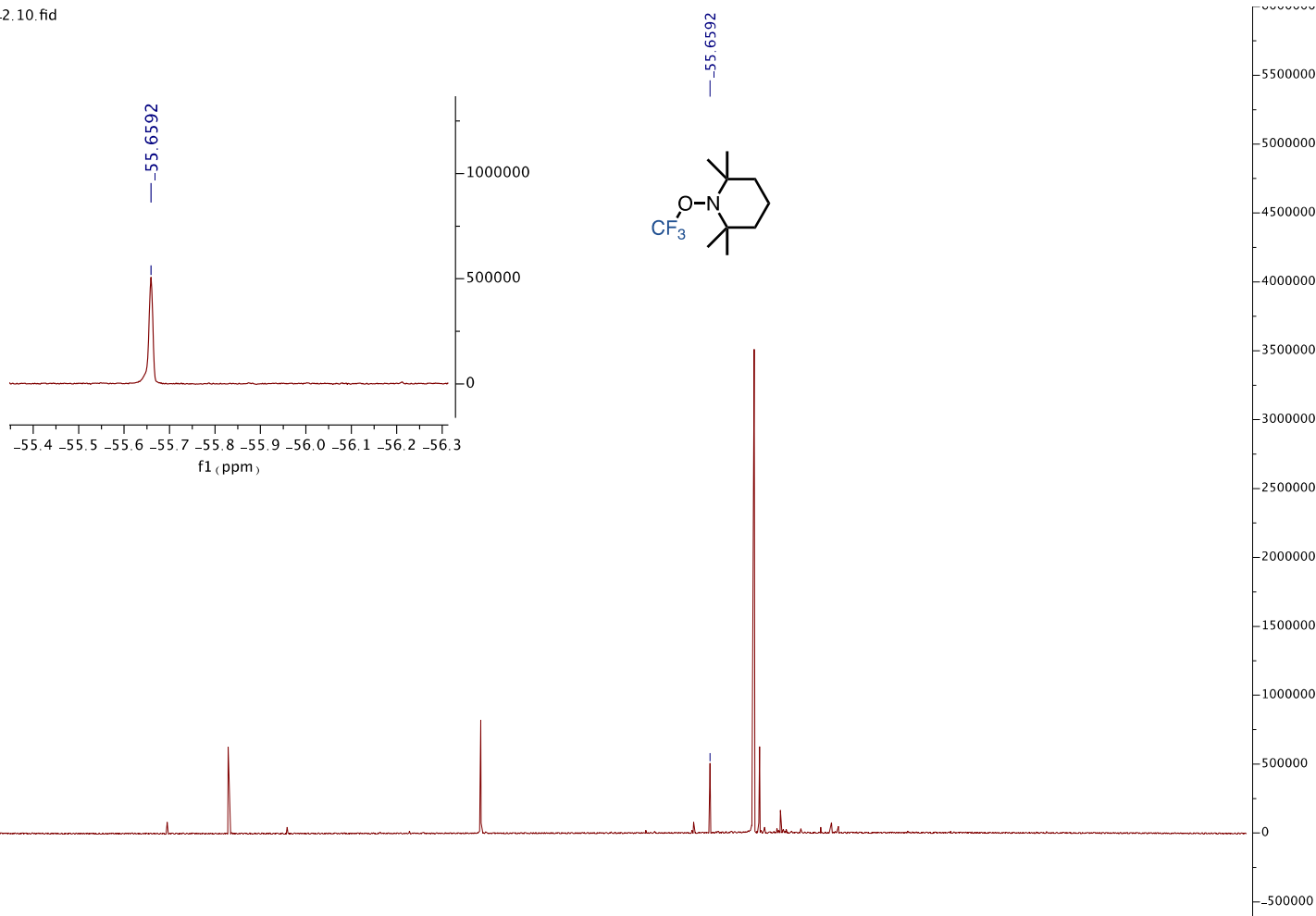

15

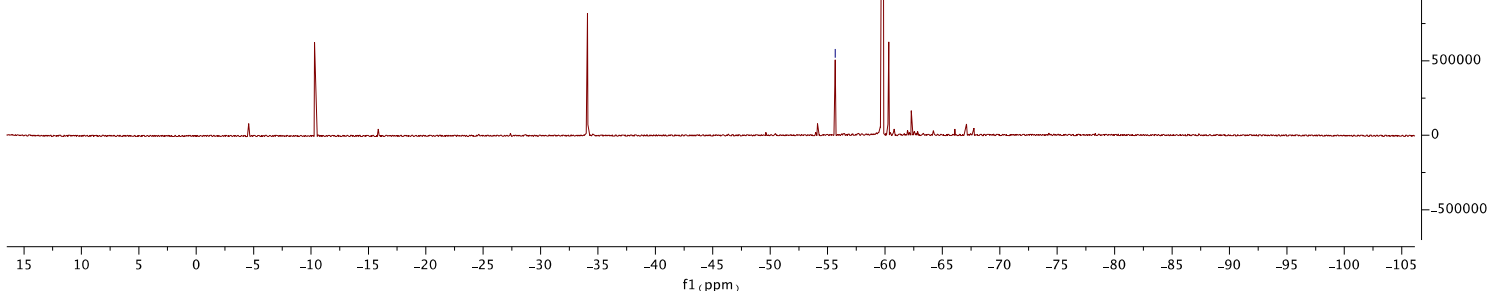

Figure S1. ${ }^{19} \mathrm{~F}$ NMR of the crude reaction mixture 
(b) Reaction with 2.5 equiv of 2
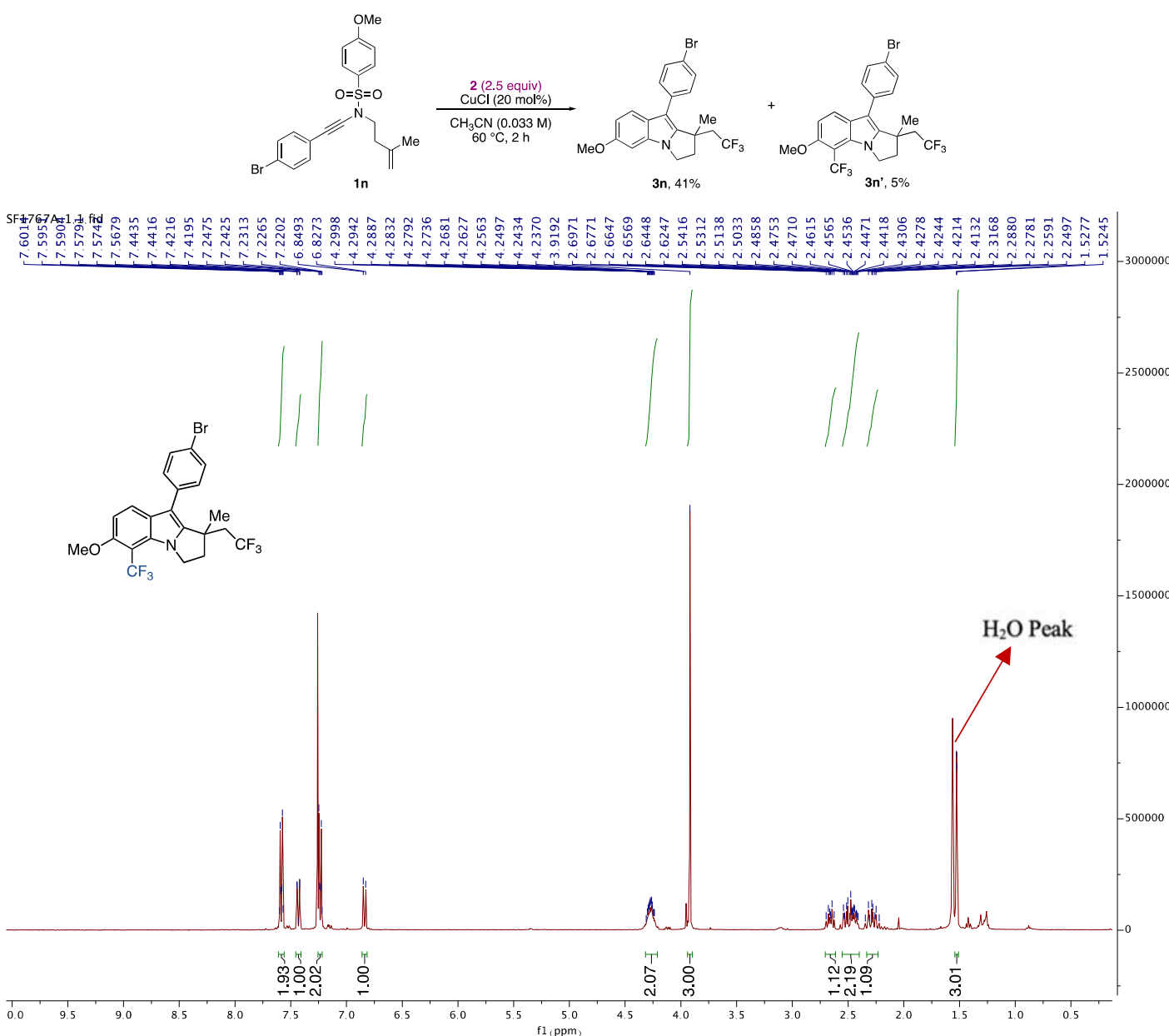

Figure S2. 'H NMR data of compound 3n'

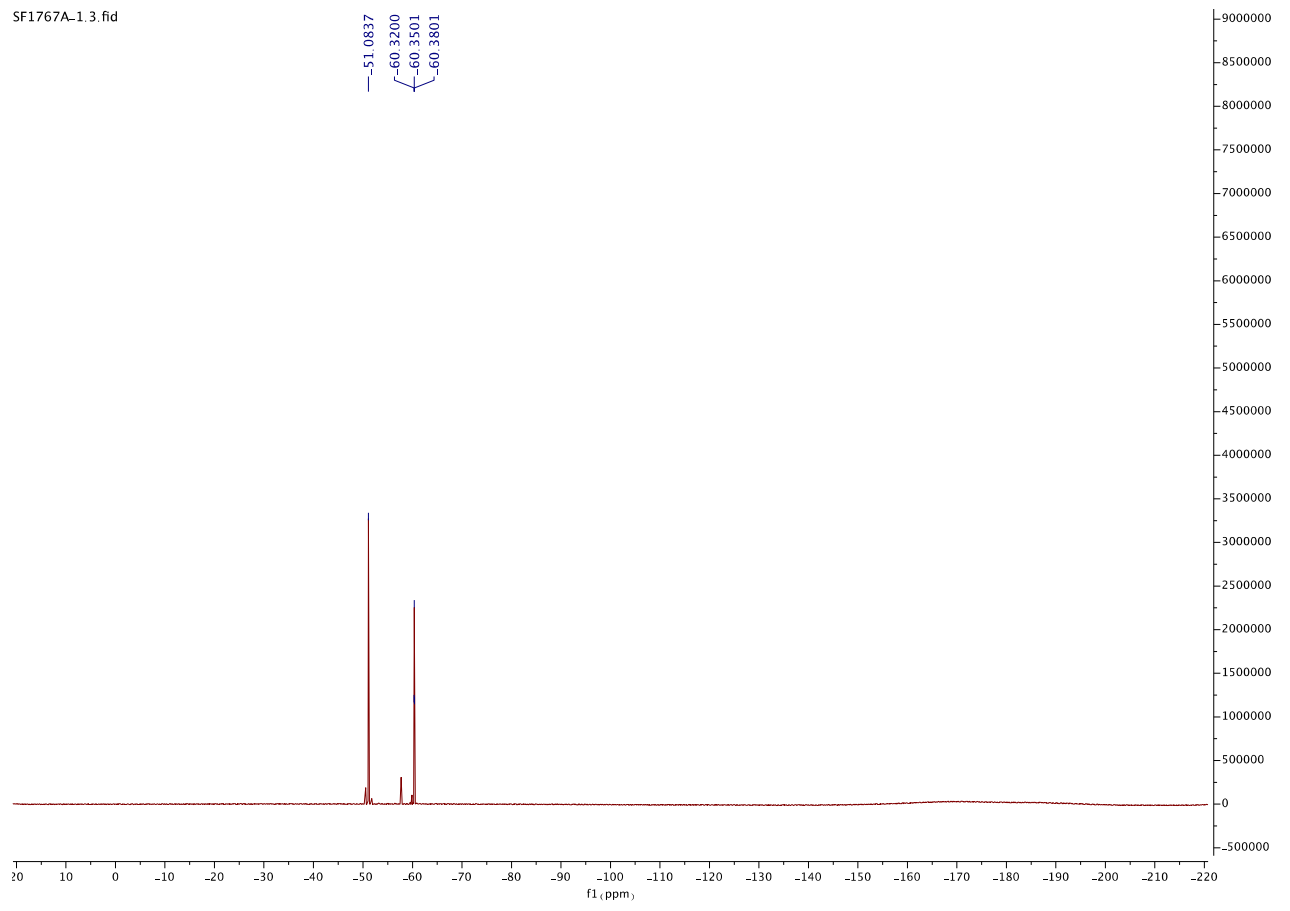

Figure S3. ${ }^{19} \mathrm{~F}$ NMR data of compound 3n' 
3n', Light yellow solid. ${ }^{1} \mathrm{H}$ NMR (400 MHz, $\left.\mathrm{CDCl}_{3}\right) \delta 7.61-7.56(\mathrm{~m}, 2 \mathrm{H}), 7.43(\mathrm{~d}, J=8.8 \mathrm{~Hz}, 1 \mathrm{H})$, $7.25-7.21(\mathrm{~m}, 2 \mathrm{H}), 6.84(\mathrm{~d}, J=8.8 \mathrm{~Hz}, 1 \mathrm{H}), 4.32-4.22(\mathrm{~m}, 2 \mathrm{H}), 3.92(\mathrm{~s}, 3 \mathrm{H}), 2.71-2.61(\mathrm{~m}, 1 \mathrm{H})$, $2.56-2.40(\mathrm{~m}, 2 \mathrm{H}), 2.35-2.23(\mathrm{~m}, 1 \mathrm{H}), 1.53(\mathrm{~s}, 3 \mathrm{H}) .{ }^{19} \mathrm{~F} \mathrm{NMR}\left(376 \mathrm{MHz}, \mathrm{CDCl}_{3}\right) \delta-51.08(\mathrm{~s}, 3 \mathrm{~F}),-$ $60.30--60.40(\mathrm{t}, J=11.3 \mathrm{~Hz}, 3 \mathrm{~F})$. HRMS ESI $[\mathrm{M}+\mathrm{H}]^{+}$calculated for $\mathrm{C}_{22} \mathrm{H}_{20} \mathrm{~F}_{3} \mathrm{~N}_{2} \mathrm{O}_{4} 433.1375$, found 433.1376.

\section{b. DFT Calculations of Radical and Cationic Pathways for Arylation Step}
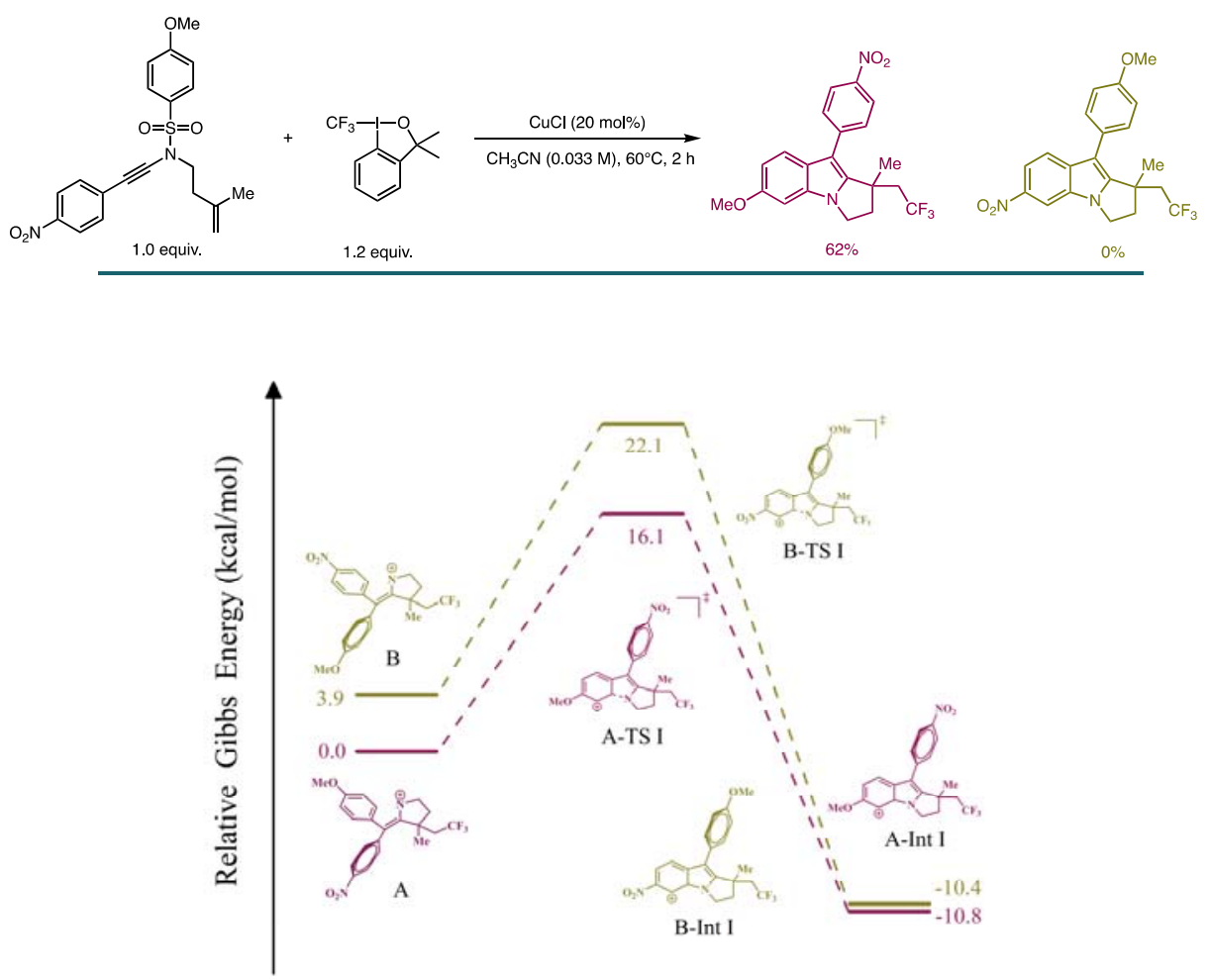

Figure S4. Aza-Nazarov type cyclization

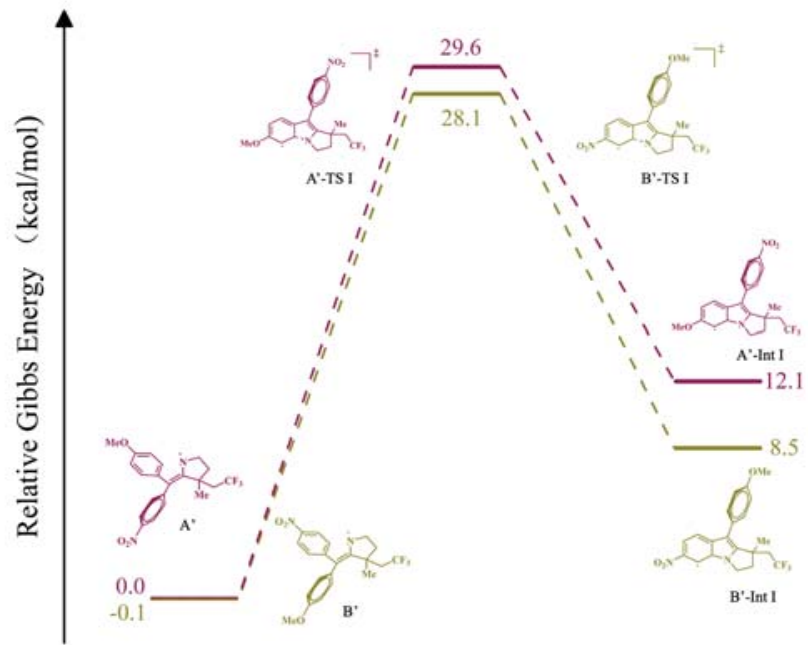

Figure S5. N-centered radical cyclization 

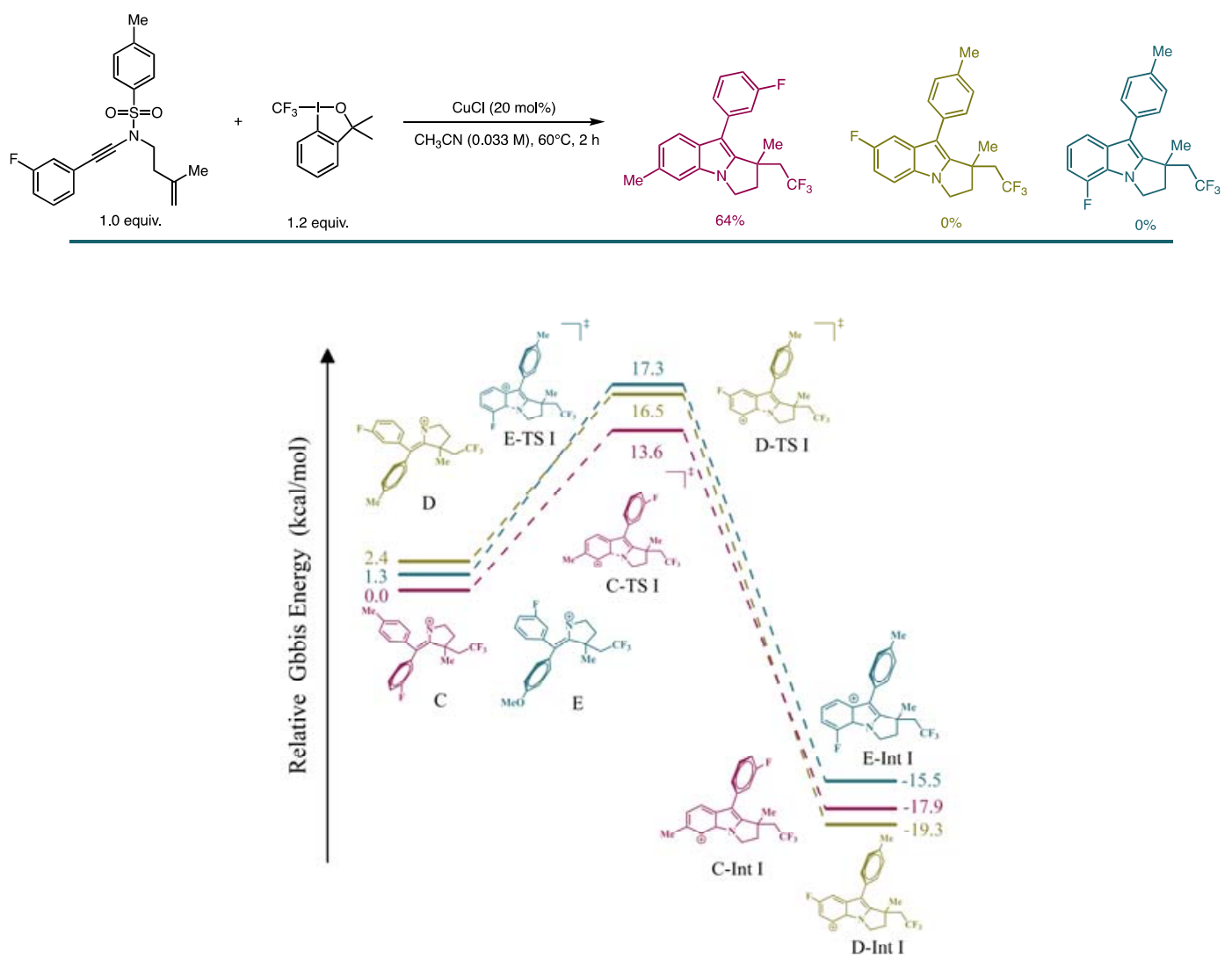

Figure S6. Aza-Nazarov type cyclization

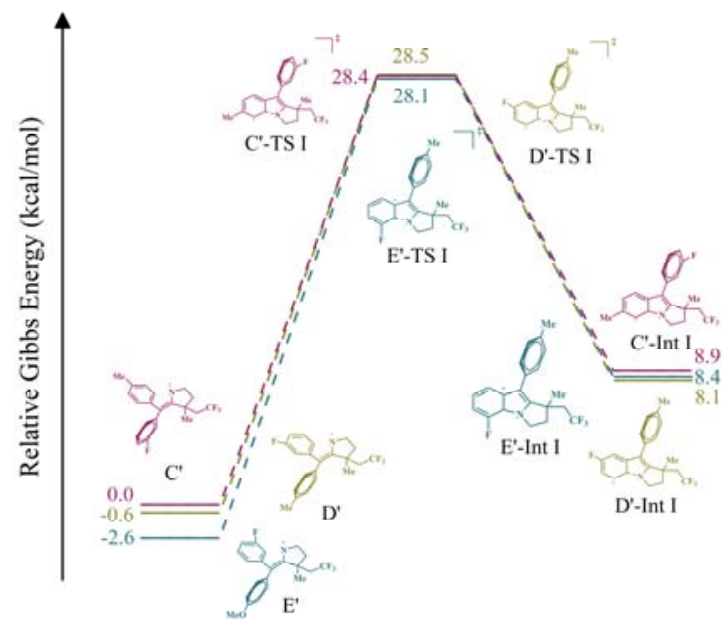

Figure S7. N-centered radical cyclization

\section{Computational details}

All calculations were performed Gaussian 16 software package. All intermediate and transition state geometric optimizations were optimized at the M06-2XD3/def2-SVP level in acetonitrile using SMD solvation model. An aza-Nazarov type cyclization of the cation generated on single-electron oxidation 
of the N-centered radical is the favoured pathway (Figure S4, S6), which correlates well to the experimentally outcomes.

\section{Calculated Cartesian Coordinates}

Structure 1. A

\begin{tabular}{|c|c|c|c|}
\hline & 0.12550300 & 3.57463500 & -2.79057100 \\
\hline & 2.03156000 & 3.16350300 & -1.89605500 \\
\hline & 0.71047100 & 4.59806300 & -0.99267100 \\
\hline & -0.63886700 & 1.33763100 & 2.51783200 \\
\hline C & 2.99550000 & -2.51246400 & -0.04744600 \\
\hline & 2.81787500 & -1.96783500 & 1.22058700 \\
\hline & 3.56663000 & -2.12175800 & 1.99575000 \\
\hline & 1.67467600 & -1.21709100 & 1.45800000 \\
\hline & 1.51272900 & -0.76465600 & 2.43789700 \\
\hline & 0.71300500 & -1.05805400 & 0.44516100 \\
\hline & -0.47802100 & -0.23861100 & 0.71904800 \\
\hline & -1.76889000 & -0.63144400 & 0.40415400 \\
\hline & -2.06339900 & -1.98497200 & 0.00671900 \\
\hline & -1.26275300 & -2.72477300 & -0.01297000 \\
\hline & -3.33662200 & -2.38218200 & -0.28759700 \\
\hline & -3.53658900 & -3.41748900 & -0.55806100 \\
\hline & -4.39502700 & -1.43155400 & -0.20890300 \\
\hline & 2.07834900 & -2.35173400 & -1.07890400 \\
\hline & 2.26929200 & -2.78616900 & -2.05879700 \\
\hline & 0.92041500 & -1.62555100 & -0.82311500 \\
\hline & 0.19222000 & -1.46789300 & -1.62035200 \\
\hline & -2.86861400 & 0.30050500 & 0.49386200 \\
\hline & -4.13667200 & -0.08491500 & 0.18666500 \\
\hline & -4.97666800 & 0.60846700 & 0.22671800 \\
\hline & -0.22301000 & 1.08330000 & 1.34478700 \\
\hline & 0.57099900 & 2.19760200 & 0.63960300 \\
\hline & 0.13937900 & 3.37471900 & 1.54502800 \\
\hline & 0.92418600 & 4.13492400 & 1.64145800 \\
\hline & -0.75813400 & 3.85262700 & 1.12533600 \\
\hline & -0.21558500 & 2.69511700 & 2.87140400 \\
\hline & 0.64648700 & 2.61492400 & 3.55387500 \\
\hline & -1.01965900 & 3.19825500 & 3.42491800 \\
\hline & 2.08322700 & 1.94242800 & 0.74708900 \\
\hline
\end{tabular}




$\begin{array}{llll}\mathrm{H} & 2.35642200 & 1.69508400 & 1.78353700 \\ \mathrm{H} & 2.63250800 & 2.84967200 & 0.45975000 \\ \mathrm{H} & 2.40852400 & 1.12274100 & 0.09056700 \\ \mathrm{C} & 0.09880300 & 2.31377400 & -0.81540100 \\ \mathrm{H} & 0.30083500 & 1.38146300 & -1.36779200 \\ \mathrm{H} & -0.98506800 & 2.50065900 & -0.84718700 \\ \mathrm{C} & 0.74535400 & 3.41292400 & -1.61538100 \\ \mathrm{H} & -2.67411400 & 1.33360100 & 0.78218900 \\ \mathrm{~N} & 4.22304300 & -3.29134600 & -0.31283300 \\ \mathrm{O} & 4.38388700 & -3.71938500 & -1.43039300 \\ \mathrm{O} & 4.99172100 & -3.45404600 & 0.60404100 \\ \mathrm{O} & -5.63572600 & -1.71521500 & -0.47444400 \\ \mathrm{C} & -6.02093100 & -3.03373500 & -0.87827800 \\ \mathrm{H} & -7.10063500 & -2.98859800 & -1.04523700 \\ \mathrm{H} & -5.50596500 & -3.30986600 & -1.80781200 \\ \mathrm{H} & -5.79122000 & -3.75320500 & -0.08123200\end{array}$

\section{Structure 2. A-TS 1}

F

$-3.64361800 \quad-2.38015000 \quad-1.83323700$

F $\quad-3.80705200 \quad-2.45911600 \quad 0.30439600$

F $\quad-2.70308900 \quad-3.97645700 \quad-0.74318300$

$\mathrm{N} \quad \begin{array}{llll}1.59556400 & -1.95368400 & 0.72399200\end{array}$

$\begin{array}{llll}\text { C } & -2.27864000 & 3.06309600 & 0.12489100\end{array}$

$\begin{array}{llll}\mathrm{C} & -2.04045600 & 2.32674800 & 1.28049800\end{array}$

$\mathrm{H} \quad-2.60755700 \quad 2.52890900 \quad 2.18768600$

$\begin{array}{llll}\text { C } & -1.06403000 & 1.33806500 & 1.24145900\end{array}$

$\begin{array}{llll}\mathrm{H} & -0.84386100 & 0.75690600 & 2.13802100\end{array}$

$\begin{array}{lllll}\text { C } & -0.34132600 & 1.10494600 & 0.06174900\end{array}$

$\begin{array}{llll}\mathrm{C} & 0.69218500 & 0.05436300 & 0.01480900\end{array}$

C $\quad 1.99496900 \quad 0.25824000 \quad-0.39840500$

C $\quad 2.62454600 \quad 1.55445700 \quad-0.36097300$

$\mathrm{H} \quad 2.00436500 \quad 2.45082700 \quad-0.31939200$

$\begin{array}{llll}\text { C } & 3.97056900 & 1.64559700 & -0.20969200\end{array}$

$\mathrm{H} \quad 4.43382000 \quad 2.62276400 \quad-0.08361500$

$\begin{array}{llll}\text { C } & 4.78642000 & 0.44966000 & -0.17252400\end{array}$

C $\quad \begin{array}{llll}\text { C } & -1.57658500 & 2.86219700 & -1.05811600\end{array}$

$\mathrm{H} \quad-1.79816600 \quad 3.46350500 \quad-1.93822800$ 


$\begin{array}{lrrr}\mathrm{C} & -0.59651900 & 1.87646600 & -1.08244300 \\ \mathrm{H} & -0.03692200 & 1.68776200 & -2.00021100 \\ \mathrm{C} & 2.84153300 & -0.92915000 & -0.52981500 \\ \mathrm{C} & 4.22562100 & -0.80947700 & -0.34903600 \\ \mathrm{H} & 4.88751900 & -1.66417600 & -0.48546900 \\ \mathrm{C} & 0.50045300 & -1.31953900 & 0.45251100 \\ \mathrm{C} & -0.74892200 & -2.20552600 & 0.49941500 \\ \mathrm{C} & -0.06644000 & -3.58280700 & 0.27573700 \\ \mathrm{H} & -0.61384900 & -4.39866100 & 0.76248800 \\ \mathrm{H} & -0.00967100 & -3.79514900 & -0.80198700 \\ \mathrm{C} & 1.34627400 & -3.38683400 & 0.83319200 \\ \mathrm{H} & 1.44145800 & -3.65810500 & 1.89678800 \\ \mathrm{H} & 2.12681200 & -3.93470300 & 0.28727600 \\ \mathrm{C} & -1.39340500 & -2.14230500 & 1.89458000 \\ \mathrm{H} & -0.62845600 & -2.24861900 & 2.67750900 \\ \mathrm{H} & -2.11095900 & -2.96620900 & 2.00683900 \\ \mathrm{H} & -1.92987800 & -1.19676600 & 2.05029000 \\ \mathrm{C} & -1.72148900 & -1.81705400 & -0.62217500 \\ \mathrm{H} & -2.07731900 & -0.78378200 & -0.49699000 \\ \mathrm{H} & -1.21848200 & -1.88885200 & -1.59797000 \\ \mathrm{C} & -2.96343800 & -2.66315500 & -0.71628700 \\ \mathrm{H} & 2.49642200 & -1.73395000 & -1.18564400 \\ \mathrm{O} & 6.09740800 & 0.51045100 & -0.05355900 \\ \mathrm{C} & 6.77205200 & 1.76578700 & -0.02026700 \\ \mathrm{H} & 6.47543200 & 2.34422200 & 0.86559500 \\ \mathrm{H} & 7.83889900 & 1.52895400 & 0.03766900 \\ \mathrm{H} & 6.57091000 & 2.34032800 & -0.93488600 \\ \mathrm{H} & -3.32174500 & 4.10723300 & 0.15639200 \\ \mathrm{H} & -3.50369400 & 4.74930700 & -0.85048700 \\ \mathrm{H} & -3.93292000 & 4.25884500 & 1.18718400\end{array}$

\section{Structure 3. A-Int 1}

$\begin{array}{llll}\text { F } & 3.21696900 & 2.88938200 & -1.89592100 \\ \text { F } & 3.27749900 & 3.14114100 & 0.23371600 \\ \text { F } & 1.91776200 & 4.31568100 & -0.94591100 \\ \text { N } & -1.87351800 & 1.54960100 & 0.48335800 \\ \text { C } & 2.81614900 & -2.81191500 & 0.07258100\end{array}$




\begin{tabular}{|c|c|c|c|}
\hline $\mathrm{C}$ & 1.79169200 & -2.92405200 & -0.86057800 \\
\hline $\mathrm{H}$ & 1.87168900 & -3.63567100 & -1.68078200 \\
\hline $\mathrm{C}$ & 0.67929900 & -2.10063000 & -0.72166100 \\
\hline $\mathrm{H}$ & -0.13150300 & -2.15667000 & -1.44990700 \\
\hline $\mathrm{C}$ & 0.60134600 & -1.18452400 & 0.33756300 \\
\hline $\mathrm{C}$ & -0.59043600 & -0.31868200 & 0.46430400 \\
\hline $\mathrm{C}$ & -1.90162400 & -0.72909600 & 0.56176100 \\
\hline $\mathrm{C}$ & -2.52033100 & -2.01974300 & 0.44357100 \\
\hline $\mathrm{H}$ & -1.92745400 & -2.92986600 & 0.54390800 \\
\hline $\mathrm{C}$ & -3.83062800 & -2.07069700 & 0.09228100 \\
\hline $\mathrm{H}$ & -4.29985300 & -3.04604200 & -0.03791000 \\
\hline $\mathrm{C}$ & -4.62602000 & -0.86458800 & -0.16982700 \\
\hline $\mathrm{C}$ & 2.76664600 & -1.92103900 & 1.13942800 \\
\hline $\mathrm{H}$ & 3.58707400 & -1.87424300 & 1.85372200 \\
\hline $\mathrm{C}$ & 1.64636200 & -1.10768300 & 1.26917000 \\
\hline $\mathrm{H}$ & 1.57630700 & -0.41686700 & 2.10997500 \\
\hline $\mathrm{C}$ & -2.78838700 & 0.45940400 & 0.71883400 \\
\hline $\mathrm{C}$ & -4.11667800 & 0.37053300 & 0.05277000 \\
\hline $\mathrm{H}$ & -4.72859900 & 1.26329200 & -0.08114300 \\
\hline $\mathrm{C}$ & -0.63869000 & 1.12232400 & 0.41930200 \\
\hline $\mathrm{C}$ & 0.33087900 & 2.28052000 & 0.36314900 \\
\hline $\mathrm{C}$ & -0.64229900 & 3.43609100 & -0.01999300 \\
\hline $\mathrm{H}$ & -0.32507300 & 4.39362200 & 0.40741700 \\
\hline $\mathrm{H}$ & -0.67772700 & 3.53562100 & -1.11356800 \\
\hline $\mathrm{C}$ & -2.02185100 & 3.00240100 & 0.49471400 \\
\hline $\mathrm{H}$ & -2.23194600 & 3.33622200 & 1.52125200 \\
\hline $\mathrm{H}$ & -2.85024900 & 3.30116900 & -0.15895800 \\
\hline $\mathrm{C}$ & 0.90699700 & 2.49925100 & 1.77418300 \\
\hline $\mathrm{H}$ & 0.09858000 & 2.51496500 & 2.51970900 \\
\hline $\mathrm{H}$ & 1.42242200 & 3.46806200 & 1.81285300 \\
\hline $\mathrm{H}$ & 1.62324000 & 1.71165000 & 2.04135900 \\
\hline $\mathrm{C}$ & 1.41125100 & 2.01265000 & -0.69297700 \\
\hline $\mathrm{H}$ & 1.96178600 & 1.08795300 & -0.46463900 \\
\hline $\mathrm{H}$ & 0.94869600 & 1.89906700 & -1.68395500 \\
\hline $\mathrm{C}$ & 2.45119900 & 3.09367300 & -0.81925900 \\
\hline $\mathrm{H}$ & -3.00087000 & 0.51040000 & 1.81568500 \\
\hline $\mathrm{N}$ & 4.00278500 & -3.67399100 & -0.07370100 \\
\hline
\end{tabular}




$\begin{array}{llll}\mathrm{O} & 4.01157500 & -4.46971600 & -0.98333400 \\ \mathrm{O} & 4.90054600 & -3.53574000 & 0.72353900 \\ \mathrm{O} & -5.89057400 & -0.95902400 & -0.62268000 \\ \mathrm{C} & -6.39869400 & -2.20963300 & -1.04960400 \\ \mathrm{H} & -7.38926700 & -2.00692500 & -1.47174800 \\ \mathrm{H} & -5.76337100 & -2.66072100 & -1.82728700 \\ \mathrm{H} & -6.50829800 & -2.91168100 & -0.20899800\end{array}$

\section{Structure 4. B}$$
\mathrm{F}
$$$$
\begin{array}{lll}
-1.91213200 & -3.03579500 & -2.84079300
\end{array}
$$$$
\text { F } \quad-3.51686200 \quad-2.22605900-1.67123800
$$$$
\text { F } \quad-2.50469500 \quad-4.00523500 \quad-1.01519500
$$$$
\begin{array}{lllll}
\mathrm{N} & 0.07312300 & -1.42893700 & 2.40103600
\end{array}
$$$$
\begin{array}{llll}
\text { C } & -2.27475100 & 3.65142400 & 0.07233800
\end{array}
$$$$
\begin{array}{lllll}
\mathrm{C} & -2.61581300 & 2.71421400 & 1.08869400
\end{array}
$$$$
\begin{array}{llll}
\mathrm{H} & -3.47900400 & 2.92706600 & 1.71937400
\end{array}
$$$$
\begin{array}{llll}
\text { C } & -1.85938200 & 1.59183500 & 1.26005000
\end{array}
$$$$
\begin{array}{llll}
\mathrm{H} & -2.09729100 & 0.89923400 & 2.06733600
\end{array}
$$$$
\begin{array}{lllll}
\text { C } & -0.68367700 & 1.36592600 & 0.46498400
\end{array}
$$$$
\begin{array}{lllll}
\text { C } & 0.12353600 & 0.24993400 & 0.70257400
\end{array}
$$$$
\begin{array}{lllll}
\mathrm{C} & 1.54529700 & 0.22518400 & 0.36887500
\end{array}
$$$$
\begin{array}{lllll}
\text { C } & 2.30771700 & 1.41070300 & 0.43935800
\end{array}
$$$$
\begin{array}{llll}
\mathrm{H} & 1.84793000 & 2.34006200 & 0.77570200
\end{array}
$$$$
\begin{array}{lllll}
\mathrm{C} & 3.66583800 & 1.39378800 & 0.15041100
\end{array}
$$$$
\begin{array}{llll}
\mathrm{H} & 4.26898000 & 2.29790300 & 0.21343100
\end{array}
$$$$
\begin{array}{llll}
\text { C } & 4.24549900 & 0.18292200 & -0.20734700
\end{array}
$$$$
\text { C } \quad \begin{array}{llll}
\text { C } & -1.14613700 & 3.43168500 & -0.76180100
\end{array}
$$$$
\mathrm{H} \quad \begin{array}{llll}
\mathrm{H} & -0.90952200 & 4.11966900 & -1.57140600
\end{array}
$$$$
\begin{array}{llll}
\text { C } & -0.36369600 & 2.32705200 & -0.55192400
\end{array}
$$$$
\mathrm{H} \quad 0.47589700 \quad 2.13922100 \quad-1.22207900
$$$$
\text { C } \quad 2.18518200 \quad-0.99075500 \quad 0.04603700
$$$$
\text { C } \quad 3.53702200 \quad-1.01326000 \quad-0.26287300
$$$$
\mathrm{H} \quad 4.04037800 \quad-1.93940200 \quad-0.53492700
$$$$
\begin{array}{llll}
\text { C } & -0.42483600 & -0.99195300 & 1.31664100
\end{array}
$$$$
\text { C } \quad-1.52162600 \quad-1.87292700 \quad 0.67053200
$$

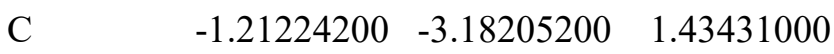$$
\mathrm{H} \quad-2.10711500 \quad-3.79775600 \quad 1.58468500
$$ 


$\begin{array}{lrrr}\mathrm{H} & -0.47247800 & -3.77046300 & 0.87048400 \\ \mathrm{C} & -0.58328700 & -2.69150800 & 2.74074200 \\ \mathrm{H} & -1.33134700 & -2.49059800 & 3.52545100 \\ \mathrm{H} & 0.15389900 & -3.38490000 & 3.16696200 \\ \mathrm{C} & -2.93089400 & -1.36976300 & 1.01961200 \\ \mathrm{H} & -3.01230800 & -1.16084300 & 2.09634000 \\ \mathrm{H} & -3.66754500 & -2.14690800 & 0.77364900 \\ \mathrm{H} & -3.19786300 & -0.46469300 & 0.45621200 \\ \mathrm{C} & -1.30530900 & -1.96740200 & -0.84582500 \\ \mathrm{H} & -1.34189100 & -0.97094900 & -1.31295400 \\ \mathrm{H} & -0.32301500 & -2.40920900 & -1.06561900 \\ \mathrm{C} & -2.31311800 & -2.80779400 & -1.58443600 \\ \mathrm{H} & 1.62045100 & -1.92222900 & 0.01642100 \\ \mathrm{~N} & 5.69057100 & 0.16038000 & -0.52316300 \\ \mathrm{O} & 6.29186600 & 1.20523800 & -0.46194000 \\ \mathrm{O} & 6.17950500 & -0.90106800 & -0.82500000 \\ \mathrm{O} & -3.05859000 & 4.69037600 & -0.03457700 \\ \mathrm{C} & -2.79106600 & 5.70434400 & -1.00566700 \\ \mathrm{H} & -3.56907500 & 6.46074000 & -0.86915700 \\ \mathrm{H} & -1.80096900 & 6.14655700 & -0.83017800 \\ \mathrm{H} & -2.84856600 & 5.28525400 & -2.01937600\end{array}$

\section{Structure 5. B-TS 1}

$\begin{array}{lrrr}\text { F } & -4.16632700 & -1.82443200 & -1.75020400 \\ \text { F } & -4.24928300 & -2.20078300 & 0.36034400 \\ \text { F } & -3.22888400 & -3.57843700 & -0.93570600 \\ \text { N } & 1.16818600 & -1.91545300 & 0.68284300 \\ \text { C } & -2.41685200 & 3.41642800 & 0.33188400 \\ \text { C } & -2.41457100 & 2.42828300 & 1.34086500 \\ \text { H } & -3.12465200 & 2.52320200 & 2.16274000 \\ \text { C } & -1.52534100 & 1.37834400 & 1.27971500 \\ \text { H } & -1.51466100 & 0.63625900 & 2.07905600 \\ \text { C } & -0.59062900 & 1.28318300 & 0.22155600 \\ \text { C } & 0.35862700 & 0.19951200 & 0.14456600 \\ \text { C } & 1.70457800 & 0.35311100 & -0.26217800 \\ \text { C } & 2.43186700 & 1.56256700 & -0.03812300 \\ \text { H } & 1.89282700 & 2.47714400 & 0.21013400\end{array}$




\begin{tabular}{|c|c|c|c|}
\hline $\mathrm{C}$ & 3.80036100 & 1.54085600 & 0.00428100 \\
\hline $\mathrm{H}$ & 4.37730000 & 2.43389100 & 0.23684200 \\
\hline $\mathrm{C}$ & 4.46407100 & 0.30487200 & -0.19500100 \\
\hline $\mathrm{C}$ & -1.49712500 & 3.33636200 & -0.73007800 \\
\hline 11 & -1.49091100 & 4.08087800 & -1.52475200 \\
\hline 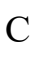 & -0.59302100 & 2.28666000 & -0.76957000 \\
\hline 11 & 0.10368000 & 2.21715000 & -1.60729100 \\
\hline . & 2.41782000 & -0.85915000 & -0.57485700 \\
\hline $\mathrm{C}$ & 3.83502200 & -0.87561400 & -0.47704600 \\
\hline $\mathrm{H}$ & 4.40250200 & -1.77844300 & -0.69710600 \\
\hline $\mathrm{C}$ & 0.10559300 & -1.19479200 & 0.49054400 \\
\hline $\mathrm{C}$ & -1.18750000 & -2.03294800 & 0.46833200 \\
\hline $\mathrm{C}$ & -0.56509600 & -3.39008600 & 0.04105000 \\
\hline $\mathrm{H}$ & -1.15282600 & -4.24425600 & 0.39641600 \\
\hline $\mathrm{H}$ & -0.50148900 & -3.43833000 & -1.05644300 \\
\hline$C$ & 0.84196500 & -3.33352900 & 0.63219200 \\
\hline $\mathrm{H}$ & 0.90324700 & -3.71682700 & 1.66408500 \\
\hline$\Pi$ & 1.60872900 & -3.85998500 & 0.04705500 \\
\hline$C$ & -1.78444900 & -2.15774600 & 1.88179400 \\
\hline $\mathrm{H}$ & -0.99754700 & -2.37513000 & 2.61815600 \\
\hline $\mathrm{H}$ & -2.50437800 & -2.98694700 & 1.89983700 \\
\hline $\mathrm{H}$ & -2.31237600 & -1.24593400 & 2.18902000 \\
\hline $\mathrm{C}$ & -2.18522500 & -1.47742100 & -0.55557500 \\
\hline $\mathrm{H}$ & -2.51285500 & -0.46314000 & -0.28776400 \\
\hline$T$ & -1.71814300 & -1.43086900 & -1.55044400 \\
\hline $\mathrm{C}$ & -3.45175700 & -2.27691900 & -0.71328700 \\
\hline $\mathrm{H}$ & 1.97343000 & -1.58362400 & -1.26154600 \\
\hline $\mathrm{N}$ & 5.94781400 & 0.30386200 & -0.13969600 \\
\hline $\mathrm{O}$ & 6.49942800 & 1.37514000 & -0.08150900 \\
\hline $\mathrm{O}$ & 6.50677800 & -0.76455600 & -0.15464300 \\
\hline 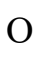 & -3.31864700 & 4.38407200 & 0.46140300 \\
\hline $\mathrm{C}$ & -3.37216400 & 5.41573100 & -0.51089500 \\
\hline $\mathrm{H}$ & -3.60184200 & 5.00609200 & -1.50588500 \\
\hline $\mathrm{H}$ & -4.17667800 & 6.08948500 & -0.19875400 \\
\hline $\mathrm{H}$ & -2.42243400 & 5.96991100 & -0.54832700 \\
\hline
\end{tabular}




\begin{tabular}{|c|c|c|c|}
\hline $\mathrm{F}$ & -3.76085600 & -2.35636200 & -1.90340400 \\
\hline $\mathrm{F}$ & -3.84216100 & -2.76518500 & 0.20090000 \\
\hline $\mathrm{F}$ & -2.57044700 & -3.94223600 & -1.07065300 \\
\hline $\mathrm{N}$ & 1.40455100 & -1.59813300 & 0.55986300 \\
\hline $\mathrm{C}$ & -2.88934600 & 3.23521600 & 0.18946600 \\
\hline $\mathrm{C}$ & -1.78930000 & 3.27432600 & -0.67674400 \\
\hline $\mathrm{H}$ & -1.72285100 & 4.01904400 & -1.46869100 \\
\hline $\mathrm{C}$ & -0.76439700 & 2.33921200 & -0.53082000 \\
\hline $\mathrm{H}$ & 0.08135900 & 2.36632000 & -1.22136500 \\
\hline $\mathrm{C}$ & -0.81124300 & 1.35829500 & 0.46544500 \\
\hline $\mathrm{C}$ & 0.29469600 & 0.38850400 & 0.59174600 \\
\hline $\mathrm{C}$ & 1.63464700 & 0.67167800 & 0.69668100 \\
\hline $\mathrm{C}$ & 2.36786300 & 1.91155500 & 0.62164900 \\
\hline $\mathrm{H}$ & 1.86036400 & 2.86307800 & 0.78586400 \\
\hline $\mathrm{C}$ & 3.66711600 & 1.86456100 & 0.24175200 \\
\hline $\mathrm{H}$ & 4.25637500 & 2.77322900 & 0.12928600 \\
\hline $\mathrm{C}$ & 4.29025600 & 0.59042100 & -0.09535600 \\
\hline $\mathrm{C}$ & -2.94188300 & 2.25708900 & 1.19771100 \\
\hline $\mathrm{H}$ & -3.80079600 & 2.24788000 & 1.87001600 \\
\hline $\mathrm{C}$ & -1.91669500 & 1.33491800 & 1.33323300 \\
\hline $\mathrm{H}$ & -1.96304500 & 0.59480000 & 2.13366600 \\
\hline $\mathrm{C}$ & 2.41258400 & -0.60194200 & 0.80679500 \\
\hline $\mathrm{C}$ & 3.71480900 & -0.60873200 & 0.08345100 \\
\hline $\mathrm{H}$ & 4.21323600 & -1.54341600 & -0.17749100 \\
\hline $\mathrm{C}$ & 0.21594100 & -1.05759800 & 0.50677000 \\
\hline $\mathrm{C}$ & -0.85175700 & -2.12058800 & 0.39776100 \\
\hline $\mathrm{C}$ & 0.01655300 & -3.34035400 & -0.03613000 \\
\hline $\mathrm{H}$ & -0.39086600 & -4.28485800 & 0.34063800 \\
\hline $\mathrm{H}$ & 0.05596500 & -3.38827400 & -1.13311900 \\
\hline $\mathrm{C}$ & 1.42331700 & -3.05935100 & 0.50834600 \\
\hline $\mathrm{H}$ & 1.59090500 & -3.45226000 & 1.52145500 \\
\hline $\mathrm{H}$ & 2.22941700 & -3.40144900 & -0.15158800 \\
\hline $\mathrm{C}$ & -1.45031600 & -2.34990600 & 1.79823200 \\
\hline $\mathrm{H}$ & -0.64973000 & -2.47332500 & 2.54207100 \\
\hline $\mathrm{H}$ & -2.05203400 & -3.26807400 & 1.79125800 \\
\hline $\mathrm{H}$ & -2.09215600 & -1.51340200 & 2.10263300 \\
\hline $\mathrm{C}$ & -1.89778700 & -1.70755900 & -0.6461780 \\
\hline
\end{tabular}




$\begin{array}{lrrr}\mathrm{H} & -2.37894500 & -0.75996100 & -0.36310900 \\ \mathrm{H} & -1.41660300 & -1.56730500 & -1.62490400 \\ \mathrm{C} & -3.01335200 & -2.69772500 & -0.84895200 \\ \mathrm{H} & 2.69210800 & -0.70937100 & 1.88323700 \\ \mathrm{O} & -3.92730900 & 4.08560200 & 0.12893900 \\ \mathrm{C} & -3.91742100 & 5.09274600 & -0.86160100 \\ \mathrm{H} & -3.89926100 & 4.65860700 & -1.87363800 \\ \mathrm{H} & -4.84211200 & 5.66594300 & -0.73201000 \\ \mathrm{H} & -3.05390200 & 5.76531500 & -0.73909700 \\ \mathrm{~N} & 5.63308200 & 0.63130600 & -0.70897000 \\ \mathrm{O} & 6.08662500 & 1.71952400 & -0.97064800 \\ \mathrm{O} & 6.19250900 & -0.41753500 & -0.91735600\end{array}$

\section{Structure 7. A'}

$\begin{array}{lrrr}\text { F } & 0.03281400 & 3.49340700 & -2.84600300 \\ \text { F } & 1.93106600 & 3.27259300 & -1.87196200 \\ \text { F } & 0.46411200 & 4.62145500 & -1.06731800 \\ \text { N } & -0.73483600 & 1.34517100 & 2.50294600 \\ \text { C } & 3.15294500 & -2.44527200 & -0.03515600 \\ \text { C } & 3.06212100 & -1.67164400 & 1.12096200 \\ \mathrm{H} & 3.89361600 & -1.64007700 & 1.82339400 \\ \mathrm{C} & 1.89621200 & -0.95867700 & 1.35197900 \\ \mathrm{H} & 1.80508900 & -0.35971100 & 2.25948600 \\ \mathrm{C} & 0.80980300 & -1.01602200 & 0.44820000 \\ \mathrm{C} & -0.39433200 & -0.24411500 & 0.71698800 \\ \mathrm{C} & -1.72195000 & -0.70556400 & 0.38868200 \\ \mathrm{C} & -2.01095500 & -2.07169400 & 0.16082300 \\ \mathrm{H} & -1.21836600 & -2.81489300 & 0.25474000 \\ \mathrm{C} & -3.29312500 & -2.51493000 & -0.13923800 \\ \mathrm{H} & -3.46479400 & -3.57984800 & -0.29124800 \\ \mathrm{C} & -4.34877900 & -1.59402200 & -0.22413900 \\ \mathrm{C} & 2.11226200 & -2.52174000 & -0.96018200 \\ \mathrm{H} & 2.22774800 & -3.12099500 & -1.86202400 \\ \mathrm{C} & 0.94748200 & -1.81205400 & -0.71322500 \\ \mathrm{H} & 0.13960100 & -1.84433500 & -1.44518300 \\ \mathrm{C} & -2.81552700 & 0.20070400 & 0.31592400 \\ \mathrm{C} & -4.09085400 & -0.22974000 & 0.01157300\end{array}$




$\begin{array}{lrrr}\mathrm{H} & -4.92142500 & 0.47458200 & -0.05441300 \\ \mathrm{C} & -0.24730100 & 1.08873700 & 1.35396600 \\ \mathrm{C} & 0.44346800 & 2.27995500 & 0.65165600 \\ \mathrm{C} & -0.16102900 & 3.42850500 & 1.48756500 \\ \mathrm{H} & 0.51917500 & 4.28416300 & 1.58553900 \\ \mathrm{H} & -1.09121300 & 3.77959200 & 1.01515200 \\ \mathrm{C} & -0.49053000 & 2.75229800 & 2.82259200 \\ \mathrm{H} & 0.34424600 & 2.80687700 & 3.54177200 \\ \mathrm{H} & -1.37044600 & 3.18124200 & 3.32210500 \\ \mathrm{C} & 1.96928300 & 2.22302000 & 0.83166300 \\ \mathrm{H} & 2.22362800 & 2.05330400 & 1.88832000 \\ \mathrm{H} & 2.41873900 & 3.17873300 & 0.52658800 \\ \mathrm{H} & 2.42341100 & 1.42416800 & 0.22905500 \\ \mathrm{C} & 0.03598200 & 2.30025400 & -0.82660700 \\ \mathrm{H} & 0.34192900 & 1.36842900 & -1.32803400 \\ \mathrm{H} & -1.05737000 & 2.38557100 & -0.91506400 \\ \mathrm{C} & 0.61838200 & 3.42099600 & -1.64326500 \\ \mathrm{H} & -2.64672000 & 1.26435700 & 0.49096000 \\ \mathrm{~N} & 4.38360200 & -3.19804900 & -0.28917800 \\ \mathrm{O} & 4.43138800 & -3.88868700 & -1.28285000 \\ \mathrm{O} & 5.28924700 & -3.09038800 & 0.50758000 \\ \mathrm{O} & -5.61674800 & -1.92328600 & -0.51118000 \\ \mathrm{C} & -5.92618200 & -3.28228600 & -0.74560100 \\ \mathrm{H} & -6.99950500 & -3.32458600 & -0.96099200 \\ \mathrm{H} & -5.36694500 & -3.67575400 & -1.60881300 \\ & -5.70904200 & -3.89910400 & 0.14047500 \\ \mathrm{H} & & & \\ \mathrm{H} & & & \end{array}$

\section{Structure 8. A'-TS 1}

$\begin{array}{lrrr}\text { F } & -3.90760400 & -2.42950100 & -1.37964900 \\ \text { F } & -3.64222800 & -2.86251300 & 0.70472200 \\ \text { F } & -2.64426600 & -4.05862300 & -0.77548800 \\ \text { N } & 1.68617200 & -1.88664800 & 0.37732200 \\ \text { C } & -2.38341200 & 3.12744300 & 0.05453000 \\ \text { C } & -2.40263000 & 2.13138900 & 1.02788600 \\ \text { H } & -3.16263300 & 2.14465600 & 1.80775400 \\ \text { C } & -1.42651600 & 1.14547400 & 0.99006800 \\ \text { H } & -1.41145700 & 0.38285200 & 1.76873200\end{array}$




\begin{tabular}{|c|c|c|c|}
\hline $\mathrm{C}$ & -0.44031100 & 1.13010200 & -0.01729100 \\
\hline $\mathrm{C}$ & 0.60525100 & 0.10942500 & -0.06896400 \\
\hline $\mathrm{C}$ & 1.98835700 & 0.42224900 & -0.34174300 \\
\hline $\mathrm{C}$ & 2.64072600 & 1.61417800 & 0.00221500 \\
\hline $\mathrm{H}$ & 2.04573500 & 2.49360800 & 0.25965200 \\
\hline $\mathrm{C}$ & 4.02427900 & 1.67607500 & 0.11926400 \\
\hline $\mathrm{H}$ & 4.49885800 & 2.60745800 & 0.42232900 \\
\hline $\mathrm{C}$ & 4.79603500 & 0.50461100 & -0.10903400 \\
\hline $\mathrm{C}$ & -1.41380900 & 3.16424200 & -0.94613000 \\
\hline $\mathrm{H}$ & -1.42901200 & 3.95817600 & -1.69132800 \\
\hline $\mathrm{C}$ & -0.44867400 & 2.16845000 & -0.97349900 \\
\hline $\mathrm{H}$ & 0.30845100 & 2.17756700 & -1.75929800 \\
\hline $\mathrm{C}$ & 2.78275000 & -0.75749200 & -0.66610000 \\
\hline $\mathrm{C}$ & 4.19550400 & -0.67917000 & -0.51539300 \\
\hline $\mathrm{H}$ & 4.83338200 & -1.51494500 & -0.80517100 \\
\hline $\mathrm{C}$ & 0.51829000 & -1.25879600 & 0.27758000 \\
\hline $\mathrm{C}$ & -0.64181700 & -2.25703800 & 0.40758800 \\
\hline $\mathrm{C}$ & 0.06893300 & -3.52464900 & -0.14173200 \\
\hline $\mathrm{H}$ & -0.36841400 & -4.45006500 & 0.25324300 \\
\hline $\mathrm{H}$ & -0.01729700 & -3.54114200 & -1.23888300 \\
\hline $\mathrm{C}$ & 1.53623200 & -3.32613500 & 0.25308800 \\
\hline $\mathrm{H}$ & 1.79120300 & -3.80863600 & 1.21077200 \\
\hline $\mathrm{H}$ & 2.23932000 & -3.71248300 & -0.50171700 \\
\hline $\mathrm{C}$ & -1.00502000 & -2.46808500 & 1.88701300 \\
\hline $\mathrm{H}$ & -0.09432200 & -2.62895000 & 2.48198800 \\
\hline $\mathrm{H}$ & -1.64252900 & -3.35592400 & 1.99698700 \\
\hline $\mathrm{H}$ & -1.54412100 & -1.60786400 & 2.30671800 \\
\hline $\mathrm{C}$ & -1.84135200 & -1.84055400 & -0.45244200 \\
\hline $\mathrm{H}$ & -2.26854200 & -0.88746100 & -0.11323000 \\
\hline $\mathrm{H}$ & -1.52575900 & -1.71375500 & -1.49849200 \\
\hline $\mathrm{C}$ & -2.99784800 & -2.80322300 & -0.46987100 \\
\hline $\mathrm{H}$ & 2.43462300 & -1.37797300 & -1.50380100 \\
\hline $\mathrm{O}$ & 6.13887100 & 0.49058500 & -0.00827000 \\
\hline $\mathrm{C}$ & 6.81873500 & 1.68969800 & 0.29772700 \\
\hline $\mathrm{H}$ & 6.53502800 & 2.07244700 & 1.29050600 \\
\hline $\mathrm{H}$ & 7.88766900 & 1.44790700 & 0.30037400 \\
\hline $\mathrm{H}$ & 6.62707400 & 2.46642100 & -0.45911100 \\
\hline
\end{tabular}




$\begin{array}{llll}\mathrm{N} & -3.40812900 & 4.17370000 & 0.08930600 \\ \mathrm{O} & -3.35919200 & 5.04422100 & -0.75116200 \\ \mathrm{O} & -4.25204300 & 4.11335800 & 0.95577700\end{array}$

\section{Structure9. A'-Int 1}

$\mathrm{F}$

$3.26570100 \quad 2.91121800-1.86322800$

$\mathrm{F}$

$3.28791600 \quad 3.18920200 \quad 0.26366100$

F

$\begin{array}{llll}1.93126400 & 4.32975900 & -0.95232000\end{array}$

$\mathrm{N} \quad \begin{array}{llll}-1.87800900 & 1.53496000 & 0.41018400\end{array}$

$\begin{array}{llll}\mathrm{C} & 2.83350700 & -2.85294900 & 0.07495500\end{array}$

C $\quad 1.70942900 \quad-3.09035600 \quad-0.71178200$

$\mathrm{H} \quad 1.70747700 \quad-3.91125300 \quad-1.42741900$

C $\quad 0.61057100 \quad-2.25266100 \quad-0.57209000$

$\mathrm{H} \quad-0.26396500 \quad-2.41331000 \quad-1.20320100$

C $\quad 0.62226300 \quad-1.18202700 \quad 0.34392600$

$\begin{array}{llll}\text { C } & -0.55518600 & -0.30897200 & 0.47336800\end{array}$

$\begin{array}{llll}\text { C } & -1.92097900 & -0.75367300 & 0.51277000\end{array}$

C $\quad-2.53523800 \quad-1.99138600 \quad 0.34674600$

$\mathrm{H} \quad-1.94497100 \quad-2.91002900 \quad 0.34124100$

C $\quad-3.90887400 \quad-2.05910500 \quad 0.09697700$

$\mathrm{H} \quad-4.37909000 \quad-3.03250600 \quad-0.03020700$

C $\quad-4.66589200 \quad-0.85438600 \quad-0.13665700$

C $\quad 2.88532200 \quad-1.80983300 \quad 0.99467000$

$\mathrm{H} \quad 3.77713200 \quad-1.65952800 \quad 1.60126600$

C $\quad 1.77431500 \quad-0.98628400 \quad 1.12775400$

$\mathrm{H} \quad 1.79036400 \quad-0.18549400 \quad 1.86685400$

$\begin{array}{lllll}\text { C } & -2.79266000 & 0.46085700 & 0.73782700\end{array}$

C $\quad-4.11765300 \quad 0.38256500 \quad 0.05660200$

$\mathrm{H} \quad-4.71563900 \quad 1.28031100 \quad-0.10959600$

$\begin{array}{lllll}\text { C } & -0.60689300 & 1.08731000 & 0.41429400\end{array}$

$\begin{array}{lllll}\mathrm{C} & 0.34242400 & 2.27300300 & 0.35137000\end{array}$

C $\quad-0.64051900 \quad 3.40293300 \quad-0.07674000$

$\mathrm{H} \quad-0.32228400 \quad 4.38562100 \quad 0.29062800$

$\mathrm{H} \quad-0.69655600 \quad 3.44192500 \quad-1.17439600$

$\begin{array}{llll}\mathrm{C} & -2.00299200 & 2.97519800 & 0.48191300\end{array}$

$\mathrm{H} \quad-2.15603800 \quad 3.30934200 \quad 1.52321500$

$\mathrm{H} \quad-2.84516300 \quad 3.33143200 \quad-0.12627900$ 


$\begin{array}{llll}\mathrm{C} & 0.88362100 & 2.57076500 & 1.76096000 \\ \mathrm{H} & 0.05833600 & 2.58993400 & 2.48794200 \\ \mathrm{H} & 1.37236700 & 3.55493200 & 1.77799900 \\ \mathrm{H} & 1.61431300 & 1.81857300 & 2.08516500 \\ \mathrm{C} & 1.45211500 & 2.02065400 & -0.67737500 \\ \mathrm{H} & 2.01684200 & 1.10994100 & -0.43077600 \\ \mathrm{H} & 1.01156600 & 1.88506000 & -1.67559800 \\ \mathrm{C} & 2.47659200 & 3.11505700 & -0.80100400 \\ \mathrm{H} & -2.96415300 & 0.53505100 & 1.84643700 \\ \mathrm{~N} & 3.99965800 & -3.73175600 & -0.06849800 \\ \mathrm{O} & 3.92735700 & -4.64484400 & -0.85972500 \\ \mathrm{O} & 4.97333300 & -3.49716100 & 0.61120300 \\ \mathrm{O} & -5.95064700 & -0.90062500 & -0.57769900 \\ \mathrm{C} & -6.49335500 & -2.13386100 & -0.98888400 \\ \mathrm{H} & -7.49181100 & -1.91711800 & -1.38741500 \\ \mathrm{H} & -5.88779900 & -2.60433000 & -1.78032600 \\ \mathrm{H} & -6.59624600 & -2.83865800 & -0.14818100\end{array}$

\section{Structure 10. B'}

$\begin{array}{lrrr}\text { F } & -0.86123500 & -3.26453700 & -2.85744500 \\ \text { F } & -2.72602200 & -2.69891800 & -1.96066200 \\ \text { F } & -1.57331000 & -4.30416100 & -1.11583200 \\ \text { N } & 0.04888300 & -1.34793900 & 2.55733600 \\ \text { C } & -3.00606300 & 3.15118600 & 0.08913700 \\ \text { C } & -2.98379700 & 2.37976700 & 1.26537200 \\ \text { H } & -3.78046700 & 2.52306000 & 1.99671800 \\ \text { C } & -1.97054200 & 1.46332500 & 1.47844800 \\ \text { H } & -1.96435200 & 0.87192800 & 2.39664100 \\ \text { C } & -0.92750300 & 1.29116200 & 0.54013000 \\ \text { C } & 0.11272900 & 0.29553600 & 0.78647600 \\ \text { C } & 1.49748000 & 0.48875200 & 0.46506900 \\ \text { C } & 2.02645400 & 1.77699600 & 0.17119900 \\ \text { H } & 1.37945800 & 2.65223700 & 0.21707400 \\ \text { C } & 3.36360700 & 1.95323400 & -0.13345700 \\ \text { H } & 3.76583800 & 2.94276600 & -0.34546400 \\ \mathrm{C} & 4.20659300 & 0.83967200 & -0.15150300 \\ \mathrm{C} & -1.99002700 & 2.98558500 & -0.86375300\end{array}$




$\begin{array}{llll}\mathrm{H} & -1.99264000 & 3.55722300 & -1.79075000 \\ \mathrm{C} & -0.96777800 & 2.06970500 & -0.63073400 \\ \mathrm{H} & -0.19954900 & 1.93580700 & -1.39486600 \\ \mathrm{C} & 2.40234600 & -0.61097400 & 0.45619800 \\ \mathrm{C} & 3.73850200 & -0.44264000 & 0.14568000 \\ \mathrm{H} & 4.42311300 & -1.28926400 & 0.12703400 \\ \mathrm{C} & -0.31026900 & -0.99427300 & 1.38820300 \\ \mathrm{C} & -1.19899200 & -2.00685100 & 0.63354700 \\ \mathrm{C} & -0.89835900 & -3.26026500 & 1.48303700 \\ \mathrm{H} & -1.75101500 & -3.94919600 & 1.53601700 \\ \mathrm{H} & -0.04327900 & -3.80260400 & 1.05167700 \\ \mathrm{C} & -0.50086000 & -2.67463900 & 2.84256100 \\ \mathrm{H} & -1.36305200 & -2.55504900 & 3.52007500 \\ \mathrm{H} & 0.24369700 & -3.28085800 & 3.37704400 \\ \mathrm{C} & -2.68192200 & -1.61570200 & 0.74068000 \\ \mathrm{H} & -2.94501700 & -1.39969500 & 1.78672100 \\ \mathrm{H} & -3.31514200 & -2.44643300 & 0.39831700 \\ \mathrm{H} & -2.91606300 & -0.73086300 & 0.13207200 \\ \mathrm{C} & -0.72994700 & -2.10607300 & -0.82317000 \\ \mathrm{H} & -0.82534100 & -1.13199900 & -1.32877800 \\ \mathrm{H} & 0.32950400 & -2.40117800 & -0.85905000 \\ \mathrm{H} & -1.47560400 & -3.09225400 & -1.67946900 \\ \mathrm{C} & 2.03745600 & -1.61231500 & 0.68504800 \\ \mathrm{H} & -4.18325900 & 4.18212500 & -2.11353400 \\ \mathrm{~N} & -4.091669300 & 1.02184400 & -0.47983900 \\ \mathrm{O} & 6.00057800 & 2.14174700 & -0.74119700 \\ \mathrm{H} & 6.33328600 & 0.04388900 & -0.47555700 \\ \mathrm{H} & -4.02865100 & 4.00991100 & -0.04342200 \\ \mathrm{H} & -3.21417100 & 5.45715200 & -1.30047500 \\ \mathrm{H} & 4.80525500 & -1.20959900 \\ & & 5.42498000 & -1.11146200 \\ & & & \end{array}$

\section{Structure 11. B'-TS 1}

$\begin{array}{llll}\text { F } & -4.40838800 & -1.87010100 & -1.28255300 \\ \text { F } & -4.09889500 & -2.44090900 & 0.76216400 \\ \text { F } & -3.26674000 & -3.63085000 & -0.82196900 \\ \text { N } & 1.26403600 & -1.89015100 & 0.27726000\end{array}$




\begin{tabular}{|c|c|c|c|}
\hline $\mathrm{C}$ & -2.50842000 & 3.41122200 & 0.28573100 \\
\hline $\mathrm{C}$ & -2.42412900 & 2.42999100 & 1.28736300 \\
\hline $\mathrm{H}$ & -3.08660400 & 2.50857900 & 2.15067200 \\
\hline $\mathrm{C}$ & -1.50585300 & 1.39708000 & 1.17988100 \\
\hline $\mathrm{H}$ & -1.43645900 & 0.65841000 & 1.98028400 \\
\hline $\mathrm{C}$ & -0.64861300 & 1.29400800 & 0.06770800 \\
\hline $\mathrm{C}$ & 0.33544000 & 0.20888700 & -0.06360300 \\
\hline $\mathrm{C}$ & 1.72366300 & 0.43956400 & -0.33468400 \\
\hline $\mathrm{C}$ & 2.43286200 & 1.60858400 & -0.00135200 \\
\hline $\mathrm{H}$ & 1.87820400 & 2.51341100 & 0.25406000 \\
\hline $\mathrm{C}$ & 3.81451800 & 1.59058900 & 0.11568900 \\
\hline $\mathrm{H}$ & 4.37365200 & 2.47190900 & 0.42247100 \\
\hline $\mathrm{C}$ & 4.48492700 & 0.37687000 & -0.11222200 \\
\hline $\mathrm{C}$ & -1.64871000 & 3.34013500 & -0.81859800 \\
\hline $\mathrm{H}$ & -1.68722600 & 4.08849500 & -1.60940100 \\
\hline $\mathrm{C}$ & -0.73392900 & 2.29169300 & -0.91387600 \\
\hline $\mathrm{H}$ & -0.07606200 & 2.24196800 & -1.78472900 \\
\hline $\mathrm{C}$ & 2.44320300 & -0.77356800 & -0.70224900 \\
\hline $\mathrm{C}$ & 3.85983800 & -0.78258300 & -0.53420000 \\
\hline $\mathrm{H}$ & 4.45008700 & -1.65437100 & -0.81037700 \\
\hline $\mathrm{C}$ & 0.15587200 & -1.16443900 & 0.22847500 \\
\hline $\mathrm{C}$ & -1.08110400 & -2.05708800 & 0.39009500 \\
\hline $\mathrm{C}$ & -0.50539600 & -3.37493200 & -0.19816500 \\
\hline $\mathrm{H}$ & -1.00338700 & -4.26382000 & 0.20905800 \\
\hline $\mathrm{H}$ & -0.63892700 & -3.37332200 & -1.29041500 \\
\hline $\mathrm{C}$ & 0.99085400 & -3.30948900 & 0.13422900 \\
\hline $\mathrm{H}$ & 1.24740800 & -3.82952400 & 1.07136000 \\
\hline $\mathrm{H}$ & 1.62550300 & -3.73566600 & -0.65852900 \\
\hline $\mathrm{C}$ & -1.41071900 & -2.24648200 & 1.88022000 \\
\hline $\mathrm{H}$ & -0.49802900 & -2.48804600 & 2.44420300 \\
\hline $\mathrm{H}$ & -2.11867400 & -3.07616500 & 2.00977300 \\
\hline $\mathrm{H}$ & -1.85920600 & -1.34325400 & 2.31568400 \\
\hline $\mathrm{C}$ & -2.26377800 & -1.51092000 & -0.41936200 \\
\hline $\mathrm{H}$ & -2.59211300 & -0.53639600 & -0.03321200 \\
\hline $\mathrm{H}$ & -1.96794800 & -1.37277300 & -1.46989300 \\
\hline $\mathrm{C}$ & -3.49904400 & -2.36936900 & -0.43486500 \\
\hline $\mathrm{H}$ & 2.06714600 & -1.34402000 & -1.56204300 \\
\hline
\end{tabular}




$\begin{array}{llll}\mathrm{N} & 5.94530000 & 0.35790800 & 0.03985400 \\ \mathrm{O} & 6.51100500 & 1.40909100 & 0.24926700 \\ \mathrm{O} & 6.51359900 & -0.70835100 & -0.04897400 \\ \mathrm{O} & -3.42773100 & 4.37725400 & 0.46771100 \\ \mathrm{C} & -3.54305200 & 5.38796500 & -0.51063700 \\ \mathrm{H} & -3.81774900 & 4.96774300 & -1.49128700 \\ \mathrm{H} & -4.33865200 & 6.06114200 & -0.17226200 \\ \mathrm{H} & -2.60566800 & 5.95789100 & -0.61149000\end{array}$

\section{Structure 12. B'-Int 1}

$\mathrm{F}$

$\begin{array}{lll}-3.76068300 & -2.38216800 & -1.89900500\end{array}$

F $\quad \begin{array}{llll}-3.84920300 & -2.74496300 & 0.21315100\end{array}$

F $\quad-2.57817700 \quad-3.95354400 \quad-1.02942600$

$\mathrm{N} \quad 1.43750900 \quad-1.58030700 \quad 0.49879300$

$\begin{array}{lllll}\mathrm{C} & -2.93643300 & 3.21168600 & 0.19214700\end{array}$

C $\quad-1.85256100 \quad 3.23899900 \quad-0.69409200$

$\mathrm{H} \quad-1.80641200 \quad 3.96759700 \quad-1.50267400$

$\begin{array}{llll}\text { C } & -0.81838200 & 2.31234100 & -0.54882300\end{array}$

$\begin{array}{llll}\mathrm{H} & 0.01214900 & 2.33372000 & -1.25785300\end{array}$

$\begin{array}{lllll}\text { C } & -0.82937000 & 1.34913900 & 0.46750400\end{array}$

$\begin{array}{lllll}\text { C } & 0.27960800 & 0.37706500 & 0.58852300\end{array}$

$\begin{array}{llll}\mathrm{C} & 1.66458800 & 0.70019500 & 0.64477500\end{array}$

$\begin{array}{llll}\mathrm{C} & 2.36522000 & 1.90222100 & 0.50848100\end{array}$

$\mathrm{H} \quad 1.83586200 \quad 2.85656500 \quad 0.54468400$

$\begin{array}{llll}\text { C } & 3.72657400 & 1.87195000 & 0.21532200\end{array}$

$\begin{array}{llll}\mathrm{H} & 4.30189500 & 2.78554600 & 0.08535500\end{array}$

$\begin{array}{lllll}\text { C } & 4.33376300 & 0.61675300 & -0.08565500\end{array}$

$\begin{array}{llll}\text { C } & -2.95922000 & 2.25611700 & 1.21990800\end{array}$

$\mathrm{H} \quad \begin{array}{llll}\mathrm{H} & 3.80442900 & 2.25340300 & 1.90992700\end{array}$

C $\quad \begin{array}{llll}-1.91878500 & 1.34738800 & 1.35620900\end{array}$

$\mathrm{H} \quad-1.94190900 \quad 0.62798900 \quad 2.17671100$

$\begin{array}{llll}\text { C } & 2.43898500 & -0.59057400 & 0.82425000\end{array}$

$\begin{array}{llll}\text { C } & 3.72568400 & -0.59580400 & 0.06888500\end{array}$

$\mathrm{H} \quad 4.20443600 \quad-1.52524200 \quad-0.23599600$

C $\quad 0.21546600 \quad-1.01781500 \quad 0.49394300$

$\begin{array}{llll}\text { C } & -0.84293400 & -2.09863500 & 0.39804900\end{array}$

$\begin{array}{lllll}\text { C } & 0.03123300 & -3.31865700 & -0.01906500\end{array}$ 


$\begin{array}{llll}\mathrm{H} & -0.38542200 & -4.26659800 & 0.34129800 \\ \mathrm{H} & 0.09849000 & -3.36214800 & -1.11579900 \\ \mathrm{C} & 1.42351400 & -3.02871100 & 0.55887700 \\ \mathrm{H} & 1.53120300 & -3.38092300 & 1.59920600 \\ \mathrm{H} & 2.23470100 & -3.45878200 & -0.04301800 \\ \mathrm{C} & -1.44707100 & -2.33563700 & 1.79301000 \\ \mathrm{H} & -0.64718000 & -2.44859600 & 2.53961700 \\ \mathrm{H} & -2.04614700 & -3.25673000 & 1.79528900 \\ \mathrm{H} & -2.09197800 & -1.50044500 & 2.09697700 \\ \mathrm{C} & -1.89571900 & -1.71260600 & -0.64909300 \\ \mathrm{H} & -2.37459700 & -0.75830100 & -0.38317500 \\ \mathrm{H} & -1.41762900 & -1.58927300 & -1.63174100 \\ \mathrm{C} & -3.01406300 & -2.70117600 & -0.83450200 \\ \mathrm{H} & 2.66609300 & -0.70205800 & 1.91743800 \\ \mathrm{O} & -3.98438900 & 4.05723400 & 0.13350700 \\ \mathrm{C} & -3.99350700 & 5.04339900 & -0.87510100 \\ \mathrm{H} & -3.99066100 & 4.59169500 & -1.88002800 \\ \mathrm{H} & -4.91728000 & 5.61798500 & -0.74272600 \\ \mathrm{H} & -3.13009000 & 5.72154800 & -0.78203500 \\ \mathrm{~N} & 5.67886600 & 0.63959300 & -0.68098300 \\ \mathrm{O} & 6.13955400 & -0.40098100 & -1.09552900 \\ \mathrm{O} & 6.26574900 & 1.69853300 & -0.72046600\end{array}$

\section{Structure 13. C}

$\begin{array}{llll}\text { F } & 2.62436500 & -1.78113400 & 2.79215200 \\ \text { F } & 3.56704000 & -0.11742400 & 1.82093100 \\ \text { F } & 3.74588200 & -2.08844800 & 0.98285400 \\ \text { N } & 0.31960700 & -1.04032100 & -2.49362300 \\ \text { C } & -0.09867900 & 4.40719300 & -0.18485300 \\ \text { C } & 0.32128500 & 3.78433300 & -1.36098400 \\ \text { H } & 0.79304900 & 4.37565100 & -2.14600200 \\ \mathrm{C} & 0.15591700 & 2.41479600 & -1.52597600 \\ \text { H } & 0.48691000 & 1.92390900 & -2.44226100 \\ \mathrm{C} & -0.48681500 & 1.66006000 & -0.52270100 \\ \mathrm{C} & -0.66531800 & 0.23271500 & -0.72186100 \\ \mathrm{C} & -1.81816500 & -0.47399200 & -0.33204300 \\ \mathrm{C} & -3.04219700 & 0.20424900 & -0.04094400\end{array}$




\begin{tabular}{|c|c|c|c|}
\hline $\mathrm{H}$ & -3.10831100 & 1.28455600 & -0.16851500 \\
\hline $\mathrm{C}$ & -4.16265300 & -0.50786200 & 0.32546600 \\
\hline $\mathrm{H}$ & -5.09945600 & 0.01477900 & 0.52299300 \\
\hline $\mathrm{C}$ & -4.12320600 & -1.91384100 & 0.42658800 \\
\hline $\mathrm{C}$ & -0.70458500 & 3.64278600 & 0.80795300 \\
\hline $\mathrm{C}$ & -0.92393500 & 2.28439400 & 0.66316700 \\
\hline $\mathrm{H}$ & -1.37581100 & 1.71822300 & 1.47874900 \\
\hline $\mathrm{C}$ & -1.79863200 & -1.90313400 & -0.25802100 \\
\hline $\mathrm{C}$ & -2.92205400 & -2.59516700 & 0.12672900 \\
\hline $\mathrm{H}$ & -2.89281900 & -3.68300300 & 0.20601300 \\
\hline $\mathrm{C}$ & 0.45766500 & -0.50745000 & -1.34919100 \\
\hline $\mathrm{C}$ & 1.83208500 & -0.67381200 & -0.67140400 \\
\hline $\mathrm{C}$ & 2.35939600 & -1.83717400 & -1.54400700 \\
\hline $\mathrm{H}$ & 3.44765500 & -1.79754700 & -1.67466400 \\
\hline $\mathrm{H}$ & 2.09925400 & -2.79703300 & -1.07386400 \\
\hline $\mathrm{C}$ & 1.58478400 & -1.68332100 & -2.85665300 \\
\hline $\mathrm{H}$ & 2.09995000 & -1.03285700 & -3.58245800 \\
\hline $\mathrm{H}$ & 1.37919400 & -2.63484800 & -3.36492400 \\
\hline $\mathrm{C}$ & 2.69218100 & 0.58627400 & -0.85193800 \\
\hline $\mathrm{H}$ & 2.68764800 & 0.90611000 & -1.90402700 \\
\hline $\mathrm{H}$ & 3.73135400 & 0.36577700 & -0.57086000 \\
\hline $\mathrm{H}$ & 2.33728600 & 1.41768100 & -0.22654500 \\
\hline $\mathrm{C}$ & 1.62988700 & -1.03612800 & 0.80586300 \\
\hline $\mathrm{H}$ & 1.08029000 & -0.23836200 & 1.33129200 \\
\hline $\mathrm{H}$ & 1.04454700 & -1.96382900 & 0.89099000 \\
\hline $\mathrm{C}$ & 2.89452700 & -1.25321300 & 1.59247900 \\
\hline $\mathrm{H}$ & -0.87498800 & -2.44288700 & -0.46887100 \\
\hline $\mathrm{H}$ & 0.04604400 & 5.47590500 & -0.01908600 \\
\hline $\mathrm{C}$ & -5.33332900 & -2.68925800 & 0.82284000 \\
\hline $\mathrm{H}$ & -5.09491000 & -3.35058100 & 1.66957000 \\
\hline $\mathrm{H}$ & -6.16876500 & -2.03335200 & 1.09269400 \\
\hline $\mathrm{H}$ & -5.64154200 & -3.34169500 & -0.00977700 \\
\hline $\mathrm{F}$ & -1.08204100 & 4.23822400 & 1.93871500 \\
\hline
\end{tabular}

\section{Structure 14. C-TS 1}

$\mathrm{F}$

$-4.10555400 \quad-0.36084800 \quad-1.82765400$

F $\quad-4.32943400 \quad-0.59253200 \quad 0.29335900$ 


\begin{tabular}{|c|c|c|c|}
\hline $\mathrm{F}$ & -3.79223800 & -2.28372400 & -0.91879200 \\
\hline T & 0.90943500 & -2.03989600 & 0.67792100 \\
\hline $\mathrm{C}$ & -1.04589900 & 4.05411600 & 0.76938600 \\
\hline $\mathrm{C}$ & -1.09110900 & 3.10089300 & 1.78622000 \\
\hline $\mathrm{H}$ & -1.58577300 & 3.34093000 & 2.72808100 \\
\hline$y$ & -0.50939500 & 1.84796100 & 1.60626000 \\
\hline $\mathrm{H}$ & -0.52866900 & 1.11228200 & 2.41136100 \\
\hline $\mathrm{C}$ & 0.13846300 & 1.54419700 & 0.39879300 \\
\hline $\mathrm{C}$ & 0.75417800 & 0.22686100 & 0.19191200 \\
\hline $\mathrm{C}$ & 2.05367300 & 0.03049300 & -0.26703000 \\
\hline 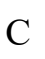 & 3.08591400 & 1.01902000 & -0.10653500 \\
\hline $\mathrm{H}$ & 2.81077100 & 2.04987600 & 0.11914300 \\
\hline 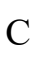 & 4.38878000 & 0.62313300 & -0.08860900 \\
\hline $\mathrm{H}$ & 5.17746300 & 1.35192300 & 0.10771500 \\
\hline $\mathrm{C}$ & 4.76299700 & -0.75427000 & -0.28213600 \\
\hline $\mathrm{C}$ & -0.39469600 & 3.73450500 & -0.41641100 \\
\hline $\mathrm{C}$ & 0.20695500 & 2.50299200 & -0.62362600 \\
\hline $\mathrm{H}$ & 0.69744800 & 2.29039900 & -1.57519000 \\
\hline $\mathrm{C}$ & 2.43726500 & -1.32707400 & -0.60805100 \\
\hline $\mathrm{C}$ & 3.79360400 & -1.70465900 & -0.53553400 \\
\hline $\mathrm{H}$ & 4.08215800 & -2.72481700 & -0.79211200 \\
\hline $\mathrm{C}$ & 0.10708700 & -1.03897400 & 0.49162600 \\
\hline $\mathrm{C}$ & -1.37583600 & -1.43919100 & 0.45689700 \\
\hline $\mathrm{C}$ & -1.20072300 & -2.93056800 & 0.06234900 \\
\hline $\mathrm{H}$ & -2.01302400 & -3.55854500 & 0.44687900 \\
\hline I & -1.17809700 & -3.02147400 & -1.03378400 \\
\hline $\mathrm{C}$ & 0.16987600 & -3.29487300 & 0.63445400 \\
\hline $\mathrm{H}$ & 0.12739700 & -3.68363300 & 1.66514700 \\
\hline $\mathrm{H}$ & 0.73198200 & -4.02715200 & 0.03819400 \\
\hline $\mathrm{C}$ & -1.99815400 & -1.32405300 & 1.85830600 \\
\hline $\mathrm{H}$ & -1.33470200 & -1.76985000 & 2.61355300 \\
\hline $\mathrm{H}$ & -2.95331500 & -1.86611200 & 1.88174000 \\
\hline $\mathrm{H}$ & -2.19214200 & -0.27803400 & 2.12974500 \\
\hline $\mathrm{C}$ & -2.13167300 & -0.61709100 & -0.59528400 \\
\hline $\mathrm{H}$ & -2.11379100 & 0.45506700 & -0.35070900 \\
\hline $\mathrm{H}$ & -1.66550500 & -0.74961200 & -1.58284600 \\
\hline $\mathrm{C}$ & -3.58648600 & -0.97049300 & -0.7555 \\
\hline
\end{tabular}




$\begin{array}{lrrr}\mathrm{H} & 1.79176100 & -1.90460000 & -1.27416400 \\ \mathrm{C} & 6.21527100 & -1.10245400 & -0.27083800 \\ \mathrm{H} & 6.74903100 & -0.52438300 & -1.04071600 \\ \mathrm{H} & 6.65336100 & -0.81875400 & 0.69885500 \\ \mathrm{H} & 6.37867100 & -2.17293900 & -0.44125800 \\ \mathrm{H} & -1.50350200 & 5.03796300 & 0.88108500 \\ \mathrm{~F} & -0.34812100 & 4.64353800 & -1.39307900\end{array}$

\section{Structure 15. C-Int 1}

$\mathrm{F}$

$\begin{array}{lll}-4.22557400 & -0.47081100 & -1.70798300\end{array}$

$\begin{array}{llll}-4.31497600 & -0.91518000 & 0.38917300\end{array}$

$\begin{array}{llll}-3.67733700 & -2.42575500 & -1.00011100\end{array}$

$\mathrm{N} \quad 0.99387500 \quad-1.84919300 \quad 0.35616200$

C $\quad-1.14691200 \quad 4.25375000 \quad 0.41193300$

$\begin{array}{llll}\text { C } & -0.22556800 & 3.86629500 & -0.55171500\end{array}$

$\begin{array}{llll}\text { C } & 0.39169900 & 2.62303800 & -0.53600600\end{array}$

$\mathrm{H} \quad 1.09719900 \quad 2.36005500 \quad-1.32593200$

$\begin{array}{lllll}\text { C } & 0.07492300 & 1.72615100 & 0.49229900\end{array}$

$\begin{array}{lllll}\mathrm{C} & 0.73060600 & 0.39913000 & 0.51314600\end{array}$

$\begin{array}{lllll}\mathrm{C} & 2.08485200 & 0.14722700 & 0.53280800\end{array}$

$\begin{array}{lllll}\text { C } & 3.22976000 & 1.00958700 & 0.42460600\end{array}$

$\mathrm{H} \quad 3.13462500 \quad 2.08063100 \quad 0.60916800$

$\begin{array}{lllll}\text { C } & 4.38617600 & 0.46421100 & -0.03190500\end{array}$

$\mathrm{H} \quad 5.25918000 \quad 1.10623000 \quad-0.17416900$

$\begin{array}{llll}\text { C } & 4.52143500 & -0.94602200 & -0.42240200\end{array}$

C $\quad-1.45014000 \quad 3.35610100 \quad 1.43502600$

$\begin{array}{llll}\mathrm{H} & -2.16399800 & 3.64320700 & 2.20827700\end{array}$

C $\quad-0.84399600 \quad 2.10117500 \quad 1.48202500$

$\mathrm{H} \quad-1.07063200 \quad 1.41504000 \quad 2.29854300$

$\begin{array}{lllll}\mathrm{C} & 2.32049400 & -1.32558400 & 0.56637500\end{array}$

C $\quad 3.50724700 \quad-1.80793900 \quad-0.20129000$

$\mathrm{H} \quad 3.58666600 \quad-2.87117000 \quad-0.43812900$

$\begin{array}{llll}\text { C } & 0.10079000 & -0.89345500 & 0.39963100\end{array}$

$\begin{array}{lllll}\text { C } & -1.29742400 & -1.46443800 & 0.35469100\end{array}$

C $\quad-0.99176200 \quad-2.90001900 \quad-0.16837800$

$\mathrm{H} \quad-1.70648900 \quad-3.63528800 \quad 0.21738000$

$\mathrm{H} \quad-1.04042300 \quad-2.90627200 \quad-1.26610600$ 


$\begin{array}{lrrr}\mathrm{C} & 0.44659500 & -3.20068300 & 0.27595200 \\ \mathrm{H} & 0.50985800 & -3.67792100 & 1.26472800 \\ \mathrm{H} & 1.01532400 & -3.79533700 & -0.44907000 \\ \mathrm{C} & -1.84320500 & -1.51625200 & 1.79359800 \\ \mathrm{H} & -1.10375600 & -1.97199200 & 2.46836900 \\ \mathrm{H} & -2.75077000 & -2.13370700 & 1.81868500 \\ \mathrm{H} & -2.09250100 & -0.51405100 & 2.16497500 \\ \mathrm{C} & -2.17512900 & -0.63604900 & -0.59191300 \\ \mathrm{H} & -2.23752300 & 0.40803600 & -0.25160600 \\ \mathrm{H} & -1.74606100 & -0.64042400 & -1.60439100 \\ \mathrm{C} & -3.59532400 & -1.11798200 & -0.72225200 \\ \mathrm{H} & 2.55567500 & -1.55849200 & 1.63365800 \\ \mathrm{C} & 5.81301400 & -1.36576400 & -1.06103900 \\ \mathrm{H} & 5.79275000 & -2.42609600 & -1.34235800 \\ \mathrm{H} & 6.01024400 & -0.76248900 & -1.96004100 \\ \mathrm{H} & 6.65285000 & -1.19946000 & -0.36924400 \\ \mathrm{H} & -1.60712200 & 5.24068700 & 0.35188400 \\ \mathrm{~F} & 0.07555300 & 4.71711100 & -1.53873400\end{array}$

\section{Structure 16. D}

\begin{tabular}{|c|c|c|c|}
\hline $\mathrm{F}$ & -3.30348600 & -0.78560400 & 3700 \\
\hline $\mathrm{F}$ & -3.64997400 & 1.00118800 & -1.58299200 \\
\hline $\mathrm{F}$ & -4.32326700 & -0.89538300 & -0.82735700 \\
\hline $\mathrm{N}$ & -0.52246600 & -1.12100600 & 2.38541500 \\
\hline $\mathrm{C}$ & 1.71058400 & 3.87862100 & -0.11338500 \\
\hline C & 0.99781400 & 3.49161100 & 1.03649700 \\
\hline $\mathrm{C}$ & 0.64237500 & 2.17179000 & 1.23576200 \\
\hline $\mathrm{H}$ & 0.10957500 & 1.87987300 & 2.14194400 \\
\hline $\mathrm{C}$ & 1.04556100 & 1.17304200 & 0.30971500 \\
\hline $\mathrm{C}$ & 0.71222400 & -0.18715300 & 0.56339200 \\
\hline $\mathrm{C}$ & 1.53364900 & -1.29099500 & 0.14861600 \\
\hline $\mathrm{C}$ & 2.92023600 & -1.10472600 & -0.08012000 \\
\hline $\mathrm{C}$ & 3.68041700 & -2.19130700 & -0.46033700 \\
\hline$C$ & 3.12852100 & -3.45995300 & -0.63199600 \\
\hline $\mathrm{C}$ & 2.08070700 & 2.89517400 & -1.05392000 \\
\hline$C$ & 1.77571200 & 1.56639500 & -0.84745100 \\
\hline $\mathbf{I}$ & 2.03243300 & 0.82447900 & -1.60473600 \\
\hline
\end{tabular}




$\begin{array}{lrrr}\mathrm{C} & 0.97234400 & -2.58569900 & 0.00801700 \\ \mathrm{C} & 1.76581500 & -3.64902200 & -0.39687500 \\ \mathrm{C} & -0.55356800 & -0.51041300 & 1.27154300 \\ \mathrm{C} & -1.95553500 & -0.19571300 & 0.70232100 \\ \mathrm{C} & -2.75884100 & -1.19334500 & 1.56959600 \\ \mathrm{H} & -3.77585200 & -0.83858500 & 1.77594500 \\ \mathrm{H} & -2.82503700 & -2.16162000 & 1.05125000 \\ \mathrm{C} & -1.89975100 & -1.34514600 & 2.82767800 \\ \mathrm{H} & -2.13764100 & -0.59568900 & 3.60049300 \\ \mathrm{H} & -1.97504900 & -2.33291700 & 3.30150000 \\ \mathrm{C} & -2.37228600 & 1.24986800 & 1.01014600 \\ \mathrm{H} & -2.20167600 & 1.48314900 & 2.07122700 \\ \mathrm{H} & -3.44476700 & 1.37378500 & 0.80645900 \\ \mathrm{H} & -1.82409400 & 1.97526500 & 0.39221500 \\ \mathrm{C} & -1.98636500 & -0.50098600 & -0.80136400 \\ \mathrm{H} & -1.26885100 & 0.13394700 & -1.34507500 \\ \mathrm{H} & -1.71405200 & -1.55091400 & -0.98168600 \\ \mathrm{C} & -3.31711600 & -0.29179700 & -1.47329700 \\ \mathrm{H} & -0.09076800 & -2.74311800 & 0.18811900 \\ \mathrm{H} & 2.60806900 & 3.19607400 & -1.96094100 \\ \mathrm{H} & 3.77738400 & -4.28541900 & -0.92965200 \\ \mathrm{H} & 0.72081200 & 4.24594000 & 1.77434900 \\ \mathrm{C} & 2.05749900 & 5.31120600 & -0.35964000 \\ \mathrm{H} & 3.13070000 & 5.41252800 & -0.57887100 \\ \mathrm{H} & 1.51513300 & 5.67639500 & -1.24612500 \\ \mathrm{H} & 1.79915400 & 5.94418000 & 0.49736900 \\ \mathrm{H} & 1.32836000 & -4.63895500 & -0.52543800 \\ & 3.41212500 & -0.14856600 & 0.09787800 \\ \mathrm{H} & 4.98798600 & -2.03036900 & -0.65179300\end{array}$

\section{Structure 17. D-TS 1}

$\begin{array}{llll}\text { F } & -4.03299500 & -0.12105900 & -1.83801300 \\ \text { F } & -4.32079500 & -0.33958500 & 0.27683500 \\ \text { F } & -3.89883500 & -2.06545700 & -0.93245900 \\ \text { N } & 0.75428700 & -2.22095900 & 0.81623900 \\ \text { C } & -0.45733400 & 4.06880900 & 0.19114000 \\ \text { C } & -0.71403800 & 3.21739900 & 1.27815800\end{array}$




\begin{tabular}{|c|c|c|c|}
\hline $\mathrm{H}$ & -1.24583500 & 3.60571900 & 2.14912000 \\
\hline $\mathrm{C}$ & -0.29712100 & 1.89365500 & 1.26386800 \\
\hline $\mathrm{H}$ & -0.48374500 & 1.25452800 & 2.12832800 \\
\hline $\mathrm{C}$ & 0.41094600 & 1.38515400 & 0.15957300 \\
\hline $\mathrm{C}$ & 0.85513100 & -0.00188300 & 0.12245300 \\
\hline $\mathrm{C}$ & 2.12948400 & -0.40935600 & -0.31154500 \\
\hline $\mathrm{C}$ & 3.27188400 & 0.44404400 & -0.22198700 \\
\hline $\mathrm{C}$ & 4.50353400 & -0.14030800 & -0.22787000 \\
\hline $\mathrm{C}$ & 4.69266400 & -1.54480900 & -0.34851900 \\
\hline $\mathrm{C}$ & 0.25394500 & 3.55825600 & -0.90487500 \\
\hline $\mathrm{C}$ & 0.69175900 & 2.24015800 & -0.92239100 \\
\hline $\mathrm{H}$ & 1.23006400 & 1.85479900 & -1.79087800 \\
\hline $\mathrm{C}$ & 2.30980000 & -1.82073400 & -0.56524600 \\
\hline $\mathrm{C}$ & 3.61414700 & -2.37529600 & -0.51418600 \\
\hline $\mathrm{C}$ & 0.07237000 & -1.14723000 & 0.54414500 \\
\hline $\mathrm{C}$ & -1.44870900 & -1.38342900 & 0.52604800 \\
\hline $\mathrm{C}$ & -1.43313500 & -2.90282700 & 0.20974000 \\
\hline $\mathrm{H}$ & -2.32050600 & -3.41601700 & 0.59843300 \\
\hline $\mathrm{H}$ & -1.38930100 & -3.05363900 & -0.87933200 \\
\hline $\mathrm{C}$ & -0.12786900 & -3.37975500 & 0.84458900 \\
\hline $\mathrm{H}$ & -0.23539800 & -3.67850900 & 1.90066800 \\
\hline $\mathrm{H}$ & 0.35700600 & -4.21694300 & 0.32349800 \\
\hline $\mathrm{C}$ & -2.05681100 & -1.14097400 & 1.91782600 \\
\hline $\mathrm{H}$ & -1.44703000 & -1.62056900 & 2.69701500 \\
\hline $\mathrm{H}$ & -3.06438200 & -1.57599100 & 1.95837900 \\
\hline $\mathrm{H}$ & -2.13872900 & -0.06993100 & 2.14441000 \\
\hline $\mathrm{C}$ & -2.11423400 & -0.53947300 & -0.56839100 \\
\hline $\mathrm{H}$ & -2.01390300 & 0.53483700 & -0.35665100 \\
\hline $\mathrm{H}$ & -1.63874700 & -0.74009700 & -1.54013500 \\
\hline $\mathrm{C}$ & -3.58910500 & -0.77447100 & -0.75782100 \\
\hline $\mathrm{H}$ & 1.56743100 & -2.35319500 & -1.16394900 \\
\hline $\mathrm{H}$ & 0.46415100 & 4.20705600 & -1.75749700 \\
\hline $\mathrm{H}$ & 5.71465000 & -1.92859400 & -0.34956600 \\
\hline $\mathrm{C}$ & -0.95452700 & 5.48498600 & 0.19431800 \\
\hline $\mathrm{H}$ & -1.98826200 & 5.52265400 & -0.18534300 \\
\hline $\mathrm{H}$ & -0.96225000 & 5.89976300 & 1.21115000 \\
\hline $\mathrm{H}$ & -0.33923500 & 6.12448900 & -0.45203800 \\
\hline
\end{tabular}




$\begin{array}{llll}\mathrm{H} & 3.75741600 & -3.44063800 & -0.69432200 \\ \mathrm{H} & 3.17126800 & 1.51203400 & -0.02680500 \\ \mathrm{~F} & 5.59136400 & 0.60759700 & -0.07106300\end{array}$

\section{Structure 18. D-Int 1}

$\mathrm{F}$

$-4.19017600 \quad-0.44971500 \quad-1.68899400$

$\mathrm{F}$

$\begin{array}{lll}-4.25086700 & -0.71621300 & 0.43878600\end{array}$

$\mathrm{F}$

$-3.77208300-2.37897700 \quad-0.83586600$

$\mathrm{N}$

$\begin{array}{lll}0.96996400 & -2.06625200 & 0.33286800\end{array}$

$\begin{array}{llll}\text { C } & -0.76604900 & 4.18062800 & 0.08971500\end{array}$

C $\quad 0.09093800 \quad 3.68221100 \quad-0.90196800$

C $\quad 0.61913600 \quad 2.39745800 \quad-0.81678600$

$\mathrm{H} \quad 1.27364400 \quad 2.02187800 \quad-1.60654900$

$\begin{array}{llll}\text { C } & 0.30344300 & 1.56997200 & 0.27133900\end{array}$

$\begin{array}{llll}\text { C } & 0.86841300 & 0.20470300 & 0.34589200\end{array}$

$\begin{array}{llll}\text { C } & 2.20380800 & -0.14241900 & 0.32404500\end{array}$

$\begin{array}{llll}\text { C } & 3.38865800 & 0.63359000 & 0.12161700\end{array}$

$\begin{array}{llll}\text { C } & 4.47650600 & -0.04656900 & -0.31937500\end{array}$

C $\quad 4.51267100 \quad-1.47429800 \quad-0.59935600$

$\begin{array}{llll}\text { C } & -1.06854800 & 3.35248700 & 1.17694300\end{array}$

$\mathrm{H} \quad \begin{array}{llll}\mathrm{H} & -1.72414300 & 3.72395700 & 1.96767100\end{array}$

$\begin{array}{llll}\text { C } & -0.54070600 & 2.06538700 & 1.27203800\end{array}$

$\mathrm{H} \quad-0.77290600 \quad 1.44630400 \quad 2.14026100$

$\begin{array}{lllll}\text { C } & 2.33474800 & -1.62753200 & 0.45918800\end{array}$

$\begin{array}{llll}\text { C } & 3.45740100 & -2.25125700 & -0.30242600\end{array}$

$\begin{array}{llll}\text { C } & 0.15056700 & -1.04297800 & 0.34197400\end{array}$

$\begin{array}{llll}\text { C } & -1.28631500 & -1.51008800 & 0.37465100\end{array}$

C $\quad-1.10372600 \quad-2.99859500 \quad-0.04923300$

$\mathrm{H} \quad-1.85659000 \quad-3.64837500 \quad 0.41057500$

$\mathrm{H} \quad-1.19129200 \quad-3.08065000 \quad-1.14147600$

C $\quad 0.32301100 \quad-3.37501200 \quad 0.37300400$

$\mathrm{H} \quad 0.38522200 \quad-3.78102100 \quad 1.39317500$

$\mathrm{H} \quad 0.81885800 \quad-4.06411500 \quad-0.32121100$

C $\quad-1.79656300 \quad-1.41622700 \quad 1.82446600$

$\mathrm{H} \quad-1.07368000 \quad-1.87361100 \quad 2.51579900$

$\mathrm{H} \quad \begin{array}{llll}\mathrm{H} & -2.74487700 & -1.96235000 & 1.91508300\end{array}$

$\mathrm{H} \quad \begin{array}{llll}\mathrm{H} & -1.96397600 & -0.37351500 & 2.12390300\end{array}$ 


$\begin{array}{lrrr}\mathrm{C} & -2.12602400 & -0.68741200 & -0.61158800 \\ \mathrm{H} & -2.09435200 & 0.38104700 & -0.35229900 \\ \mathrm{H} & -1.73002500 & -0.80294600 & -1.63084400 \\ \mathrm{C} & -3.58216300 & -1.06361200 & -0.66857900 \\ \mathrm{H} & 2.60490300 & -1.79890100 & 1.52949800 \\ \mathrm{H} & 5.41650000 & -1.87446200 & -1.06024900 \\ \mathrm{H} & 0.34294700 & 4.31168900 & -1.75881900 \\ \mathrm{C} & -1.34252900 & 5.56538400 & -0.02503100 \\ \mathrm{H} & -0.54727000 & 6.31078400 & -0.17242900 \\ \mathrm{H} & -2.01703600 & 5.63500200 & -0.89211800 \\ \mathrm{H} & -1.91134700 & 5.83579000 & 0.87403100 \\ \mathrm{H} & 3.45605800 & -3.32805500 & -0.47953300 \\ \mathrm{H} & 3.39416300 & 1.72176600 & 0.19265900 \\ \mathrm{~F} & 5.59594800 & 0.60808700 & -0.58730200\end{array}$

\section{Structure 19. E}

$\begin{array}{lrrr}\text { F } & 2.12467500 & -2.56669700 & -2.60150800 \\ \text { F } & 0.59012300 & -3.62638800 & -1.54258000 \\ \text { F } & 2.54723700 & -3.42592900 & -0.67634000 \\ \text { N } & 0.97577800 & 0.16532000 & 2.40575400 \\ \text { C } & -4.40560600 & -0.11729600 & -0.24015900 \\ \text { C } & -3.77274200 & -0.54823000 & 0.94424400 \\ \text { C } & -2.44241800 & -0.27005900 & 1.17479800 \\ \text { H } & -1.96697600 & -0.59200000 & 2.10246500 \\ \text { C } & -1.70208100 & 0.51134800 & 0.24478700 \\ \text { C } & -0.34170700 & 0.82322200 & 0.52193300 \\ \text { C } & 0.29273400 & 2.03892000 & 0.09272900 \\ \text { C } & -0.47617800 & 3.19924400 & -0.17926300 \\ \text { H } & -1.55471600 & 3.18746500 & -0.02629700 \\ \text { C } & 0.15169700 & 4.37778800 & -0.55813400 \\ \text { H } & -0.43925300 & 5.27374400 & -0.74811400 \\ \text { C } & 1.54058700 & 4.42812900 & -0.67699100 \\ \text { C } & -3.66553100 & 0.62177200 & -1.18149700 \\ \text { C } & -2.34482300 & 0.95132600 & -0.94428600 \\ \text { H } & -1.77275400 & 1.48755700 & -1.70254800 \\ \text { C } & 1.70657900 & 2.10540200 & 0.00900100 \\ \text { C } & 2.29176800 & 3.28881200 & -0.38787700\end{array}$




$\begin{array}{llll}\mathrm{C} & 0.48647500 & -0.15226200 & 1.27734600 \\ \mathrm{C} & 0.84582600 & -1.55828600 & 0.74469000 \\ \mathrm{C} & 2.05560100 & -1.82711600 & 1.67078100 \\ \mathrm{H} & 2.17028400 & -2.89257900 & 1.90397800 \\ \mathrm{H} & 2.97744800 & -1.47434800 & 1.18490700 \\ \mathrm{C} & 1.76495500 & -0.96357300 & 2.90118300 \\ \mathrm{H} & 1.16633400 & -1.49132400 & 3.66186000 \\ \mathrm{H} & 2.66616800 & -0.58714300 & 3.40322300 \\ \mathrm{C} & -0.28154500 & -2.56468800 & 1.01696000 \\ \mathrm{H} & -0.60978100 & -2.50095900 & 2.06453200 \\ \mathrm{H} & 0.08731100 & -3.58475000 & 0.84229700 \\ \mathrm{H} & -1.14703800 & -2.39969200 & 0.35973700 \\ \mathrm{C} & 1.20145200 & -1.47555300 & -0.74607400 \\ \mathrm{H} & 0.34465500 & -1.10847900 & -1.33391800 \\ \mathrm{H} & 2.04050100 & -0.78111100 & -0.89796200 \\ \mathrm{C} & 1.61294200 & -2.77613600 & -1.38279700 \\ \mathrm{H} & 2.33869100 & 1.24199400 & 0.21745800 \\ \mathrm{H} & -4.14113000 & 0.92729000 & -2.11471800 \\ \mathrm{H} & 2.05701200 & 5.34236900 & -0.97479200 \\ \mathrm{H} & -4.34552300 & -1.11486700 & 1.68044400 \\ \mathrm{~F} & 3.61719500 & 3.35213000 & -0.49453400 \\ \mathrm{C} & -5.84395400 & -0.44488800 & -0.47876600 \\ \mathrm{H} & -6.47394600 & 0.16055000 & 0.19288000 \\ \mathrm{H} & -6.14000600 & -0.23661500 & -1.51382600 \\ \mathrm{H} & -6.04500100 & -1.49966200 & -0.24285900 \\ & & & \\ \mathrm{H} & & & \end{array}$

\section{Structure 20. E-TS 1}

$\begin{array}{lrrr}\text { F } & -3.57978300 & -1.71264900 & -1.86105200 \\ \text { F } & -3.79053100 & -1.87985900 & 0.26736400 \\ \text { F } & -2.68135800 & -3.36480500 & -0.82016700 \\ \text { N } & 1.60483100 & -1.47927000 & 0.86221700 \\ \text { C } & -2.14690800 & 3.69156400 & 0.12058700 \\ \text { C } & -2.02589800 & 2.83049700 & 1.22523100 \\ \text { H } & -2.68003300 & 2.97196100 & 2.08820500 \\ \text { C } & -1.08828300 & 1.80923200 & 1.23554200 \\ \text { H } & -0.99032300 & 1.16589900 & 2.11156700 \\ \text { C } & -0.22455300 & 1.62922700 & 0.13784700\end{array}$




\begin{tabular}{|c|c|c|c|}
\hline $\mathrm{C}$ & 0.76619800 & 0.56215400 & 0.12486300 \\
\hline $\mathrm{C}$ & 2.10099900 & 0.73285900 & -0.28351900 \\
\hline $\mathrm{C}$ & 2.78075700 & 1.98770700 & -0.16616000 \\
\hline $\mathrm{H}$ & 2.20214700 & 2.89792800 & -0.00892500 \\
\hline $\mathrm{C}$ & 4.14843800 & 2.00577100 & -0.10318300 \\
\hline $\mathrm{H}$ & 4.67729300 & 2.94596100 & 0.05451400 \\
\hline $\mathrm{C}$ & 4.90345800 & 0.80253200 & -0.20240700 \\
\hline $\mathrm{C}$ & -1.28286800 & 3.50979400 & -0.96769400 \\
\hline $\mathrm{C}$ & -0.32554000 & 2.50179000 & -0.96032300 \\
\hline $\mathrm{H}$ & 0.33037000 & 2.36400500 & -1.82229800 \\
\hline $\mathrm{C}$ & 2.86209200 & -0.47761900 & -0.48234700 \\
\hline $\mathrm{C}$ & 4.26667200 & -0.39684800 & -0.36966400 \\
\hline $\mathrm{C}$ & 0.53604400 & -0.80537700 & 0.55939000 \\
\hline $\mathrm{C}$ & -0.73778600 & -1.66853600 & 0.53894100 \\
\hline $\mathrm{C}$ & -0.07140200 & -3.04549900 & 0.27095500 \\
\hline $\mathrm{H}$ & -0.65914700 & -3.87554000 & 0.67985000 \\
\hline $\mathrm{H}$ & 0.04206400 & -3.19627900 & -0.81301300 \\
\hline $\mathrm{C}$ & 1.30737500 & -2.90403700 & 0.91444100 \\
\hline $\mathrm{H}$ & 1.33271900 & -3.20116100 & 1.97582400 \\
\hline $\mathrm{H}$ & 2.11089800 & -3.45684300 & 0.40785600 \\
\hline $\mathrm{C}$ & -1.41794800 & -1.66883200 & 1.91897700 \\
\hline $\mathrm{H}$ & -0.67530700 & -1.80631500 & 2.71802800 \\
\hline $\mathrm{H}$ & -2.13301500 & -2.50082300 & 1.97295500 \\
\hline $\mathrm{H}$ & -1.96747900 & -0.73663000 & 2.10280500 \\
\hline $\mathrm{C}$ & -1.67902500 & -1.22249600 & -0.58748400 \\
\hline $\mathrm{H}$ & -2.02877200 & -0.19198200 & -0.43052000 \\
\hline $\mathrm{H}$ & -1.15410400 & -1.26059600 & -1.55355300 \\
\hline $\mathrm{C}$ & -2.92719700 & -2.05060500 & -0.74291000 \\
\hline $\mathrm{H}$ & 2.47373100 & -1.29098600 & -1.10062200 \\
\hline $\mathrm{H}$ & -1.36601400 & 4.16802300 & -1.83458100 \\
\hline $\mathrm{H}$ & 5.99481800 & 0.82570600 & -0.19428800 \\
\hline $\mathrm{F}$ & 4.96484800 & -1.51757200 & -0.54421400 \\
\hline $\mathrm{C}$ & -3.17956400 & 4.78062500 & 0.12330100 \\
\hline $\mathrm{H}$ & -3.16574700 & 5.34843000 & -0.81532500 \\
\hline $\mathrm{H}$ & -4.18551900 & 4.35824100 & 0.26524600 \\
\hline $\mathrm{H}$ & -3.00133400 & 5.47643600 & 0.95706800 \\
\hline
\end{tabular}




\section{Structure 21. E-Int 1}

F

F

F

$\mathrm{N}$

C

C

C

$\mathrm{H}$

C

C

C

C

$\mathrm{H}$

C

$\mathrm{H}$

C

C

$\mathrm{H}$

$\mathrm{C}$

$\mathrm{H}$

$\mathrm{C}$

C

C

C

C

$\mathrm{H}$

$\mathrm{H}$

$\mathrm{C}$

$\mathrm{H}$

$\mathrm{H}$

C

$\mathrm{H}$

$\mathrm{H}$

$\mathrm{H}$

C

$\mathrm{H}$ $\begin{array}{lll}-3.44358300 & -2.29965200 & -1.63253600\end{array}$

$\begin{array}{lll}-3.35868100 & -2.49908100 & 0.50175700\end{array}$

$\begin{array}{lll}-2.13267600 & -3.75262800 & -0.74085000\end{array}$

$\begin{array}{lll}1.85352900 & -1.12783400 & 0.33857700\end{array}$

$\begin{array}{lll}-2.70461700 & 3.47607800 & 0.00003000\end{array}$

$\begin{array}{lll}-1.68570800 & 3.46156400 & -0.96329800\end{array}$

$\begin{array}{lll}-0.60081000 & 2.59776000 & -0.84935800\end{array}$

$\begin{array}{llll}0.17596200 & 2.59070800 & -1.61726600\end{array}$

$\begin{array}{lll}-0.50438000 & 1.71855500 & 0.23981900\end{array}$

$\begin{array}{lll}0.65474900 & 0.80573600 & 0.34423700\end{array}$

$\begin{array}{lll}1.98665500 & 1.16031900 & 0.33678400\end{array}$

$\begin{array}{lll}2.64647900 & 2.41512000 & 0.10392900\end{array}$

$\begin{array}{llll}2.09863800 & 3.35229100 & 0.20661200\end{array}$

$\begin{array}{llll}3.91543900 & 2.38114700 & -0.38375600\end{array}$

$\begin{array}{llll}4.42148300 & 3.31512900 & -0.63397500\end{array}$

$\begin{array}{llll}4.62843000 & 1.14053800 & -0.66014800\end{array}$

$\begin{array}{lll}-2.59740200 & 2.60056500 & 1.08688800\end{array}$

$\begin{array}{lll}-3.37491200 & 2.60015600 & 1.85399600\end{array}$

$\begin{array}{lll}-1.51220100 & 1.73385000 & 1.21055700\end{array}$

$\begin{array}{lll}-1.44106400 & 1.07418300 & 2.07667900\end{array}$

$\begin{array}{lll}2.82265700 & -0.07414200 & 0.47881600\end{array}$

$\begin{array}{llll}4.07729400 & -0.03417100 & -0.32658900\end{array}$

$\begin{array}{lll}0.63797100 & -0.63706200 & 0.34709100\end{array}$

$\begin{array}{lll}-0.38577300 & -1.74633900 & 0.40418100\end{array}$

$\begin{array}{llll}0.49964100 & -2.96575900 & 0.00871900\end{array}$

$\begin{array}{lll}0.16296100 & -3.88803200 & 0.49473700\end{array}$

$\begin{array}{llll}0.45979900 & -3.10936600 & -1.07980100\end{array}$

$\begin{array}{lll}1.92897600 & -2.58767600 & 0.41749500\end{array}$

$2.18311400-2.88000200 \quad 1.44666900$

$\begin{array}{llll}2.69638700 & -2.96514200 & -0.26731600\end{array}$

$\begin{array}{lll}-0.86800900 & -1.87632200 & 1.86105600\end{array}$

$\begin{array}{lll}-0.00831600 & -1.90903400 & 2.54592600\end{array}$

$\begin{array}{lll}-1.43019300 & -2.81210300 & 1.97830700\end{array}$

$\begin{array}{lll}-1.51784800 & -1.03872000 & 2.14511600\end{array}$

$\begin{array}{lll}-1.52384900 & -1.46557700 & -0.58573100\end{array}$

$\begin{array}{lll}-2.02020100 & -0.51266300 & -0.35007600\end{array}$ 


$\begin{array}{lrrr}\mathrm{H} & -1.12556400 & -1.40060700 & -1.60855400 \\ \mathrm{C} & -2.60962800 & -2.50805600 & -0.60853800 \\ \mathrm{H} & 3.16629900 & -0.10807300 & 1.53939100 \\ \mathrm{H} & 5.60959200 & 1.16128400 & -1.13486100 \\ \mathrm{H} & -1.74893100 & 4.13585400 & -1.82075100 \\ \mathrm{C} & -3.87173800 & 4.41497300 & -0.13787400 \\ \mathrm{H} & -3.53526300 & 5.46228600 & -0.10501100 \\ \mathrm{H} & -4.38022300 & 4.26821100 & -1.10226900 \\ \mathrm{H} & -4.60250500 & 4.26266800 & 0.66689300 \\ \mathrm{~F} & 4.67172000 & -1.20095000 & -0.52285200\end{array}$

\section{Structure 22. C'}

$\begin{array}{llrl}\text { F } & 3.13073500 & -0.63026800 & 2.80706000 \\ \text { F } & 4.05260600 & 0.11593400 & 1.01953300 \\ \text { F } & 3.77120900 & -1.99878300 & 1.27830800 \\ \text { N } & -0.09411100 & -1.98029500 & -1.85339800 \\ \text { C } & 0.44744300 & 4.14674600 & -0.69979400 \\ \mathrm{C} & 0.71114100 & 3.32327200 & -1.79337100 \\ \mathrm{H} & 1.20480500 & 3.73893000 & -2.67312500 \\ \mathrm{C} & 0.34495000 & 1.97817600 & -1.77348700 \\ \mathrm{H} & 0.54387500 & 1.34635500 & -2.64072200 \\ \mathrm{C} & -0.30282400 & 1.42570300 & -0.65436400 \\ \mathrm{C} & -0.69309200 & 0.00650200 & -0.64580300 \\ \mathrm{C} & -2.02163000 & -0.39336600 & -0.21894200 \\ \mathrm{C} & -3.09605800 & 0.52606500 & -0.20411500 \\ \mathrm{H} & -2.93603600 & 1.54859400 & -0.54878400 \\ \mathrm{C} & -4.36738700 & 0.14539800 & 0.21154300 \\ \mathrm{H} & -5.17662000 & 0.87921900 & 0.19712700 \\ \mathrm{C} & -4.63388600 & -1.16144600 & 0.63901200 \\ \mathrm{C} & -0.19462100 & 3.58868700 & 0.40026800 \\ \mathrm{C} & -0.57868800 & 2.25880800 & 0.44618500 \\ \mathrm{H} & -1.07243300 & 1.87478000 & 1.34058300 \\ \mathrm{C} & -2.30111000 & -1.71551000 & 0.21068800 \\ \mathrm{C} & -3.57042600 & -2.08056100 & 0.63057600 \\ \mathrm{H} & -3.74797600 & -3.10485700 & 0.96786100 \\ \mathrm{C} & 0.26646100 & -1.01227500 & -1.09331200 \\ \mathrm{C} & 1.74285000 & -1.08274000 & -0.62420400\end{array}$




$\begin{array}{lrrr}\mathrm{C} & 1.99427800 & -2.58371100 & -0.88967700 \\ \mathrm{H} & 3.04381900 & -2.80238100 & -1.12392100 \\ \mathrm{H} & 1.70885400 & -3.16905900 & -0.00196100 \\ \mathrm{C} & 1.02848700 & -2.89554600 & -2.03413700 \\ \mathrm{H} & 1.47843300 & -2.71471400 & -3.02595000 \\ \mathrm{H} & 0.67099400 & -3.93515500 & -2.03255200 \\ \mathrm{C} & 2.67425600 & -0.23280400 & -1.50519600 \\ \mathrm{H} & 2.44213100 & -0.38596800 & -2.56976800 \\ \mathrm{H} & 3.71868200 & -0.53451800 & -1.34142600 \\ \mathrm{H} & 2.59082200 & 0.83812800 & -1.27961100 \\ \mathrm{C} & 1.82985300 & -0.69745200 & 0.85786700 \\ \mathrm{H} & 1.51306900 & 0.34523500 & 1.01180000 \\ \mathrm{H} & 1.16434600 & -1.34140900 & 1.45264900 \\ \mathrm{C} & 3.19468700 & -0.80582300 & 1.48005400 \\ \mathrm{H} & -1.50059900 & -2.45513900 & 0.22739200 \\ \mathrm{H} & 0.72503900 & 5.20128400 & -0.68561000 \\ \mathrm{C} & -6.00467100 & -1.58052500 & 1.09041400 \\ \mathrm{H} & -5.97598300 & -1.97379500 & 2.11799300 \\ \mathrm{H} & -6.70909600 & -0.73906000 & 1.06122100 \\ \mathrm{H} & -6.39918700 & -2.38398600 & 0.44953700 \\ \mathrm{~F} & -0.44879800 & 4.36689400 & 1.46063600\end{array}$

\section{Structure 23. C'-TS 1}

$\begin{array}{lrrr}\text { F } & -4.44859800 & -0.14251200 & -1.30725100 \\ \text { F } & -4.37006400 & -0.82634500 & 0.72451500 \\ \text { F } & -3.94306500 & -2.18725200 & -0.88287700 \\ \text { N } & 0.90713200 & -2.03106600 & 0.27950400 \\ \text { C } & -0.93546200 & 4.21126300 & 0.58330100 \\ \text { C } & -1.26126600 & 3.19244700 & 1.47857000 \\ \text { H } & -1.92069300 & 3.40749500 & 2.32095500 \\ \text { C } & -0.74032200 & 1.90982900 & 1.31737700 \\ \text { H } & -0.97980500 & 1.13183700 & 2.04281400 \\ \text { C } & 0.11011500 & 1.60631900 & 0.23922900 \\ \text { C } & 0.68806900 & 0.26753100 & 0.04954900 \\ \text { C } & 2.09178100 & 0.05760600 & -0.21673500 \\ \text { C } & 3.14295700 & 0.88078200 & 0.22068200 \\ \text { H } & 2.91757300 & 1.89131500 & 0.57028500\end{array}$




$\begin{array}{lrrr}\mathrm{C} & 4.43965400 & 0.39261200 & 0.29449900 \\ \mathrm{H} & 5.24514400 & 1.03380000 & 0.65903000 \\ \mathrm{C} & 4.73114300 & -0.95241600 & -0.05940000 \\ \mathrm{C} & -0.07727600 & 3.90419200 & -0.46555500 \\ \mathrm{C} & 0.44982500 & 2.63633700 & -0.65553900 \\ \mathrm{H} & 1.11049100 & 2.45360100 & -1.50488300 \\ \mathrm{C} & 2.38286900 & -1.28793400 & -0.67764000 \\ \mathrm{C} & 3.72419100 & -1.76736600 & -0.54652200 \\ \mathrm{H} & 3.96691400 & -2.76874700 & -0.90923200 \\ \mathrm{C} & 0.07973400 & -0.98255000 & 0.26542000 \\ \mathrm{C} & -1.37859000 & -1.45366300 & 0.34983600 \\ \mathrm{C} & -1.22632000 & -2.83635400 & -0.34210600 \\ \mathrm{H} & -1.99190800 & -3.55150000 & -0.01558300 \\ \mathrm{H} & -1.31659200 & -2.70766700 & -1.43152700 \\ \mathrm{C} & 0.20275800 & -3.27120700 & 0.00656600 \\ \mathrm{H} & 0.24573800 & -3.92633700 & 0.89219000 \\ \mathrm{H} & 0.69569500 & -3.81111200 & -0.81808900 \\ \mathrm{H} & -1.79421800 & -1.64788000 & 1.81749300 \\ \mathrm{H} & -1.01787800 & -2.20704400 & 2.35975200 \\ \mathrm{H} & -2.72864400 & -2.22292500 & 1.87583100 \\ \mathrm{H} & -1.32332400 & 5.22509700 & 0.68772800 \\ \mathrm{H} & -1.95056200 & -0.68837200 & 2.32930900 \\ \mathrm{H} & -2.31514100 & -0.51389900 & -0.41901200 \\ \mathrm{H} & -2.31744000 & 0.49365300 & 0.01766600 \\ \mathrm{H} & -1.98155500 & -0.42539900 & -1.46339900 \\ \mathrm{H} & -3.76088500 & -0.92689700 & -0.46634200 \\ \mathrm{H} & 1.83205400 & -1.65094300 & -1.55639700 \\ \mathrm{H} & 6.14742900 & -1.44868600 & 0.04930700 \\ \mathrm{H} & 6.82867300 & -0.82460100 & -0.54881700 \\ \mathrm{H} & & -1.87035900 & -1.33463200\end{array}$

\section{Structure 24. C'-Int 1}

F $\begin{array}{lll}-4.26805400 & -0.47390100 & -1.72876000\end{array}$

F $\quad-4.35559100 \quad-0.86850300 \quad 0.37802300$

F $\quad-3.73122900 \quad-2.41535900 \quad-0.97727800$ 


\begin{tabular}{|c|c|c|c|}
\hline $\mathrm{N}$ & 0.98064400 & -1.85279700 & 0.27124100 \\
\hline $\mathrm{C}$ & -1.10211100 & 4.30054200 & 0.38917500 \\
\hline $\mathrm{C}$ & -0.08280700 & 3.93783500 & -0.48155000 \\
\hline $\mathrm{C}$ & 0.50889500 & 2.68333700 & -0.46245900 \\
\hline $\mathrm{H}$ & 1.28938900 & 2.45195400 & -1.18866200 \\
\hline $\mathrm{C}$ & 0.07239800 & 1.73051000 & 0.47268800 \\
\hline $\mathrm{C}$ & 0.69799700 & 0.39201800 & 0.49720400 \\
\hline $\mathrm{C}$ & 2.10820100 & 0.13404100 & 0.47394100 \\
\hline $\mathrm{C}$ & 3.23059700 & 0.94839000 & 0.31356200 \\
\hline $\mathrm{H}$ & 3.13865200 & 2.03534300 & 0.37707700 \\
\hline $\mathrm{C}$ & 4.45190700 & 0.36812400 & -0.02398000 \\
\hline $\mathrm{H}$ & 5.33290100 & 0.99848100 & -0.16304100 \\
\hline $\mathrm{C}$ & 4.54686200 & -1.03383800 & -0.37025400 \\
\hline $\mathrm{C}$ & -1.53106600 & 3.35469900 & 1.31951000 \\
\hline $\mathrm{H}$ & -2.32452100 & 3.61358900 & 2.02243200 \\
\hline $\mathrm{C}$ & -0.94789000 & 2.08872900 & 1.36909900 \\
\hline $\mathrm{H}$ & -1.27544200 & 1.37094300 & 2.12124500 \\
\hline $\mathrm{C}$ & 2.30464500 & -1.36067400 & 0.59403300 \\
\hline $\mathrm{C}$ & 3.48859400 & -1.86643700 & -0.16828900 \\
\hline $\mathrm{H}$ & 3.53995400 & -2.92580700 & -0.43337700 \\
\hline $\mathrm{C}$ & 0.07690100 & -0.85051100 & 0.37765200 \\
\hline $\mathrm{C}$ & -1.32587700 & -1.43041100 & 0.34682600 \\
\hline $\mathrm{C}$ & -1.02746300 & -2.87586700 & -0.14992400 \\
\hline $\mathrm{H}$ & -1.76483300 & -3.59897300 & 0.21849900 \\
\hline $\mathrm{H}$ & -1.03915000 & -2.89548900 & -1.24956900 \\
\hline $\mathrm{C}$ & 0.39416200 & -3.17428400 & 0.34300500 \\
\hline $\mathrm{H}$ & 0.40683300 & -3.56224700 & 1.37743900 \\
\hline $\mathrm{H}$ & 0.92804700 & -3.88494600 & -0.30191600 \\
\hline $\mathrm{C}$ & -1.88800300 & -1.48402600 & 1.77820200 \\
\hline $\mathrm{H}$ & -1.14723900 & -1.92674300 & 2.46033600 \\
\hline $\mathrm{H}$ & -2.79208500 & -2.10814300 & 1.80832500 \\
\hline $\mathrm{H}$ & -2.14640700 & -0.48347000 & 2.14882800 \\
\hline $\mathrm{C}$ & -2.21339700 & -0.62732600 & -0.61280800 \\
\hline $\mathrm{H}$ & -2.27040600 & 0.42583800 & -0.30002000 \\
\hline $\mathrm{H}$ & -1.78919100 & -0.65641300 & -1.62696300 \\
\hline $\mathrm{C}$ & -3.63586600 & -1.10165200 & -0.72893900 \\
\hline $\mathrm{H}$ & 2.46731600 & -1.58888100 & 1.68266600 \\
\hline
\end{tabular}




$\begin{array}{llll}\mathrm{C} & 5.82845900 & -1.52187900 & -0.99281300 \\ \mathrm{H} & 5.77263100 & -2.58991400 & -1.24245300 \\ \mathrm{H} & 6.06074300 & -0.95972900 & -1.91053700 \\ \mathrm{H} & 6.67415200 & -1.37237600 & -0.30343500 \\ \mathrm{H} & -1.53538200 & 5.29963400 & 0.33123300 \\ \mathrm{~F} & 0.34139700 & 4.83098600 & -1.38643900\end{array}$

\section{Structure 25. D'}$$
\mathrm{F}
$$$$
-3.11869500 \quad-0.85722800 \quad-2.81071300
$$$$
\text { F } \quad \begin{array}{llll}
-3.75724500 & 0.60175200 & -1.37382600
\end{array}
$$$$
\text { F } \quad \begin{array}{llll}
-4.09561100 & -1.48767200 & -1.00261100
\end{array}
$$$$
\mathrm{N} \quad \quad \quad-0.28713700 \quad-1.64193600 \quad 2.20772900
$$$$
\begin{array}{lllll}
\mathrm{C} & 0.68399900 & 4.06915400 & 0.09910200
\end{array}
$$$$
\begin{array}{lllll}
\text { C } & 0.29291300 & 3.46260400 & 1.29839800
\end{array}
$$$$
\begin{array}{lllll}
\text { C } & 0.31388300 & 2.07720000 & 1.44420000
\end{array}
$$$$
\begin{array}{llll}
\mathrm{H} & 0.00794600 & 1.62643300 & 2.39108400
\end{array}
$$$$
\begin{array}{lllll}
\text { C } & 0.74067100 & 1.24574000 & 0.39479000
\end{array}
$$$$
\begin{array}{llll}
\text { C } & 0.74730900 & -0.21213800 & 0.56679100
\end{array}
$$$$
\text { C } \quad 1.85668800 \quad-1.03299600 \quad 0.12852100
$$$$
\begin{array}{llll}
\text { C } & 3.12004200 & -0.45821000 & -0.15005300
\end{array}
$$$$
\text { C } \quad 4.16156500 \quad-1.26535200 \quad-0.56991000
$$$$
\text { C } \quad 4.03207300 \quad-2.63930600 \quad-0.74074600
$$$$
\begin{array}{llll}
\text { C } & 1.10458800 & 3.23936000 & -0.95295300
\end{array}
$$$$
\begin{array}{llll}
\text { C } & 1.13982600 & 1.85794700 & -0.81090400
\end{array}
$$$$
\mathrm{H} \quad \begin{array}{llll}
1.45895200 & 1.23531200 & -1.64966300
\end{array}
$$$$
\text { C } \quad 1.71982500 \quad-2.43510000 \quad-0.03765000
$$$$
\text { C } \quad 2.78774600 \quad-3.21295600 \quad-0.46648900
$$$$
\begin{array}{llll}
\text { C } & -0.41627800 & -0.85163500 & 1.21189600
\end{array}
$$$$
\begin{array}{llll}
\text { C } & -1.86562400 & -0.68626100 & 0.69521000
\end{array}
$$$$
\text { C } \quad \begin{array}{llll}
-2.48671600 & -1.94418600 & 1.34109300
\end{array}
$$$$
\mathrm{H} \quad \begin{array}{llll}
\mathrm{H} & -3.54852400 & -1.81412500 & 1.58604700
\end{array}
$$$$
\mathrm{H} \quad \begin{array}{llll}
\mathrm{H} & -2.39329100 & -2.79831600 & 0.65303500
\end{array}
$$$$
\begin{array}{llll}
\text { C } & -1.59852100 & -2.17937200 & 2.56606600
\end{array}
$$$$
\mathrm{H} \quad \begin{array}{llll}
\mathrm{H} & -1.96426000 & -1.64754900 & 3.46115700
\end{array}
$$$$
\mathrm{H} \quad-1.50771100 \quad-3.23975500 \quad 2.84094700
$$$$
\begin{array}{llll}
\text { C } & -2.53138500 & 0.58297400 & 1.25178800
\end{array}
$$$$
\mathrm{H} \quad-2.35895500 \quad 0.66169600 \quad 2.33551800
$$ 


$\begin{array}{lrrr}\mathrm{H} & -3.61723600 & 0.53824600 & 1.08548200 \\ \mathrm{H} & -2.14894800 & 1.49257500 & 0.76978700 \\ \mathrm{C} & -1.85778000 & -0.71416800 & -0.83849800 \\ \mathrm{H} & -1.26379000 & 0.12150100 & -1.24035300 \\ \mathrm{H} & -1.40417100 & -1.65128600 & -1.19499700 \\ \mathrm{C} & -3.20650200 & -0.61461500 & -1.49590200 \\ \mathrm{H} & 0.75946800 & -2.90920800 & 0.16248400 \\ \mathrm{H} & 1.40452400 & 3.69014200 & -1.90221200 \\ \mathrm{H} & 4.88611100 & -3.22890400 & -1.07517800 \\ \mathrm{H} & -0.03359700 & 4.08655100 & 2.13350200 \\ \mathrm{C} & 0.65999800 & 5.56313800 & -0.07135200 \\ \mathrm{H} & 1.66897500 & 5.94875700 & -0.28272100 \\ \mathrm{H} & 0.02067700 & 5.84994700 & -0.91986000 \\ \mathrm{H} & 0.28243500 & 6.06170000 & 0.83084700 \\ \mathrm{H} & 2.65309000 & -4.28808500 & -0.59538000 \\ \mathrm{H} & 3.30693200 & 0.60718900 & -0.01549500 \\ \mathrm{~F} & 5.35059700 & -0.69687300 & -0.81379800\end{array}$

\section{Structure 26. D'-TS 1}

$\begin{array}{lrrr}\text { F } & -4.36224800 & 0.03157700 & -1.30414200 \\ \text { F } & -4.31421200 & -0.52595300 & 0.76662900 \\ \text { F } & -4.04374700 & -2.02061300 & -0.75354000 \\ \text { N } & 0.81195300 & -2.25597300 & 0.37288100 \\ \text { C } & -0.42721700 & 4.15231900 & 0.06785800 \\ \text { C } & -0.78534700 & 3.26000600 & 1.08433300 \\ \text { H } & -1.37678000 & 3.61994600 & 1.92956900 \\ \text { C } & -0.38779900 & 1.92365300 & 1.04572300 \\ \text { H } & -0.65828100 & 1.25719100 & 1.86679700 \\ \text { C } & 0.36966200 & 1.42493900 & -0.02494700 \\ \text { C } & 0.81040300 & 0.02197800 & -0.08963100 \\ \text { C } & 2.18060600 & -0.35280700 & -0.34562000 \\ \text { C } & 3.30657500 & 0.41792700 & -0.02306100 \\ \text { C } & 4.53807100 & -0.20669300 & 0.07129500 \\ \text { C } & 4.70247600 & -1.58481000 & -0.13169500 \\ \text { C } & 0.35048300 & 3.66030000 & -0.99173000 \\ \text { C } & 0.74315300 & 2.32724600 & -1.03848400 \\ \text { H } & 1.34085500 & 1.96715700 & -1.87928000\end{array}$




$\begin{array}{lrrr}\mathrm{C} & 2.32581800 & -1.76350100 & -0.67000400 \\ \mathrm{C} & 3.61977500 & -2.35508800 & -0.51350200 \\ \mathrm{C} & 0.09288900 & -1.13287300 & 0.25881400 \\ \mathrm{C} & -1.40082600 & -1.44230300 & 0.41544700 \\ \mathrm{C} & -1.40603900 & -2.89041700 & -0.14726700 \\ \mathrm{H} & -2.23118400 & -3.49053500 & 0.25688400 \\ \mathrm{H} & -1.51175800 & -2.85226200 & -1.24214100 \\ \mathrm{C} & -0.01928900 & -3.43709200 & 0.21696300 \\ \mathrm{H} & -0.02116500 & -4.01707300 & 1.15430300 \\ \mathrm{H} & 0.39710100 & -4.09116900 & -0.56636100 \\ \mathrm{C} & -1.78902200 & -1.45609600 & 1.90302600 \\ \mathrm{H} & -1.05781600 & -2.04123600 & 2.47955600 \\ \mathrm{H} & -2.77574500 & -1.92025600 & 2.03671500 \\ \mathrm{H} & -1.82743400 & -0.44095600 & 2.32164100 \\ \mathrm{C} & -2.25855900 & -0.48300500 & -0.41808600 \\ \mathrm{H} & -2.15797500 & 0.55144100 & -0.06318400 \\ \mathrm{H} & -1.93515400 & -0.50933700 & -1.46914200 \\ \mathrm{C} & -3.73702600 & -0.75926900 & -0.42117800 \\ \mathrm{H} & 1.72429500 & -2.15262300 & -1.50276600 \\ \mathrm{H} & 0.64721300 & 4.33737400 & -1.79682300 \\ \mathrm{H} & 5.70135300 & -2.01464800 & -0.03715300 \\ \mathrm{C} & -0.84891600 & 5.59629600 & 0.10175800 \\ \mathrm{H} & 0.02729100 & 6.26214200 & 0.09306700 \\ \mathrm{H} & -1.45510600 & 5.84902800 & -0.78141800 \\ \mathrm{H} & -1.44064100 & 5.81745300 & 0.99972400 \\ \mathrm{H} & 3.76292700 & -3.40606900 & -0.76871500 \\ \mathrm{H} & 5.61705000 & 0.52247200 & 0.40477100 \\ & 3.21353000 & 1.47461800 & 0.23500900\end{array}$

\section{Structure 27. D'-Int 1}

$\begin{array}{lrrr}\text { F } & -4.20757500 & -0.51159100 & -1.71507800 \\ \text { F } & -4.26263100 & -0.69863400 & 0.42086700 \\ \text { F } & -3.78933300 & -2.40847700 & -0.79261400 \\ \text { N } & 0.99235700 & -2.07237000 & 0.23416600 \\ \text { C } & -0.78112500 & 4.19448700 & 0.09369700 \\ \text { C } & 0.08609600 & 3.69288400 & -0.88827800 \\ \text { C } & 0.60871400 & 2.40609900 & -0.79892300\end{array}$




\begin{tabular}{|c|c|c|c|}
\hline $\mathrm{H}$ & 1.26877500 & 2.03336100 & -1.58550700 \\
\hline $\mathrm{C}$ & 0.28358000 & 1.56770100 & 0.28161300 \\
\hline $\mathrm{C}$ & 0.83910100 & 0.19750600 & 0.35450100 \\
\hline $\mathrm{C}$ & 2.23046100 & -0.13928300 & 0.29122000 \\
\hline $\mathrm{C}$ & 3.37235600 & 0.61487000 & 0.04136400 \\
\hline $\mathrm{C}$ & 4.53286500 & -0.07273300 & -0.30377100 \\
\hline $\mathrm{C}$ & 4.55759900 & -1.47192100 & -0.57236700 \\
\hline $\mathrm{C}$ & -1.09595000 & 3.36327000 & 1.17341700 \\
\hline $\mathrm{H}$ & -1.75986100 & 3.73306300 & 1.95852500 \\
\hline $\mathrm{C}$ & -0.56766000 & 2.07473000 & 1.27187300 \\
\hline $\mathrm{H}$ & -0.80740900 & 1.45859400 & 2.14019000 \\
\hline $\mathrm{C}$ & 2.35067100 & -1.63904800 & 0.48690800 \\
\hline $\mathrm{C}$ & 3.47085800 & -2.24730400 & -0.29892600 \\
\hline $\mathrm{C}$ & 0.15253100 & -1.01072000 & 0.31952500 \\
\hline $\mathrm{C}$ & -1.28247400 & -1.49930900 & 0.36906000 \\
\hline $\mathrm{C}$ & -1.09132800 & -2.99670300 & -0.01457600 \\
\hline $\mathrm{H}$ & -1.85349800 & -3.64001700 & 0.44128900 \\
\hline $\mathrm{H}$ & -1.15176200 & -3.10676400 & -1.10718300 \\
\hline $\mathrm{C}$ & 0.33053200 & -3.34298400 & 0.44690000 \\
\hline $\mathrm{H}$ & 0.36469000 & -3.63933800 & 1.51096500 \\
\hline $\mathrm{H}$ & 0.78999200 & -4.14034900 & -0.15215800 \\
\hline $\mathrm{C}$ & -1.81016600 & -1.39268500 & 1.80994600 \\
\hline $\mathrm{H}$ & -1.08514200 & -1.82875400 & 2.51303200 \\
\hline $\mathrm{H}$ & -2.75542100 & -1.94425400 & 1.91143200 \\
\hline $\mathrm{H}$ & -1.98654800 & -0.34702400 & 2.09540900 \\
\hline $\mathrm{C}$ & -2.13765600 & -0.71304400 & -0.63370900 \\
\hline $\mathrm{H}$ & -2.10487700 & 0.36361300 & -0.40827200 \\
\hline $\mathrm{H}$ & -1.74765300 & -0.85774400 & -1.65164100 \\
\hline $\mathrm{C}$ & -3.59349500 & -1.08773000 & -0.67380700 \\
\hline $\mathrm{H}$ & 2.54955700 & -1.81788900 & 1.57852000 \\
\hline $\mathrm{H}$ & 5.45213200 & -1.88569500 & -1.04268100 \\
\hline $\mathrm{H}$ & 0.34871000 & 4.32236700 & -1.74247400 \\
\hline $\mathrm{C}$ & -1.34791000 & 5.58405100 & -0.02244200 \\
\hline $\mathrm{H}$ & -0.54478200 & 6.33175500 & -0.10347000 \\
\hline $\mathrm{H}$ & -1.97054900 & 5.67939500 & -0.92510700 \\
\hline $\mathrm{H}$ & -1.96703000 & 5.83546000 & 0.84878000 \\
\hline $\mathrm{H}$ & 3.45516700 & -3.31447200 & -0.52753600 \\
\hline
\end{tabular}




\begin{tabular}{|c|c|c|}
\hline $\mathrm{H}$ & 3.35634900 & 1.70623100 \\
\hline $\mathrm{F}$ & 5.65476600 & 0.62672700 \\
\hline
\end{tabular}

\section{Structure 28. E'}

$\mathrm{F}$

$\begin{array}{lll}-2.45993800 & -2.08342400 & -2.68512900\end{array}$

F $\quad-3.63624900 \quad-1.40808200 \quad-1.02302700$

F $\quad-2.64453800 \quad-3.31231500 \quad-0.93123600$

$\mathrm{N} \quad \begin{array}{llll}0.89147400 & -1.45302200 & 2.03576100\end{array}$

C $\quad-1.76221500 \quad 3.84855900 \quad 0.03267800$

$\begin{array}{lllll}\mathrm{C} & -1.70896100 & 3.14854700 & 1.24568300\end{array}$

C $\quad-0.88915900 \quad 2.03397800 \quad 1.39749300$

$\mathrm{H} \quad-0.85752900 \quad 1.51036100 \quad 2.35549000$

$\begin{array}{lllll}\mathrm{C} & -0.08156300 & 1.58065900 & 0.33941700\end{array}$

$\begin{array}{lllll}\text { C } & 0.78180700 & 0.40336000 & 0.51336000\end{array}$

$\begin{array}{lllll}\text { C } & 2.16800300 & 0.42601000 & 0.08073300\end{array}$

$\begin{array}{llll}\text { C } & 2.84498100 & 1.65046900 & -0.14056700\end{array}$

$\begin{array}{llll}\mathrm{H} & 2.32939900 & 2.59191900 & 0.04826700\end{array}$

$\begin{array}{llll}\text { C } & 4.16944300 & 1.67273600 & -0.56353500\end{array}$

$\mathrm{H} \quad 4.67092900 \quad 2.62998500 \quad-0.71451700$

C $\quad 4.87039100 \quad 0.48680900 \quad-0.78912600$

C $\quad-0.96109700 \quad 3.39449400 \quad-1.02499700$

$\begin{array}{llll}\text { C } & -0.13056300 & 2.28847800 & -0.87634500\end{array}$

$\mathrm{H} \quad 0.47635900 \quad 1.95008800 \quad-1.71921500$

C $\quad 2.88683000 \quad-0.77281900-0.14195000$

C $\quad 4.19982300 \quad-0.71221600 \quad-0.56919100$

C $\quad 0.23595100 \quad-0.80862700 \quad 1.14296200$

C $\quad-1.10664500 \quad-1.46510800 \quad 0.73281500$

C $\quad-0.82673500 \quad-2.89525300 \quad 1.24531500$

$\mathrm{H} \quad-1.74036100 \quad-3.42428500 \quad 1.54463300$

$\mathrm{H} \quad-0.33031500 \quad-3.48085600 \quad 0.45613600$

C $\quad 0.15524400 \quad-2.66174100 \quad 2.39504000$

$\mathrm{H} \quad-0.35621300 \quad-2.49189700 \quad 3.35844300$

$\mathrm{H} \quad 0.85392600 \quad-3.49637000 \quad 2.54886300$

$\begin{array}{llll}\text { C } & -2.30158500 & -0.86061000 & 1.48914300\end{array}$

$\mathrm{H} \quad-2.06574300 \quad-0.75476700 \quad 2.55861400$

$\mathrm{H} \quad-3.17304900 \quad-1.52492500 \quad 1.40052700$

$\mathrm{H} \quad-2.58304100 \quad 0.12405700 \quad 1.09392300$ 


$\begin{array}{lrrr}\mathrm{C} & -1.27566200 & -1.37720000 & -0.78888700 \\ \mathrm{H} & -1.33075200 & -0.32782600 & -1.11628800 \\ \mathrm{H} & -0.41244000 & -1.84066600 & -1.29015100 \\ \mathrm{C} & -2.50183200 & -2.04594700 & -1.34623700 \\ \mathrm{H} & 2.42691500 & -1.74996800 & 0.00104700 \\ \mathrm{H} & -0.99448100 & 3.91655500 & -1.98407200 \\ \mathrm{H} & 5.90829900 & 0.47608200 & -1.12302400 \\ \mathrm{H} & -2.32060400 & 3.48484800 & 2.08636200 \\ \mathrm{~F} & 4.85396300 & -1.86193000 & -0.78638300 \\ \mathrm{C} & -2.63268700 & 5.06564000 & -0.11833200 \\ \mathrm{H} & -2.12884800 & 5.95487500 & 0.29316500 \\ \mathrm{H} & -2.85431500 & 5.26790900 & -1.17472900 \\ \mathrm{H} & -3.58021900 & 4.94648200 & 0.42533100\end{array}$

\section{Structure 29. E'-TS 1}

$\begin{array}{lrrr}\text { F } & -3.94225400 & -1.57924300 & -1.39205400 \\ \mathrm{~F} & -3.74249400 & -2.06462700 & 0.68793200 \\ \mathrm{~F} & -2.83005800 & -3.31900400 & -0.79931800 \\ \mathrm{~N} & 1.65195500 & -1.53236400 & 0.48066500 \\ \mathrm{C} & -2.09376600 & 3.80921900 & 0.03484300 \\ \mathrm{C} & -2.10890300 & 2.83998700 & 1.04431100 \\ \mathrm{H} & -2.83616000 & 2.92223900 & 1.85554900 \\ \mathrm{C} & -1.20212000 & 1.78081400 & 1.04255400 \\ \mathrm{H} & -1.21971400 & 1.05538000 & 1.85787100 \\ \mathrm{C} & -0.25287300 & 1.64291900 & 0.01802400 \\ \mathrm{C} & 0.72929300 & 0.54749500 & -0.00582100 \\ \mathrm{C} & 2.14214300 & 0.77731000 & -0.21258800 \\ \mathrm{C} & 2.84923600 & 1.93368700 & 0.16088800 \\ \mathrm{H} & 2.29211100 & 2.84214800 & 0.39914200 \\ \mathrm{C} & 4.22983000 & 1.89930600 & 0.31250900 \\ \mathrm{H} & 4.77278200 & 2.79164200 & 0.62541100 \\ \mathrm{C} & 4.93641000 & 0.69441800 & 0.10061000 \\ \mathrm{C} & -1.13064000 & 3.68497800 & -0.97797900 \\ \mathrm{C} & -0.22791500 & 2.62730500 & -0.98745600 \\ \mathrm{H} & 0.50787000 & 2.54984500 & -1.79134100 \\ \mathrm{C} & 2.85230500 & -0.44699500 & -0.52174900 \\ \mathrm{C} & 4.25715200 & -0.42803800 & -0.30274100\end{array}$




$\begin{array}{lrrr}\mathrm{C} & 0.53890400 & -0.80298400 & 0.33110800 \\ \mathrm{C} & -0.69847200 & -1.70287400 & 0.44128700 \\ \mathrm{C} & -0.08739400 & -3.02294600 & -0.10521100 \\ \mathrm{H} & -0.60460900 & -3.91064400 & 0.28040000 \\ \mathrm{H} & -0.16356400 & -3.02914600 & -1.20313500 \\ \mathrm{C} & 1.38840200 & -2.94998500 & 0.30810500 \\ \mathrm{H} & 1.59639400 & -3.48275000 & 1.25030700 \\ \mathrm{H} & 2.06696900 & -3.36643500 & -0.45412100 \\ \mathrm{C} & -1.09657100 & -1.87964700 & 1.91584000 \\ \mathrm{H} & -0.21071300 & -2.12274200 & 2.52047300 \\ \mathrm{H} & -1.81594400 & -2.70315700 & 2.02197600 \\ \mathrm{H} & -1.55456500 & -0.96873800 & 2.32548100 \\ \mathrm{C} & -1.84631100 & -1.18205300 & -0.43169000 \\ \mathrm{H} & -2.19336600 & -0.19872200 & -0.08760400 \\ \mathrm{H} & -1.50488000 & -1.07125900 & -1.47148400 \\ \mathrm{C} & -3.07937000 & -2.04238000 & -0.47750400 \\ \mathrm{H} & 2.52448200 & -1.06216100 & -1.37155700 \\ \mathrm{H} & -1.09277200 & 4.43177600 & -1.77510600 \\ \mathrm{H} & 6.02157000 & 0.64505300 & 0.20717200 \\ \mathrm{~F} & 4.93006600 & -1.55785100 & -0.59214800 \\ \mathrm{C} & -3.07837000 & 4.94675900 & 0.02389100 \\ \mathrm{H} & -2.57122600 & 5.90781900 & -0.14509100 \\ \mathrm{H} & -3.81187900 & 4.82031100 & -0.78780300 \\ \mathrm{H} & -3.62964200 & 5.00485100 & 0.97170300\end{array}$

\section{Structure 30. E'-Int 1}

$\begin{array}{lrrr}\text { F } & -3.39843600 & -2.37911800 & -1.67283300 \\ \text { F } & -3.36565500 & -2.48049100 & 0.46941100 \\ \text { F } & -2.11121900 & -3.78966500 & -0.68443500 \\ \text { N } & 1.89268100 & -1.10626200 & 0.21192600 \\ \text { C } & -2.76910000 & 3.43643300 & 0.02604500 \\ \text { C } & -1.80714900 & 3.36135800 & -0.99236800 \\ \text { C } & -0.70955700 & 2.51255500 & -0.88476300 \\ \text { H } & 0.01874700 & 2.46079200 & -1.69741400 \\ \text { C } & -0.53229100 & 1.70329100 & 0.25036000 \\ \text { C } & 0.63365700 & 0.79318800 & 0.33889700 \\ \text { C } & 2.00915900 & 1.19357300 & 0.26586400\end{array}$




\begin{tabular}{|c|c|c|c|}
\hline $\mathrm{C}$ & 2.62294300 & 2.40418500 & -0.05904800 \\
\hline $\mathrm{H}$ & 2.03053500 & 3.32129800 & -0.09749200 \\
\hline $\mathrm{C}$ & 3.96273900 & 2.41524900 & -0.44661100 \\
\hline $\mathrm{H}$ & 4.44983600 & 3.35118500 & -0.72082400 \\
\hline $\mathrm{C}$ & 4.68186600 & 1.18246100 & -0.62385200 \\
\hline $\mathrm{C}$ & -2.58567000 & 2.63808000 & 1.15949900 \\
\hline $\mathrm{H}$ & -3.31517300 & 2.68477100 & 1.97160500 \\
\hline $\mathrm{C}$ & -1.48292500 & 1.78926900 & 1.27493500 \\
\hline $\mathrm{H}$ & -1.35168300 & 1.19446500 & 2.18076500 \\
\hline $\mathrm{C}$ & 2.84253700 & -0.05147500 & 0.49615700 \\
\hline $\mathrm{C}$ & 4.11480800 & 0.00704300 & -0.27117200 \\
\hline $\mathrm{C}$ & 0.63293600 & -0.59331100 & 0.31034000 \\
\hline $\mathrm{C}$ & -0.37389000 & -1.72224200 & 0.40102200 \\
\hline $\mathrm{C}$ & 0.53040600 & -2.94720600 & 0.07435700 \\
\hline $\mathrm{H}$ & 0.18682400 & -3.85993600 & 0.57581600 \\
\hline $\mathrm{H}$ & 0.53101400 & -3.12561700 & -1.01084200 \\
\hline $\mathrm{C}$ & 1.93823600 & -2.52472500 & 0.51386500 \\
\hline $\mathrm{H}$ & 2.10864900 & -2.69685500 & 1.59234300 \\
\hline $\mathrm{H}$ & 2.73213100 & -3.02890900 & -0.05100200 \\
\hline $\mathrm{C}$ & -0.89370200 & -1.82256100 & 1.84500500 \\
\hline $\mathrm{H}$ & -0.05107700 & -1.82033900 & 2.55210100 \\
\hline $\mathrm{H}$ & -1.45326400 & -2.75795500 & 1.98636700 \\
\hline $\mathrm{H}$ & -1.55482500 & -0.98011600 & 2.08921100 \\
\hline $\mathrm{C}$ & -1.49951700 & -1.49827400 & -0.61803400 \\
\hline $\mathrm{H}$ & -1.99877700 & -0.53438500 & -0.43551100 \\
\hline $\mathrm{H}$ & -1.08273100 & -1.47717200 & -1.63551200 \\
\hline $\mathrm{C}$ & -2.58635400 & -2.53801300 & -0.61994200 \\
\hline $\mathrm{H}$ & 3.09588800 & -0.12601300 & 1.58808400 \\
\hline $\mathrm{H}$ & 5.67990900 & 1.17787700 & -1.06600300 \\
\hline $\mathrm{H}$ & -1.92754800 & 3.97546100 & -1.88858500 \\
\hline $\mathrm{C}$ & -3.95506700 & 4.35345000 & -0.10843300 \\
\hline $\mathrm{H}$ & -3.63234000 & 5.39876300 & -0.22765100 \\
\hline $\mathrm{H}$ & -4.55133600 & 4.09591900 & -0.99686100 \\
\hline $\mathrm{H}$ & -4.60714700 & 4.29295000 & 0.77280100 \\
\hline $\mathrm{F}$ & 4.74188700 & -1.16809300 & -0.44076700 \\
\hline
\end{tabular}


VII. Crystal Data and Structure Refinement for 3e, 3m, 3t, 10a, 10b

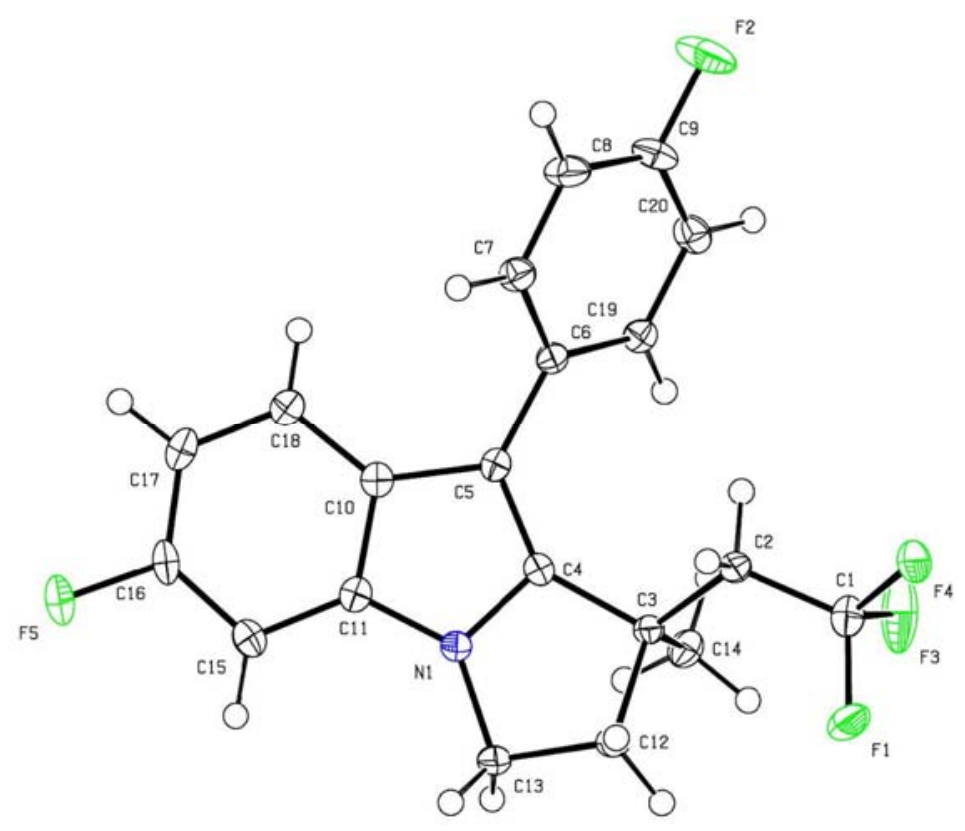

Table S8 Crystal data and structure refinement for 3e (CCDC 2043670).

Identification code

Empirical formula

Formula weight

Temperature/K

Crystal system

Space group

$\mathrm{a} / \AA$

$\mathrm{b} / \AA ̊$

$\mathrm{c} / \AA \AA$

$\alpha /{ }^{\circ}$

$\beta /{ }^{\circ}$

$\gamma /{ }^{\circ}$

Volume $/ \AA^{3}$

Z

$\rho_{\text {calcg }} / \mathrm{cm}^{3}$

$\mu / \mathrm{mm}^{-1}$

$\mathrm{F}(000)$

Crystal size $/ \mathrm{mm}^{3}$

Radiation

$2 \Theta$ range for data collection ${ }^{\circ}$

Index ranges

Reflections collected

Independent reflections cxy1386 (3e)

$\mathrm{C}_{20} \mathrm{H}_{16} \mathrm{NF}_{5}$

365.34

100.0

orthorhombic

Pbca

16.2053(8)

$11.4051(5)$

$17.4590(8)$

90

90

90

$3226.8(3)$

8

1.504

0.128

1504.0

$0.36 \times 0.29 \times 0.24$

$\operatorname{MoK} \alpha(\lambda=0.71073)$

4.666 to 55.132

$-20 \leq \mathrm{h} \leq 21,-14 \leq \mathrm{k} \leq 13,-21 \leq 1 \leq 22$

53244

$3713\left[R_{\text {int }}=0.0960, R_{\text {sigma }}=0.0401\right]$ 
Data/restraints/parameters

Goodness-of-fit on $\mathrm{F}^{2}$

Final $\mathrm{R}$ indexes $[\mathrm{I}>=2 \sigma(\mathrm{I})]$

Final $\mathrm{R}$ indexes [all data]

Largest diff. peak/hole / e $\AA^{-3}$
$3713 / 0 / 237$

1.050

$\mathrm{R}_{1}=0.0411, \mathrm{wR}_{2}=0.0961$

$\mathrm{R}_{1}=0.0627, \mathrm{wR}_{2}=0.1040$

$0.29 /-0.26$

Table S9 Fractional Atomic Coordinates $\left(\times 10^{4}\right)$ and Equivalent Isotropic Displacement Parameters $\left(\AA^{2} \times 10^{3}\right)$ for $3 \mathrm{e} . \mathrm{U}_{\text {eq }}$ is defined as $1 / 3$ of of the trace of the orthogonalised $\mathrm{U}_{\mathrm{IJ}}$ tensor.

\begin{tabular}{lrr} 
Atom & \multicolumn{2}{c}{$\boldsymbol{y}$} \\
F1 & $1675.1(7)$ & $3824.4(11)$ \\
F2 & $6379.3(7)$ & $6011.2(10)$ \\
F3 & $2794.6(9)$ & $2840.8(10)$ \\
F4 & $2401.8(6)$ & $3992.7(9)$ \\
F5 & $3979.0(7)$ & $9644.4(9)$ \\
N1 & $3127.6(8)$ & $6414.3(12)$ \\
C1 & $2450.6(11)$ & $3893.3(15)$ \\
C2 & $2931.4(10)$ & $4883.0(14)$ \\
C3 & $2960.4(10)$ & $4926.1(13)$ \\
C4 & $3484.6(10)$ & $5948.8(13)$ \\
C5 & $4191.8(10)$ & $6556.5(13)$ \\
C6 & $4764.9(9)$ & $6391.1(14)$ \\
C7 & $5039.0(10)$ & $7362.7(15)$ \\
C8 & $5589.1(11)$ & $7245.6(16)$ \\
C9 & $5855.1(11)$ & $6135.5(17)$ \\
C10 & $4268.4(10)$ & $7453.8(14)$ \\
C11 & $7326.3(13)$ \\
C12 & $3593.2(10)$ & $5259.8(14)$ \\
C13 & $2115.2(10)$ & $5874.3(14)$ \\
C14 & $2341.7(10)$ & $3780.1(14)$ \\
C15 & $3285.0(11)$ & $8048.4(14)$ \\
C16 & $3478.1(10)$ & $8897.4(14)$ \\
C17 & $4068.4(11)$ & $9053.7(15)$ \\
C18 & $4746.0(11)$ & $8332.4(14)$ \\
C19 & $4852.4(10)$ & $5285.7(14)$ \\
C20 & $5067.1(10)$ &
\end{tabular}

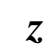

6395.3(6)

7791.9(7)

6525.8(7)

7418.4(5)

2893.3(6)

4508.4(7)

6651.0(9)

6307.9(9)

5426.8(8)

5157.4(8)

5335.7(8)

5980.8(9)

6398.0(9)

7004.0(10)

7190.3(10)

4750.5(9)

4239.4(9)

5057.0(9)

4300.9(9)

5067.4(9)

3601.4(9)

3498.8(9)

3971.8(10)

4602.1(9)

6188.0(9)

6798.5(9)
$\mathbf{U}(\mathbf{e q})$

35.9(3)

40.5(3)

48.1(4)

26.9(3)

27.7(3)

15.1(3)

21.5(4)

15.5(3)

14.1(3)

13.7(3)

14.1(3)

14.6(3)

19.0(4)

24.9(4)

24.7(4)

15.0(3)

15.1(3)

17.1(3)

17.8(3)

19.6(4)

18.3(3)

19.5(4)

21.0(4)

17.9(3)

16.4(3)

21.0(4)

Table S10 Anisotropic Displacement Parameters $\left(\AA^{2} \times 10^{3}\right)$ for 3e. The Anisotropic displacement factor exponent takes the form: $-2 \pi^{2}\left[h^{2} a * 2 U_{11}+2 h k a * b * U_{12}+\ldots\right]$.

\begin{tabular}{lrrrrrr} 
Atom & \multicolumn{1}{c}{$\mathbf{U}_{\mathbf{1 1}}$} & $\mathbf{U}_{\mathbf{2 2}}$ & \multicolumn{1}{c}{$\mathbf{U}_{\mathbf{3 3}}$} & \multicolumn{1}{c}{$\mathbf{U}_{\mathbf{2 3}}$} & \multicolumn{1}{c}{$\mathbf{U}_{\mathbf{1 3}}$} & \multicolumn{1}{c}{$\mathbf{U}_{\mathbf{1 2}}$} \\
F1 & $29.2(6)$ & $50.0(7)$ & $28.5(6)$ & $7.0(5)$ & $-3.5(5)$ & $-23.1(5)$ \\
F2 & $40.4(7)$ & $41.0(7)$ & $40.1(7)$ & $-5.8(5)$ & $-26.2(6)$ & $11.2(6)$ \\
F3 & $79.0(10)$ & $17.3(5)$ & $47.9(7)$ & $9.8(5)$ & $27.5(7)$ & $10.5(6)$ \\
F4 & $29.1(6)$ & $33.8(6)$ & $17.7(5)$ & $7.4(4)$ & $2.4(4)$ & $-5.1(5)$
\end{tabular}




$\begin{array}{lrrrrrr}\text { F5 } & 35.8(6) & 24.6(5) & 22.8(5) & 11.7(4) & 3.2(4) & 1.5(5) \\ \text { N1 } & 15.9(7) & 15.0(6) & 14.3(6) & 0.2(5) & -1.2(5) & -1.3(5) \\ \text { C1 } & 25.4(9) & 18.8(8) & 20.3(8) & 1.6(7) & 3.4(7) & -0.8(7) \\ \text { C2 } & 14.1(8) & 16.5(8) & 16.0(7) & 0.4(6) & 0.3(6) & -0.3(6) \\ \text { C3 } & 13.0(8) & 15.4(7) & 14.0(7) & 0.0(6) & -0.6(6) & -0.8(6) \\ \text { C4 } & 15.0(8) & 13.6(7) & 12.4(7) & -0.9(6) & 1.7(6) & 2.7(6) \\ \text { C5 } & 15.8(8) & 11.7(7) & 14.8(7) & -2.2(6) & 2.3(6) & 0.9(6) \\ \text { C6 } & 10.4(7) & 17.1(7) & 16.1(7) & 0.6(6) & 2.1(6) & 0.5(6) \\ \text { C7 } & 16.6(8) & 16.6(8) & 23.9(8) & -0.8(7) & -1.8(7) & 1.5(6) \\ \text { C8 } & 21.8(9) & 23.2(9) & 29.8(9) & -6.0(8) & -8.5(7) & -1.3(7) \\ \text { C9 } & 18.3(9) & 32.9(10) & 22.7(8) & -1.0(7) & -8.5(7) & 4.3(8) \\ \text { C10 } & 15.3(8) & 14.1(7) & 15.5(7) & -1.5(6) & 3.0(6) & 3.0(6) \\ \text { C11 } & 17.1(8) & 12.9(7) & 15.4(7) & -2.5(6) & 3.6(6) & 1.2(6) \\ \text { C12 } & 15.3(8) & 18.8(8) & 17.1(7) & -0.8(6) & -1.6(6) & -1.7(6) \\ \text { C13 } & 18.5(9) & 18.1(8) & 16.9(7) & -0.9(6) & -4.1(6) & -3.1(7) \\ \text { C14 } & 21.1(9) & 17.0(8) & 20.8(8) & -2.0(6) & 2.8(7) & -2.0(7) \\ \text { C15 } & 21.5(9) & 18.6(8) & 14.7(7) & -0.7(6) & 1.2(6) & 3.8(7) \\ \text { C16 } & 27.5(9) & 14.6(8) & 16.2(8) & 5.0(6) & 6.2(7) & 4.1(7) \\ \text { C17 } & 21.3(9) & 15.5(8) & 26.0(9) & 2.4(7) & 6.6(7) & -2.1(7) \\ \text { C18 } & 16.4(8) & 15.9(8) & 21.3(8) & -0.5(6) & 2.0(6) & 0.6(6) \\ \text { C19 } & 14.2(8) & 16.8(8) & 18.2(7) & -0.7(6) & 2.0(6) & 1.1(6) \\ \text { C20 } & 17.8(9) & 22.3(9) & 23.0(8) & 2.5(7) & -0.4(7) & 6.6(7) \\ & & & & & & \end{array}$

Table S11 Bond Lengths for $3 \mathrm{e}$.

\begin{tabular}{llrlll}
\multicolumn{2}{c}{ Atom Atom } & Length/ $\AA$ & \multicolumn{2}{c}{ Atom Atom } & Length/ $\boldsymbol{\AA}$ \\
F1 & C1 & $1.336(2)$ & C5 & C10 & $1.452(2)$ \\
F2 & C9 & $1.3583(19)$ & C6 & C7 & $1.398(2)$ \\
F3 & C1 & $1.341(2)$ & C6 & C19 & $1.400(2)$ \\
F4 & C1 & $1.3470(19)$ & C7 & C8 & $1.390(2)$ \\
F5 & C16 & $1.3654(18)$ & C8 & C9 & $1.376(3)$ \\
N1 & C4 & $1.3785(19)$ & C9 & C20 & $1.373(2)$ \\
N1 & C11 & $1.368(2)$ & C10 & C11 & $1.419(2)$ \\
N1 & C13 & $1.460(2)$ & C10 & C18 & $1.402(2)$ \\
C1 & C2 & $1.497(2)$ & C11 & C15 & $1.398(2)$ \\
C2 & C3 & $1.540(2)$ & C12 & C13 & $1.539(2)$ \\
C3 & C4 & $1.518(2)$ & C15 & C16 & $1.373(2)$ \\
C3 & C12 & $1.561(2)$ & C16 & C17 & $1.385(2)$ \\
C3 & C14 & $1.542(2)$ & C17 & C18 & $1.385(2)$ \\
C4 & C5 & $1.375(2)$ & C19 & C20 & $1.388(2)$ \\
C5 & C6 & $1.472(2)$ & & &
\end{tabular}

Table S12 Bond Angles for $3 e$. 


\begin{tabular}{|c|c|c|c|c|c|c|c|}
\hline \multicolumn{3}{|c|}{ Atom Atom Atom } & \multirow{2}{*}{$\begin{array}{l}\text { Angle }^{\circ} \\
\quad 114.05(13)\end{array}$} & \multicolumn{3}{|c|}{ Atom Atom Atom } & \multirow{2}{*}{$\begin{array}{l}\text { Angle }^{\circ} \\
\quad 117.89(14)\end{array}$} \\
\hline $\mathrm{C} 4$ & N1 & $\mathrm{C} 13$ & & $\mathrm{C} 7$ & C6 & $\mathrm{C} 19$ & \\
\hline $\mathrm{C} 11$ & N1 & $\mathrm{C} 4$ & $110.09(13)$ & C19 & C6 & $\mathrm{C} 5$ & $122.27(14)$ \\
\hline $\mathrm{C} 11$ & N1 & $\mathrm{C} 13$ & $135.76(13)$ & $\mathrm{C} 8$ & $\mathrm{C} 7$ & C6 & $121.60(16)$ \\
\hline $\mathrm{F} 1$ & $\mathrm{C} 1$ & F3 & $106.49(15)$ & $\mathrm{C} 9$ & $\mathrm{C} 8$ & $\mathrm{C} 7$ & $117.97(16)$ \\
\hline F1 & $\mathrm{C} 1$ & $\mathrm{~F} 4$ & $106.39(14)$ & $\mathrm{F} 2$ & C9 & $\mathrm{C} 8$ & $118.33(16)$ \\
\hline $\mathrm{F} 1$ & $\mathrm{C} 1$ & $\mathrm{C} 2$ & $113.60(14)$ & $\mathrm{F} 2$ & C9 & $\mathrm{C} 20$ & $118.85(16)$ \\
\hline F3 & $\mathrm{C} 1$ & $\mathrm{~F} 4$ & $105.18(13)$ & $\mathrm{C} 20$ & C9 & $\mathrm{C} 8$ & $122.82(15)$ \\
\hline F3 & $\mathrm{C} 1$ & $\mathrm{C} 2$ & $113.16(14)$ & $\mathrm{C} 11$ & $\mathrm{C} 10$ & $\mathrm{C} 5$ & $107.72(14)$ \\
\hline F4 & $\mathrm{C} 1$ & $\mathrm{C} 2$ & $111.42(14)$ & $\mathrm{C} 18$ & $\mathrm{C} 10$ & $\mathrm{C} 5$ & $133.75(15)$ \\
\hline $\mathrm{C} 1$ & $\mathrm{C} 2$ & $\mathrm{C} 3$ & $116.09(13)$ & $\mathrm{C} 18$ & $\mathrm{C} 10$ & $\mathrm{C} 11$ & $118.52(14)$ \\
\hline $\mathrm{C} 2$ & $\mathrm{C} 3$ & $\mathrm{C} 12$ & $113.21(13)$ & N1 & $\mathrm{C} 11$ & $\mathrm{C} 10$ & $106.69(13)$ \\
\hline $\mathrm{C} 2$ & $\mathrm{C} 3$ & $\mathrm{C} 14$ & $112.94(13)$ & N1 & $\mathrm{C} 11$ & $\mathrm{C} 15$ & $130.39(15)$ \\
\hline $\mathrm{C} 4$ & $\mathrm{C} 3$ & $\mathrm{C} 2$ & $110.57(12)$ & C15 & C11 & $\mathrm{C} 10$ & $122.92(15)$ \\
\hline $\mathrm{C} 4$ & $\mathrm{C} 3$ & $\mathrm{C} 12$ & $100.11(12)$ & $\mathrm{C} 13$ & $\mathrm{C} 12$ & C3 & $104.86(12)$ \\
\hline $\mathrm{C} 4$ & $\mathrm{C} 3$ & $\mathrm{C} 14$ & $109.53(12)$ & N1 & $\mathrm{C} 13$ & $\mathrm{C} 12$ & $100.79(12)$ \\
\hline C14 & $\mathrm{C} 3$ & $\mathrm{C} 12$ & $109.73(13)$ & $\mathrm{C} 16$ & $\mathrm{C} 15$ & $\mathrm{C} 11$ & $115.25(15)$ \\
\hline N1 & $\mathrm{C} 4$ & $\mathrm{C} 3$ & $108.41(13)$ & F5 & $\mathrm{C} 16$ & C15 & $117.85(15)$ \\
\hline C5 & $\mathrm{C} 4$ & N1 & $109.98(13)$ & F5 & $\mathrm{C} 16$ & $\mathrm{C} 17$ & $117.73(15)$ \\
\hline $\mathrm{C} 5$ & $\mathrm{C} 4$ & $\mathrm{C} 3$ & $141.60(14)$ & $\mathrm{C} 15$ & $\mathrm{C} 16$ & $\mathrm{C} 17$ & $124.42(15)$ \\
\hline $\mathrm{C} 4$ & $\mathrm{C} 5$ & C6 & $129.39(14)$ & $\mathrm{C} 18$ & $\mathrm{C} 17$ & $\mathrm{C} 16$ & $119.73(15)$ \\
\hline $\mathrm{C} 4$ & C5 & $\mathrm{C} 10$ & $105.50(13)$ & C17 & C18 & $\mathrm{C} 10$ & $119.16(16)$ \\
\hline $\mathrm{C} 10$ & $\mathrm{C} 5$ & C6 & $125.10(14)$ & $\mathrm{C} 20$ & C19 & C6 & $121.18(15)$ \\
\hline $\mathrm{C} 7$ & C6 & $\mathrm{C} 5$ & $119.82(14)$ & C9 & $\mathrm{C} 20$ & C19 & $118.51(15)$ \\
\hline
\end{tabular}

Table S13 Torsion Angles for $3 \mathrm{e}$.

\begin{tabular}{|c|c|c|c|c|}
\hline A B & $\mathbf{C}$ & Angle ${ }^{\circ}$ & $\mathbf{A}$ & Angle $/^{\circ}$ \\
\hline $\mathrm{F} 1 \mathrm{C} 1$ & $\mathrm{C} 2 \mathrm{C} 3$ & $-54.38(19)$ & C6 C5 C10C18 & $2.2(3)$ \\
\hline F2 C9 & C20C19 & $179.51(15)$ & $\begin{array}{lllll}\mathrm{C} 6 & \mathrm{C} 7 & \mathrm{C} 8 & \mathrm{C} 9\end{array}$ & $-0.6(3)$ \\
\hline $\mathrm{F} 3 \mathrm{C} 1$ & $\mathrm{C} 2 \mathrm{C} 3$ & $67.2(2)$ & C6 C19C20C9 & $-1.2(2)$ \\
\hline $\mathrm{F} 4 \mathrm{C} 1$ & $\mathrm{C} 2 \mathrm{C} 3$ & $-174.51(14)$ & C7 C6 C19C20 & $1.4(2)$ \\
\hline F5 C1 & $5 \mathrm{C} 17 \mathrm{C} 18$ & $178.64(14)$ & $\begin{array}{llll}\mathrm{C} 7 & \mathrm{C} 8 & \mathrm{C} 9 & \mathrm{~F} 2\end{array}$ & $-178.66(16)$ \\
\hline $\mathrm{N} 1 \mathrm{C} 4$ & $\mathrm{C} 5 \mathrm{C} 6$ & $178.79(15)$ & $\begin{array}{llll}\mathrm{C} 7 & \mathrm{C} 8 & \mathrm{C} 9 & \mathrm{C} 20\end{array}$ & $0.8(3)$ \\
\hline $\mathrm{N} 1 \mathrm{C} 4$ & C5 C10 & $-0.11(17)$ & C8 C9 C20C19 & $0.1(3)$ \\
\hline $\mathrm{N} 1 \mathrm{C} 1$ & C $15 \mathrm{C} 16$ & $179.55(15)$ & $\mathrm{C} 10 \mathrm{C} 5 \mathrm{C} 6 \mathrm{C} 7$ & $44.6(2)$ \\
\hline $\mathrm{C} 1 \mathrm{C} 2$ & $\mathrm{C} 3 \mathrm{C} 4$ & $-178.48(13)$ & C10C5 C6 C19 & $-133.92(17)$ \\
\hline $\mathrm{C} 1 \mathrm{C} 2$ & $\mathrm{C} 3 \mathrm{C} 12$ & $70.12(18)$ & $\mathrm{C} 10 \mathrm{C} 11 \mathrm{C} 15 \mathrm{C} 16$ & $0.1(2)$ \\
\hline $\mathrm{C} 1 \mathrm{C} 2$ & C3 C14 & $-55.36(19)$ & C11N1 C4 C3 & $-178.81(12)$ \\
\hline $\mathrm{C} 2 \mathrm{C} 3$ & $\mathrm{C} 4 \mathrm{~N} 1$ & $-143.02(13)$ & C11N1 C4 C5 & $0.70(17)$ \\
\hline $\mathrm{C} 2 \mathrm{C} 3$ & $\mathrm{C} 4 \mathrm{C} 5$ & $37.7(3)$ & C11N1 C13C12 & $-158.59(17)$ \\
\hline $\mathrm{C} 2 \mathrm{C} 3$ & $\mathrm{C} 12 \mathrm{C} 13$ & $151.10(13)$ & $\mathrm{C} 11 \mathrm{C} 10 \mathrm{C} 18 \mathrm{C} 17$ & $0.9(2)$ \\
\hline C3 C4 & C5 C6 & $-2.0(3)$ & C11 C15C16F5 & $-178.70(14)$ \\
\hline C3 C4 & C5 C10 & $179.13(18)$ & $\mathrm{C} 11 \mathrm{C} 15 \mathrm{C} 16 \mathrm{C} 17$ & $0.8(2)$ \\
\hline
\end{tabular}




\begin{tabular}{|c|c|c|c|}
\hline $\mathrm{C} 3 \mathrm{C} 12 \mathrm{C} 13 \mathrm{~N} 1$ & $-31.11(15)$ & $\mathrm{C} 12 \mathrm{C} 3 \mathrm{C} 4 \mathrm{~N} 1$ & $-23.39(15)$ \\
\hline $\mathrm{C} 4 \mathrm{~N} 1 \mathrm{C} 11 \mathrm{C} 10$ & $-0.99(17)$ & $\mathrm{C} 12 \mathrm{C} 3 \mathrm{C} 4 \mathrm{C} 5$ & $157.4(2)$ \\
\hline C4N1 C11C15 & $179.53(16)$ & C13N1 C4 C3 & $4.26(17)$ \\
\hline $\mathrm{C} 4 \mathrm{~N} 1 \mathrm{C} 13 \mathrm{C} 12$ & $17.27(17)$ & $\mathrm{C} 13 \mathrm{~N} 1 \mathrm{C} 4 \mathrm{C} 5$ & $-176.23(13)$ \\
\hline C4C3 C12C13 & $33.41(14)$ & C13N1 C11C10 & $175.00(16)$ \\
\hline $\mathrm{C} 4 \mathrm{C} 5 \mathrm{C} 6 \mathrm{C} 7$ & $-134.09(17)$ & C13N1 C11C15 & $-4.5(3)$ \\
\hline C4 C5 C6 C19 & $47.4(2)$ & $\mathrm{C} 14 \mathrm{C} 3 \mathrm{C} 4 \mathrm{~N} 1$ & $91.89(15)$ \\
\hline C4C5 C10C11 & $-0.48(17)$ & $\mathrm{C} 14 \mathrm{C} 3 \mathrm{C} 4 \mathrm{C} 5$ & $-87.4(2)$ \\
\hline C4C5 C10C18 & $-178.79(17)$ & C14C3 C12C13 & $-81.72(15)$ \\
\hline $\mathrm{C} 5 \mathrm{C} 6 \mathrm{C} 7 \mathrm{C} 8$ & $-179.09(15)$ & C15C16C17C18 & $-0.8(3)$ \\
\hline C5C6 C19C20 & $179.94(15)$ & $\mathrm{C} 16 \mathrm{C} 17 \mathrm{C} 18 \mathrm{C} 10$ & $-0.1(2)$ \\
\hline C5 C10C11N1 & $0.90(17)$ & $\mathrm{C} 18 \mathrm{C} 10 \mathrm{C} 11 \mathrm{~N} 1$ & $179.50(13)$ \\
\hline C5 C10C11 C15 & $-179.57(14)$ & C18C10C11 C15 & $-1.0(2)$ \\
\hline C5 C10 C18C17 & $179.07(16)$ & C19C6 C7 C8 & $-0.5(2)$ \\
\hline C6 C5 C10C11 & $-179.45(14)$ & & \\
\hline
\end{tabular}

Table S14 Hydrogen Atom Coordinates $\left(\AA \times 10^{4}\right)$ and Isotropic Displacement Parameters $\left(\AA^{2} \times 10^{3}\right)$ for $3 e$.

$\begin{array}{lrrrr}\text { Atom } & \boldsymbol{x} & \boldsymbol{y} & \boldsymbol{z} & \mathbf{U}(\mathbf{e q}) \\ \text { H2A } & 3504.87 & 4838.06 & 6501.14 & 19 \\ \text { H2B } & 2692.87 & 5629.23 & 6494.66 & 19 \\ \text { H7 } & 4844.57 & 8121.18 & 6263.84 & 23 \\ \text { H8 } & 5775.7 & 7911.46 & 7281.28 & 30 \\ \text { H12A } & 1779.3 & 4550.54 & 4958.87 & 20 \\ \text { H12B } & 1800.69 & 5793.92 & 5395.58 & 20 \\ \text { H13A } & 2407.28 & 5305.37 & 3876.99 & 21 \\ \text { H13B } & 1925.37 & 6469.77 & 4156.39 & 21 \\ \text { H14A } & 3359.82 & 3890.81 & 4515.16 & 29 \\ \text { H14B } & 2886.56 & 3148.46 & 5157.09 & 29 \\ \text { H14C } & 3814.83 & 3572.97 & 5301.75 & 22 \\ \text { H15 } & 3023.64 & 7958.05 & 3262.75 & 21 \\ \text { H17 } & 5136.08 & 9653.25 & 3864.12 & 20 \\ \text { H18 } & 5315.11 & 8431.39 & 4929.92 & 25 \\ \text { H19 } & 4899.05 & 4614.72 & 5905.82 & 6941.94 \\ \text { H20 } & 5802.77 & 4398.21 & & \end{array}$




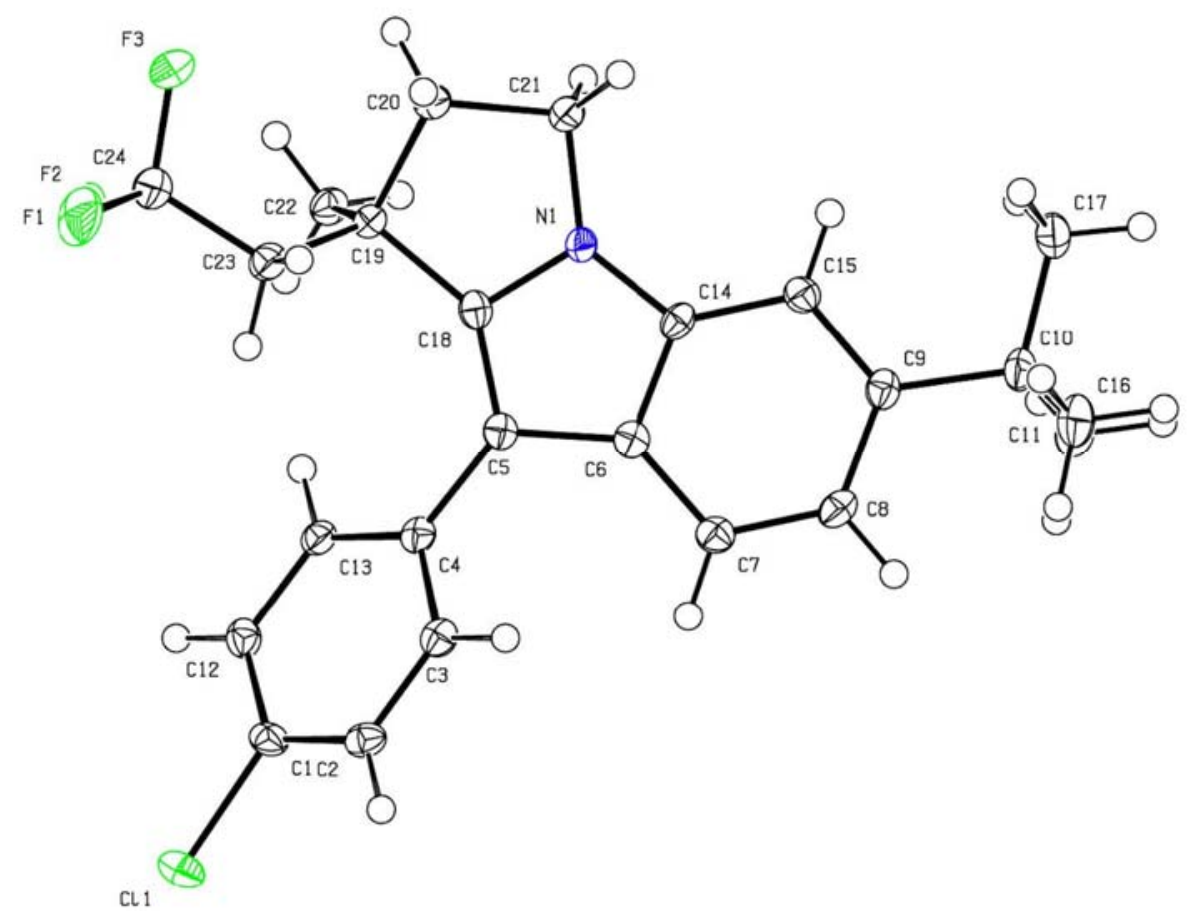

Table S15 Crystal data and structure refinement for 3m (CCDC 2043671).

Identification code

Empirical formula

Formula weight

Temperature/K

Crystal system

Space group

$\mathrm{a} / \AA$

$\mathrm{b} / \AA$

$\mathrm{c} / \AA$

$\alpha /{ }^{\circ}$

$\beta /{ }^{\circ}$

$\gamma /{ }^{\circ}$

Volume $/ \AA^{3}$

Z

$\rho_{\text {calcg }} / \mathrm{cm}^{3}$

$\mu / \mathrm{mm}^{-1}$

$\mathrm{F}(000)$

Crystal size $/ \mathrm{mm}^{3}$

Radiation

$2 \Theta$ range for data collection $/{ }^{\circ}$

Index ranges

Reflections collected

Independent reflections

Data/restraints/parameters

Goodness-of-fit on $\mathrm{F}^{2}$ cxy3029_0m (3m)

$\mathrm{C}_{24} \mathrm{H}_{25} \mathrm{ClF}_{3} \mathrm{~N}$

419.90

100.0

monoclinic

$\mathrm{P} 21 / \mathrm{c}$

$5.9225(3)$

$14.7827(8)$

23.7371(12)

90

90.671(2)

90

2078.05(19)

4

1.342

1.940

880.0

$0.35 \times 0.31 \times 0.29$

$\mathrm{CuK} \alpha(\lambda=1.54178)$

7.044 to 144.442

$-7 \leq \mathrm{h} \leq 7,-18 \leq \mathrm{k} \leq 18,-29 \leq 1 \leq 29$

53982

$4089\left[\mathrm{R}_{\text {int }}=0.0397, \mathrm{R}_{\text {sigma }}=0.0202\right]$

$4089 / 0 / 267$

1.243 
Final $\mathrm{R}$ indexes $[\mathrm{I}>=2 \sigma(\mathrm{I})]$

Final $\mathrm{R}$ indexes [all data]

Largest diff. peak/hole / e $\AA^{-3}$

$$
\begin{aligned}
& \mathrm{R}_{1}=0.0562, \mathrm{wR}_{2}=0.1306 \\
& \mathrm{R}_{1}=0.0569, \mathrm{wR}_{2}=0.1308 \\
& 0.31 /-0.31
\end{aligned}
$$

Table S16 Fractional Atomic Coordinates $\left(\times 10^{4}\right)$ and Equivalent Isotropic Displacement Parameters $\left(\AA^{2} \times 10^{3}\right)$ for $3 \mathrm{~m}$. $U_{\text {eq }}$ is defined as $1 / 3$ of of the trace of the orthogonalised $U_{I J}$ tensor.

\begin{tabular}{lrrrr} 
Atom & \multicolumn{1}{r}{$\boldsymbol{y}$} & $\boldsymbol{Z}$ & $\mathbf{U}(\mathbf{e q})$ \\
C11 & $-3224.4(11)$ & $219.1(4)$ & $1532.3(3)$ & $23.42(18)$ \\
F1 & $1197(3)$ & $4815.9(14)$ & $570.7(8)$ & $37.3(5)$ \\
F2 & $3806(3)$ & $3866.7(12)$ & $362.8(7)$ & $32.1(4)$ \\
F3 & $4706(3)$ & $5175.2(12)$ & $691.6(7)$ & $34.6(4)$ \\
N1 & $3855.8(14)$ & $2584.7(8)$ & $15.3(4)$ \\
C1 & $6430(4)$ & $1023.4(17)$ & $1780.2(11)$ & $17.6(5)$ \\
C2 & $-1309(4)$ & $1429.3(17)$ & $2297.3(11)$ & $18.0(5)$ \\
C3 & $-1664(4)$ & $2032.6(17)$ & $2508.3(11)$ & $16.8(5)$ \\
C4 & $-57(4)$ & $2234.1(16)$ & $2212.3(10)$ & $14.8(5)$ \\
C5 & $1918(4)$ & $2839.2(17)$ & $2455.4(10)$ & $14.6(5)$ \\
C6 & $2816.0(17)$ & $3039.0(10)$ & $15.4(5)$ \\
C7 & $2295.3(17)$ & $3514.9(11)$ & $17.3(5)$ \\
C8 & $2427.3(18)$ & $4011.0(11)$ & $18.5(5)$ \\
C9 & $3642(4)$ & $4071.8(17)$ & $4063.4(10)$ & $16.9(5)$ \\
C10 & $3170.3(19)$ & $4634.8(10)$ & $19.5(5)$ \\
C11 & $2261(2)$ & $4815.6(12)$ & $24.9(6)$ \\
C12 & $3875(4)$ & $1212.8(17)$ & $1469.4(11)$ & $17.7(5)$ \\
C13 & $5069(4)$ & $1812.7(17)$ & $1687.4(10)$ & $16.0(5)$ \\
C14 & $6832(4)$ & $3452.9(17)$ & $3098.6(10)$ & $15.1(5)$ \\
C15 & $8059(5)$ & $3589.2(17)$ & $3602.2(10)$ & $16.8(5)$ \\
C16 & $3491(2)$ & $5076.3(11)$ & $26.6(6)$ \\
C17 & $3863(2)$ & $4610.4(12)$ & $25.0(6)$ \\
C18 & $3488.4(17)$ & $2200.1(10)$ & $15.4(5)$ \\
C19 & $3926.3(17)$ & $1629.4(10)$ & $15.9(5)$ \\
C20 & $4800.6(18)$ & $1812.8(11)$ & $18.6(5)$ \\
C21 & $4567.9(18)$ & $2369.3(10)$ & $18.5(5)$ \\
C22 & $3328.1(18)$ & $1274.8(11)$ & $17.9(5)$ \\
C23 & $4130.6(18)$ & $1331.7(11)$ & $18.8(5)$ \\
C24 & $4500(2)$ & $740.8(12)$ & $23.8(6)$
\end{tabular}

Table S17 Anisotropic Displacement Parameters $\left(\AA^{2} \times 10^{3}\right)$ for $3 \mathrm{~m}$. The Anisotropic displacement factor exponent takes the form: $-2 \pi^{2}\left[h^{2} a * 2 U_{11}+2 h k a * b * U_{12}+\ldots\right]$.

\begin{tabular}{lcrrrrr} 
Atom & $\mathbf{U}_{\mathbf{1 1}}$ & $\mathbf{U}_{\mathbf{2 2}}$ & $\mathbf{U}_{\mathbf{3 3}}$ & $\mathbf{U}_{\mathbf{2 3}}$ & $\mathbf{U}_{\mathbf{1 3}}$ & \multicolumn{1}{c}{$\mathbf{U}_{\mathbf{1 2}}$} \\
C11 & $21.2(3)$ & $18.8(3)$ & $30.3(4)$ & $-3.2(3)$ & $-2.0(2)$ & $-5.6(2)$ \\
F1 & $33.1(10)$ & $46.3(11)$ & $32.1(10)$ & $14.9(8)$ & $-10.3(8)$ & $5.7(8)$ \\
F2 & $42.2(10)$ & $37.2(10)$ & $16.9(8)$ & $-0.8(7)$ & $-3.0(7)$ & $-3.0(8)$
\end{tabular}




$\begin{array}{lrrrrrr}\text { F3 } & 44.2(11) & 32.3(10) & 27.0(9) & 13.2(7) & -6.0(8) & -15.5(8) \\ \text { N1 } & 18.9(10) & 15.3(10) & 11.6(10) & -0.8(8) & -0.3(8) & -2.0(8) \\ \text { C1 } & 18.2(12) & 11.3(12) & 23.2(13) & 2.4(10) & -4.4(10) & -1.4(9) \\ \text { C2 } & 16.3(12) & 15.3(12) & 22.5(13) & 4.1(10) & 1.5(10) & 0.9(10) \\ \text { C3 } & 19.1(12) & 14.3(12) & 16.9(12) & 1.1(9) & 0.7(9) & 3.0(10) \\ \text { C4 } & 18.3(12) & 10.0(11) & 15.9(12) & 1.5(9) & -1.1(9) & 3.4(9) \\ \text { C5 } & 15.8(12) & 13.7(11) & 14.2(11) & -1.8(9) & 0.9(9) & 2.3(9) \\ \text { C6 } & 17.0(12) & 13.1(12) & 16.3(12) & -1.6(9) & 1.2(9) & 3.0(9) \\ \text { C7 } & 20.2(12) & 12.8(12) & 18.8(12) & -1.3(10) & 0.7(10) & 0.5(9) \\ \text { C8 } & 23.4(13) & 15.9(12) & 16.4(12) & 3.3(10) & 2.8(10) & 1.2(10) \\ \text { C9 } & 21.3(12) & 15.1(12) & 14.3(12) & -1.7(9) & -0.2(10) & 2.9(10) \\ \text { C10 } & 23.6(13) & 22.6(13) & 12.3(12) & -0.8(10) & -1.6(10) & 0.3(11) \\ \text { C11 } & 26.8(14) & 27.2(15) & 20.5(13) & 4.0(11) & -3.4(11) & -0.2(12) \\ \text { C12 } & 21.1(13) & 16.1(12) & 15.7(12) & -1.2(10) & -1.5(10) & 1.1(10) \\ \text { C13 } & 18.5(12) & 15.0(12) & 14.6(11) & 2.8(9) & 0.6(9) & -0.3(10) \\ \text { C14 } & 18.9(12) & 14.0(12) & 12.4(11) & 0.6(9) & 3.3(9) & 3.1(9) \\ \text { C15 } & 19.1(12) & 14.6(12) & 16.8(12) & -2.2(10) & 0.4(9) & -0.9(10) \\ \text { C16 } & 32.5(15) & 31.2(16) & 16.0(13) & -3.7(11) & -1.3(11) & 3.2(12) \\ \text { C17 } & 31.2(15) & 25.4(14) & 18.3(13) & 1.2(11) & -7.3(11) & -3.2(12) \\ \text { C18 } & 16.7(12) & 15.4(12) & 14.1(12) & -3.0(9) & -0.2(9) & 2.2(9) \\ \text { C19 } & 18.2(12) & 15.9(12) & 13.7(11) & 1.9(9) & 0.1(9) & -2.0(10) \\ \text { C20 } & 22.1(13) & 16.6(13) & 17.0(12) & 1.3(10) & 0.4(10) & -3.2(10) \\ \text { C21 } & 23.2(13) & 17.7(13) & 14.6(12) & 0.8(10) & 0.5(10) & -3.8(10) \\ \text { C22 } & 19.5(12) & 17.8(13) & 16.3(12) & -0.6(10) & 3.3(10) & 0.0(10) \\ \text { C23 } & 19.5(13) & 19.4(13) & 17.7(12) & 3.2(10) & 0.3(10) & -0.3(10) \\ \text { C24 } & 24.5(14) & 24.8(14) & 21.8(14) & 4.9(11) & -3.7(11) & -2.5(11)\end{array}$

Table S18 Bond Lengths for 3m.

Atom Atom Length $/ \AA$ Atom Atom Length $/ \AA$

$\begin{array}{llllll}\mathrm{C} 11 & \mathrm{C} 1 & 1.741(3) & \mathrm{C} 6 & \mathrm{C} 14 & 1.410(4) \\ \mathrm{F} 1 & \mathrm{C} 24 & 1.343(3) & \mathrm{C} 7 & \mathrm{C} 8 & 1.380(4) \\ \mathrm{F} 2 & \mathrm{C} 24 & 1.345(3) & \mathrm{C} 8 & \mathrm{C} 9 & 1.418(4) \\ \mathrm{F} 3 & \mathrm{C} 24 & 1.337(3) & \mathrm{C} 9 & \mathrm{C} 10 & 1.538(3) \\ \mathrm{N} 1 & \mathrm{C} 14 & 1.367(3) & \mathrm{C} 9 & \mathrm{C} 15 & 1.379(4) \\ \mathrm{N} 1 & \mathrm{C} 18 & 1.370(3) & \mathrm{C} 10 & \mathrm{C} 11 & 1.530(4) \\ \mathrm{N} 1 & \mathrm{C} 21 & 1.450(3) & \mathrm{C} 10 & \mathrm{C} 16 & 1.542(4) \\ \mathrm{C} 1 & \mathrm{C} 2 & 1.385(4) & \mathrm{C} 10 & \mathrm{C} 17 & 1.533(4) \\ \mathrm{C} 1 & \mathrm{C} 12 & 1.387(4) & \mathrm{C} 12 & \mathrm{C} 13 & 1.391(4) \\ \mathrm{C} 2 & \mathrm{C} 3 & 1.393(4) & \mathrm{C} 14 & \mathrm{C} 15 & 1.402(3) \\ \mathrm{C} 3 & \mathrm{C} 4 & 1.404(4) & \mathrm{C} 18 & \mathrm{C} 19 & 1.523(3) \\ \mathrm{C} 4 & \mathrm{C} 5 & 1.470(3) & \mathrm{C} 19 & \mathrm{C} 20 & 1.558(3) \\ \mathrm{C} 4 & \mathrm{C} 13 & 1.405(3) & \mathrm{C} 19 & \mathrm{C} 22 & 1.544(3) \\ \mathrm{C} 5 & \mathrm{C} 6 & 1.454(3) & \mathrm{C} 19 & \mathrm{C} 23 & 1.539(3)\end{array}$




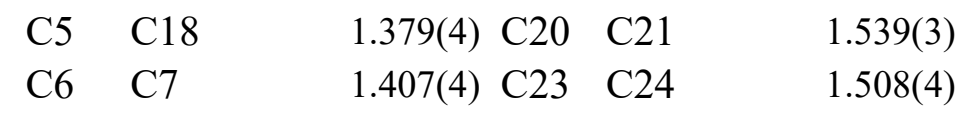

Table S19 Bond Angles for $3 \mathrm{~m}$.

\begin{tabular}{lllllllr} 
Atom Atom Atom & \multicolumn{1}{l}{ Angle ${ }^{\circ}$} & \multicolumn{2}{c}{ Atom Atom Atom } & Angle $^{\circ}$ \\
C14 & N1 & C18 & $110.2(2)$ & C17 & C10 & C9 & $11.9(2)$ \\
C14 & N1 & C21 & $134.6(2)$ & C17 & C10 & C16 & $108.4(2)$ \\
C18 & N1 & C21 & $115.2(2)$ & C1 & C12 & C13 & $119.1(2)$ \\
C2 & C1 & C11 & $119.4(2)$ & C12 & C13 & C4 & $121.7(2)$ \\
C2 & C1 & C12 & $121.2(2)$ & N1 & C14 & C6 & $106.9(2)$ \\
C12 & C1 & C11 & $119.4(2)$ & N1 & C14 & C15 & $129.6(2)$ \\
C1 & C2 & C3 & $119.1(2)$ & C15 & C14 & C6 & $123.5(2)$ \\
C2 & C3 & C4 & $121.7(2)$ & C9 & C15 & C14 & $118.2(2)$ \\
C3 & C4 & C5 & $120.8(2)$ & N1 & C18 & C5 & $110.0(2)$ \\
C3 & C4 & C13 & $117.2(2)$ & N1 & C18 & C19 & $108.5(2)$ \\
C13 & C4 & C5 & $122.0(2)$ & C5 & C18 & C19 & $141.5(2)$ \\
C6 & C5 & C4 & $124.7(2)$ & C18 & C19 & C20 & $100.72(19)$ \\
C18 & C5 & C4 & $130.0(2)$ & C18 & C19 & C22 & $110.2(2)$ \\
C18 & C5 & C6 & $105.2(2)$ & C18 & C19 & C23 & $110.2(2)$ \\
C7 & C6 & C5 & $134.9(2)$ & C22 & C19 & C20 & $109.3(2)$ \\
C7 & C6 & C14 & $117.4(2)$ & C23 & C19 & C20 & $112.6(2)$ \\
C14 & C6 & C5 & $107.7(2)$ & C23 & C19 & C22 & $113.1(2)$ \\
C8 & C7 & C6 & $119.2(2)$ & C21 & C20 & C19 & $106.1(2)$ \\
C7 & C8 & C9 & $122.6(2)$ & N1 & C21 & C20 & $101.1(2)$ \\
C8 & C9 & C10 & $118.7(2)$ & C24 & C23 & C19 & $116.5(2)$ \\
C15 & C9 & C8 & $119.1(2)$ & F1 & C24 & F2 & $106.1(2)$ \\
C15 & C9 & C10 & $122.2(2)$ & F1 & C24 & C23 & $110.6(2)$ \\
C9 & C10 & C16 & $108.7(2)$ & F2 & C24 & C23 & $112.6(2)$ \\
C11 & C10 & C9 & $110.1(2)$ & F3 & C24 & F1 & $107.4(2)$ \\
C11 & C10 & C16 & $109.7(2)$ & F3 & C24 & F2 & $106.6(2)$ \\
C11 & C10 & C17 & $108.0(2)$ & F3 & C24 & C23 & $113.0(2)$
\end{tabular}

Table S20 Torsion Angles for $\mathbf{3 m}$.

\begin{tabular}{|c|c|c|c|c|}
\hline A B C & Angle $/^{\circ}$ & $\mathbf{A}$ & D & Angle ${ }^{\circ}$ \\
\hline $\mathrm{C} 11 \mathrm{C} 1 \mathrm{C} 2 \mathrm{C} 3$ & $176.26(19)$ & $\mathrm{C} 8 \mathrm{C} 9$ & $\mathrm{C} 10 \mathrm{C} 16$ & $-63.0(3)$ \\
\hline $\mathrm{C} 11 \mathrm{C} 1 \mathrm{C} 12 \mathrm{C} 13$ & $-175.89(19)$ & $\mathrm{C} 8 \mathrm{C} 9$ & $\mathrm{C} 10 \mathrm{C} 17$ & $177.4(2)$ \\
\hline N1 C14C15C9 & $-178.2(2)$ & $\mathrm{C} 8 \mathrm{C} 9$ & C15C14 & $0.0(4)$ \\
\hline N1 C18C19C20 & $-19.3(3)$ & $\mathrm{C} 10 \mathrm{C} 9$ & C15C14 & $-179.9(2)$ \\
\hline N1 C18C19C22 & $96.1(2)$ & $\mathrm{C} 12 \mathrm{C} 1$ & $\mathrm{C} 2 \mathrm{C} 3$ & $-0.5(4)$ \\
\hline N1 C18C19C23 & $-138.4(2)$ & $\mathrm{C} 13 \mathrm{C} 4$ & $\mathrm{C} 5 \mathrm{C} 6$ & $-135.1(3)$ \\
\hline $\mathrm{C} 1 \mathrm{C} 2 \mathrm{C} 3 \mathrm{C} 4$ & $-0.5(4)$ & $\mathrm{C} 13 \mathrm{C} 4$ & C5 C18 & $40.5(4)$ \\
\hline $\mathrm{C} 1 \mathrm{C} 12 \mathrm{C} 13 \mathrm{C} 4$ & $-0.3(4)$ & C14N1 & $\mathrm{C} 18 \mathrm{C} 5$ & $1.1(3)$ \\
\hline
\end{tabular}




\begin{tabular}{|c|c|c|c|}
\hline $\mathrm{C} 2 \mathrm{C} 1 \mathrm{C} 12 \mathrm{C} 13$ & $0.9(4)$ & C14N1 C18C19 & $-177.6(2)$ \\
\hline $\mathrm{C} 2 \mathrm{C} 3 \mathrm{C} 4 \mathrm{C} 5$ & $-177.1(2)$ & C14N1 C21 C20 & $-164.2(3)$ \\
\hline C2 C3 C4 C13 & $1.0(4)$ & C14C6 C7 C8 & $0.0(4)$ \\
\hline $\mathrm{C} 3 \mathrm{C} 4 \mathrm{C} 5 \mathrm{C} 6$ & $42.9(4)$ & $\mathrm{C} 15 \mathrm{C} 9 \mathrm{C} 10 \mathrm{C} 11$ & $-122.8(3)$ \\
\hline C3 C4 C5 C18 & $-141.5(3)$ & $\mathrm{C} 15 \mathrm{C} 9 \mathrm{C} 10 \mathrm{C} 16$ & $117.0(3)$ \\
\hline C3 C4 C13C12 & $-0.6(4)$ & $\mathrm{C} 15 \mathrm{C} 9 \mathrm{C} 10 \mathrm{C} 17$ & $-2.7(4)$ \\
\hline C4 C5 C6 C7 & $-0.6(4)$ & C18N1 C14C6 & $-1.5(3)$ \\
\hline C4 C5 C6 C14 & $175.9(2)$ & C18N1 C14C15 & $177.0(3)$ \\
\hline $\mathrm{C} 4 \mathrm{C} 5 \mathrm{C} 18 \mathrm{~N} 1$ & $-176.6(2)$ & C18N1 C21C20 & $15.2(3)$ \\
\hline C4 C5 C18C19 & $1.5(5)$ & C18C5 C6 C7 & $-177.1(3)$ \\
\hline C5 C4 C13C12 & 177.4(2) & C18C5 C6 C14 & $-0.6(3)$ \\
\hline C5 $\mathrm{C} 6 \quad \mathrm{C} 7 \mathrm{C} 8$ & $176.3(3)$ & C18C19C20C21 & $28.2(2)$ \\
\hline C5 C6 C14N1 & $1.2(3)$ & C18C19C23 C24 & $-175.4(2)$ \\
\hline C5 C6 C14C15 & $-177.4(2)$ & C19C20C21 N1 & $-26.7(3)$ \\
\hline C5 C18C19C20 & $162.6(3)$ & C19C23 C24F1 & $-167.7(2)$ \\
\hline C5 C18C19C22 & $-82.0(4)$ & $\mathrm{C} 19 \mathrm{C} 23 \mathrm{C} 24 \mathrm{~F} 2$ & $73.7(3)$ \\
\hline C5 C18C19C23 & $43.5(4)$ & C19C23 C24F3 & $-47.2(3)$ \\
\hline C6 C5 C18N1 & $-0.3(3)$ & C20C19C23 C24 & $73.1(3)$ \\
\hline C6 C5 C18C19 & 177.7(3) & $\mathrm{C} 21 \mathrm{~N} 1 \mathrm{C} 14 \mathrm{C} 6$ & $178.0(3)$ \\
\hline C6 $\mathrm{C} 7 \quad \mathrm{C} 8 \quad \mathrm{C} 9$ & $0.1(4)$ & C21N1 C14C15 & $-3.6(5)$ \\
\hline C6 C14C15C9 & $0.1(4)$ & $\mathrm{C} 21 \mathrm{~N} 1 \mathrm{C} 18 \mathrm{C} 5$ & $-178.4(2)$ \\
\hline C7 C6 C14N1 & $178.5(2)$ & C21 N1 C18C19 & $2.9(3)$ \\
\hline C7 C6 C14C15 & $-0.1(4)$ & $\mathrm{C} 22 \mathrm{C} 19 \mathrm{C} 20 \mathrm{C} 21$ & $-87.8(2)$ \\
\hline C7 C8 C9 C10 & $179.8(2)$ & C22 C19C23 C24 & $-51.5(3)$ \\
\hline C7 C8 C9 C15 & $-0.1(4)$ & C23 C19C20C21 & $145.5(2)$ \\
\hline C8 C9 C10C11 & $57.2(3)$ & & \\
\hline
\end{tabular}

Table S21 Hydrogen Atom Coordinates $\left(\AA \times 10^{4}\right)$ and Isotropic Displacement Parameters $\left(\AA^{2} \times \mathbf{1 0}^{3}\right)$ for $3 \mathrm{~m}$.

$\begin{array}{lrrrr}\text { Atom } & \boldsymbol{x} & \boldsymbol{y} & \boldsymbol{z} & \mathbf{U}(\mathbf{e q}) \\ \text { H2 } & -2985.58 & 1298.34 & 2505.24 & 22 \\ \text { H3 } & -304.94 & 2315.03 & 2861.7 & 20 \\ \text { H7 } & 2701.34 & 1857.79 & 3495 & 21 \\ \text { H8 } & 4694.55 & 2072.03 & 4330.09 & 22 \\ \text { H11A } & 10168.29 & 2062.97 & 4536.02 & 37 \\ \text { H11B } & 7862.21 & 1810.42 & 4843.58 & 37 \\ \text { H11C } & 9822.79 & 2328.15 & 5182.96 & 21 \\ \text { H12 } & 819.5 & 936.65 & 1112.72 & 19 \\ \text { H13 } & 3511.06 & 1940.75 & 1475.57 & 20 \\ \text { H15 } & 8562.05 & 4026.23 & 3624.95 & 40 \\ \text { H16A } & 5129 & 3045.4 & 5106.67 & 40 \\ \text { H16B } & 5711.14 & 4075.43 & 4960.21 & 40 \\ \text { H16C } & 7108.72 & 3557.17 & 5442.61 & \end{array}$




$\begin{array}{lrrrr}\text { H17A } & 10727.54 & 3903.16 & 4980.43 & 38 \\ \text { H17B } & 9368.48 & 4456.33 & 4506.48 & 38 \\ \text { H17C } & 11081.82 & 3672.28 & 4328.75 & 38 \\ \text { H20A } & 7739.89 & 4980.66 & 1522.41 & 22 \\ \text { H20B } & 5565.91 & 5304.71 & 1868.81 & 22 \\ \text { H21A } & 9419.41 & 4347.99 & 2303.25 & 22 \\ \text { H21B } & 7921.62 & 5093.9 & 2627.22 & 22 \\ \text { H22A } & 8225.99 & 3131.47 & 1508.23 & 27 \\ \text { H22B } & 7491.65 & 3676.31 & 953.06 & 27 \\ \text { H22C } & 6109.54 & 2797.06 & 1138.25 & 23 \\ \text { H23A } & 2197.89 & 3565.62 & 1320.22 & 23 \\ \text { H23B } & 2258.69 & 4570.61 & 1564.5 & \end{array}$

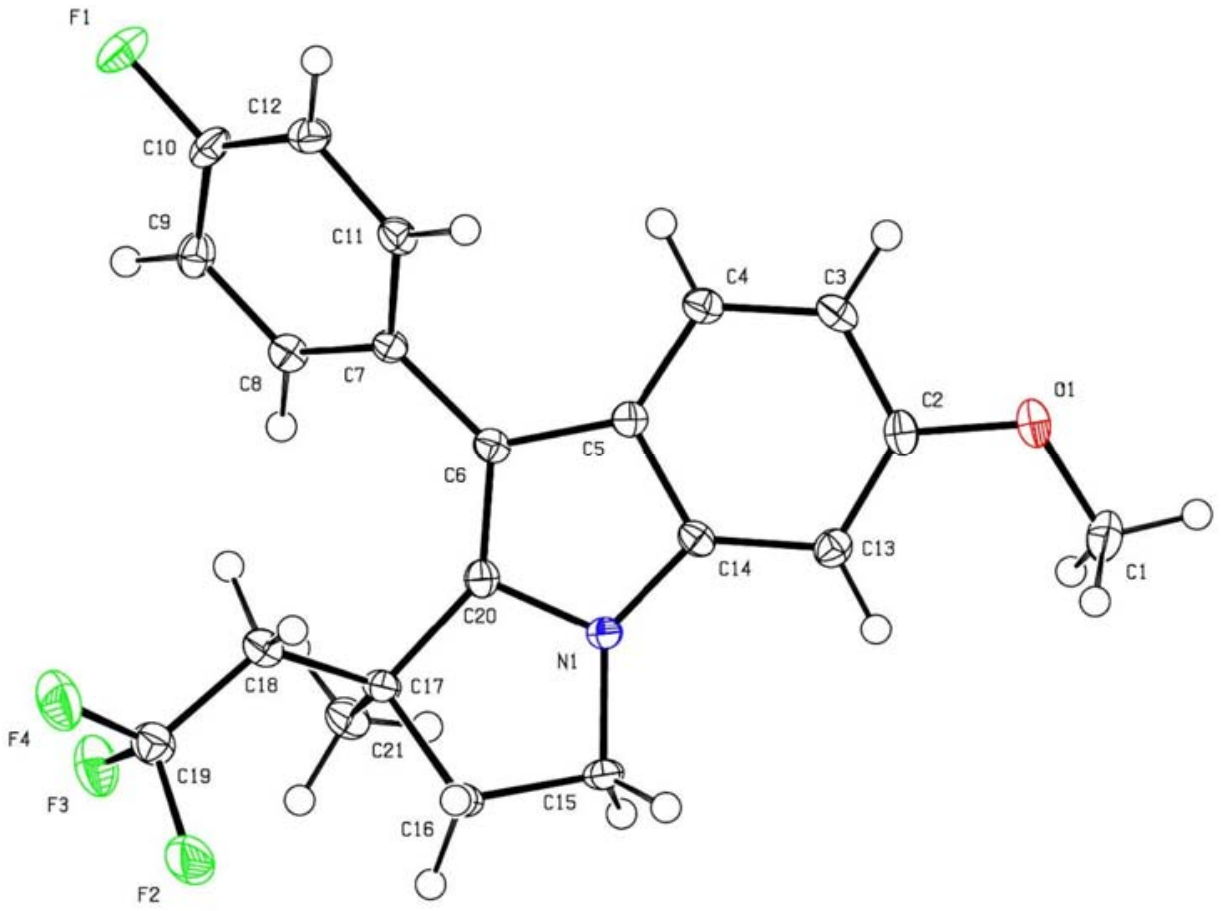

Table S22 Crystal data and structure refinement for 3t (2043669).

Identification code

cxy1273_0m (6a)

Empirical formula

$\mathrm{C}_{21} \mathrm{H}_{19} \mathrm{~F}_{4} \mathrm{NO}$

Formula weight

377.37

Temperature/K

100.0

Crystal system

monoclinic

Space group

$\mathrm{P} 21 / \mathrm{c}$

$\mathrm{a} / \AA ̊$

9.8850(4)

$\mathrm{b} / \AA$

15.8544(7)

$\mathrm{c} / \AA \AA$

$11.3937(6)$

$\alpha /{ }^{\circ}$

90

$\beta /{ }^{\circ}$

$106.016(2)$

$\gamma /{ }^{\circ}$

90 


$\begin{array}{ll}\text { Volume } / \AA^{3} & 1716.32(14) \\ \mathrm{Z} & 4 \\ \rho_{\text {calcg }} / \mathrm{cm}^{3} & 1.460 \\ \mu / \mathrm{mm}^{-1} & 0.119 \\ \mathrm{~F}(000) & 784.0 \\ \text { Crystal size } / \mathrm{mm}^{3} & 0.35 \times 0.34 \times 0.29 \\ \text { Radiation } & \mathrm{MoK} \alpha(\lambda=0.71073) \\ 2 \Theta \text { range for data collection } /{ }^{\circ} & 4.52 \text { to } 54.976 \\ \text { Index ranges } & -12 \leq \mathrm{h} \leq 12,-20 \leq \mathrm{k} \leq 20,-13 \leq 1 \leq 14 \\ \text { Reflections collected } & 16025 \\ \text { Independent reflections } & 3927\left[\mathrm{R}_{\text {int }}=0.0678, \mathrm{R}_{\text {sigma }}=0.0519\right] \\ \text { Data/restraints/parameters } & 3927 / 0 / 247 \\ \text { Goodness-of-fit on } \mathrm{F}^{2} & 1.041 \\ \text { Final R indexes }[\mathrm{I}>=2 \sigma(\mathrm{I})] & \mathrm{R}_{1}=0.0481, \mathrm{wR}_{2}=0.1192 \\ \text { Final R indexes }[\text { all data }] & \mathrm{R}_{1}=0.0744, \mathrm{wR}_{2}=0.1292 \\ \text { Largest diff. peak/hole } / \mathrm{e} \AA^{-3} & 0.59 /-0.34\end{array}$

Table S23 Fractional Atomic Coordinates $\left(\times 10^{4}\right)$ and Equivalent Isotropic Displacement Parameters $\left(\AA^{2} \times 10^{3}\right)$ for $3 t$. $U_{\text {eq }}$ is defined as $1 / 3$ of of the trace of the orthogonalised $\mathrm{U}_{\mathrm{IJ}}$ tensor.

\begin{tabular}{lrrrr} 
Atom & \multicolumn{1}{r}{$\boldsymbol{y}$} & \multicolumn{1}{l}{$\boldsymbol{U}(\mathbf{e q})$} \\
F1 & $10075.6(13)$ & $6567.2(8)$ & $5423.4(12)$ & $27.6(3)$ \\
F2 & $7557.2(16)$ & $1532.5(9)$ & $6547.1(13)$ & $39.4(4)$ \\
F3 & $7789.9(14)$ & $2601.8(9)$ & $7731.1(11)$ & $30.9(3)$ \\
F4 & $2291.5(9)$ & $6908.3(12)$ & $32.7(3)$ \\
O1 & $9432.7(13)$ & $4339.5(10)$ & $-608.6(12)$ & $23.2(3)$ \\
N1 & $1193.7(15)$ & $3208.9(10)$ & $3316.0(14)$ & $14.2(3)$ \\
C1 & $4099.4(16)$ & $3830.1(15)$ & $-649(2)$ & $27.7(5)$ \\
C2 & $-3(2)$ & $4286.4(13)$ & $427.7(16)$ & $17.2(4)$ \\
C3 & $2303.8(19)$ & $4933.1(13)$ & $533.1(17)$ & $17.3(4)$ \\
C4 & $3306(2)$ & $4977.2(12)$ & $1528.0(16)$ & $15.8(4)$ \\
C5 & $4473(2)$ & $4366.9(12)$ & $2450.4(16)$ & $13.7(4)$ \\
C6 & $4675.0(19)$ & $4244.0(12)$ & $3610.3(16)$ & $13.2(4)$ \\
C7 & $5726.4(19)$ & $4817.9(12)$ & $4113.0(16)$ & $13.4(4)$ \\
C8 & $6915.9(19)$ & $5088.2(13)$ & $5333.4(17)$ & $15.9(4)$ \\
C9 & $7269(2)$ & $5665.1(13)$ & $5784.4(17)$ & $18.2(4)$ \\
C10 & $8347(2)$ & $5986.9(13)$ & $4993.7(18)$ & $18.0(4)$ \\
C11 & $5153.2(12)$ & $3358.0(16)$ & $15.9(4)$ \\
C12 & $7687.4(19)$ & $5744.5(13)$ & $3785.0(17)$ & $18.0(4)$ \\
C13 & $8739(2)$ & $3662.9(13)$ & $1306.1(16)$ & $15.8(4)$ \\
C14 & $2459.8(19)$ & $3712.8(12)$ & $2306.8(16)$ & $13.6(4)$ \\
C15 & $3663.7(19)$ & $2408.1(12)$ & $3713.7(17)$ & $16.4(4)$ \\
C16 & $3647(2)$ & $2117.9(13)$ & $4652.9(17)$ & $18.2(4)$ \\
C17 & $5013(2)$ & $2945.3(12)$ & $5198.5(16)$ & $14.8(4)$ \\
C18 & $2878.6(13)$ & $5610.0(17)$ & $18.6(4)$ \\
& $5771.3(19)$ & & &
\end{tabular}




$\begin{array}{lrlll}\mathrm{C} 19 & 8027(2) & 2328.4(14) & 6694.4(18) & 21.0(4) \\ \mathrm{C} 20 & 5319.5(19) & 3525.1(12) & 4098.8(16) & 13.9(4) \\ \mathrm{C} 21 & 5153(2) & 3260.7(13) & 6229.8(17) & 19.9(4)\end{array}$

Table S24 Anisotropic Displacement Parameters $\left(\AA^{2} \times 10^{3}\right)$ for 3t. The Anisotropic displacement factor exponent takes the form: $-2 \pi^{2}\left[h^{2} a * 2 U_{11}+2 h k a * b * U_{12}+\ldots\right]$.

\begin{tabular}{lrrrrrr} 
Atom & \multicolumn{1}{c}{$\mathbf{U}_{\mathbf{1 1}}$} & \multicolumn{1}{c}{$\mathbf{U}_{\mathbf{2 2}}$} & \multicolumn{1}{c}{$\mathbf{U}_{\mathbf{3 3}}$} & \multicolumn{1}{c}{$\mathbf{U}_{\mathbf{2 3}}$} & \multicolumn{1}{c}{$\mathbf{U}_{\mathbf{1 3}}$} & \multicolumn{1}{c}{$\mathbf{U}_{\mathbf{1 2}}$} \\
F1 & $23.8(6)$ & $25.9(7)$ & $32.7(7)$ & $-8.2(6)$ & $7.1(5)$ & $-12.7(5)$ \\
F2 & $53.0(9)$ & $17.9(7)$ & $34.4(8)$ & $5.6(6)$ & $-9.8(7)$ & $-4.4(7)$ \\
F3 & $31.3(7)$ & $43.0(9)$ & $16.3(6)$ & $0.3(6)$ & $2.9(5)$ & $3.2(6)$ \\
F4 & $25.1(6)$ & $41.7(9)$ & $28.5(7)$ & $8.1(6)$ & $2.9(5)$ & $8.6(6)$ \\
O1 & $22.2(7)$ & $27.6(8)$ & $15.1(6)$ & $2.5(6)$ & $-2.8(6)$ & $-1.0(6)$ \\
N1 & $15.4(7)$ & $13.6(8)$ & $13.2(7)$ & $0.6(6)$ & $3.5(6)$ & $-2.4(6)$ \\
C1 & $19.5(10)$ & $29.0(12)$ & $27.5(11)$ & $1.3(9)$ & $-5.5(8)$ & $-2.9(9)$ \\
C2 & $16.4(9)$ & $21.1(10)$ & $12.6(8)$ & $-1.7(7)$ & $1.7(7)$ & $2.5(8)$ \\
C3 & $22.2(9)$ & $15.7(10)$ & $14.3(8)$ & $3.8(7)$ & $5.7(7)$ & $2.3(8)$ \\
C4 & $19.6(9)$ & $13.8(9)$ & $14.8(8)$ & $0.2(7)$ & $6.4(7)$ & $0.0(8)$ \\
C5 & $15.3(8)$ & $13.3(9)$ & $13.1(8)$ & $-1.1(7)$ & $5.1(7)$ & $-0.2(7)$ \\
C6 & $16.6(8)$ & $12.8(9)$ & $11.0(8)$ & $-0.8(7)$ & $5.1(7)$ & $0.4(7)$ \\
C7 & $13.7(8)$ & $10.0(9)$ & $15.6(8)$ & $0.7(7)$ & $2.7(7)$ & $1.5(7)$ \\
C8 & $17.4(9)$ & $15.6(10)$ & $15.3(9)$ & $-0.1(7)$ & $5.4(7)$ & $1.1(7)$ \\
C9 & $17.7(9)$ & $18.0(10)$ & $17.4(9)$ & $-4.9(7)$ & $2.3(7)$ & $0.9(8)$ \\
C10 & $14.3(8)$ & $13.5(9)$ & $24.8(10)$ & $-5.1(8)$ & $2.8(7)$ & $-2.8(8)$ \\
C11 & $19.5(9)$ & $14.9(10)$ & $13.3(8)$ & $0.6(7)$ & $4.7(7)$ & $1.1(7)$ \\
C12 & $17.7(9)$ & $16.7(10)$ & $20.7(9)$ & $2.3(7)$ & $7.3(8)$ & $-1.5(8)$ \\
C13 & $16.4(8)$ & $16.3(9)$ & $15.1(8)$ & $-2.3(7)$ & $5.0(7)$ & $-1.7(7)$ \\
C14 & $16.7(8)$ & $13.3(9)$ & $11.7(8)$ & $0.7(7)$ & $5.3(7)$ & $2.1(7)$ \\
C15 & $19.3(9)$ & $13.7(9)$ & $16.6(8)$ & $1.1(7)$ & $5.6(7)$ & $-5.2(8)$ \\
C16 & $23.1(9)$ & $14.5(9)$ & $17.3(9)$ & $2.4(7)$ & $6.2(8)$ & $-2.2(8)$ \\
C17 & $17.3(9)$ & $12.8(9)$ & $14.4(8)$ & $2.1(7)$ & $4.7(7)$ & $-1.2(7)$ \\
C18 & $20.0(9)$ & $18.9(10)$ & $16.7(9)$ & $3.7(8)$ & $4.6(7)$ & $-0.3(8)$ \\
C19 & $23.9(10)$ & $18.7(10)$ & $20.1(9)$ & $0.9(8)$ & $5.4(8)$ & $0.8(8)$ \\
C20 & $14.7(8)$ & $14.8(9)$ & $12.0(8)$ & $-1.6(7)$ & $3.6(7)$ & $0.4(7)$ \\
C21 & $24.4(10)$ & $19.5(10)$ & $17.2(9)$ & $1.4(8)$ & $8.0(8)$ & $1.6(8)$
\end{tabular}

Table S25 Bond Lengths for 3t.

\begin{tabular}{llrllr}
\multicolumn{2}{c}{ Atom Atom } & Length $/ \AA$ & \multicolumn{2}{c}{ Atom Atom } & Length/ $\boldsymbol{\AA}$ \\
F1 & C10 & $1.358(2)$ & C6 & C7 & $1.472(3)$ \\
F2 & C19 & $1.339(3)$ & C6 & C20 & $1.376(3)$ \\
F3 & C19 & $1.338(2)$ & C7 & C8 & $1.404(2)$ \\
F4 & C19 & $1.344(2)$ & C7 & C11 & $1.399(2)$ \\
O1 & C1 & $1.423(3)$ & C8 & C9 & $1.391(3)$
\end{tabular}




$\begin{array}{llllll}\mathrm{O} 1 & \mathrm{C} 2 & 1.375(2) & \mathrm{C} 9 & \mathrm{C} 10 & 1.377(3) \\ \mathrm{N} 1 & \mathrm{C} 14 & 1.368(2) & \mathrm{C} 10 & \mathrm{C} 12 & 1.380(3) \\ \mathrm{N} 1 & \mathrm{C} 15 & 1.459(2) & \mathrm{C} 11 & \mathrm{C} 12 & 1.388(3) \\ \mathrm{N} 1 & \mathrm{C} 20 & 1.381(2) & \mathrm{C} 13 & \mathrm{C} 14 & 1.405(3) \\ \mathrm{C} 2 & \mathrm{C} 3 & 1.408(3) & \mathrm{C} 15 & \mathrm{C} 16 & 1.544(3) \\ \mathrm{C} 2 & \mathrm{C} 13 & 1.385(3) & \mathrm{C} 16 & \mathrm{C} 17 & 1.553(3) \\ \mathrm{C} 3 & \mathrm{C} 4 & 1.378(3) & \mathrm{C} 17 & \mathrm{C} 18 & 1.534(3) \\ \mathrm{C} 4 & \mathrm{C} 5 & 1.402(3) & \mathrm{C} 17 & \mathrm{C} 20 & 1.518(3) \\ \mathrm{C} 5 & \mathrm{C} 6 & 1.451(2) & \mathrm{C} 17 & \mathrm{C} 21 & 1.550(3) \\ \mathrm{C} 5 & \mathrm{C} 14 & 1.418(3) & \mathrm{C} 18 & \mathrm{C} 19 & 1.503(3)\end{array}$

Table S26 Bond Angles for 3t.

\begin{tabular}{llllllll} 
Atom Atom Atom & Angle $^{\circ}$ & \multicolumn{1}{c}{ Atom Atom Atom } & Angle $^{\circ}$ \\
C2 & O1 & C1 & $116.90(16)$ & C10 & C12 & C11 & $118.38(17)$ \\
C14 & N1 & C15 & $135.76(16)$ & C2 & C13 & C14 & $116.54(18)$ \\
C14 & N1 & C20 & $110.31(15)$ & N1 & C14 & C5 & $106.35(16)$ \\
C20 & N1 & C15 & $113.78(15)$ & N1 & C14 & C13 & $130.45(18)$ \\
O1 & C2 & C3 & $113.88(17)$ & C13 & C14 & C5 & $123.17(17)$ \\
O1 & C2 & C13 & $124.59(18)$ & N1 & C15 & C16 & $100.94(15)$ \\
C13 & C2 & C3 & $121.52(17)$ & C15 & C16 & C17 & $105.01(16)$ \\
C4 & C3 & C2 & $121.11(18)$ & C18 & C17 & C16 & $114.17(16)$ \\
C3 & C4 & C5 & $119.72(18)$ & C18 & C17 & C21 & $112.57(16)$ \\
C4 & C5 & C6 & $133.91(18)$ & C20 & C17 & C16 & $100.46(14)$ \\
C4 & C5 & C14 & $117.91(17)$ & C20 & C17 & C18 & $109.77(15)$ \\
C14 & C5 & C6 & $108.16(16)$ & C20 & C17 & C21 & $110.04(15)$ \\
C5 & C6 & C7 & $124.45(16)$ & C21 & C17 & C16 & $109.17(15)$ \\
C20 & C6 & C5 & $105.29(16)$ & C19 & C18 & C17 & $117.43(16)$ \\
C20 & C6 & C7 & $130.22(17)$ & F2 & C19 & F4 & $106.68(18)$ \\
C8 & C7 & C6 & $121.84(16)$ & F2 & C19 & C18 & $112.95(17)$ \\
C11 & C7 & C6 & $120.39(16)$ & F3 & C19 & F2 & $106.08(17)$ \\
C11 & C7 & C8 & $117.66(17)$ & F3 & C19 & F4 & $106.14(16)$ \\
C9 & C8 & C7 & $121.49(17)$ & F3 & C19 & C18 & $113.55(17)$ \\
C10 & C9 & C8 & $118.32(18)$ & F4 & C19 & C18 & $110.94(16)$ \\
F1 & C10 & C9 & $118.46(17)$ & N1 & C20 & C17 & $108.59(15)$ \\
F1 & C10 & C12 & $119.04(17)$ & C6 & C20 & N1 & $109.88(16)$ \\
C9 & C10 & C12 & $122.50(18)$ & C6 & C20 & C17 & $141.52(17)$ \\
C12 & C11 & C7 & $121.61(17)$ & & & &
\end{tabular}

Table S27 Torsion Angles for 3t.
A B C D
Angle/ ${ }^{\circ}$
A $\quad$ B $\quad$ C $\quad$ D
Angle $/^{\circ}$
F1 C10C12C11 -179.79(17) $\mathrm{C} 11 \mathrm{C} 7 \mathrm{C} 8 \mathrm{C} 9$
$-0.3(3)$
O1C2 C3 C4 $179.08(17) \mathrm{C} 13 \mathrm{C} 2 \quad \mathrm{C} 3 \mathrm{C} 4$
$-1.1(3)$ 


\begin{tabular}{|c|c|c|c|}
\hline $\mathrm{O} 1 \mathrm{C} 2 \mathrm{C} 13 \mathrm{C} 14$ & $-179.84(17)$ & C14N1 C15C16 & $157.5(2)$ \\
\hline $\mathrm{N} 1 \mathrm{C} 15 \mathrm{C} 16 \mathrm{C} 17$ & $30.72(17)$ & $\mathrm{C} 14 \mathrm{~N} 1 \mathrm{C} 20 \mathrm{C} 6$ & $-0.8(2)$ \\
\hline $\mathrm{C} 1 \mathrm{O} 1 \mathrm{C} 2 \mathrm{C} 3$ & $-166.37(17)$ & $\mathrm{C} 14 \mathrm{~N} 1 \mathrm{C} 20 \mathrm{C} 17$ & $-179.57(15)$ \\
\hline $\mathrm{C} 1 \mathrm{O} 1 \mathrm{C} 2 \mathrm{C} 13$ & $13.8(3)$ & $\mathrm{C} 14 \mathrm{C} 5 \mathrm{C} 6 \mathrm{C} 7$ & $-177.05(16)$ \\
\hline $\mathrm{C} 2 \mathrm{C} 3 \mathrm{C} 4 \mathrm{C} 5$ & $0.4(3)$ & C14C5 C6 C20 & $0.8(2)$ \\
\hline $\mathrm{C} 2 \mathrm{C} 13 \mathrm{C} 14 \mathrm{~N} 1$ & $178.90(18)$ & $\mathrm{C} 15 \mathrm{~N} 1 \mathrm{C} 14 \mathrm{C} 5$ & $-173.78(19)$ \\
\hline $\mathrm{C} 2 \mathrm{C} 13 \mathrm{C} 14 \mathrm{C} 5$ & $1.1(3)$ & $\mathrm{C} 15 \mathrm{~N} 1 \mathrm{C} 14 \mathrm{C} 13$ & $8.2(3)$ \\
\hline C3 C2 C13C14 & $0.3(3)$ & C15N1 C20C6 & $175.46(15)$ \\
\hline $\mathrm{C} 3 \mathrm{C} 4 \mathrm{C} 5 \mathrm{C} 6$ & $-177.34(19)$ & $\mathrm{C} 15 \mathrm{~N} 1 \mathrm{C} 20 \mathrm{C} 17$ & $-3.4(2)$ \\
\hline $\mathrm{C} 3 \mathrm{C} 4 \mathrm{C} 5 \mathrm{C} 14$ & $1.0(3)$ & $\mathrm{C} 15 \mathrm{C} 16 \mathrm{C} 17 \mathrm{C} 18$ & $-149.87(15)$ \\
\hline $\mathrm{C} 4 \mathrm{C} 5 \mathrm{C} 6 \mathrm{C} 7$ & $1.4(3)$ & C15C16C17C20 & $-32.49(17)$ \\
\hline C4 C5 C6 C20 & $179.26(19)$ & $\mathrm{C} 15 \mathrm{C} 16 \mathrm{C} 17 \mathrm{C} 21$ & $83.17(17)$ \\
\hline C4 C5 C14N1 & $180.00(16)$ & $\mathrm{C} 16 \mathrm{C} 17 \mathrm{C} 18 \mathrm{C} 19$ & $-68.2(2)$ \\
\hline $\mathrm{C} 4 \mathrm{C} 5 \mathrm{C} 14 \mathrm{C} 13$ & $-1.8(3)$ & $\mathrm{C} 16 \mathrm{C} 17 \mathrm{C} 20 \mathrm{~N} 1$ & $22.40(18)$ \\
\hline $\begin{array}{lll}\mathrm{C} 5 \mathrm{C} 6 & \mathrm{C} 7 & \mathrm{C} 8\end{array}$ & $133.50(19)$ & $\mathrm{C} 16 \mathrm{C} 17 \mathrm{C} 20 \mathrm{C} 6$ & $-155.8(2)$ \\
\hline C5 C6 C7 C11 & $-42.6(3)$ & C17C18C19F2 & $56.8(2)$ \\
\hline C5 C6 C20N1 & $-0.1(2)$ & C17C18C19F3 & $-64.0(2)$ \\
\hline C5C6 C20C17 & $178.1(2)$ & C17C18C19F4 & $176.52(17)$ \\
\hline C6 C5 C14N1 & $-1.3(2)$ & $\mathrm{C} 18 \mathrm{C} 17 \mathrm{C} 20 \mathrm{~N} 1$ & $142.98(16)$ \\
\hline C6C5 C14C13 & $176.96(17)$ & C18C17C20C6 & $-35.2(3)$ \\
\hline $\mathrm{C} 6 \mathrm{C} 7 \mathrm{C} 8 \mathrm{C} 9$ & $-176.54(18)$ & $\mathrm{C} 20 \mathrm{~N} 1 \mathrm{C} 14 \mathrm{C} 5$ & $1.3(2)$ \\
\hline C6C7 C11 C12 & $175.04(18)$ & $\mathrm{C} 20 \mathrm{~N} 1 \mathrm{C} 14 \mathrm{C} 13$ & $-176.81(18)$ \\
\hline C7 C6 C20N1 & $177.65(17)$ & $\mathrm{C} 20 \mathrm{~N} 1 \mathrm{C} 15 \mathrm{C} 16$ & $-17.46(19)$ \\
\hline C7 C6 C20C17 & $-4.2(4)$ & C20C6 C7 C8 & $-43.8(3)$ \\
\hline C7 C8 C9 C10 & $1.6(3)$ & C20C6 C7 C11 & $140.1(2)$ \\
\hline C 7 C 11 C 12 C 10 & $1.4(3)$ & C20C17C18C19 & $179.96(17)$ \\
\hline $\mathrm{C} 8 \mathrm{C} 7 \mathrm{C} 11 \mathrm{C} 12$ & $-1.2(3)$ & C21 C17C18C19 & $57.0(2)$ \\
\hline C8 C9 C10F1 & $178.29(17)$ & $\mathrm{C} 21 \mathrm{C} 17 \mathrm{C} 20 \mathrm{~N} 1$ & $-92.61(18)$ \\
\hline C8C9 C10C12 & $-1.4(3)$ & C21 C17C20C6 & $89.2(3)$ \\
\hline C9 C10C12C11 & $-0.1(3)$ & & \\
\hline
\end{tabular}

Table S28 Hydrogen Atom Coordinates $\left(\AA \times 10^{4}\right)$ and Isotropic Displacement Parameters $\left(\AA^{2} \times 10^{3}\right)$ for $3 t$.

$\begin{array}{lrrrr}\text { Atom } & \boldsymbol{x} & \boldsymbol{y} & \boldsymbol{z} & \mathbf{U}(\mathbf{e q}) \\ \text { H1A } & 237.66 & 3234.95 & -708.3 & 42 \\ \text { H1B } & -774.11 & 3985.87 & -1361.76 & 42 \\ \text { H1C } & -296.63 & 3918.57 & 96.23 & 42 \\ \text { H3 } & 3176.21 & 5346.13 & -92.3 & 21 \\ \text { H4 } & 5137.08 & 5419.33 & 1588.56 & 19 \\ \text { H8 } & 6759.76 & 4871.99 & 5863.1 & 19 \\ \text { H9 } & 8593.26 & 5833.02 & 6616.96 & 22 \\ \text { H11 } & 7475.97 & 4971.5 & 2532.84 & 19 \\ \text { H12 } & 9238.31 & 5976.96 & 3258.26 & 22\end{array}$




$\begin{array}{llrrr}\text { H13 } & 1787.56 & 3224.11 & 1235.71 & 19 \\ \text { H15A } & 3347.82 & 2004.31 & 3027.97 & 20 \\ \text { H15B } & 2871.05 & 2487.37 & 4097.58 & 20 \\ \text { H16A } & 5605.87 & 1784.84 & 4250.55 & 22 \\ \text { H16B } & 4796.77 & 1768.81 & 5298.5 & 22 \\ \text { H18A } & 7696.83 & 2667.32 & 4911.38 & 22 \\ \text { H18B } & 7767.46 & 3454.62 & 5796.18 & 22 \\ \text { H21A } & 5652.67 & 3770.84 & 6598.46 & 30 \\ \text { H21B } & 5262.78 & 2821.48 & 6854.39 & 30 \\ \text { H21C } & 4151.01 & 3389.17 & 5888.78 & 30\end{array}$

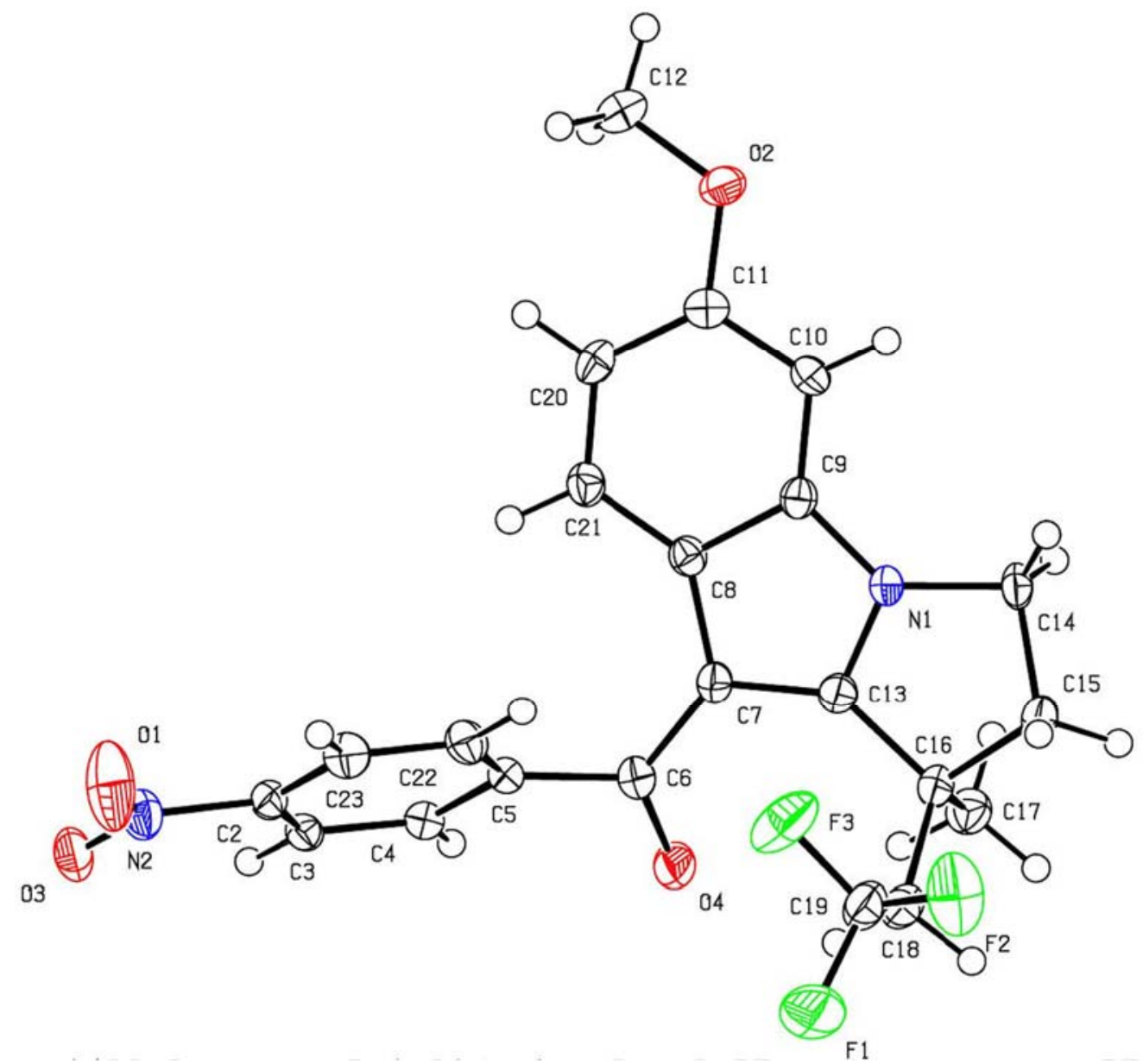

Table S29 Crystal data and structure refinement for 10a (CCDC 2043672).

Identification code

Empirical formula

Formula weight

Temperature/K

Crystal system

Space group

$\mathrm{a} / \AA$ cxy1428_0m (10a)

$\mathrm{C}_{22} \mathrm{H}_{19} \mathrm{~F}_{3} \mathrm{~N}_{2} \mathrm{O}_{4}$

432.39

100

monoclinic

$\mathrm{P} 21 / \mathrm{c}$

9.4606(4) 


$$
\begin{aligned}
& \mathrm{b} / \AA ̊ \\
& \mathrm{c} / \AA \quad 13.2571(6) \\
& \alpha /^{\circ} \quad 90 \\
& \beta /{ }^{\circ} \quad 104.405(2) \\
& \gamma /{ }^{\circ} \quad 90 \\
& \text { Volume } / \AA^{3} \quad \text { 1937.43(16) } \\
& \mathrm{Z} \quad 4 \\
& \rho_{\text {calc }} / \mathrm{cm}^{3} \quad 1.482 \\
& \mu / \mathrm{mm}^{-1} \quad 0.122 \\
& \mathrm{~F}(000) \quad 896.0 \\
& \text { Crystal size } / \mathrm{mm}^{3} \quad 0.34 \times 0.29 \times 0.08 \\
& \text { Radiation } \quad \operatorname{MoK} \alpha(\lambda=0.71073) \\
& 2 \Theta \text { range for data collection/ }{ }^{\circ} \quad 4.446 \text { to } 56.702 \\
& \text { Index ranges } \\
& \text { Reflections collected } \\
& \text { Independent reflections } \\
& \text { Data/restraints/parameters } \\
& \text { Goodness-of-fit on } \mathrm{F}^{2} \\
& -12 \leq \mathrm{h} \leq 12,-21 \leq \mathrm{k} \leq 21,-14 \leq 1 \leq 17 \\
& 28293 \\
& 4816\left[\mathrm{R}_{\text {int }}=0.0602, \mathrm{R}_{\text {sigma }}=0.0471\right] \\
& 4816 / 0 / 283 \\
& 1.032 \\
& \text { Final } \mathrm{R} \text { indexes }[\mathrm{I}>=2 \sigma(\mathrm{I})] \quad \mathrm{R}_{1}=0.0538, \mathrm{wR}_{2}=0.1241 \\
& \text { Final } \mathrm{R} \text { indexes [all data] } \quad \mathrm{R}_{1}=0.0900, \mathrm{wR}_{2}=0.1418 \\
& \text { Largest diff. peak/hole / e } \AA^{-3} \quad 0.58 /-0.40
\end{aligned}
$$

Table S30 Fractional Atomic Coordinates $\left(\times 10^{4}\right)$ and Equivalent Isotropic Displacement Parameters $\left(\AA^{2} \times 10^{3}\right)$ for $10 a$. $U_{\text {eq }}$ is defined as $1 / 3$ of of the trace of the orthogonalised $U_{I J}$ tensor.

\begin{tabular}{lrrrr} 
Atom & \multicolumn{1}{l}{$\boldsymbol{x}$} & $\boldsymbol{Z}$ & $\mathbf{U}(\mathbf{e q})$ \\
F1 & $370.7(16)$ & $3447.4(11)$ & $4417.5(11)$ & $50.6(4)$ \\
F2 & $-39.6(18)$ & $4198.9(11)$ & $5642.1(13)$ & $54.8(5)$ \\
F3 & $2078.1(15)$ & $4194.3(10)$ & $5343.2(12)$ & $50.4(4)$ \\
O1 & $7693(2)$ & $4033.9(12)$ & $2188.8(16)$ & $55.8(6)$ \\
O2 & $5463.4(15)$ & $2745.9(9)$ & $9293.3(12)$ & $26.0(3)$ \\
O3 & $8380.7(18)$ & $2156.9(10)$ & $2312.8(12)$ & $34.7(4)$ \\
O4 & $4298.7(18)$ & $4083.8(10)$ & $5815.6(13)$ & $33.8(4)$ \\
N1 & $4174.1(17)$ & $3344.2(12)$ & $7960.0(13)$ & $20.0(4)$ \\
N2 & $7770(2)$ & $3227.0(13)$ & $2595.4(14)$ & $29.1(4)$ \\
C2 & $7096(2)$ & $2445.0(12)$ & $3471.4(15)$ & $21.6(4)$ \\
C3 & $7186(2)$ & $2331.7(13)$ & $4743.8(15)$ & $20.2(4)$ \\
C4 & $6530(2)$ & $2998.5(12)$ & $5090.1(15)$ & $20.7(4)$ \\
C5 & $5821(2)$ & $2824.9(13)$ & $5887.7(16)$ & $23.5(4)$ \\
C6 & $4973(2)$ & $3441.6(13)$ & $6684.8(16)$ & $21.2(4)$ \\
C7 & $4935(2)$ & $4057.9(12)$ & $7178.4(15)$ & $19.2(4)$ \\
C8 & $6025(2)$ & $4430.3(12)$ & $7993.6(15)$ & $19.2(4)$ \\
C9 & $5522(2)$ & $5010.2(12)$ & $8685.5(15)$ & $19.8(4)$ \\
C10 & $6312(2)$ & $5218.6(12)$ & $8567.4(15)$ & $21.5(4)$ \\
C11 & $7686(2)$ & &
\end{tabular}




$\begin{array}{lrrrr}\mathrm{C} 12 & 9947(2) & 5919.0(14) & 9280.6(18) & 29.6(5) \\ \mathrm{C} 13 & 3833(2) & 3492.9(12) & 7203.7(15) & 20.8(4) \\ \mathrm{C} 14 & 3137(2) & 4134.9(13) & 8616.2(16) & 22.6(4) \\ \mathrm{C} 15 & 3749.6(14) & 7879.9(17) & 27.6(5) \\ \mathrm{C} 16 & 1781(2) & 3103.8(13) & 7203.6(15) & 22.2(4) \\ \mathrm{C} 17 & 2376(2) & 2250.7(14) & 7761.6(17) & 27.3(5) \\ \mathrm{C} 18 & 2631(2) & 2966.4(14) & 6115.0(17) & 26.7(5) \\ \mathrm{C} 19 & 1369(2) & 3705.5(16) & 5407.8(17) & 31.0(5) \\ \mathrm{C} 20 & 968(2) & 4870.6(13) & 7761.0(16) & 23.7(4) \\ \mathrm{C} 21 & 8213(2) & 4297.7(13) & 7072.4(16) & 22.5(4) \\ \mathrm{C} 22 & 7398(2) & 3779.9(13) & 4617.7(16) & 24.9(4) \\ \mathrm{C} 23 & 5769(2) & 3901.4(13) & 3790.6(16) & 25.4(5)\end{array}$

Table S31 Anisotropic Displacement Parameters $\left(\AA^{2} \times 10^{3}\right)$ for 10a. The Anisotropic displacement factor exponent takes the form: $-2 \pi^{2}\left[h^{2} a * 2 U_{11}+2 h k a * b * U_{12}+\ldots\right]$.

\begin{tabular}{lrrrrrr} 
Atom & \multicolumn{1}{c}{$\mathbf{U}_{\mathbf{1 1}}$} & \multicolumn{1}{c}{$\mathbf{U}_{\mathbf{2 2}}$} & \multicolumn{1}{c}{$\mathbf{U}_{\mathbf{3 3}}$} & \multicolumn{1}{c}{$\mathbf{U}_{\mathbf{2 3}}$} & \multicolumn{1}{c}{$\mathbf{U}_{\mathbf{1 3}}$} & \multicolumn{1}{c}{$\mathbf{U}_{\mathbf{1 2}}$} \\
F1 & $41.2(8)$ & $72.2(11)$ & $30.4(8)$ & $4.9(7)$ & $-6.0(6)$ & $-6.1(8)$ \\
F2 & $56.3(10)$ & $61.9(11)$ & $50.8(10)$ & $20.3(8)$ & $21.8(8)$ & $26.6(8)$ \\
F3 & $31.5(8)$ & $62.9(10)$ & $49.7(9)$ & $27.9(8)$ & $-3.0(6)$ & $-14.8(7)$ \\
O1 & $81.2(15)$ & $46.0(11)$ & $56.0(12)$ & $28.6(10)$ & $46.8(11)$ & $19.3(10)$ \\
O2 & $20.8(7)$ & $26.5(8)$ & $29.9(8)$ & $-4.8(6)$ & $4.6(6)$ & $-4.1(6)$ \\
O3 & $42.1(9)$ & $36.4(9)$ & $32.7(9)$ & $-6.6(7)$ & $22.9(7)$ & $-5.3(7)$ \\
O4 & $39.2(9)$ & $30.2(9)$ & $38.9(9)$ & $-11.3(7)$ & $22.5(7)$ & $-13.6(7)$ \\
N1 & $17.9(8)$ & $23.5(9)$ & $20.3(8)$ & $-2.9(7)$ & $8.1(6)$ & $0.0(7)$ \\
N2 & $31.1(10)$ & $34.6(11)$ & $24.3(9)$ & $2.1(8)$ & $12.1(8)$ & $-1.2(8)$ \\
C2 & $19.5(10)$ & $28.5(11)$ & $16.8(9)$ & $-0.4(8)$ & $4.6(7)$ & $-2.3(8)$ \\
C3 & $17.9(9)$ & $23.3(10)$ & $19.3(10)$ & $-4.6(8)$ & $4.5(7)$ & $0.6(8)$ \\
C4 & $20.3(10)$ & $22.2(10)$ & $19.5(10)$ & $-2.0(8)$ & $4.8(8)$ & $-1.7(8)$ \\
C5 & $18.5(9)$ & $24.0(10)$ & $18.1(10)$ & $-4.1(8)$ & $5.7(7)$ & $-2.8(8)$ \\
C6 & $22.9(10)$ & $26.1(11)$ & $23.3(10)$ & $-3.4(8)$ & $9.3(8)$ & $-0.7(8)$ \\
C7 & $19.7(9)$ & $23.4(10)$ & $22.7(10)$ & $-2.1(8)$ & $9.2(8)$ & $-0.7(8)$ \\
C8 & $19.4(9)$ & $19.2(9)$ & $19.3(9)$ & $-0.3(8)$ & $5.4(7)$ & $2.4(8)$ \\
C9 & $18.1(9)$ & $19.7(9)$ & $20.4(10)$ & $1.8(8)$ & $6.0(7)$ & $2.3(7)$ \\
C10 & $22.8(10)$ & $18.6(10)$ & $18.1(9)$ & $-0.6(7)$ & $5.3(8)$ & $2.2(8)$ \\
C11 & $22.0(10)$ & $18.6(9)$ & $22.2(10)$ & $0.8(8)$ & $2.3(8)$ & $0.7(8)$ \\
C12 & $22.0(10)$ & $28.3(11)$ & $36.1(12)$ & $0.0(9)$ & $2.9(9)$ & $-4.9(9)$ \\
C13 & $19.6(9)$ & $22.6(10)$ & $20.5(10)$ & $0.8(8)$ & $5.6(7)$ & $0.2(8)$ \\
C14 & $22.5(10)$ & $26.4(11)$ & $23.0(10)$ & $-1.2(8)$ & $13.7(8)$ & $1.3(8)$ \\
C15 & $22.7(10)$ & $32.4(12)$ & $31.4(12)$ & $-3.4(9)$ & $13.9(9)$ & $-1.6(9)$ \\
C16 & $19.4(10)$ & $25.9(10)$ & $22.4(10)$ & $-0.4(8)$ & $7.5(8)$ & $-1.2(8)$ \\
C17 & $27.7(11)$ & $26.6(11)$ & $27.9(11)$ & $1.5(9)$ & $7.5(9)$ & $-2.3(9)$ \\
C18 & $20.7(10)$ & $31.5(12)$ & $28.3(11)$ & $0.8(9)$ & $6.9(8)$ & $-4.5(9)$ \\
C19 & $20.9(10)$ & $43.8(14)$ & $28.1(12)$ & $3.7(10)$ & $6.0(9)$ & $-4.6(10)$ \\
& & & & & &
\end{tabular}




$\begin{array}{lrllrrr}\mathrm{C} 20 & 17.1(9) & 23.8(10) & 31.3(11) & 2.3(9) & 8.5(8) & 0.6(8) \\ \mathrm{C} 21 & 22.3(10) & 23.0(10) & 24.1(10) & -1.1(8) & 9.2(8) & 2.0(8) \\ \mathrm{C} 22 & 25.4(10) & 22.9(10) & 27.5(11) & -4.8(9) & 8.7(8) & 2.9(8) \\ \mathrm{C} 23 & 27.9(11) & 23.2(11) & 24.4(11) & 4.2(8) & 5.0(8) & 0.7(8)\end{array}$

Table S32 Bond Lengths for 10a.

Atom Atom Length $/ \AA$ Atom Atom Length $/ \AA$

$\begin{array}{llllll}\mathrm{F} 1 & \mathrm{C} 19 & 1.358(3) & \mathrm{C} 5 & \mathrm{C} 22 & 1.390(3) \\ \mathrm{F} 2 & \mathrm{C} 19 & 1.331(3) & \mathrm{C} 6 & \mathrm{C} 7 & 1.451(3) \\ \mathrm{F} 3 & \mathrm{C} 19 & 1.327(3) & \mathrm{C} 7 & \mathrm{C} 8 & 1.457(3) \\ \mathrm{O} 1 & \mathrm{~N} 2 & 1.219(2) & \mathrm{C} 7 & \mathrm{C} 13 & 1.387(3) \\ \mathrm{O} 2 & \mathrm{C} 11 & 1.377(2) & \mathrm{C} 8 & \mathrm{C} 9 & 1.415(3) \\ \mathrm{O} 2 & \mathrm{C} 12 & 1.427(2) & \mathrm{C} 8 & \mathrm{C} 21 & 1.395(3) \\ \mathrm{O} 3 & \mathrm{~N} 2 & 1.222(2) & \mathrm{C} 9 & \mathrm{C} 10 & 1.384(3) \\ \mathrm{O} 4 & \mathrm{C} 6 & 1.233(3) & \mathrm{C} 10 & \mathrm{C} 11 & 1.387(3) \\ \mathrm{N} 1 & \mathrm{C} 9 & 1.380(2) & \mathrm{C} 11 & \mathrm{C} 20 & 1.402(3) \\ \mathrm{N} 1 & \mathrm{C} 13 & 1.355(3) & \mathrm{C} 13 & \mathrm{C} 16 & 1.512(3) \\ \mathrm{N} 1 & \mathrm{C} 14 & 1.467(2) & \mathrm{C} 14 & \mathrm{C} 15 & 1.534(3) \\ \mathrm{N} 2 & \mathrm{C} 2 & 1.469(3) & \mathrm{C} 15 & \mathrm{C} 16 & 1.560(3) \\ \mathrm{C} 2 & \mathrm{C} 3 & 1.383(3) & \mathrm{C} 16 & \mathrm{C} 17 & 1.539(3) \\ \mathrm{C} 2 & \mathrm{C} 23 & 1.382(3) & \mathrm{C} 16 & \mathrm{C} 18 & 1.534(3) \\ \mathrm{C} 3 & \mathrm{C} 4 & 1.379(3) & \mathrm{C} 18 & \mathrm{C} 19 & 1.495(3) \\ \mathrm{C} 4 & \mathrm{C} 5 & 1.395(3) & \mathrm{C} 20 & \mathrm{C} 21 & 1.383(3) \\ \mathrm{C} 5 & \mathrm{C} 6 & 1.503(3) & \mathrm{C} 22 & \mathrm{C} 23 & 1.385(3)\end{array}$

Table S33 Bond Angles for 10a.

\begin{tabular}{llllllll} 
Atom Atom Atom & \multicolumn{1}{c}{ Angle $^{\circ}$} & \multicolumn{3}{c}{ Atom Atom Atom } & Angle $/^{\circ}$ \\
C11 & O2 & C12 & $116.92(16)$ & C9 & C10 & C11 & $116.88(18)$ \\
C9 & N1 & C14 & $135.04(17)$ & O2 & C11 & C10 & $115.32(18)$ \\
C13 & N1 & C9 & $110.50(16)$ & O2 & C11 & C20 & $123.88(18)$ \\
C13 & N1 & C14 & $113.97(16)$ & C10 & C11 & C20 & $120.81(18)$ \\
O1 & N2 & O3 & $123.38(19)$ & N1 & C13 & C7 & $109.77(17)$ \\
O1 & N2 & C2 & $118.30(19)$ & N1 & C13 & C16 & $109.55(17)$ \\
O3 & N2 & C2 & $118.31(18)$ & C7 & C13 & C16 & $140.67(19)$ \\
C3 & C2 & N2 & $118.31(18)$ & N1 & C14 & C15 & $100.21(15)$ \\
C23 & C2 & N2 & $118.51(19)$ & C14 & C15 & C16 & $105.44(16)$ \\
C23 & C2 & C3 & $123.18(19)$ & C13 & C16 & C15 & $100.86(16)$ \\
C4 & C3 & C2 & $118.27(18)$ & C13 & C16 & C17 & $109.21(16)$ \\
C3 & C4 & C5 & $120.19(19)$ & C13 & C16 & C18 & $114.31(17)$ \\
C4 & C5 & C6 & $118.40(18)$ & C17 & C16 & C15 & $110.08(17)$ \\
C22 & C5 & C4 & $120.05(18)$ & C18 & C16 & C15 & $113.99(17)$ \\
C22 & C5 & C6 & $121.15(18)$ & C18 & C16 & C17 & $108.19(17)$
\end{tabular}




$\begin{array}{lllllllr}\text { O4 } & \text { C6 } & \text { C5 } & 117.45(18) & \text { C19 } & \text { C18 } & \text { C16 } & 118.58(18) \\ \text { O4 } & \text { C6 } & \text { C7 } & 122.38(19) & \text { F1 } & \text { C19 } & \text { C18 } & 110.3(2) \\ \text { C7 } & \text { C6 } & \text { C5 } & 120.14(18) & \text { F2 } & \text { C19 } & \text { F1 } & 104.76(18) \\ \text { C6 } & \text { C7 } & \text { C8 } & 129.89(18) & \text { F2 } & \text { C19 } & \text { C18 } & 113.85(19) \\ \text { C13 } & \text { C7 } & \text { C6 } & 124.04(18) & \text { F3 } & \text { C19 } & \text { F1 } & 105.16(18) \\ \text { C13 } & \text { C7 } & \text { C8 } & 105.82(17) & \text { F3 } & \text { C19 } & \text { F2 } & 107.0(2) \\ \text { C9 } & \text { C8 } & \text { C7 } & 106.96(17) & \text { F3 } & \text { C19 } & \text { C18 } & 114.97(18) \\ \text { C21 } & \text { C8 } & \text { C7 } & 135.47(18) & \text { C21 } & \text { C20 } & \text { C11 } & 121.30(18) \\ \text { C21 } & \text { C8 } & \text { C9 } & 117.40(18) & \text { C20 } & \text { C21 } & \text { C8 } & 119.66(19) \\ \text { N1 } & \text { C9 } & \text { C8 } & 106.88(17) & \text { C23 } & \text { C22 } & \text { C5 } & 120.56(19) \\ \text { N1 } & \text { C9 } & \text { C10 } & 129.17(18) & \text { C2 } & \text { C23 } & \text { C22 } & 117.74(19) \\ \text { C10 } & \text { C9 } & \text { C8 } & 123.92(18) & & & & \end{array}$

Table S34 Torsion Angles for 10a.

\begin{tabular}{|c|c|c|c|}
\hline $\begin{array}{llll}\mathbf{A} & \mathbf{B} & \mathbf{C} & \mathbf{D}\end{array}$ & Angle $/^{\circ}$ & D & Angle $/^{\circ}$ \\
\hline $\mathrm{O} 1 \mathrm{~N} 2 \mathrm{C} 2 \mathrm{C} 3$ & $-179.9(2)$ & C8 C7 C13C16 & $-179.9(2)$ \\
\hline $\mathrm{O} 1 \mathrm{~N} 2 \mathrm{C} 2 \mathrm{C} 23$ & $0.4(3)$ & C8 C9 C10C11 & $-1.1(3)$ \\
\hline $\mathrm{O} 2 \mathrm{C} 11 \mathrm{C} 20 \mathrm{C} 21$ & $178.09(18)$ & C9 N1 C13C7 & $-1.6(2)$ \\
\hline $\mathrm{O} 3 \mathrm{~N} 2 \mathrm{C} 2 \mathrm{C} 3$ & $0.0(3)$ & C9 N1 C13C16 & $178.26(16)$ \\
\hline $\mathrm{O} 3 \mathrm{~N} 2 \mathrm{C} 2 \mathrm{C} 23$ & $-179.68(19)$ & C9 N1 C14C15 & 166.1(2) \\
\hline $\mathrm{O} 4 \mathrm{C} 6 \mathrm{C} 7 \mathrm{C} 8$ & $151.4(2)$ & C9 C8 C21 C20 & $0.8(3)$ \\
\hline O4C6 C7 C13 & $-22.0(3)$ & $\mathrm{C} 9 \mathrm{C} 10 \mathrm{C} 11 \mathrm{O} 2$ & $-177.63(17)$ \\
\hline N1C9 C10C11 & $176.75(19)$ & C9 C10C11 C20 & $1.8(3)$ \\
\hline N1 C13C16C15 & $15.0(2)$ & $\mathrm{C} 10 \mathrm{C} 11 \mathrm{C} 20 \mathrm{C} 21$ & $-1.3(3)$ \\
\hline N1 C13C16C17 & $-100.96(19)$ & $\mathrm{C} 11 \mathrm{C} 20 \mathrm{C} 21 \mathrm{C} 8$ & $-0.1(3)$ \\
\hline N1 C13 C16C18 & $137.70(18)$ & $\mathrm{C} 12 \mathrm{O} 2 \mathrm{C} 11 \mathrm{C} 10$ & $172.14(17)$ \\
\hline N1 C14C15C16 & $30.8(2)$ & $\mathrm{C} 12 \mathrm{O} 2 \mathrm{C} 11 \mathrm{C} 20$ & $-7.3(3)$ \\
\hline $\mathrm{N} 2 \mathrm{C} 2 \mathrm{C} 3 \mathrm{C} 4$ & $-178.88(17)$ & C13N1 C9 C8 & $2.6(2)$ \\
\hline $\mathrm{N} 2 \mathrm{C} 2 \mathrm{C} 23 \mathrm{C} 22$ & $-179.95(18)$ & C13N1 C9 C10 & $-175.5(2)$ \\
\hline $\mathrm{C} 2 \mathrm{C} 3 \mathrm{C} 4 \mathrm{C} 5$ & $-1.0(3)$ & $\mathrm{C} 13 \mathrm{~N} 1 \mathrm{C} 14 \mathrm{C} 15$ & $-22.8(2)$ \\
\hline C3 C2 C23 C22 & $0.4(3)$ & C13C7 C8 C9 & $1.6(2)$ \\
\hline $\mathrm{C} 3 \mathrm{C} 4 \mathrm{C} 5 \mathrm{C} 6$ & $172.98(18)$ & $\mathrm{C} 13 \mathrm{C} 7 \mathrm{C} 8 \mathrm{C} 21$ & $176.5(2)$ \\
\hline $\mathrm{C} 3 \mathrm{C} 4 \mathrm{C} 5 \mathrm{C} 22$ & $0.1(3)$ & C13C16C18C19 & $-57.4(3)$ \\
\hline $\mathrm{C} 4 \mathrm{C} 5 \quad \mathrm{C} 6 \quad \mathrm{O} 4$ & $-37.3(3)$ & C14N1 C9 C8 & $173.9(2)$ \\
\hline $\mathrm{C} 4 \mathrm{C} 5 \mathrm{C} 6 \mathrm{C} 7$ & $144.49(19)$ & C14N1 C9 C10 & $-4.2(4)$ \\
\hline $\mathrm{C} 4 \mathrm{C} 5 \mathrm{C} 22 \mathrm{C} 23$ & $1.1(3)$ & C14N1 C13C7 & $-174.88(17)$ \\
\hline $\mathrm{C} 5 \mathrm{C} 6 \mathrm{C} 7 \mathrm{C} 8$ & $-30.4(3)$ & $\mathrm{C} 14 \mathrm{~N} 1 \mathrm{C} 13 \mathrm{C} 16$ & $5.0(2)$ \\
\hline C5 C6 C7 C13 & $156.1(2)$ & $\mathrm{C} 14 \mathrm{C} 15 \mathrm{C} 16 \mathrm{C} 13$ & $-28.4(2)$ \\
\hline $\mathrm{C} 5 \mathrm{C} 22 \mathrm{C} 23 \mathrm{C} 2$ & $-1.3(3)$ & $\mathrm{C} 14 \mathrm{C} 15 \mathrm{C} 16 \mathrm{C} 17$ & $86.9(2)$ \\
\hline C6C5 C22C23 & $-171.58(18)$ & $\mathrm{C} 14 \mathrm{C} 15 \mathrm{C} 16 \mathrm{C} 18$ & $-151.35(17)$ \\
\hline $\mathrm{C} 6 \mathrm{C} 7 \mathrm{C} 8 \mathrm{C} 9$ & $-172.8(2)$ & $\mathrm{C} 15 \mathrm{C} 16 \mathrm{C} 18 \mathrm{C} 19$ & $57.9(2)$ \\
\hline C6 C7 C8 C21 & $2.1(4)$ & C16C18C19F1 & $165.83(18)$ \\
\hline C6 C7 C13N1 & $174.75(18)$ & C16C18C19F2 & $-76.7(2)$ \\
\hline
\end{tabular}




\begin{tabular}{|c|c|c|c|}
\hline C6C7 C13C16 & $-5.1(4)$ & C16C18C19F3 & $47.2(3)$ \\
\hline C7 C8 C9 N1 & $-2.5(2)$ & $\mathrm{C} 17 \mathrm{C} 16 \mathrm{C} 18 \mathrm{C} 19$ & $-179.28(18)$ \\
\hline C7 C8 C9 C10 & $175.68(18)$ & C21 C8 C9 N1 & $-178.48(17)$ \\
\hline C7 C8 C $21 \mathrm{C} 20$ & $-173.6(2)$ & C21 C8 C9 C10 & $-0.3(3)$ \\
\hline C 7 C 13 C $16 C 15$ & $-165.2(3)$ & $\mathrm{C} 22 \mathrm{C} 5 \mathrm{C} 6 \mathrm{O} 4$ & $135.5(2)$ \\
\hline C7 C13C16C17 & $78.9(3)$ & $\mathrm{C} 22 \mathrm{C} 5 \mathrm{C} 6 \mathrm{C} 7$ & $-42.7(3)$ \\
\hline C7 C13C16C18 & $-42.5(4)$ & $\mathrm{C} 23 \mathrm{C} 2 \mathrm{C} 3 \mathrm{C} 4$ & $0.8(3)$ \\
\hline C8 C7 C13N1 & $0.0(2)$ & & \\
\hline
\end{tabular}

Table S35 Hydrogen Atom Coordinates $\left(\AA \times 10^{4}\right)$ and Isotropic Displacement Parameters $\left(\AA^{2} \times 10^{3}\right)$ for $10 a$.

\begin{tabular}{lrrrr} 
Atom & \multicolumn{1}{c}{$\boldsymbol{y}$} & $\boldsymbol{z}$ & $\mathbf{U}(\mathbf{e q})$ \\
H3 & 7685.37 & 1997.54 & 3699.41 & 24 \\
H4 & 6562.29 & 1797.97 & 5066.92 & 25 \\
H10 & 5932.33 & 5254.12 & 9216.66 & 24 \\
H12A & 10465.65 & 5382.56 & 9331.41 & 44 \\
H12B & 10413.63 & 6275.27 & 9872.19 & 44 \\
H12C & 9982.04 & 6199.47 & 8629.42 & 44 \\
H14A & 2964.01 & 4722.09 & 8795.95 & 27 \\
H14B & 3466.96 & 3802.47 & 9262.71 & 33 \\
H15A & 1194.22 & 4187.28 & 7435.43 & 33 \\
H15B & 1163.96 & 3466.86 & 8280.14 & 41 \\
H17A & 3106.89 & 1868.34 & 7369.99 & 41 \\
H17B & 1692.27 & 2013.68 & 7804.85 & 31 \\
H17C & 3256.35 & 2326.92 & 8465.25 & 32 \\
H18A & 1838.34 & 2546.93 & 5753.53 & 28 \\
H18B & 452.42 & 2713.06 & 6204.08 & 27 \\
H20 & 9148.14 & 5031.46 & 7686.1 & 30 \\
H21 & 7771.97 & 4068.55 & 6529.88 & 31 \\
H22 & 5299.45 & 4234.43 & 4864.32 & 3453.89 \\
H23 & 6350.8 & 4430.15 & &
\end{tabular}




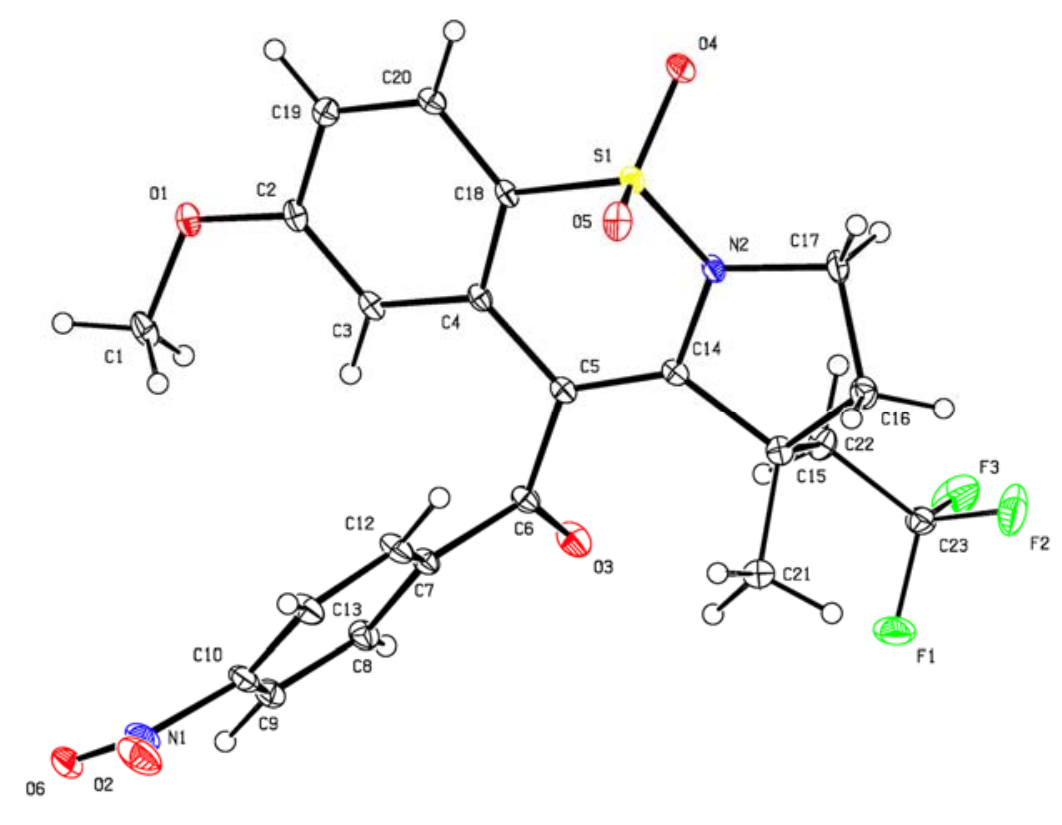

Table S36 Crystal data and structure refinement for 10b (CCDC 2043674).

Identification code

Empirical formula

Formula weight

Temperature/K

Crystal system

Space group

$\mathrm{a} / \AA$

$\mathrm{b} / \AA$

$\mathrm{c} / \AA$

$\alpha /{ }^{\circ}$

$\beta /{ }^{\circ}$

$\gamma /{ }^{\circ}$

Volume/ $\AA^{3}$

Z

$\rho_{\text {calc } g / \mathrm{cm}^{3}}$

$\mu / \mathrm{mm}^{-1}$

$\mathrm{F}(000)$

Crystal size $/ \mathrm{mm}^{3}$

Radiation

$2 \Theta$ range for data collection ${ }^{\circ}$

Index ranges

Reflections collected

Independent reflections

Data/restraints/parameters

Goodness-of-fit on $\mathrm{F}^{2}$ cxy1463_0m (10b)

$\mathrm{C}_{22} \mathrm{H}_{19} \mathrm{~F}_{3} \mathrm{~N}_{2} \mathrm{O}_{6} \mathrm{~S}$

496.45

100

triclinic

P-1

$7.3756(3)$

$12.0877(6)$

$13.0747(6)$

$108.314(2)$

$105.9730(10)$

$97.148(2)$

$1035.10(8)$

2

1.593

0.229

512.0

$0.38 \times 0.36 \times 0.15$

$\operatorname{MoK} \alpha(\lambda=0.71073)$

5.768 to 56.88

$-9 \leq \mathrm{h} \leq 9,-16 \leq \mathrm{k} \leq 16,-17 \leq 1 \leq 17$

15506

$5200\left[\mathrm{R}_{\text {int }}=0.0306, \mathrm{R}_{\text {sigma }}=0.0331\right]$

$5200 / 5 / 327$

1.058 
Final $\mathrm{R}$ indexes $[\mathrm{I}>=2 \sigma(\mathrm{I})] \quad \mathrm{R}_{1}=0.0486, \mathrm{wR}_{2}=0.1202$

Final $\mathrm{R}$ indexes [all data] $\quad \mathrm{R}_{1}=0.0585, \mathrm{wR}_{2}=0.1262$

Largest diff. peak/hole / e $\AA^{-3} \quad 0.66 /-0.49$

Table S37 Fractional Atomic Coordinates $\left(\times 10^{4}\right)$ and Equivalent Isotropic

Displacement Parameters $\left(\AA^{2} \times 10^{3}\right)$ for $10 \mathrm{~b}$. $U_{\text {eq }}$ is defined as $1 / 3$ of of the trace of the orthogonalised $\mathrm{U}_{\mathrm{IJ}}$ tensor.

\begin{tabular}{|c|c|c|c|c|}
\hline Atom & $x$ & $y$ & $\mathbf{z}$ & $\mathbf{U}(\mathbf{e q})$ \\
\hline S1 & $6093.8(6)$ & $5431.1(4)$ & $2327.6(4)$ & $15.11(13)$ \\
\hline $\mathrm{F} 1$ & $-2457(2)$ & $984.9(13)$ & $-370.4(17)$ & $44.7(5)$ \\
\hline $\mathrm{F} 2$ & $-2437(2)$ & $2367.0(18)$ & $-1051.5(15)$ & $46.4(5)$ \\
\hline F3 & $-3699(2)$ & $2444.5(16)$ & $249.2(16)$ & $46.0(5)$ \\
\hline $\mathrm{O} 1$ & $8451(2)$ & $4563.8(13)$ & $6588.1(12)$ & $21.0(3)$ \\
\hline $\mathrm{O} 2$ & 9878(3) & $-1108.4(16)$ & $3120.2(19)$ & $40.6(5)$ \\
\hline $\mathrm{O} 3$ & $1767(2)$ & $1608.5(14)$ & $2463.0(14)$ & $26.0(3)$ \\
\hline $\mathrm{O} 4$ & $5980(2)$ & $6649.0(13)$ & $2499.7(13)$ & $23.5(3)$ \\
\hline $\mathrm{O} 5$ & $7356(2)$ & $4976.7(14)$ & $1723.4(12)$ & $22.6(3)$ \\
\hline O6 & $8715(3)$ & $-1245.5(15)$ & $4448.5(16)$ & $37.1(4)$ \\
\hline $\mathrm{N} 2$ & $3875(2)$ & $4588.8(14)$ & $1647.8(14)$ & $15.9(3)$ \\
\hline $\mathrm{C} 1$ & $8025(3)$ & $3374(2)$ & 6589.3(19) & $24.9(4)$ \\
\hline $\mathrm{C} 2$ & $7749(3)$ & $4716.3(18)$ & $5580.5(16)$ & $16.1(4)$ \\
\hline $\mathrm{C} 3$ & $6366(3)$ & $3852.9(17)$ & $4615.5(16)$ & $15.6(4)$ \\
\hline $\mathrm{C} 4$ & $5750(3)$ & $4045.1(16)$ & $3579.9(16)$ & $14.0(3)$ \\
\hline $\mathrm{C} 5$ & 4208(3) & $3163.3(16)$ & $2574.8(16)$ & $14.5(3)$ \\
\hline C6 & $3476(3)$ & $1975.3(17)$ & $2624.1(17)$ & $16.8(4)$ \\
\hline $\mathrm{C} 7$ & 4877(3) & $1220.0(17)$ & $2879.4(17)$ & $17.0(4)$ \\
\hline $\mathrm{C} 8$ & $4406(3)$ & $392.0(18)$ & $3360.5(18)$ & $20.6(4)$ \\
\hline C9 & $5683(3)$ & $-309.3(19)$ & $3624.3(19)$ & $23.9(4)$ \\
\hline $\mathrm{C} 10$ & 7367(3) & $-189.0(18)$ & $3356(2)$ & $23.7(4)$ \\
\hline N1 & $8762(3)$ & $-906.0(17)$ & $3660.5(19)$ & $30.5(4)$ \\
\hline C12 & 6593(3) & $1322.4(18)$ & $2624.3(19)$ & $22.0(4)$ \\
\hline C13 & 7841(3) & $591.9(19)$ & $2839(2)$ & $24.5(4)$ \\
\hline $\mathrm{C} 14$ & $3307(3)$ & $3462.0(16)$ & $1675.2(16)$ & $14.7(4)$ \\
\hline $\mathrm{C} 15$ & 1596(3) & $2740.9(18)$ & $564.6(16)$ & $17.4(4)$ \\
\hline $\mathrm{C} 16$ & 1883(3) & $3440.3(19)$ & $-206.8(18)$ & $21.8(4)$ \\
\hline C17 & 2703(3) & 4734.6(19) & $602.1(17)$ & $20.5(4)$ \\
\hline $\mathrm{C} 18$ & $6577(3)$ & $5161.1(16)$ & $3588.7(16)$ & $14.0(3)$ \\
\hline C19 & $8501(3)$ & $5830.5(17)$ & $5569.1(16)$ & $17.0(4)$ \\
\hline $\mathrm{C} 20$ & 7912(3) & $6052.3(17)$ & $4572.2(16)$ & $15.9(4)$ \\
\hline $\mathrm{C} 21$ & 1664(4) & $1430(2)$ & $-2(2)$ & $21.1(5)$ \\
\hline $\mathrm{C} 22$ & $-291(3)$ & $2861.7(19)$ & $854.9(17)$ & $20.5(4)$ \\
\hline $\mathrm{C} 23$ & $-2198(3)$ & $2162(2)$ & $-87(2)$ & $21.7(4)$ \\
\hline $\mathrm{F} 1 \mathrm{~A}$ & $1200(30)$ & $-127(14)$ & $-1260(20)$ & $44.7(5)$ \\
\hline $\mathrm{C} 23 \mathrm{~A}$ & $1140(30)$ & $1033(17)$ & $-987(18)$ & $21.7(4)$ \\
\hline F2A & $2140(30)$ & $1450(20)$ & $-1551(17)$ & $46.4(5)$ \\
\hline
\end{tabular}




$\begin{array}{llrrr}\text { F3A } & -740(20) & 1070(19) & -1371(19) & 46.0(5) \\ \text { C21A } & 2120(60) & 1520(20) & 290(20) & 21.1(5)\end{array}$

Table S38 Anisotropic Displacement Parameters $\left(\AA^{2} \times 10^{3}\right)$ for $10 b$. The Anisotropic displacement factor exponent takes the form: $-2 \pi^{2}\left[h^{2} a * 2 U_{11}+2 h k a * b * U_{12}+\ldots\right]$.

\begin{tabular}{|c|c|c|c|c|c|c|}
\hline Atom & $\mathbf{U}_{11}$ & $\mathbf{U}_{22}$ & $\mathbf{U}_{33}$ & $\mathbf{U}_{23}$ & $\mathbf{U}_{13}$ & $\mathbf{U}_{12}$ \\
\hline $\mathrm{S} 1$ & $14.2(2)$ & $16.5(2)$ & $18.0(2)$ & $10.77(17)$ & $5.48(17)$ & $3.14(16)$ \\
\hline F1 & $25.2(8)$ & 17.8(7) & $70.9(12)$ & $9.6(7)$ & $-4.9(8)$ & $0.1(6)$ \\
\hline $\mathrm{F} 2$ & $27.4(8)$ & $64.3(12)$ & $40.6(9)$ & $32.0(9)$ & $-7.5(7)$ & $-4.9(8)$ \\
\hline F3 & $14.7(7)$ & $50.5(11)$ & $54.7(11)$ & $0.6(8)$ & $7.5(7)$ & $3.1(7)$ \\
\hline $\mathrm{O} 1$ & $23.2(7)$ & $25.5(7)$ & $18.5(7)$ & $13.4(6)$ & $6.2(6)$ & $7.7(6)$ \\
\hline $\mathrm{O} 2$ & $29.5(9)$ & $30.1(9)$ & $70.9(14)$ & 25.3(9) & 18.4(9) & $16.0(7)$ \\
\hline $\mathrm{O} 3$ & $18.0(7)$ & 27.3(8) & 41.3(9) & $20.6(7)$ & $13.2(7)$ & $5.8(6)$ \\
\hline $\mathrm{O} 4$ & $26.6(8)$ & $18.0(7)$ & $26.5(8)$ & $13.9(6)$ & $4.1(6)$ & $2.5(6)$ \\
\hline $\mathrm{O} 5$ & $16.9(7)$ & $35.2(8)$ & $21.5(7)$ & $14.9(6)$ & $9.5(6)$ & $7.2(6)$ \\
\hline O6 & $34.7(9)$ & 21.1(8) & $46.2(10)$ & $19.0(8)$ & $-5.9(8)$ & $0.4(7)$ \\
\hline $\mathrm{N} 2$ & $13.6(7)$ & $16.9(8)$ & $19.9(8)$ & 11.7(6) & $4.0(6)$ & $3.5(6)$ \\
\hline $\mathrm{C} 1$ & $31.2(11)$ & $27.5(11)$ & $24.7(10)$ & $18.6(9)$ & $10.0(9)$ & $11.5(9)$ \\
\hline $\mathrm{C} 2$ & $15.6(8)$ & 23.2(9) & $17.3(9)$ & $11.7(7)$ & $9.5(7)$ & $10.5(7)$ \\
\hline C3 & $15.7(8)$ & 17.9(9) & $20.8(9)$ & $12.2(7)$ & $9.7(7)$ & $8.0(7)$ \\
\hline $\mathrm{C} 4$ & $12.5(8)$ & 16.6(9) & $18.2(9)$ & $9.7(7)$ & $7.6(7)$ & $6.9(7)$ \\
\hline C5 & $13.3(8)$ & $15.4(8)$ & 19.3(9) & $9.2(7)$ & $8.1(7)$ & $4.9(7)$ \\
\hline C6 & $17.5(9)$ & 16.1(9) & 21.1(9) & $9.8(7)$ & $8.9(7)$ & $4.7(7)$ \\
\hline C7 & $17.8(9)$ & 15.1(9) & 20.9(9) & $9.6(7)$ & 7.1(7) & $4.3(7)$ \\
\hline $\mathrm{C} 8$ & $19.8(9)$ & 19.3(9) & $25.8(10)$ & $12.7(8)$ & $8.3(8)$ & $2.3(7)$ \\
\hline C9 & $25.7(10)$ & $18.2(10)$ & $28.4(11)$ & $14.0(8)$ & $5.1(8)$ & $1.6(8)$ \\
\hline $\mathrm{C} 10$ & $21.3(10)$ & $14.9(9)$ & $34.6(11)$ & $12.1(8)$ & $4.6(9)$ & $5.8(7)$ \\
\hline N1 & $25.0(9)$ & 16.6(9) & $47.2(12)$ & $16.4(8)$ & $3.2(9)$ & $2.8(7)$ \\
\hline C12 & $22.3(10)$ & 18.4(9) & $34.0(11)$ & $15.7(8)$ & $14.2(9)$ & $8.0(8)$ \\
\hline C13 & $22.5(10)$ & $20.2(10)$ & $38.2(12)$ & $15.4(9)$ & 14.3(9) & $8.7(8)$ \\
\hline $\mathrm{C} 14$ & $12.2(8)$ & $15.2(8)$ & 20.4(9) & $8.8(7)$ & $7.9(7)$ & $4.2(6)$ \\
\hline $\mathrm{C} 15$ & $15.8(9)$ & 19.8(9) & $18.7(9)$ & $8.7(7)$ & $7.0(7)$ & $4.1(7)$ \\
\hline $\mathrm{C} 16$ & $21.9(10)$ & $25.0(10)$ & 20.7(9) & $12.7(8)$ & $5.9(8)$ & $4.8(8)$ \\
\hline $\mathrm{C} 17$ & $18.0(9)$ & $25.0(10)$ & $21.0(9)$ & $14.9(8)$ & $2.3(7)$ & $6.1(8)$ \\
\hline C18 & $14.0(8)$ & 16.4(9) & $17.2(8)$ & $10.5(7)$ & $7.4(7)$ & $6.6(7)$ \\
\hline C19 & $14.6(8)$ & $19.5(9)$ & $18.0(9)$ & $6.1(7)$ & $6.9(7)$ & $6.0(7)$ \\
\hline $\mathrm{C} 20$ & $14.5(8)$ & 15.6(9) & $21.5(9)$ & $8.6(7)$ & $9.2(7)$ & $5.8(7)$ \\
\hline $\mathrm{C} 21$ & $22.0(16)$ & $18.3(10)$ & $22.0(13)$ & $5.9(9)$ & 7.9(10) & $3.7(9)$ \\
\hline $\mathrm{C} 22$ & $13.2(9)$ & $25.3(10)$ & 21.2(9) & $7.3(8)$ & $5.2(7)$ & $2.8(7)$ \\
\hline $\mathrm{C} 23$ & $16.4(10)$ & $17.3(10)$ & $27.9(11)$ & $7.3(9)$ & $3.8(9)$ & $3.1(8)$ \\
\hline F1A & $25.2(8)$ & $17.8(7)$ & $70.9(12)$ & $9.6(7)$ & $-4.9(8)$ & $0.1(6)$ \\
\hline $\mathrm{C} 23 \mathrm{~A}$ & $16.4(10)$ & $17.3(10)$ & $27.9(11)$ & $7.3(9)$ & $3.8(9)$ & $3.1(8)$ \\
\hline F2A & $27.4(8)$ & $64.3(12)$ & $40.6(9)$ & $32.0(9)$ & $-7.5(7)$ & $-4.9(8)$ \\
\hline
\end{tabular}




$\begin{array}{lrrrrrr}\text { F3A } & 14.7(7) & 50.5(11) & 54.7(11) & 0.6(8) & 7.5(7) & 3.1(7) \\ \mathrm{C} 21 \mathrm{~A} & 22.0(16) & 18.3(10) & 22.0(13) & 5.9(9) & 7.9(10) & 3.7(9)\end{array}$

Table S39 Bond Lengths for $10 \mathrm{~b}$.

\begin{tabular}{|c|c|c|c|c|c|}
\hline \multicolumn{2}{|c|}{ Atom Atom } & \multirow{2}{*}{$\begin{array}{c}\text { Length/ } \AA \AA \\
1.4345(15)\end{array}$} & \multicolumn{2}{|c|}{ Atom Atom } & \multirow{2}{*}{$\begin{array}{r}\text { Length/ } \AA \\
1.500(3)\end{array}$} \\
\hline $\mathrm{S} 1$ & $\mathrm{O} 4$ & & C6 & $\mathrm{C} 7$ & \\
\hline $\mathrm{S} 1$ & $\mathrm{O} 5$ & $1.4327(15)$ & $\mathrm{C} 7$ & $\mathrm{C} 8$ & $1.396(3)$ \\
\hline $\mathrm{S} 1$ & N2 & $1.6493(16)$ & $\mathrm{C} 7$ & $\mathrm{C} 12$ & $1.395(3)$ \\
\hline $\mathrm{S} 1$ & $\mathrm{C} 18$ & $1.7310(18)$ & $\mathrm{C} 8$ & C9 & $1.389(3)$ \\
\hline $\mathrm{F} 1$ & $\mathrm{C} 23$ & $1.326(3)$ & $\mathrm{C} 9$ & $\mathrm{C} 10$ & $1.383(3)$ \\
\hline $\mathrm{F} 2$ & $\mathrm{C} 23$ & $1.330(3)$ & $\mathrm{C} 10$ & N1 & $1.477(3)$ \\
\hline F3 & $\mathrm{C} 23$ & $1.343(3)$ & $\mathrm{C} 10$ & $\mathrm{C} 13$ & $1.386(3)$ \\
\hline O1 & $\mathrm{C} 1$ & $1.434(3)$ & $\mathrm{C} 12$ & $\mathrm{C} 13$ & $1.386(3)$ \\
\hline $\mathrm{O} 1$ & $\mathrm{C} 2$ & $1.356(2)$ & $\mathrm{C} 14$ & $\mathrm{C} 15$ & $1.544(3)$ \\
\hline $\mathrm{O} 2$ & N1 & $1.224(3)$ & $\mathrm{C} 15$ & $\mathrm{C} 16$ & $1.545(3)$ \\
\hline $\mathrm{O} 3$ & C6 & $1.218(2)$ & C15 & $\mathrm{C} 21$ & $1.537(3)$ \\
\hline O6 & N1 & $1.230(3)$ & $\mathrm{C} 15$ & $\mathrm{C} 22$ & $1.552(3)$ \\
\hline $\mathrm{N} 2$ & $\mathrm{C} 14$ & $1.389(2)$ & $\mathrm{C} 15$ & $\mathrm{C} 21 \mathrm{~A}$ & $1.532(18)$ \\
\hline $\mathrm{N} 2$ & $\mathrm{C} 17$ & $1.479(2)$ & $\mathrm{C} 16$ & $\mathrm{C} 17$ & $1.518(3)$ \\
\hline $\mathrm{C} 2$ & $\mathrm{C} 3$ & $1.386(3)$ & $\mathrm{C} 18$ & $\mathrm{C} 20$ & $1.397(3)$ \\
\hline $\mathrm{C} 2$ & C19 & $1.398(3)$ & C19 & $\mathrm{C} 20$ & $1.377(3)$ \\
\hline $\mathrm{C} 3$ & $\mathrm{C} 4$ & $1.407(2)$ & $\mathrm{C} 22$ & $\mathrm{C} 23$ & $1.519(3)$ \\
\hline $\mathrm{C} 4$ & $\mathrm{C} 5$ & $1.467(3)$ & F1A & $\mathrm{C} 23 \mathrm{~A}$ & $1.344(17)$ \\
\hline $\mathrm{C} 4$ & $\mathrm{C} 18$ & $1.405(3)$ & $\mathrm{C} 23$ & $\mathrm{AF} 2 \mathrm{~A}$ & $1.337(17)$ \\
\hline $\mathrm{C} 5$ & C6 & $1.497(3)$ & $\mathrm{C} 23$ & AF3A & $1.345(17)$ \\
\hline C5 & $\mathrm{C} 14$ & $1.361(3)$ & $\mathrm{C} 23$ & $\mathrm{AC} 21 \mathrm{~A}$ & $1.512(19)$ \\
\hline
\end{tabular}

Table S40 Bond Angles for $10 \mathrm{~b}$.

\begin{tabular}{|c|c|c|c|c|c|c|c|}
\hline \multicolumn{3}{|c|}{ Atom Atom Atom } & \multirow{2}{*}{$\begin{array}{l}\text { Angle }^{\circ} \\
\qquad 107.42(9)\end{array}$} & \multicolumn{3}{|c|}{ Atom Atom Atom } & \multirow{2}{*}{$\begin{array}{l}\text { Angle }^{\circ} \\
\quad 117.83(19)\end{array}$} \\
\hline $\mathrm{O} 4$ & $\mathrm{~S} 1$ & N2 & & $\mathrm{C} 10$ & $\mathrm{C} 13$ & $\mathrm{C} 12$ & \\
\hline $\mathrm{O} 4$ & $\mathrm{~S} 1$ & $\mathrm{C} 18$ & $112.03(9)$ & N2 & $\mathrm{C} 14$ & $\mathrm{C} 15$ & $107.31(15)$ \\
\hline $\mathrm{O} 5$ & $\mathrm{~S} 1$ & $\mathrm{O} 4$ & $116.37(9)$ & C5 & $\mathrm{C} 14$ & $\mathrm{~N} 2$ & $121.44(17)$ \\
\hline $\mathrm{O} 5$ & $\mathrm{~S} 1$ & $\mathrm{~N} 2$ & $109.54(9)$ & $\mathrm{C} 5$ & $\mathrm{C} 14$ & $\mathrm{C} 15$ & $131.24(17)$ \\
\hline $\mathrm{O} 5$ & $\mathrm{~S} 1$ & $\mathrm{C} 18$ & $110.56(9)$ & $\mathrm{C} 14$ & $\mathrm{C} 15$ & $\mathrm{C} 16$ & $101.12(15)$ \\
\hline $\mathrm{N} 2$ & $\mathrm{~S} 1$ & $\mathrm{C} 18$ & $99.50(8)$ & C14 & $\mathrm{C} 15$ & $\mathrm{C} 22$ & $106.88(15)$ \\
\hline $\mathrm{C} 2$ & $\mathrm{O} 1$ & $\mathrm{C} 1$ & $117.55(16)$ & $\mathrm{C} 16$ & $\mathrm{C} 15$ & $\mathrm{C} 22$ & $111.40(16)$ \\
\hline $\mathrm{C} 14$ & $\mathrm{~N} 2$ & $\mathrm{~S} 1$ & $123.23(13)$ & $\mathrm{C} 21$ & $\mathrm{C} 15$ & $\mathrm{C} 14$ & $114.59(16)$ \\
\hline $\mathrm{C} 14$ & $\mathrm{~N} 2$ & $\mathrm{C} 17$ & $112.94(15)$ & $\mathrm{C} 21$ & $\mathrm{C} 15$ & $\mathrm{C} 16$ & $109.51(18)$ \\
\hline $\mathrm{C} 17$ & $\mathrm{~N} 2$ & $\mathrm{~S} 1$ & $118.43(13)$ & $\mathrm{C} 21$ & $\mathrm{C} 15$ & $\mathrm{C} 22$ & $112.77(18)$ \\
\hline $\mathrm{O} 1$ & $\mathrm{C} 2$ & $\mathrm{C} 3$ & $123.80(17)$ & $\mathrm{C} 21 \mathrm{~A}$ & $\mathrm{C} 15$ & $\mathrm{C} 14$ & $99.4(11)$ \\
\hline $\mathrm{O} 1$ & $\mathrm{C} 2$ & $\mathrm{C} 19$ & $115.38(17)$ & $\mathrm{C} 21 \mathrm{~A}$ & $\mathrm{C} 15$ & $\mathrm{C} 16$ & $116.6(15)$ \\
\hline C3 & $\mathrm{C} 2$ & C19 & $120.81(17)$ & $\mathrm{C} 21 \mathrm{~A}$ & $\mathrm{C} 15$ & $\mathrm{C} 22$ & $118.5(18)$ \\
\hline
\end{tabular}




$\begin{array}{lllllllr}\text { C2 } & \text { C3 } & \text { C4 } & 121.20(17) & \text { C17 } & \text { C16 } & \text { C15 } & 104.84(16) \\ \text { C3 } & \text { C4 } & \text { C5 } & 121.40(17) & \text { N2 } & \text { C17 } & \text { C16 } & 101.44(15) \\ \text { C18 } & \text { C4 } & \text { C3 } & 116.49(17) & \text { C4 } & \text { C18 } & \text { S1 } & 119.97(14) \\ \text { C18 } & \text { C4 } & \text { C5 } & 121.92(16) & \text { C20 } & \text { C18 } & \text { S1 } & 117.38(14) \\ \text { C4 } & \text { C5 } & \text { C6 } & 118.22(16) & \text { C20 } & \text { C18 } & \text { C4 } & 122.46(17) \\ \text { C14 } & \text { C5 } & \text { C4 } & 120.93(17) & \text { C20 } & \text { C19 } & \text { C2 } & 119.36(18) \\ \text { C14 } & \text { C5 } & \text { C6 } & 120.40(17) & \text { C19 } & \text { C20 } & \text { C18 } & 119.58(17) \\ \text { O3 } & \text { C6 } & \text { C5 } & 121.51(17) & \text { C23 } & \text { C22 } & \text { C15 } & 117.22(17) \\ \text { O3 } & \text { C6 } & \text { C7 } & 119.23(17) & \text { F1 } & \text { C23 } & \text { F2 } & 106.4(2) \\ \text { C5 } & \text { C6 } & \text { C7 } & 119.26(16) & \text { F1 } & \text { C23 } & \text { F3 } & 105.59(19) \\ \text { C8 } & \text { C7 } & \text { C6 } & 118.29(17) & \text { F1 } & \text { C23 } & \text { C22 } & 113.50(18) \\ \text { C12 } & \text { C7 } & \text { C6 } & 121.12(17) & \text { F2 } & \text { C23 } & \text { F3 } & 106.05(19) \\ \text { C12 } & \text { C7 } & \text { C8 } & 120.58(18) & \text { F2 } & \text { C23 } & \text { C22 } & 114.23(18) \\ \text { C9 } & \text { C8 } & \text { C7 } & 119.85(19) & \text { F3 } & \text { C23 } & \text { C22 } & 110.49(18) \\ \text { C10 } & \text { C9 } & \text { C8 } & 118.05(19) & \text { F1A } & \text { C23AF3A } & 107.2(18) \\ \text { C9 } & \text { C10 } & \text { N1 } & 118.57(19) & \text { F1A } & \text { C23AC21A } & 101.4(17) \\ \text { C9 } & \text { C10 } & \text { C13 } & 123.47(19) & \text { F2A } & \text { C23AF1A } & 106(2) \\ \text { C13 } & \text { C10 } & \text { N1 } & 117.95(19) & \text { F2A } & \text { C23AF3A } & 110.6(19) \\ \text { O2 } & \text { N1 } & \text { O6 } & 125.2(2) & \text { F2A } & \text { C23AC21A } & 114(2) \\ \text { O2 } & \text { N1 } & \text { C10 } & 117.9(2) & \text { F3A } & \text { C23AC21A } & 117(2) \\ \text { O6 } & \text { N1 } & \text { C10 } & 116.9(2) & \text { C23A C21AC15 } & 98.4(15) \\ \text { C13 } & \text { C12 } & \text { C7 } & 120.12(18) & & & & \\ & & & & & & \end{array}$

Table S41 Torsion Angles for $10 \mathrm{~b}$.

\begin{tabular}{|c|c|c|c|c|c|c|c|}
\hline A B & D & Angle $/^{\circ}$ & $\mathbf{A}$ & B & C & D & Angle $/^{\circ}$ \\
\hline S1 N2 & $\mathrm{C} 14 \mathrm{C} 5$ & $26.9(3)$ & $\mathrm{C} 5$ & $\mathrm{C} 14$ & $\mathrm{C} 15$ & C21A & $-40.1(17)$ \\
\hline $\mathrm{S} 1 \mathrm{~N} 2$ & $\mathrm{C} 14 \mathrm{C} 15$ & $-153.50(13)$ & C6 & $\mathrm{C} 5$ & C14 & $\mathrm{N} 2$ & $175.51(16)$ \\
\hline $\mathrm{S} 1 \mathrm{~N} 2$ & $\mathrm{C} 17 \mathrm{C} 16$ & $133.72(14)$ & C6 & $\mathrm{C} 5$ & C14 & $\mathrm{C} 15$ & $-3.9(3)$ \\
\hline $\mathrm{S} 1 \mathrm{C} 18$ & 3 C20 C19 & $-173.09(14)$ & C6 & $\mathrm{C} 7$ & $\mathrm{C} 8$ & C9 & $-179.01(18)$ \\
\hline $\mathrm{O} 1 \mathrm{C} 2$ & $\mathrm{C} 3 \mathrm{C} 4$ & $-177.41(16)$ & C6 & $\mathrm{C} 7$ & $\mathrm{C} 12$ & $\mathrm{C} 13$ & $-178.2(2)$ \\
\hline $\mathrm{O} 1 \mathrm{C} 2$ & C19C20 & $178.18(16)$ & $\mathrm{C} 7$ & $\mathrm{C} 8$ & C9 & $\mathrm{C} 10$ & $-2.4(3)$ \\
\hline O3 C6 & $\mathrm{C} 7 \mathrm{C} 8$ & $-24.8(3)$ & $\mathrm{C} 7$ & $\mathrm{C} 12$ & C13 & $\mathrm{C} 10$ & $-2.9(3)$ \\
\hline $\mathrm{O} 3 \mathrm{C} 6$ & C7 C12 & $154.0(2)$ & $\mathrm{C} 8$ & $\mathrm{C} 7$ & $\mathrm{C} 12$ & $\mathrm{C} 13$ & $0.5(3)$ \\
\hline $\mathrm{O} 4 \mathrm{~S} 1$ & N2 C14 & $-157.18(15)$ & $\mathrm{C} 8$ & C9 & $\mathrm{C} 10$ & N1 & $178.37(19)$ \\
\hline O4 S1 & N2 $\mathrm{C} 17$ & $50.63(17)$ & $\mathrm{C} 8$ & C9 & $\mathrm{C} 10$ & $\mathrm{C} 13$ & $0.0(3)$ \\
\hline O4 S1 & C18C4 & $144.89(15)$ & C9 & $\mathrm{C} 10$ & N1 & $\mathrm{O} 2$ & $156.5(2)$ \\
\hline O4 S1 & C $18 C 20$ & $-39.84(17)$ & C9 & $\mathrm{C} 10$ & N1 & O6 & $-24.0(3)$ \\
\hline O5 S1 & $\mathrm{N} 2 \mathrm{C} 14$ & $75.56(17)$ & C9 & $\mathrm{C} 10$ & C13 & $\mathrm{C} 12$ & $2.7(3)$ \\
\hline O5 S1 & $\mathrm{N} 2 \mathrm{C} 17$ & $-76.62(16)$ & N1 & $\mathrm{C} 10$ & C13 & $\mathrm{C} 12$ & $-175.7(2)$ \\
\hline $\mathrm{O} 5 \mathrm{~S} 1$ & $\mathrm{C} 18 \mathrm{C} 4$ & $-83.53(16)$ & $\mathrm{C} 12$ & $\mathrm{C} 7$ & $\mathrm{C} 8$ & C9 & $2.2(3)$ \\
\hline O5 S1 & $\mathrm{C} 18 \mathrm{C} 20$ & $91.74(16)$ & $\mathrm{C} 13$ & $\mathrm{C} 10$ & N1 & $\mathrm{O} 2$ & $-25.0(3)$ \\
\hline N2 S1 & $\mathrm{C} 18 \mathrm{C} 4$ & $31.61(16)$ & $\mathrm{C} 13$ & $\mathrm{C} 10$ & N1 & O6 & $154.4(2)$ \\
\hline N2S1 & C18C20 & $-153.11(15)$ & $\mathrm{C} 14$ & $\mathrm{~N} 2$ & C17 & $\mathrm{C} 16$ & $-21.2(2)$ \\
\hline
\end{tabular}




\begin{tabular}{|c|c|c|c|c|c|c|}
\hline $\mathrm{N} 2 \mathrm{C} 14 \mathrm{C} 15 \mathrm{C} 16$ & $20.74(19)$ & $\mathrm{C} 14$ & $\mathrm{C} 5$ & C6 & $\mathrm{O} 3$ & $-49.2(3)$ \\
\hline $\mathrm{N} 2 \mathrm{C} 14 \mathrm{C} 15 \mathrm{C} 21$ & $138.40(19)$ & $\mathrm{C} 14$ & $\mathrm{C} 5$ & C6 & $\mathrm{C} 7$ & $130.90(19)$ \\
\hline $\mathrm{N} 2 \mathrm{C} 14 \mathrm{C} 15 \mathrm{C} 22$ & $-95.88(18)$ & $\mathrm{C} 14$ & $\mathrm{C} 15$ & $\mathrm{C} 16$ & $\mathrm{C} 17$ & $-33.44(19)$ \\
\hline $\mathrm{N} 2 \mathrm{C} 14 \mathrm{C} 15 \mathrm{C} 21 \mathrm{~A}$ & $140.4(17)$ & $\mathrm{C} 14$ & $\mathrm{C} 15$ & $\mathrm{C} 22$ & $\mathrm{C} 23$ & $-176.25(17)$ \\
\hline $\mathrm{C} 1 \mathrm{O} 1 \mathrm{C} 2 \mathrm{C} 3$ & $12.6(3)$ & $\mathrm{C} 14$ & $\mathrm{C} 15$ & $\mathrm{C} 21 \mathrm{~A}$ & $\mathrm{C} 23 \mathrm{~A}$ & $-153(2)$ \\
\hline C1 O1 C2 C19 & $-168.12(17)$ & $\mathrm{C} 15$ & $\mathrm{C} 16$ & $\mathrm{C} 17$ & $\mathrm{~N} 2$ & $33.42(19)$ \\
\hline $\mathrm{C} 2 \mathrm{C} 3 \mathrm{C} 4 \mathrm{C} 5$ & $-176.47(17)$ & $\mathrm{C} 15$ & $\mathrm{C} 22$ & $\mathrm{C} 23$ & $\mathrm{~F} 1$ & $67.8(3)$ \\
\hline $\mathrm{C} 2 \mathrm{C} 3 \mathrm{C} 4 \mathrm{C} 18$ & $-1.4(3)$ & $\mathrm{C} 15$ & $\mathrm{C} 22$ & $\mathrm{C} 23$ & $\mathrm{~F} 2$ & $-54.4(3)$ \\
\hline C2 C19C20C18 & $-0.1(3)$ & $\mathrm{C} 15$ & $\mathrm{C} 22$ & $\mathrm{C} 23$ & F3 & $-173.86(18)$ \\
\hline C3 C2 C19C20 & $-2.5(3)$ & $\mathrm{C} 16$ & $\mathrm{C} 15$ & $\mathrm{C} 22$ & $\mathrm{C} 23$ & $74.1(2)$ \\
\hline $\mathrm{C} 3 \mathrm{C} 4 \mathrm{C} 5 \mathrm{C} 6$ & $-8.0(3)$ & $\mathrm{C} 16$ & $\mathrm{C} 15$ & $\mathrm{C} 21 \mathrm{~A}$ & $\mathrm{C} 23 \mathrm{~A}$ & $-46(3)$ \\
\hline $\mathrm{C} 3 \mathrm{C} 4 \mathrm{C} 5 \mathrm{C} 14$ & $164.41(17)$ & $\mathrm{C} 17$ & $\mathrm{~N} 2$ & $\mathrm{C} 14$ & $\mathrm{C} 5$ & $-179.52(17)$ \\
\hline C3C4 C18S1 & $173.77(13)$ & $\mathrm{C} 17$ & $\mathrm{~N} 2$ & $\mathrm{C} 14$ & $\mathrm{C} 15$ & $0.0(2)$ \\
\hline $\mathrm{C} 3 \mathrm{C} 4 \mathrm{C} 18 \mathrm{C} 20$ & $-1.2(3)$ & $\mathrm{C} 18$ & $\mathrm{~S} 1$ & $\mathrm{~N} 2$ & $\mathrm{C} 14$ & $-40.36(17)$ \\
\hline $\mathrm{C} 4 \mathrm{C} 5 \mathrm{C} 6 \mathrm{O} 3$ & $123.2(2)$ & $\mathrm{C} 18$ & $\mathrm{~S} 1$ & $\mathrm{~N} 2$ & $\mathrm{C} 17$ & $167.45(15)$ \\
\hline $\mathrm{C} 4 \mathrm{C} 5 \mathrm{C} 6 \mathrm{C} 7$ & $-56.7(2)$ & $\mathrm{C} 18$ & $\mathrm{C} 4$ & $\mathrm{C} 5$ & C6 & $177.27(16)$ \\
\hline C4 C5 C14N2 & $3.3(3)$ & $\mathrm{C} 18$ & $\mathrm{C} 4$ & $\mathrm{C} 5$ & $\mathrm{C} 14$ & $-10.3(3)$ \\
\hline C4 C5 C14C15 & $176.15(17)$ & C19 & $\mathrm{C} 2$ & $\mathrm{C} 3$ & $\mathrm{C} 4$ & $3.4(3)$ \\
\hline C4 C18 C20 C19 & $2.1(3)$ & $\mathrm{C} 21$ & $\mathrm{C} 15$ & $\mathrm{C} 16$ & $\mathrm{C} 17$ & $-154.74(17)$ \\
\hline C5C4 C18S1 & $-11.2(2)$ & $\mathrm{C} 21$ & $\mathrm{C} 15$ & $\mathrm{C} 22$ & $\mathrm{C} 23$ & $-49.5(2)$ \\
\hline $\mathrm{C} 5 \mathrm{C} 4 \mathrm{C} 18 \mathrm{C} 20$ & $173.75(17)$ & $\mathrm{C} 22$ & $\mathrm{C} 15$ & $\mathrm{C} 16$ & $\mathrm{C} 17$ & 79.81(19) \\
\hline $\mathrm{C} 5 \mathrm{C} 6 \mathrm{C} 7 \mathrm{C} 8$ & $155.07(18)$ & $\mathrm{C} 22$ & $\mathrm{C} 15$ & $\mathrm{C} 21 \mathrm{~A}$ & C $23 \mathrm{~A}$ & $92(2)$ \\
\hline C5 C6 C7 C12 & $-26.1(3)$ & F1A & $\mathrm{C} 23 \mathrm{~A}$ & $\mathrm{C} 21$ & $\mathrm{C} 15$ & $-168(2)$ \\
\hline C5 C14C15C16 & $-159.8(2)$ & $\mathrm{F} 2 \mathrm{~A}$ & $\mathrm{C} 23$ & $\mathrm{C} 21 \mathrm{~A}$ & $\mathrm{C} 15$ & $79(3)$ \\
\hline $\mathrm{C} 5 \mathrm{C} 14 \mathrm{C} 15 \mathrm{C} 21$ & $-42.1(3)$ & F3A & $\mathrm{C} 23$ & $\mathrm{C} 21$ & $\mathrm{C} 15$ & $-52(3)$ \\
\hline C5 C14C15C22 & $83.6(2)$ & $\mathrm{C} 21 \mathrm{~A}$ & $\mathrm{AC} 15$ & $\mathrm{C} 16$ & $\mathrm{C} 17$ & $-139.9(15)$ \\
\hline
\end{tabular}

Table S42 Hydrogen Atom Coordinates $\left(\AA \times 10^{4}\right)$ and Isotropic Displacement Parameters $\left(\AA^{2} \times 10^{3}\right)$ for $10 b$.

$\begin{array}{lrrrr}\text { Atom } & \boldsymbol{x} & \boldsymbol{y} & \boldsymbol{z} & \mathbf{U}(\mathbf{e q}) \\ \text { H1A } & 8308.62 & 2816.97 & 5957.48 & 37 \\ \text { H1B } & 6651.31 & 3139.5 & 6495.75 & 37 \\ \text { H1C } & 8823.97 & 3353.16 & 7315.46 & 37 \\ \text { H3 } & 5822.97 & 3118.1 & 4654.13 & 19 \\ \text { H8 } & 3214.32 & 308.22 & 3507.1 & 25 \\ \text { H9 } & 5408.75 & -855.53 & 3978.77 & 29 \\ \text { H12 } & 6905.74 & 1893.64 & 2303.12 & 26 \\ \text { H13 } & 8984.81 & 625.73 & 2637.72 & 26 \\ \text { H16A } & 2798.57 & 3148.25 & -599.61 & 26 \\ \text { H16B } & 633.77 & 3354.9 & -788.78 & 25 \\ \text { H17A } & 3517.28 & 5186.28 & 309.83 & 25 \\ \text { H17B } & 1661.43 & 5143.43 & 736.62 & 20 \\ \text { H19 } & 9410.76 & 6429.12 & 6242.53 & \end{array}$




$\begin{array}{lrrrr}\text { H20 } & 8410.21 & 6806.47 & 4553.3 & 19 \\ \text { H21A } & 2973.69 & 1394.24 & -23.82 & 32 \\ \text { H21B } & 737.84 & 1096.37 & -782.98 & 32 \\ \text { H21C } & 1324.08 & 964.99 & 438.98 & 32 \\ \text { H22A } & -355.91 & 2489.38 & 1414.22 & 31 \\ \text { H22B } & -1416.11 & 2460.66 & 157.96 & 31 \\ \text { H22C } & -291.34 & 3711.4 & 1175.98 & 31 \\ \text { H21D } & 3538.72 & 1594.24 & 509.96 & 25 \\ \text { H21E } & 1567.69 & 1027.08 & 663.19 & 25\end{array}$

Table S43 Atomic Occupancy for $10 \mathrm{~b}$.

$\begin{array}{lrlclr}\text { Atom } & \text { Occupancy } & \text { Atom } & \text { Occupancy } & \text { Atom } & \text { Occupancy } \\ \text { F1 } & 0.9218(18) & \text { F2 } & 0.9218(18) & \text { F3 } & 0.9218(18) \\ \text { C21 } & 0.9218(18) & \text { H21A } & 0.9218(18) & \text { H21B } & 0.9218(18) \\ \text { H21C } & 0.9218(18) & \text { H22B } & 0.0782(18) & \text { C23 } & 0.9218(18) \\ \text { F1A } & 0.0782(18) & \text { C23A } & 0.0782(18) & \text { F2A } & 0.0782(18) \\ \text { F3A } & 0.0782(18) & \text { C21A } & 0.0782(18) & \text { H21D } & 0.0782(18) \\ \text { H21E } & 0.0782(18) & & & \end{array}$




\section{References}

(1) Shen, Y.; Wang, C.; Chen, W.; Cui, S. Org. Chem. Front. 2018, 5, 3574-3578.

(2) Zhang, Y.; Hsung, R.-P.; Tracey, M. R.; Kurtz, K. C. M.; Vera, E. L. Org. Lett. 2004, 6, 1151-1154.

(3) Coste, A.; Karthikeyan, G.; Couty, F.; Evano, G. Angew. Chem. Int. Ed. 2009, 48, 4381-4385.

(4) Al-Rashid, Z. F.; Johnson, W. L.; Hsung, R. P.; Wei, Y.; Yao, P.-Y.; Liu, R.; Zhao, K. J. Org. Chem. 2008, 73, 8780-8784. 


\section{Spectral Data}

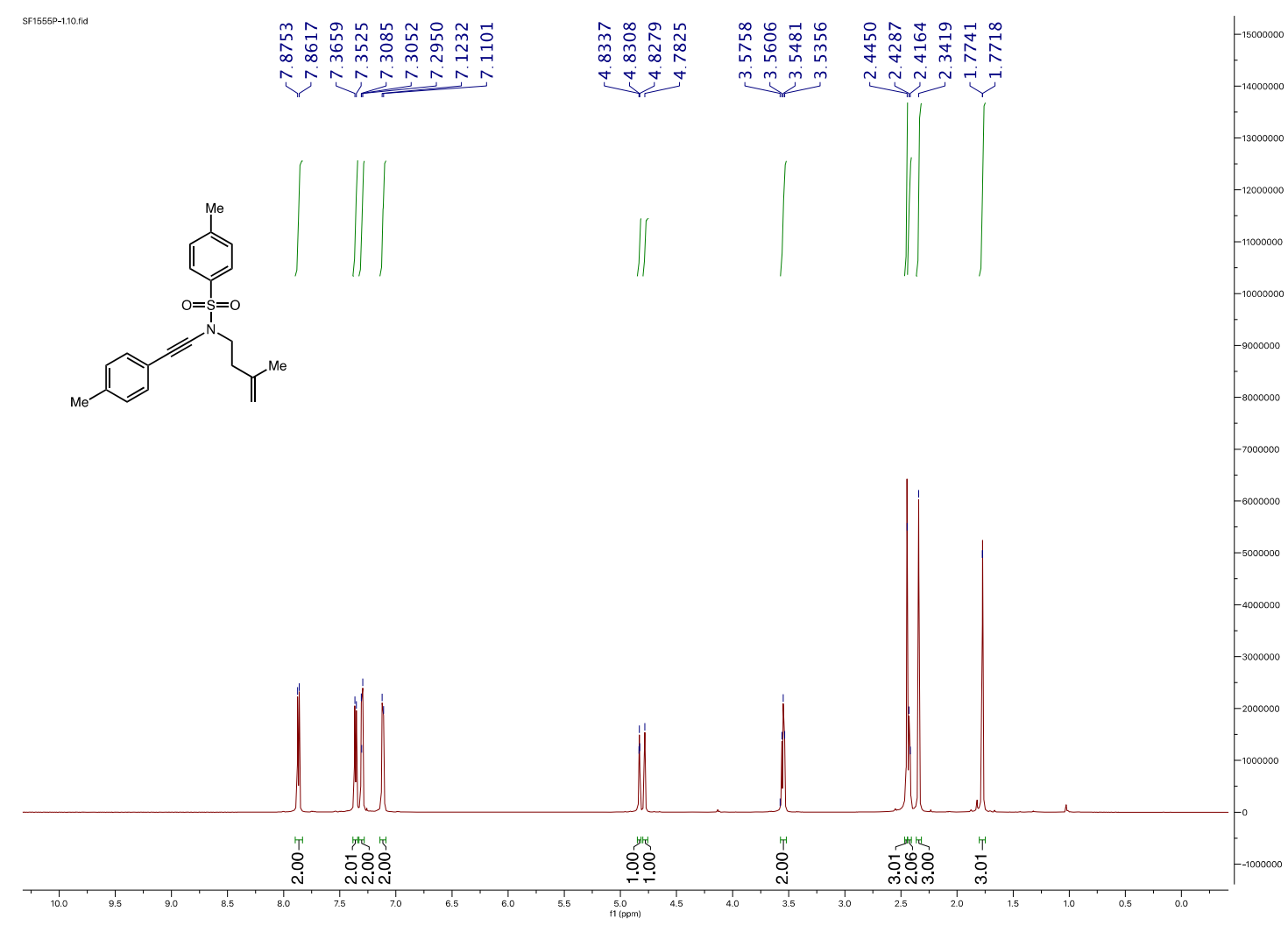

1a ${ }^{1} \mathrm{H}$ NMR $\left(600 \mathrm{MHz}, \mathrm{CDCl}_{3}\right)$

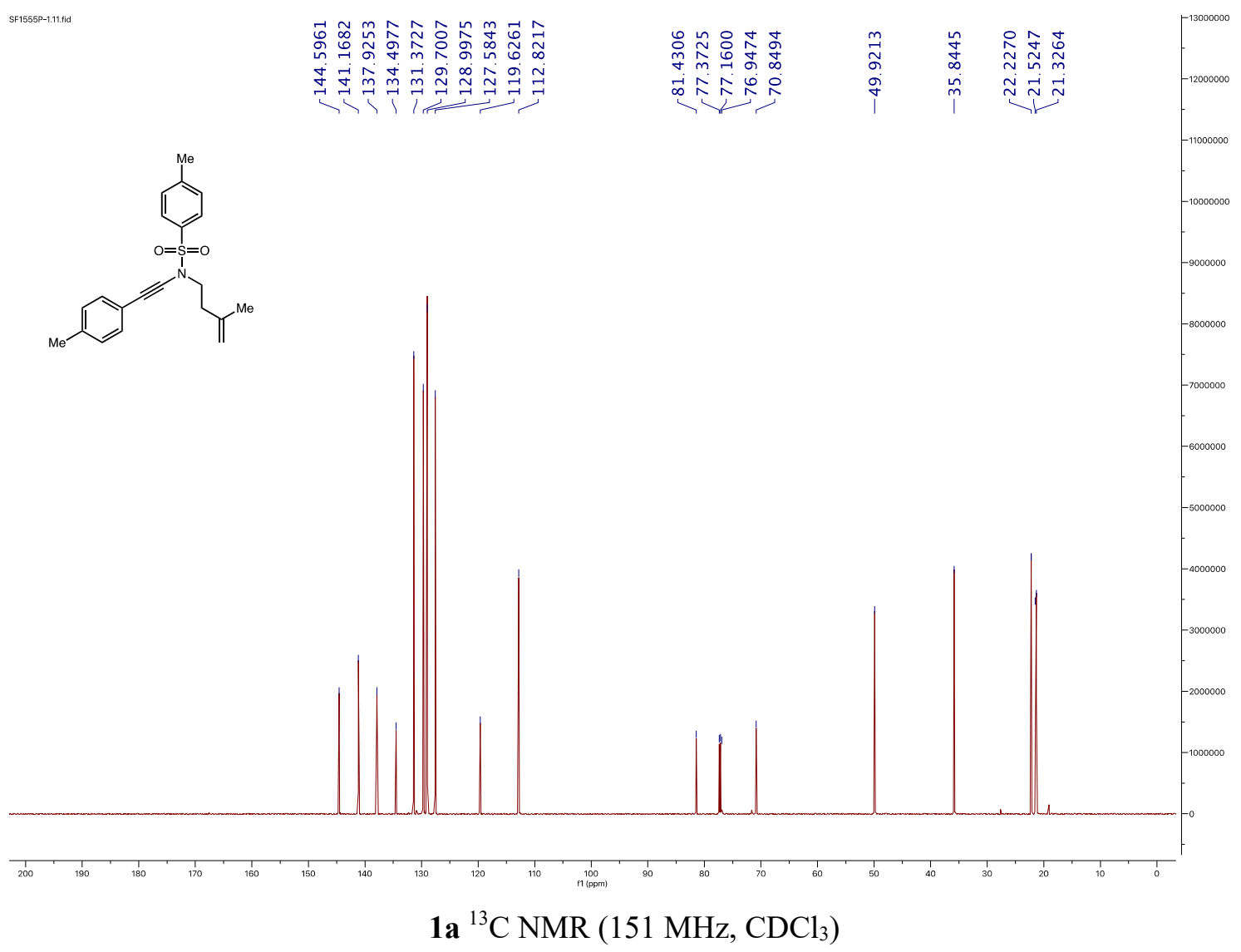




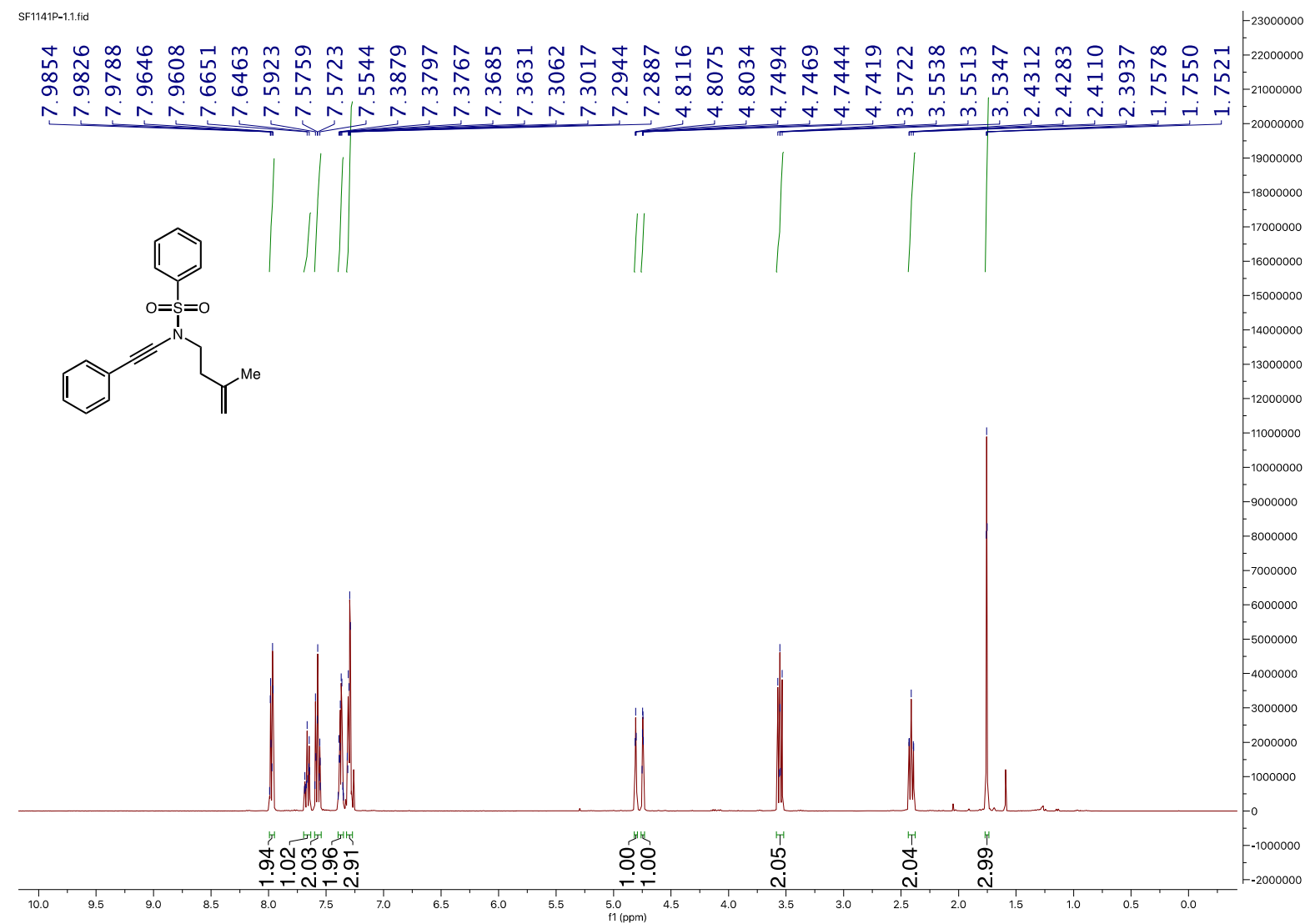

1b ${ }^{1} \mathrm{H}$ NMR (400 MHz, $\mathrm{CDCl}_{3}$ )

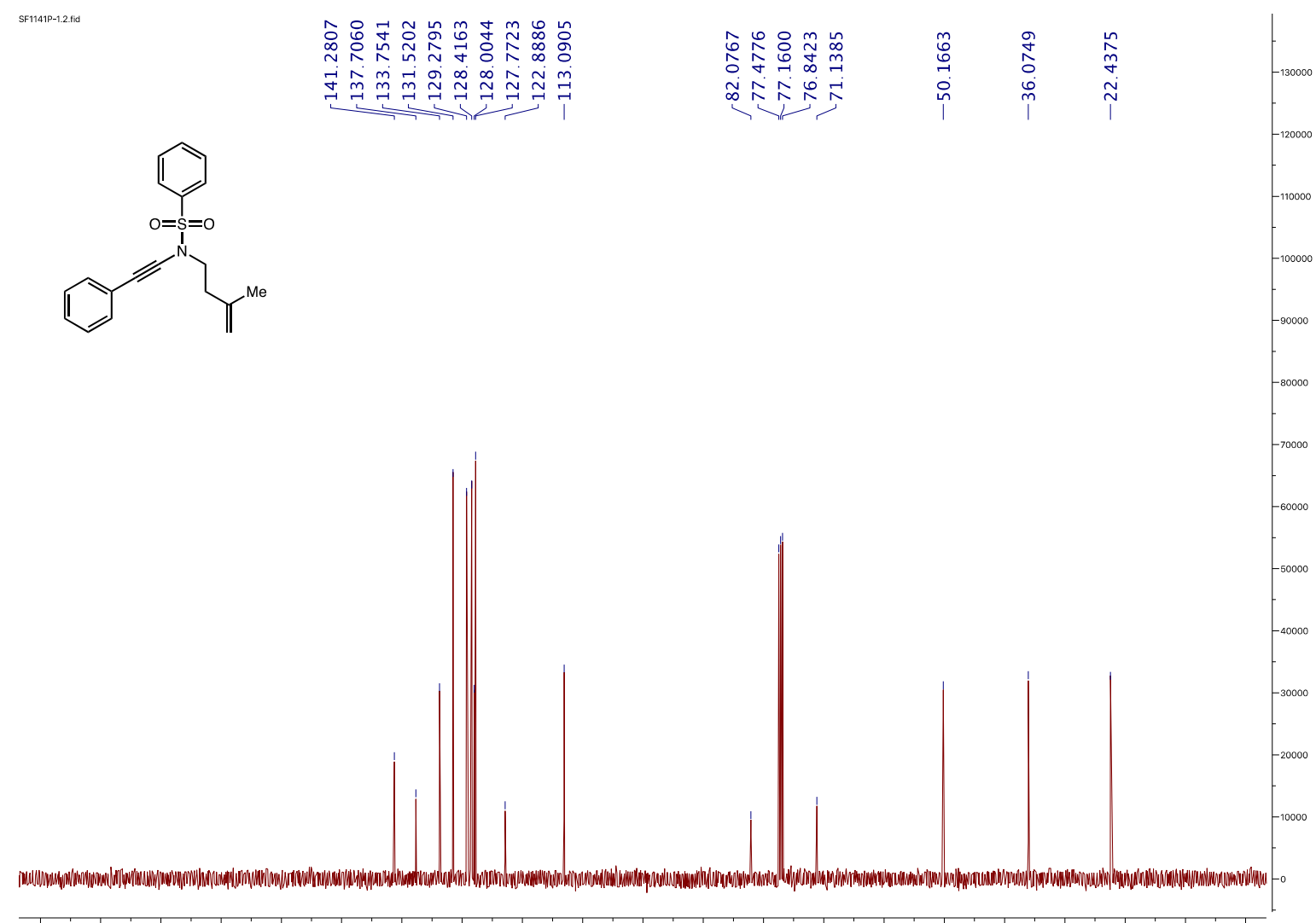

1b ${ }^{13} \mathrm{C}$ NMR (101 MHz, $\left.\mathrm{CDCl}_{3}\right)$ 


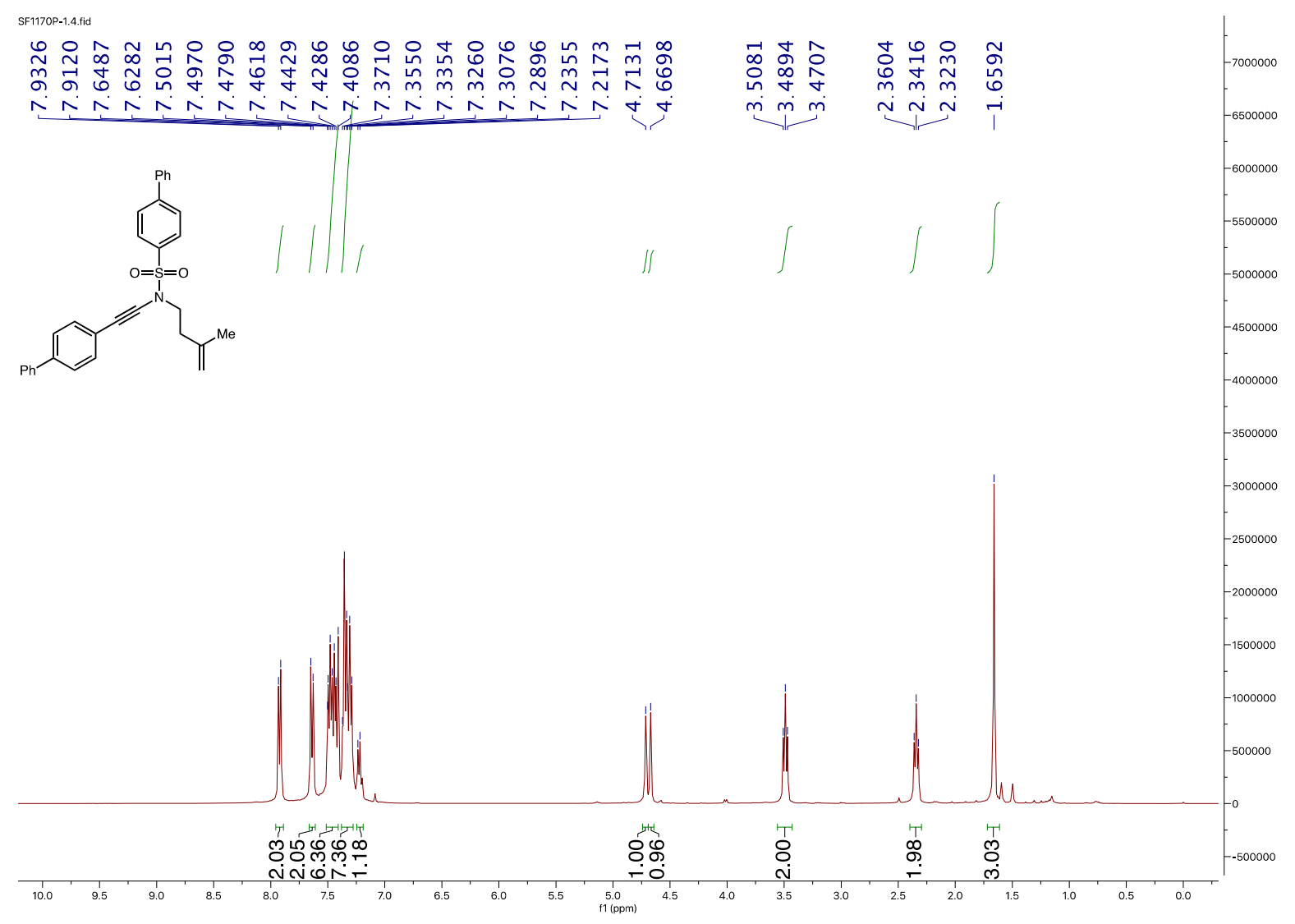

1c ${ }^{1} \mathrm{H}$ NMR (400 MHz, $\left.\mathrm{CDCl}_{3}\right)$

SF1170P-1.5.fid

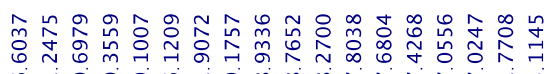

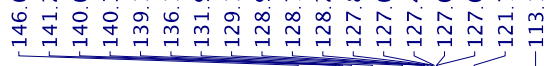

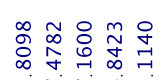

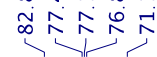

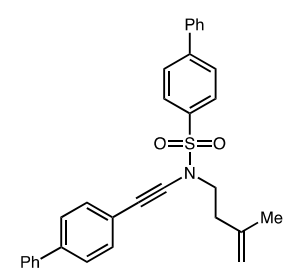




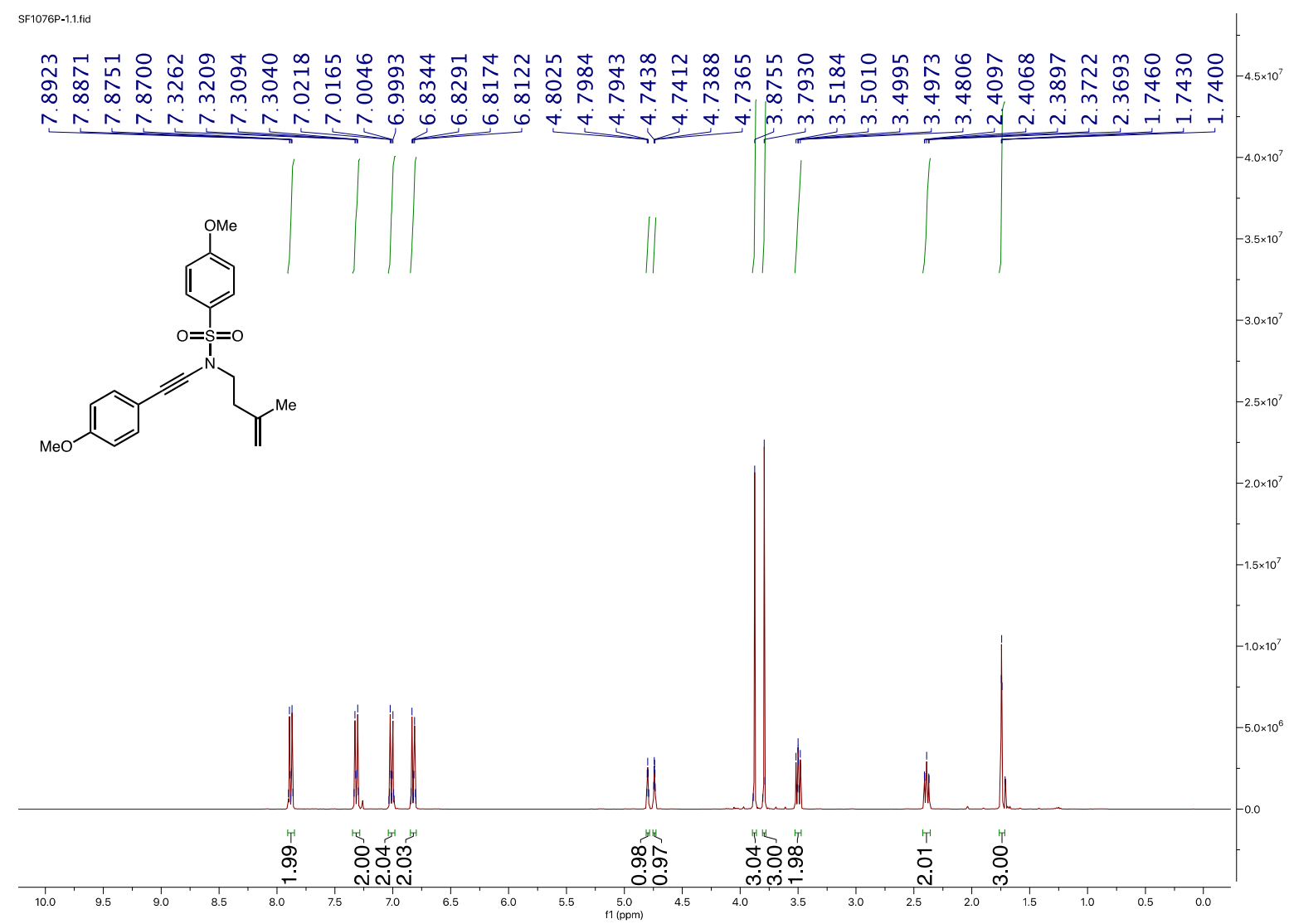

1d ${ }^{1} \mathrm{H}$ NMR (400 MHz, $\left.\mathrm{CDCl}_{3}\right)$

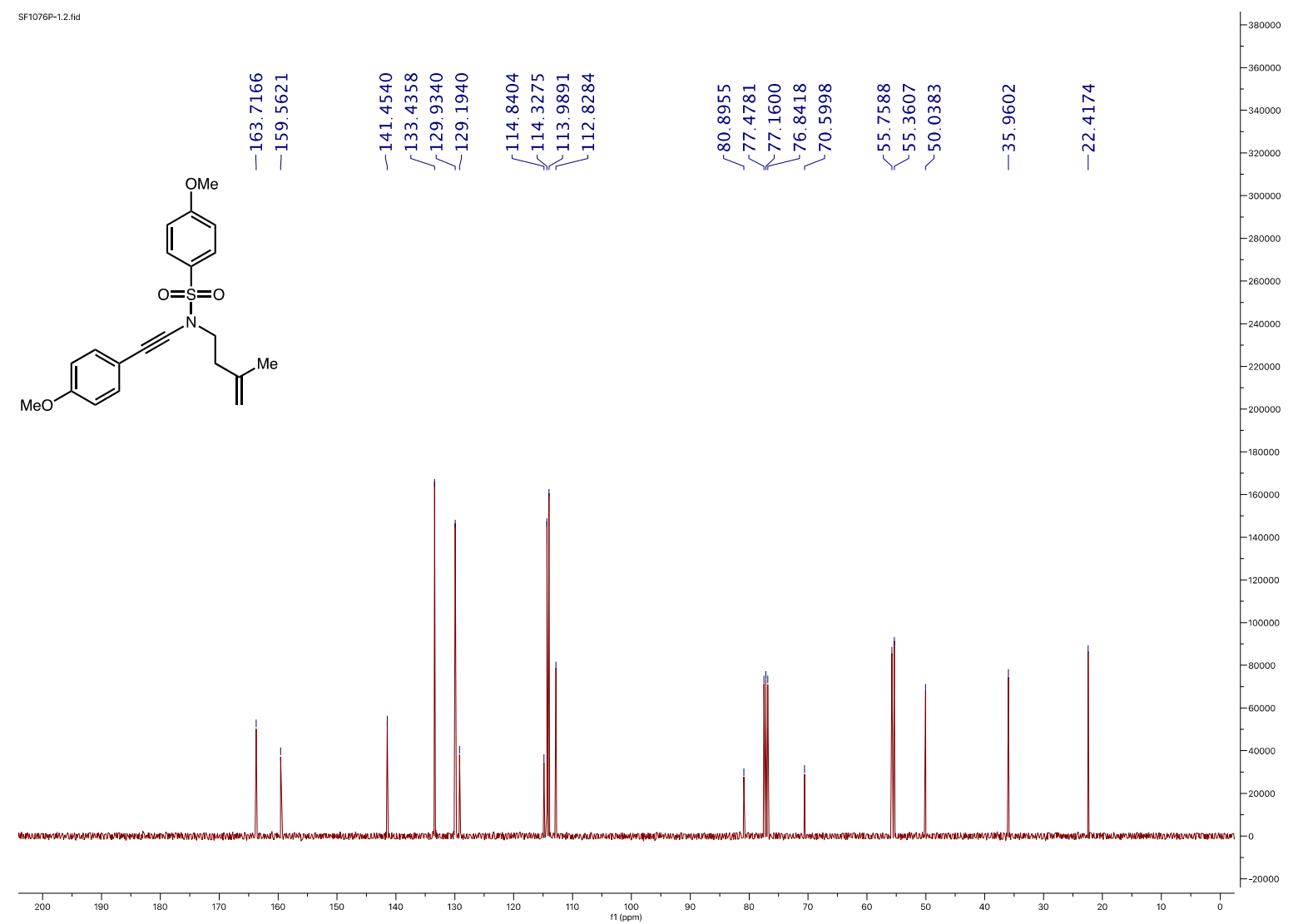

1d ${ }^{13} \mathrm{C}$ NMR $\left(101 \mathrm{MHz}, \mathrm{CDCl}_{3}\right)$ 

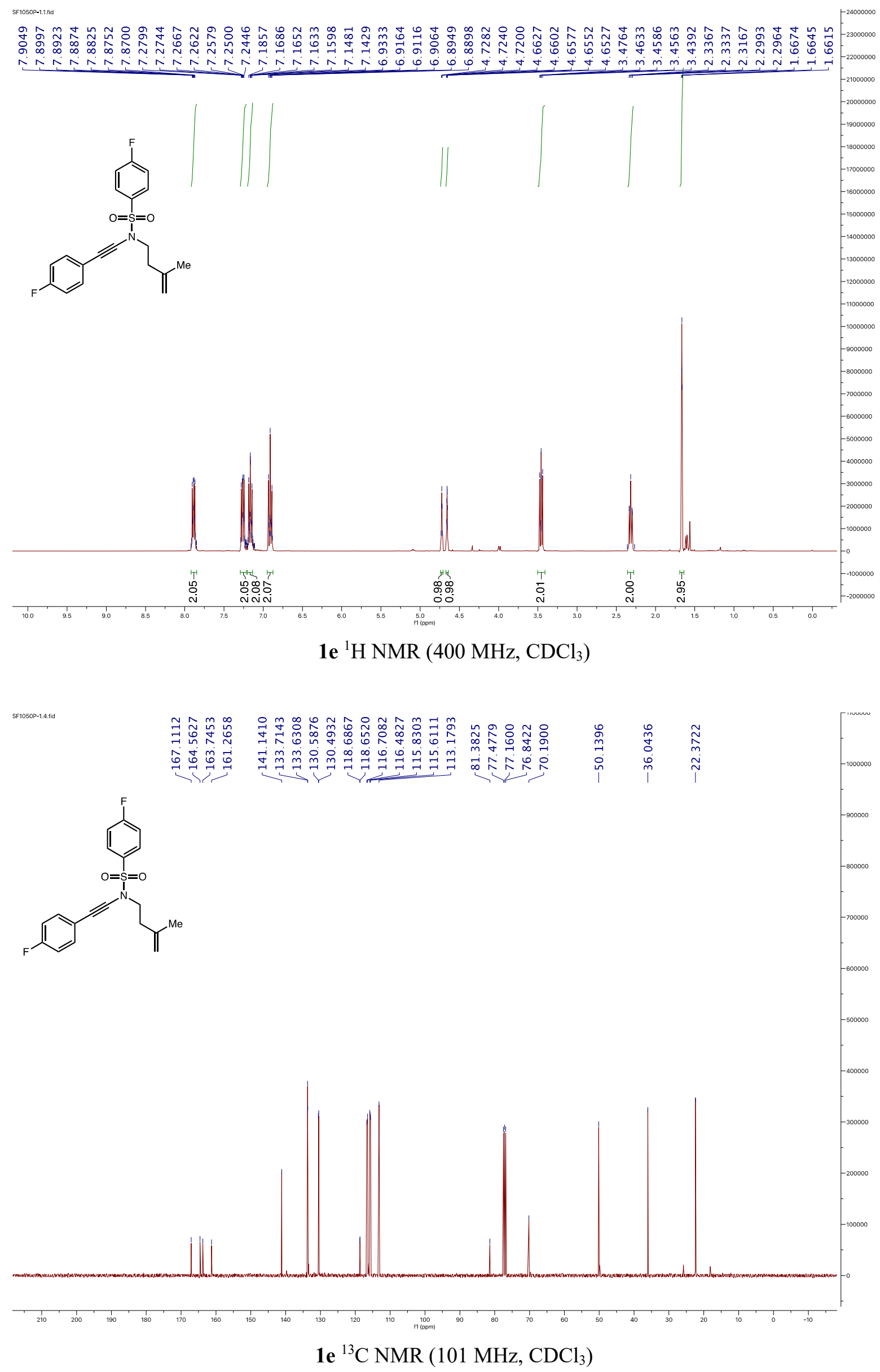

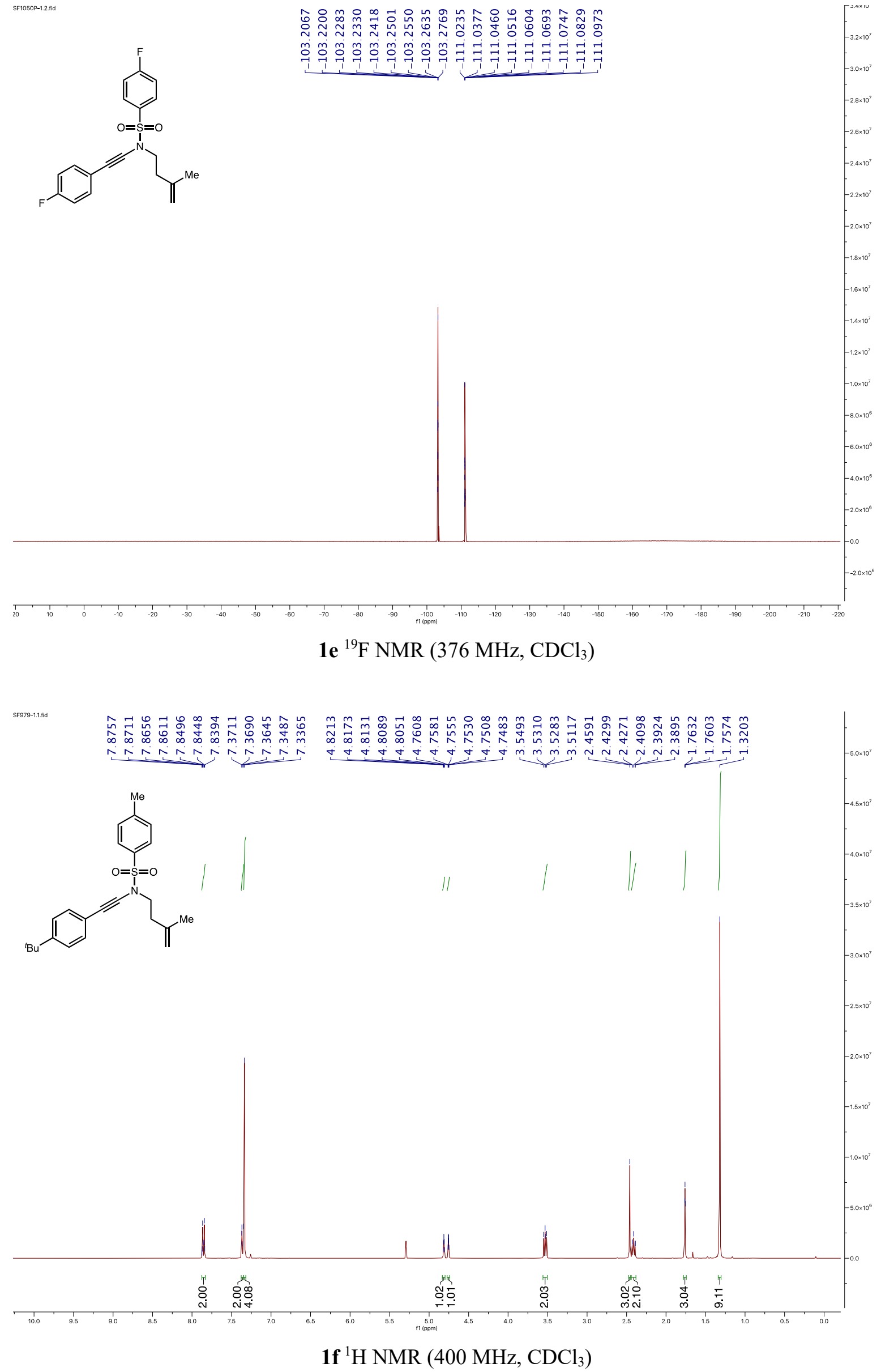

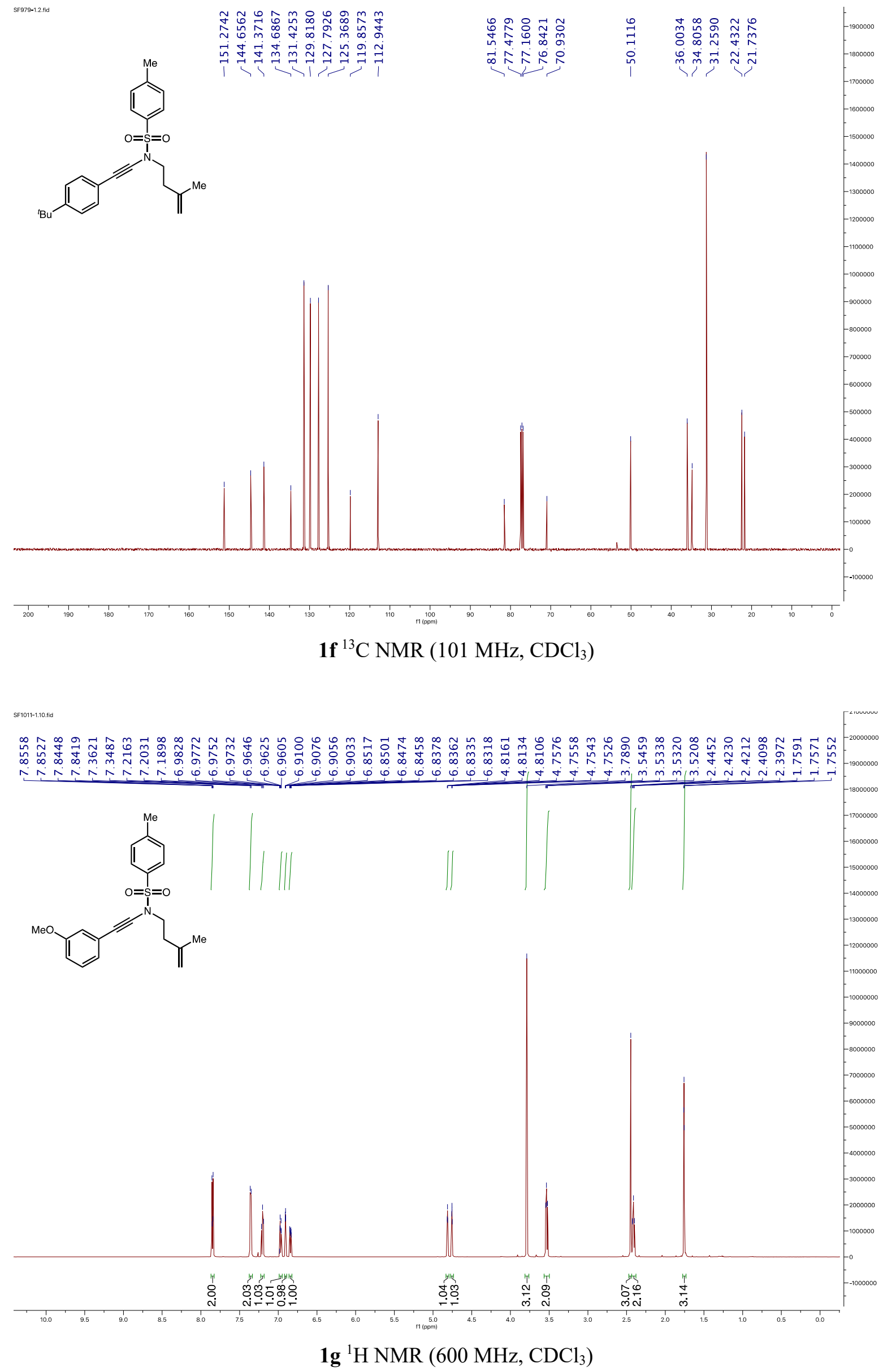

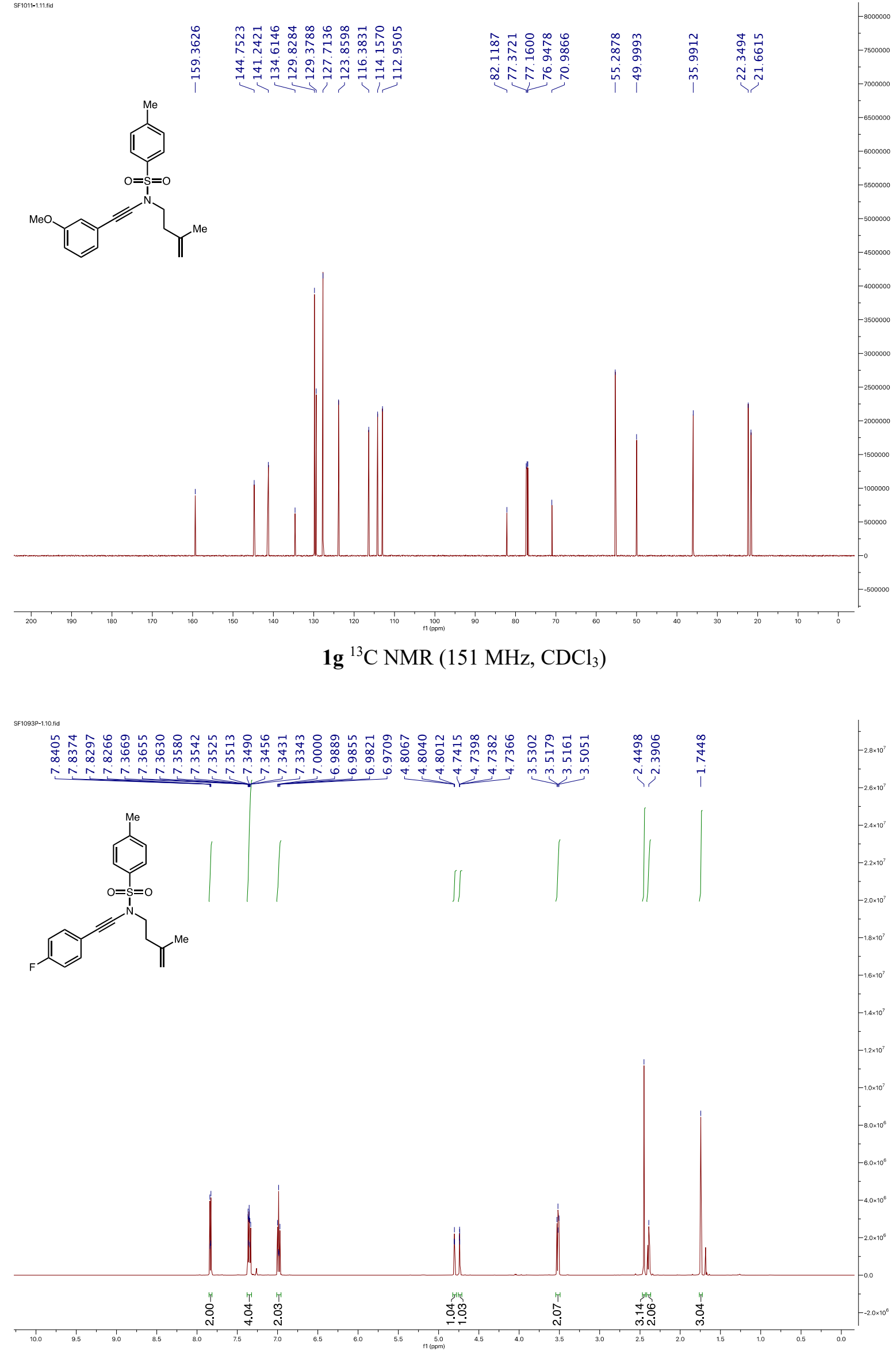

1h ${ }^{1} \mathrm{H}$ NMR (600 MHz, $\left.\mathrm{CDCl}_{3}\right)$ 


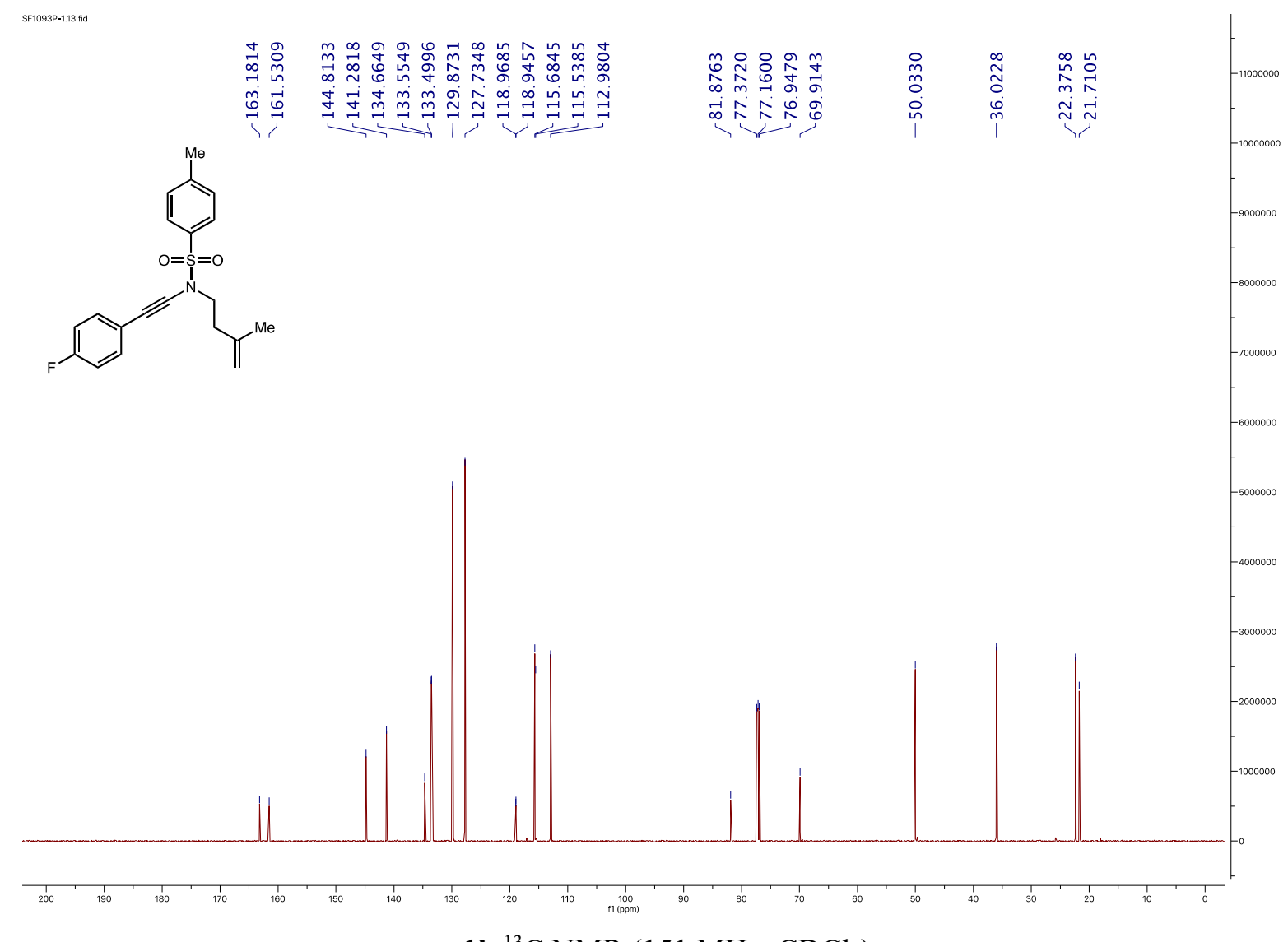

1h ${ }^{13} \mathrm{C}$ NMR (151 MHz, $\mathrm{CDCl}_{3}$ )

SF1093P-1.12.1.id

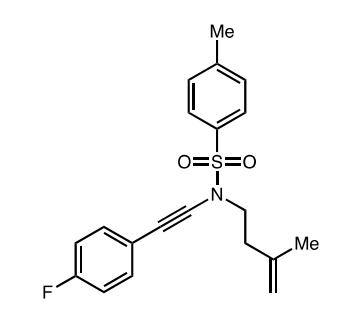

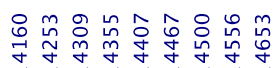

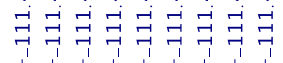

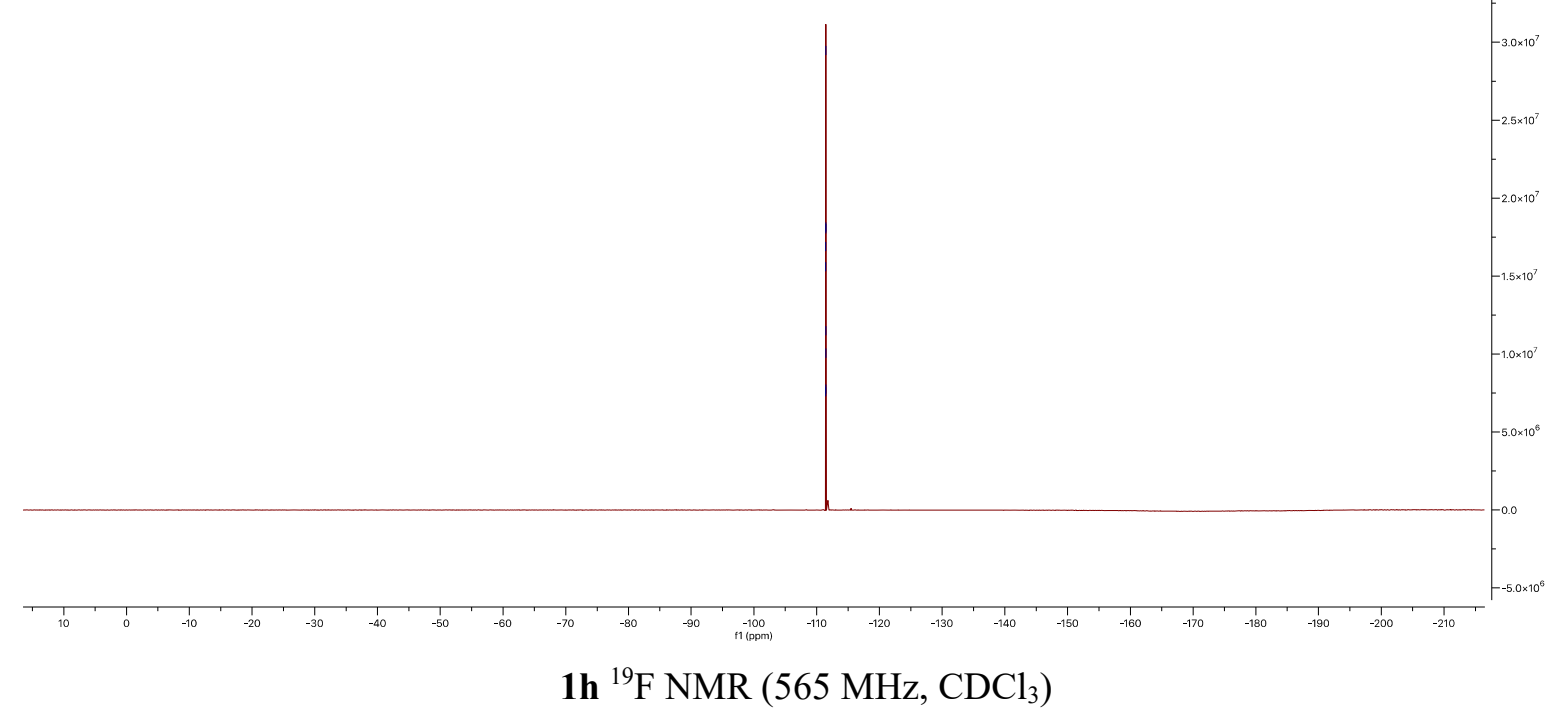



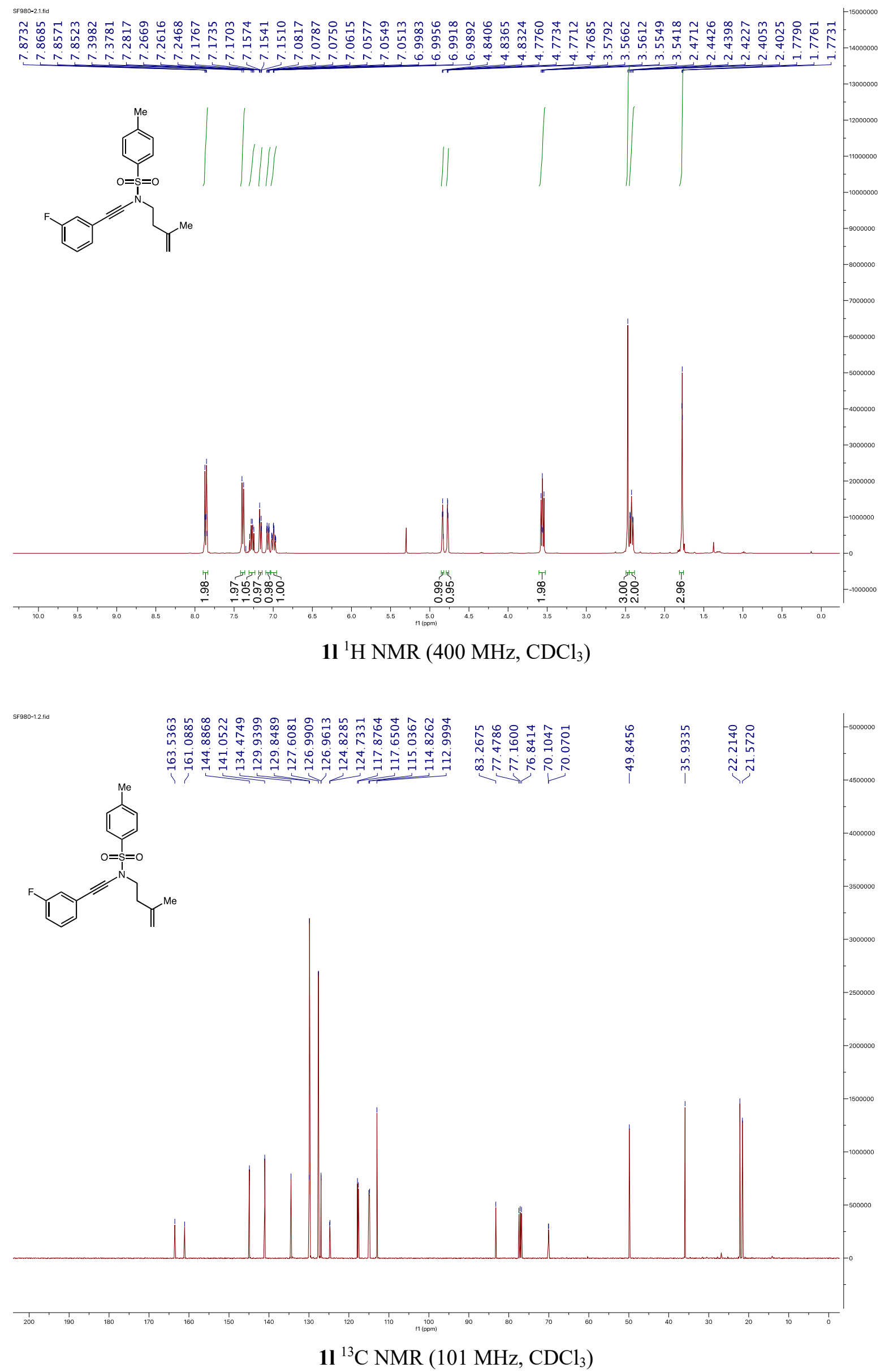

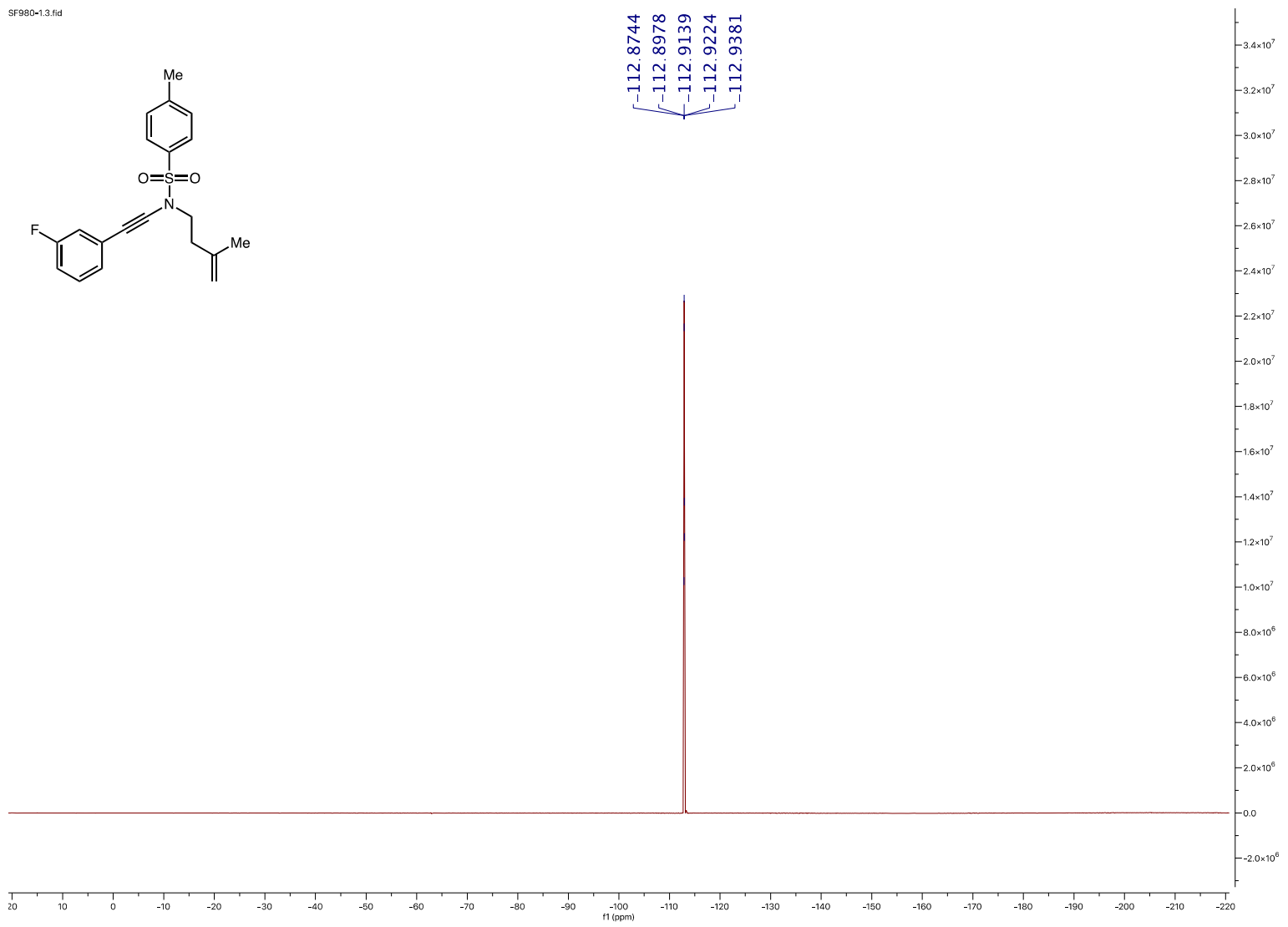

1I ${ }^{19} \mathrm{~F}$ NMR (376 MHz, $\mathrm{CDCl}_{3}$ )

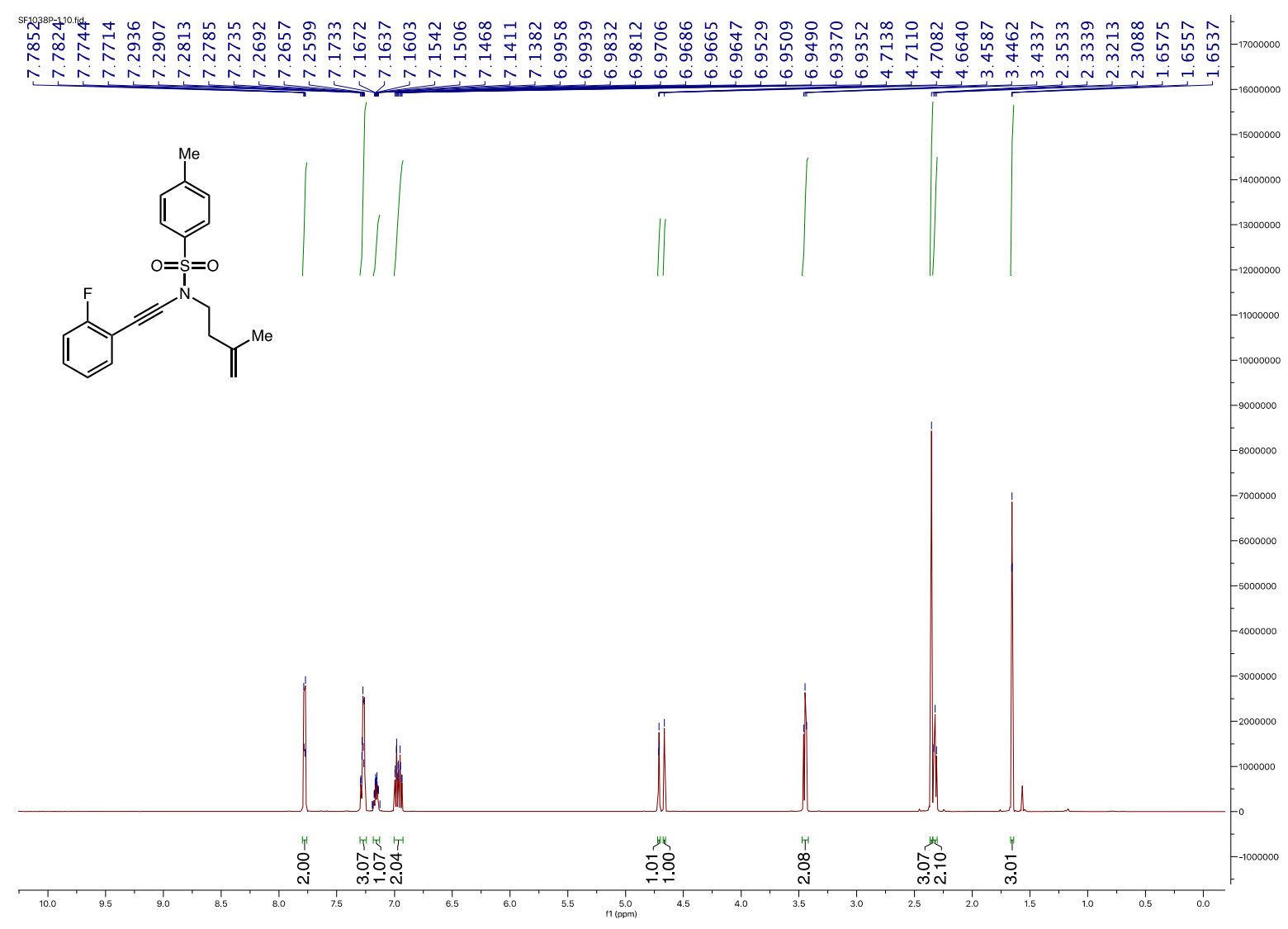

1j ${ }^{1} \mathrm{H}$ NMR (600 MHz, $\left.\mathrm{CDCl}_{3}\right)$ 


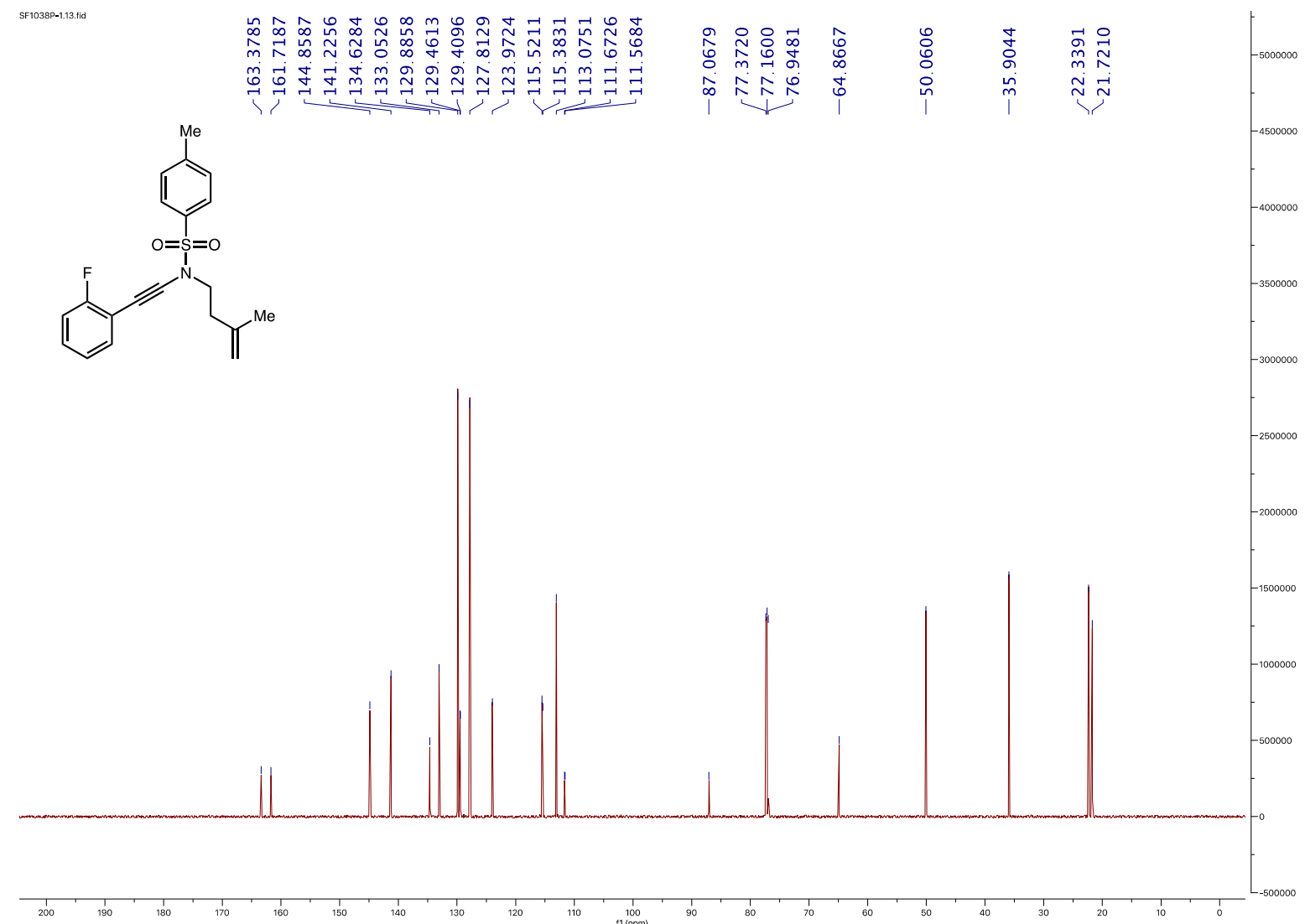

1j ${ }^{13} \mathrm{C}$ NMR (151 MHz, $\left.\mathrm{CDCl}_{3}\right)$

SF1038P-1.12:fid

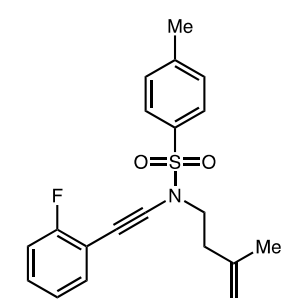

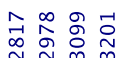

욱육육

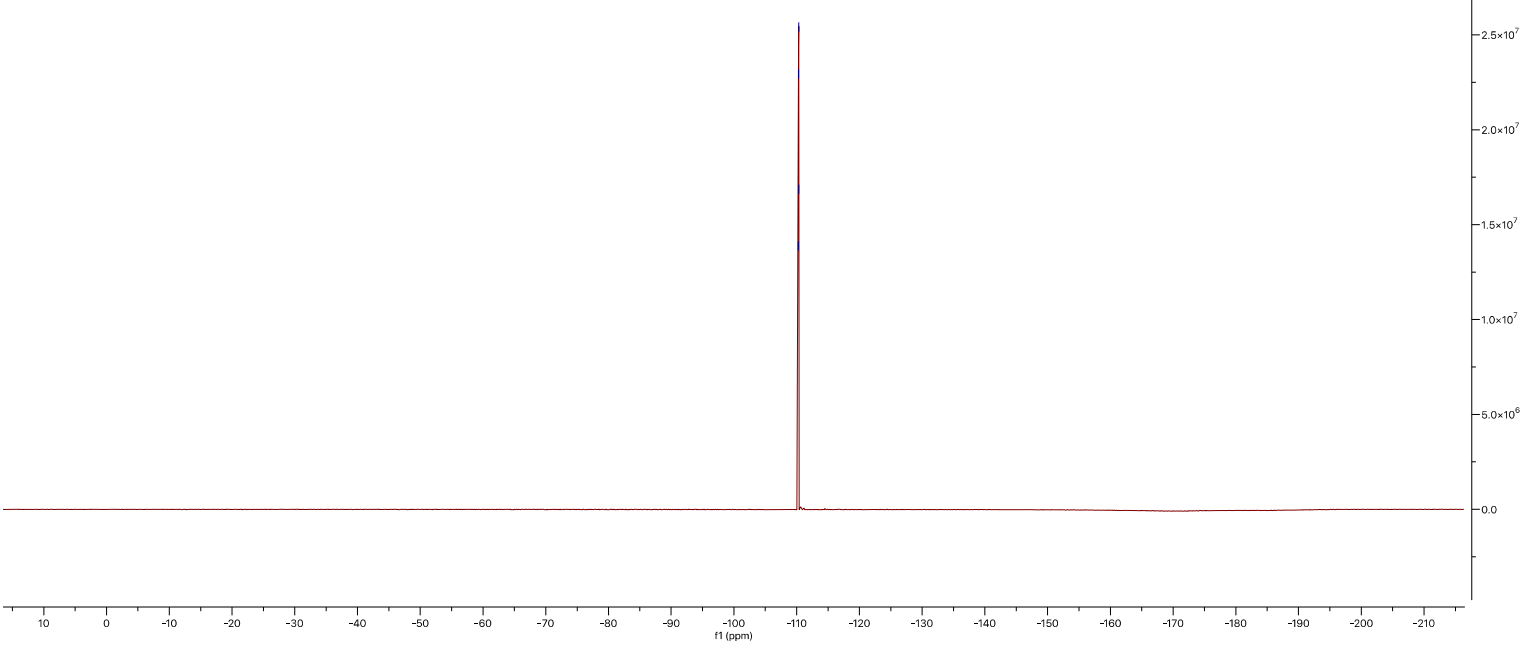

1j ${ }^{19} \mathrm{~F}$ NMR (565 MHz, $\mathrm{CDCl}_{3}$ ) 


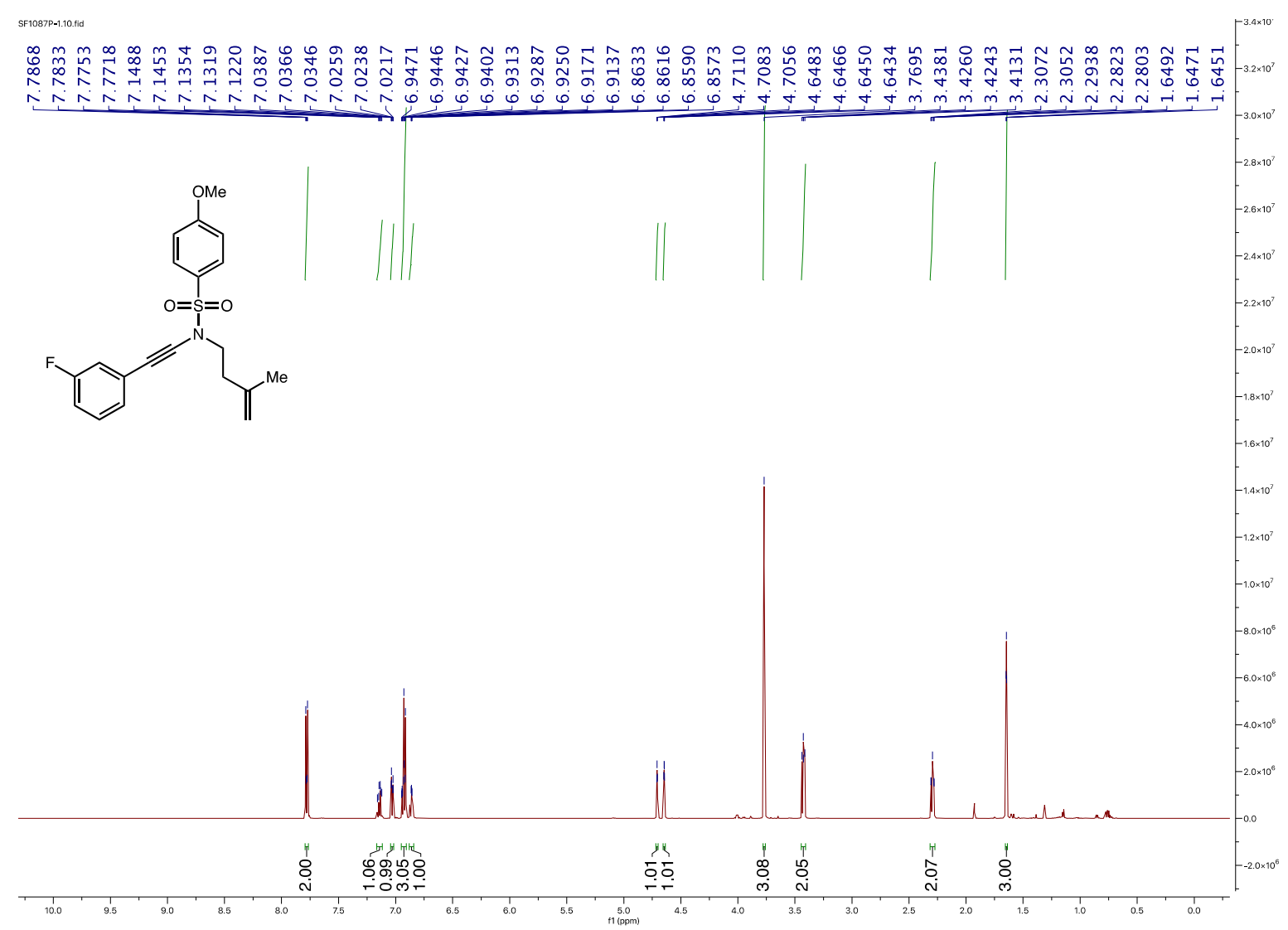

4a ${ }^{1} \mathrm{H}$ NMR (600 MHz, $\left.\mathrm{CDCl}_{3}\right)$

SF1087P-1.13.fid

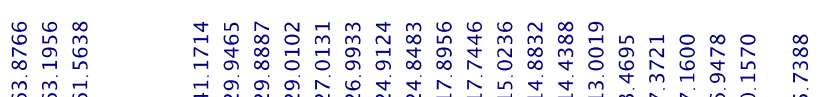

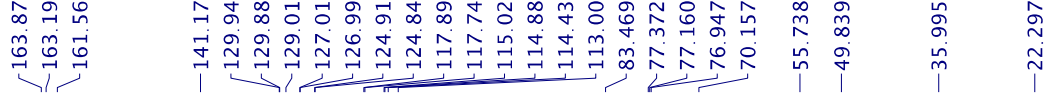
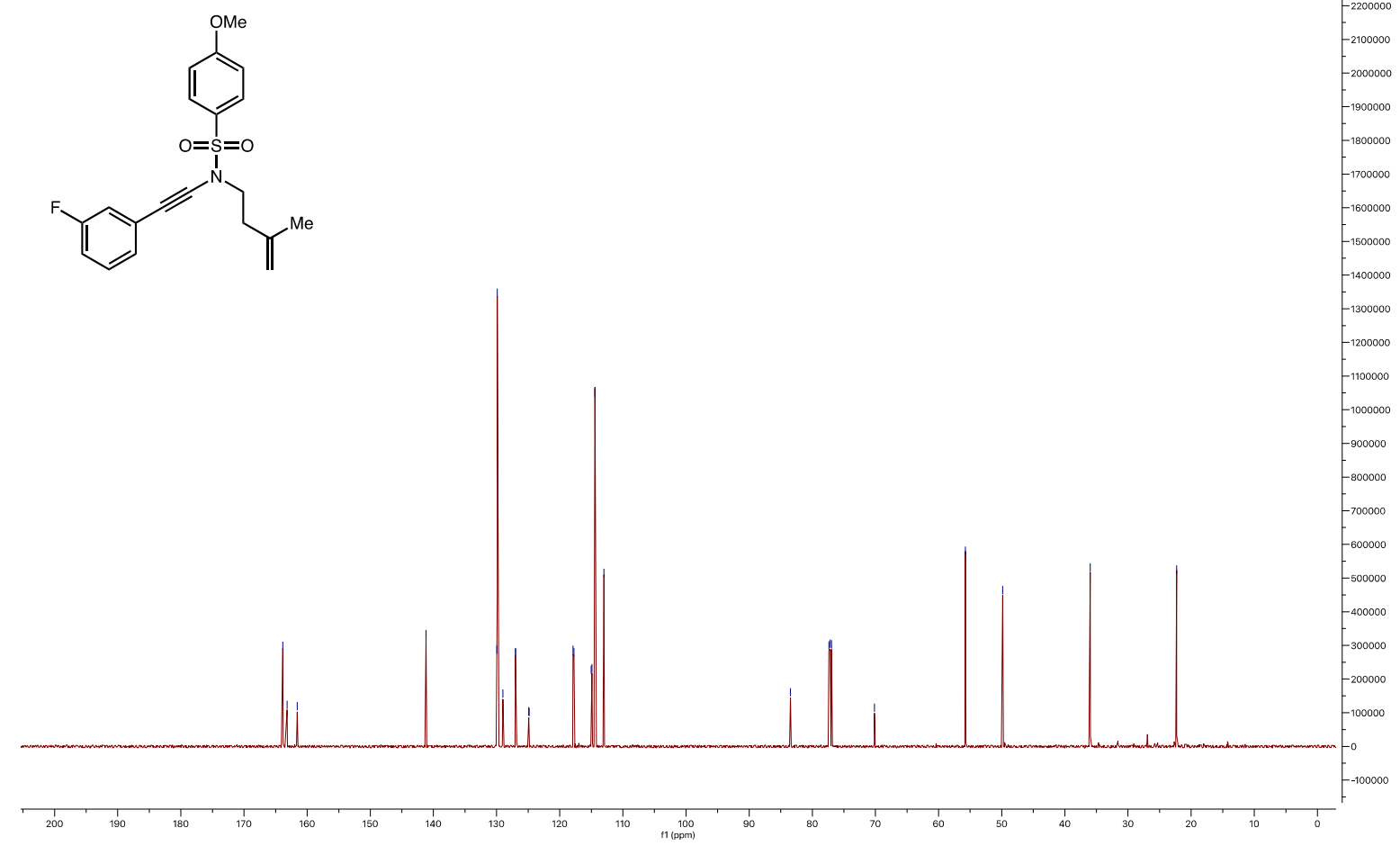

4a ${ }^{13} \mathrm{C}$ NMR (151 MHz, $\left.\mathrm{CDCl}_{3}\right)$ 


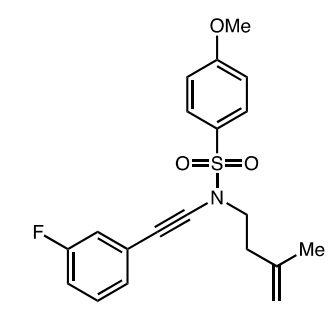

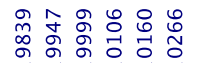

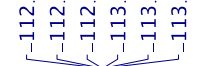

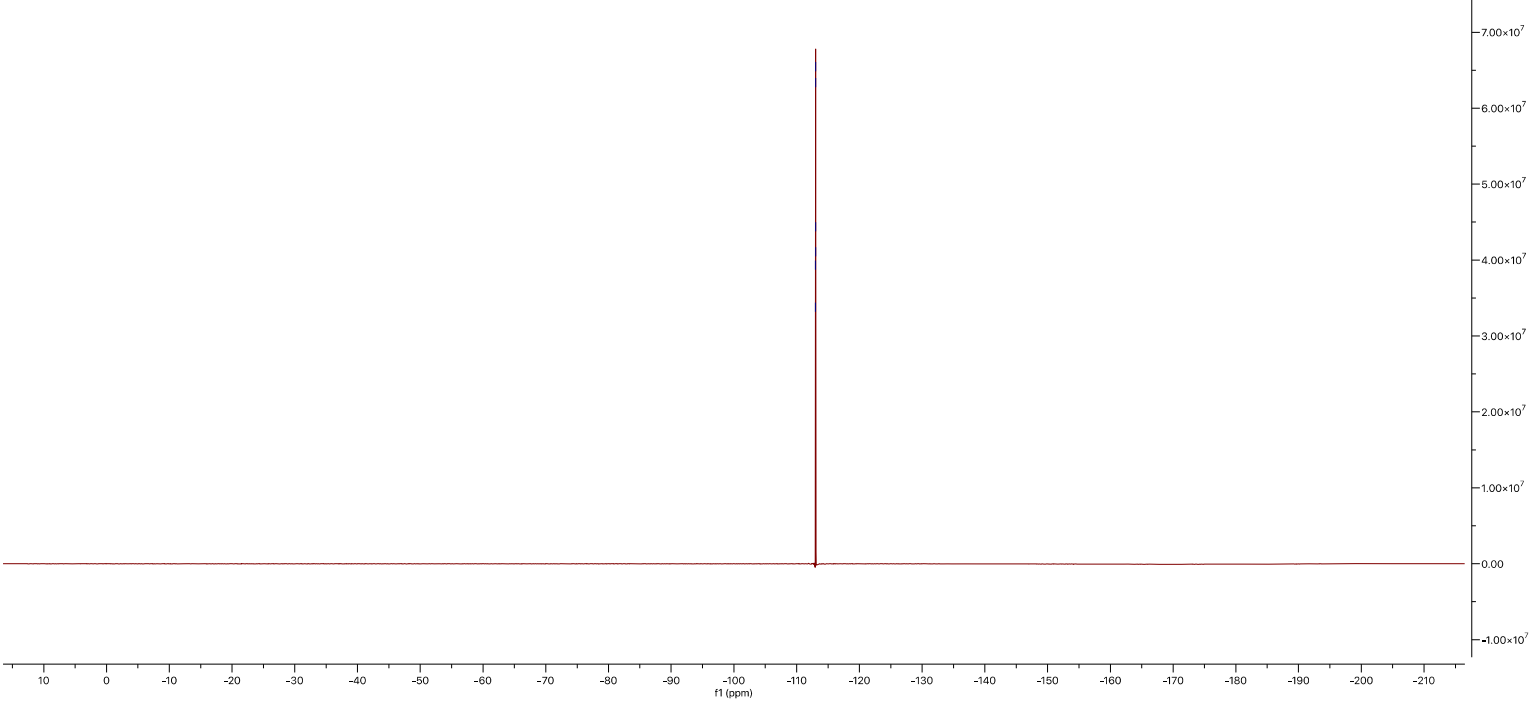

4a ${ }^{19}$ F NMR (565 MHz, $\mathrm{CDCl}_{3}$ )

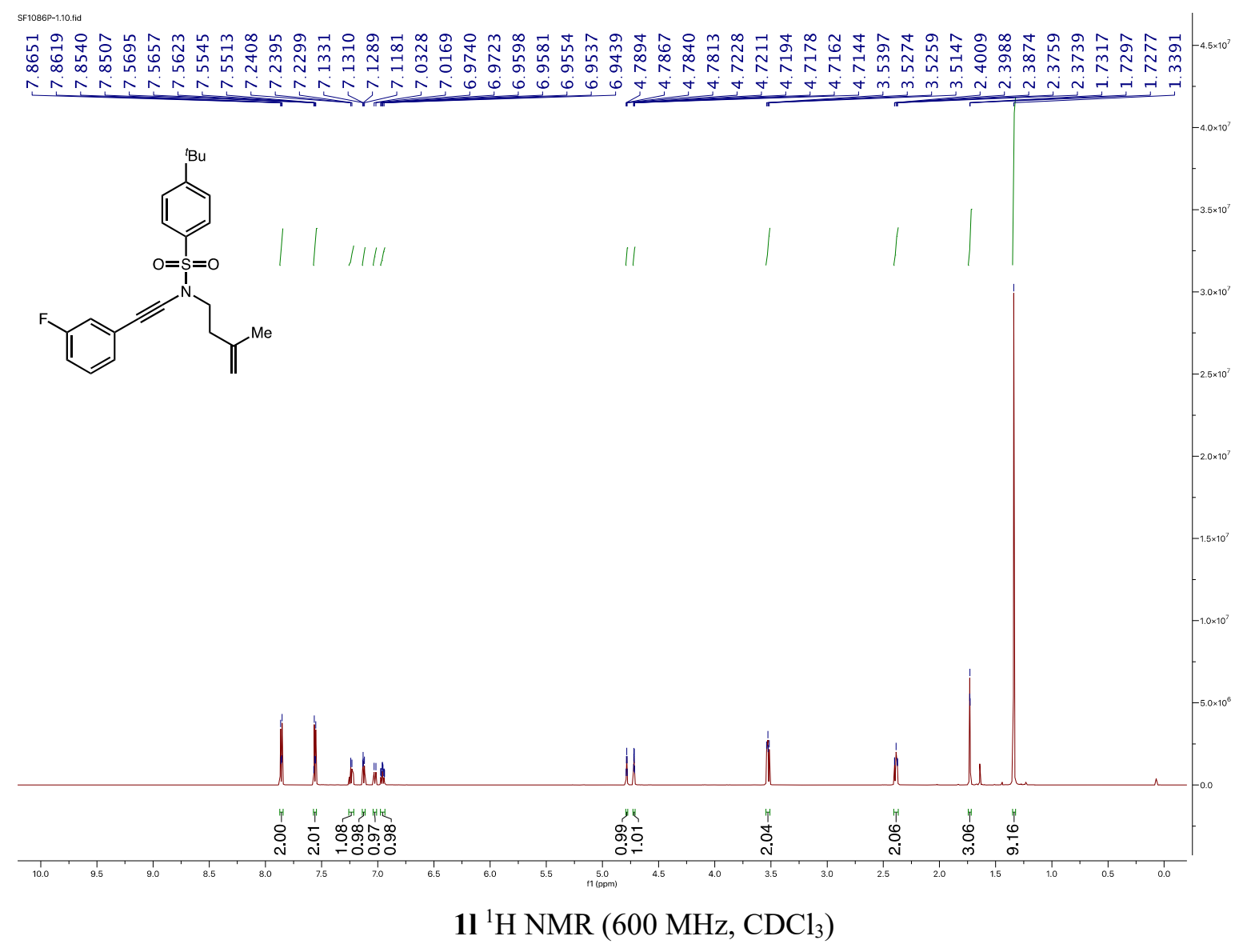



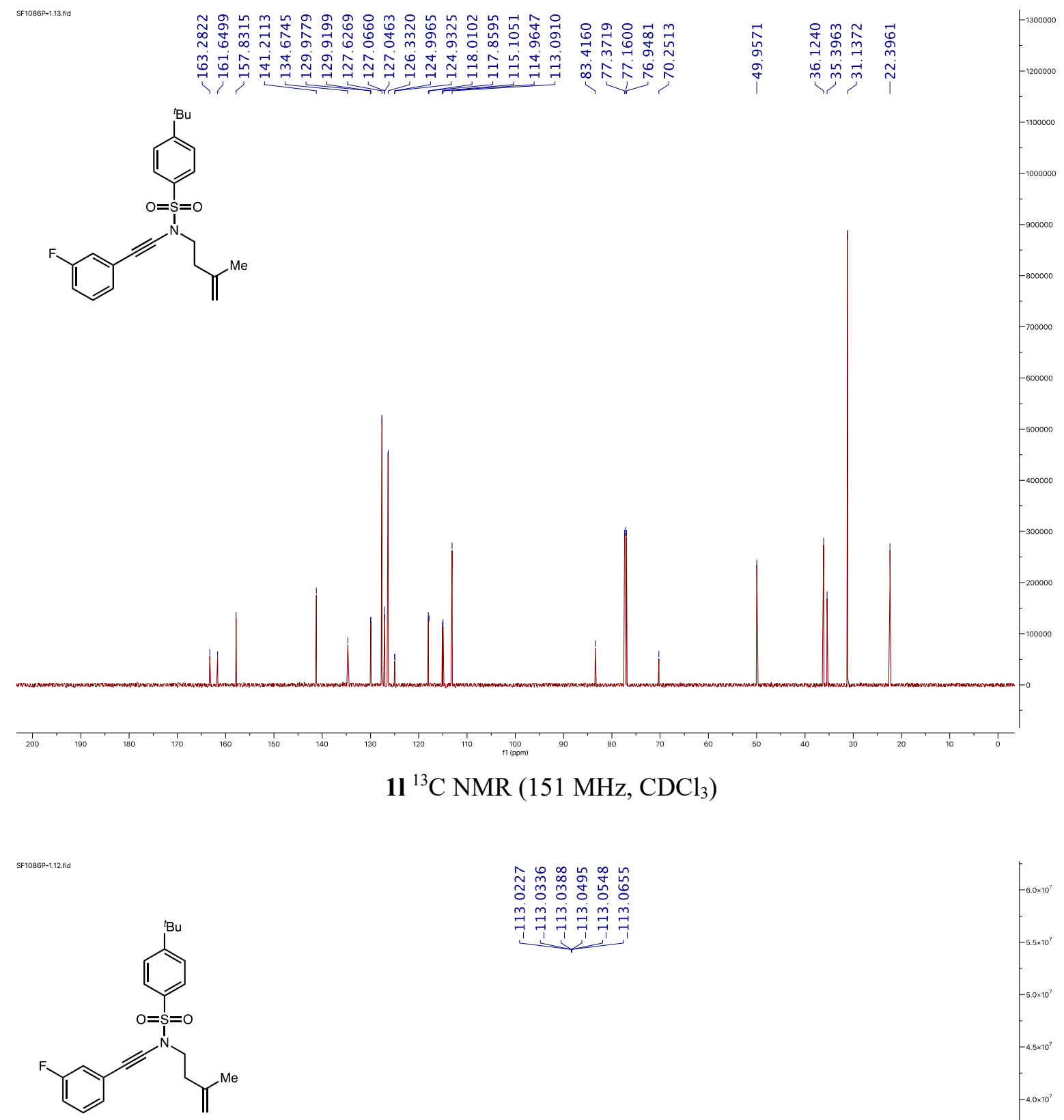

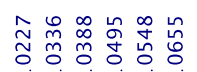

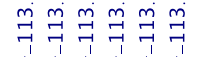

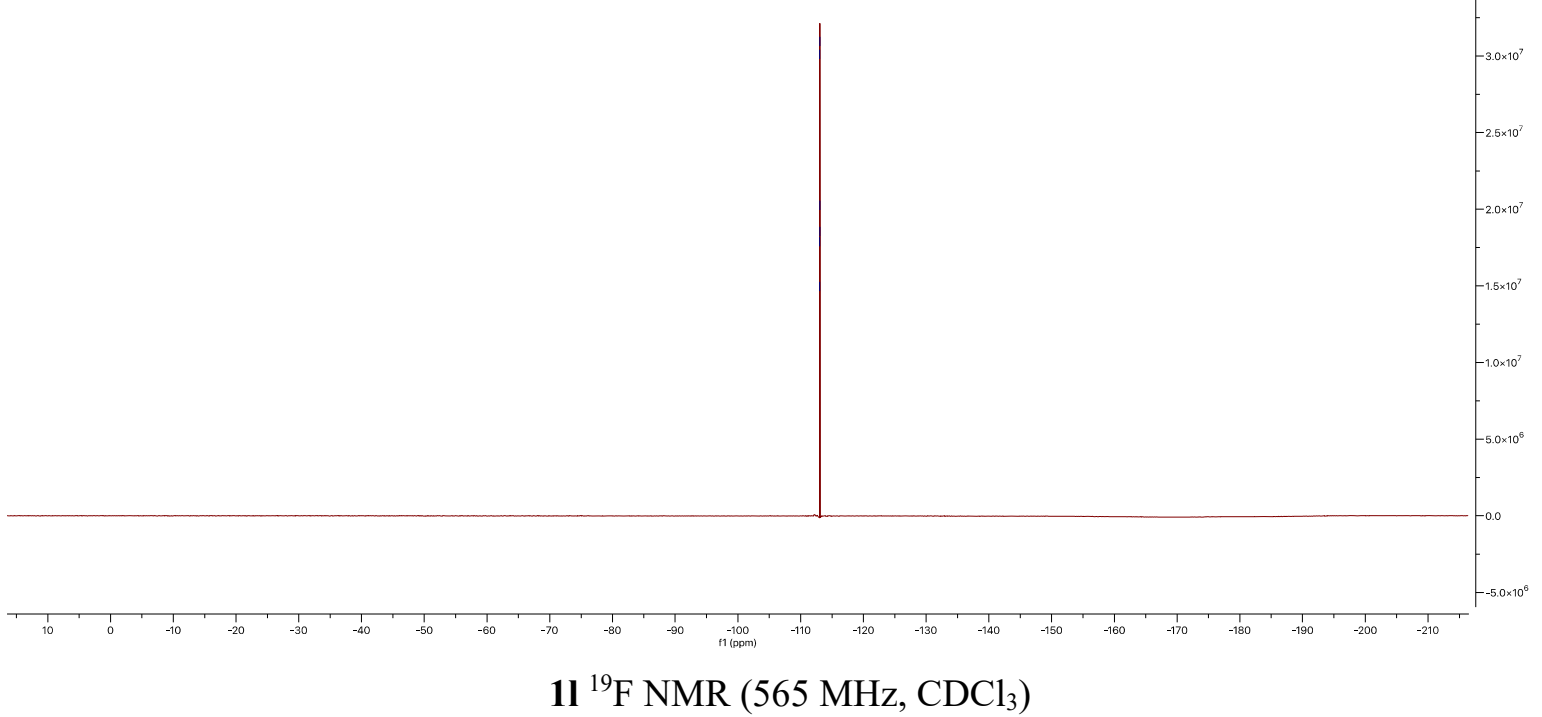




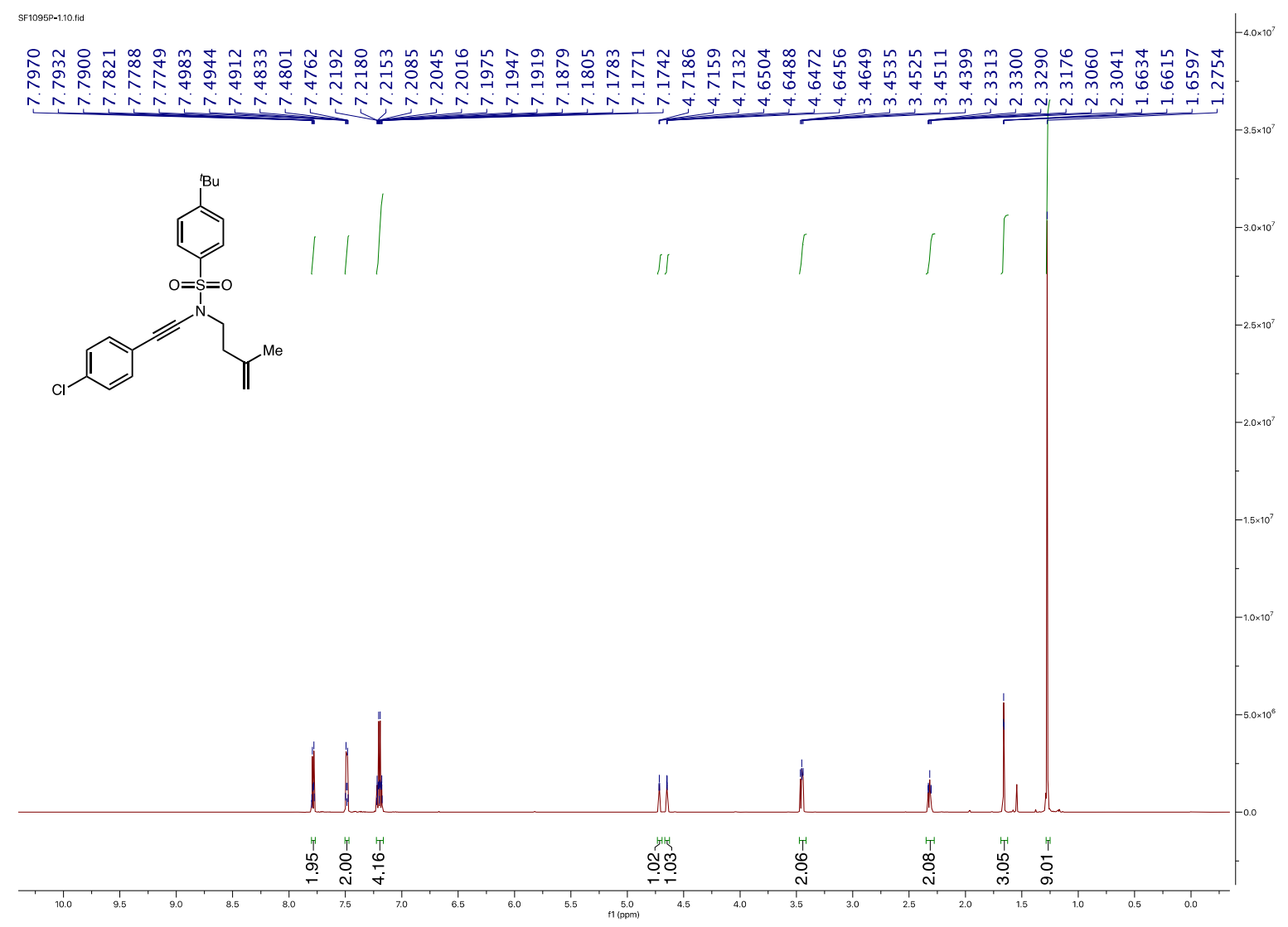

1 m ${ }^{1} \mathrm{H}$ NMR (600 MHz, $\left.\mathrm{CDCl}_{3}\right)$

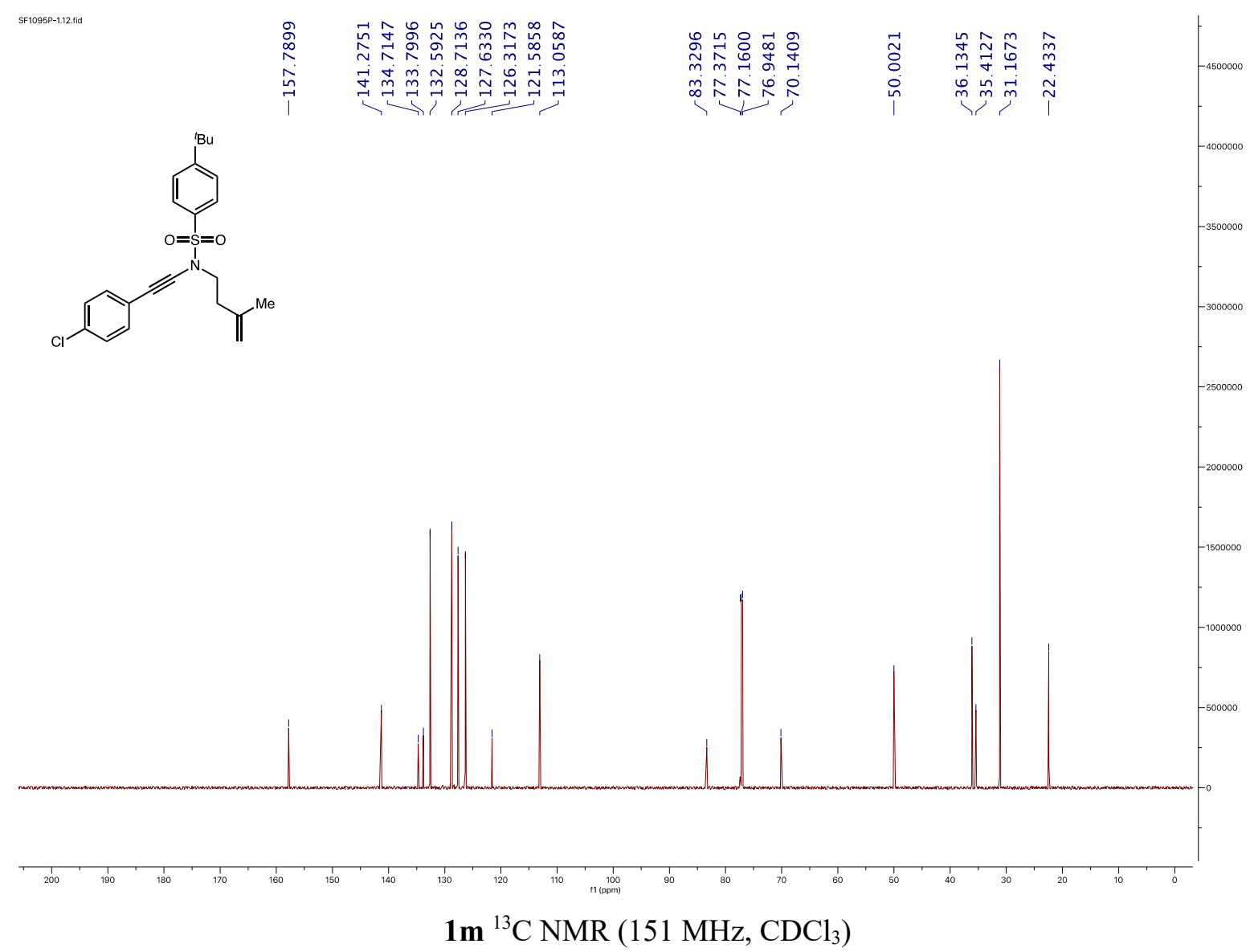




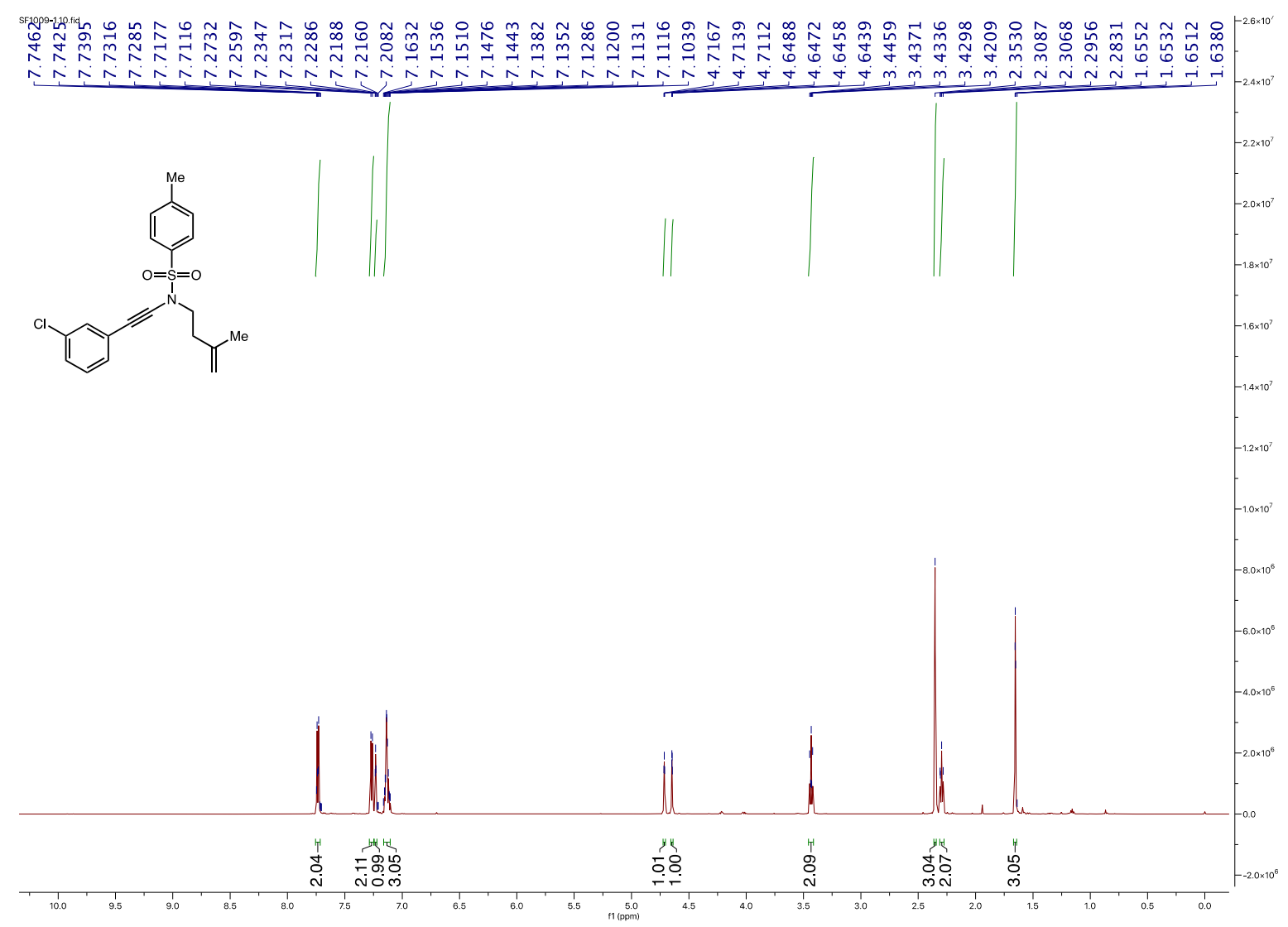

1k ${ }^{1} \mathrm{H}$ NMR (600 MHz, $\left.\mathrm{CDCl}_{3}\right)$

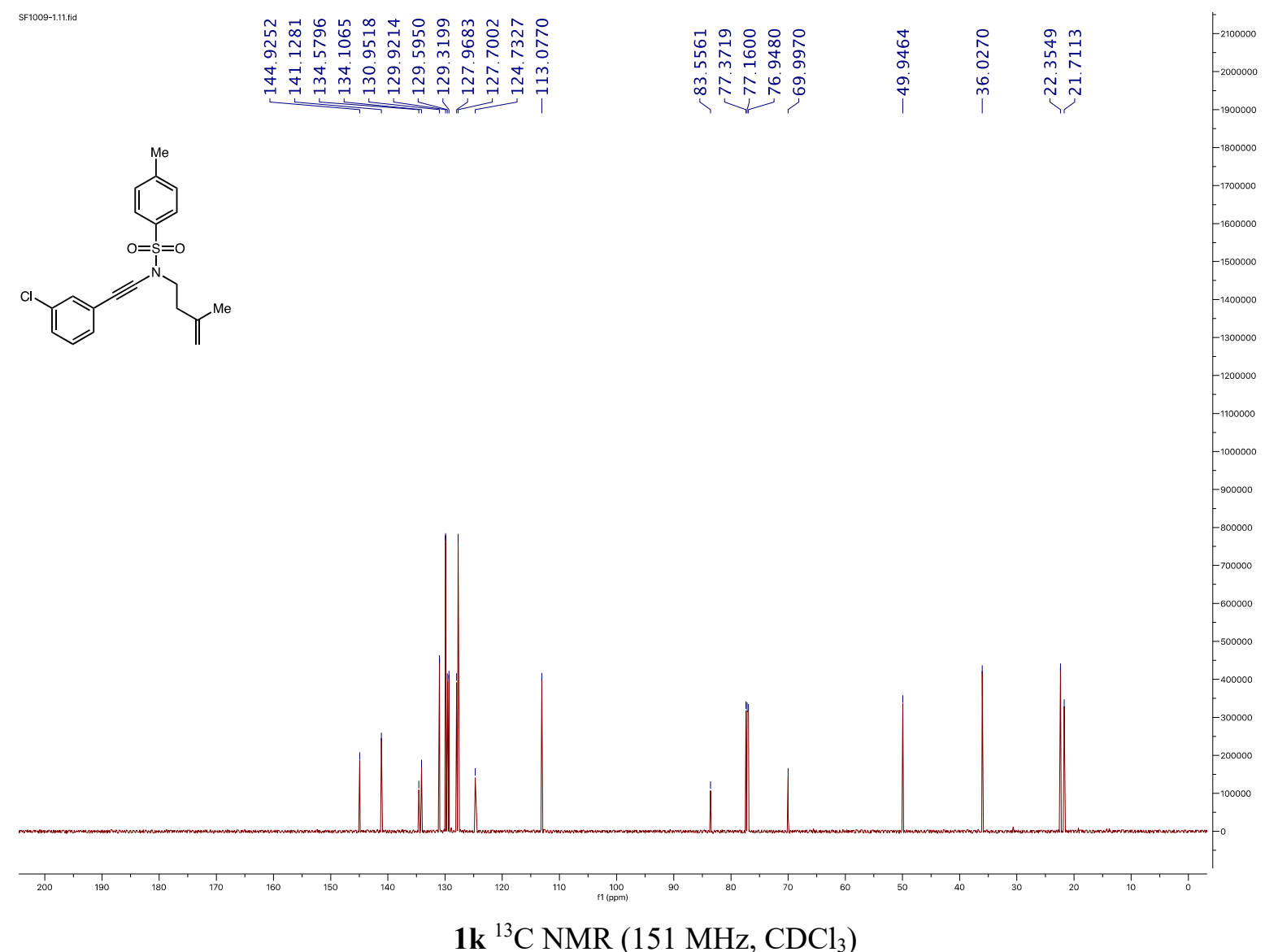



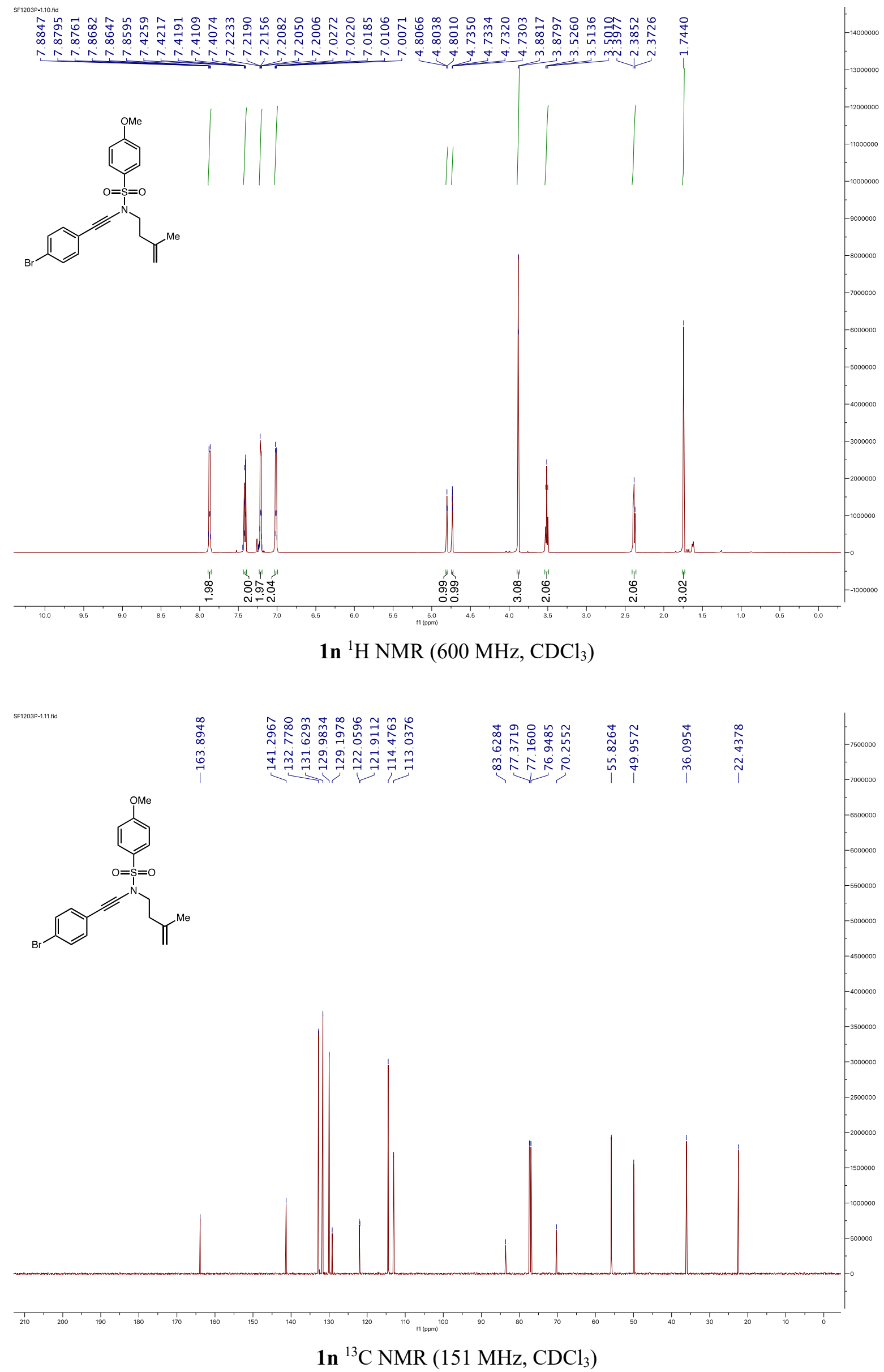

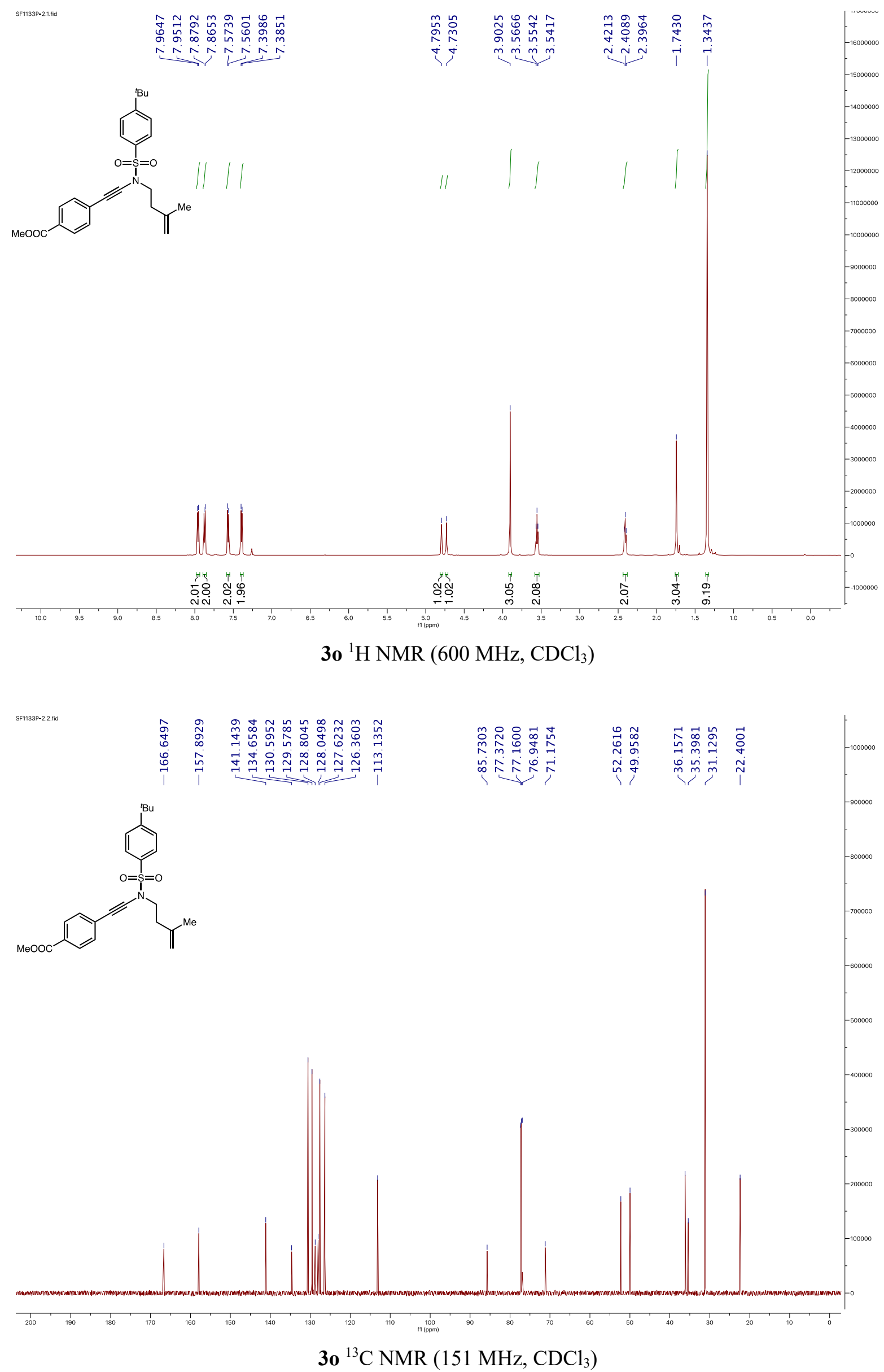


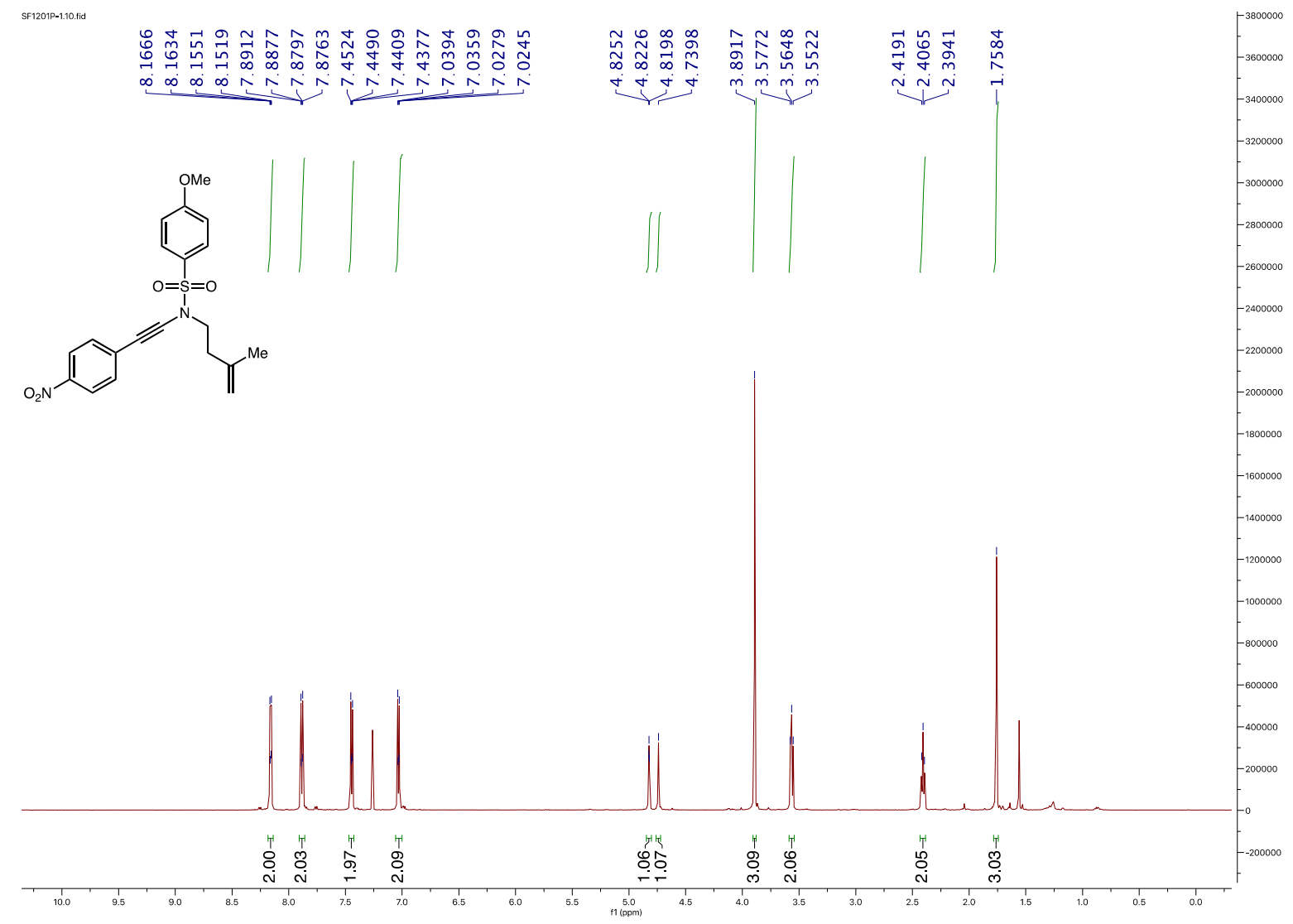

4b ${ }^{1} \mathrm{H}$ NMR (600 MHz, $\left.\mathrm{CDCl}_{3}\right)$

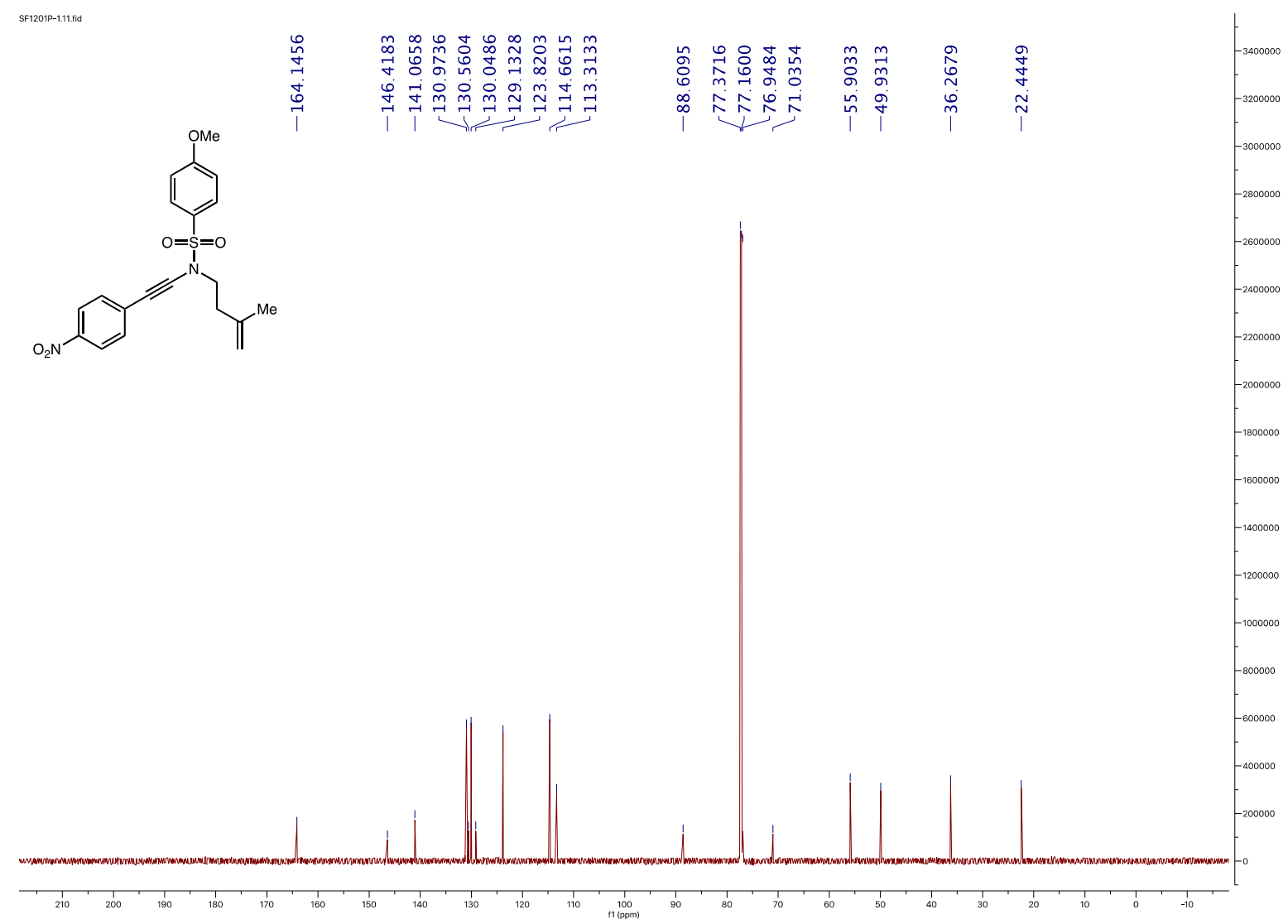

4b ${ }^{13} \mathrm{C}$ NMR (151 MHz, $\left.\mathrm{CDCl}_{3}\right)$ 

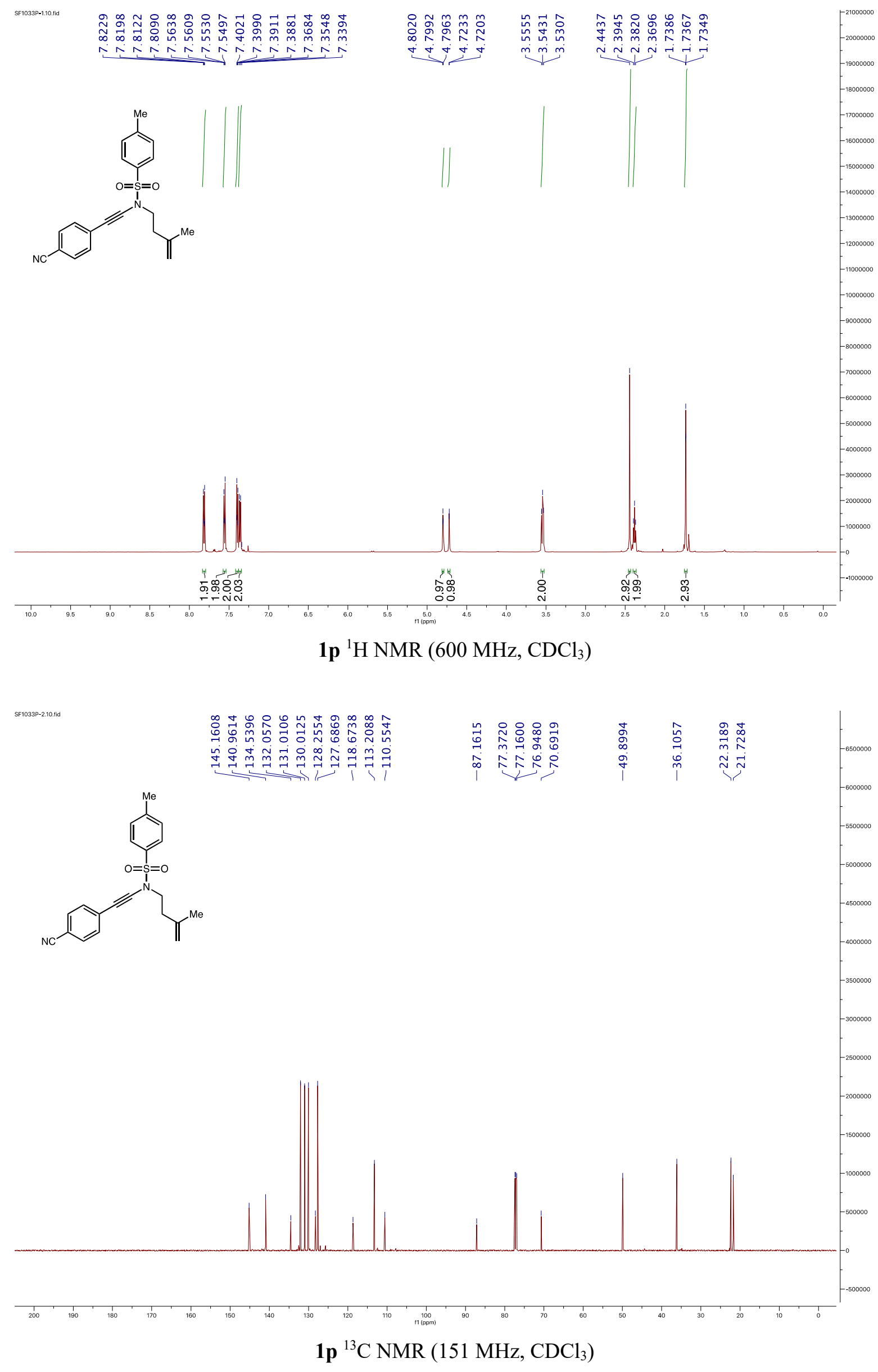


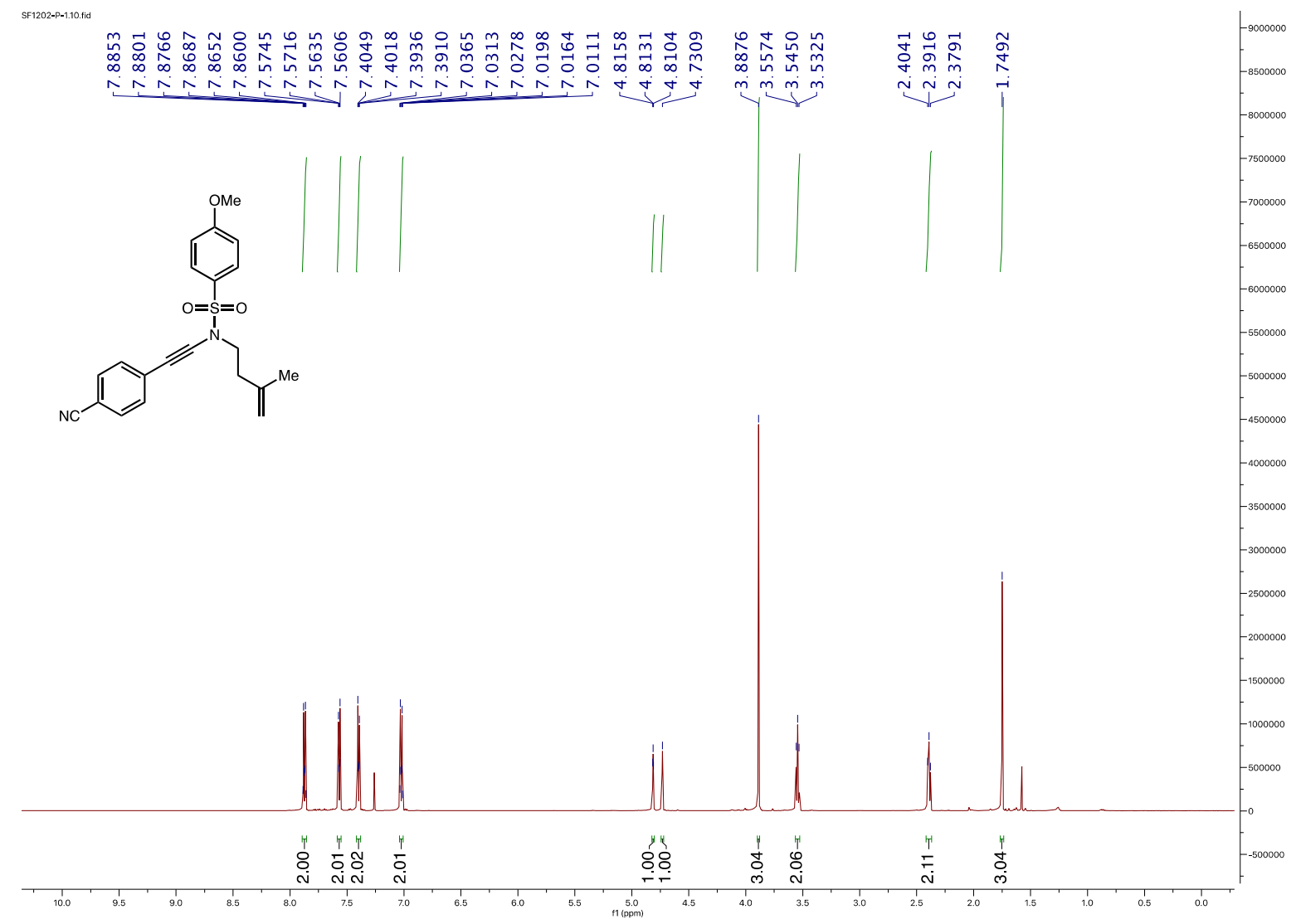

1q ${ }^{1} \mathrm{H}$ NMR $\left(600 \mathrm{MHz}, \mathrm{CDCl}_{3}\right)$

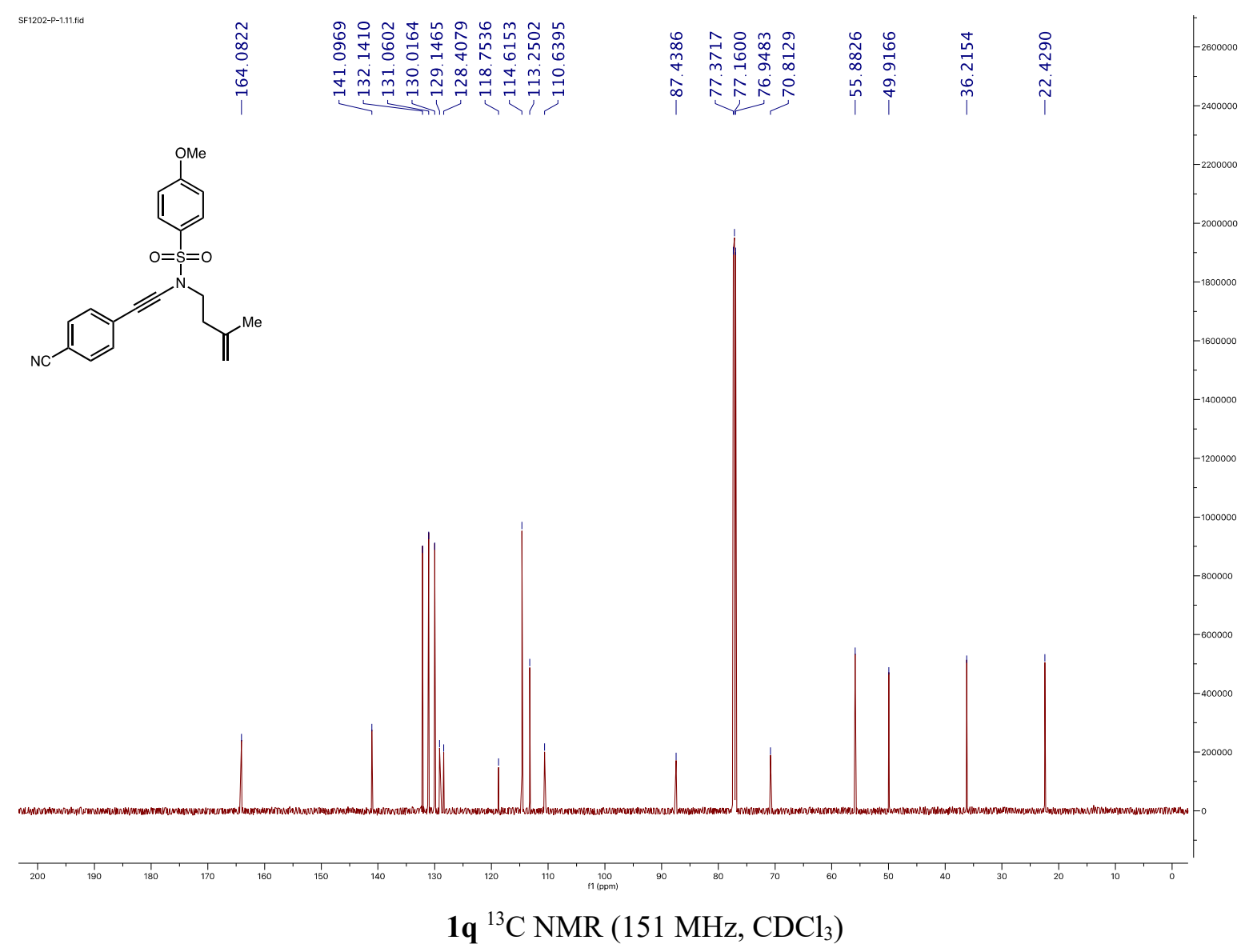




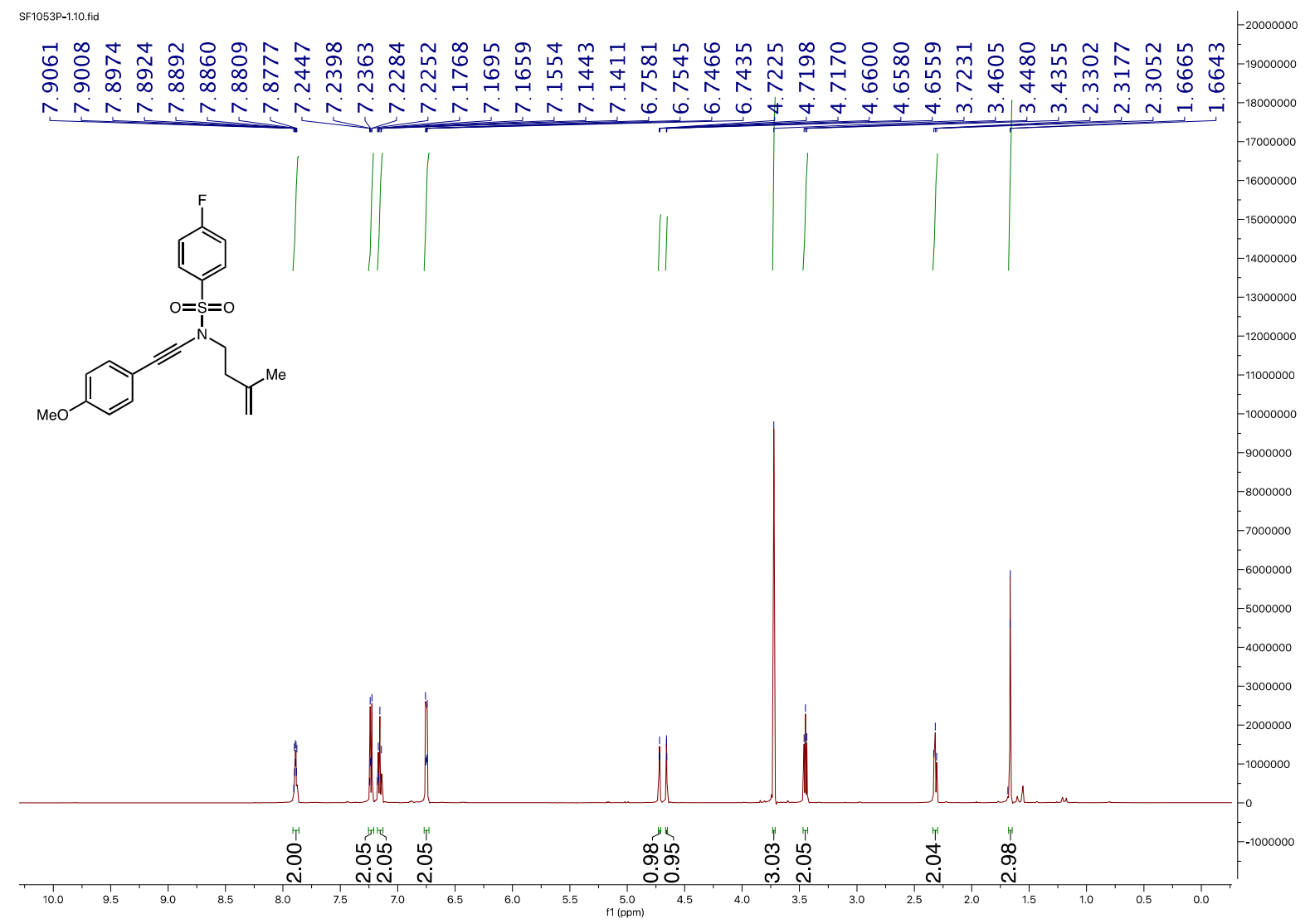

1t ${ }^{1} \mathrm{H}$ NMR (600 MHz, $\left.\mathrm{CDCl}_{3}\right)$

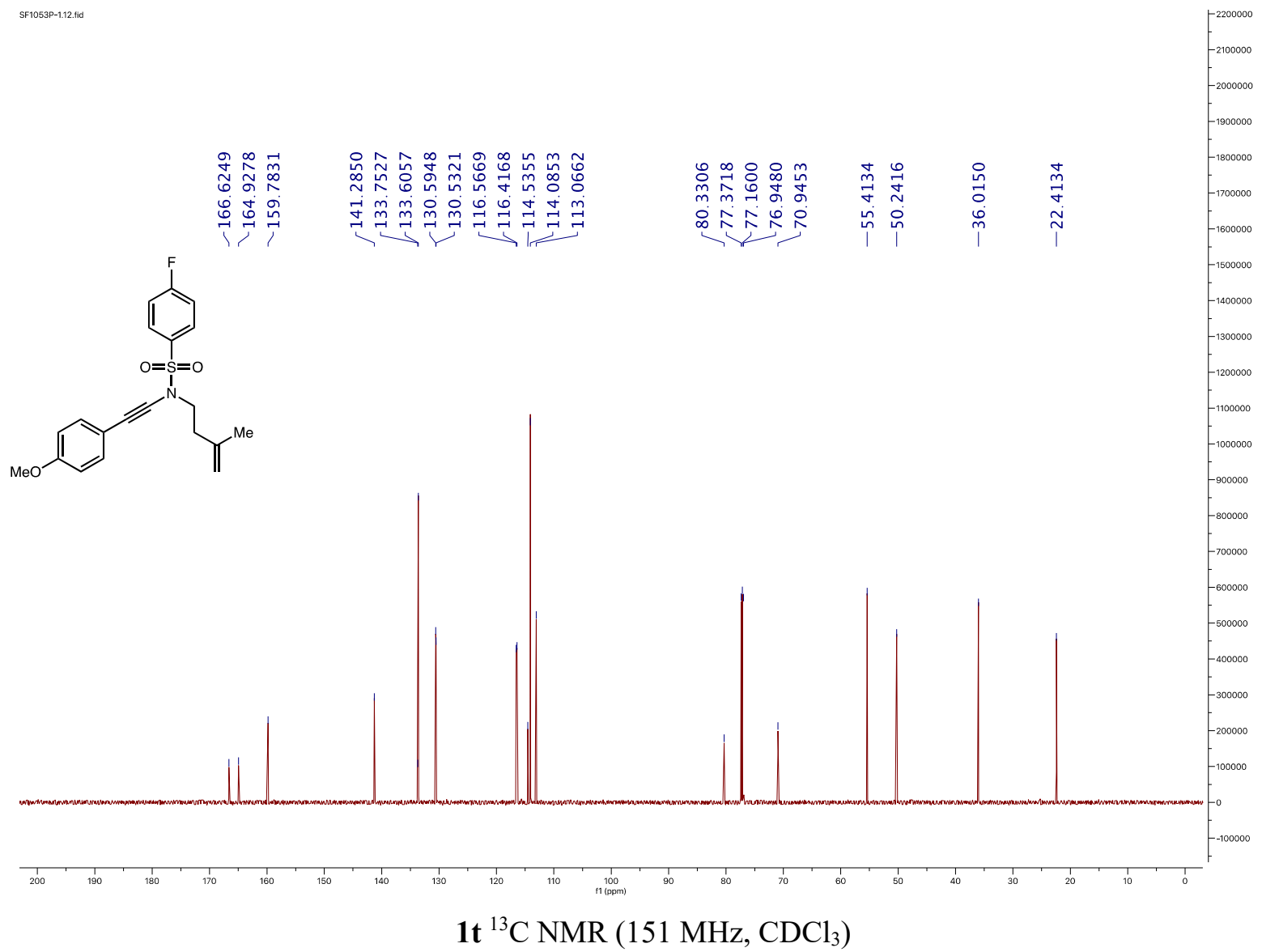




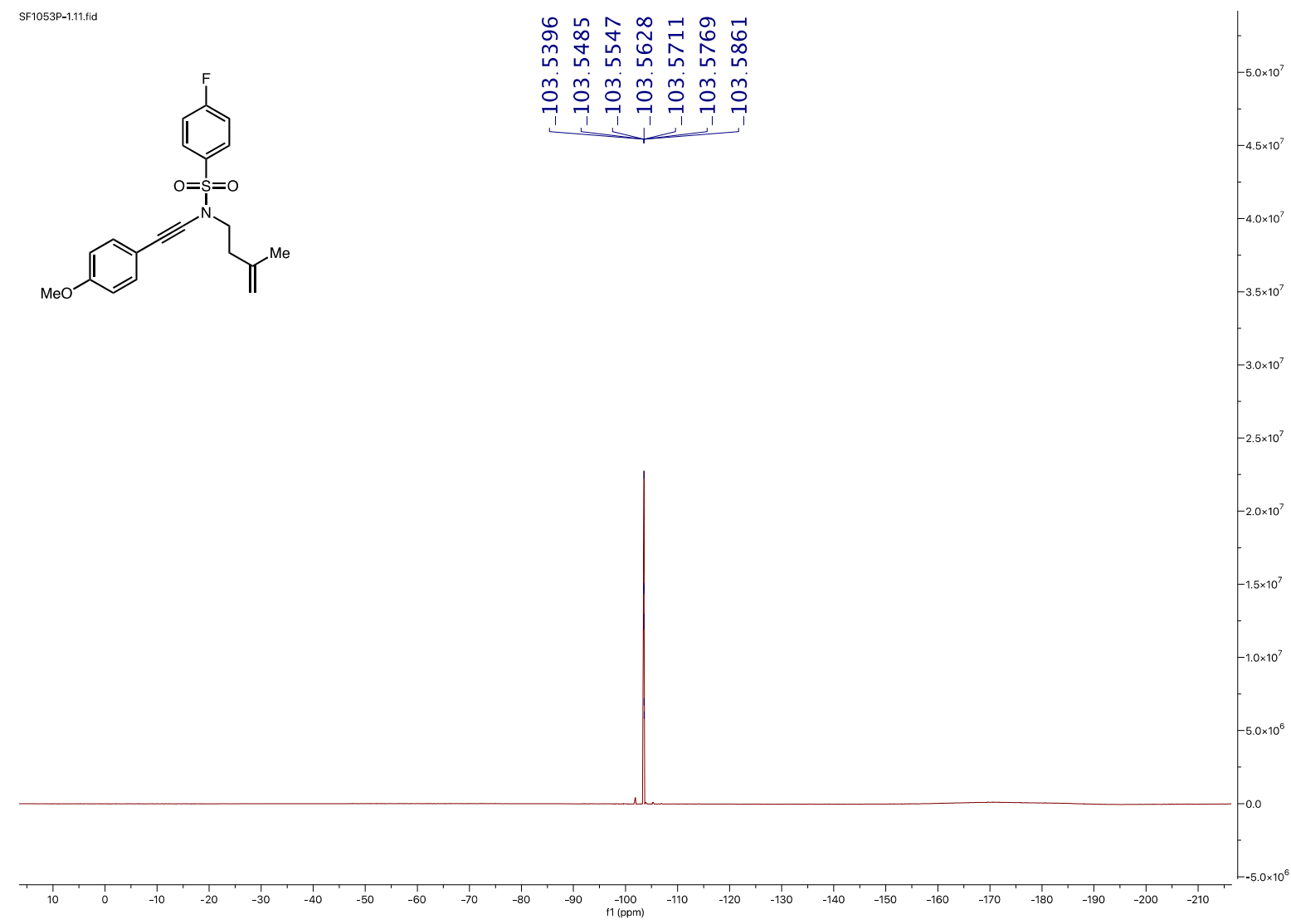

1t ${ }^{19} \mathrm{~F}$ NMR (565 MHz, $\mathrm{CDCl}_{3}$ )

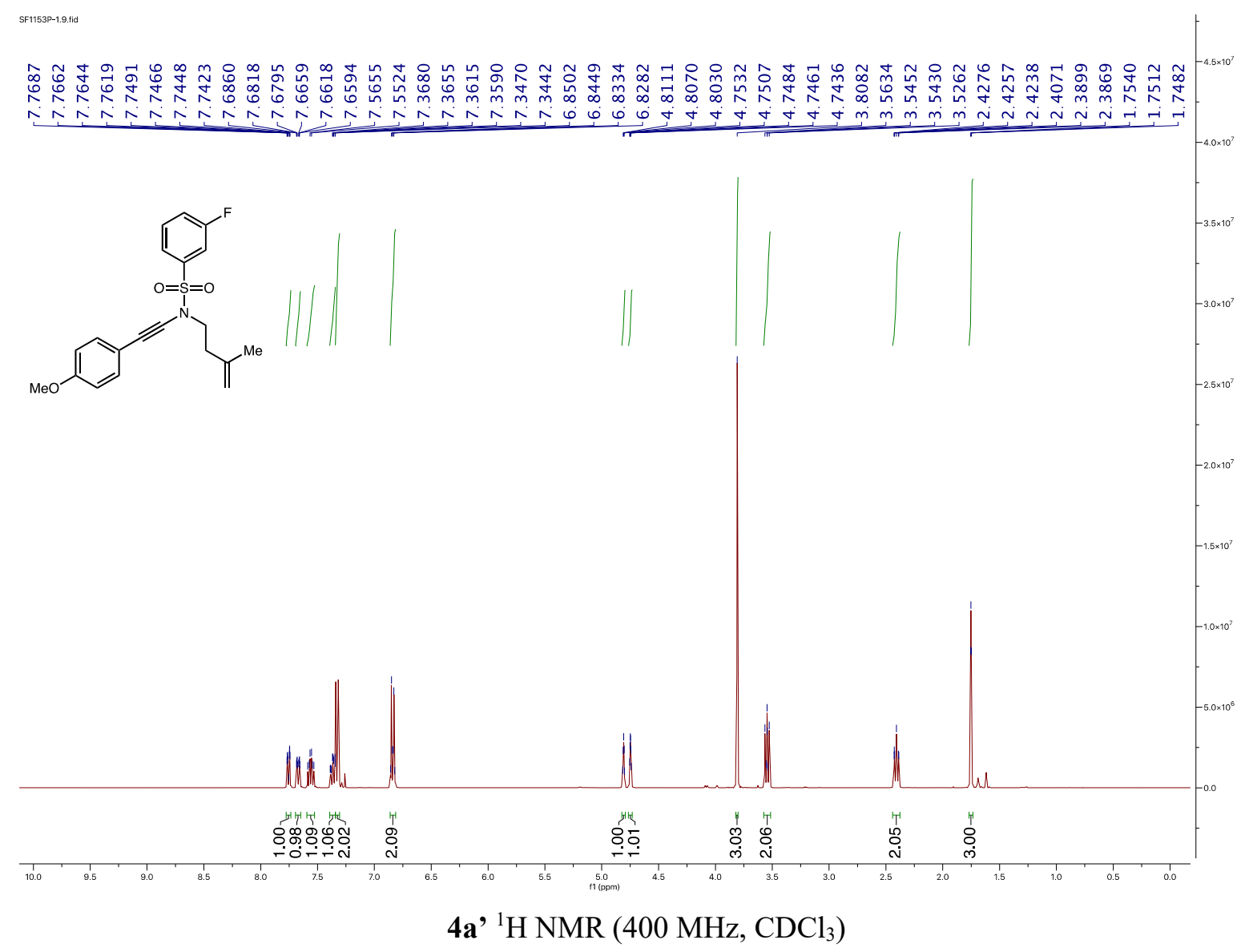




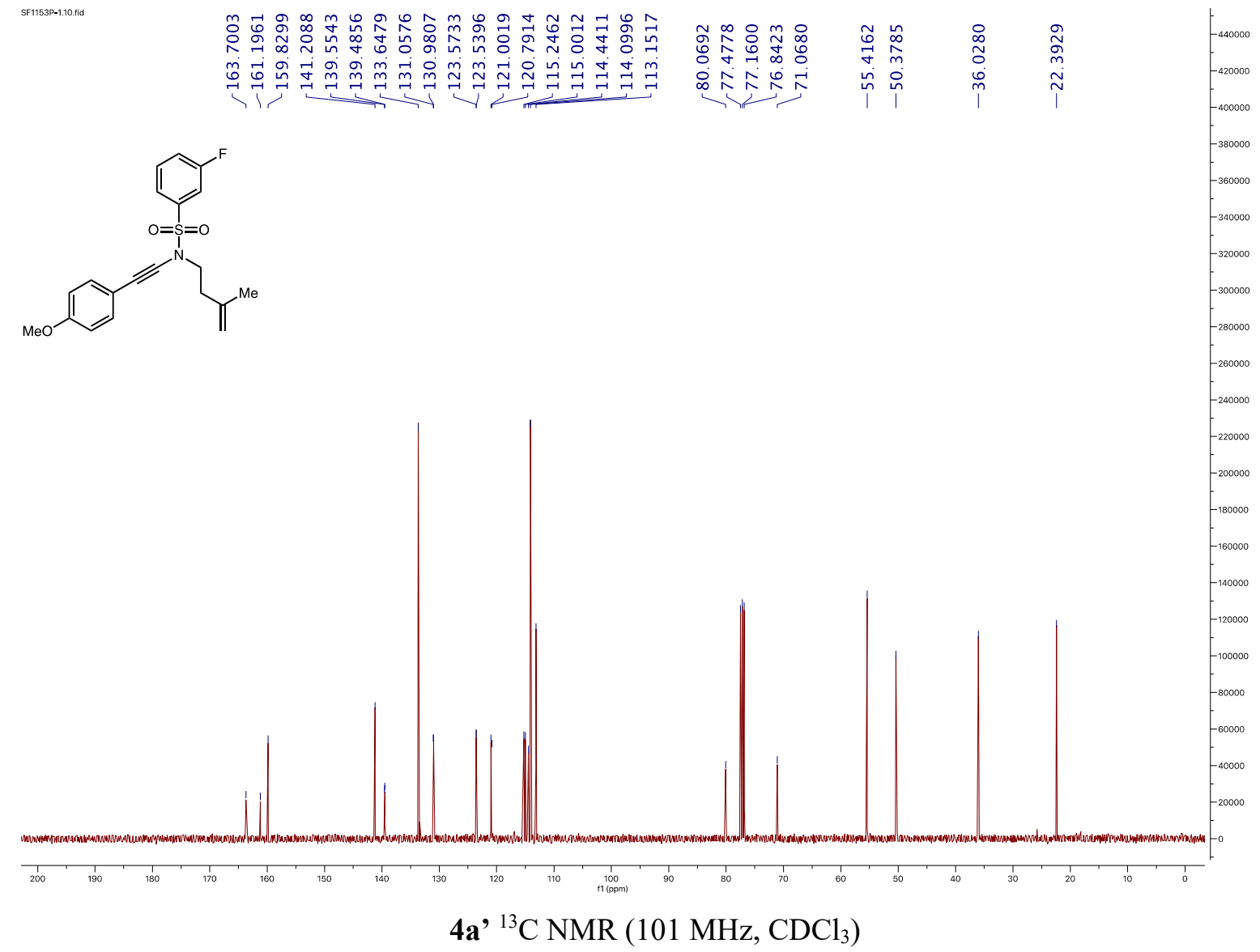

SF1153P-1111. fd

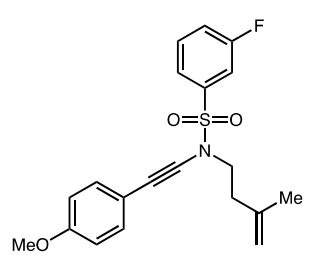

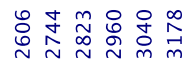

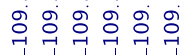

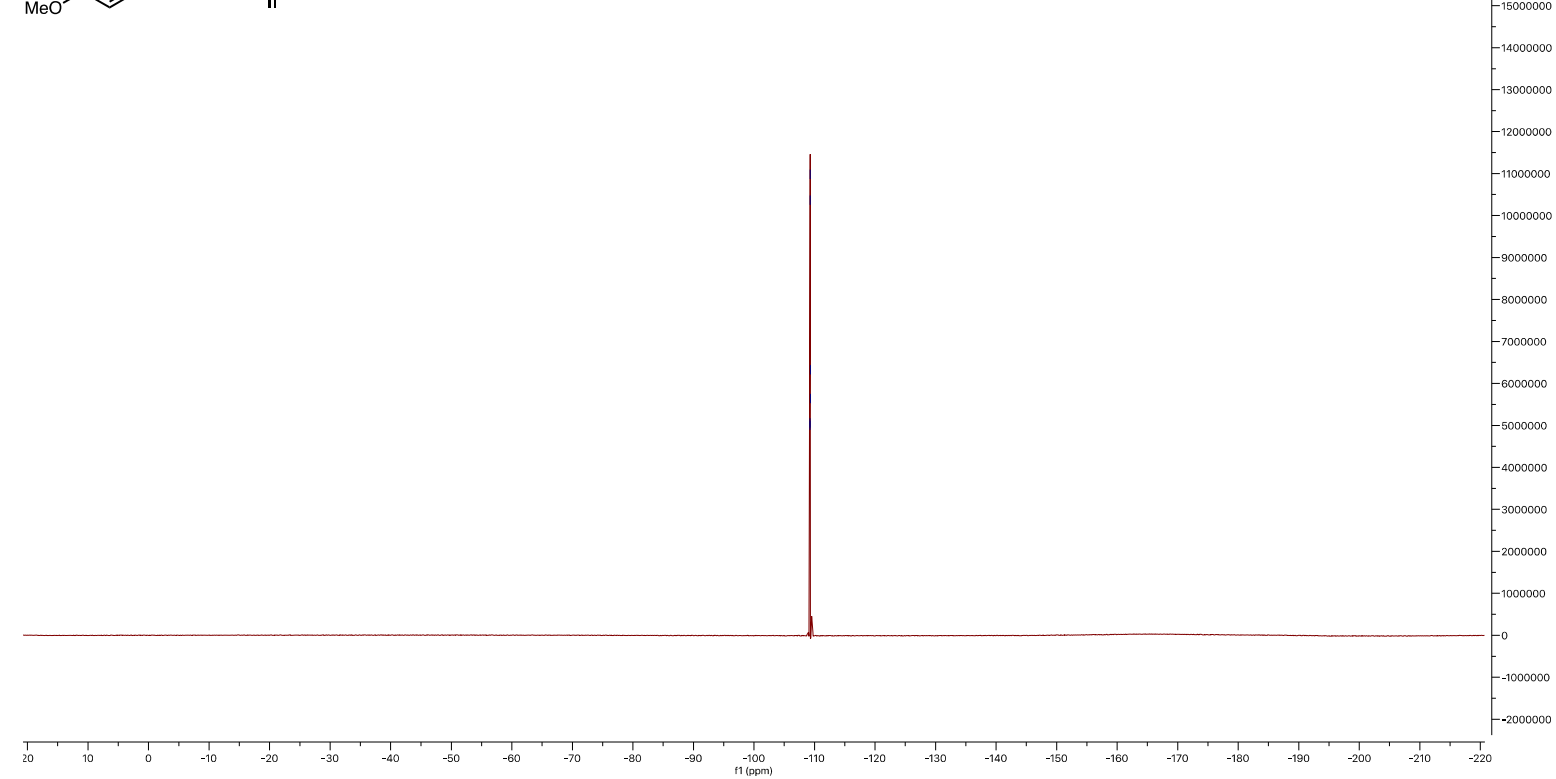

4a ${ }^{19}$ F NMR (376 MHz, $\mathrm{CDCl}_{3}$ ) 


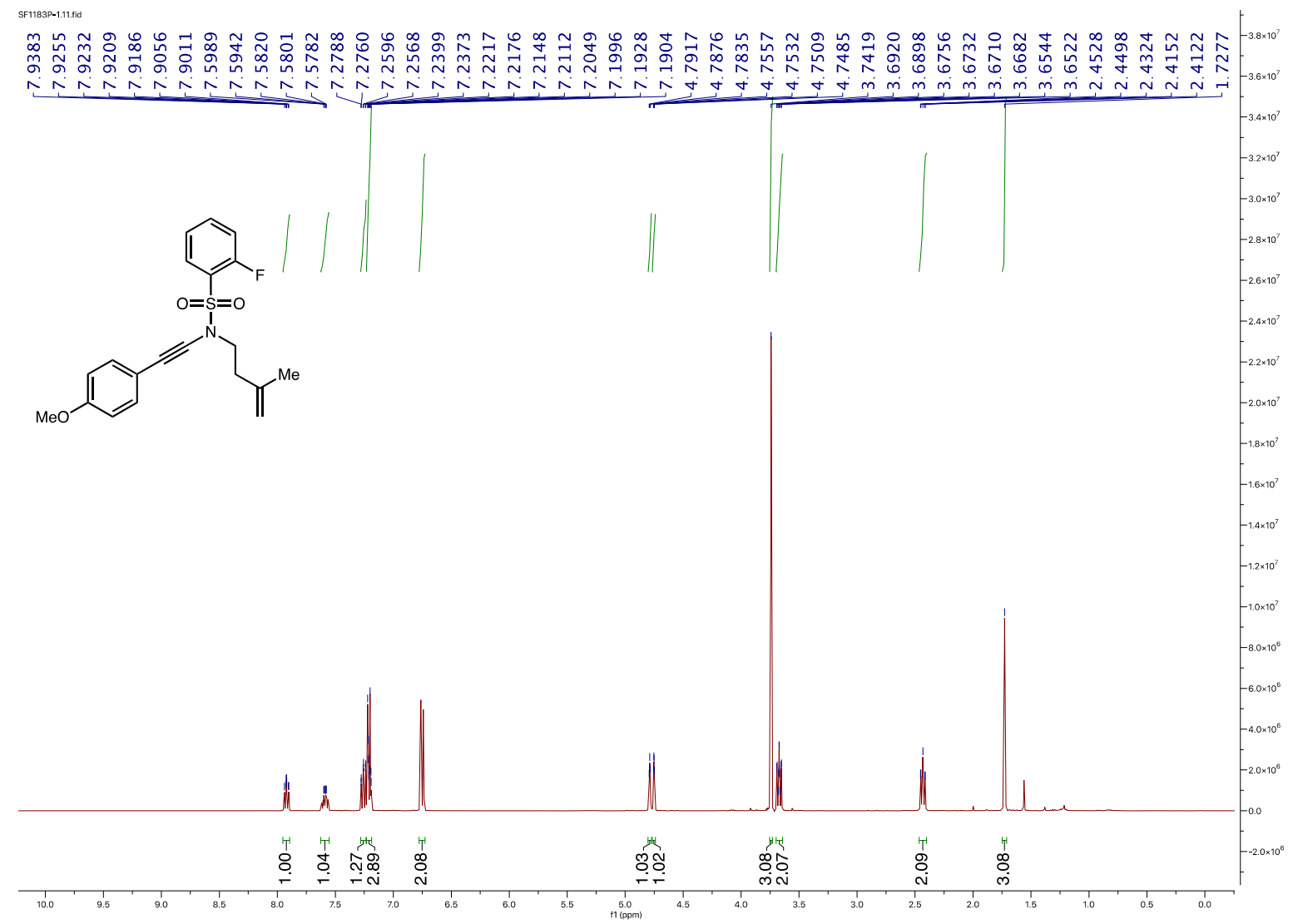

1u ${ }^{1} \mathrm{H}$ NMR (400 MHz, $\left.\mathrm{CDCl}_{3}\right)$

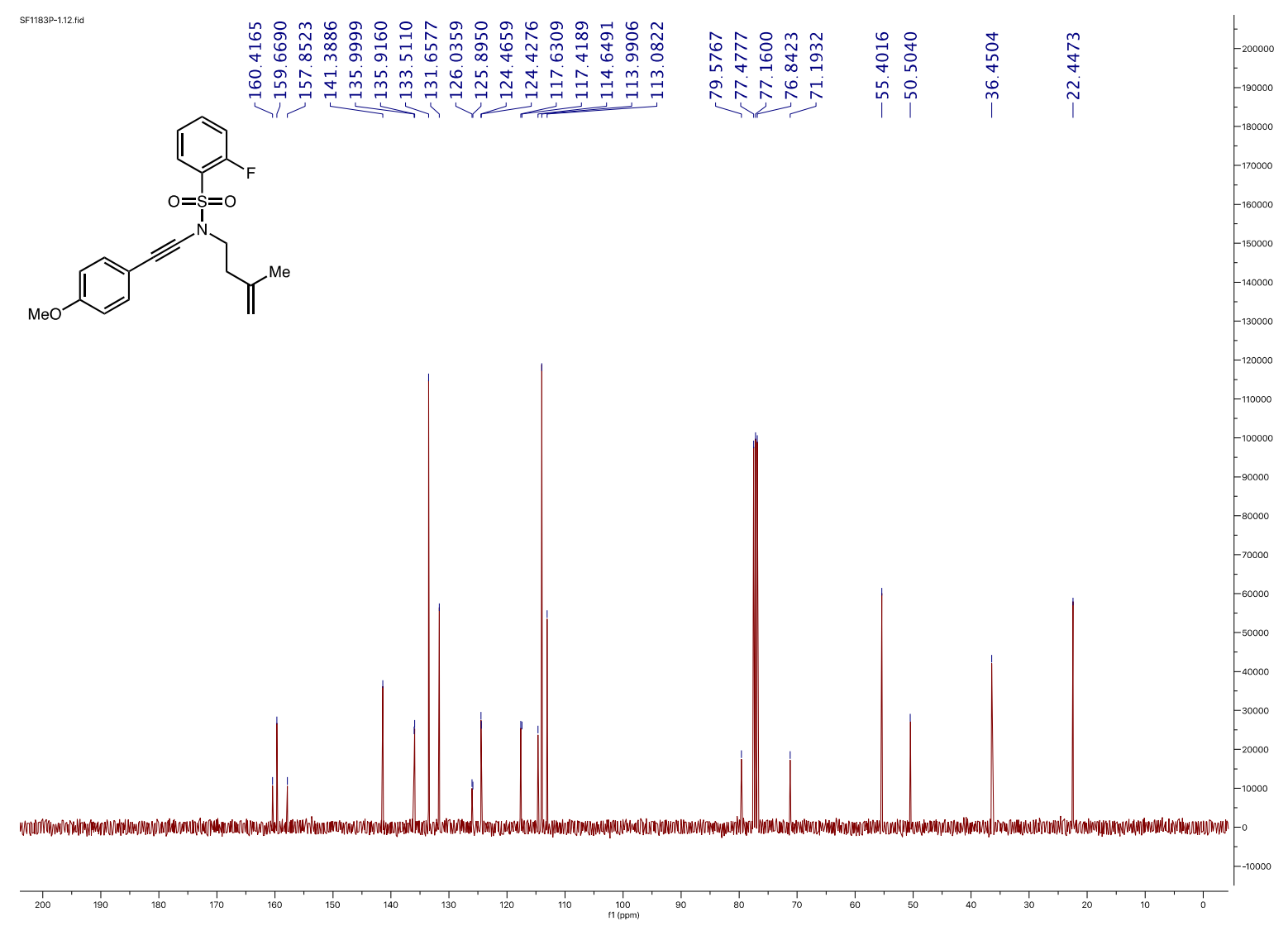

1u $\left.{ }^{13} \mathrm{C} \mathrm{NMR} \mathrm{(101} \mathrm{MHz,} \mathrm{CDCl}_{3}\right)$ 

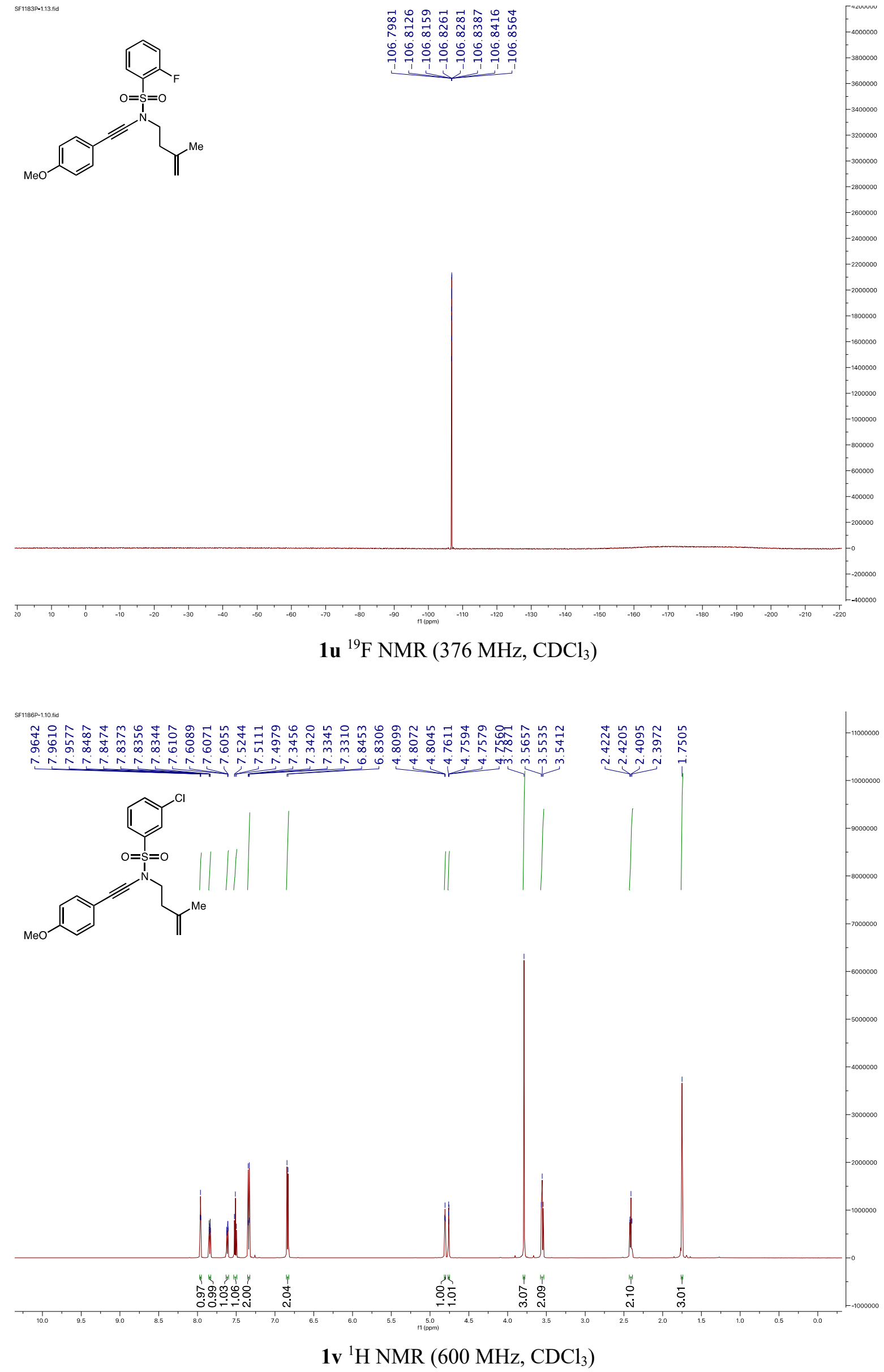

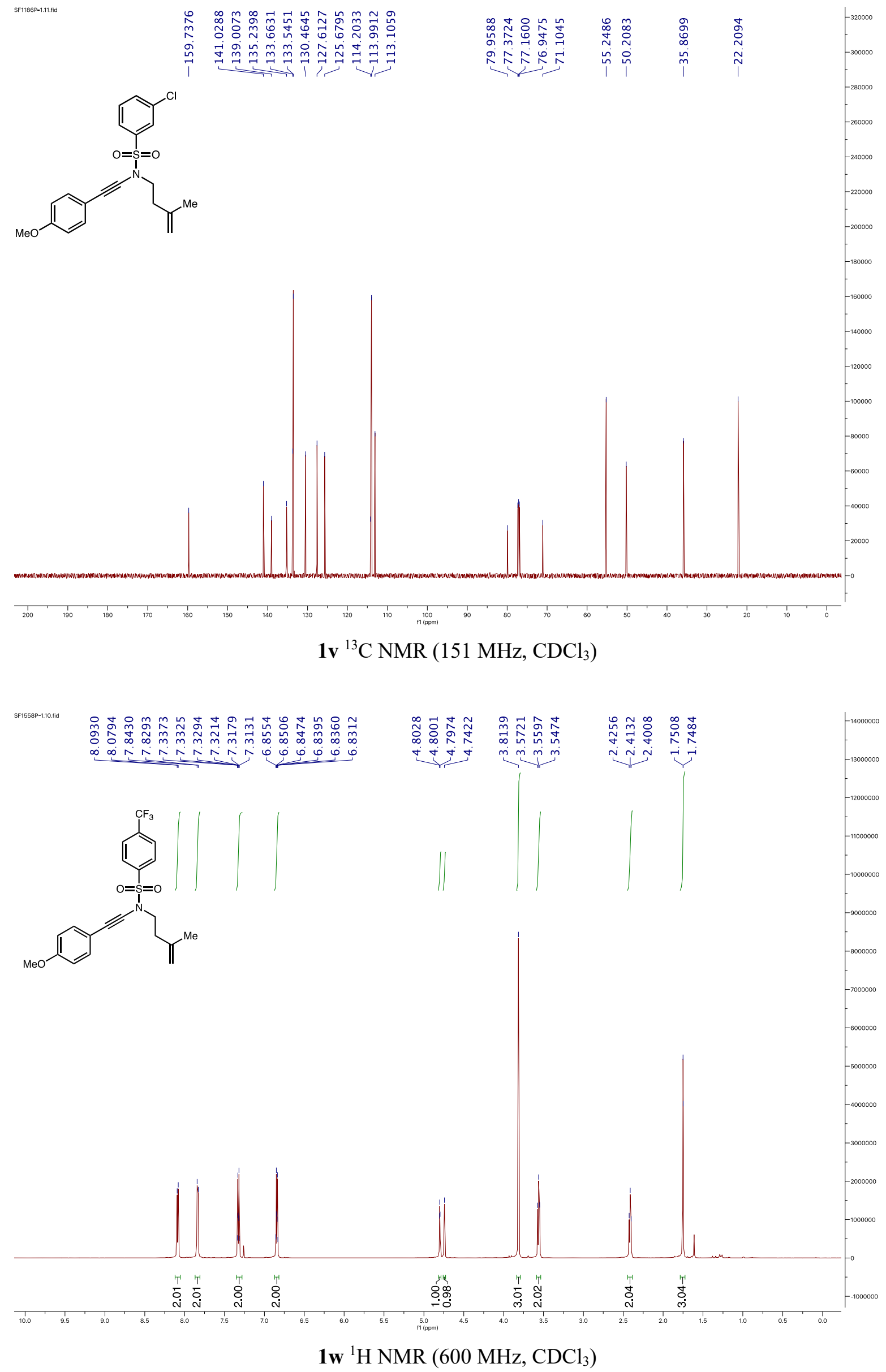


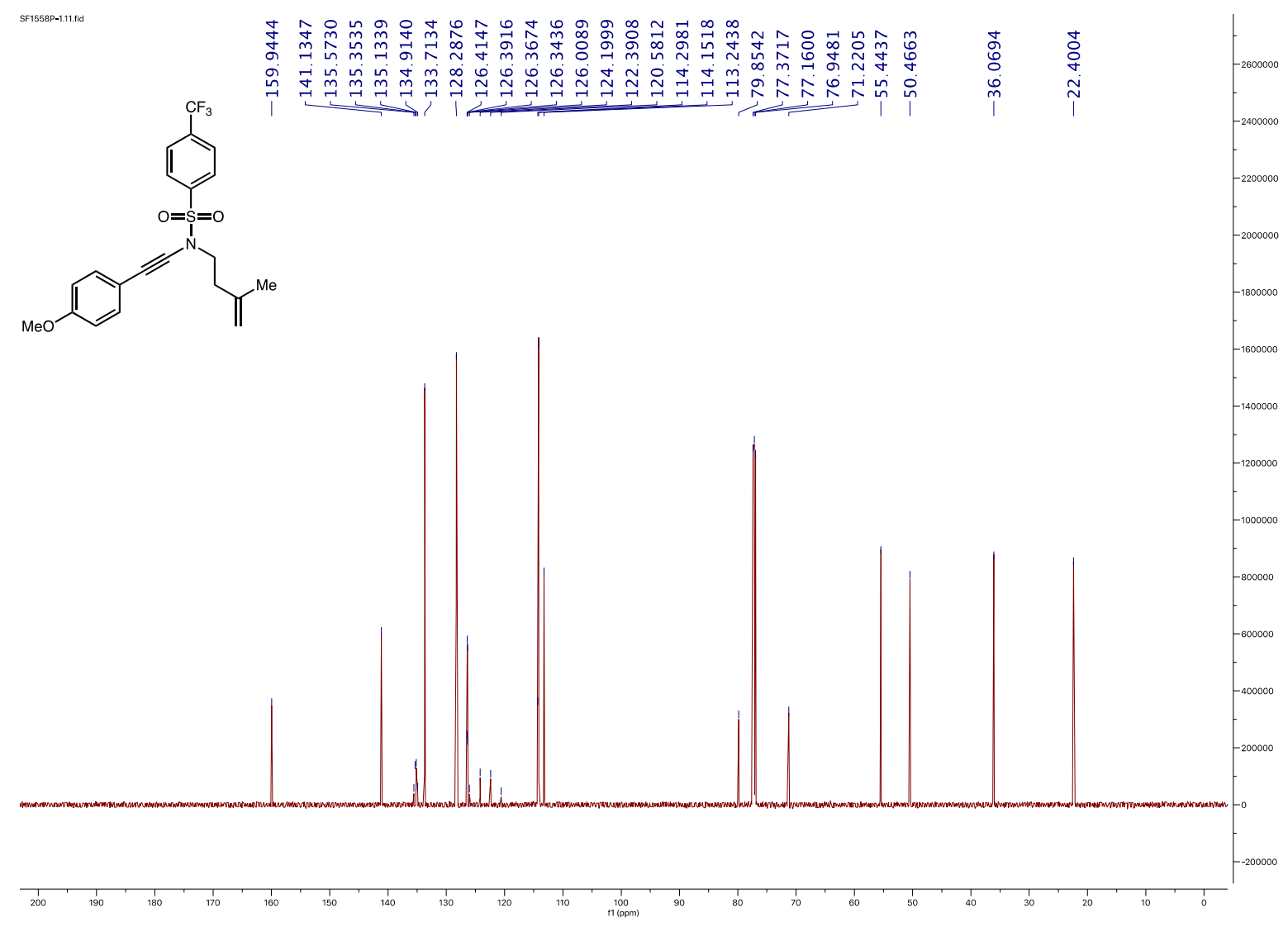

1w ${ }^{13} \mathrm{C}$ NMR (151 MHz, $\mathrm{CDCl}_{3}$ )

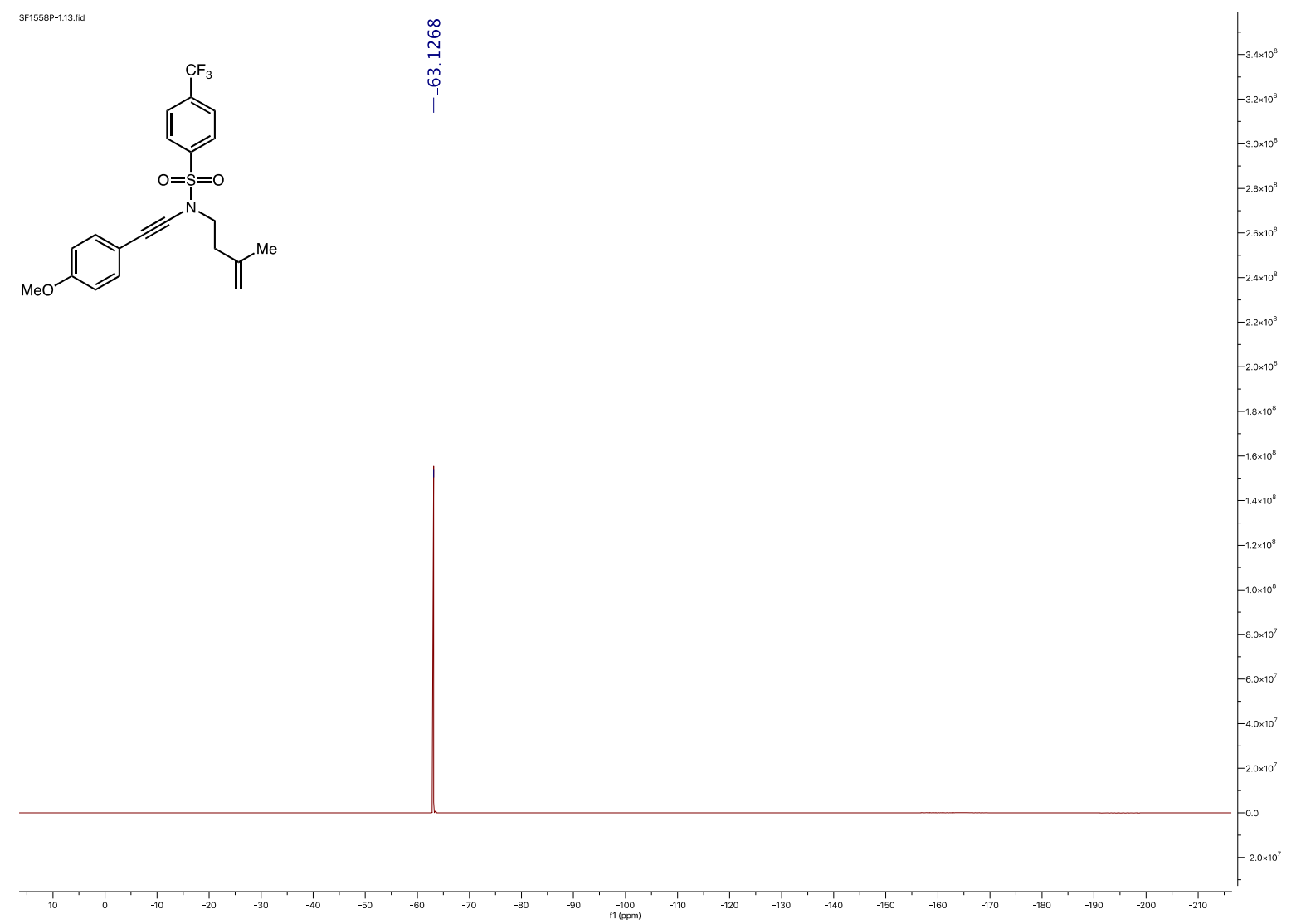

1w ${ }^{19} \mathrm{~F}$ NMR (565 MHz, $\mathrm{CDCl}_{3}$ ) 


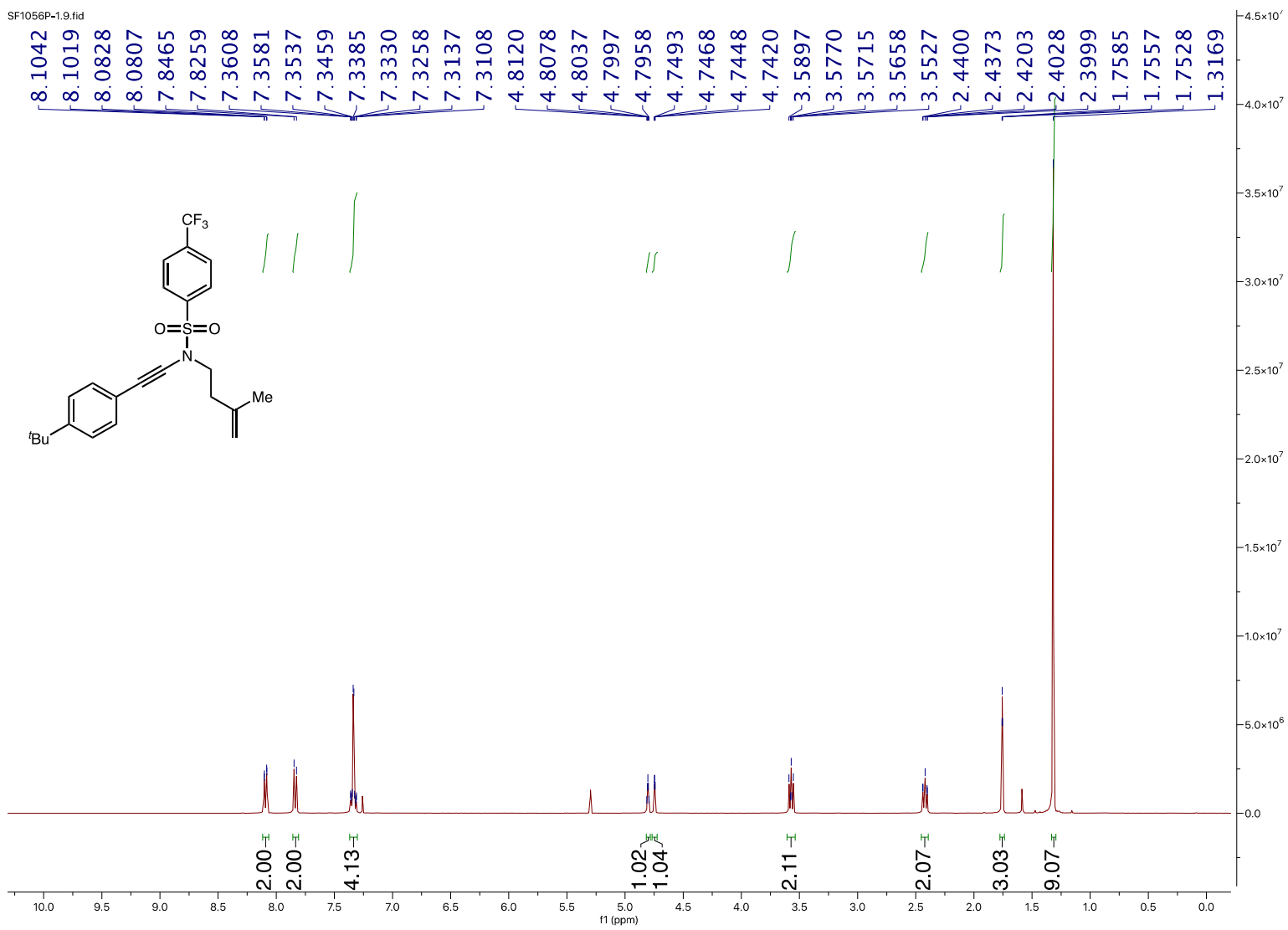

1x ${ }^{1} \mathrm{H}$ NMR (400 MHz, $\left.\mathrm{CDCl}_{3}\right)$

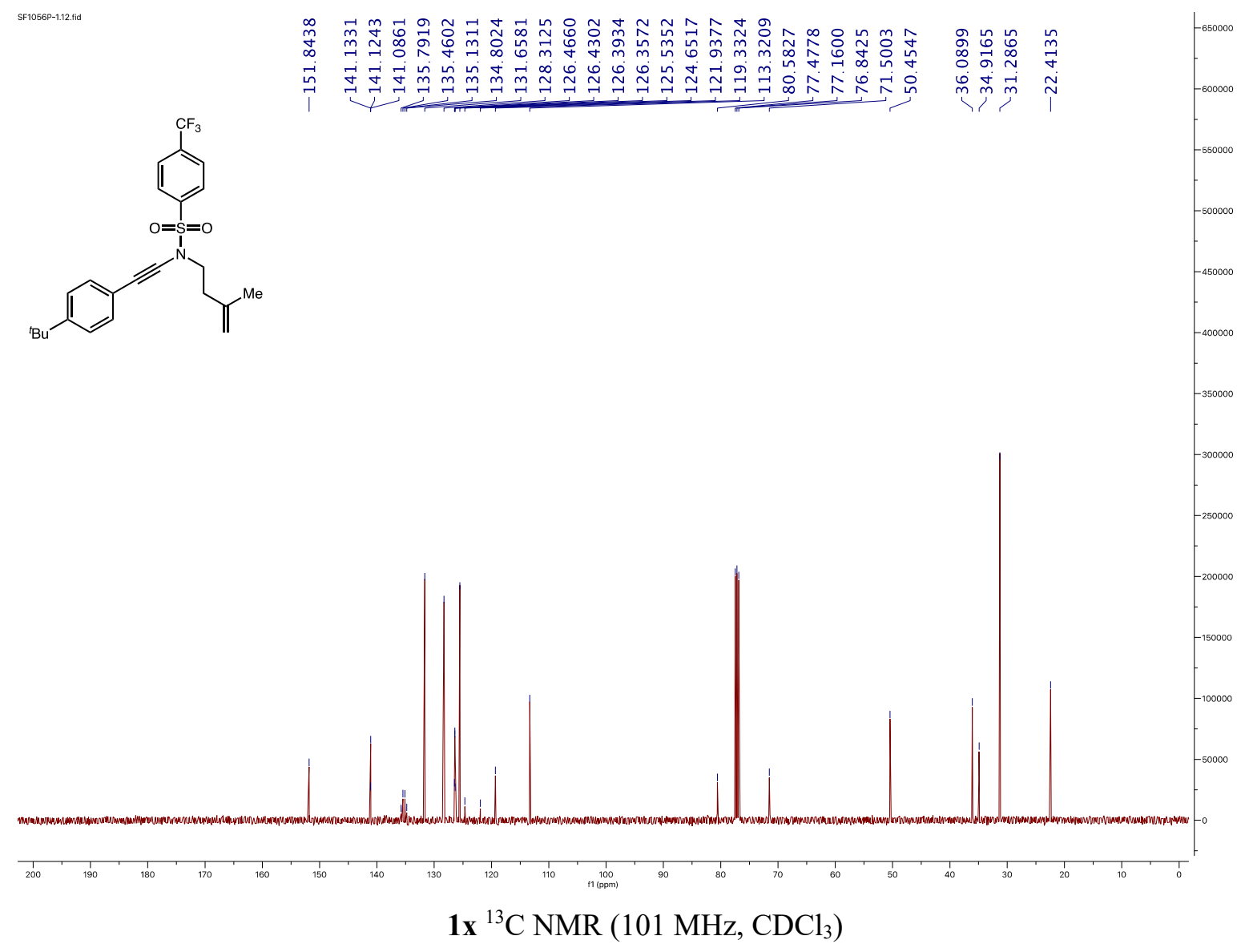




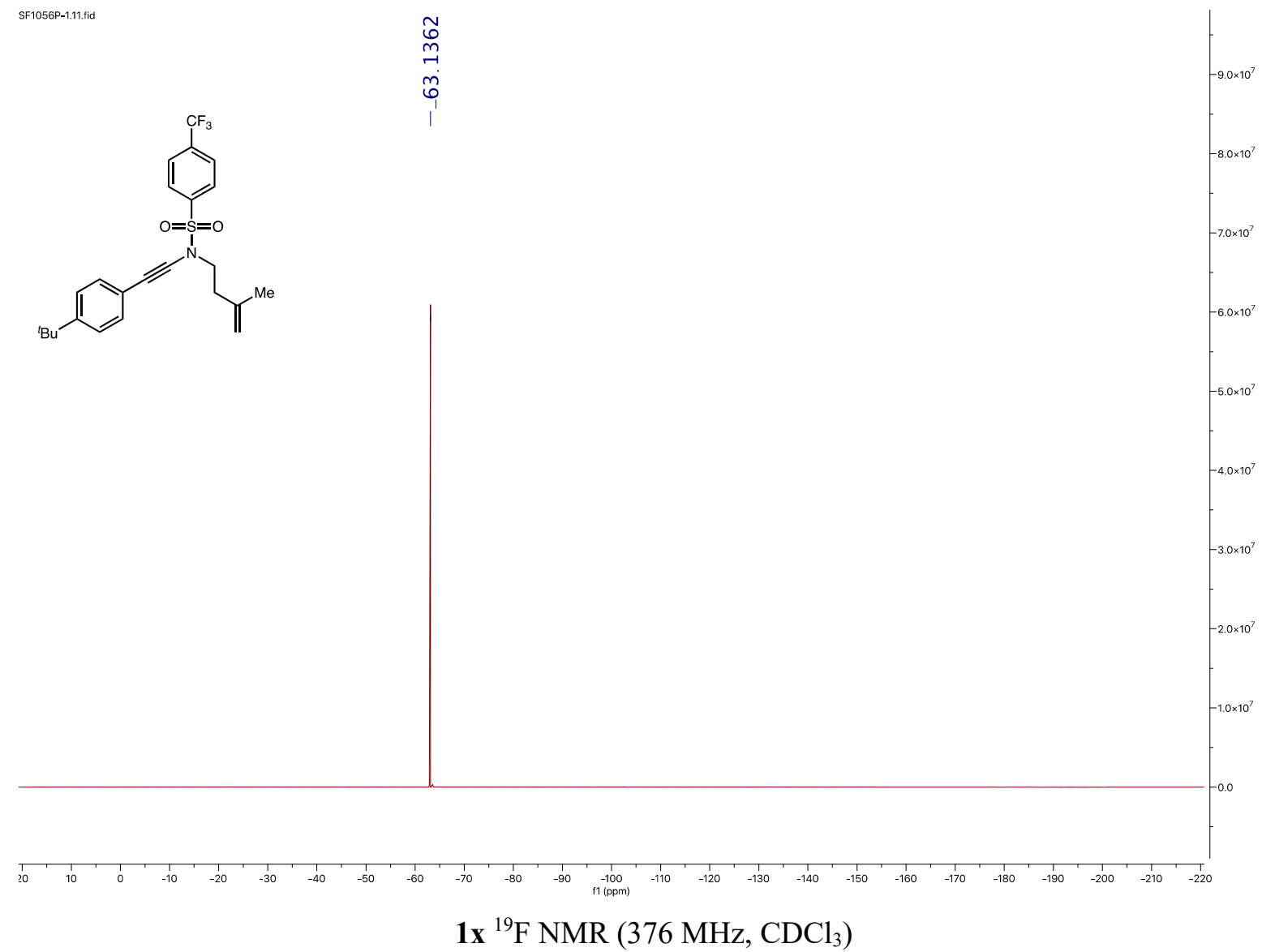

SF1161P-1.10.fid

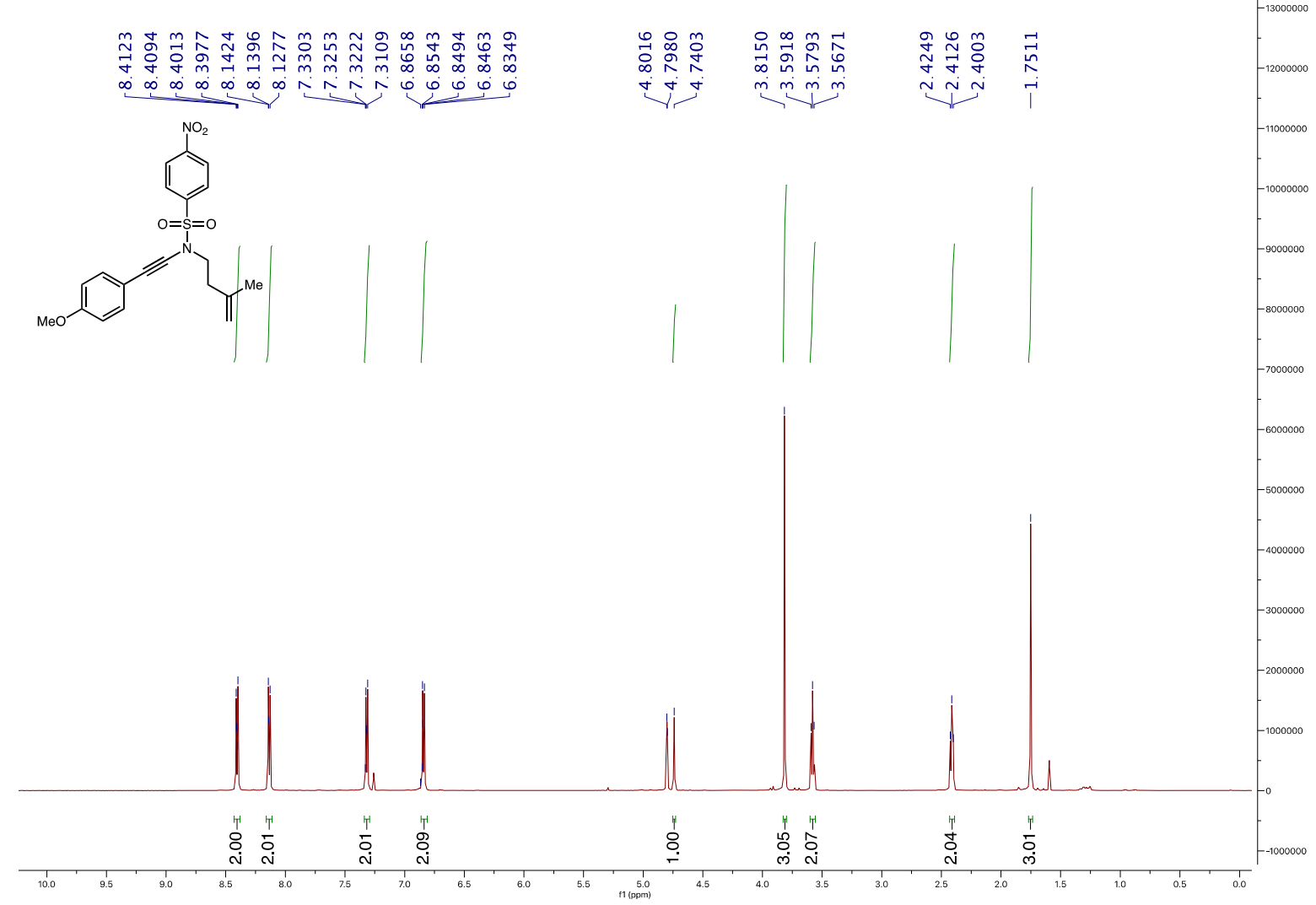

4b ${ }^{1} \mathrm{H}$ NMR (600 MHz, $\mathrm{CDCl}_{3}$ ) 


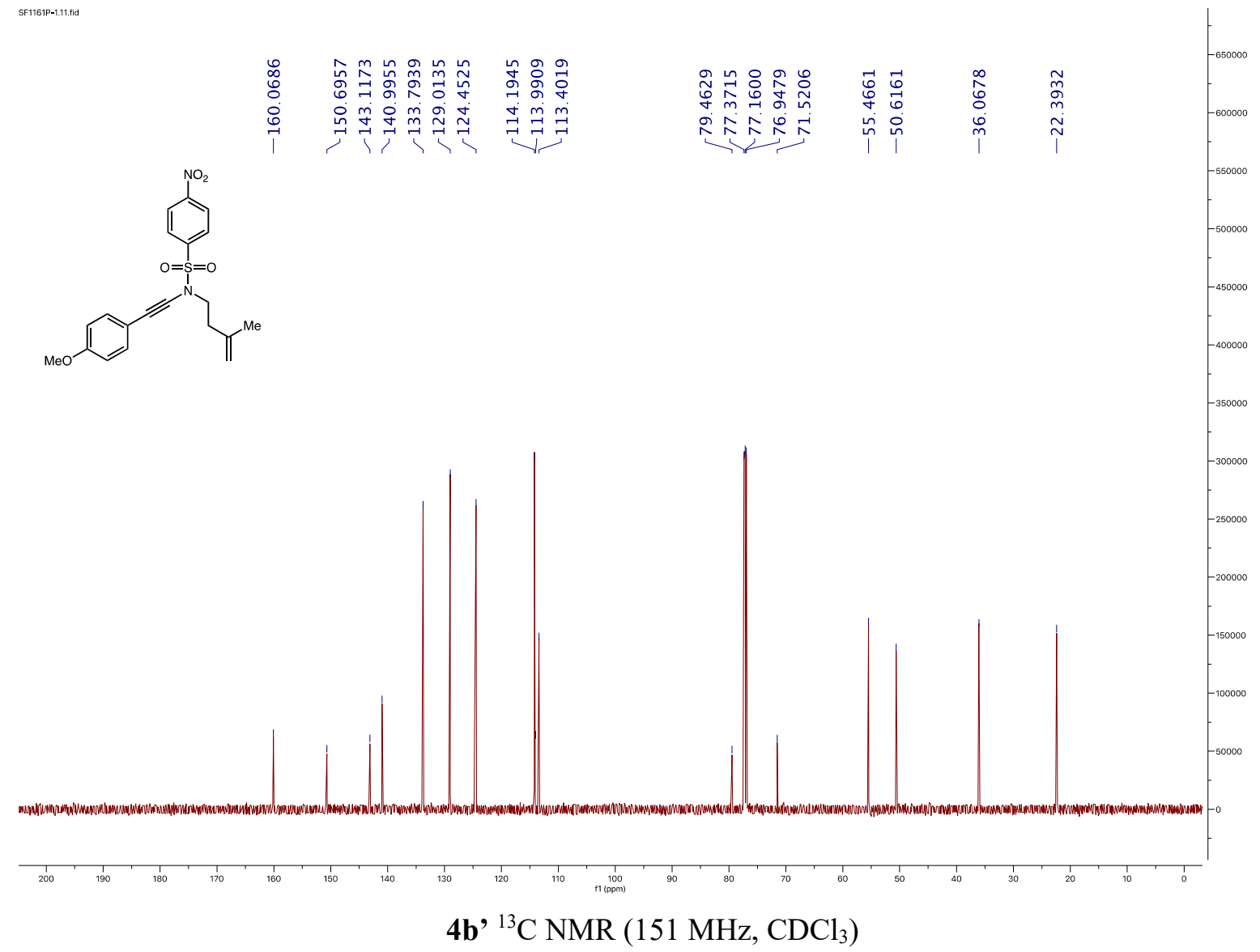

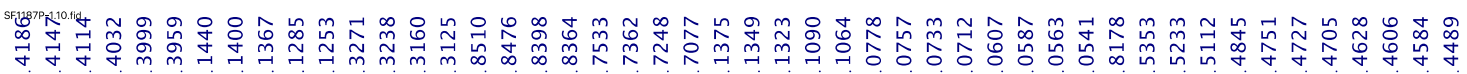

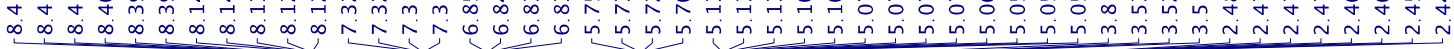
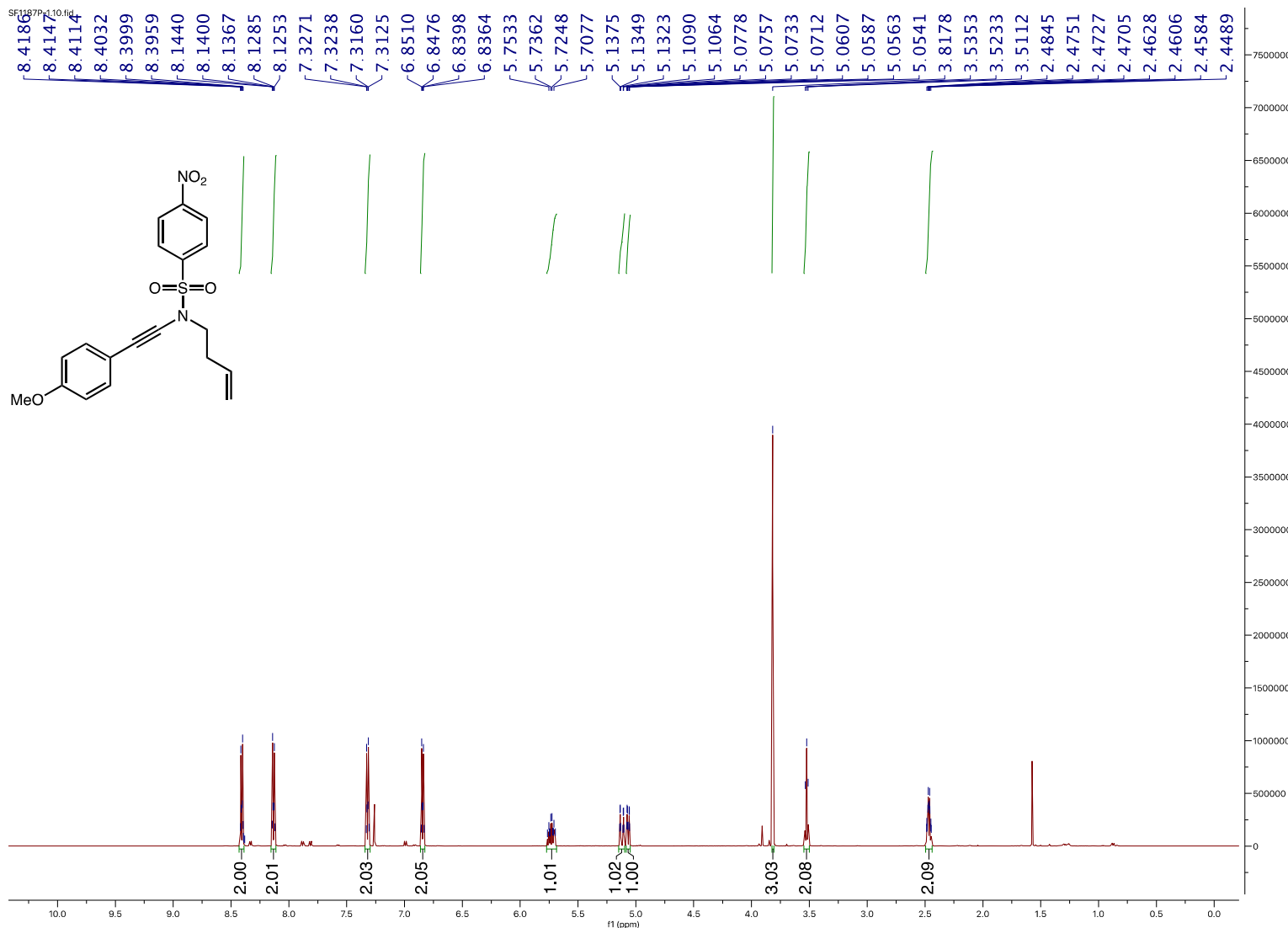

1y ${ }^{1} \mathrm{H}$ NMR (600 MHz, $\left.\mathrm{CDCl}_{3}\right)$ 

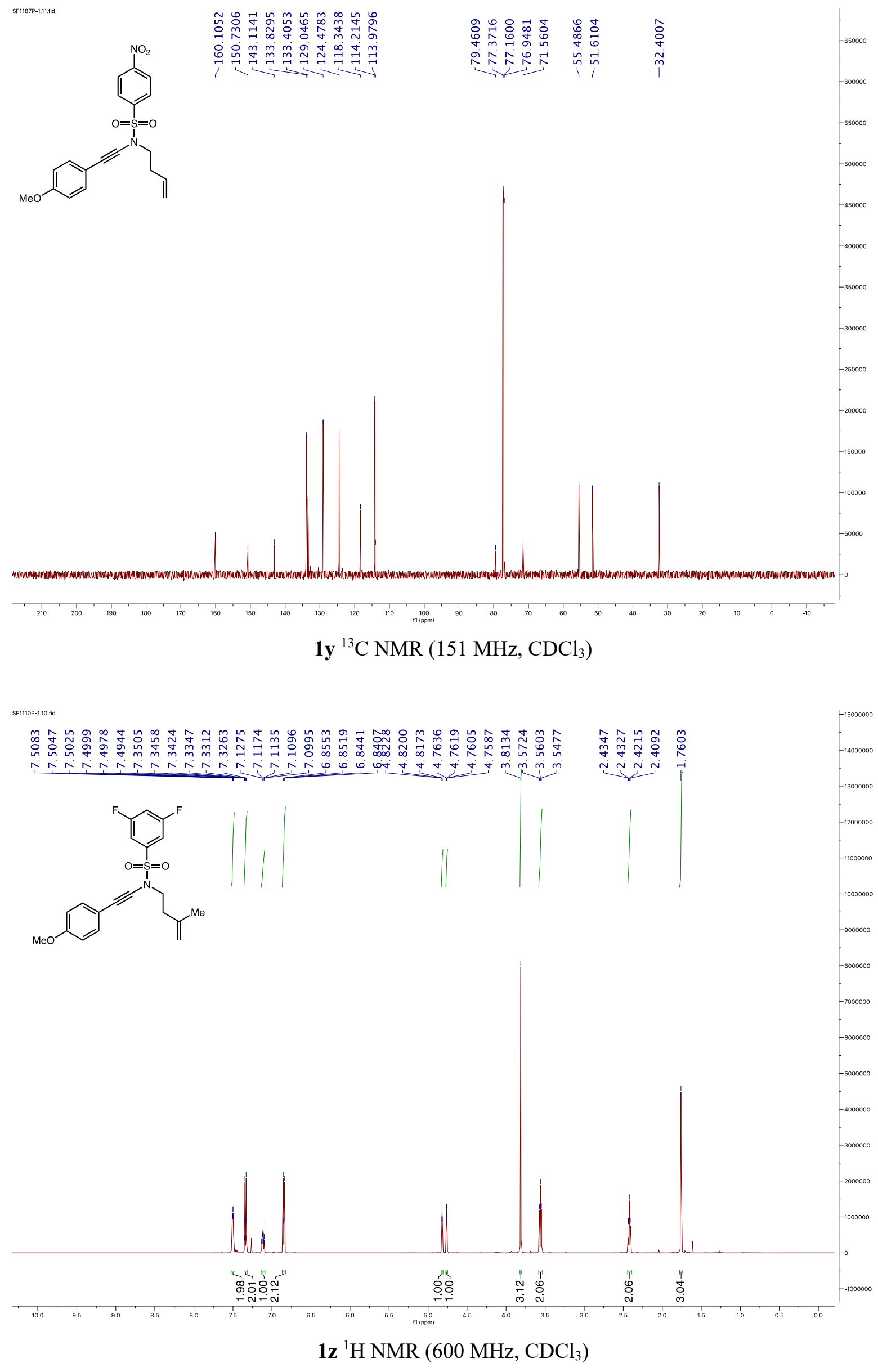

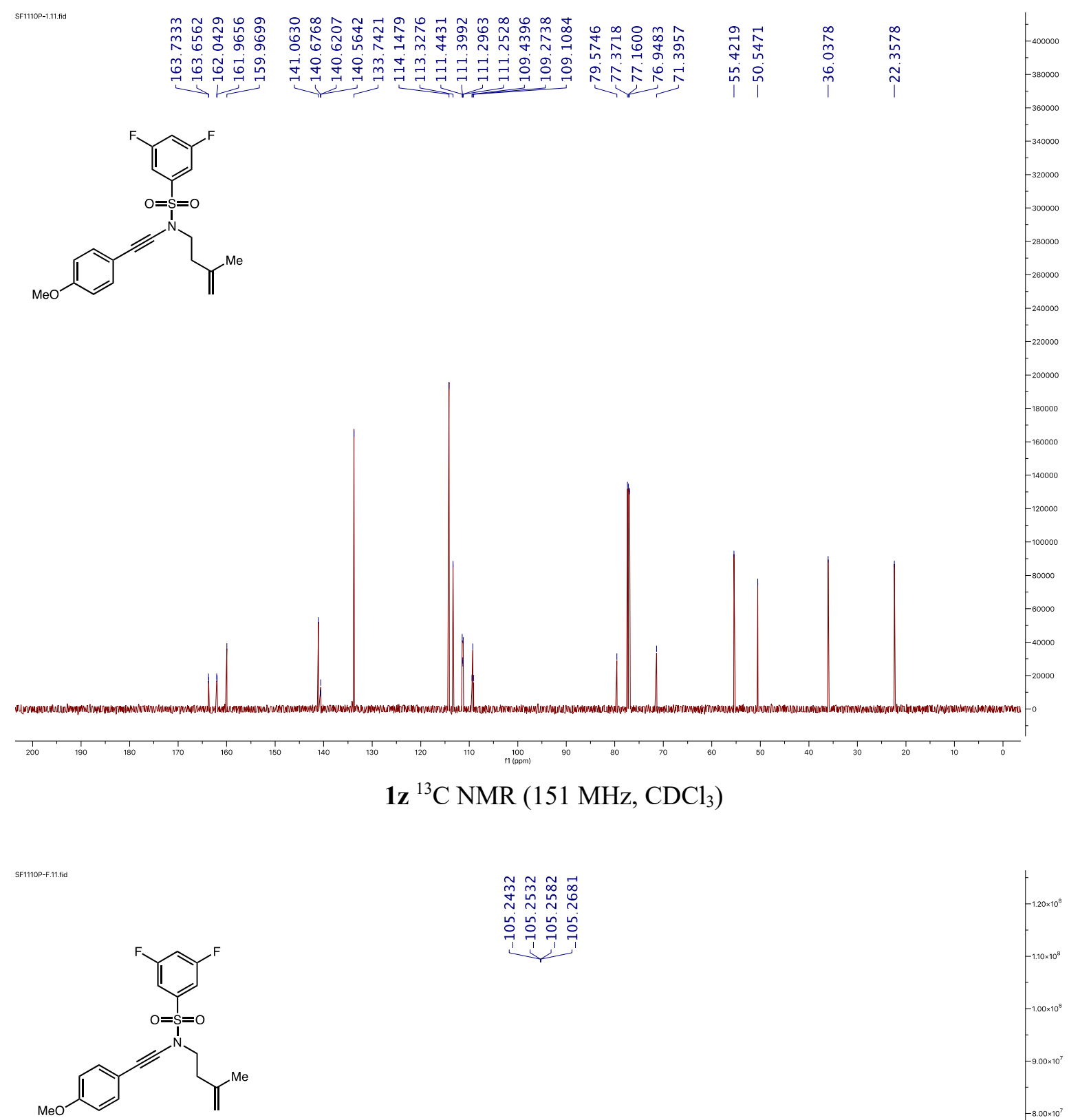

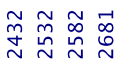

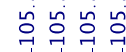

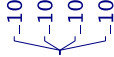

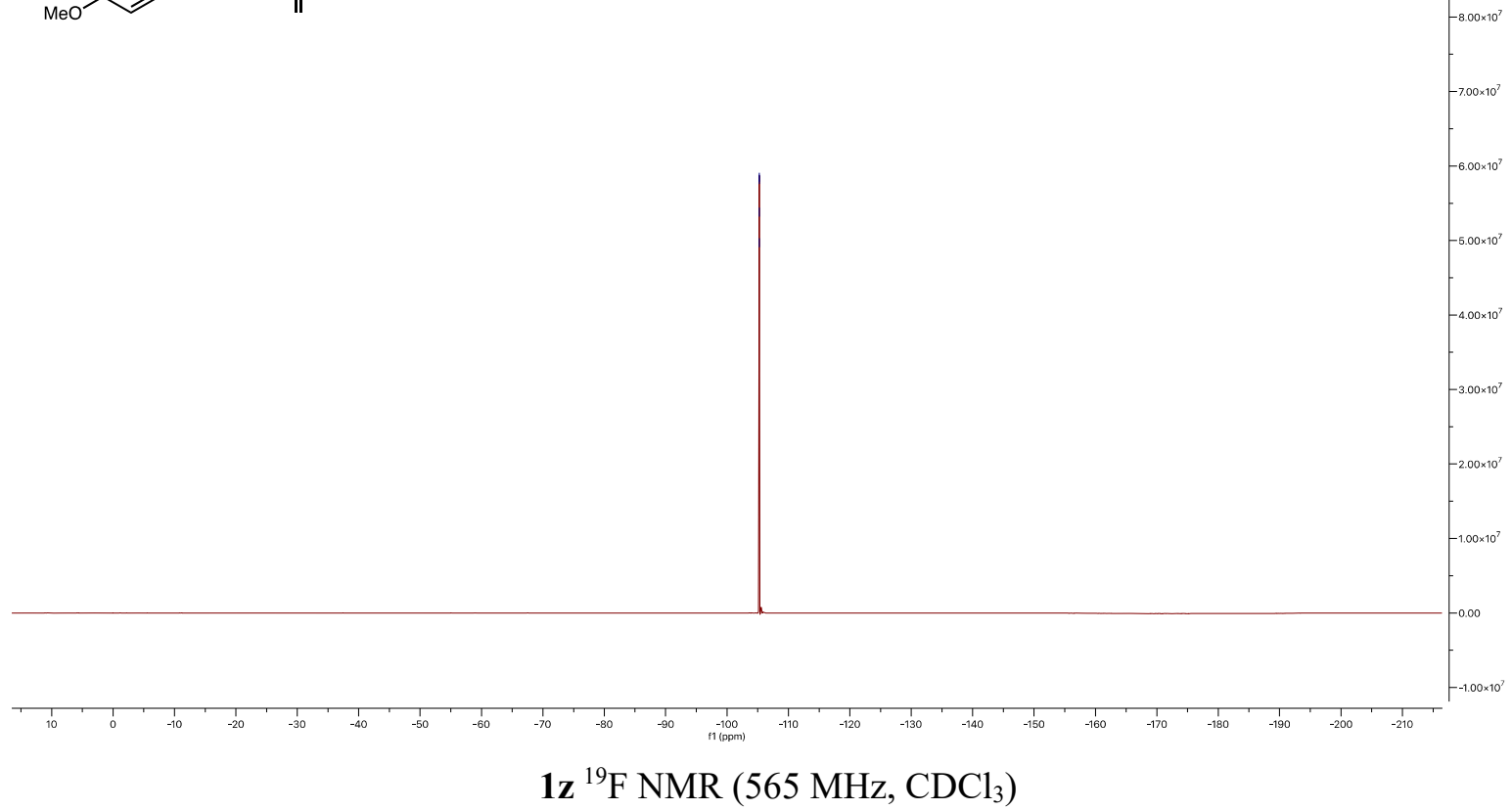



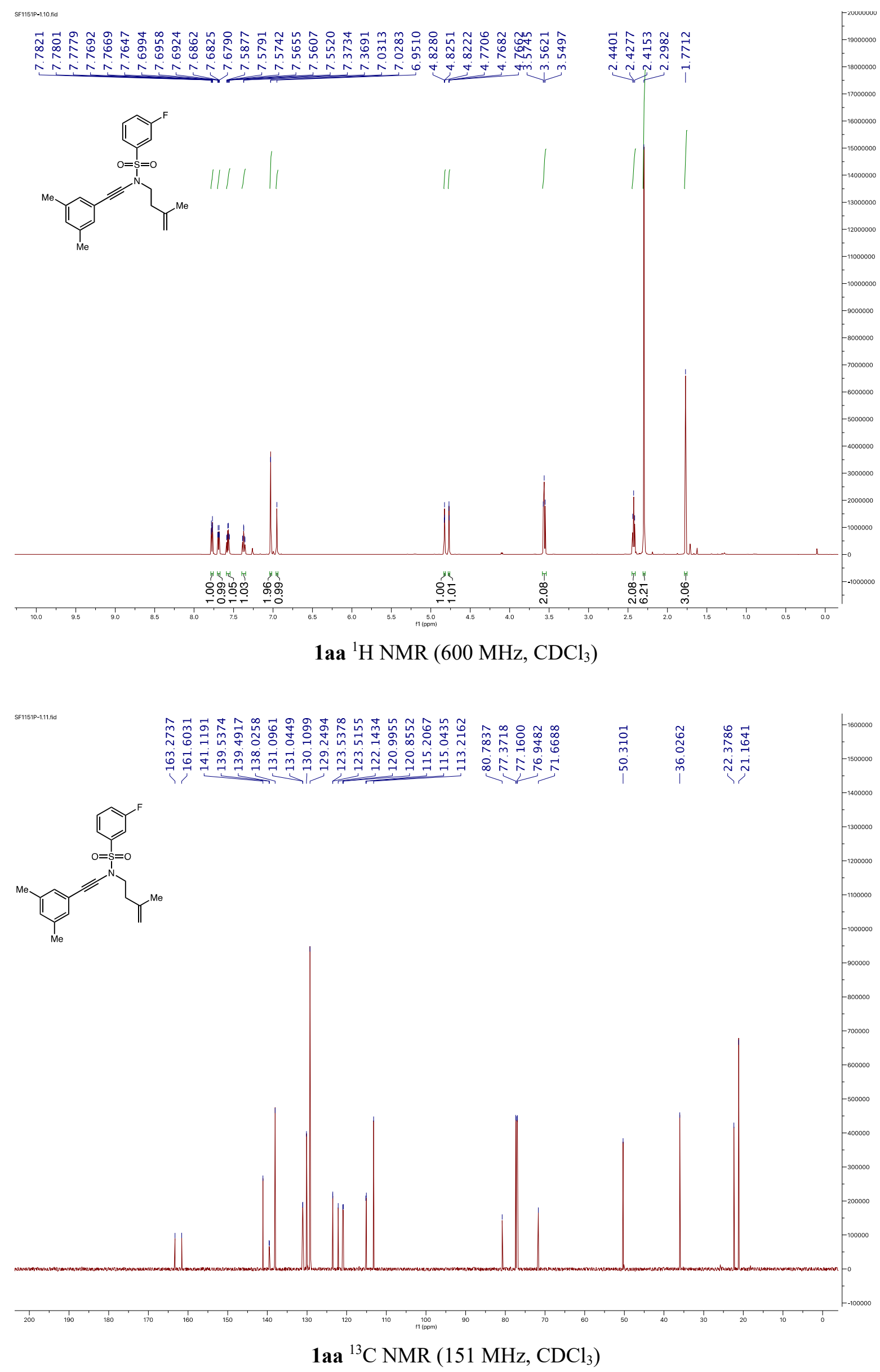

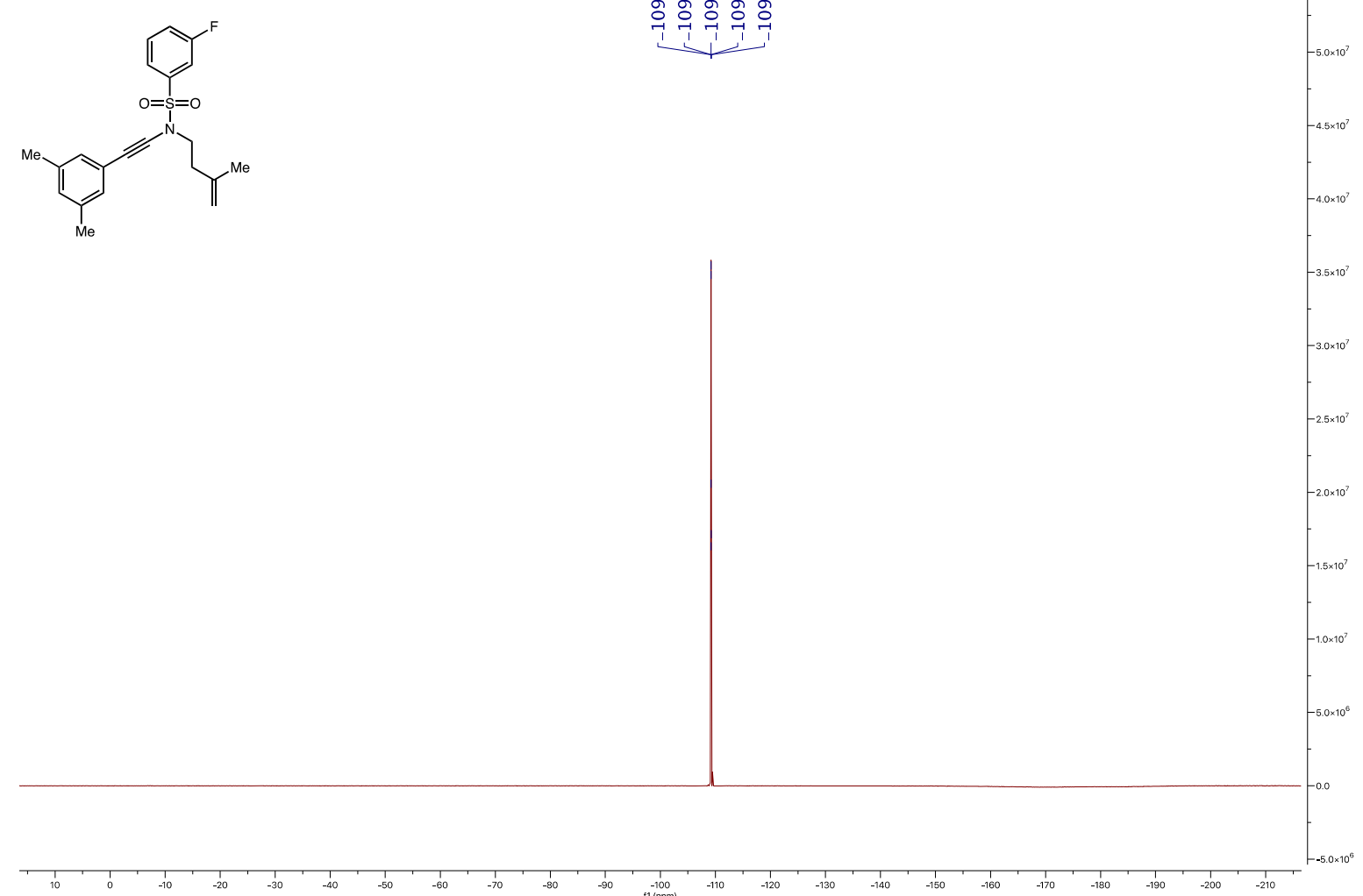

1aa ${ }^{19} \mathrm{~F}$ NMR (565 MHz, $\mathrm{CDCl}_{3}$ )

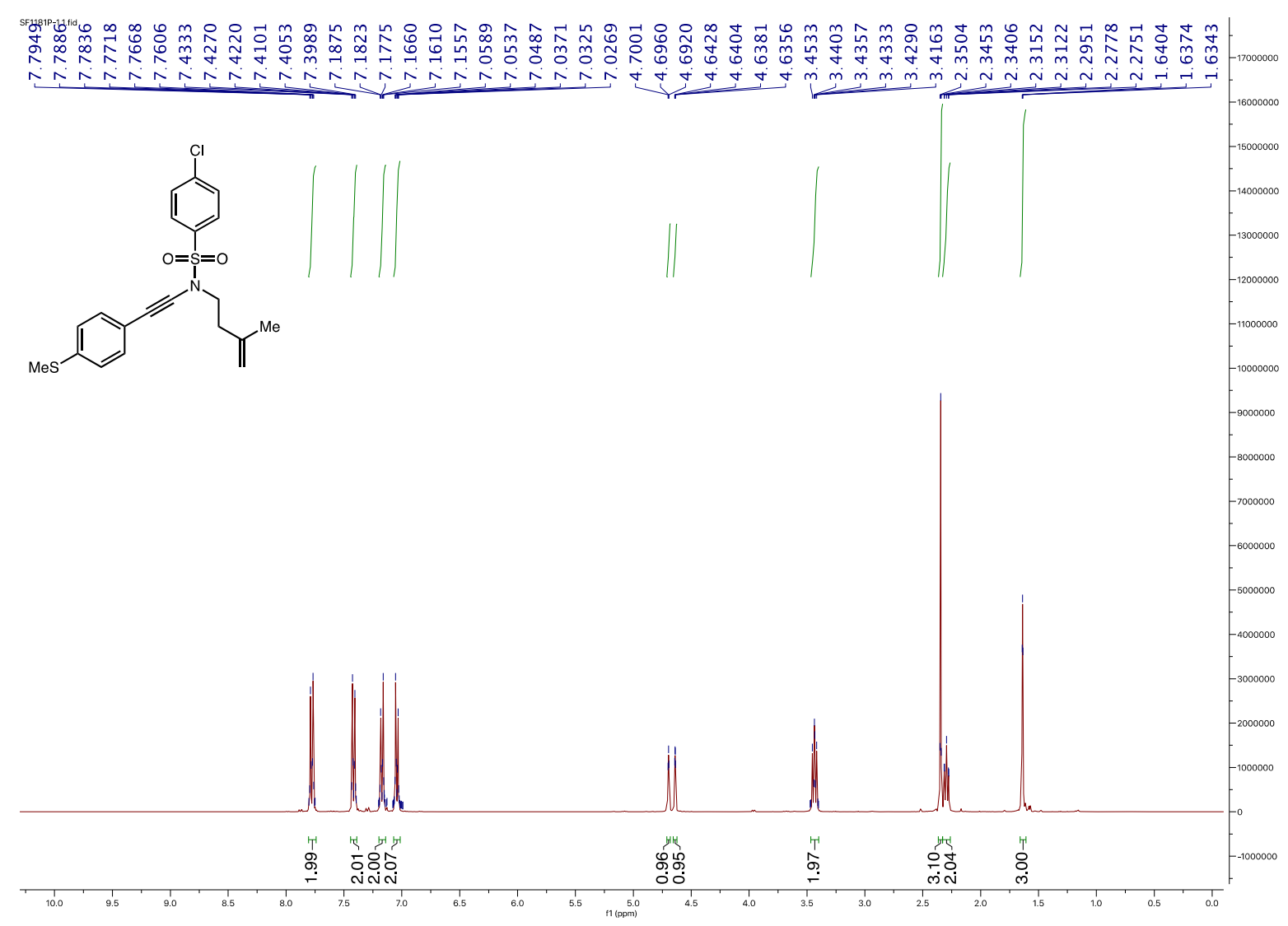

1ab ${ }^{1} \mathrm{H}$ NMR $\left(600 \mathrm{MHz}, \mathrm{CDCl}_{3}\right)$ 


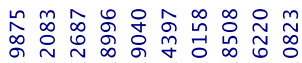

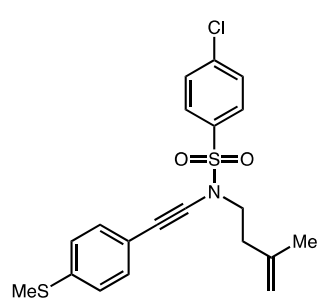

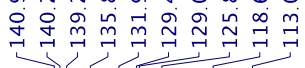

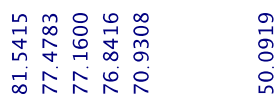

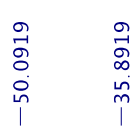

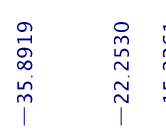

$\underset{\substack{0 \\ 0}}{\stackrel{0}{0}}$

1ab ${ }^{13} \mathrm{C}$ NMR $\left(151 \mathrm{MHz}, \mathrm{CDCl}_{3}\right)$

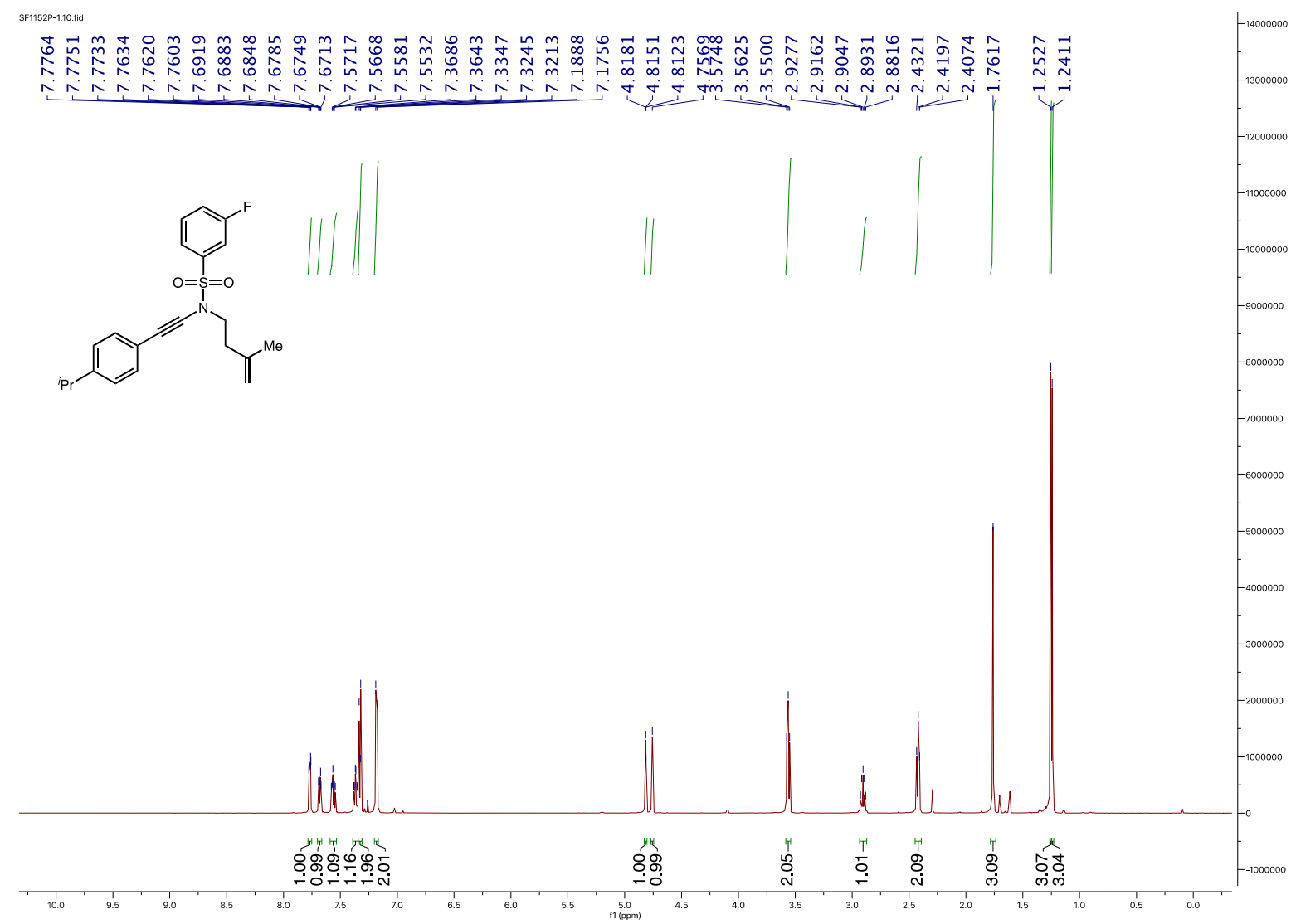

1ac ${ }^{1} \mathrm{H}$ NMR $\left(600 \mathrm{MHz}, \mathrm{CDCl}_{3}\right)$ 


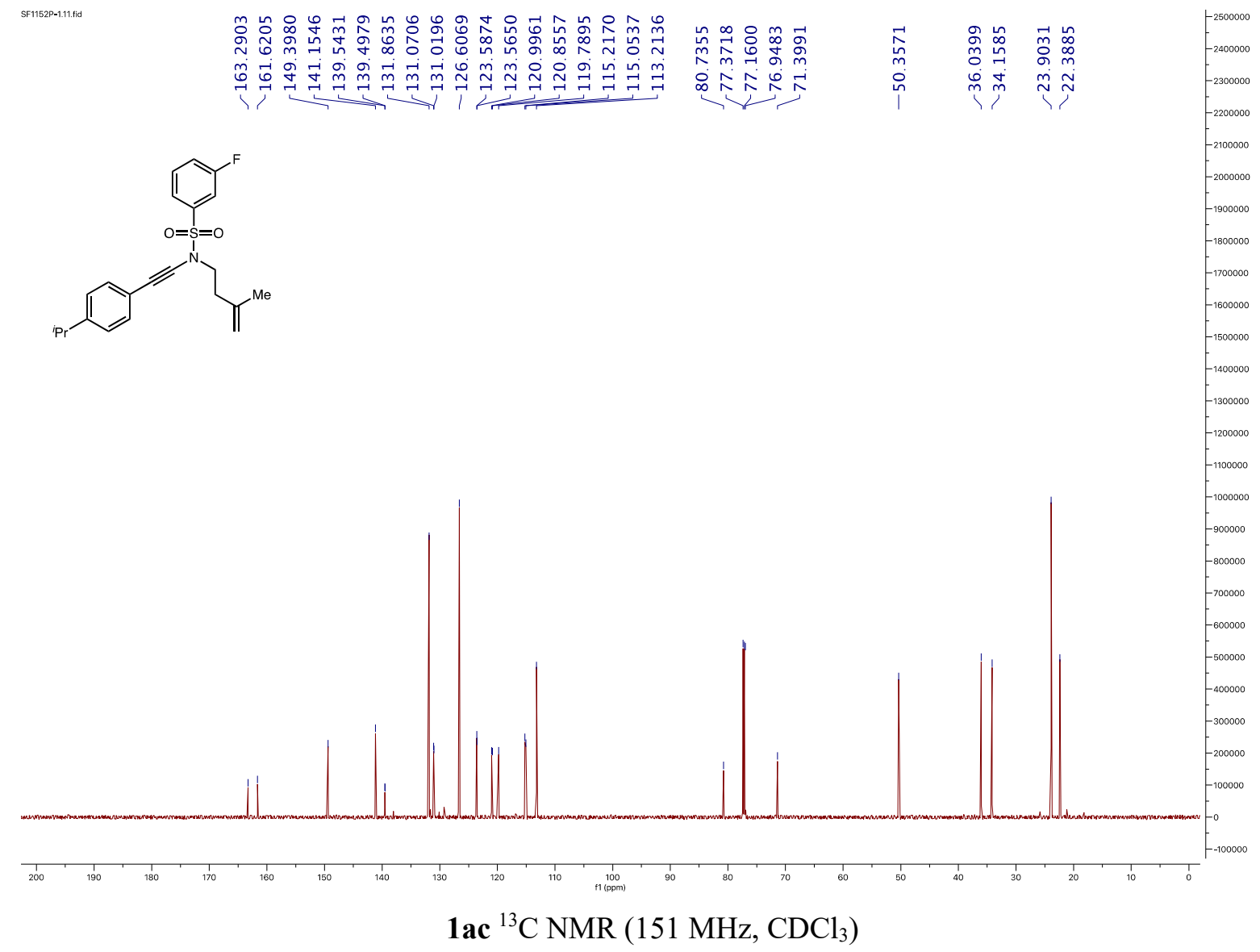

SF1152P-1.1.1.fid

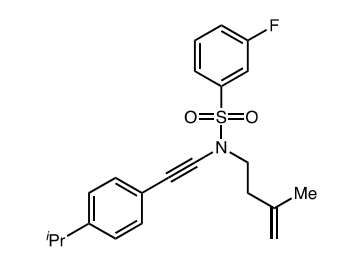

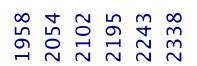

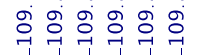

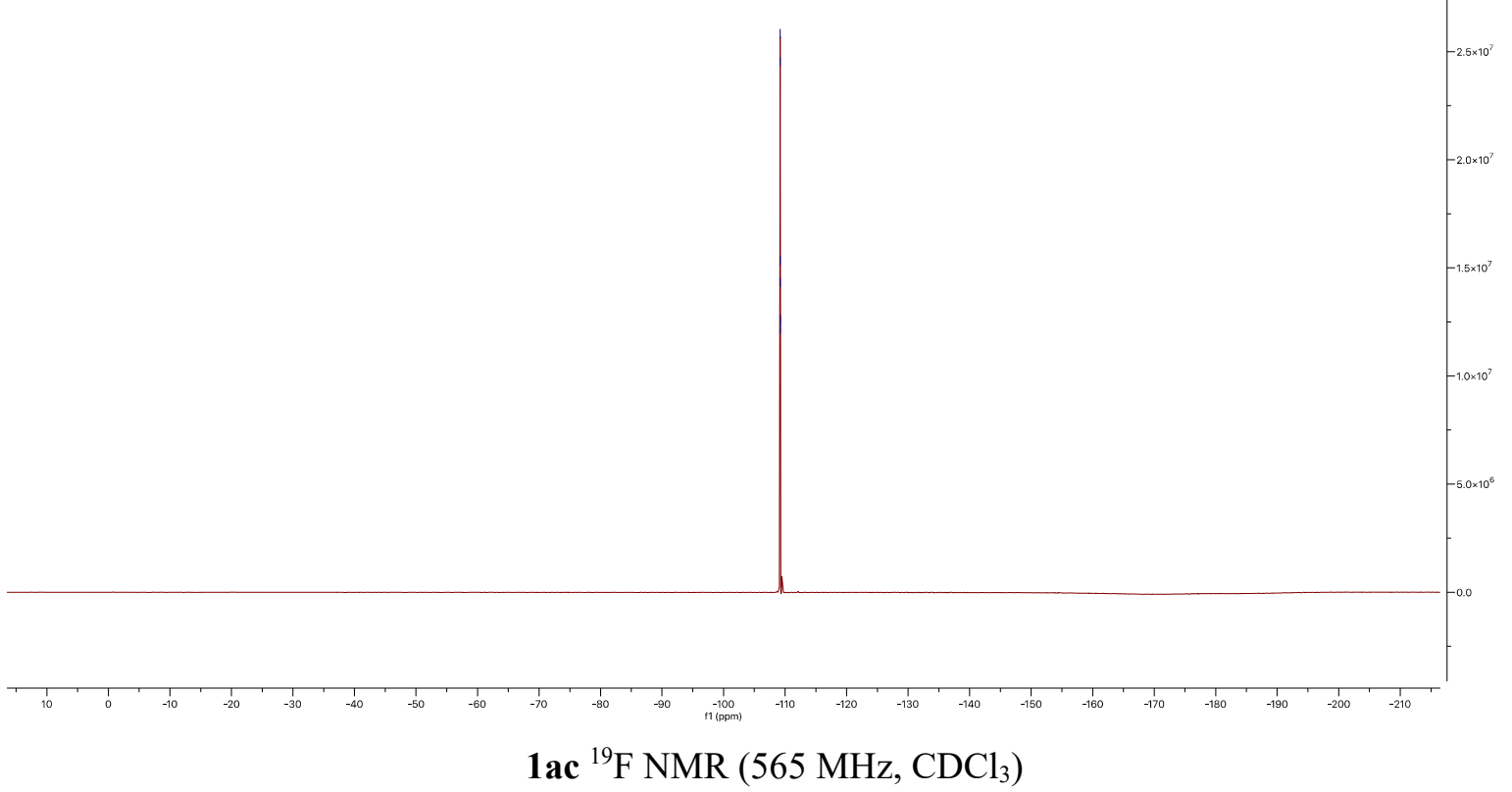



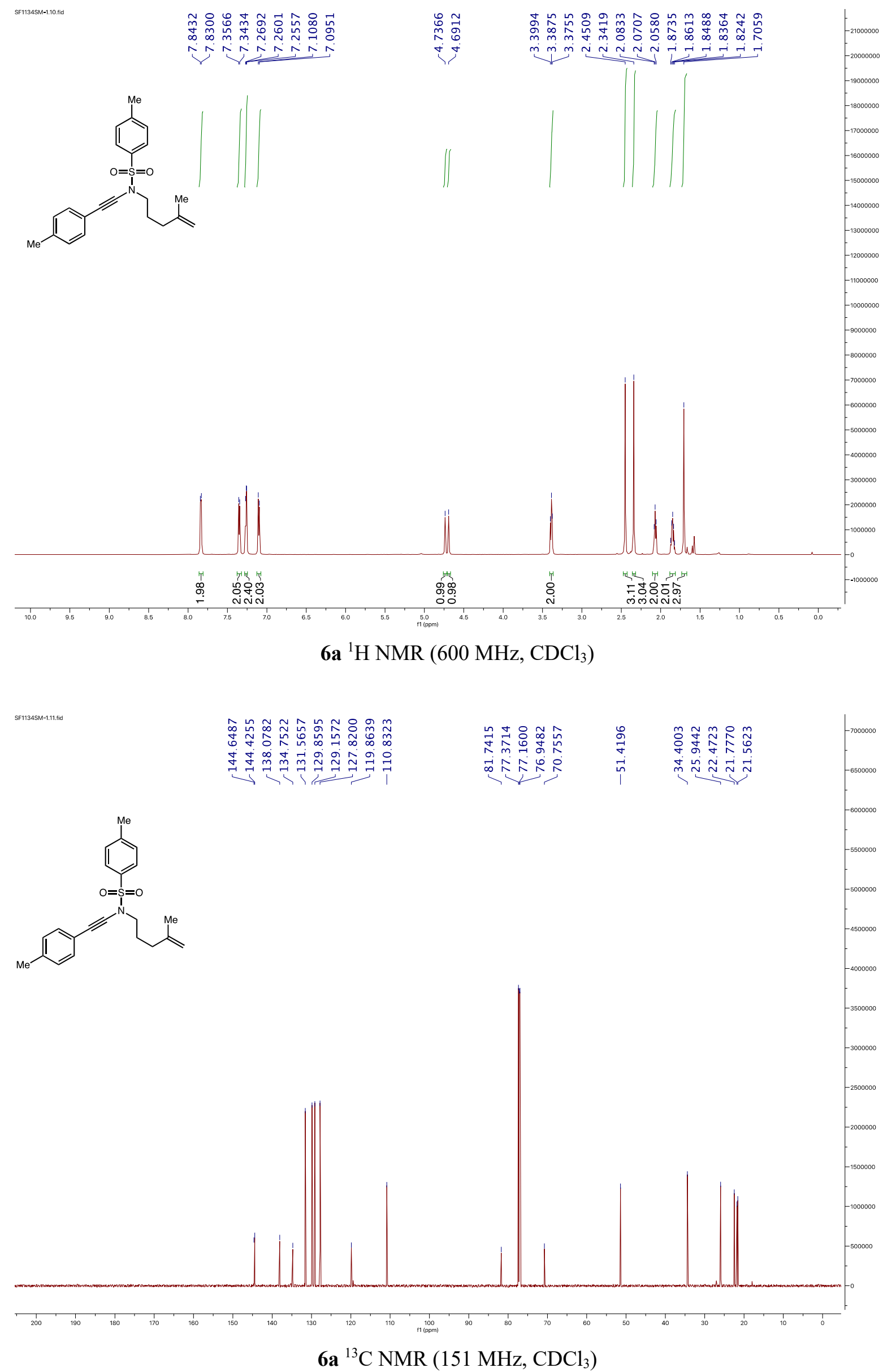


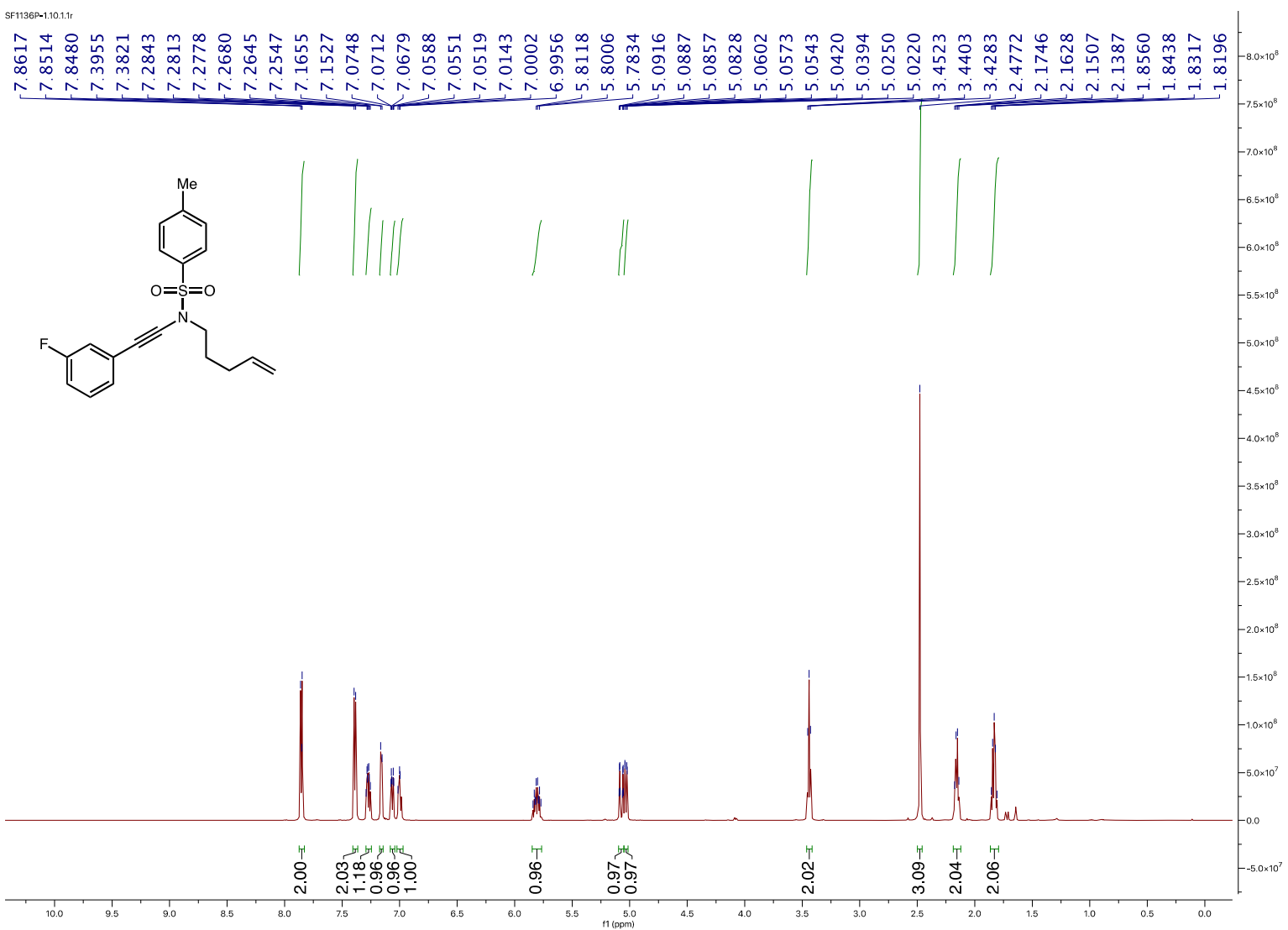

6b ${ }^{1} \mathrm{H}$ NMR (600 MHz, $\left.\mathrm{CDCl}_{3}\right)$

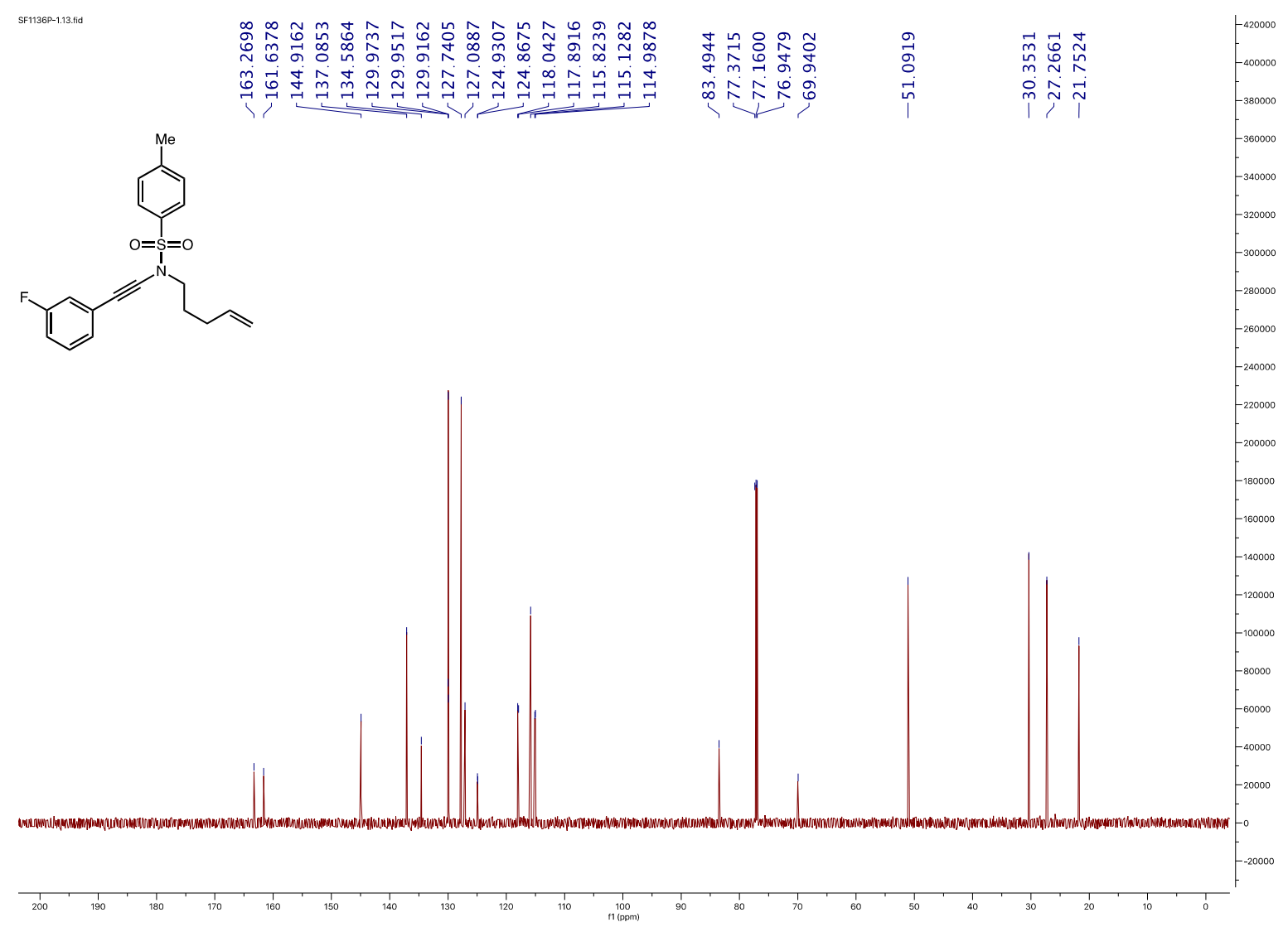

6b ${ }^{13} \mathrm{C}$ NMR (151 MHz, $\left.\mathrm{CDCl}_{3}\right)$ 


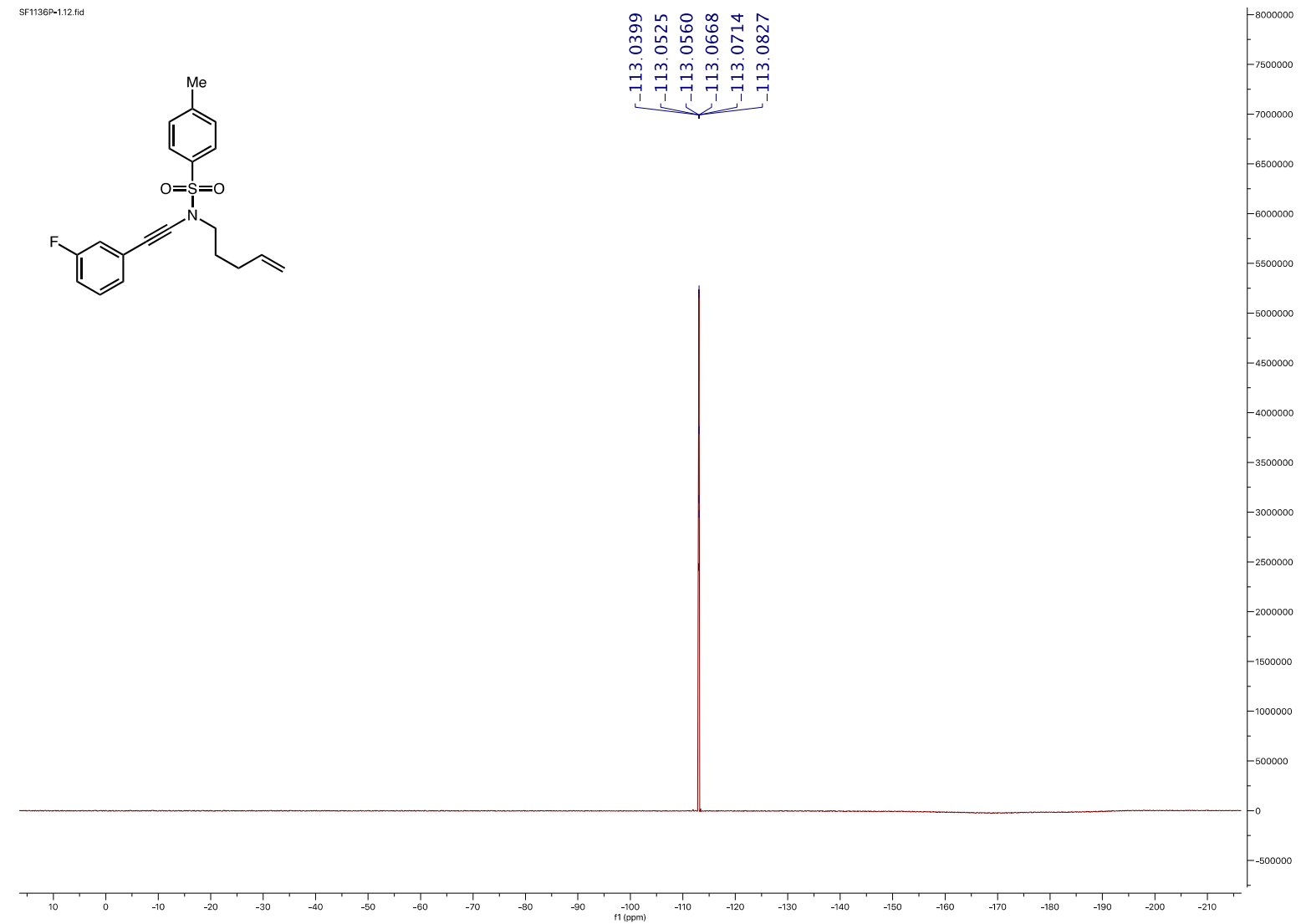

6b ${ }^{19} \mathrm{~F}$ NMR (565 MHz, $\mathrm{CDCl}_{3}$ )

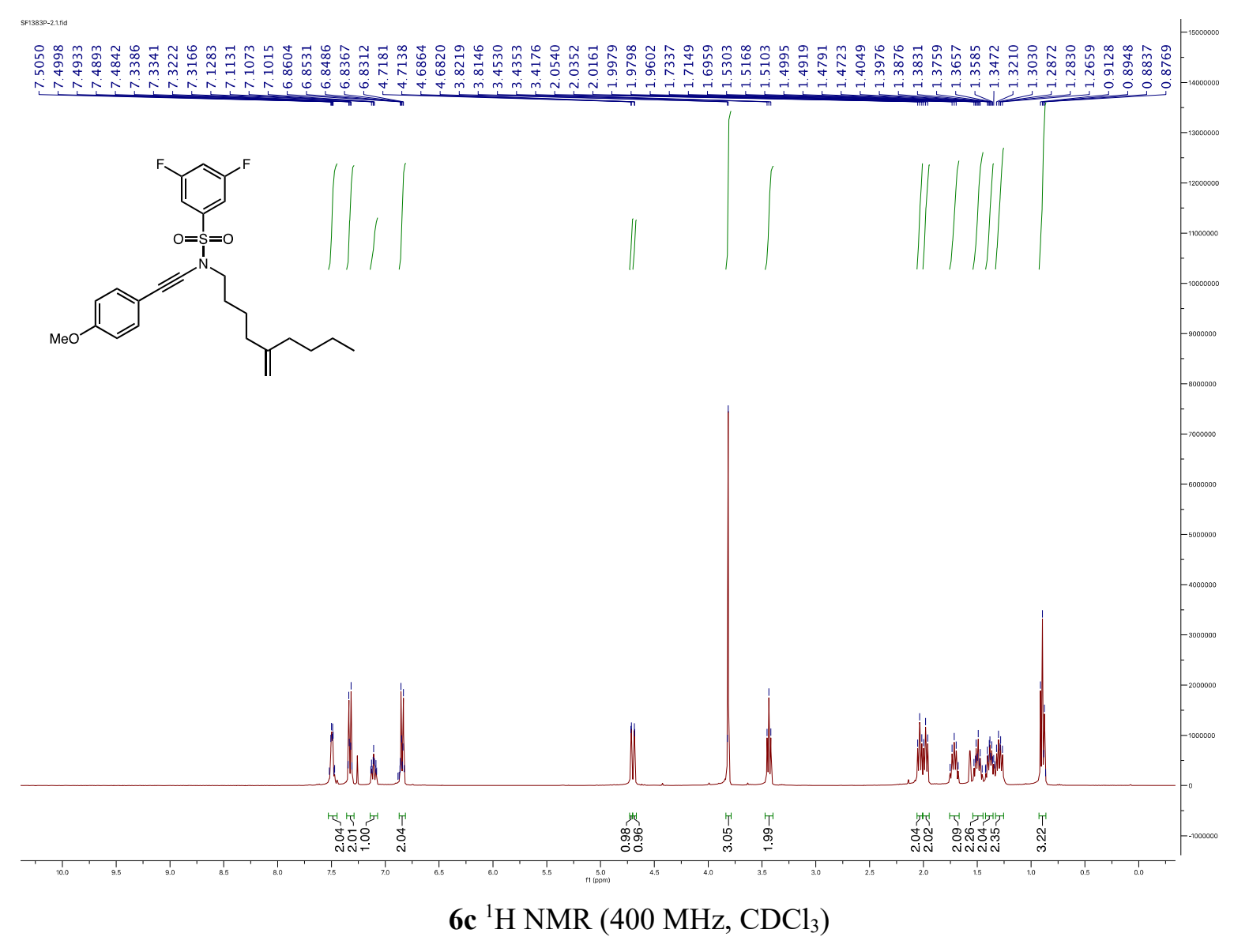




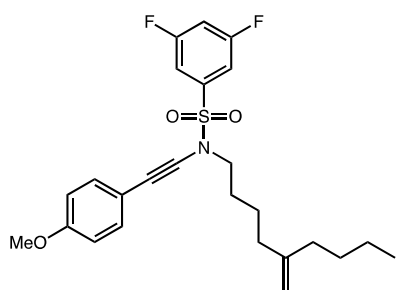

$$
\text { 6c }{ }^{13} \mathrm{C} \text { NMR }\left(101 \mathrm{MHz}, \mathrm{CDCl}_{3}\right)
$$

SF1383P-2.3.fid

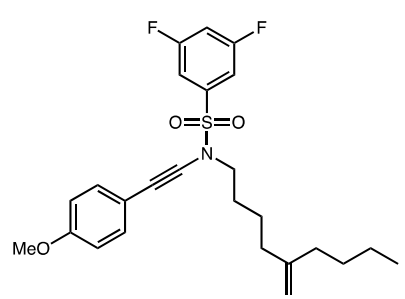

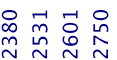

突总究总

开正

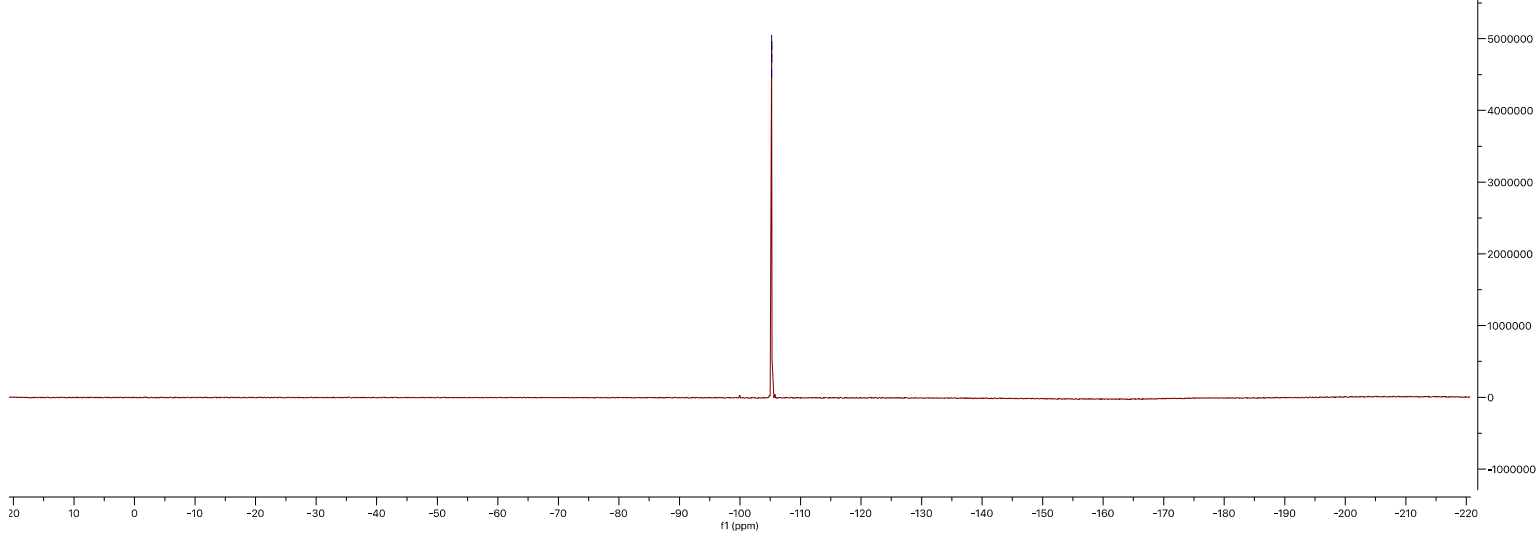

6c ${ }^{19} \mathrm{~F}$ NMR $\left(565 \mathrm{MHz}, \mathrm{CDCl}_{3}\right)$ 


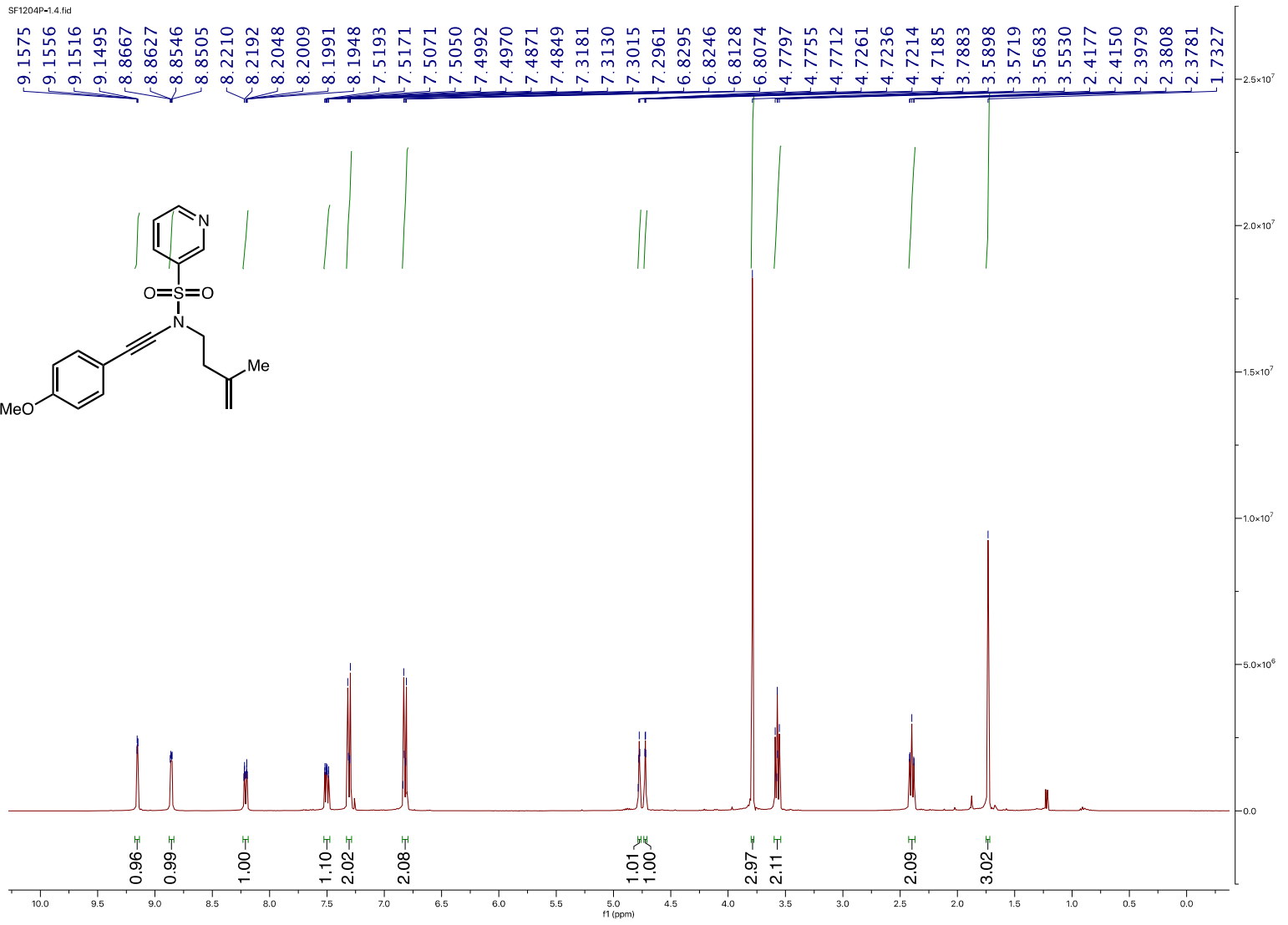

1ad ${ }^{1} \mathrm{H}$ NMR (400 MHz, $\mathrm{CDCl}_{3}$ )

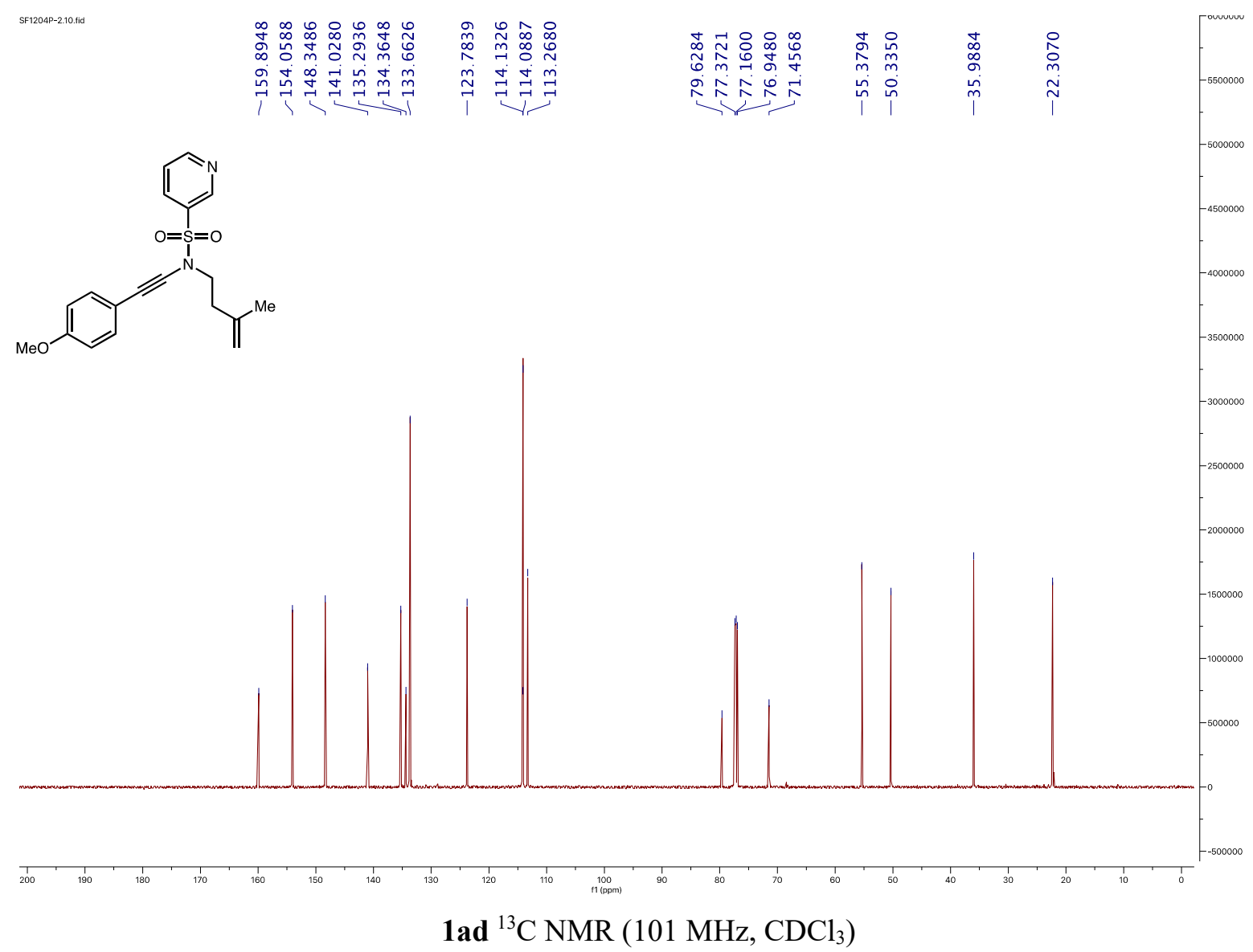




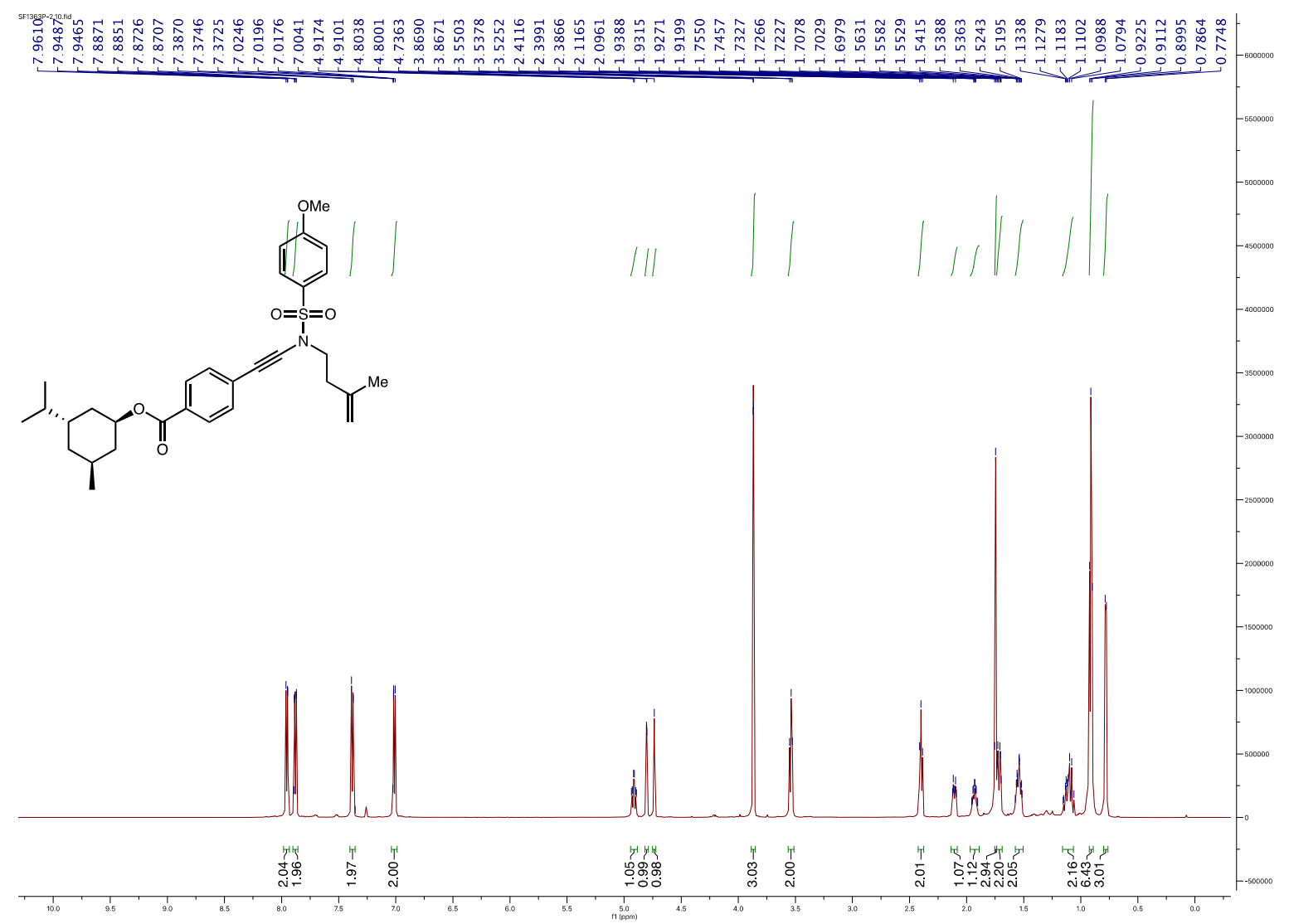

1r ${ }^{1} \mathrm{H}$ NMR $\left(600 \mathrm{MHz}, \mathrm{CDCl}_{3}\right)$

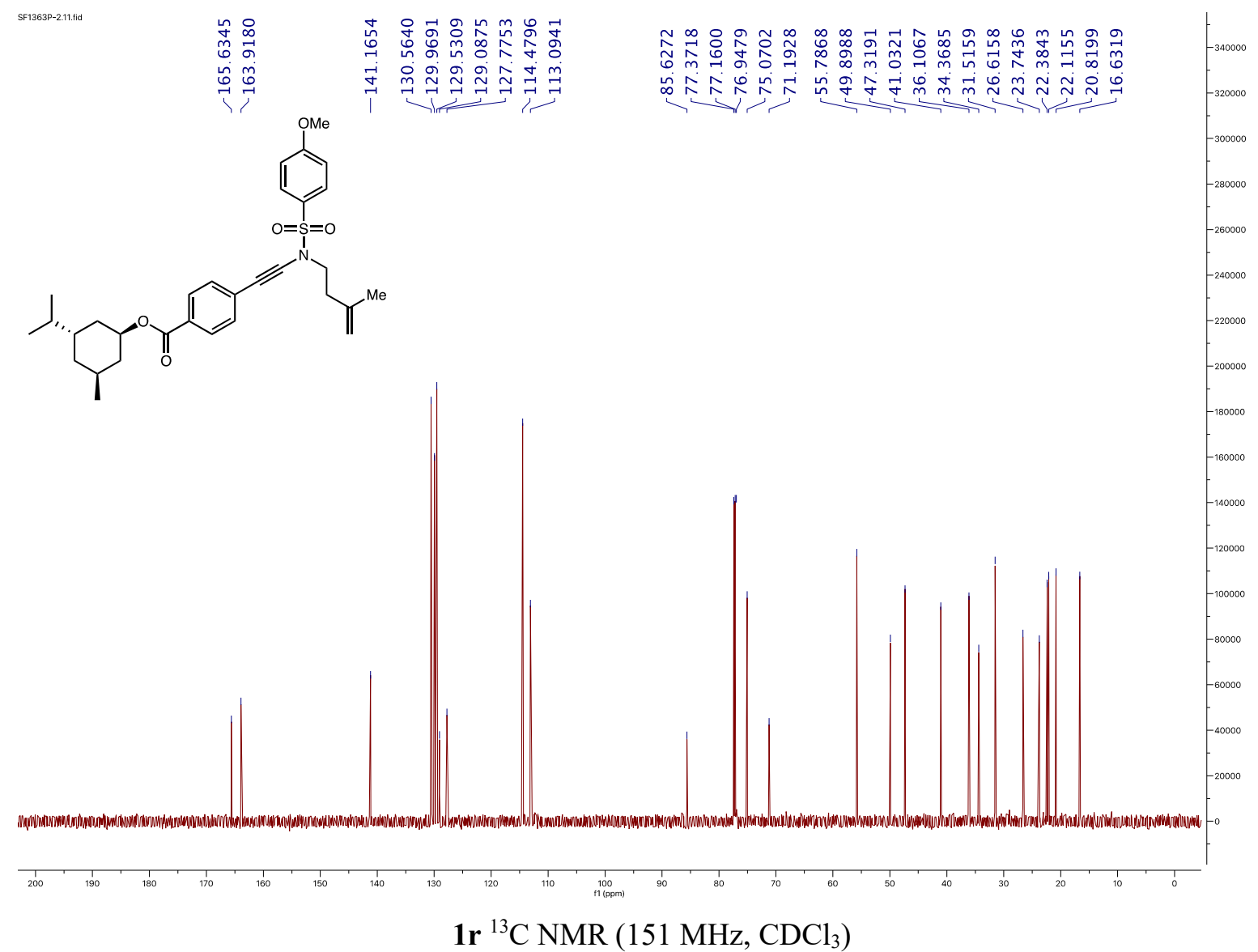




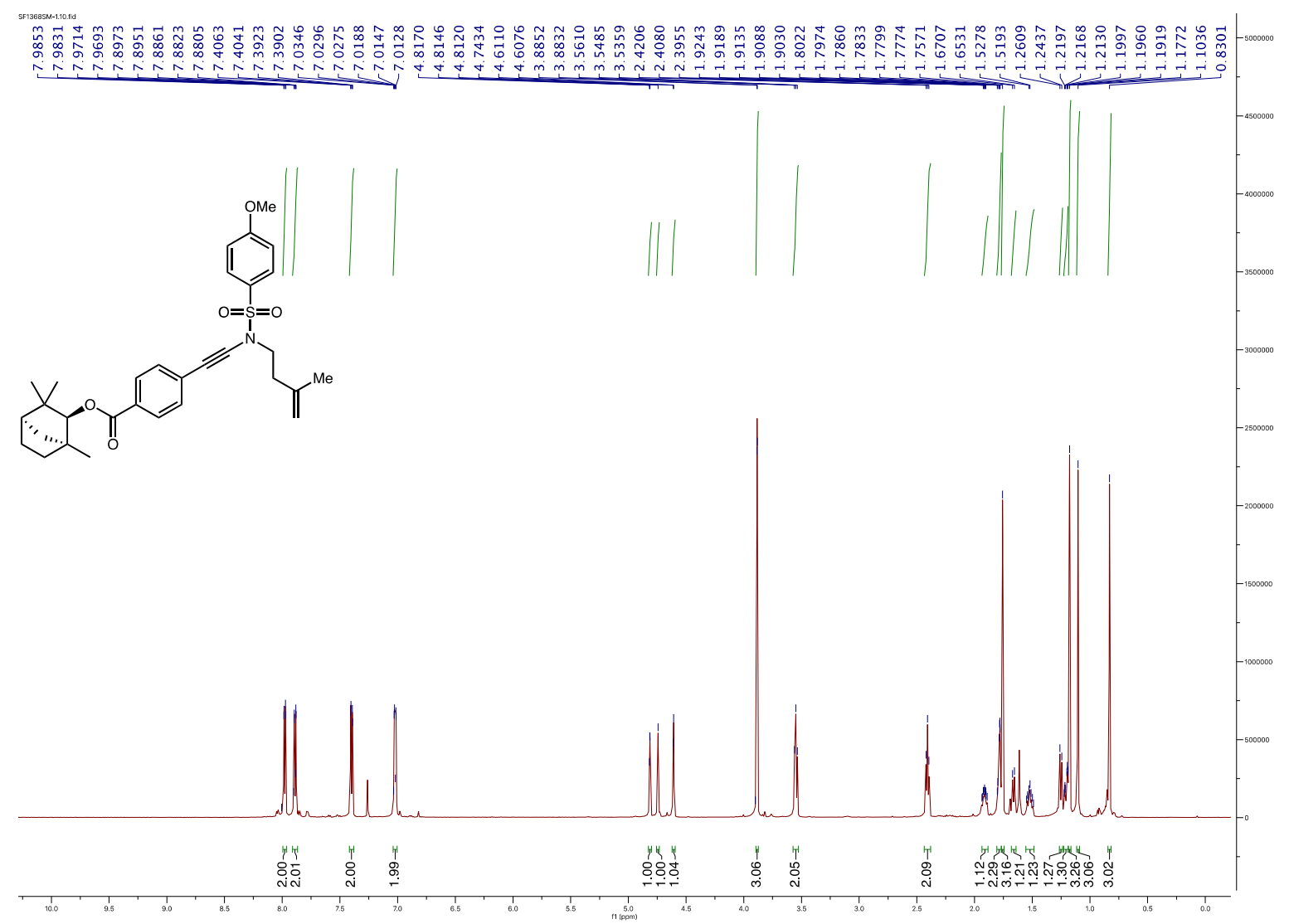

1s ${ }^{1} \mathrm{H}$ NMR (600 MHz, $\left.\mathrm{CDCl}_{3}\right)$

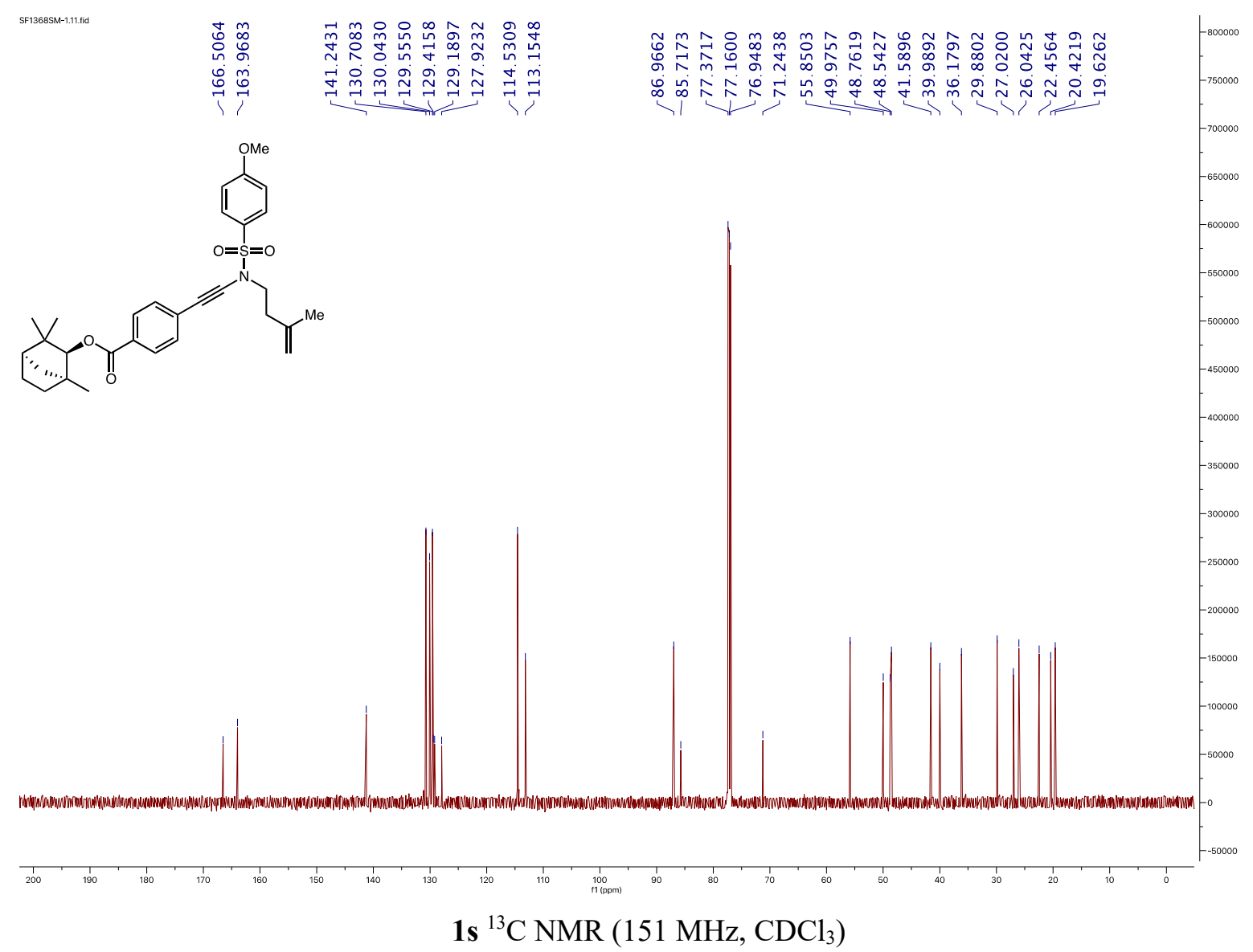




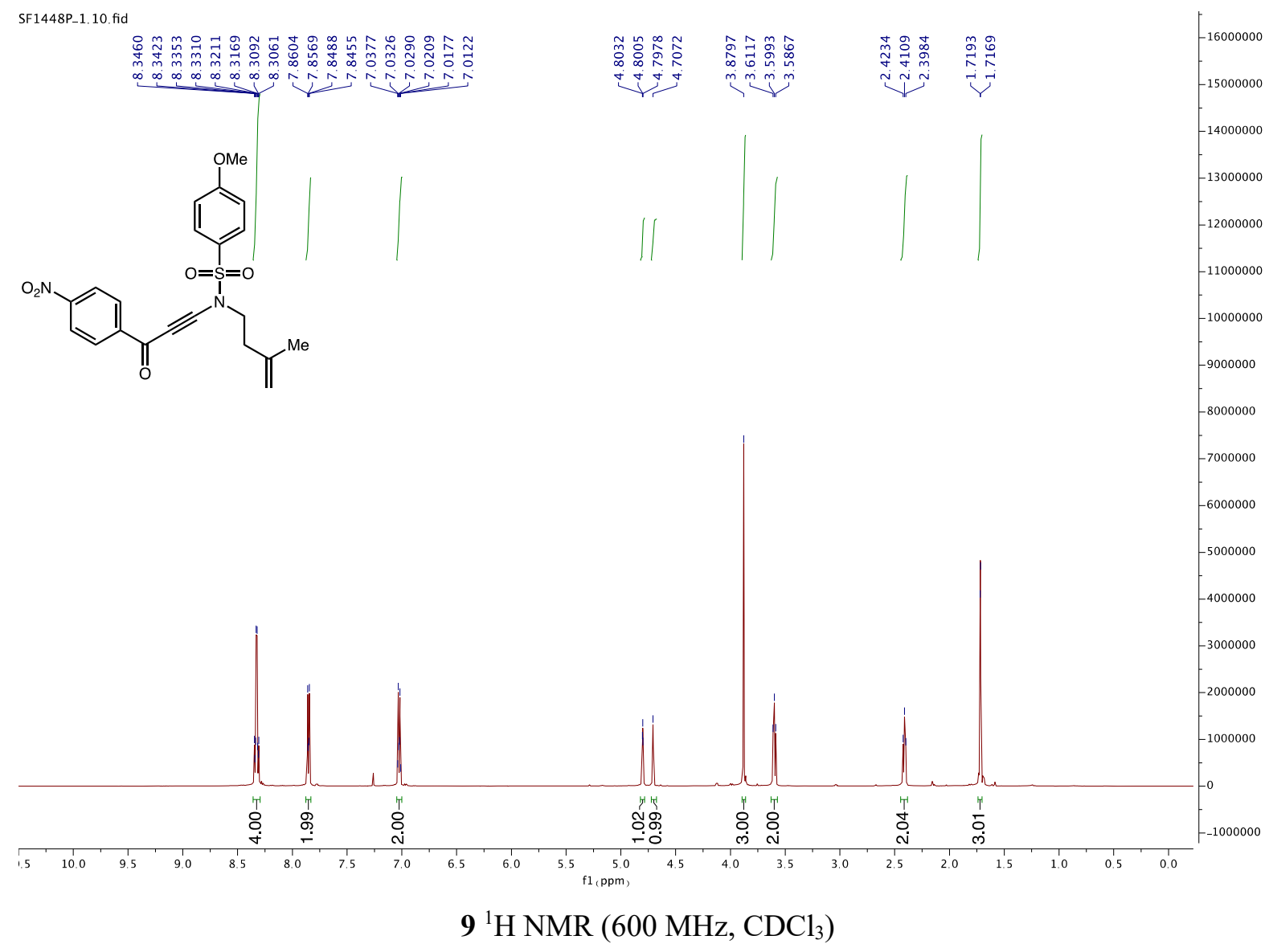

SF1448SM-1.10. fid

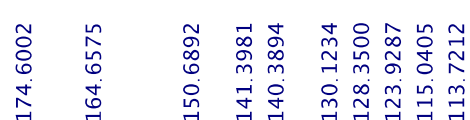

吉过

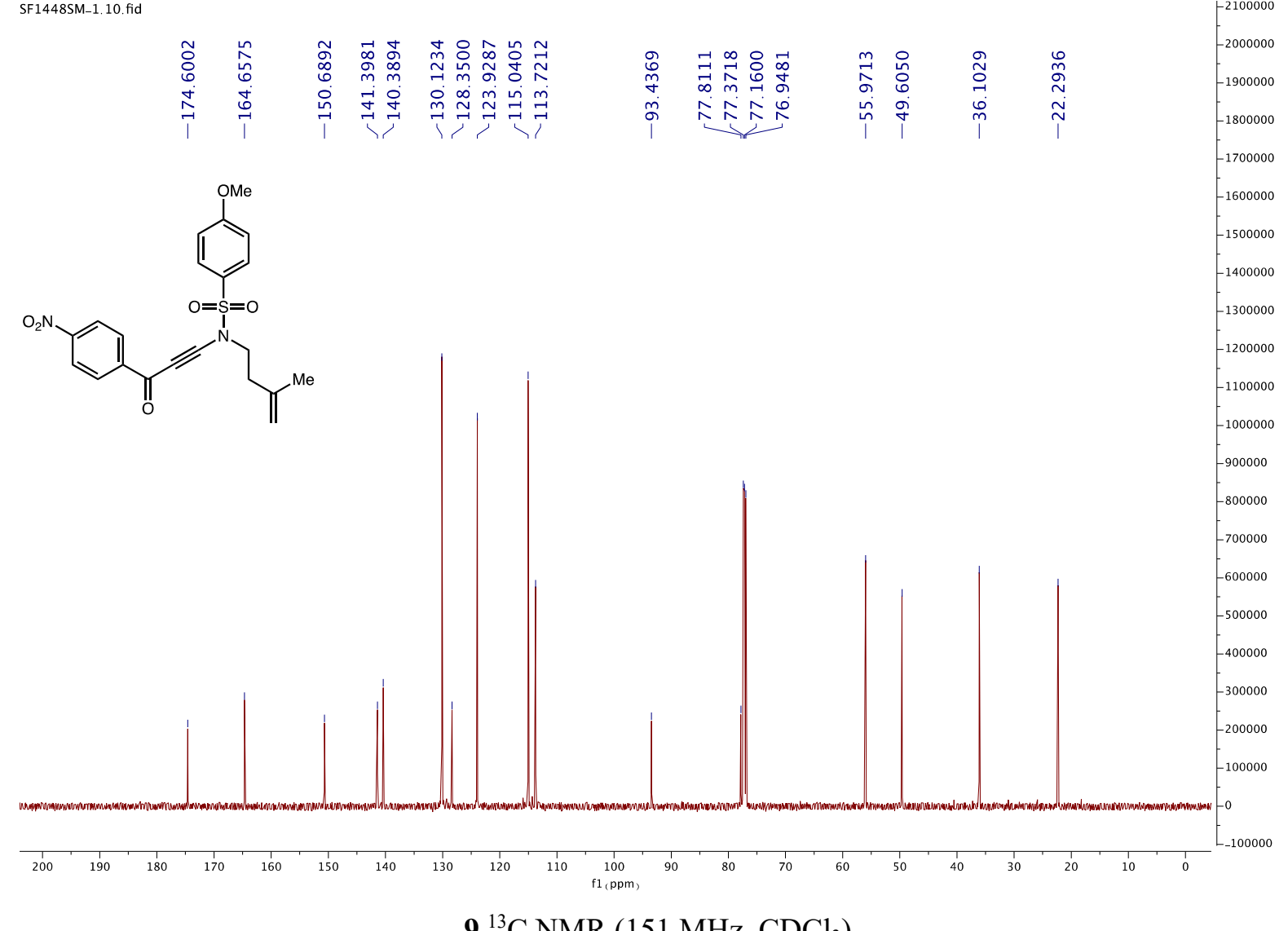

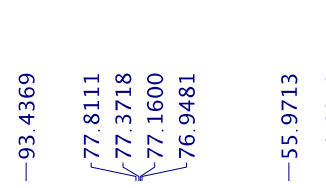

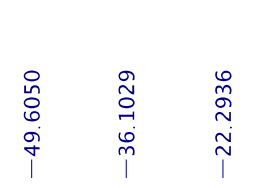

$-2100000$

$9{ }^{13} \mathrm{C}$ NMR $\left(151 \mathrm{MHz}, \mathrm{CDCl}_{3}\right)$ 


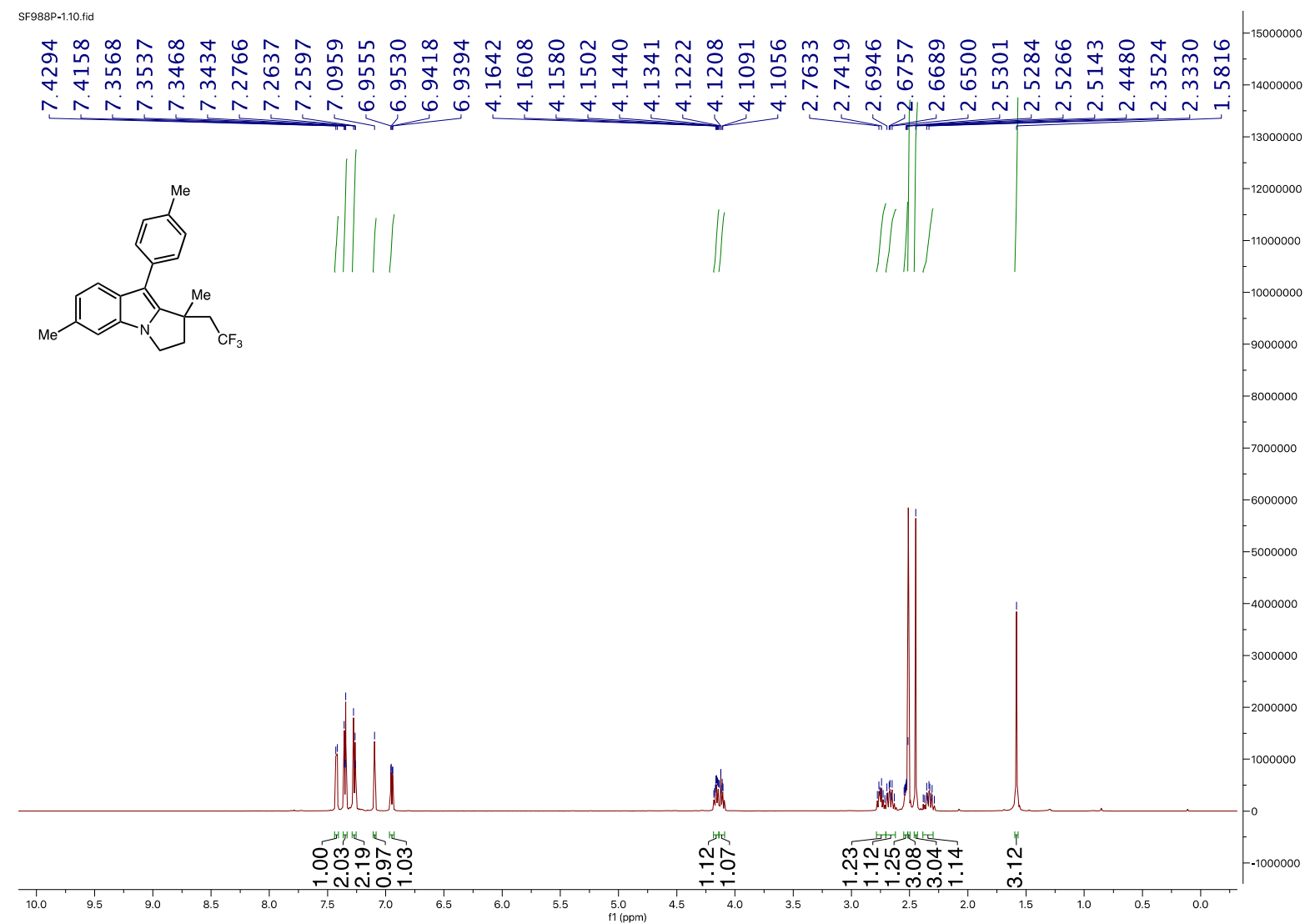

3a ${ }^{1} \mathrm{H}$ NMR $\left(600 \mathrm{MHz}, \mathrm{CDCl}_{3}\right)$

SF988-1.1.:fid
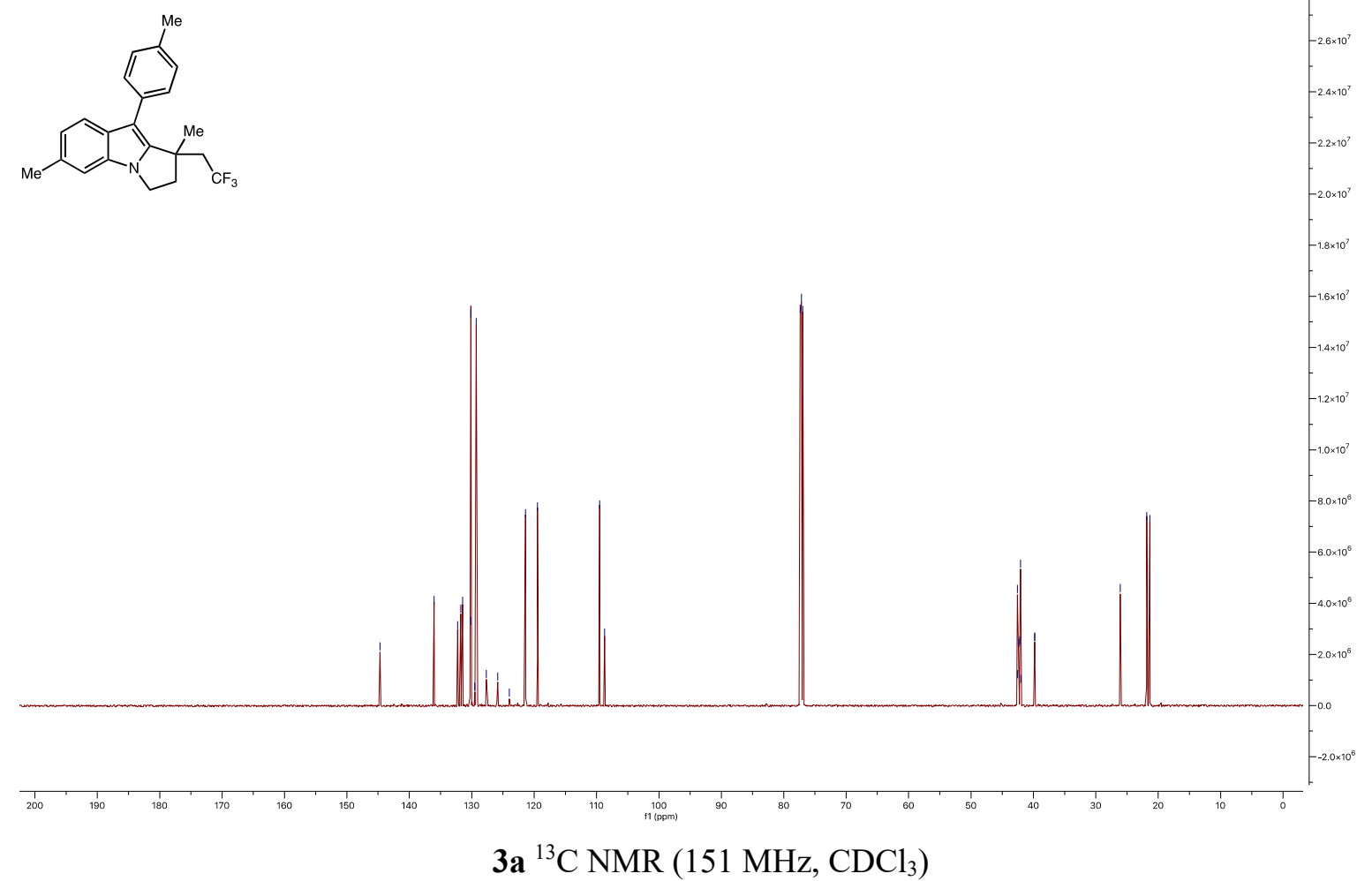

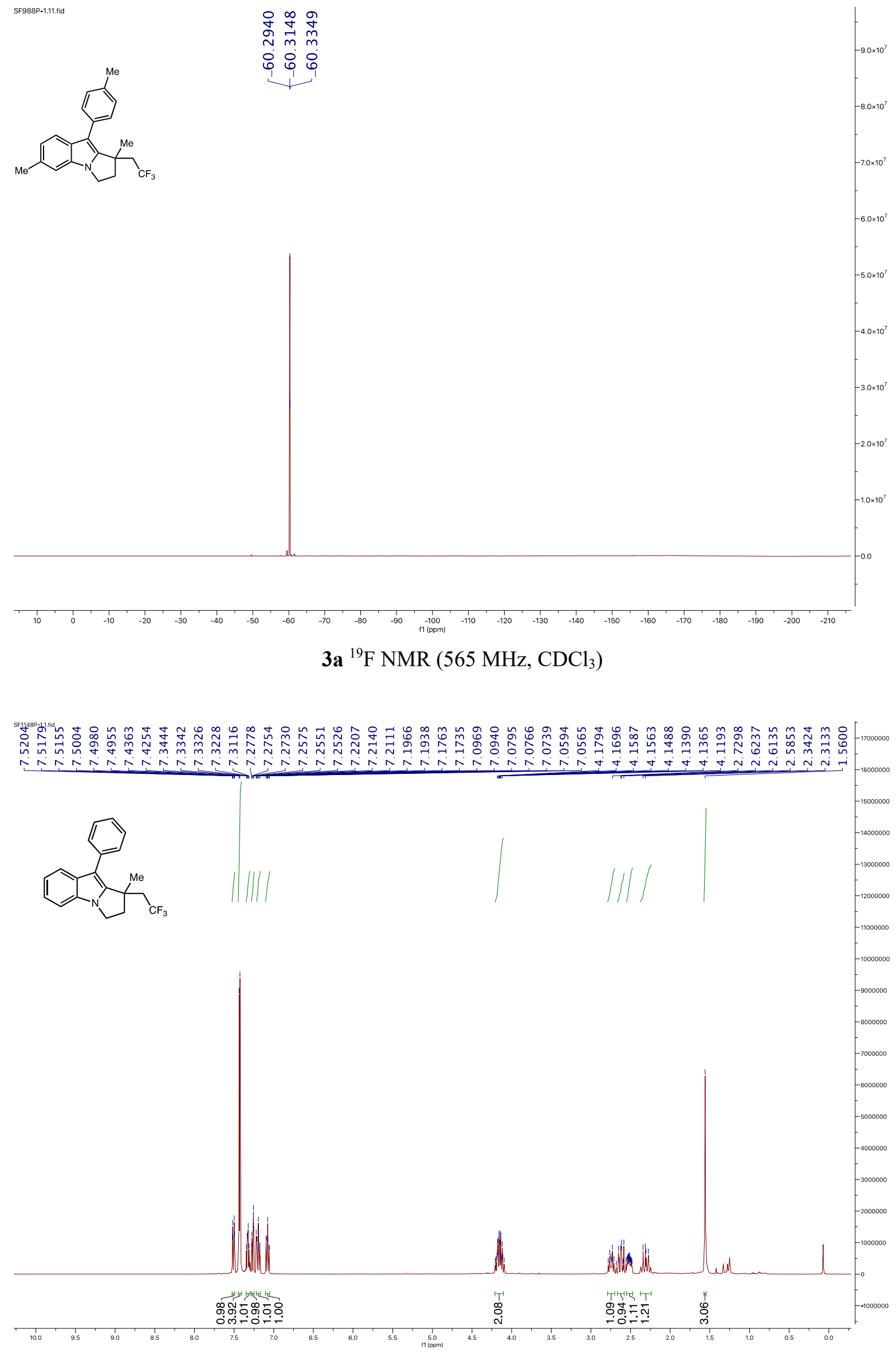

3b ${ }^{1} \mathrm{H}$ NMR (400 MHz, $\mathrm{CDCl}_{3}$ ) 


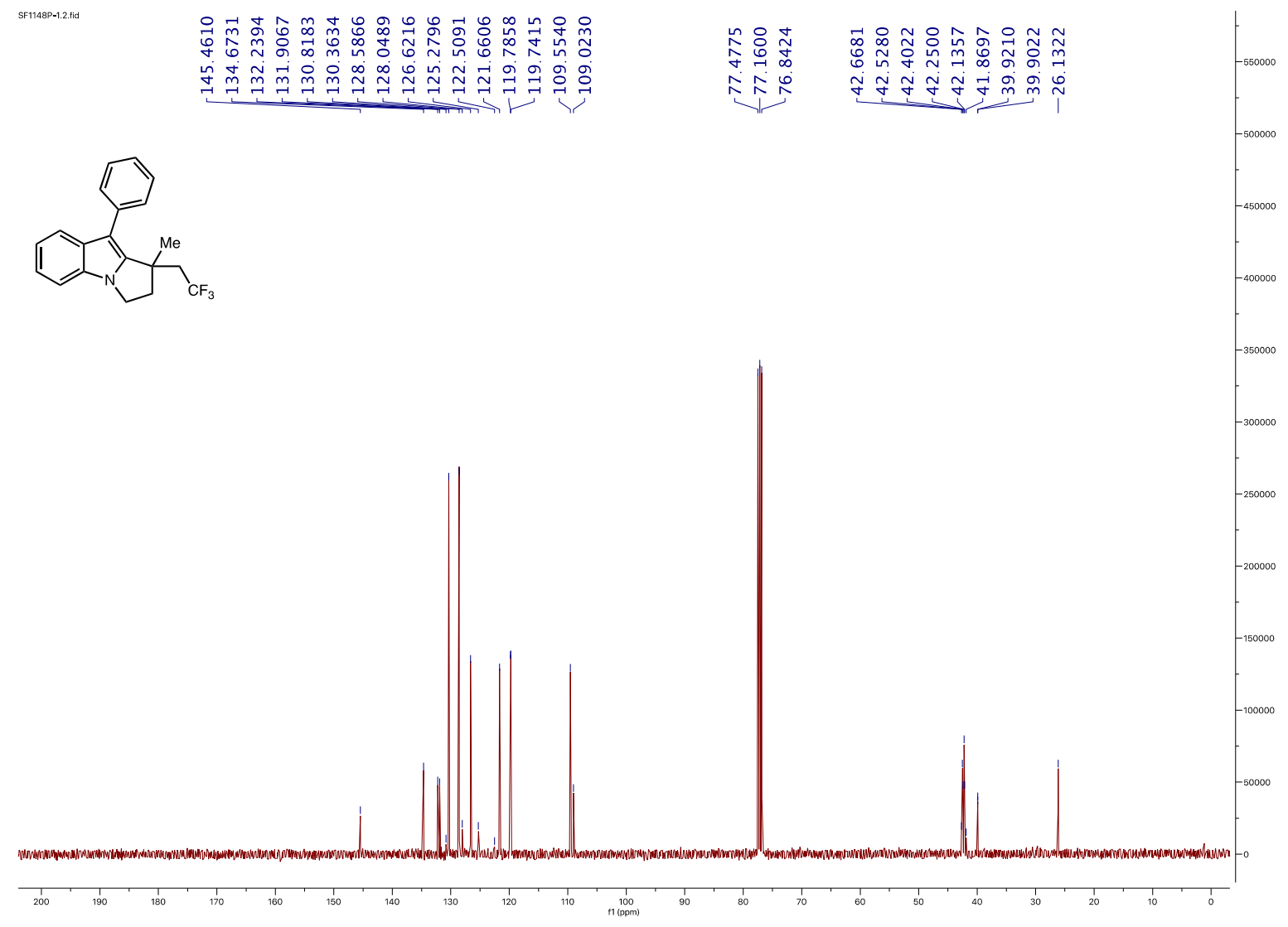

3b ${ }^{13} \mathrm{C}$ NMR (101 MHz, $\left.\mathrm{CDCl}_{3}\right)$

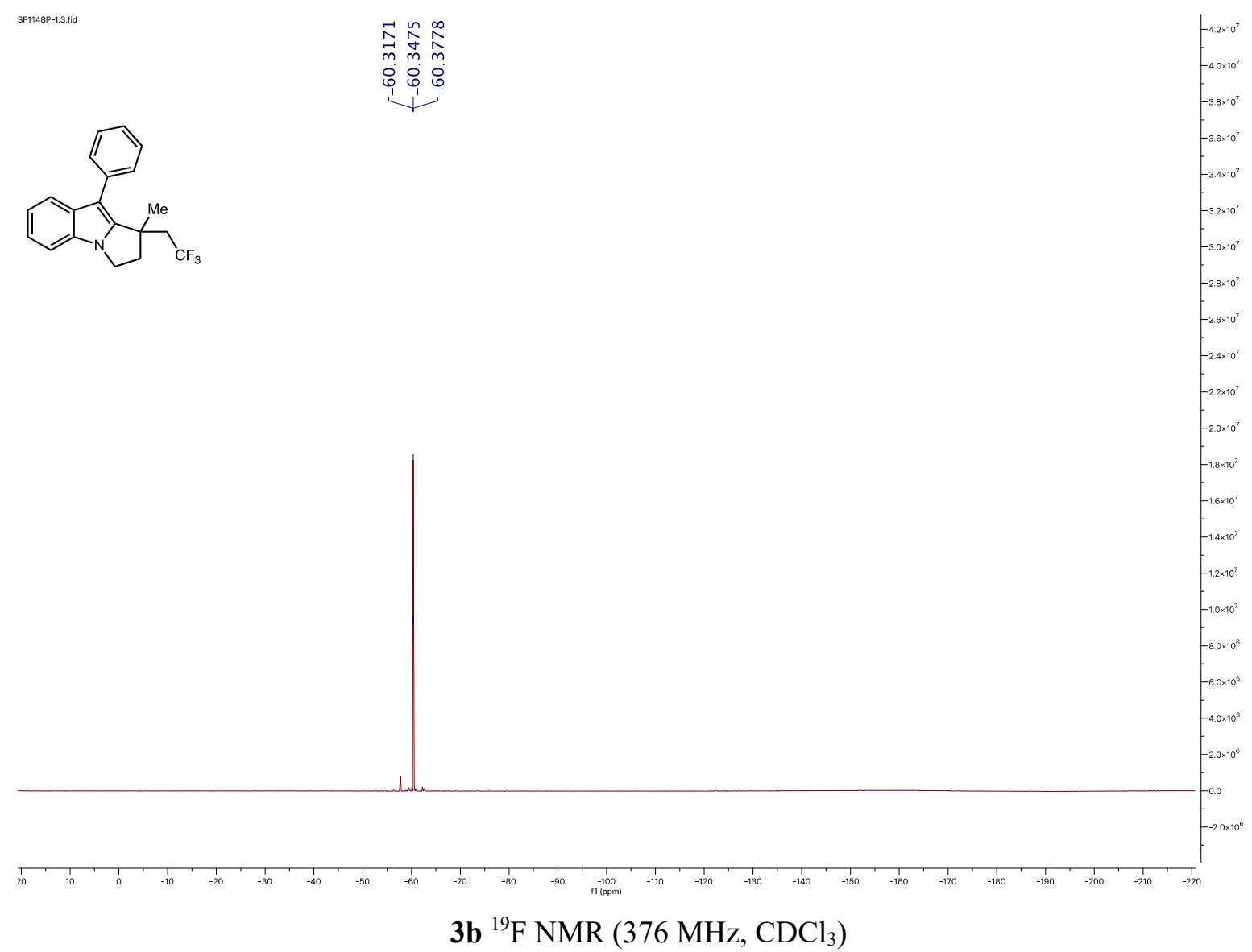




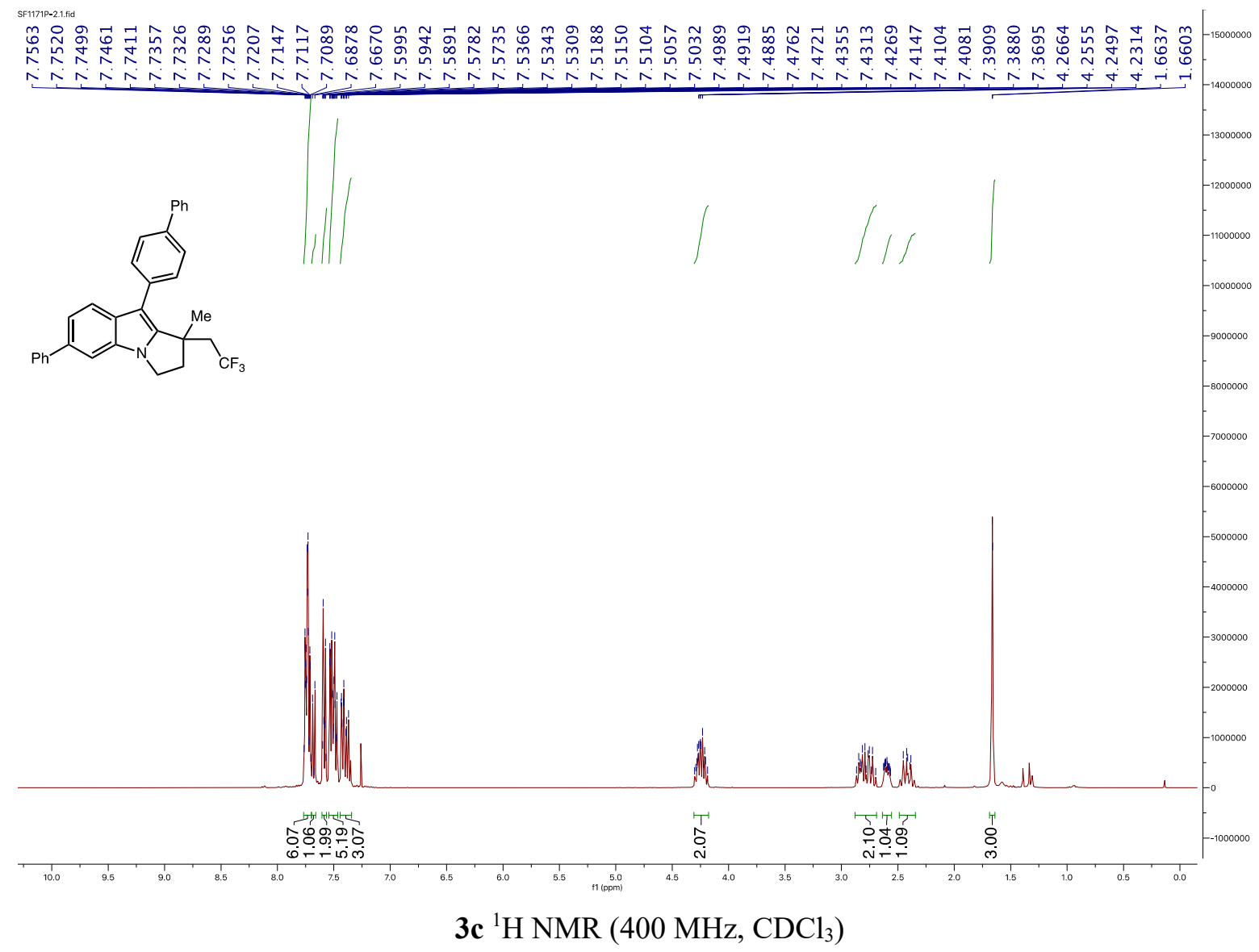

SF1717-2.2.fid

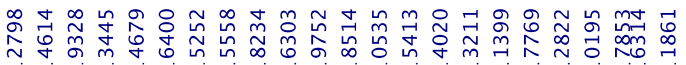

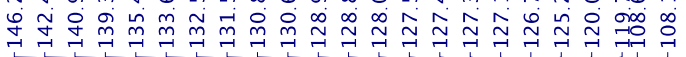

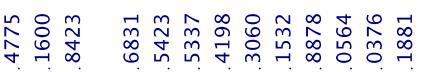

5i依<smiles></smiles>

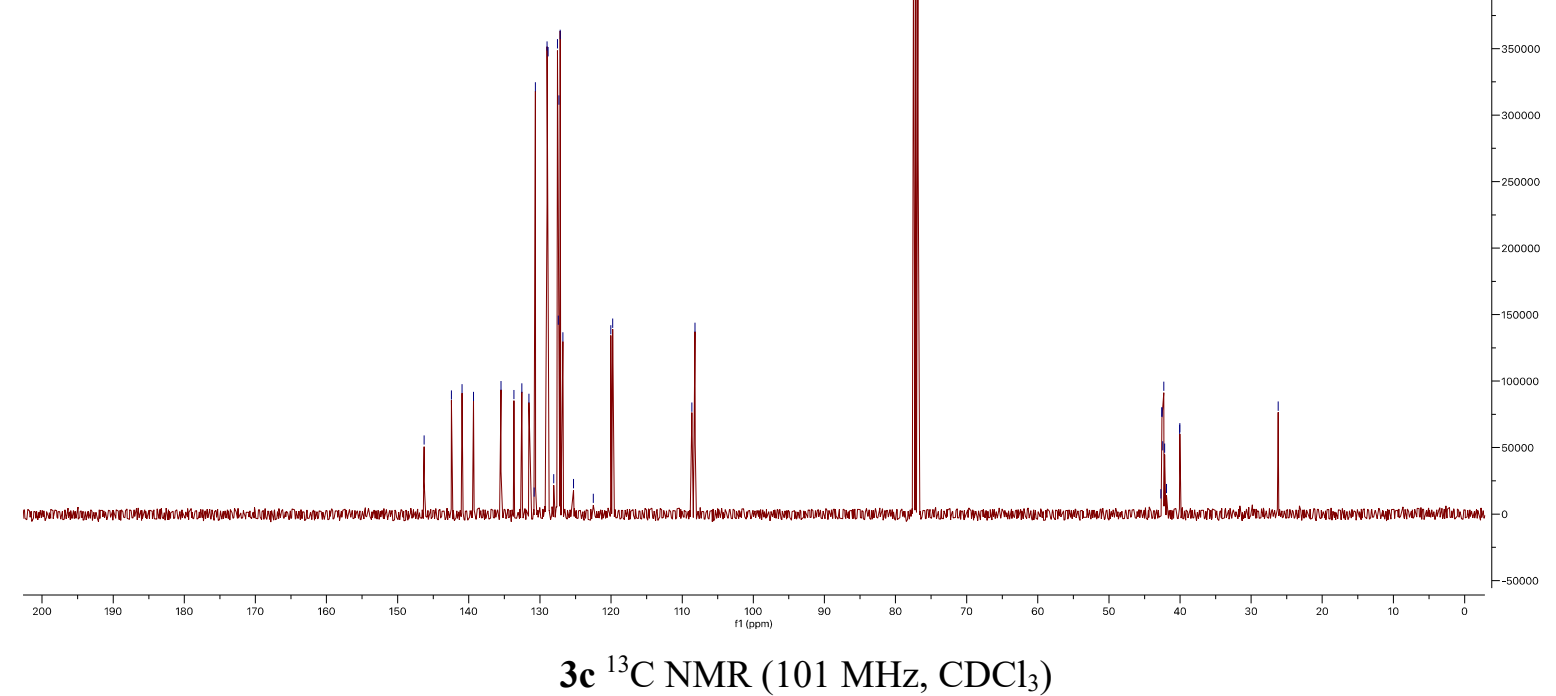



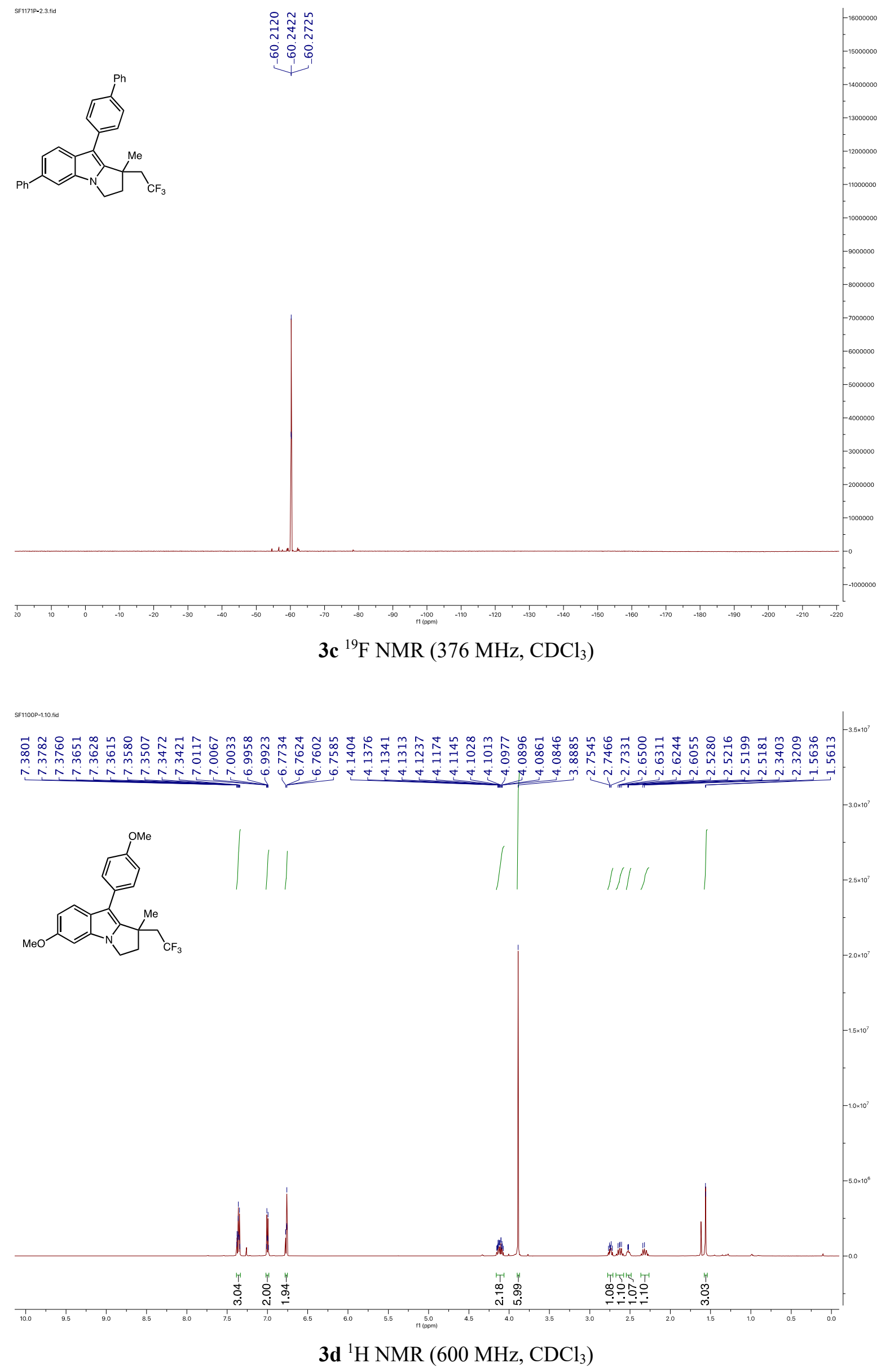


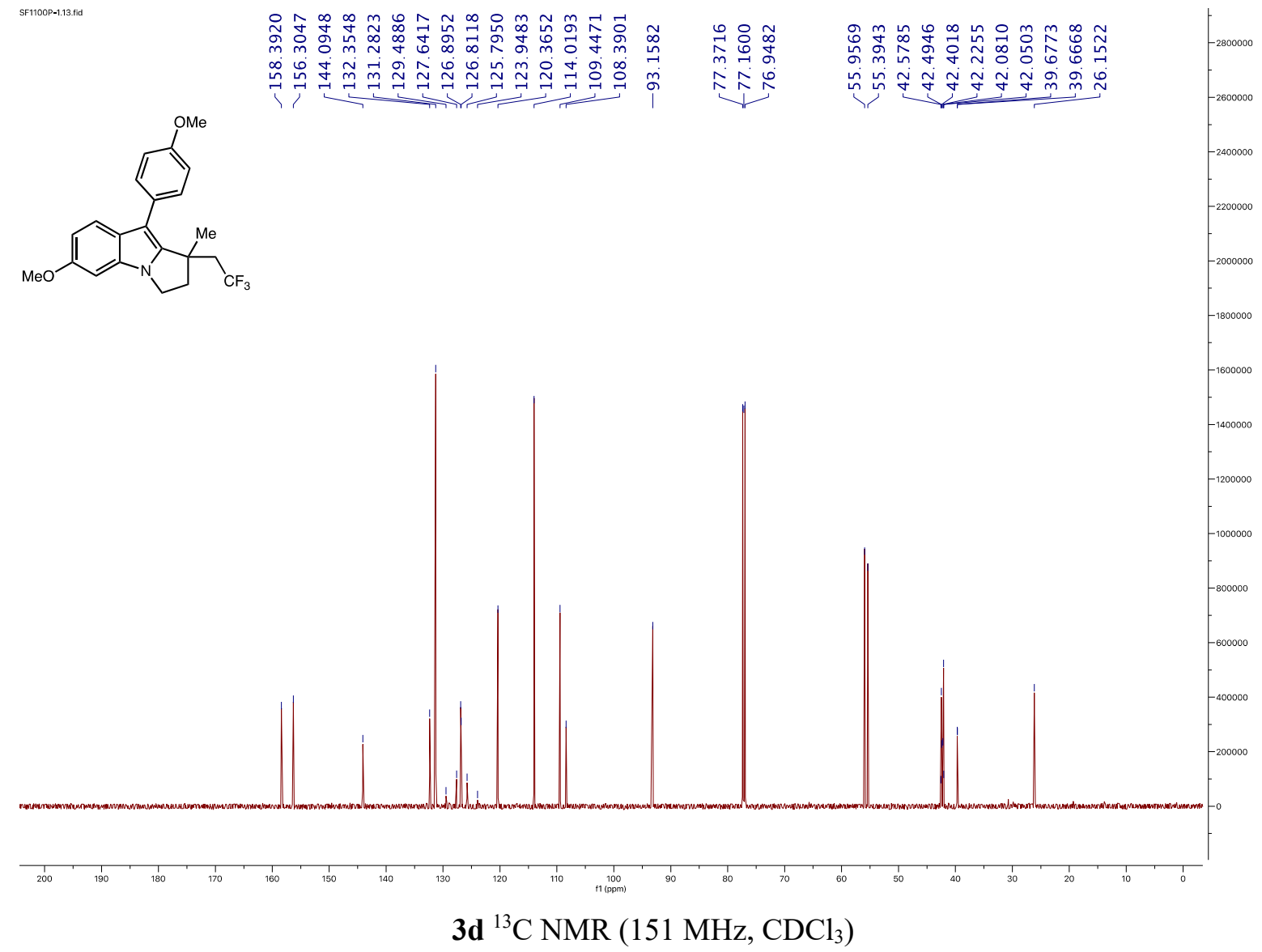

SF1100p-1.12.1id

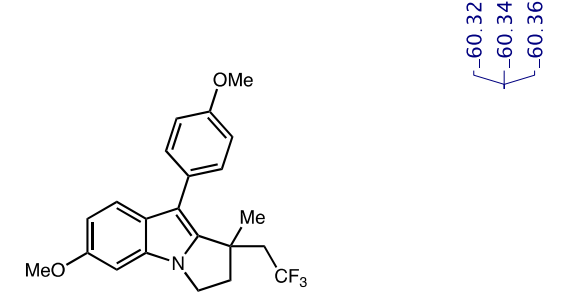

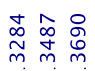

10
0

$\mathrm{Cr}_{3}$

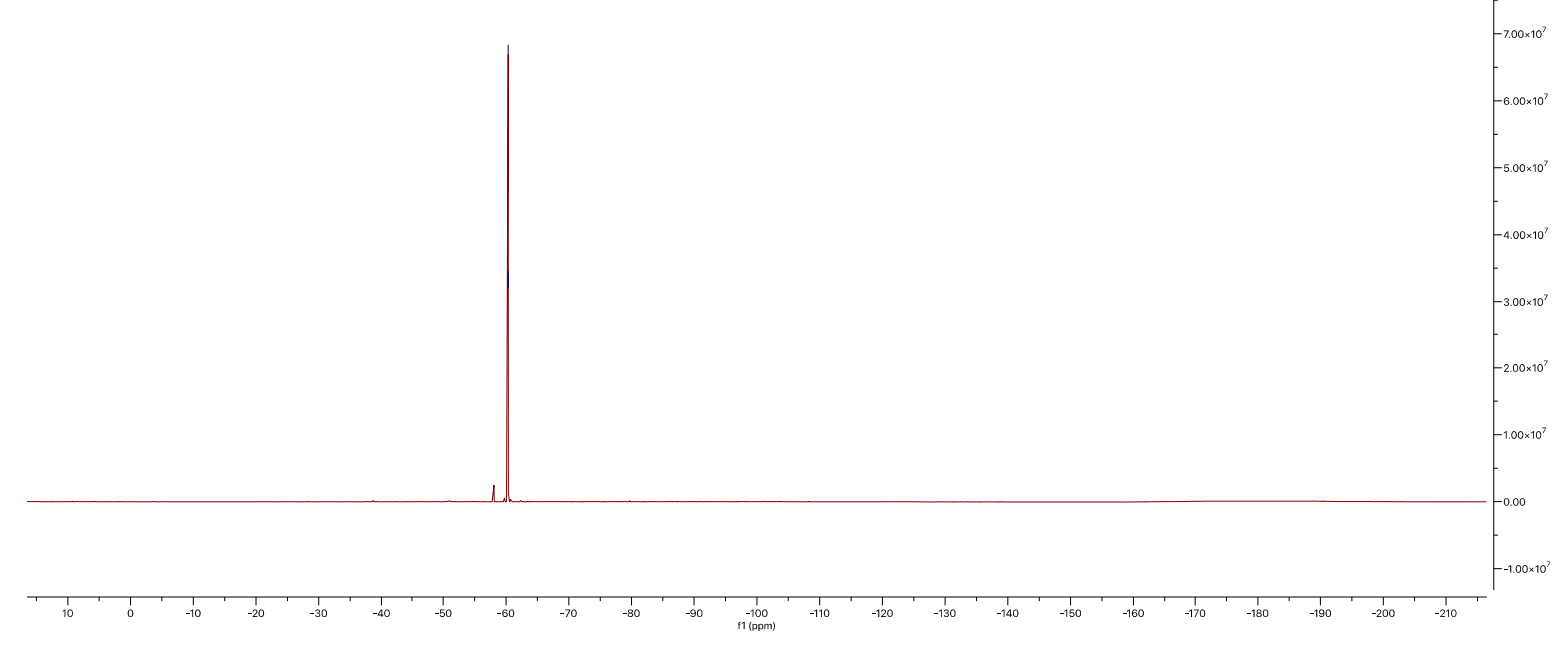

3d ${ }^{19} \mathrm{~F}$ NMR $\left(565 \mathrm{MHz}, \mathrm{CDCl}_{3}\right)$ 


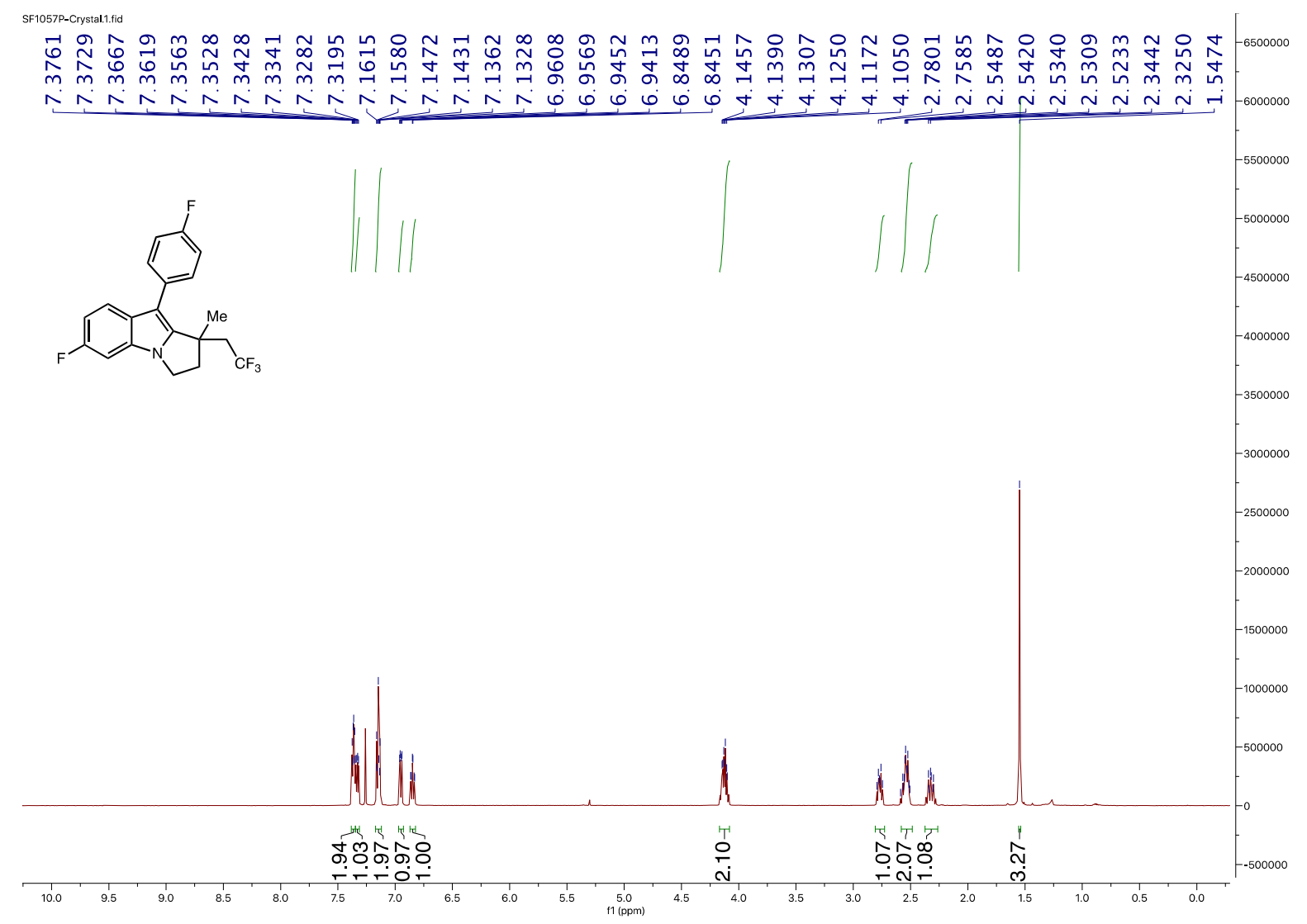

3e ${ }^{1} \mathrm{H}$ NMR (600 MHz, $\mathrm{CDCl}_{3}$ )

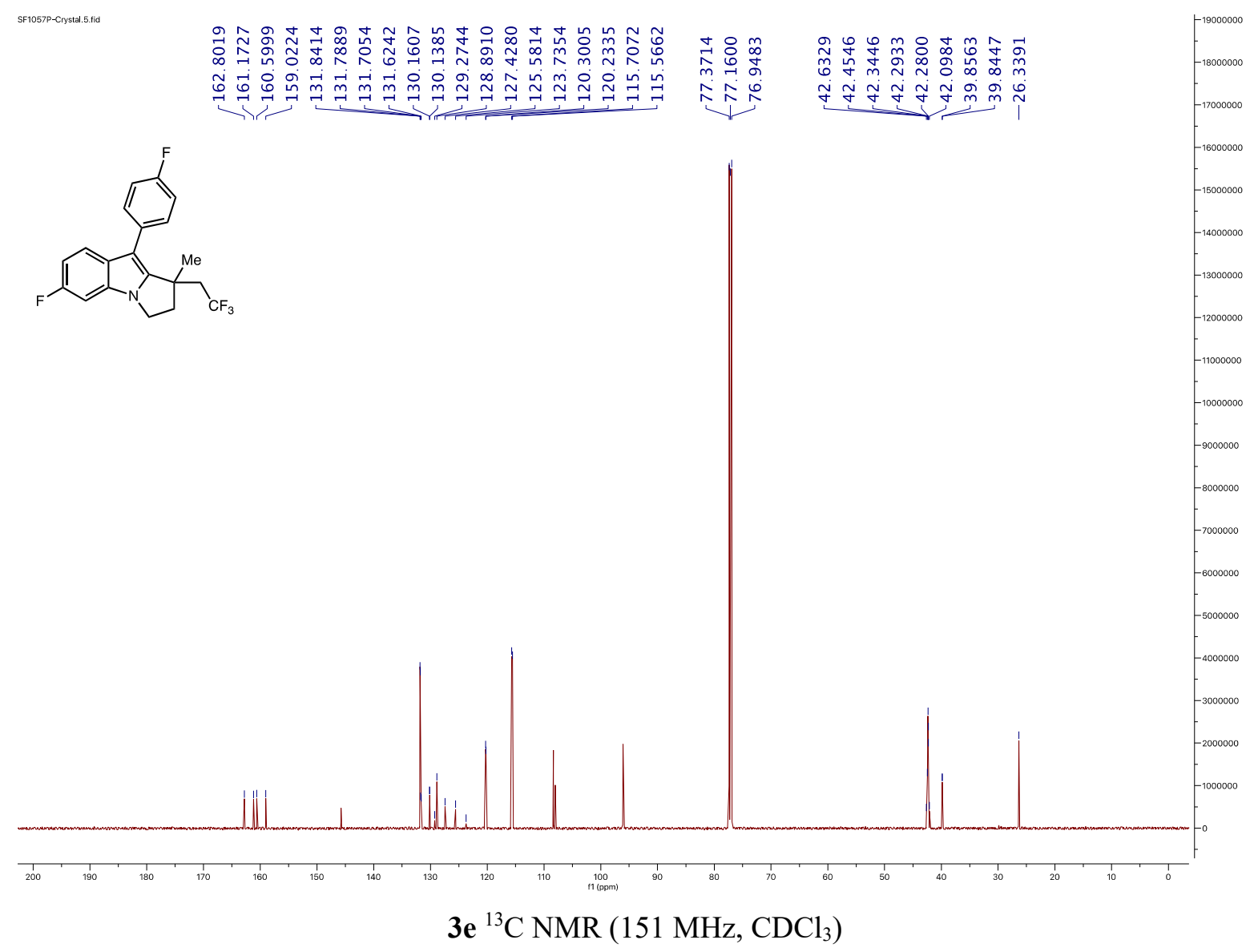




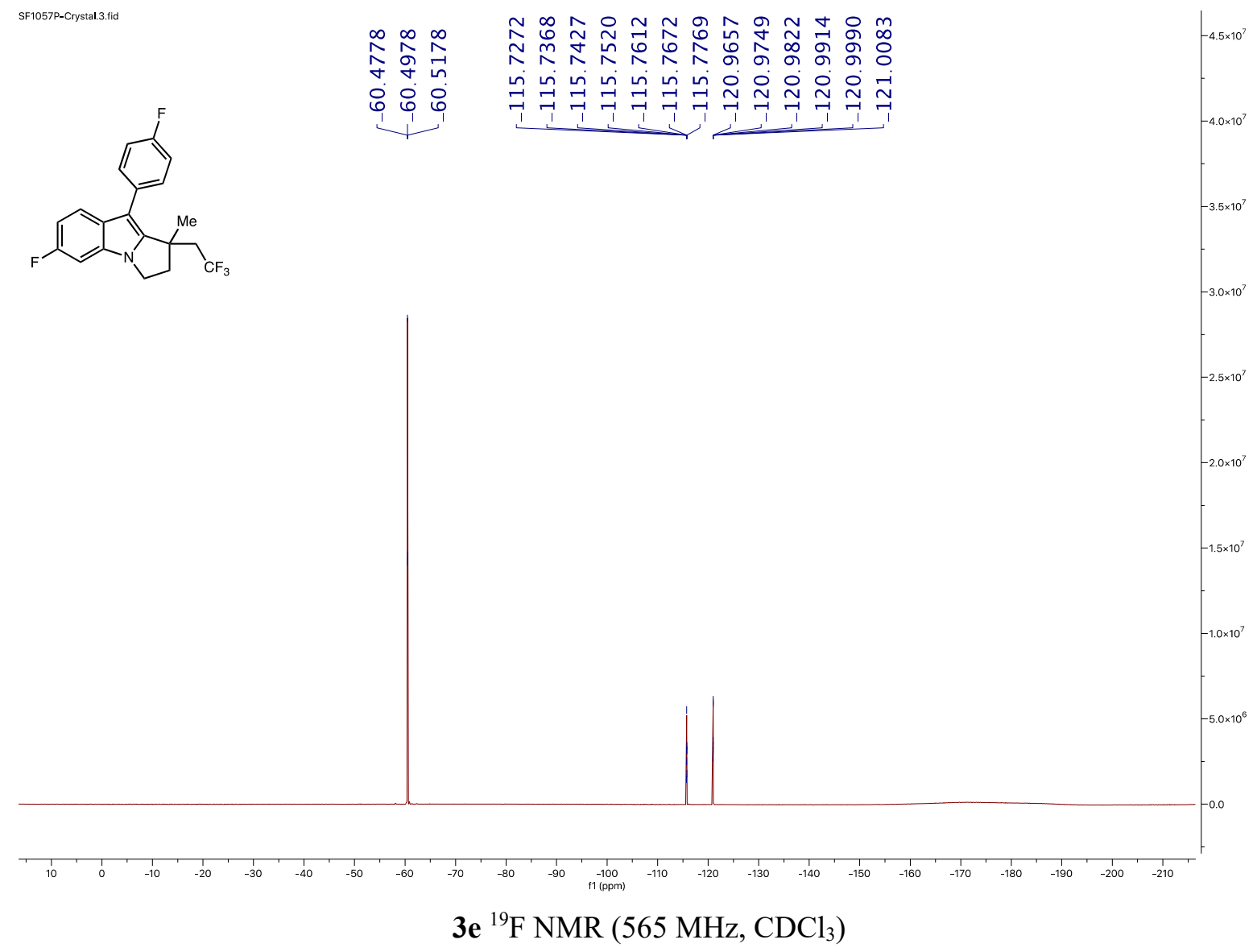

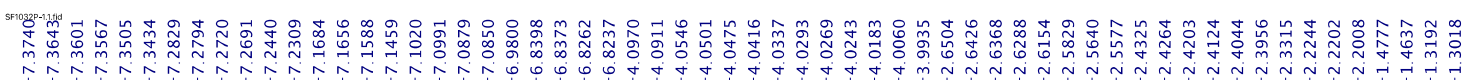
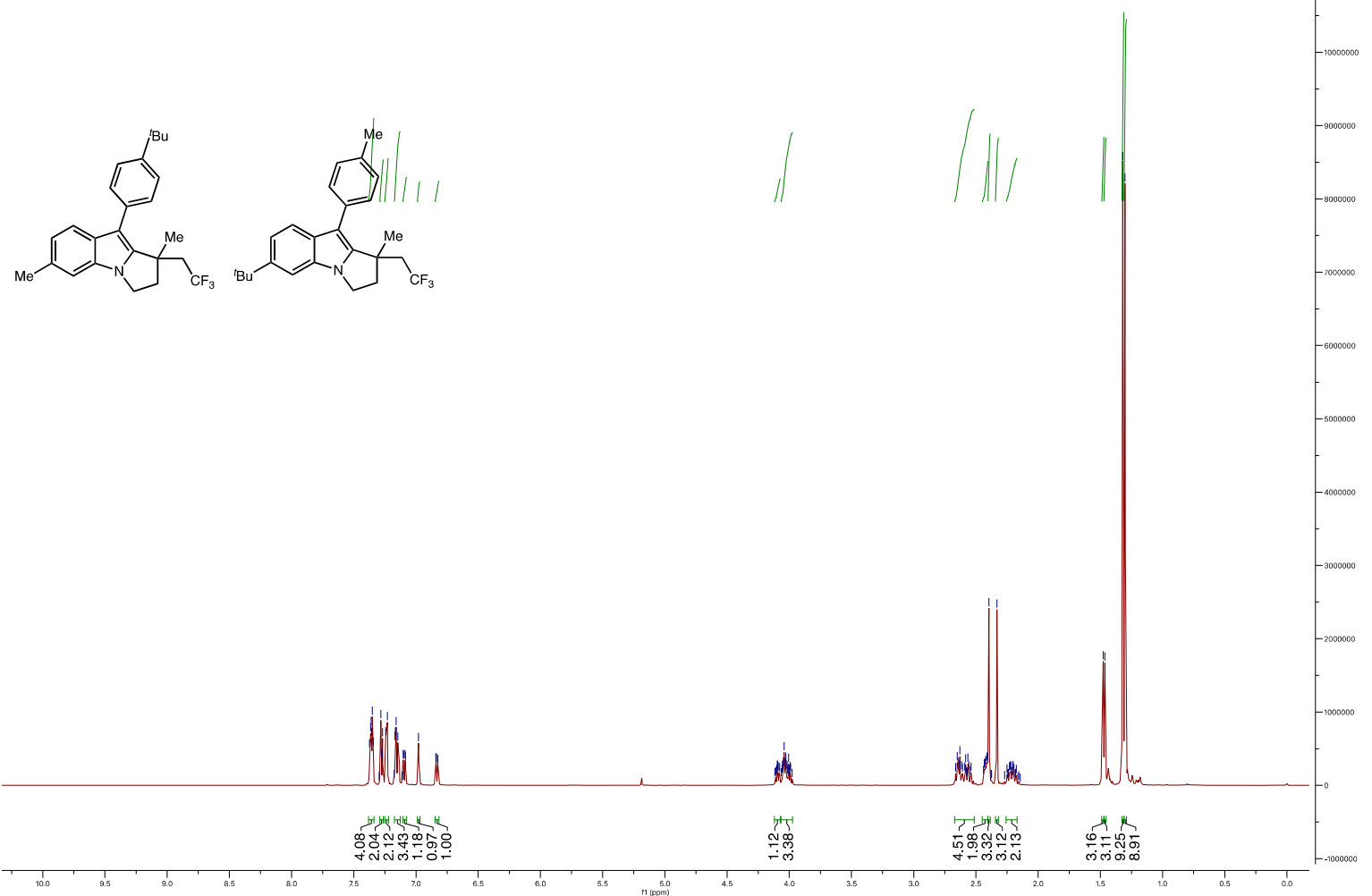

3f, 3f ${ }^{1} \mathrm{H}$ NMR $\left(600 \mathrm{MHz}, \mathrm{CDCl}_{3}\right)$ 

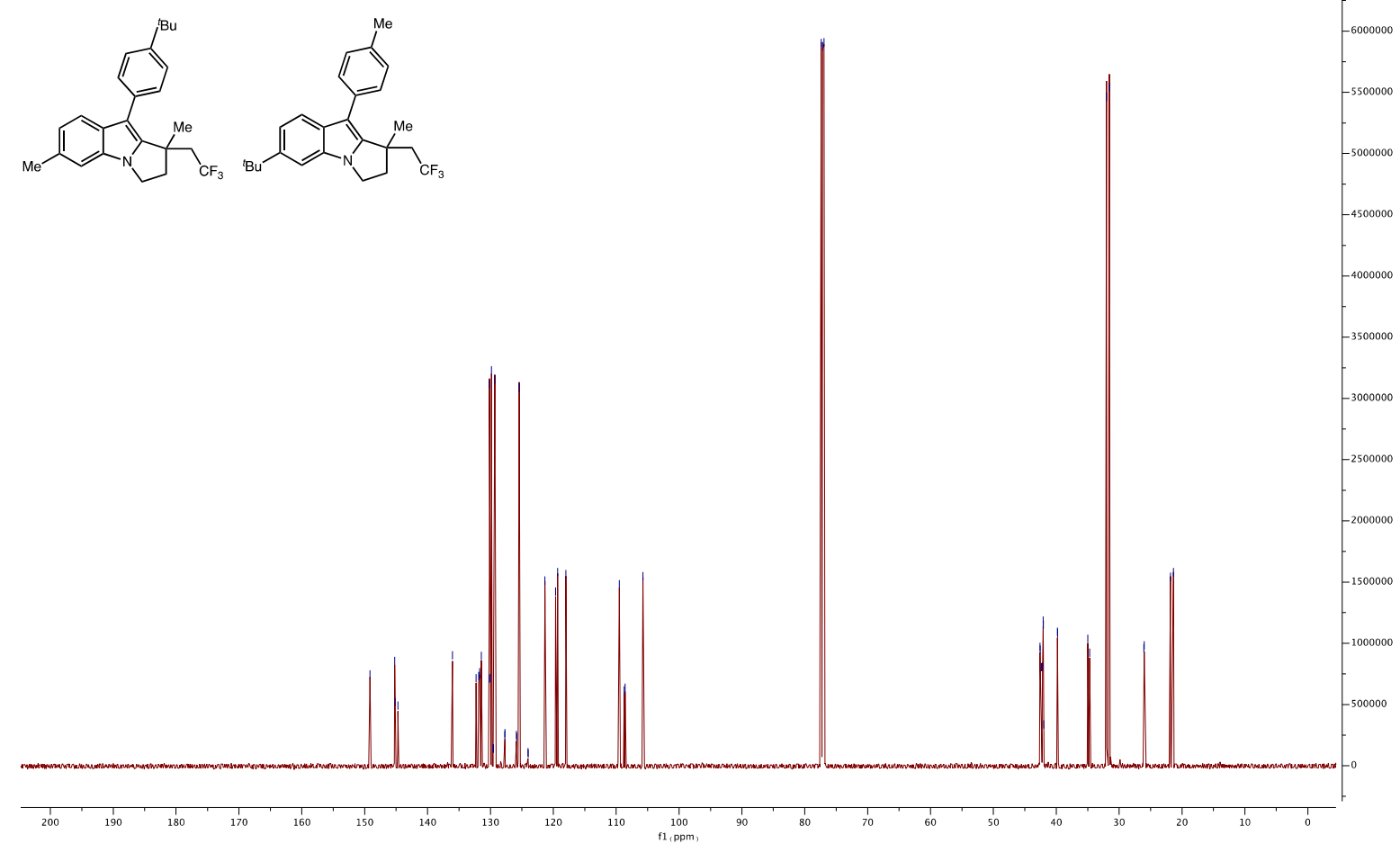

3f, 3f ${ }^{13} \mathrm{C} \mathrm{NMR}\left(151 \mathrm{MHz}, \mathrm{CDCl}_{3}\right)$
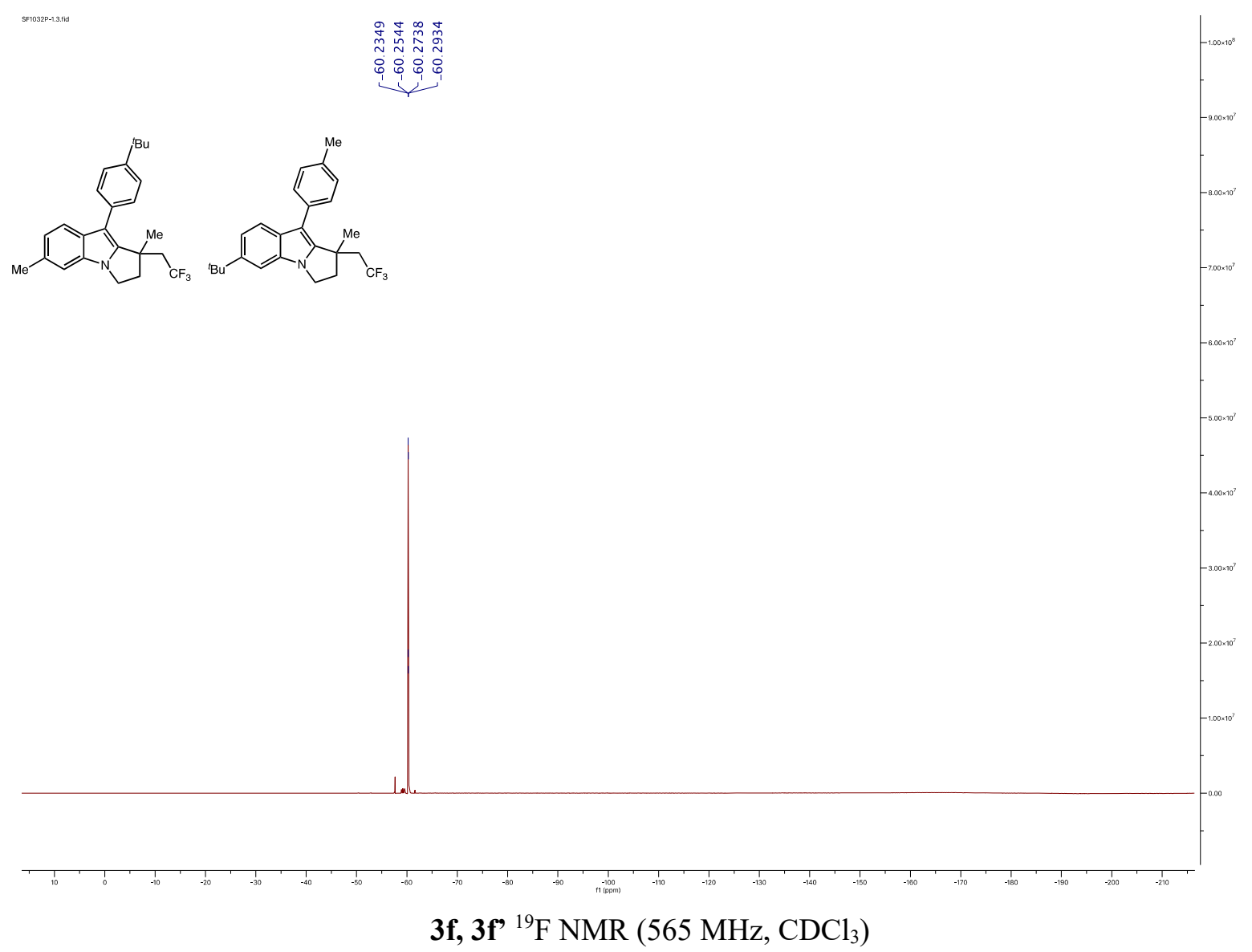


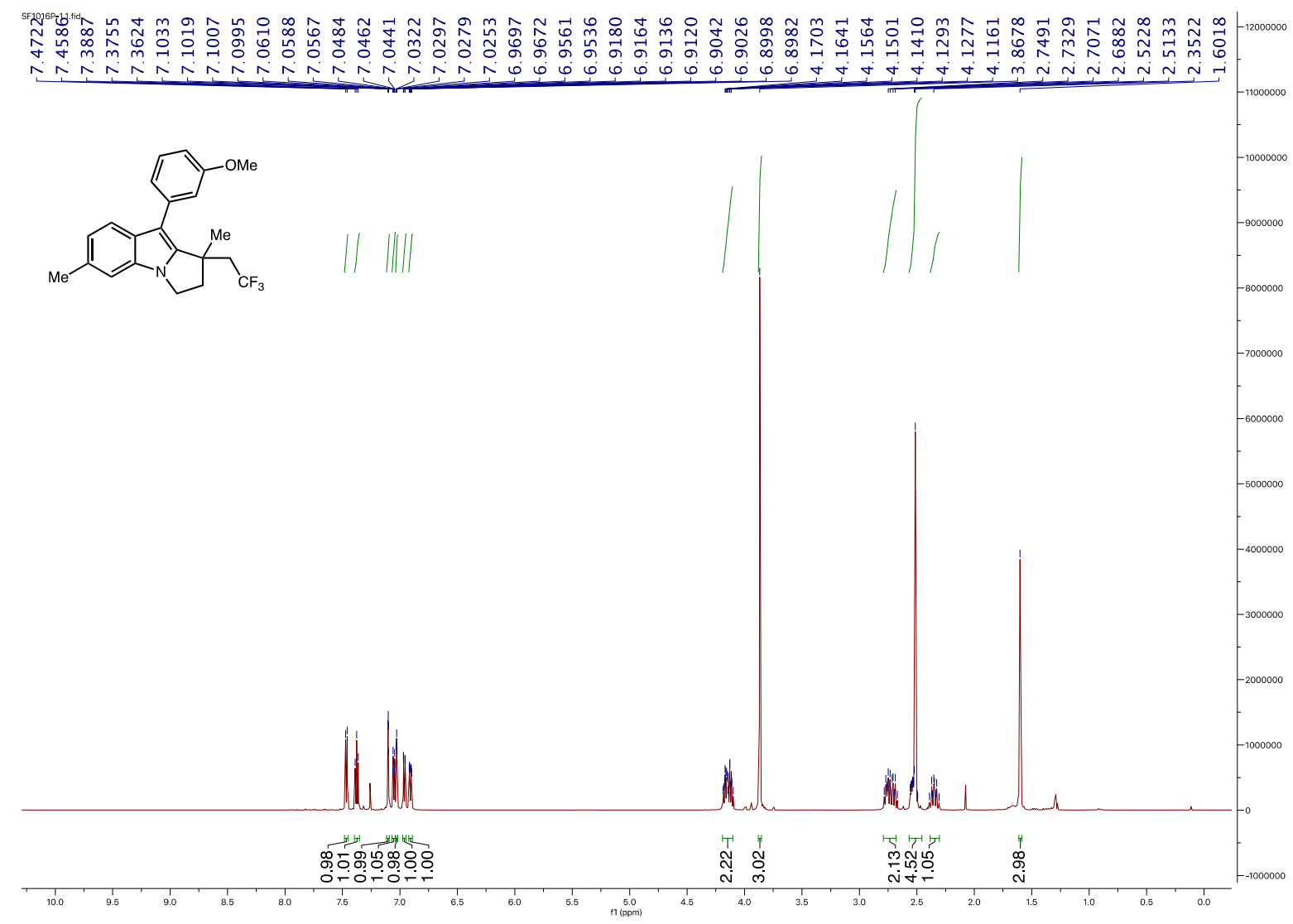

3g ${ }^{1} \mathrm{H}$ NMR (600 MHz, $\left.\mathrm{CDCl}_{3}\right)$

SF1016P-210.fid

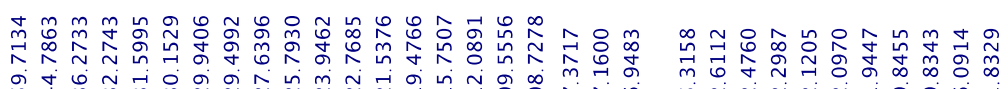

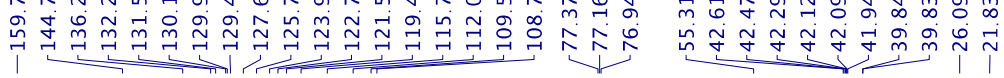

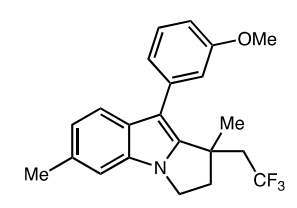



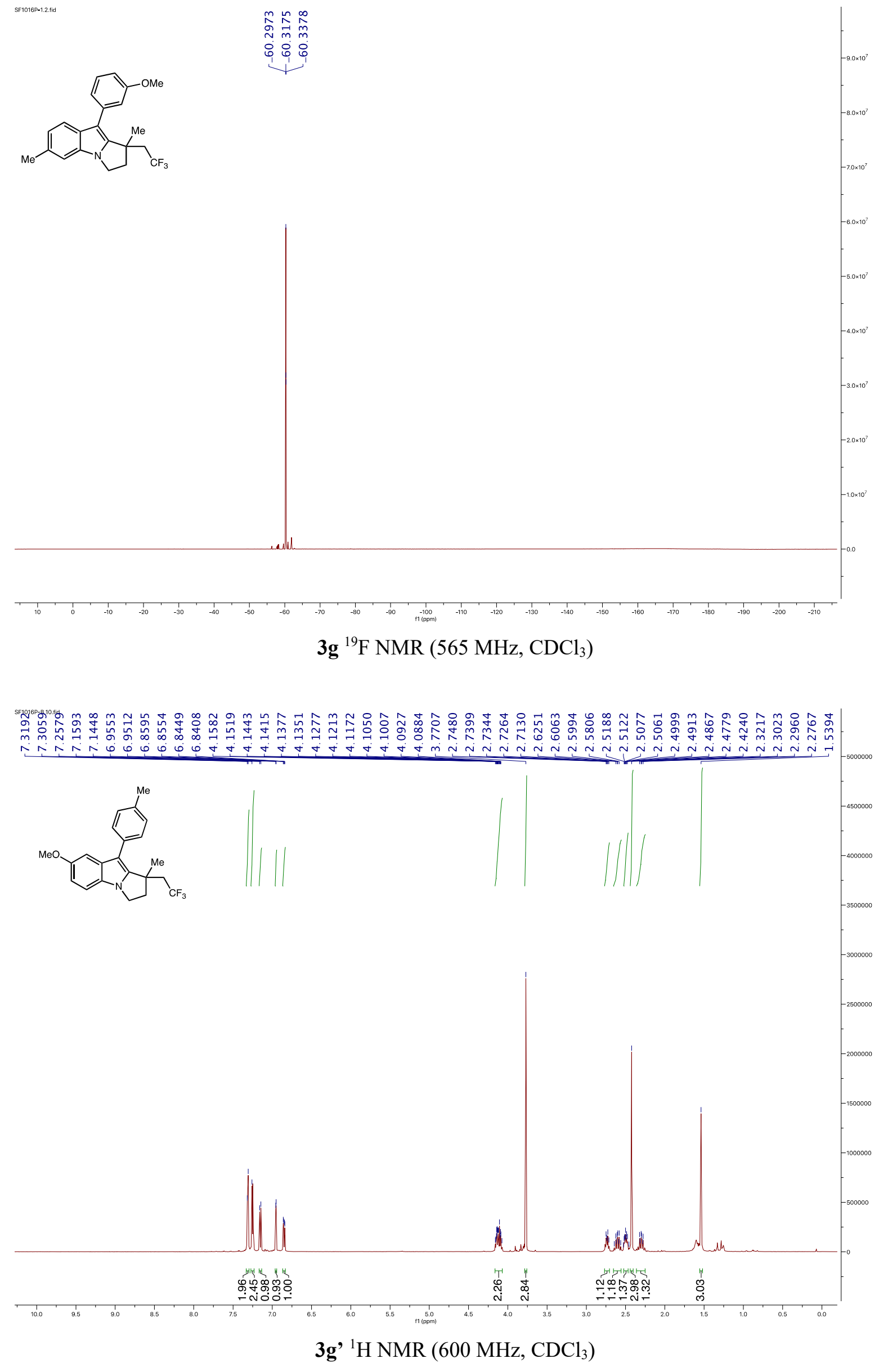

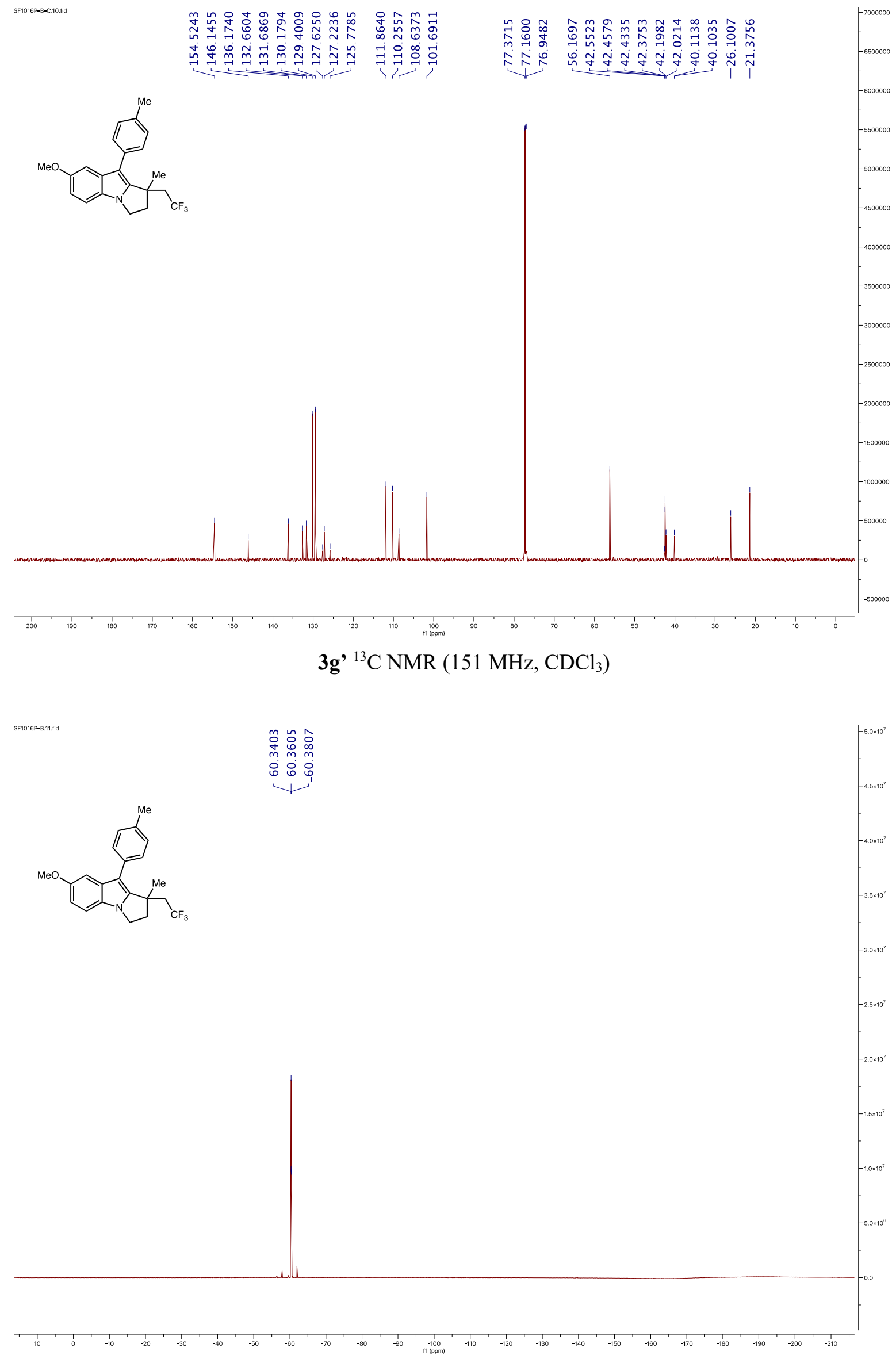

3g ${ }^{19} \mathrm{~F}$ NMR (565 MHz, $\mathrm{CDCl}_{3}$ ) 


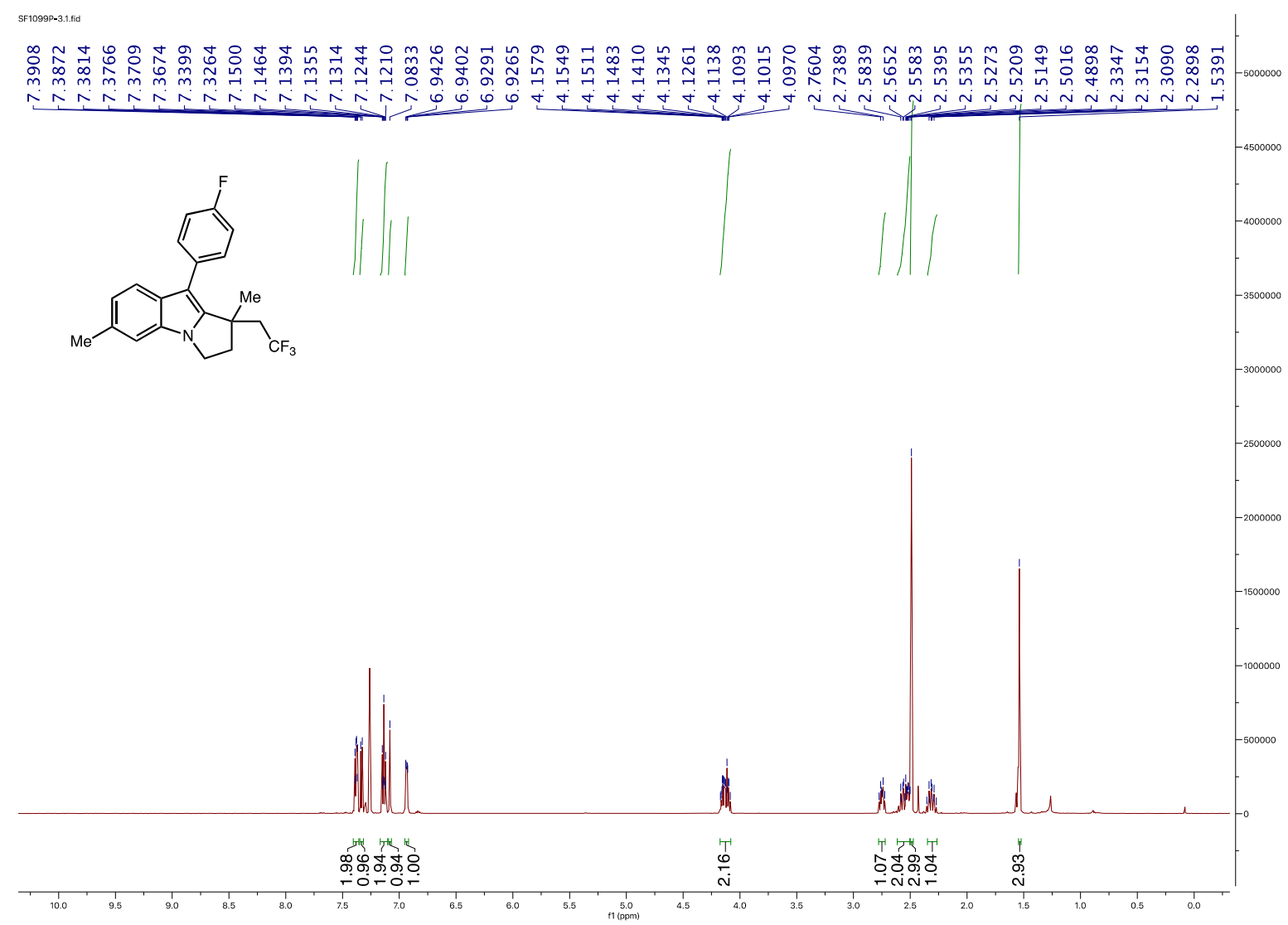

3h ${ }^{1} \mathrm{H}$ NMR (600 MHz, $\left.\mathrm{CDCl}_{3}\right)$

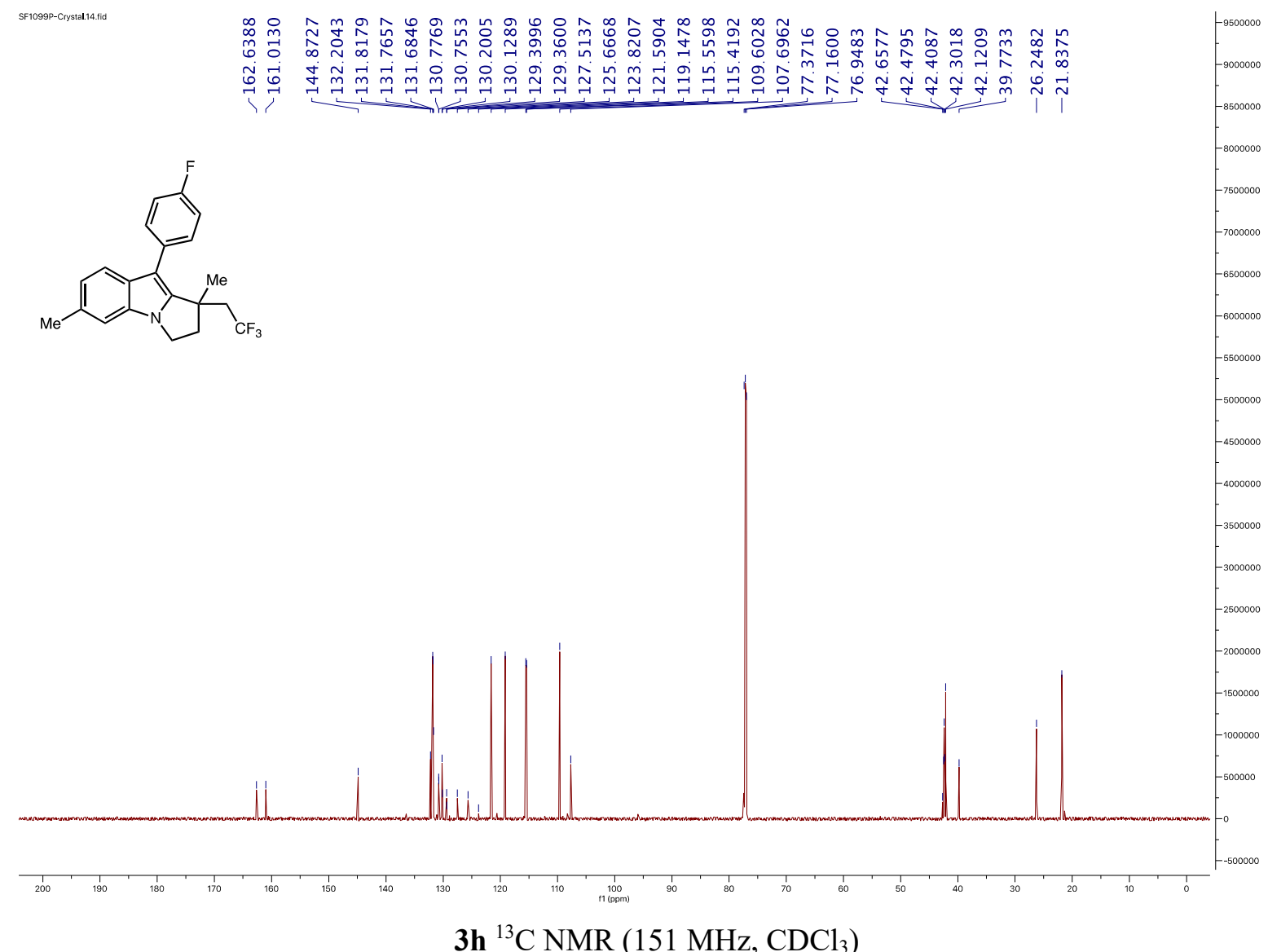



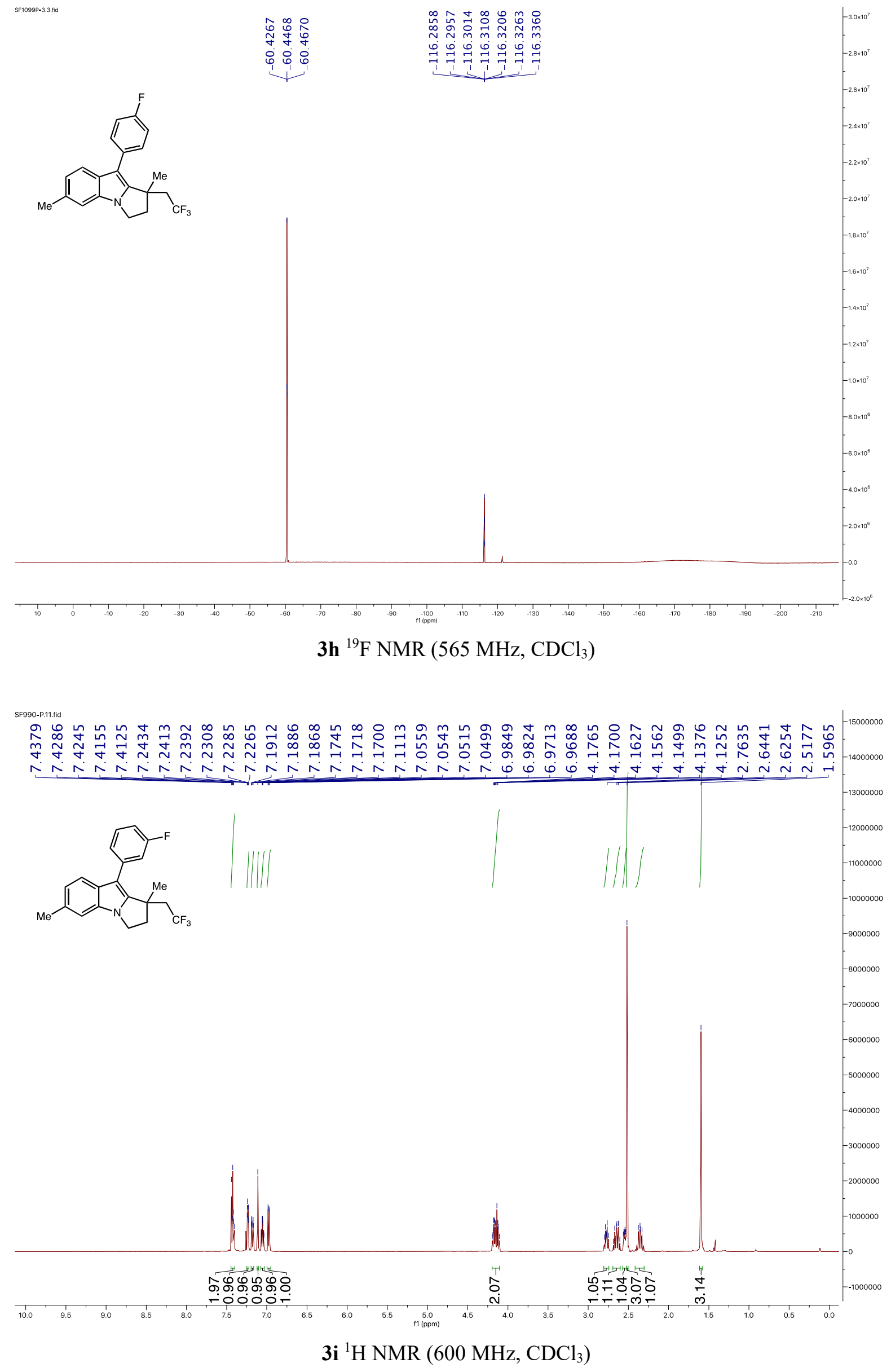

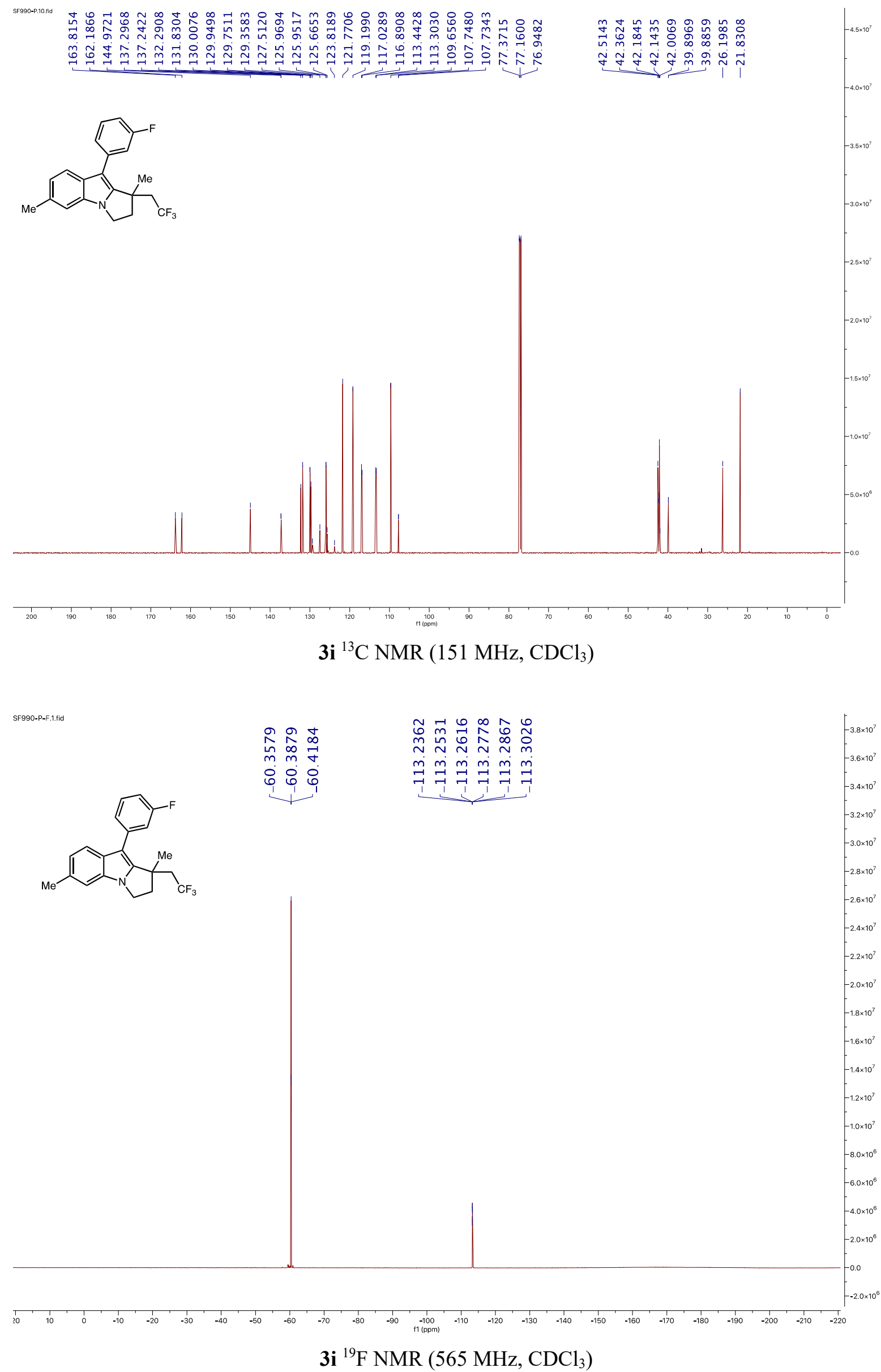


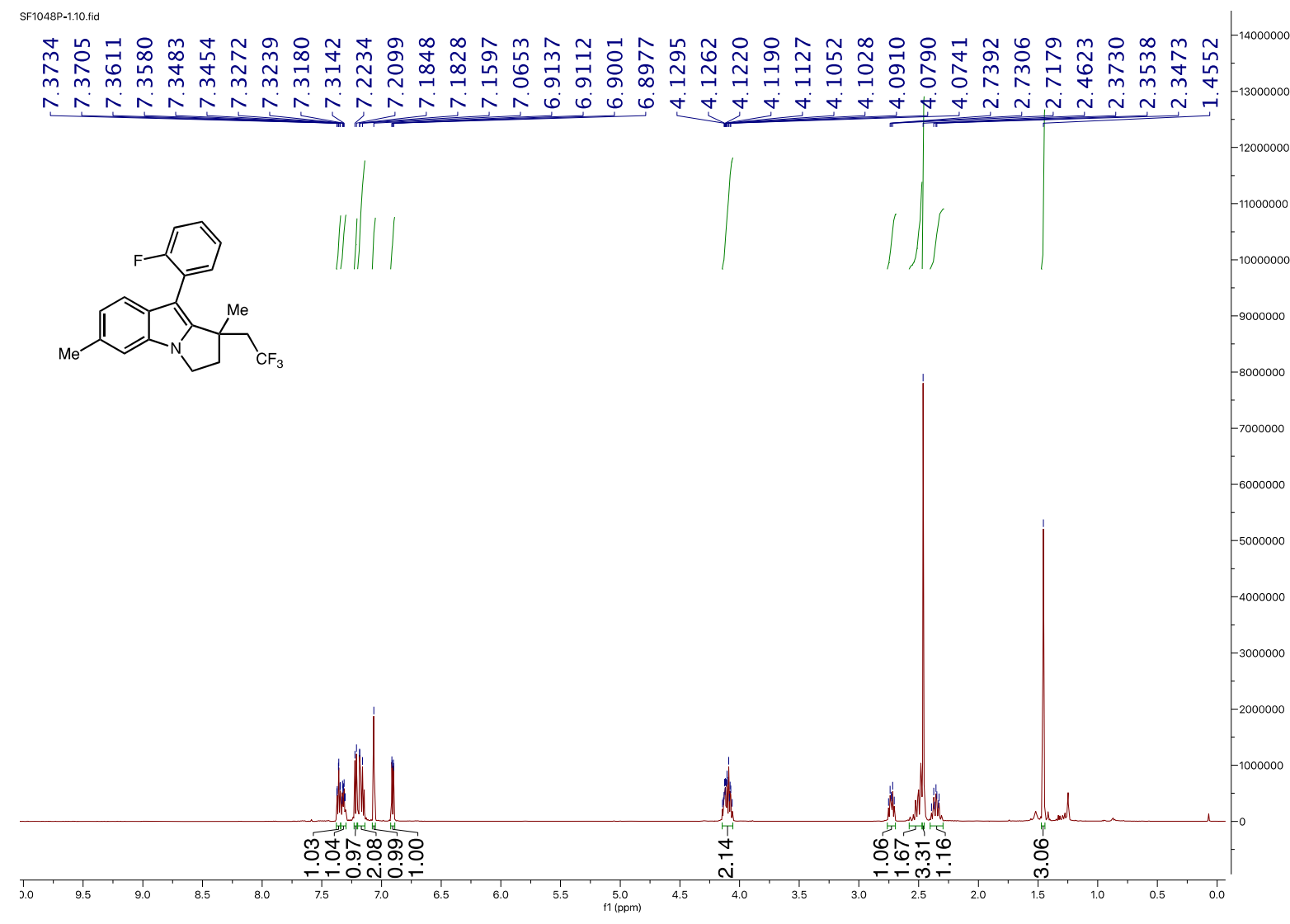

3j ${ }^{1} \mathrm{H}$ NMR (600 MHz, $\left.\mathrm{CDCl}_{3}\right)$

SF10488-113.1.id

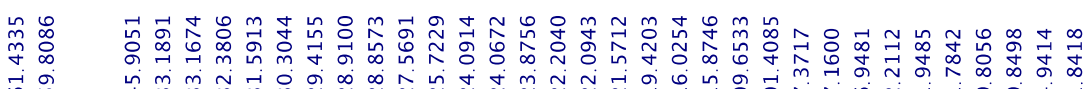

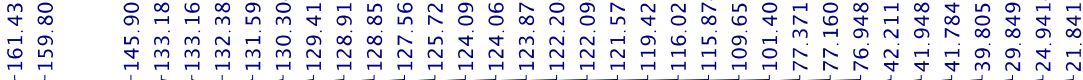
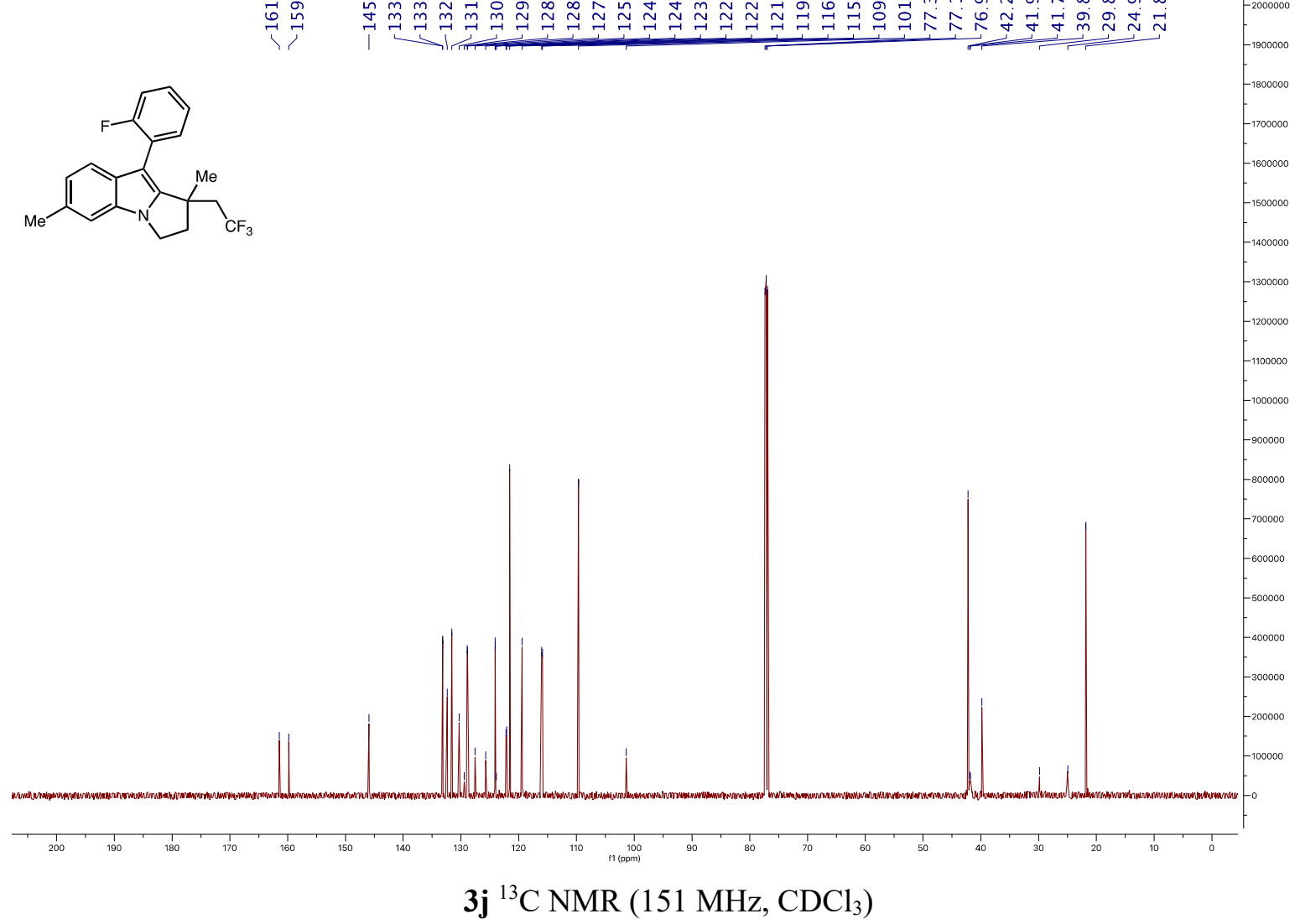


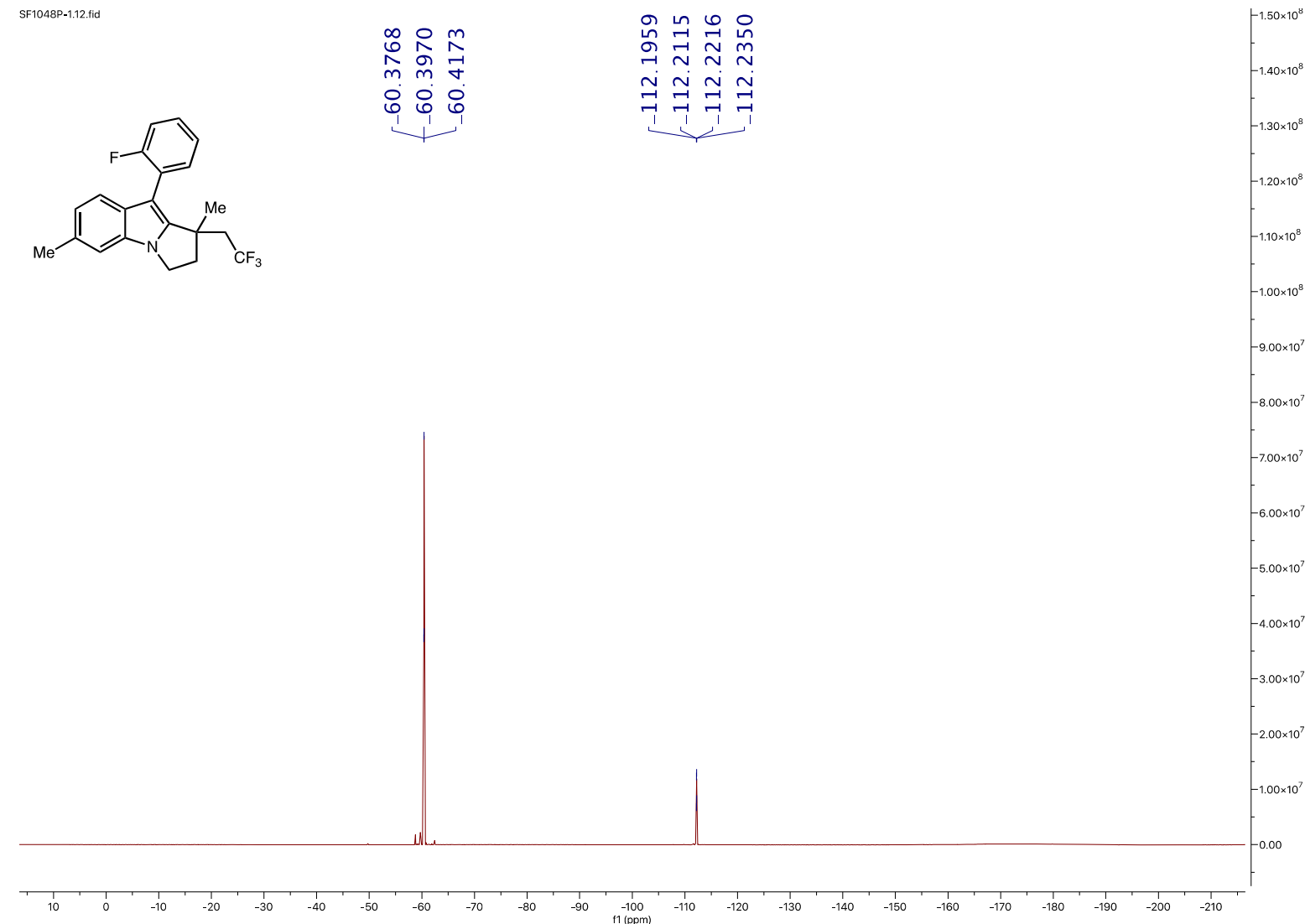

3j ${ }^{19} \mathrm{~F}$ NMR (565 MHz, $\mathrm{CDCl}_{3}$ )

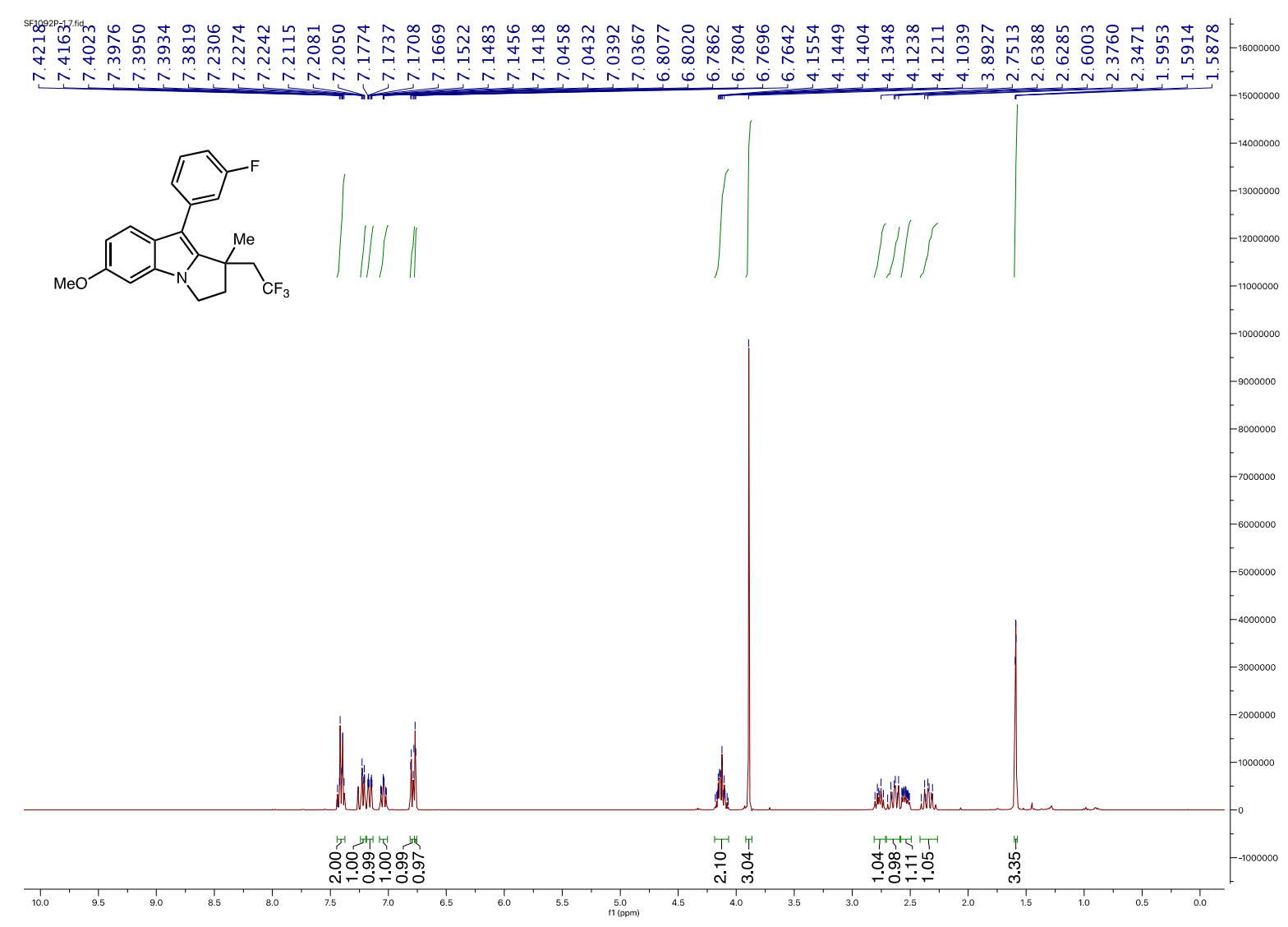

5a ${ }^{1} \mathrm{H}$ NMR (400 MHz, $\left.\mathrm{CDCl}_{3}\right)$ 

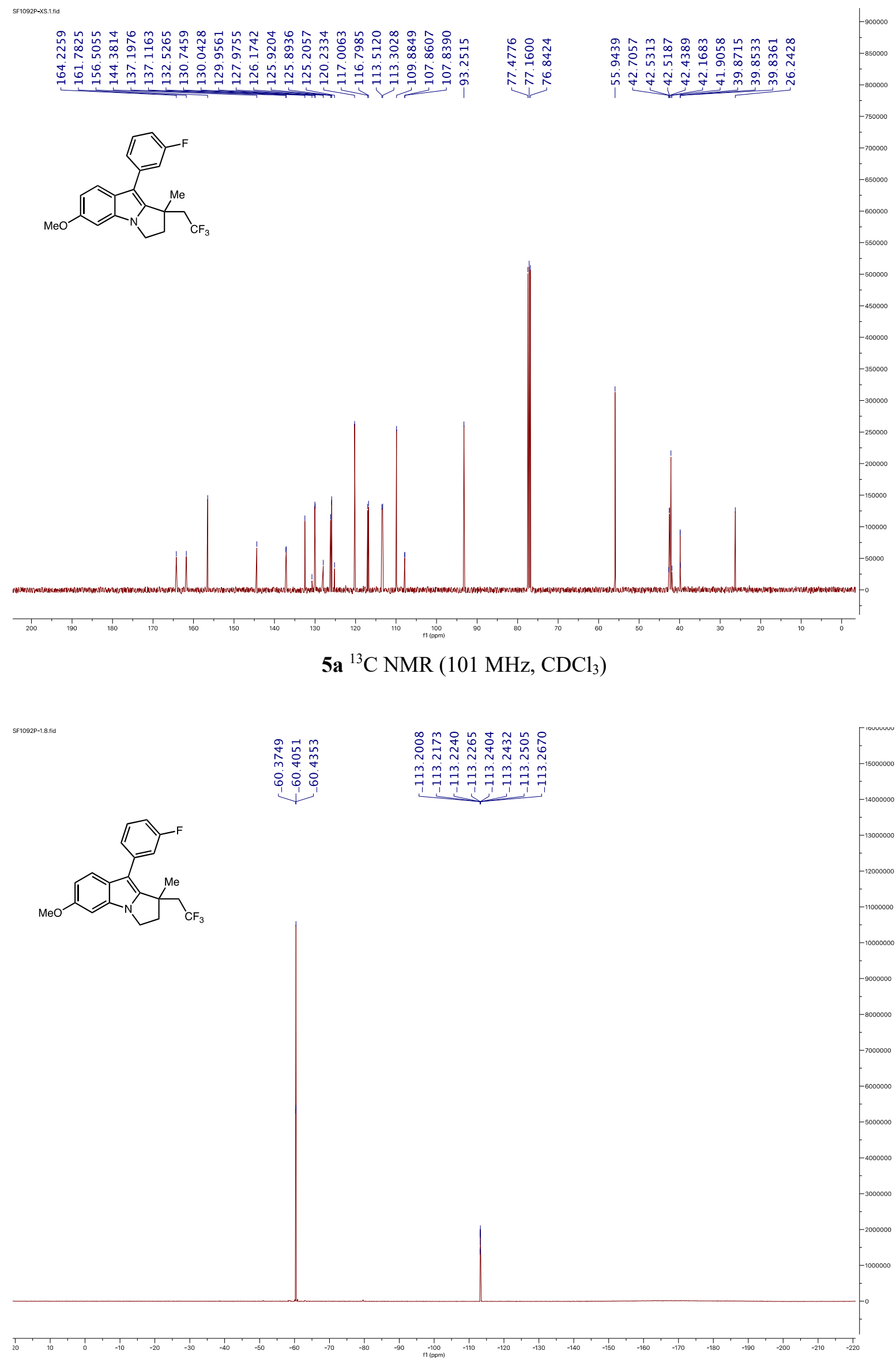

5a ${ }^{19} \mathrm{~F}$ NMR $\left(376 \mathrm{MHz}, \mathrm{CDCl}_{3}\right)$ 


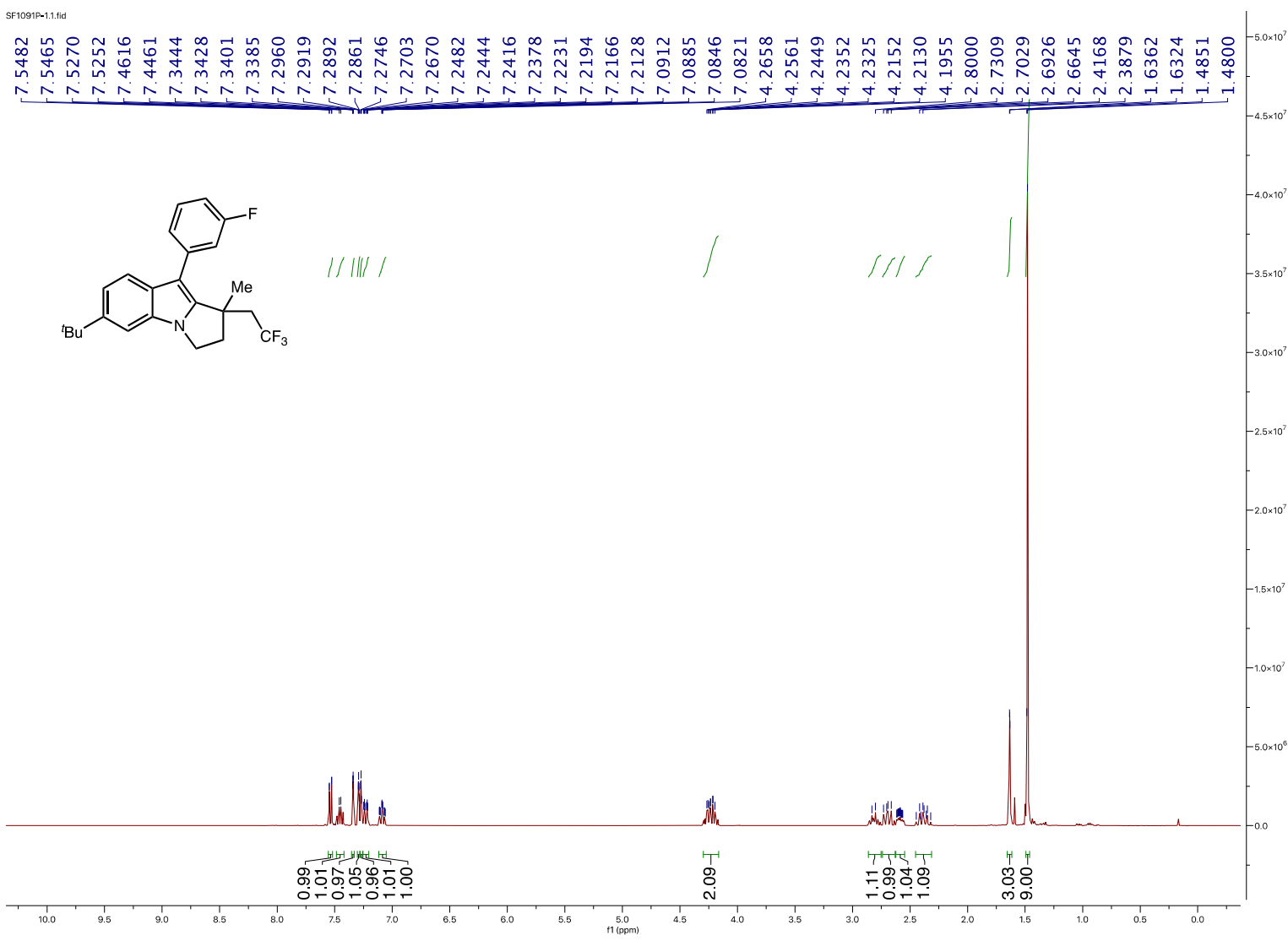

3I ${ }^{1} \mathrm{H}$ NMR (400 MHz, $\mathrm{CDCl}_{3}$ )

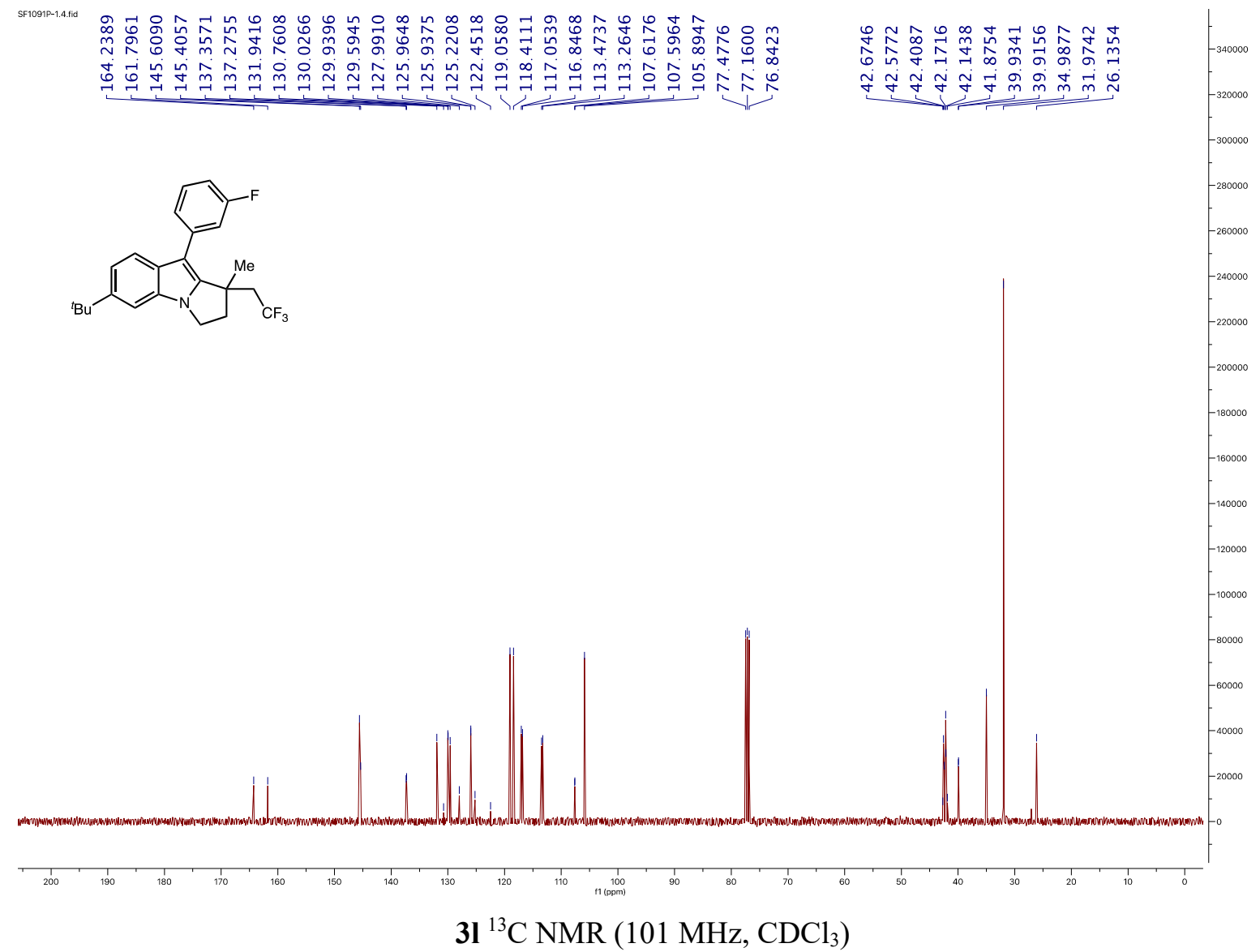



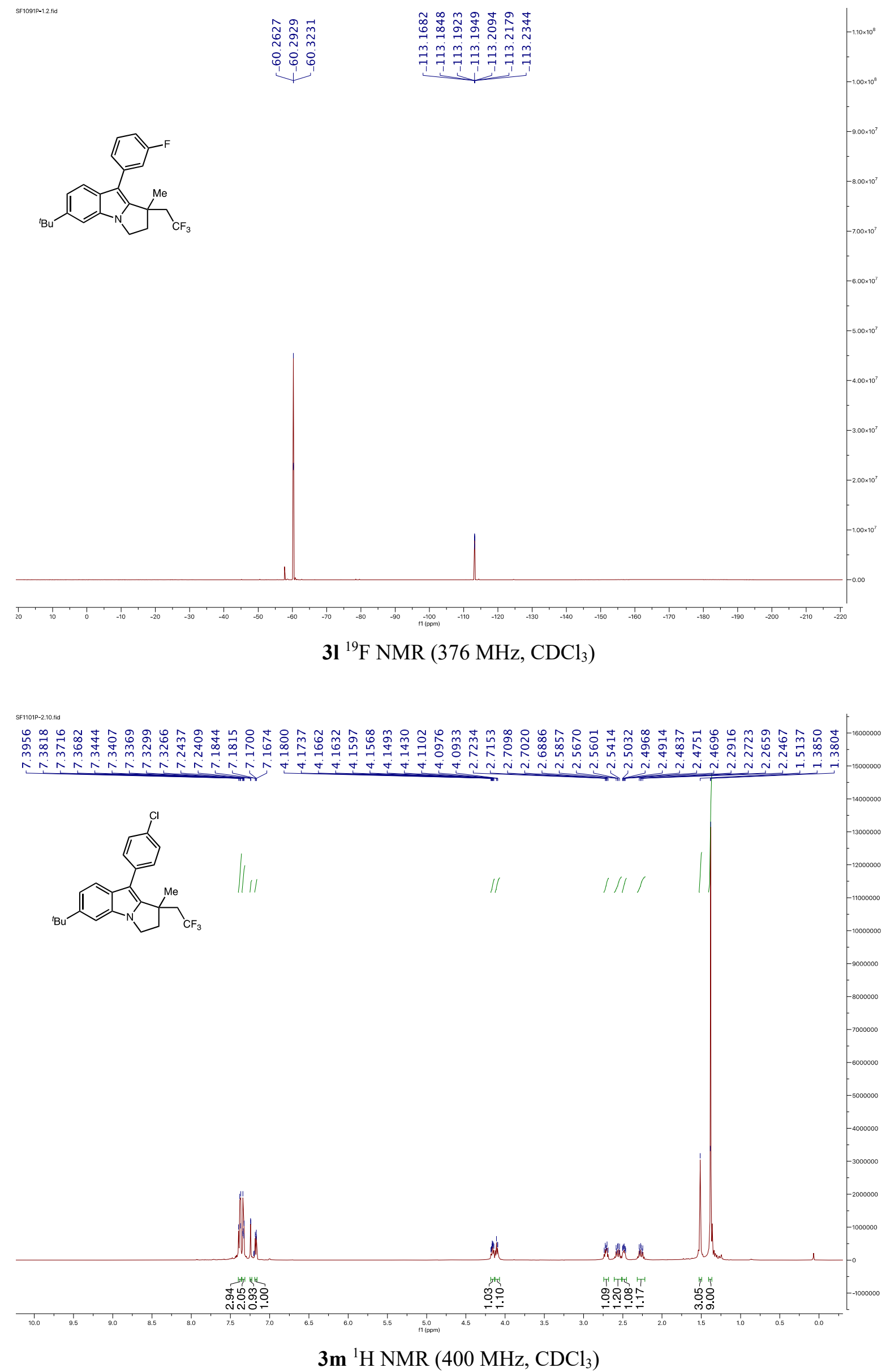

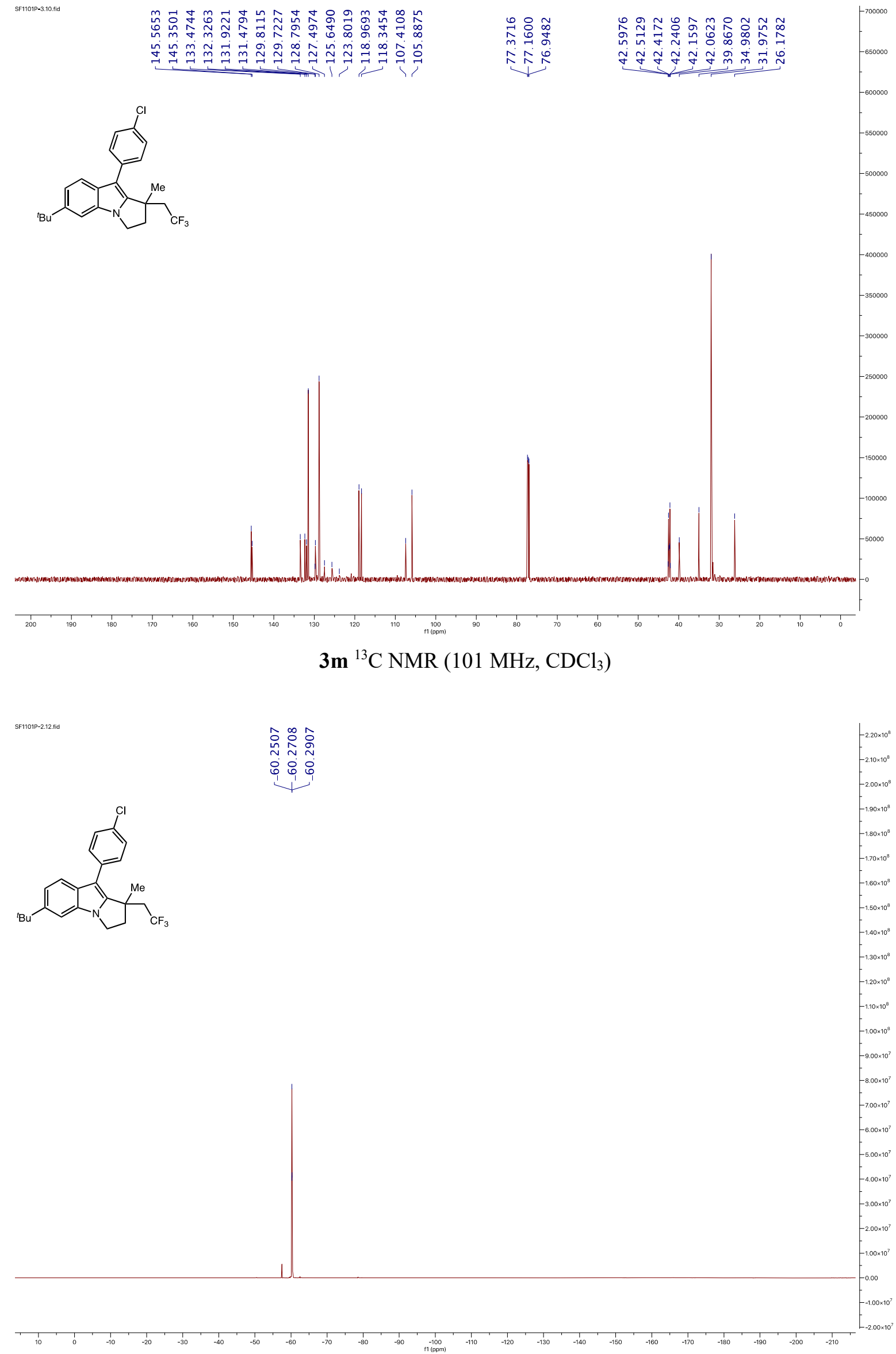

3m ${ }^{19} \mathrm{~F}$ NMR (376 MHz, $\mathrm{CDCl}_{3}$ ) 


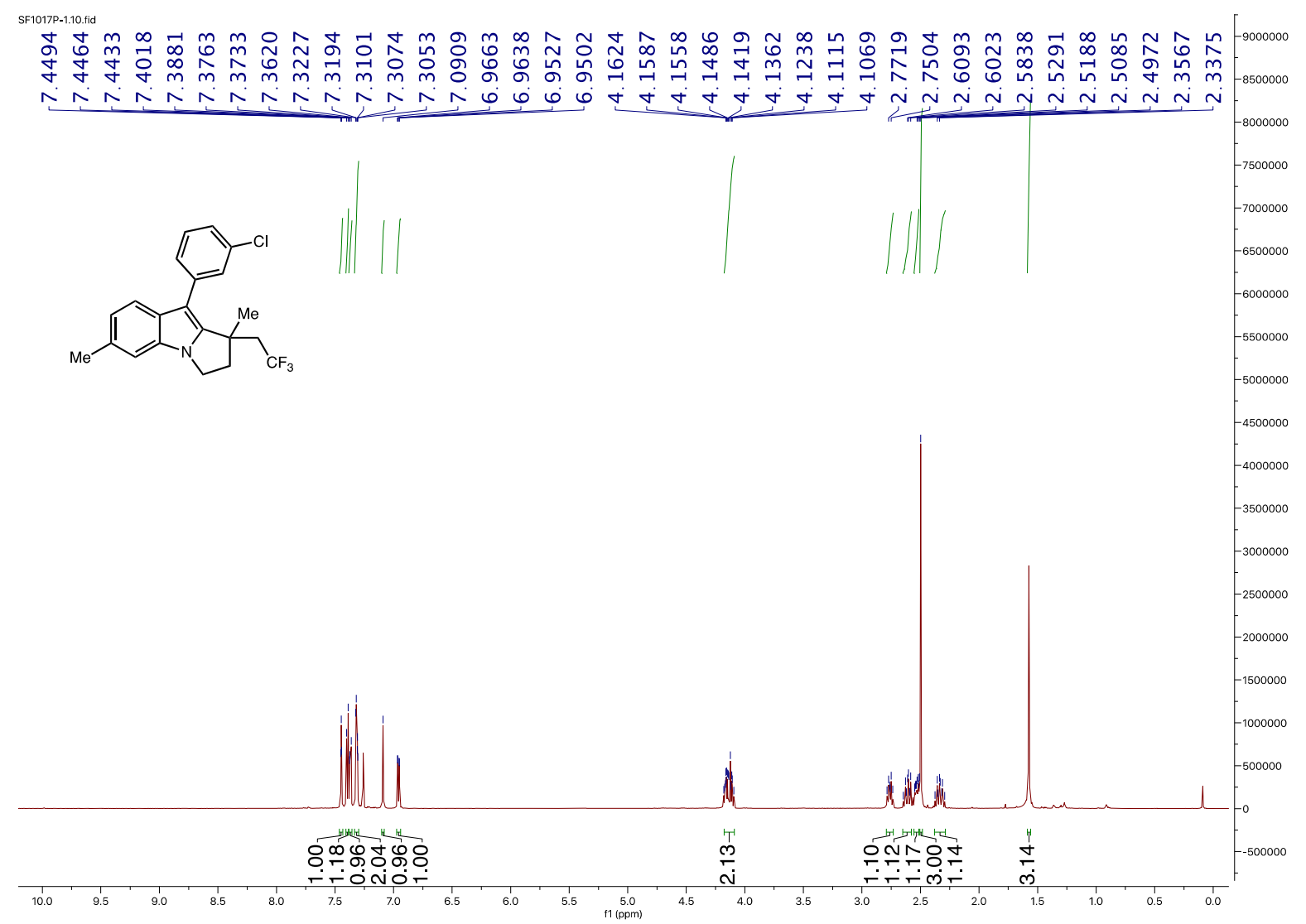

3k ${ }^{1} \mathrm{H}$ NMR (600 MHz, $\mathrm{CDCl}_{3}$ )

SF1017P-1.12.fid
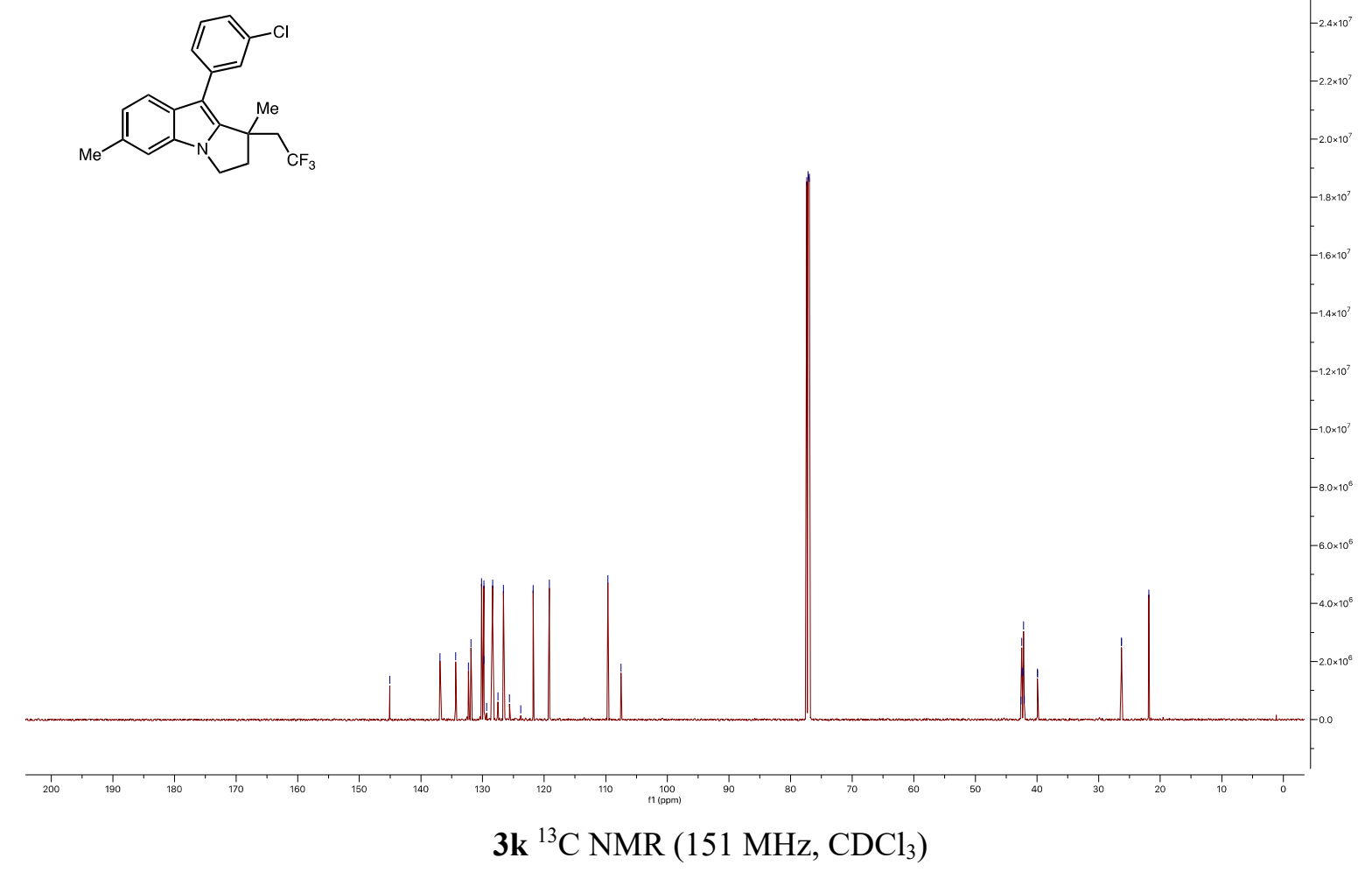

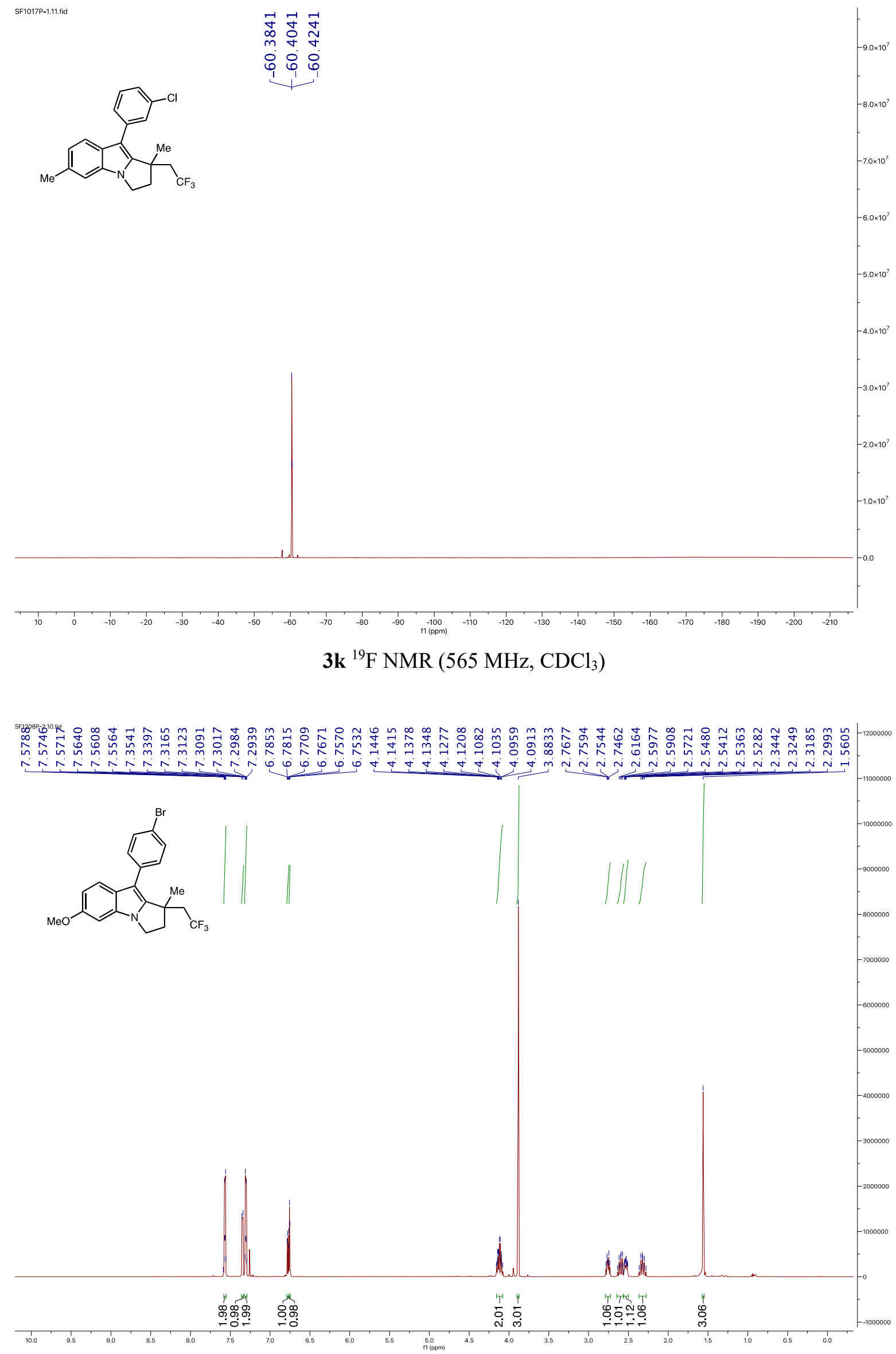

3n ${ }^{1} \mathrm{H}$ NMR (600 MHz, $\mathrm{CDCl}_{3}$ ) 

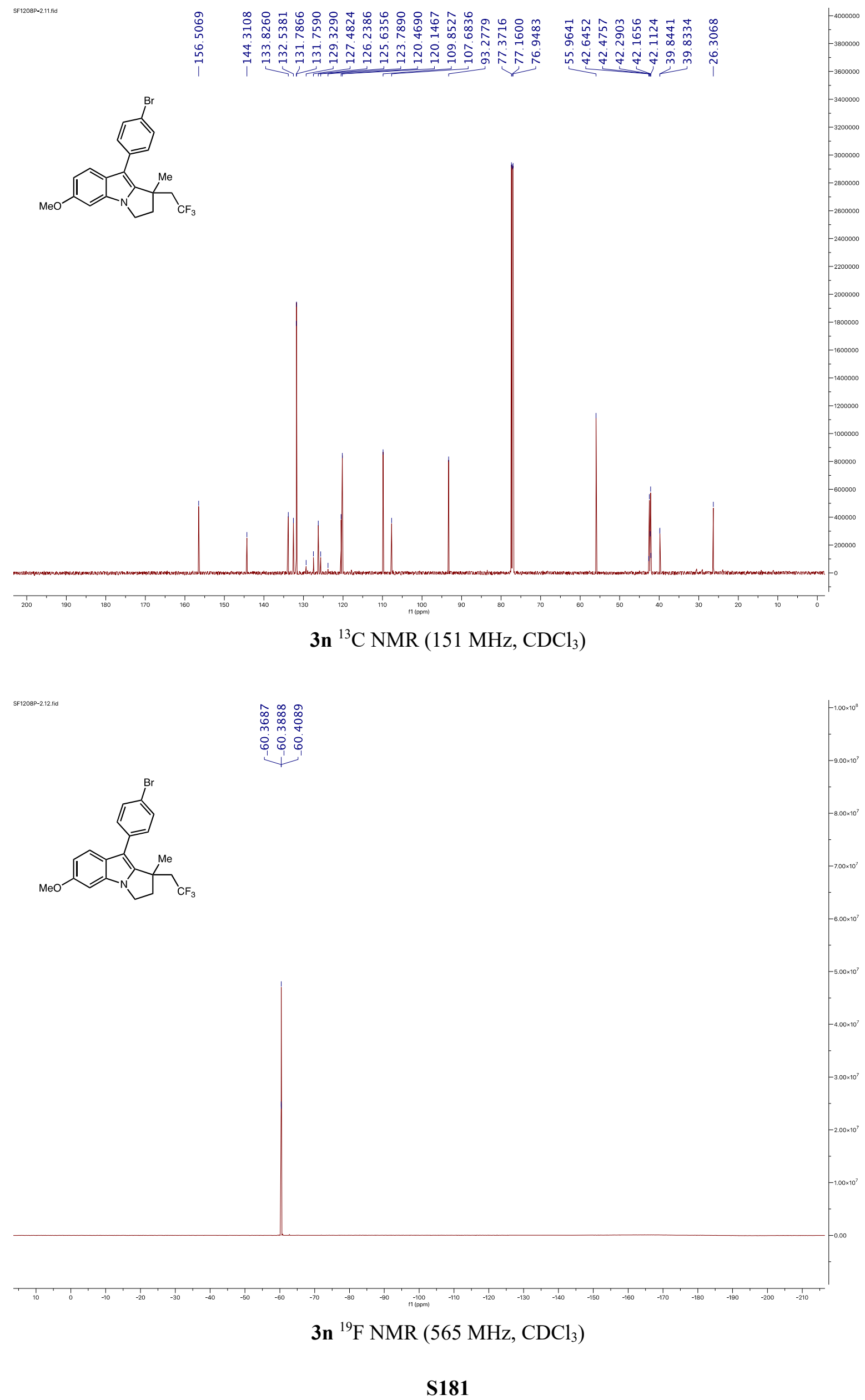

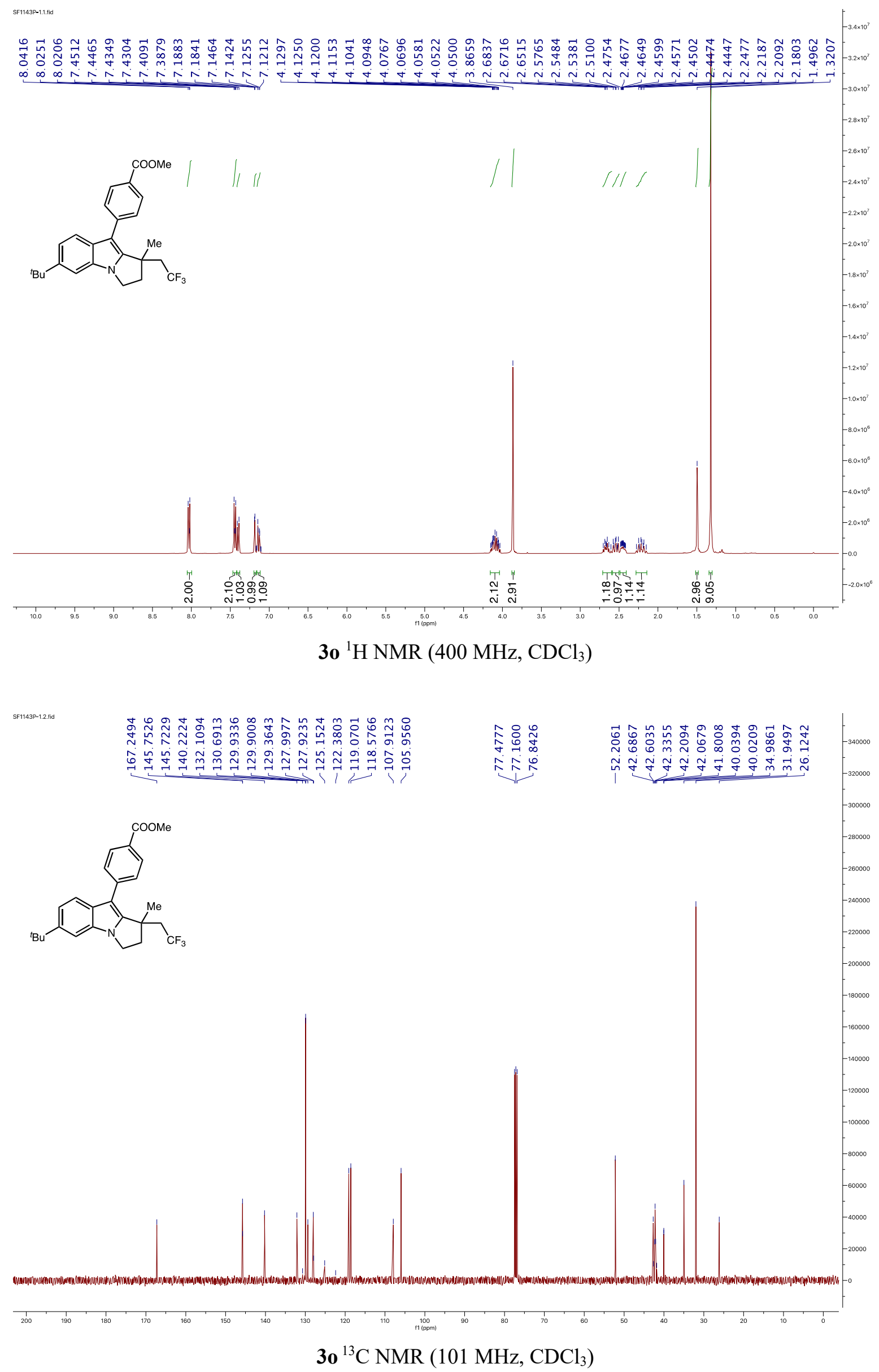

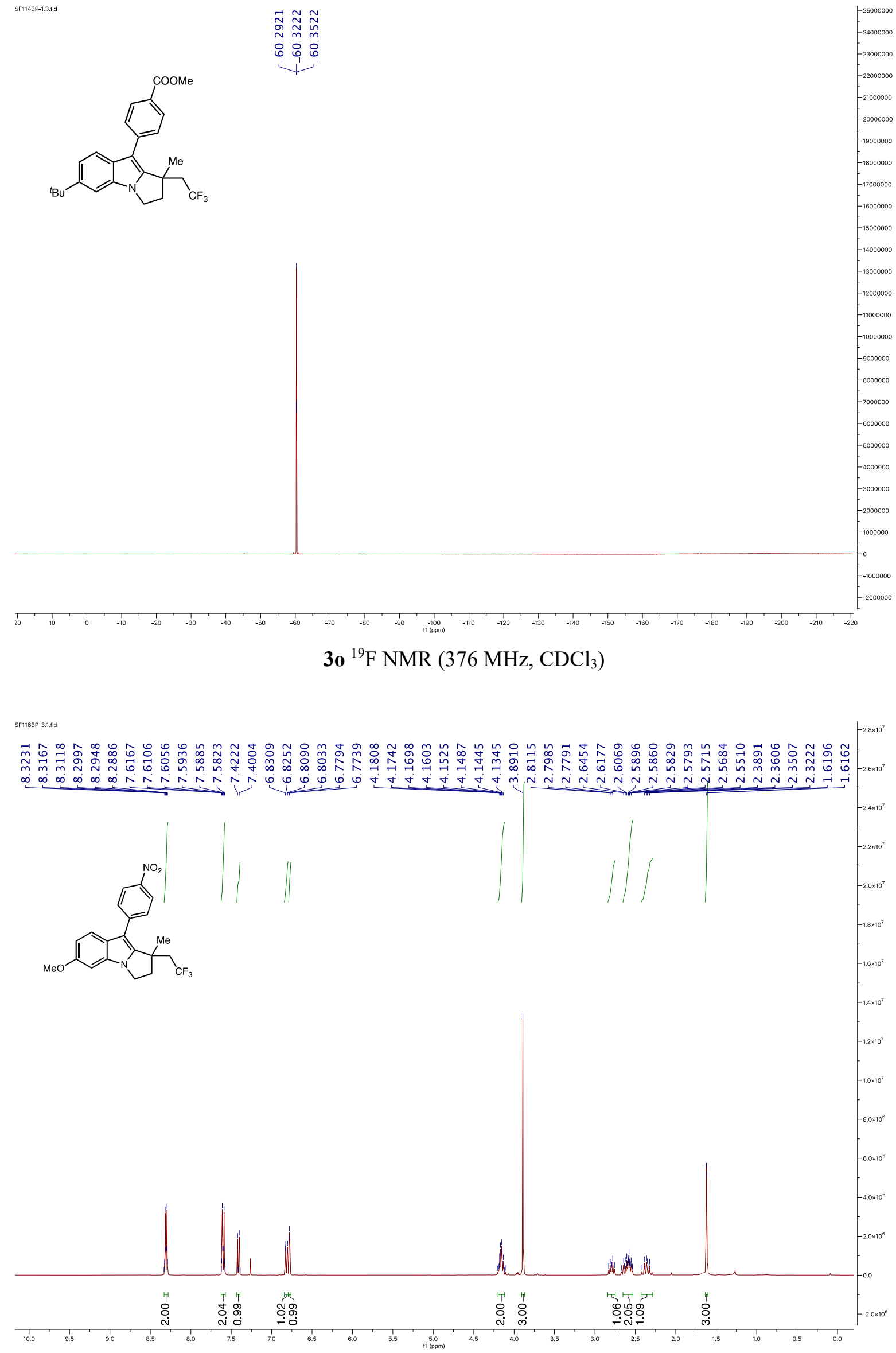

5b ${ }^{1} \mathrm{H}$ NMR (400 MHz, $\left.\mathrm{CDCl}_{3}\right)$ 


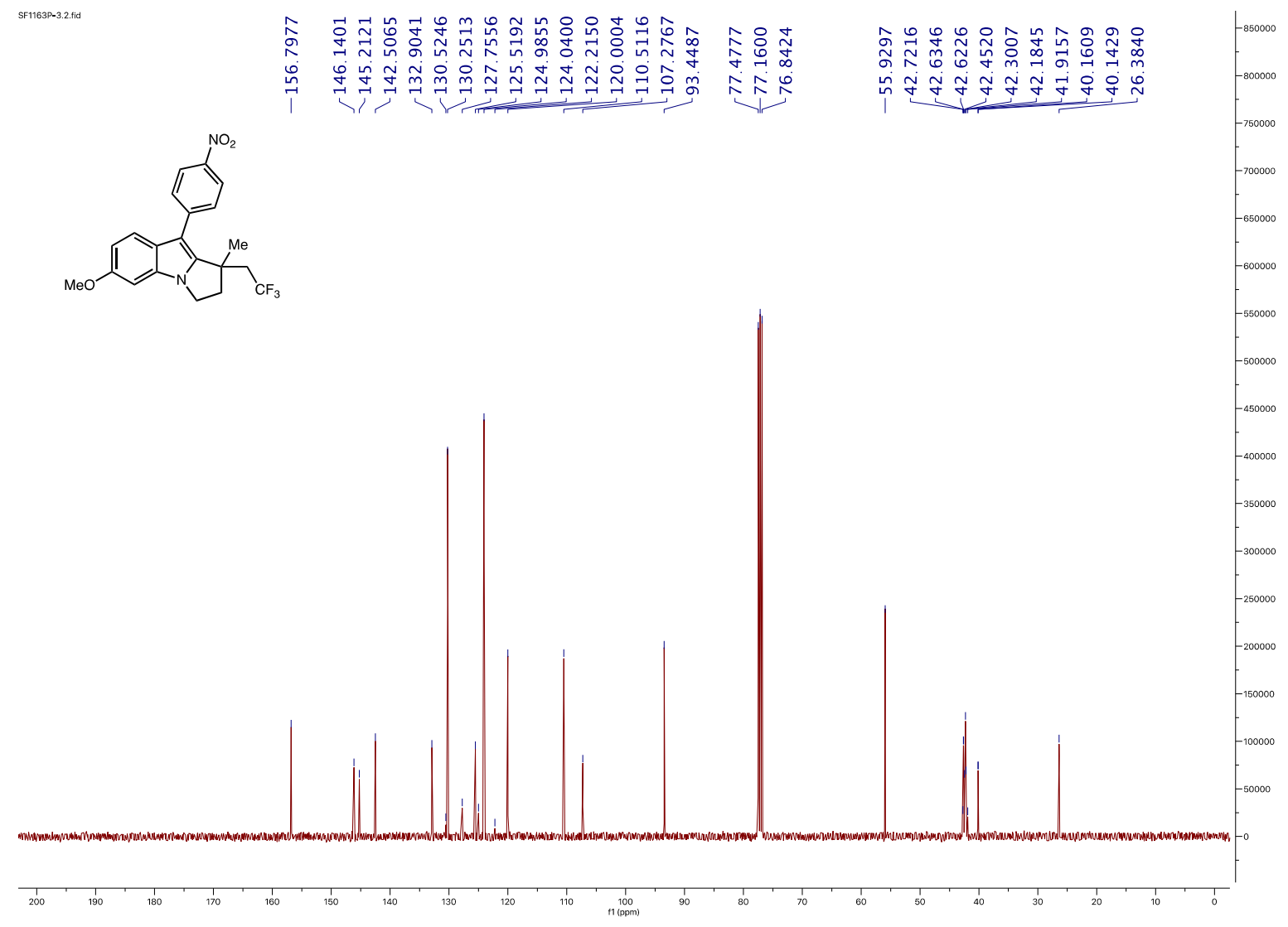

5b ${ }^{13} \mathrm{C}$ NMR (101 MHz, $\left.\mathrm{CDCl}_{3}\right)$

SF1163--3.3.fid

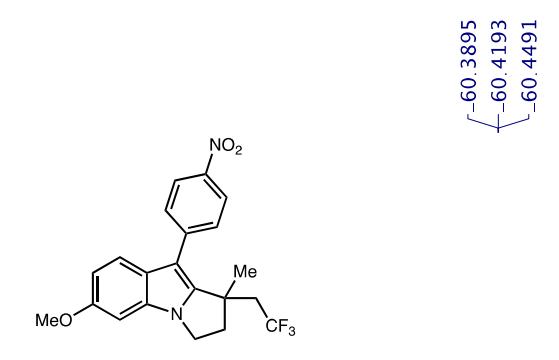

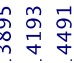

年

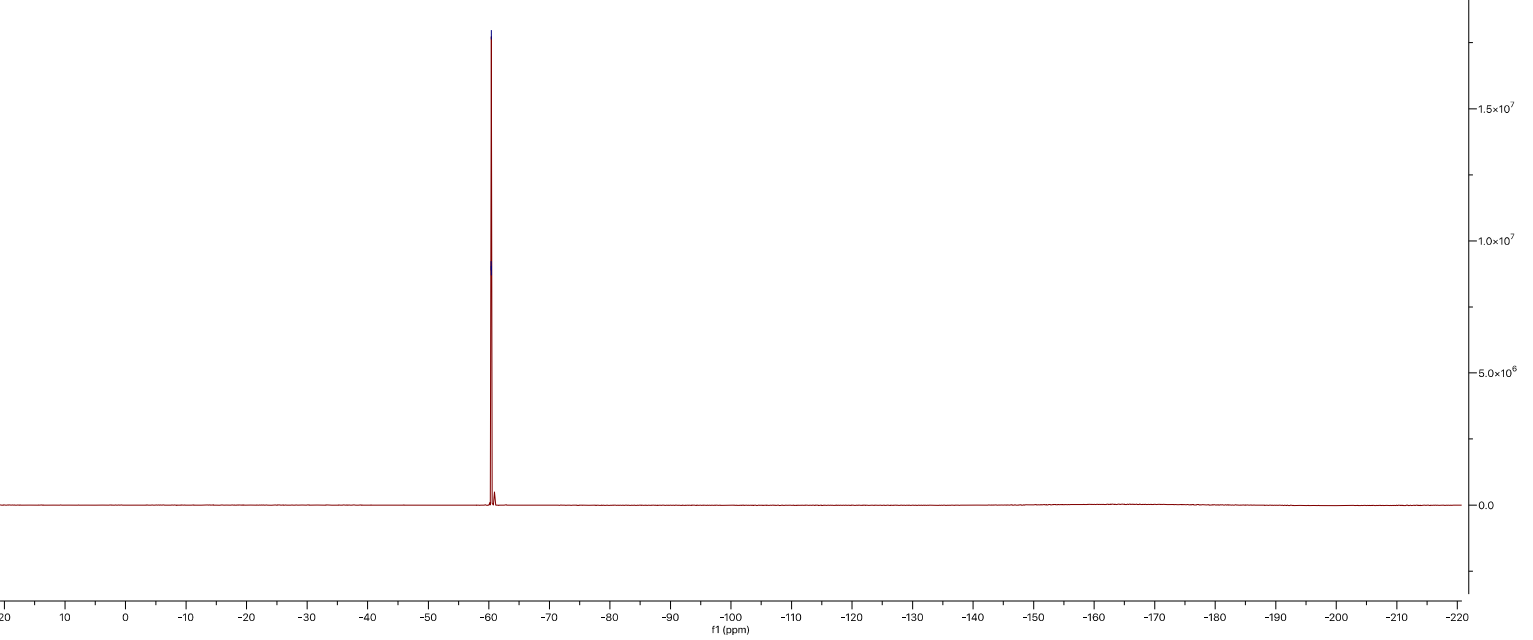

5b ${ }^{19}$ F NMR (376 MHz, $\mathrm{CDCl}_{3}$ ) 

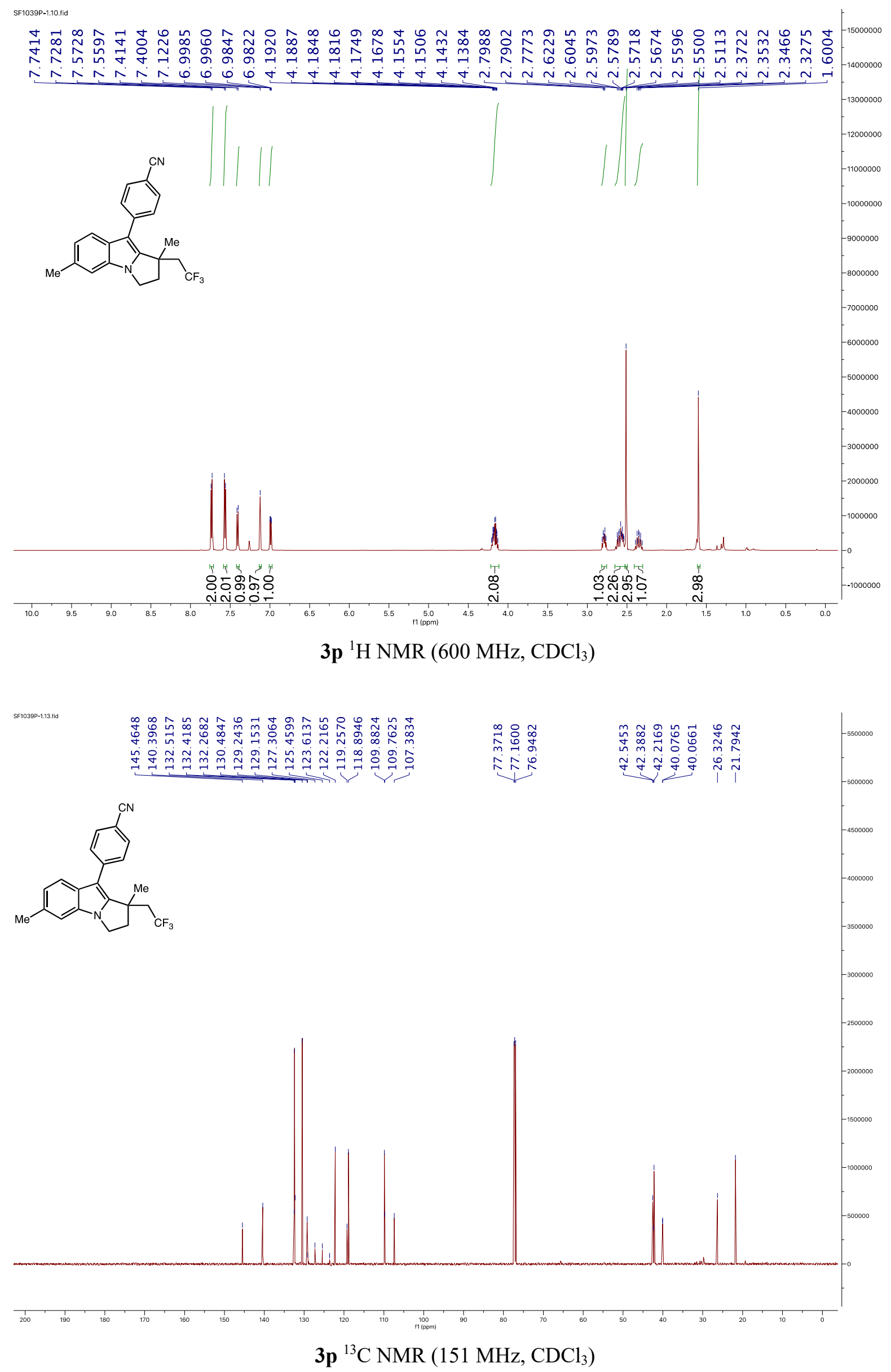

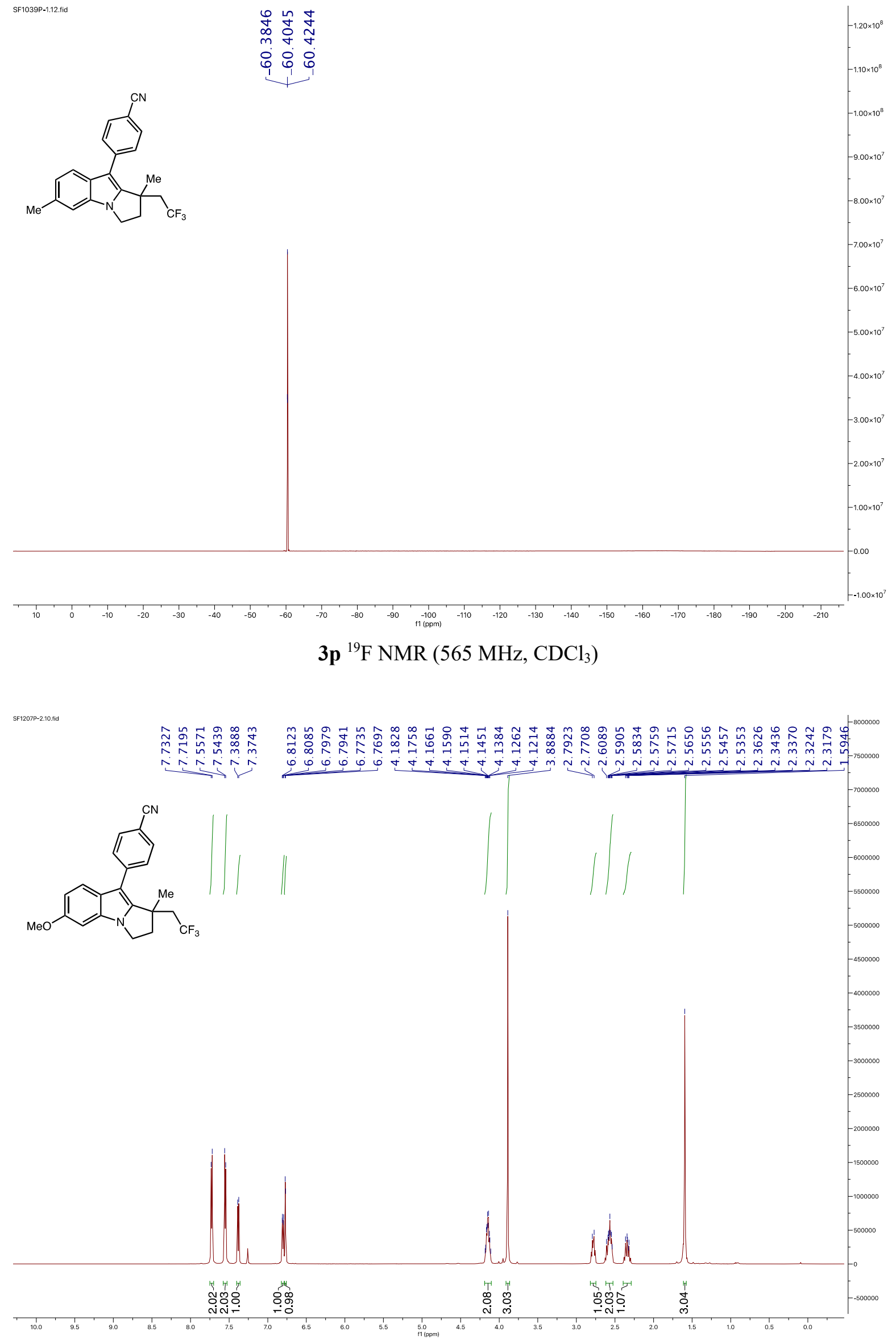

3q ${ }^{1} \mathrm{H}$ NMR $\left(600 \mathrm{MHz}, \mathrm{CDCl}_{3}\right)$ 

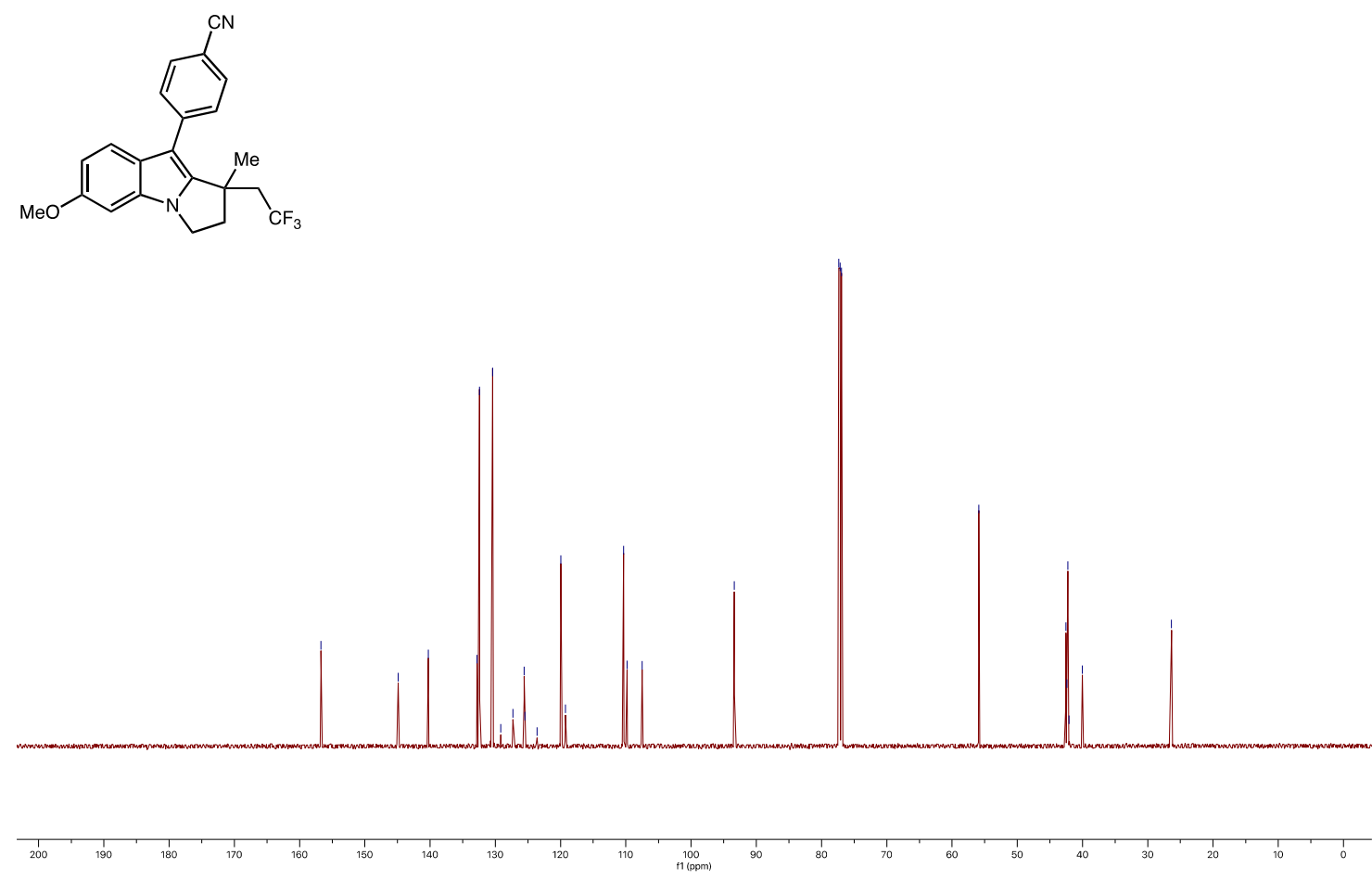

3q ${ }^{13} \mathrm{C}$ NMR (151 MHz, $\mathrm{CDCl}_{3}$ )

SF12077-2.12.fid

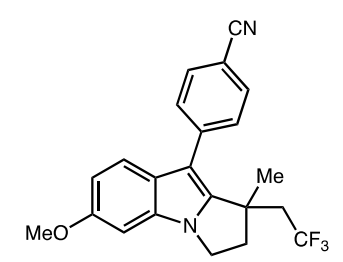

涼弯孝

$\begin{array}{lll}1 & 1 \\ 0 & 0 & 0 \\ 0 & 0 & 0 \\ 0\end{array}$

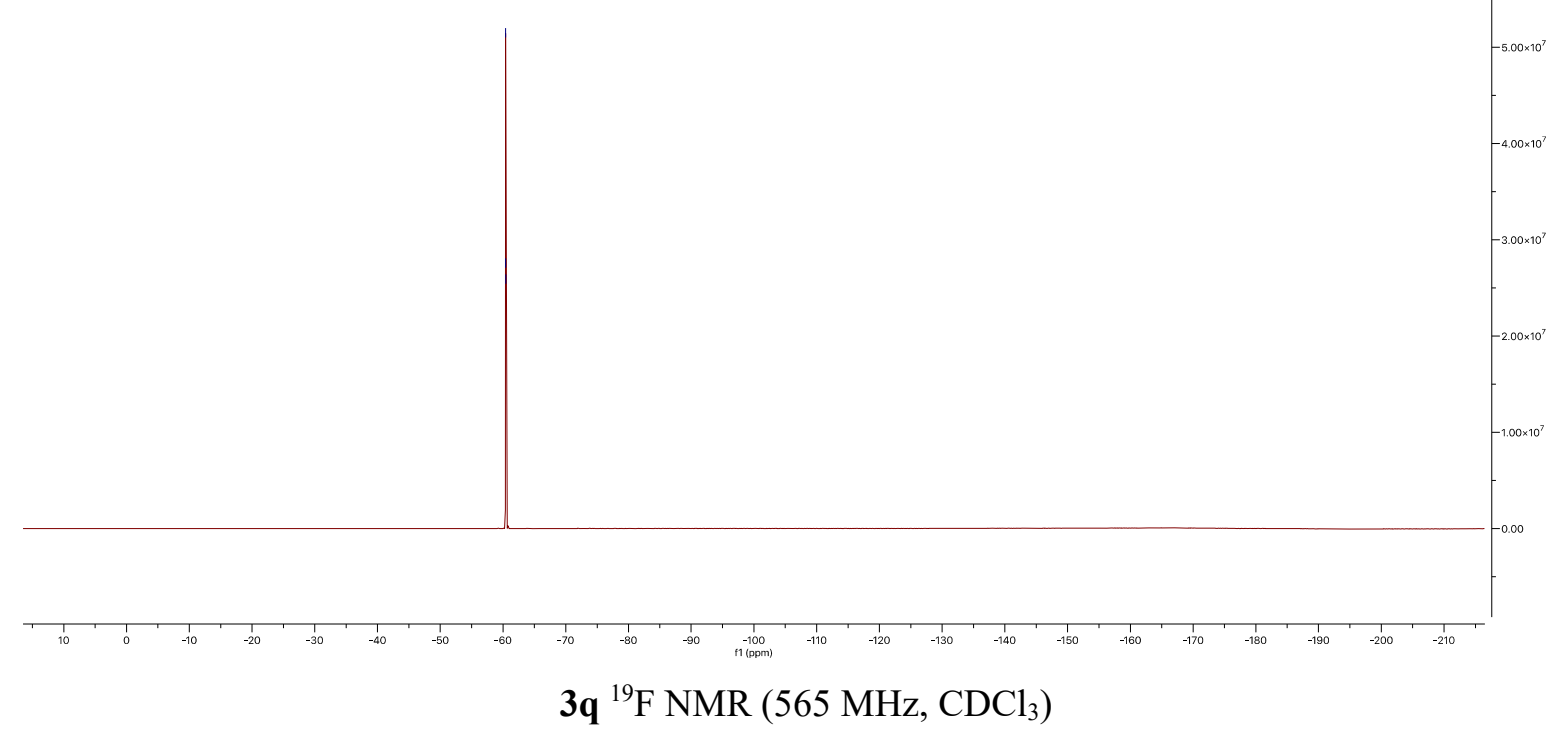



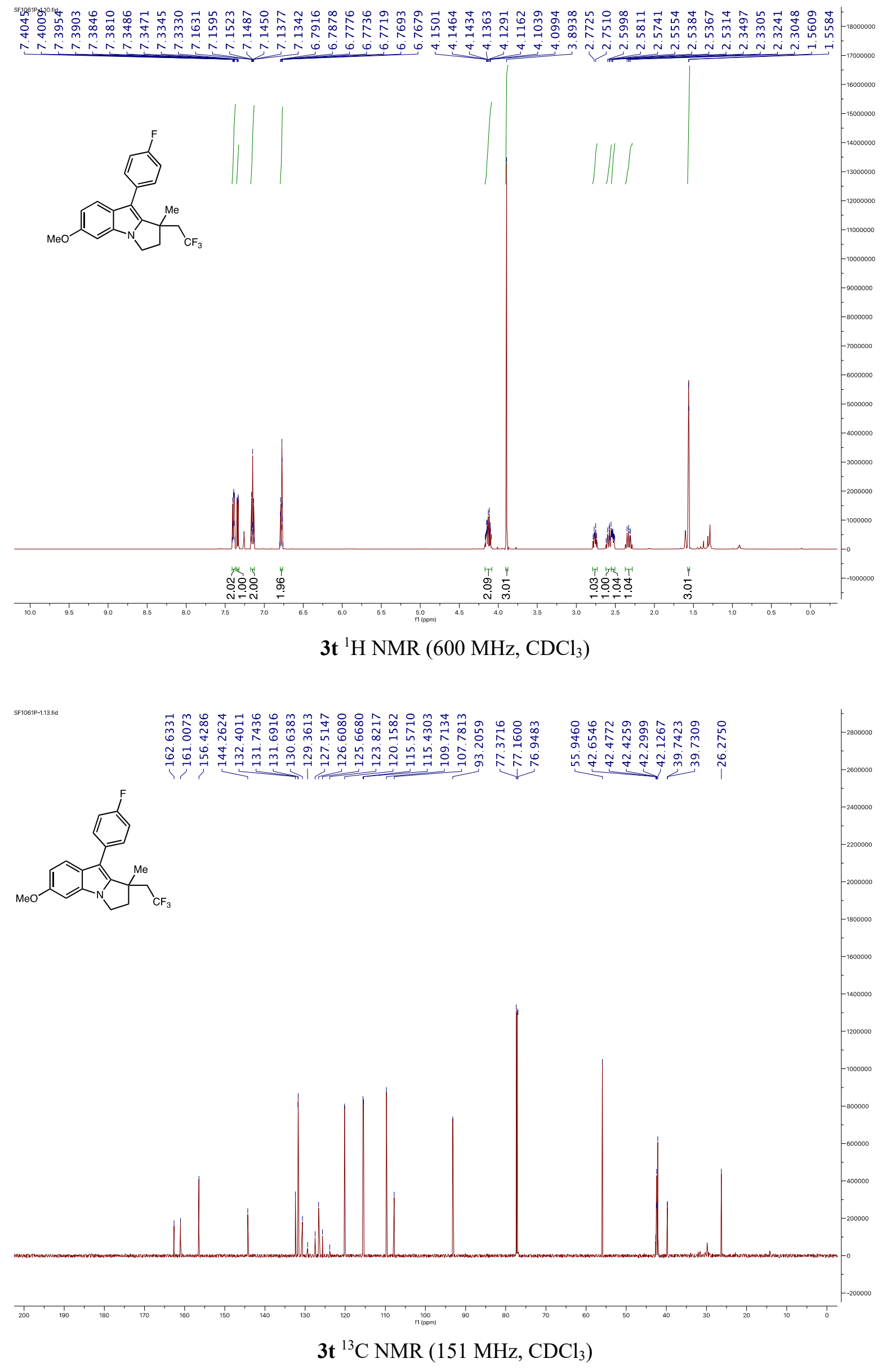

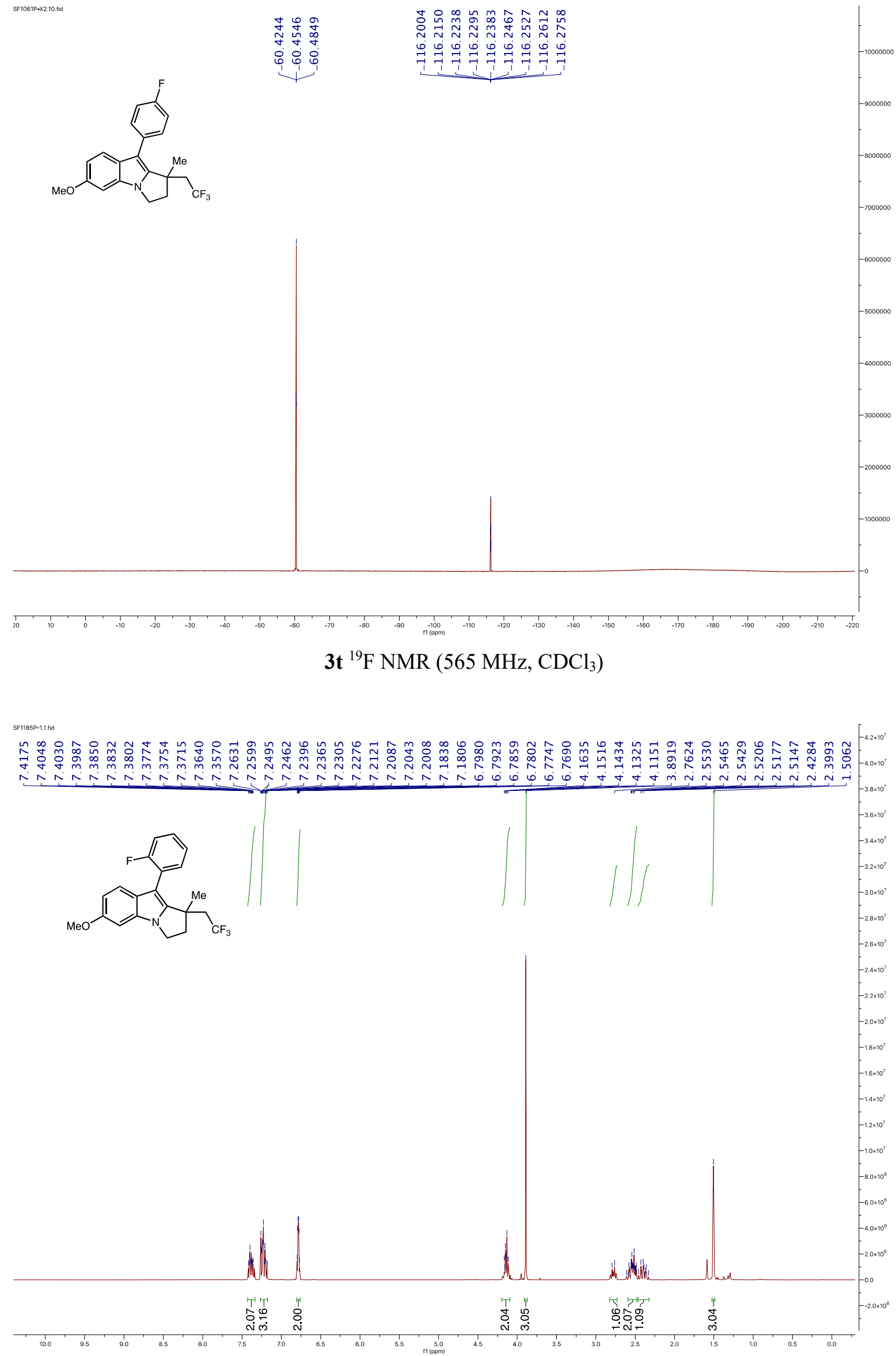

3u ${ }^{1} \mathrm{H}$ NMR $\left(400 \mathrm{MHz}, \mathrm{CDCl}_{3}\right)$ 

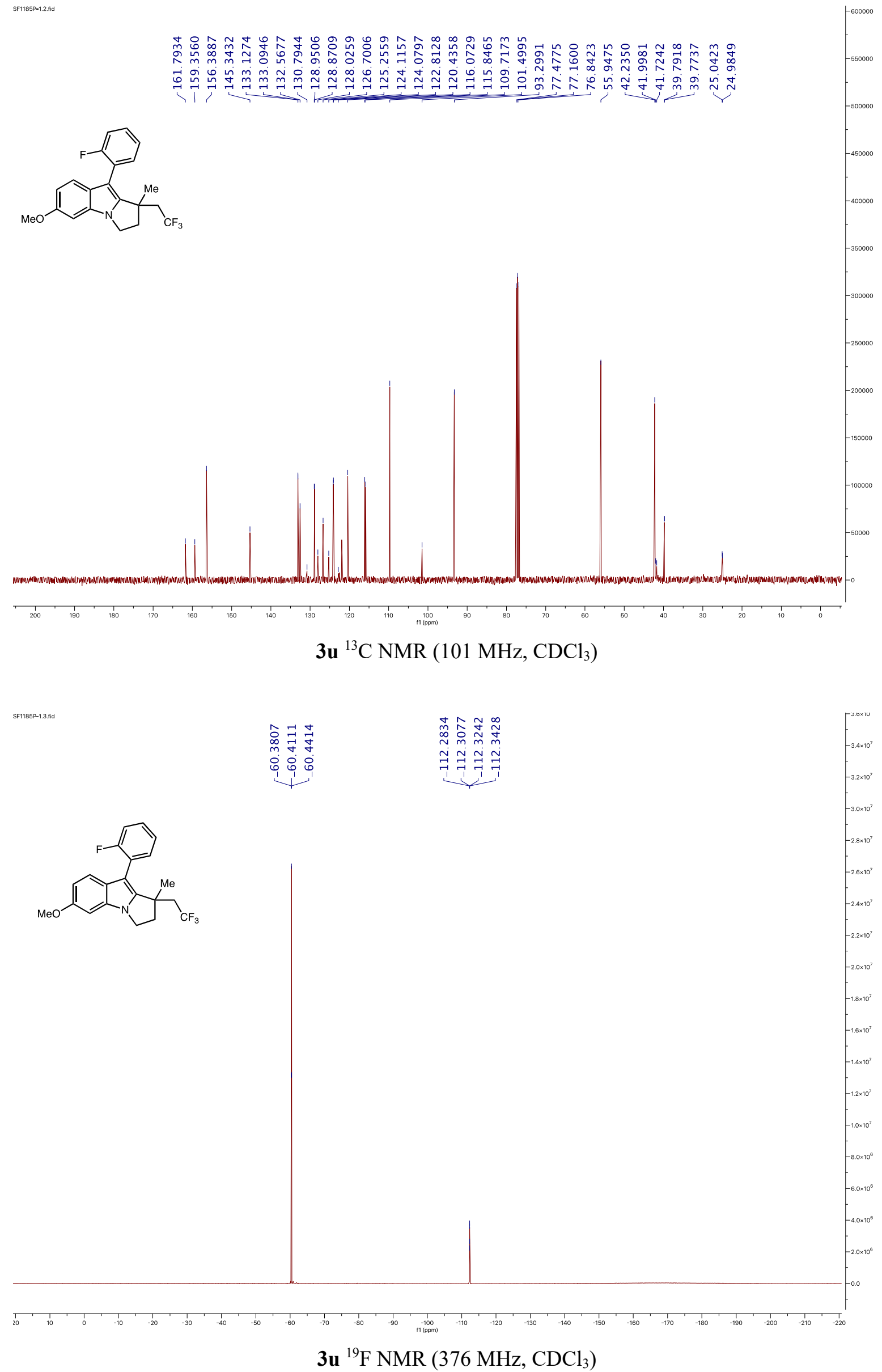


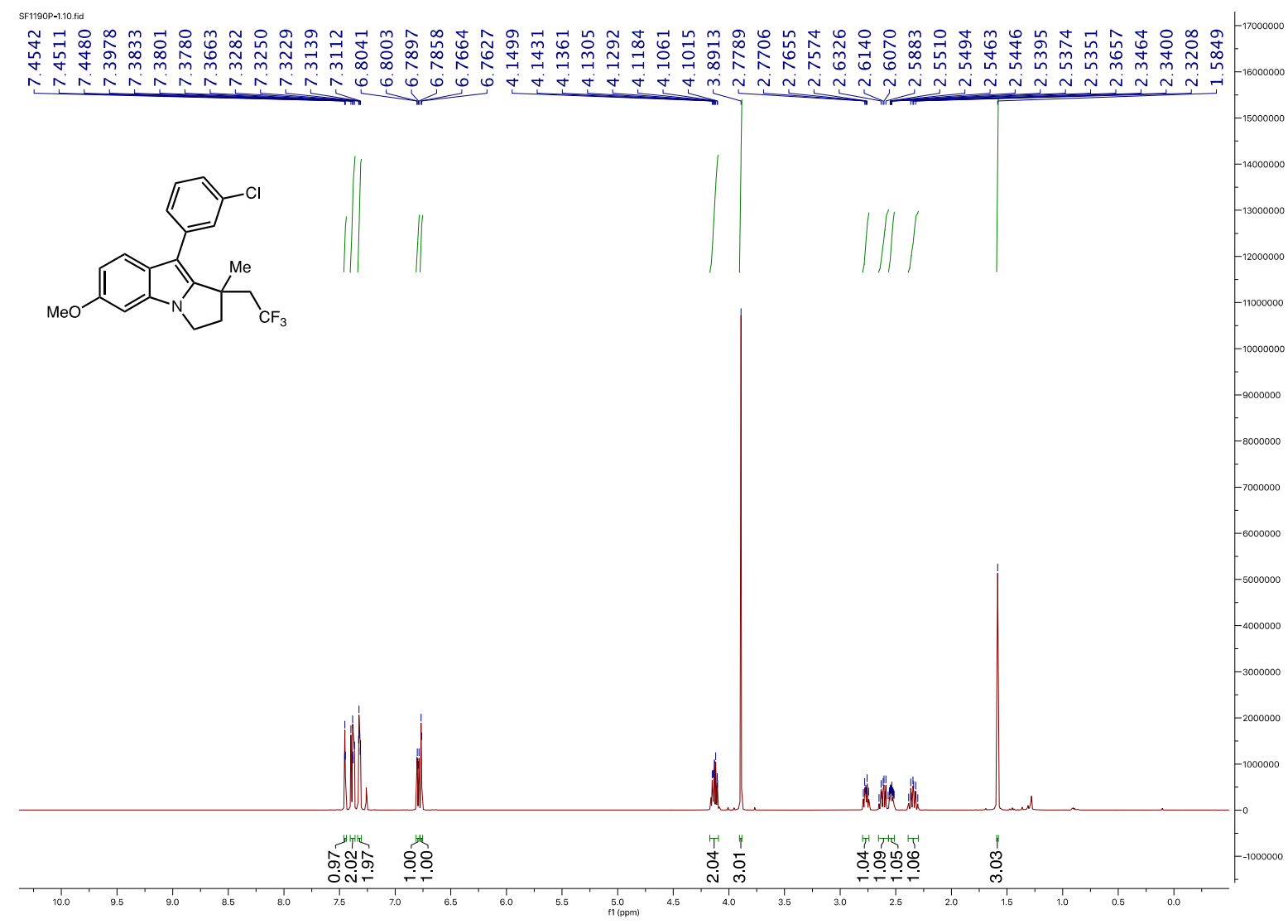

3v ${ }^{1} \mathrm{H}$ NMR $\left(600 \mathrm{MHz}, \mathrm{CDCl}_{3}\right)$

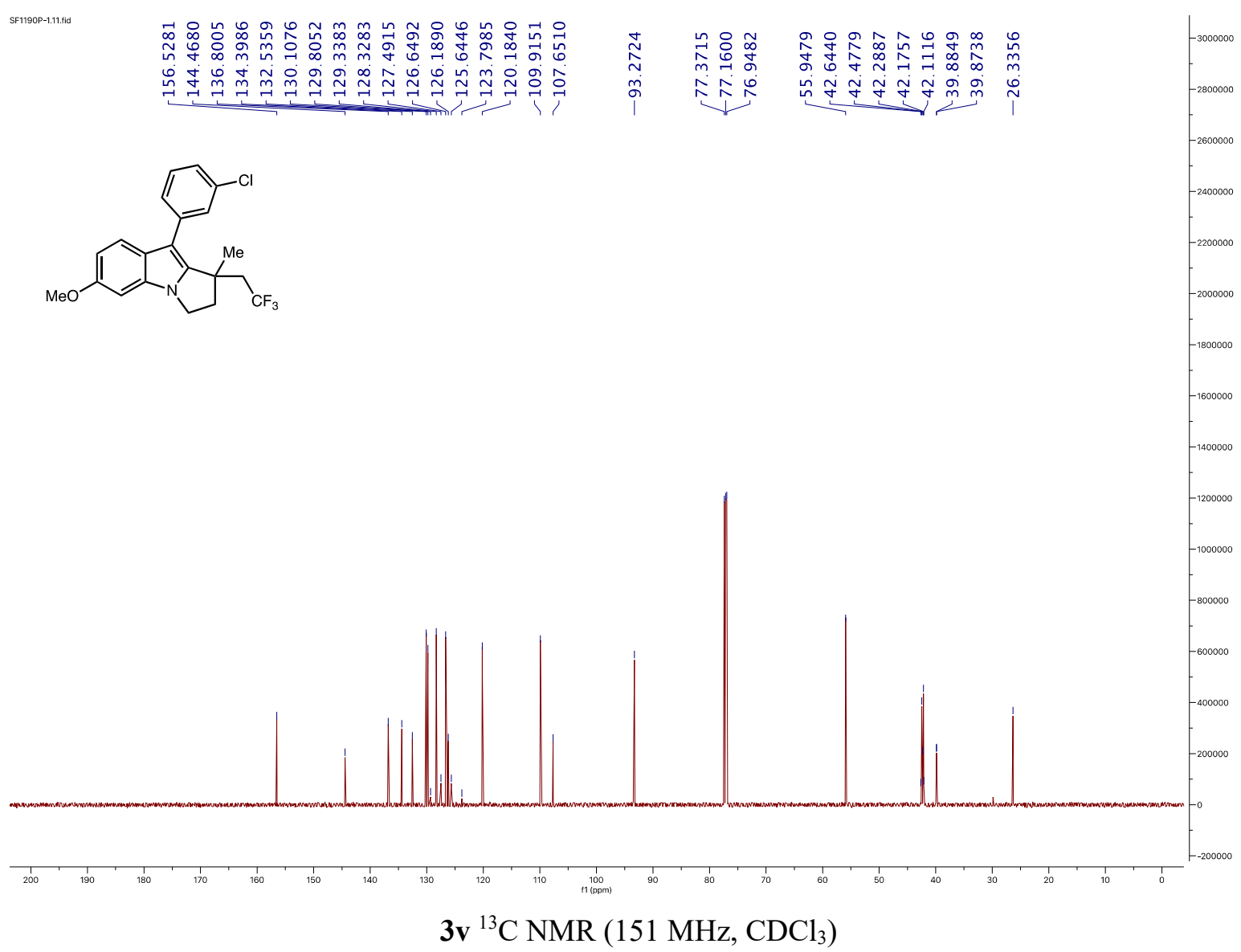



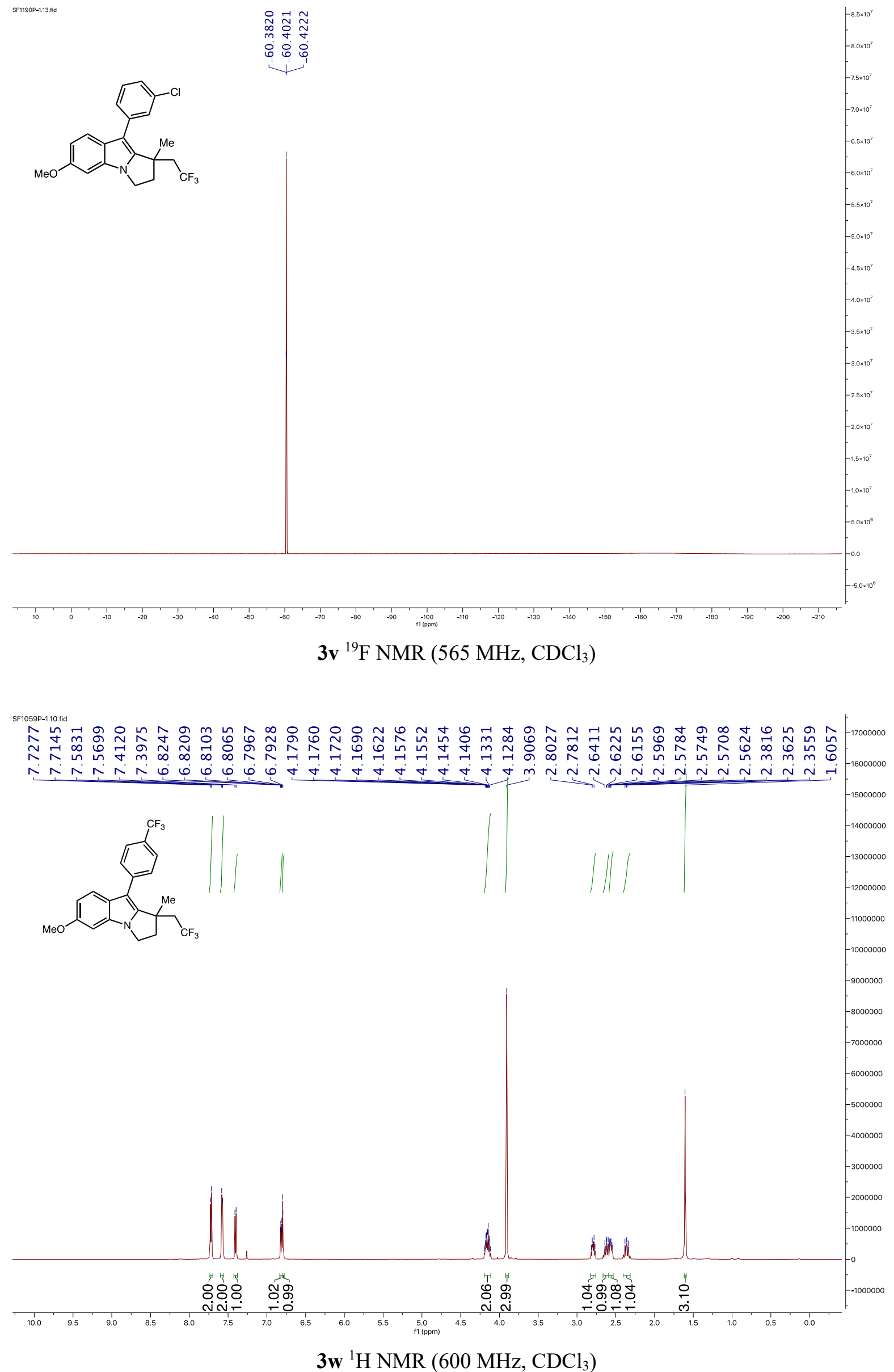
3w ${ }^{13} \mathrm{C}$ NMR (151 MHz, $\mathrm{CDCl}_{3}$ )

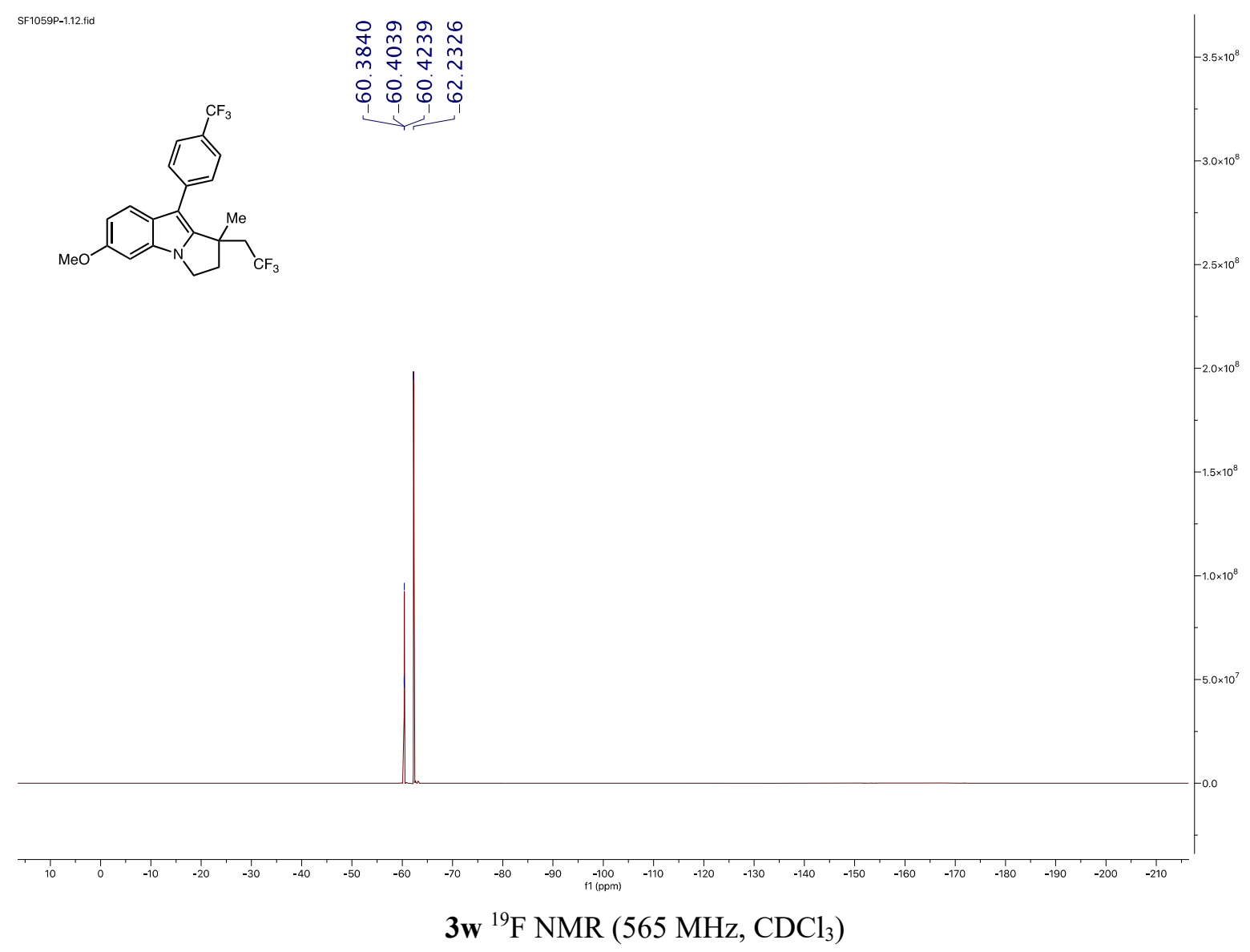




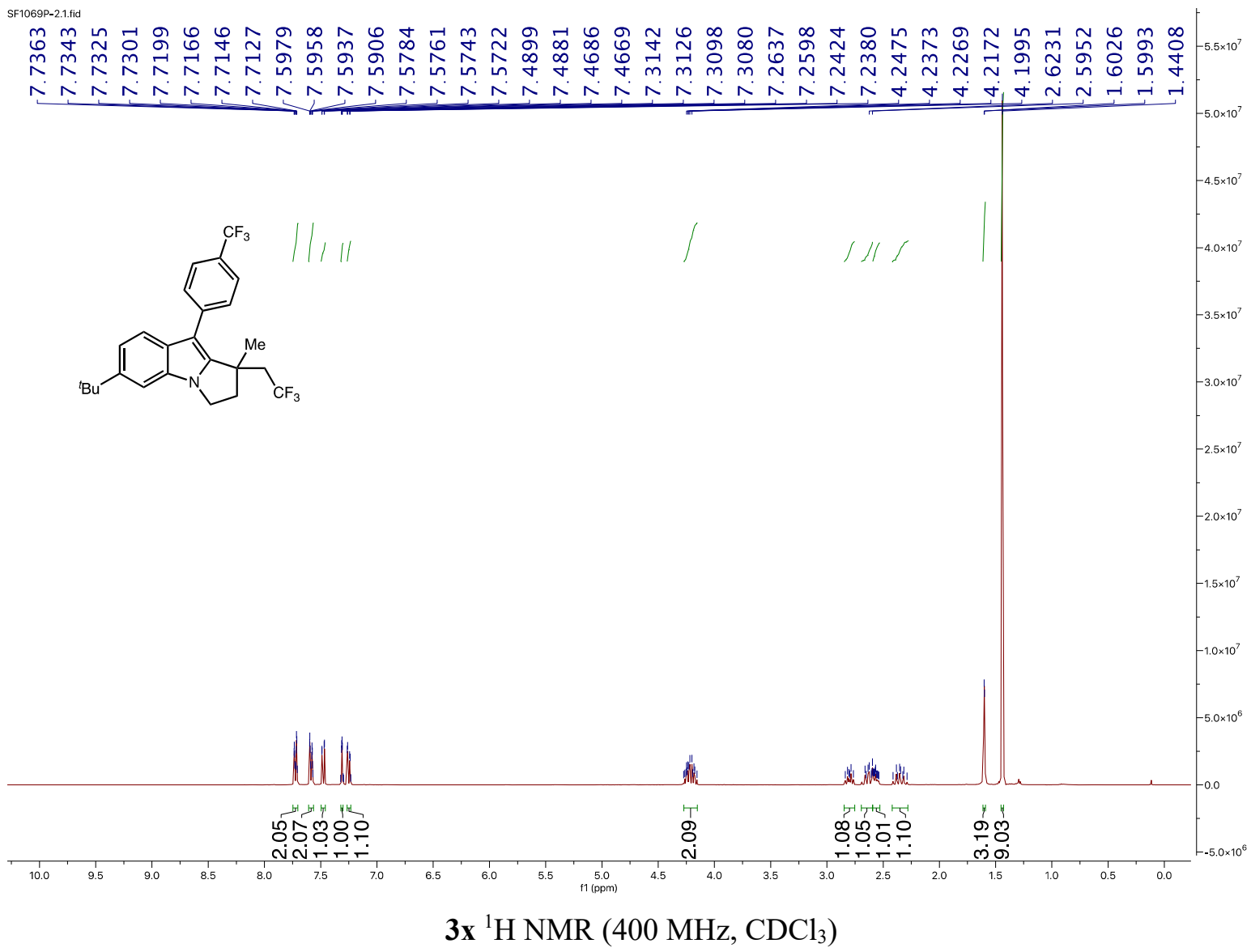

SF1069P-2.4fid

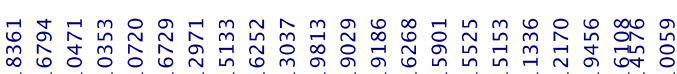

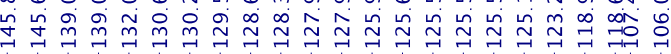

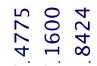

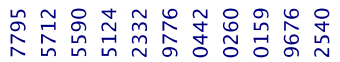

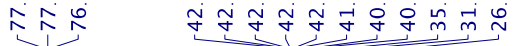

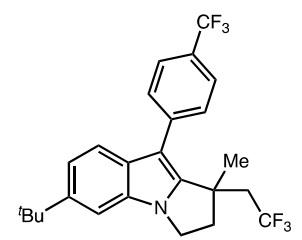
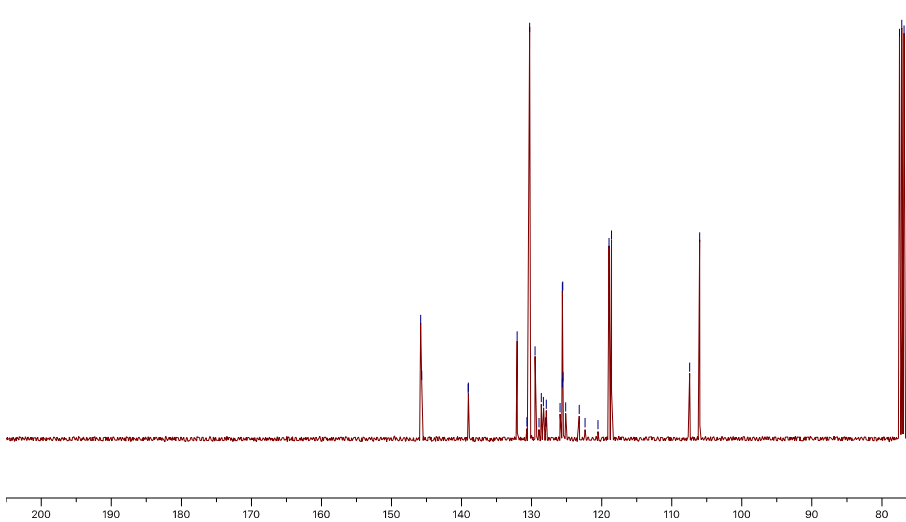

3x ${ }^{13} \mathrm{C}$ NMR (101 MHz, $\left.\mathrm{CDCl}_{3}\right)$ 

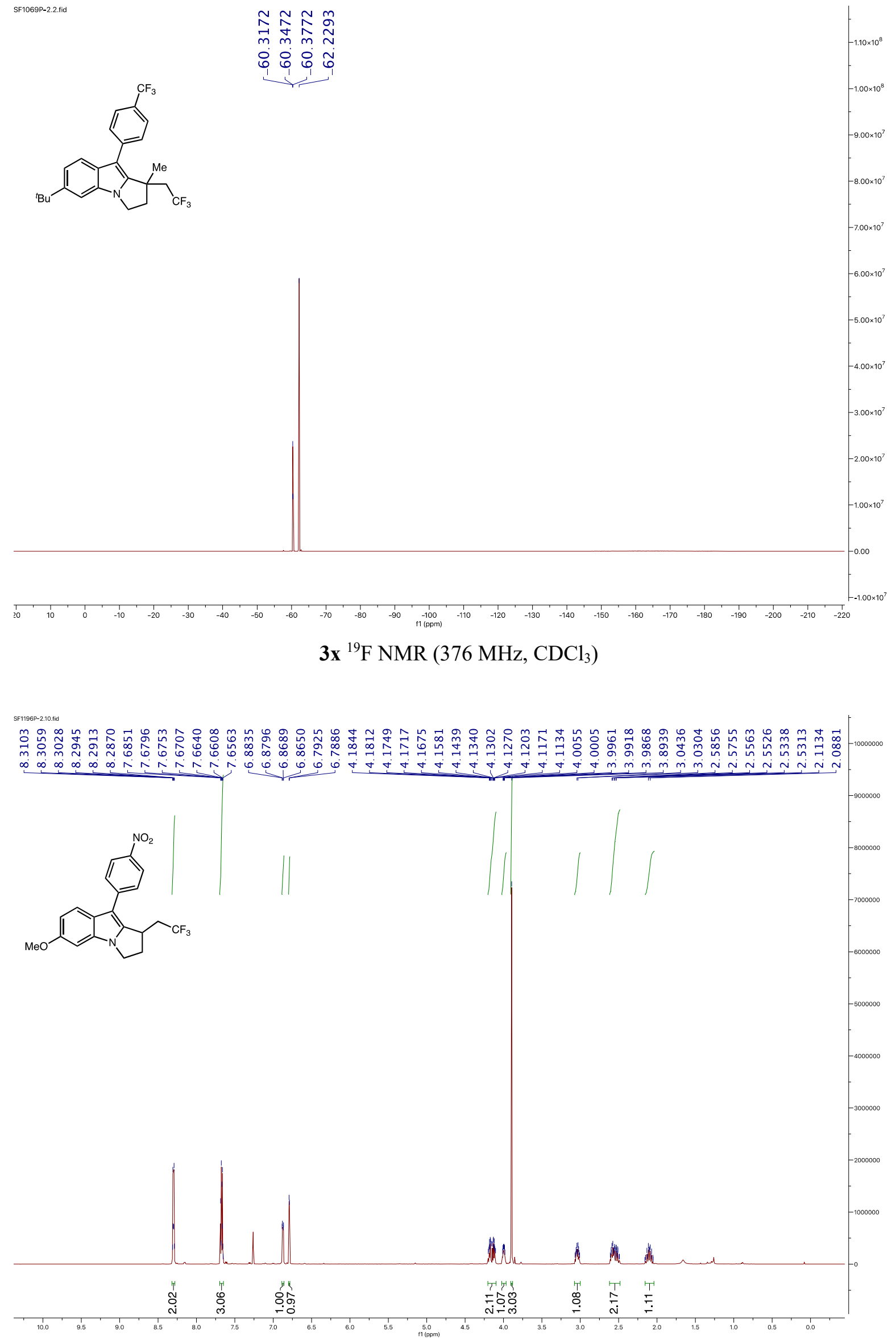

3y ${ }^{1} \mathrm{H}$ NMR (600 MHz, $\left.\mathrm{CDCl}_{3}\right)$ 


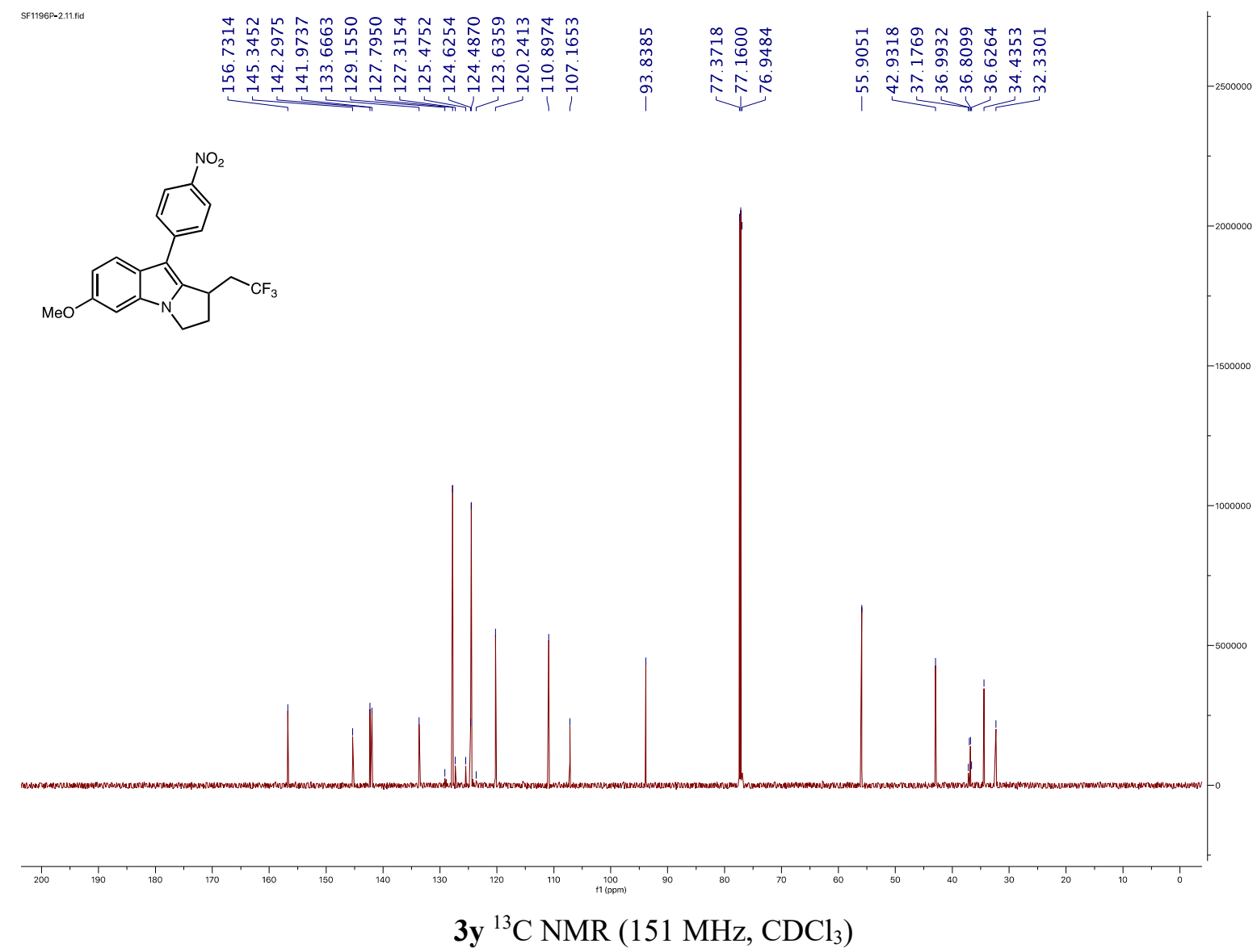

SF1196P-2.13.1. fid

\section{幽等器}

它它

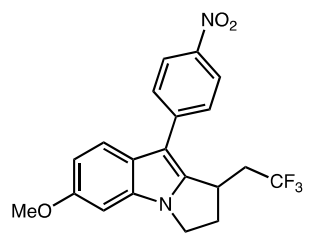



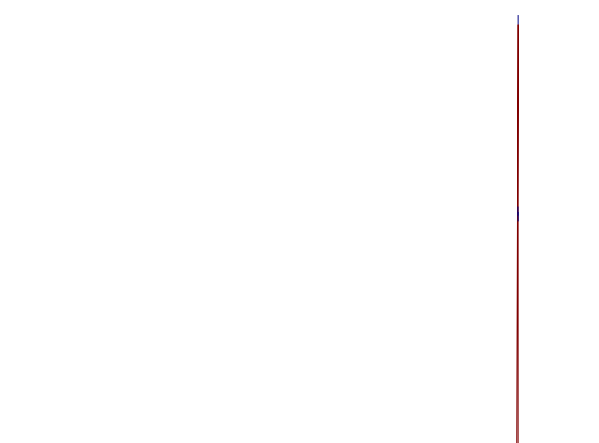

3y ${ }^{19} \mathrm{~F}$ NMR $\left(565 \mathrm{MHz}, \mathrm{CDCl}_{3}\right)$ 
SF1115P-1.20.fid

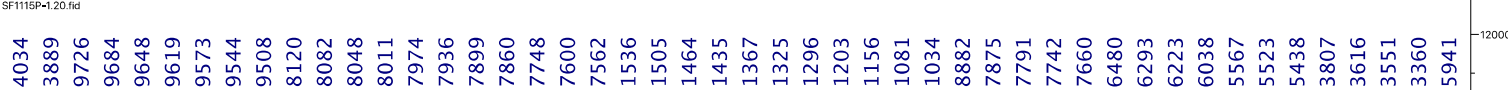

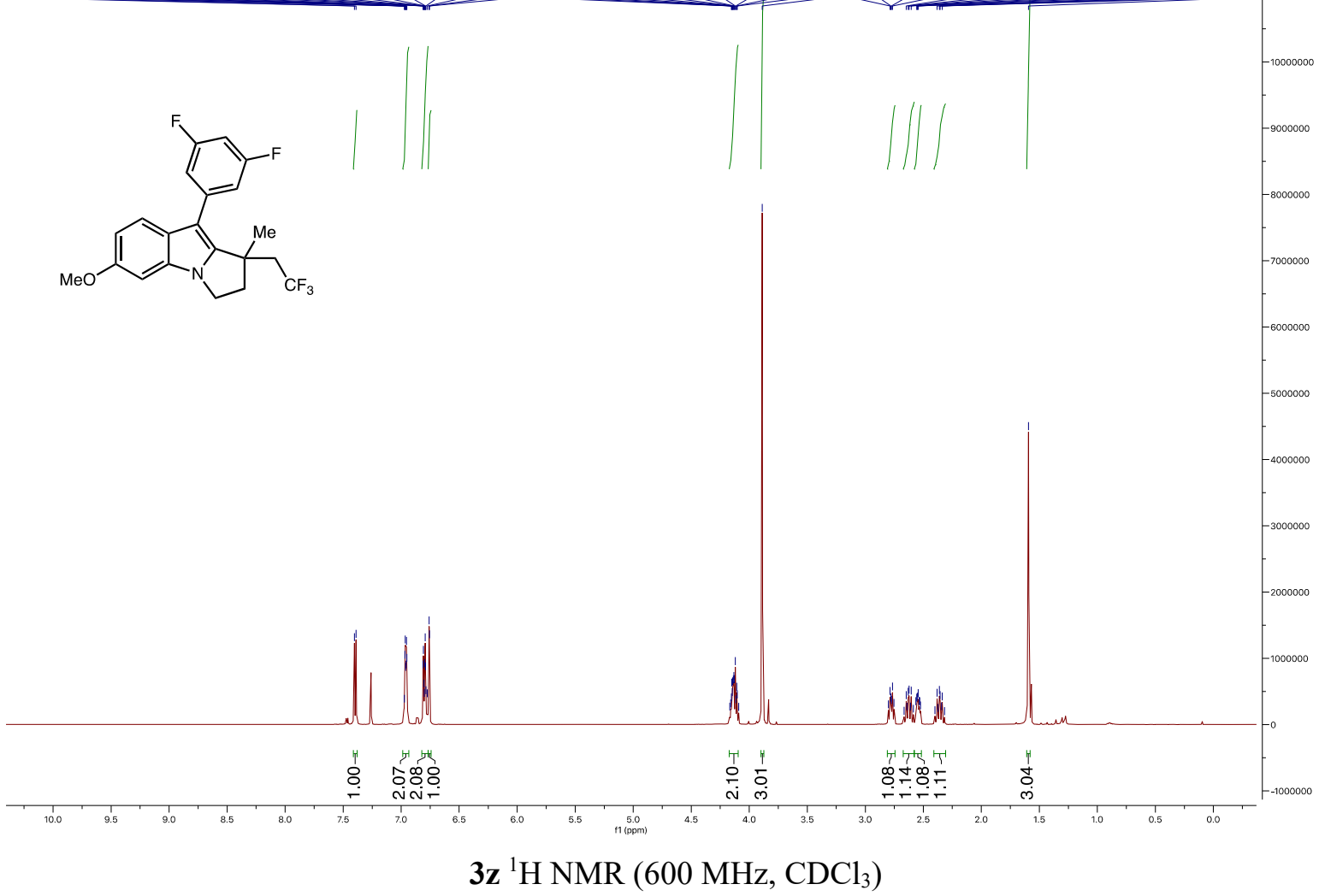

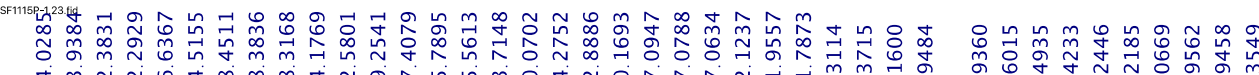

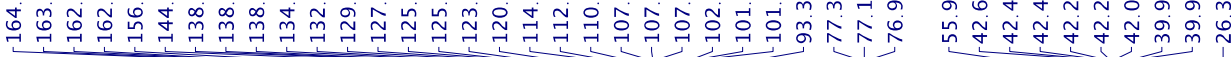
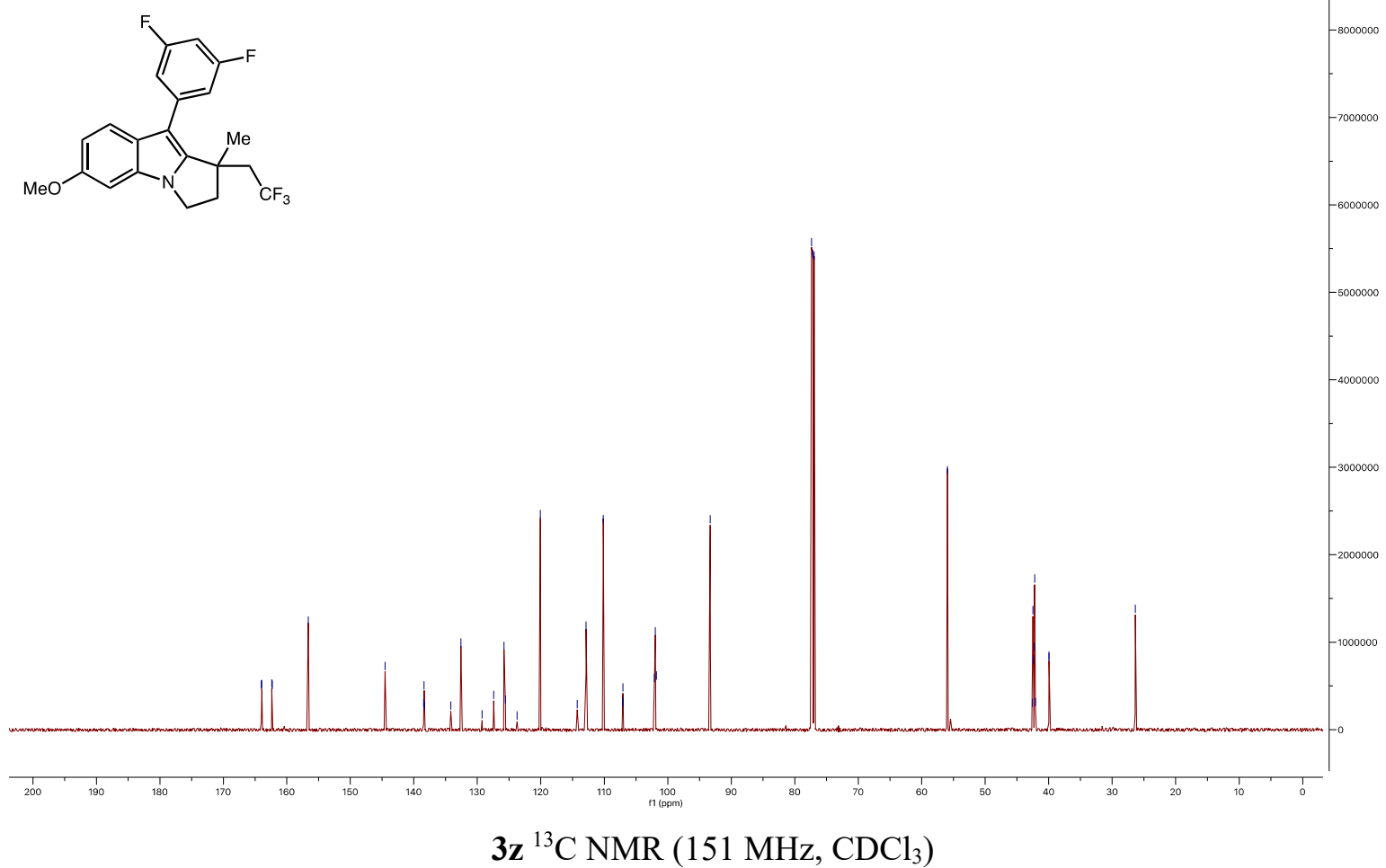

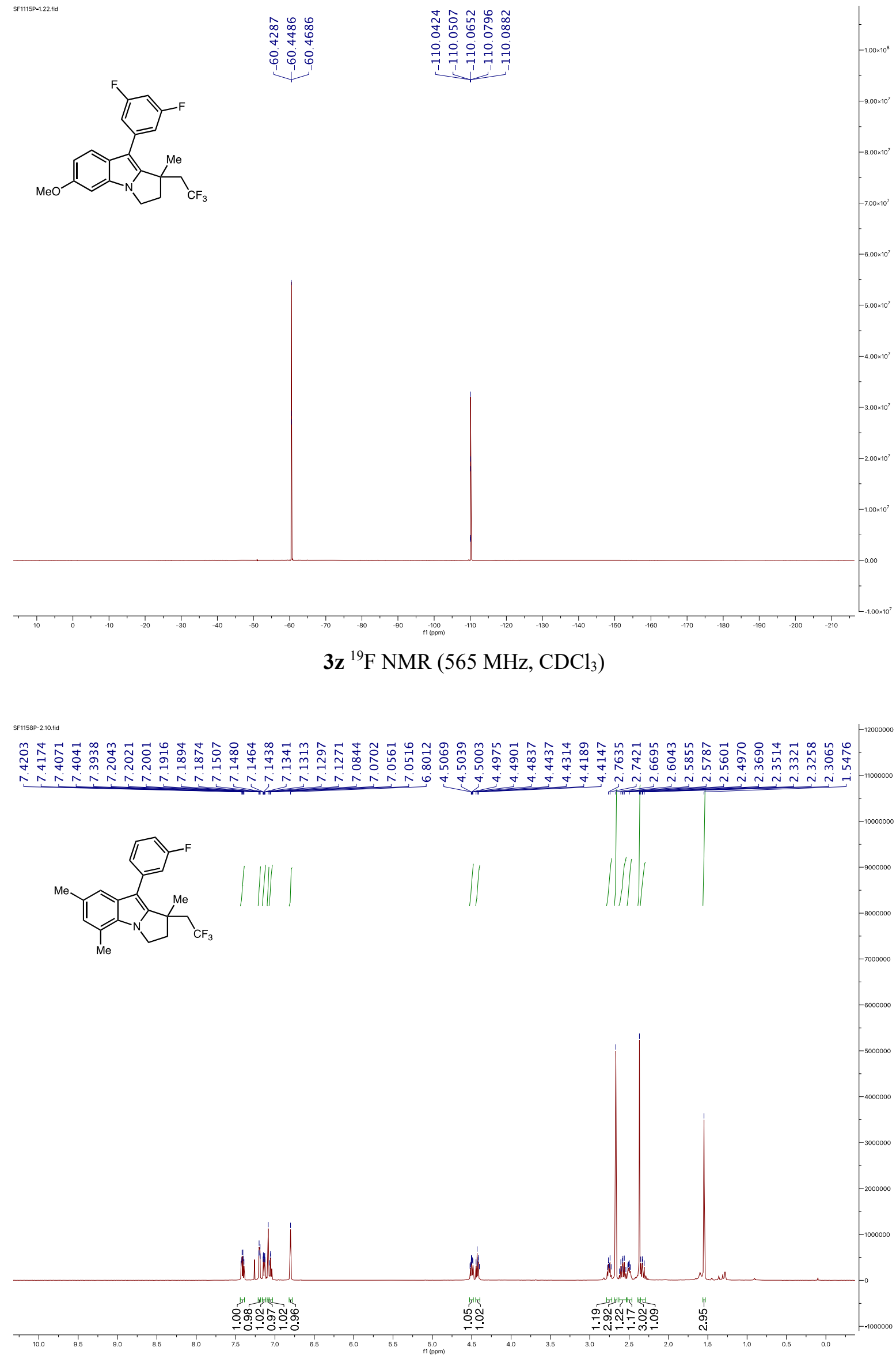

3aa ${ }^{1} \mathrm{H}$ NMR $\left(600 \mathrm{MHz}, \mathrm{CDCl}_{3}\right)$ 
<smiles>CCCC1(C)CCn2c(-c3cccc(F)c3)c3cc(C)cc(C)c3c21</smiles>

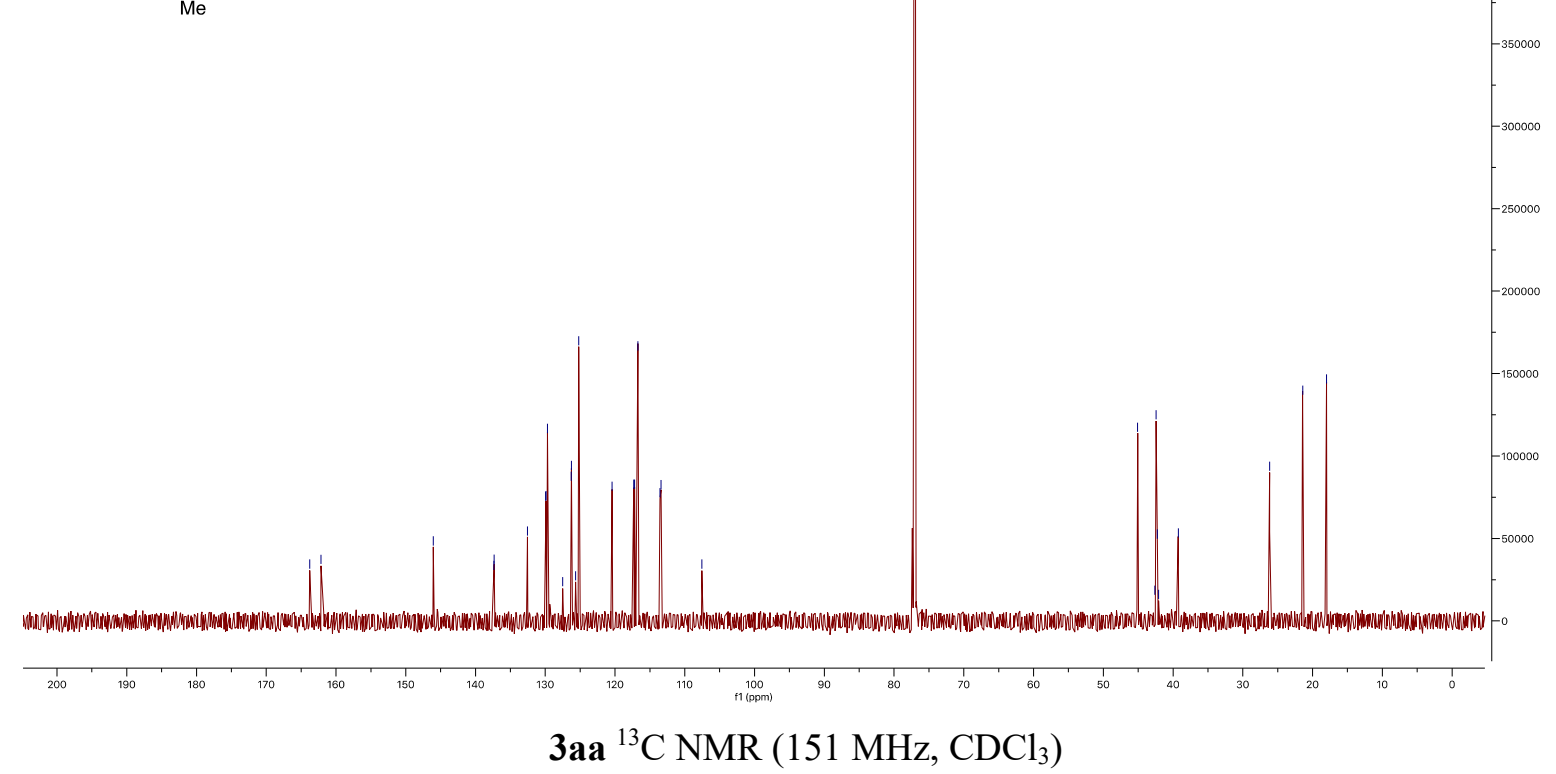
SF1158-2.21.:.id

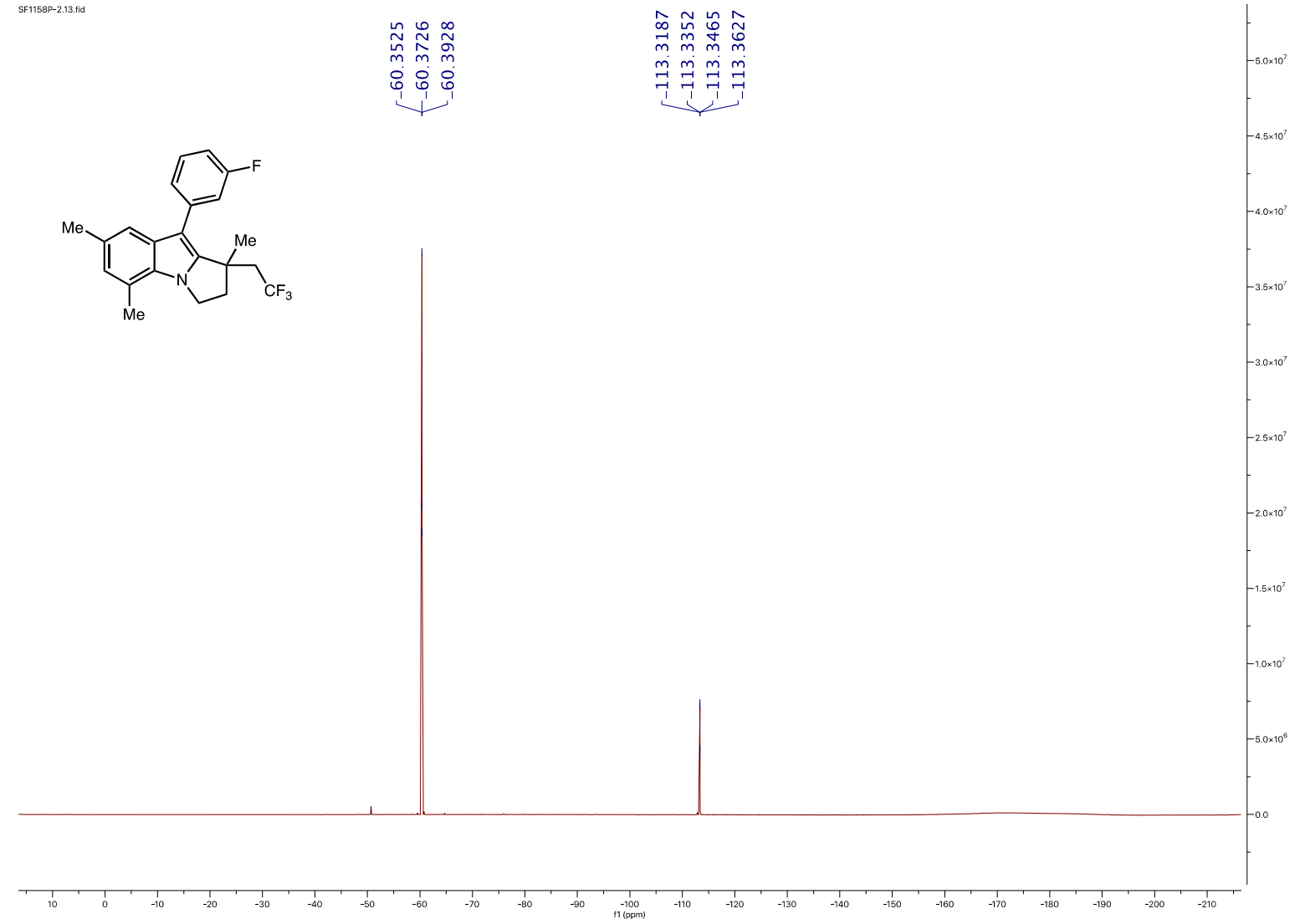

3aa ${ }^{19} \mathrm{~F}$ NMR $\left(565 \mathrm{MHz}, \mathrm{CDCl}_{3}\right)$ 


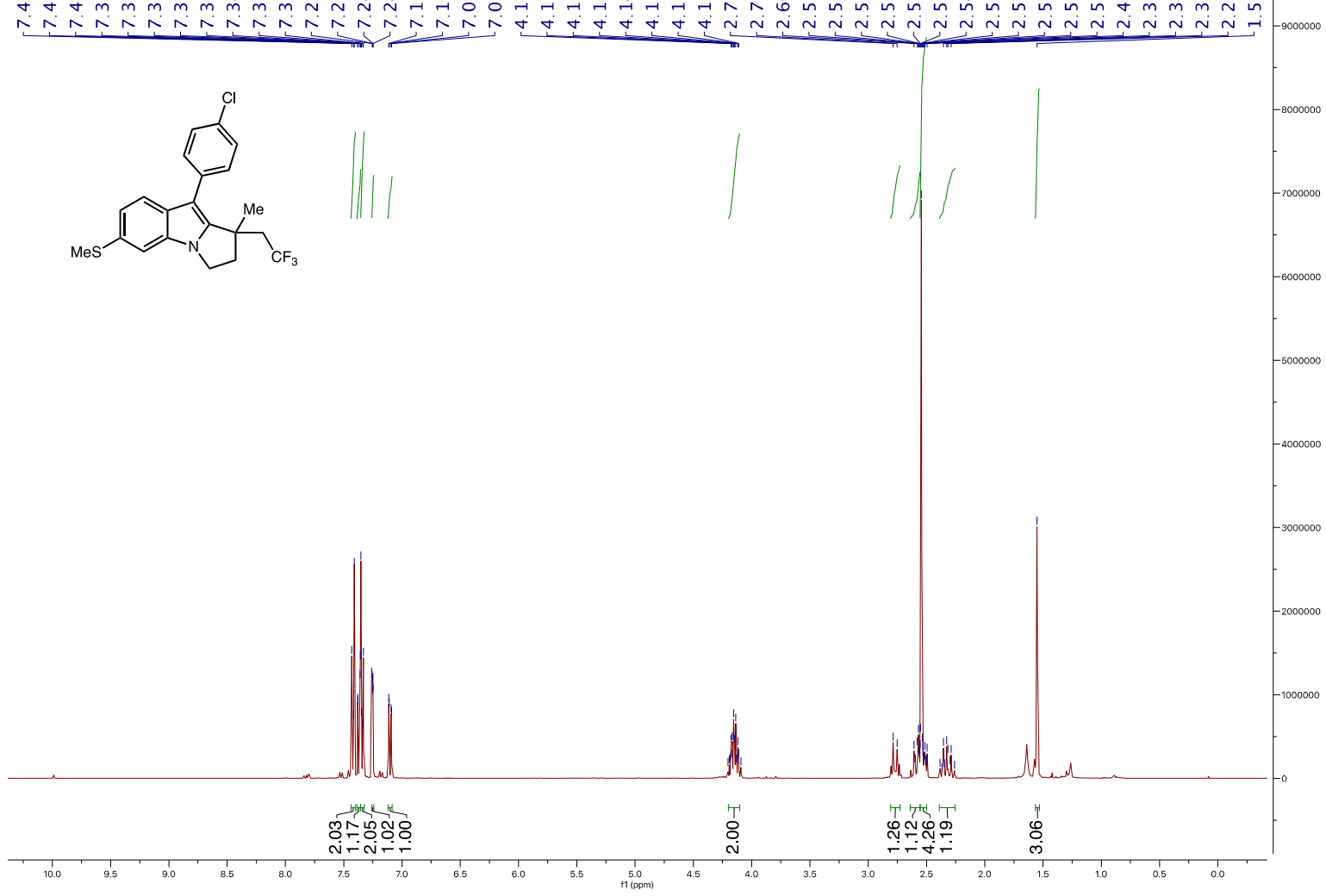

3ab ${ }^{1} \mathrm{H}$ NMR (400 MHz, $\mathrm{CDCl}_{3}$ )

SF1184P-1.5.tid
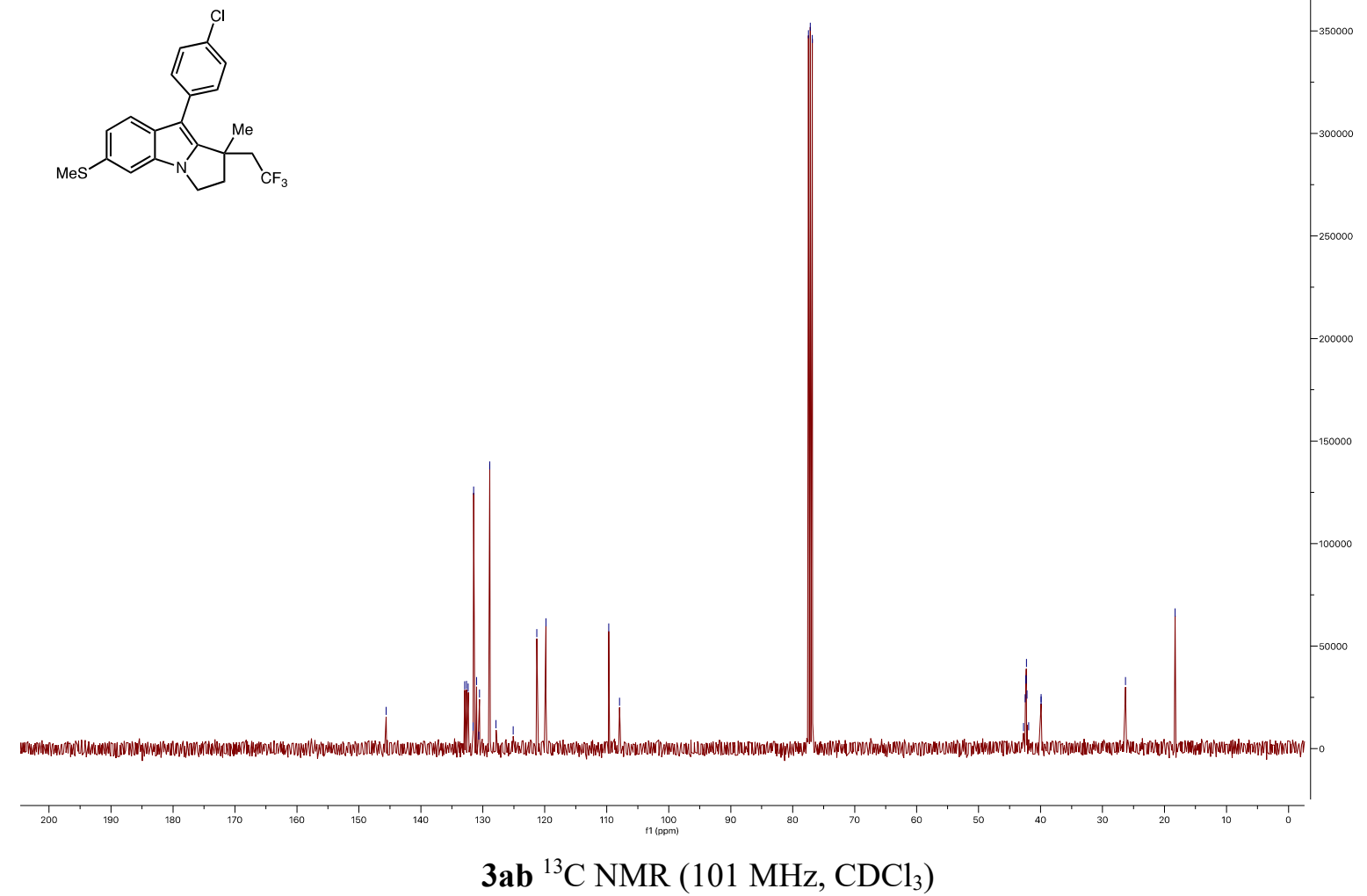


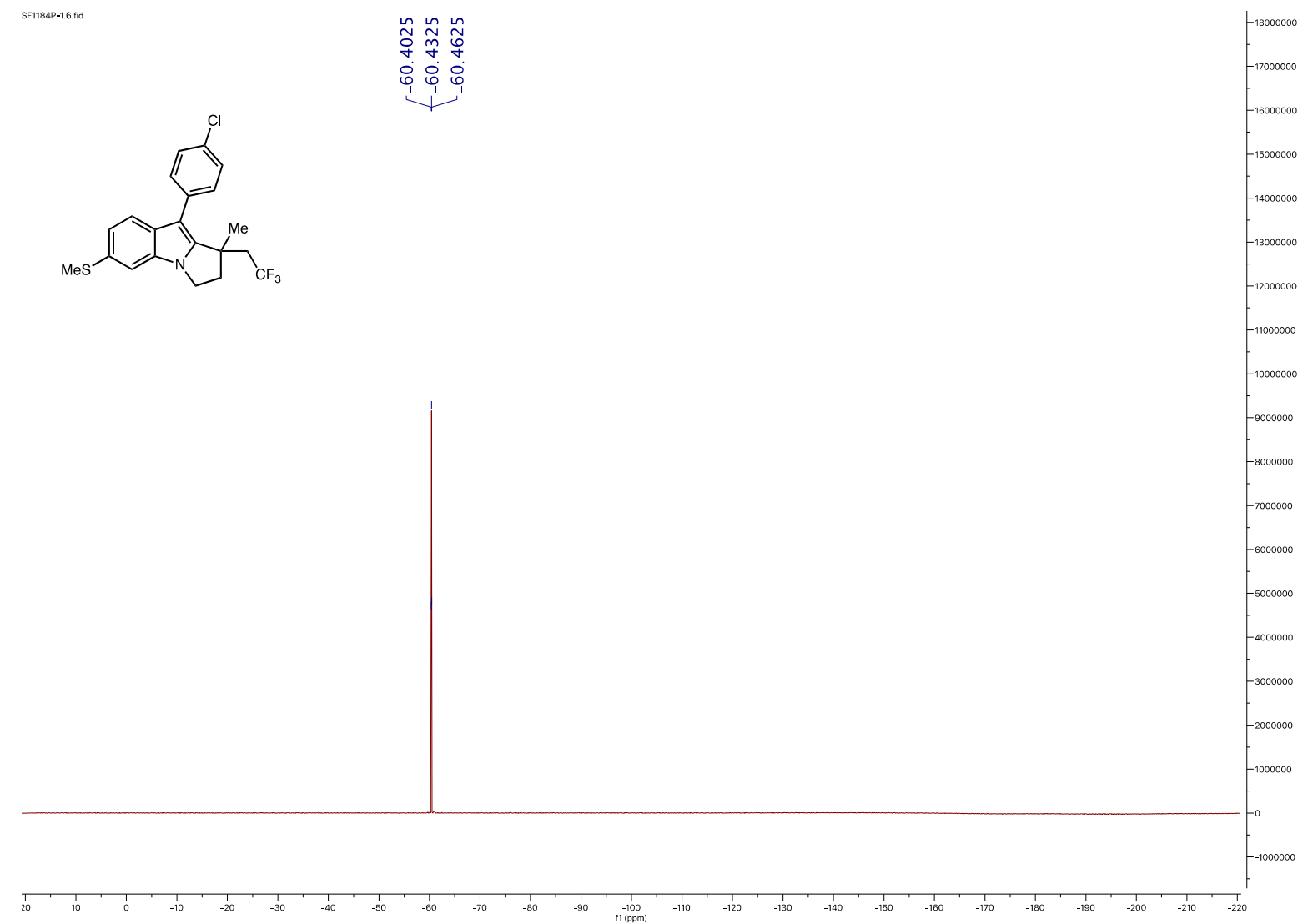

3ab ${ }^{19} \mathrm{~F}$ NMR (376 MHz, $\mathrm{CDCl}_{3}$ )

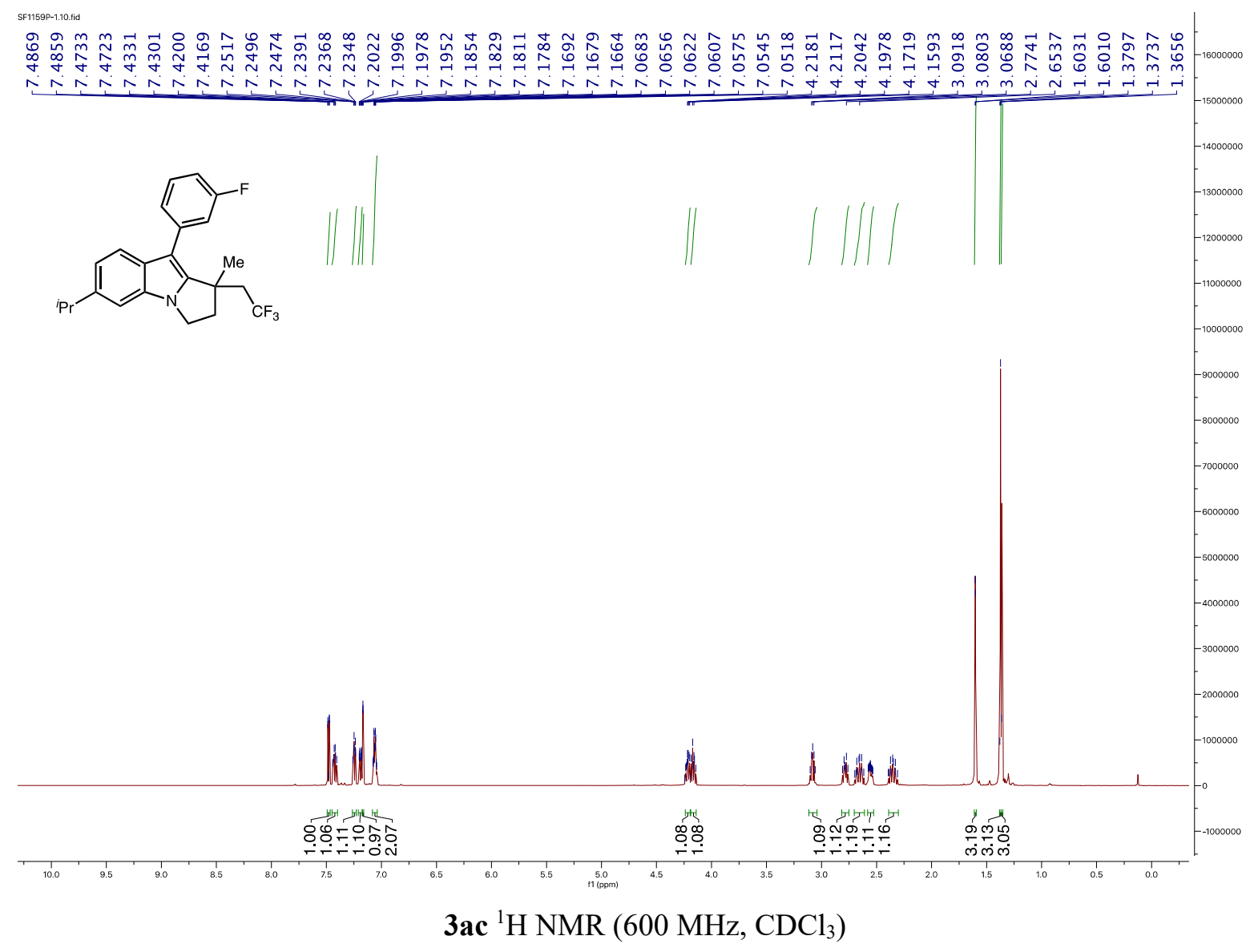



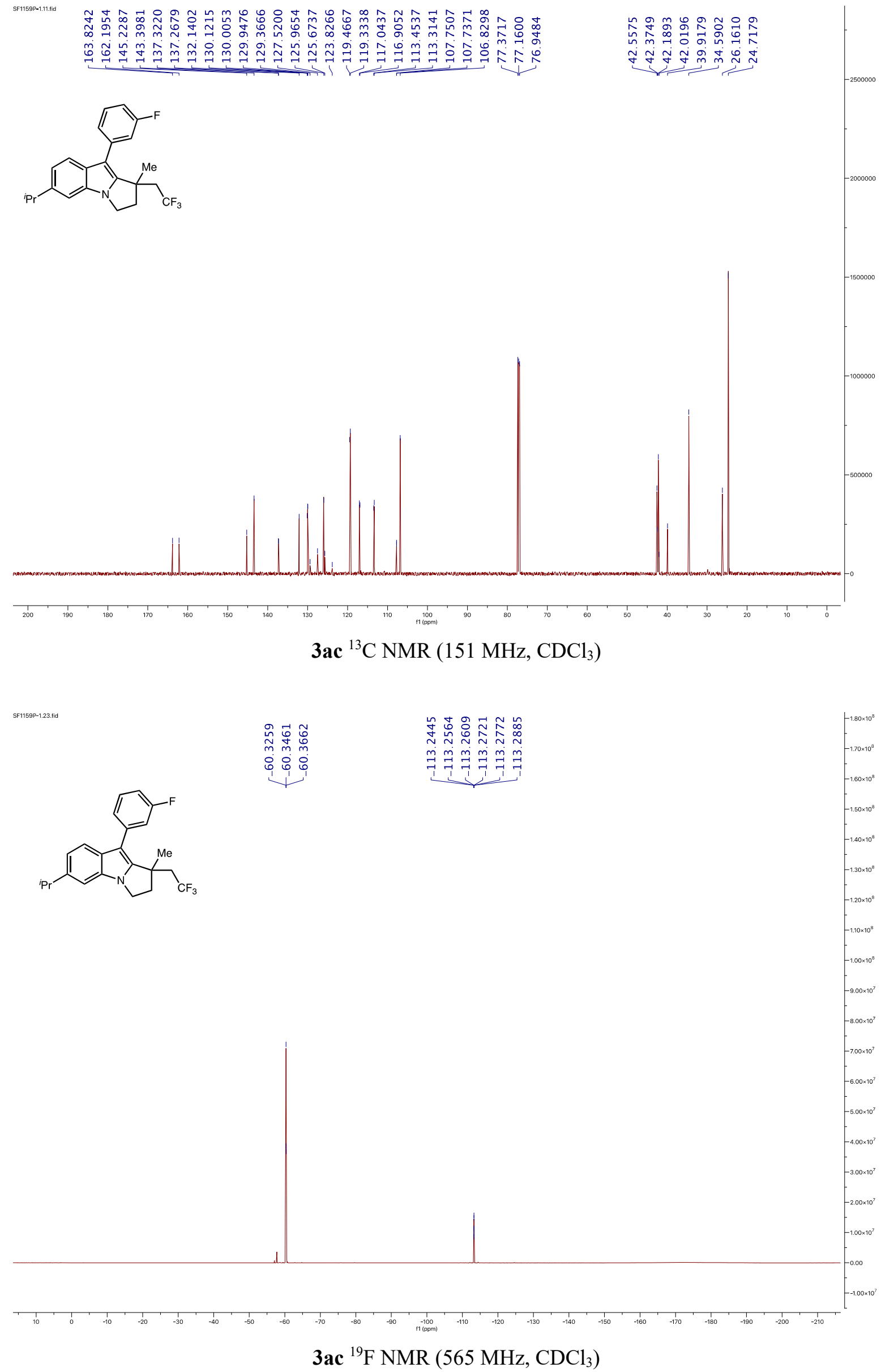


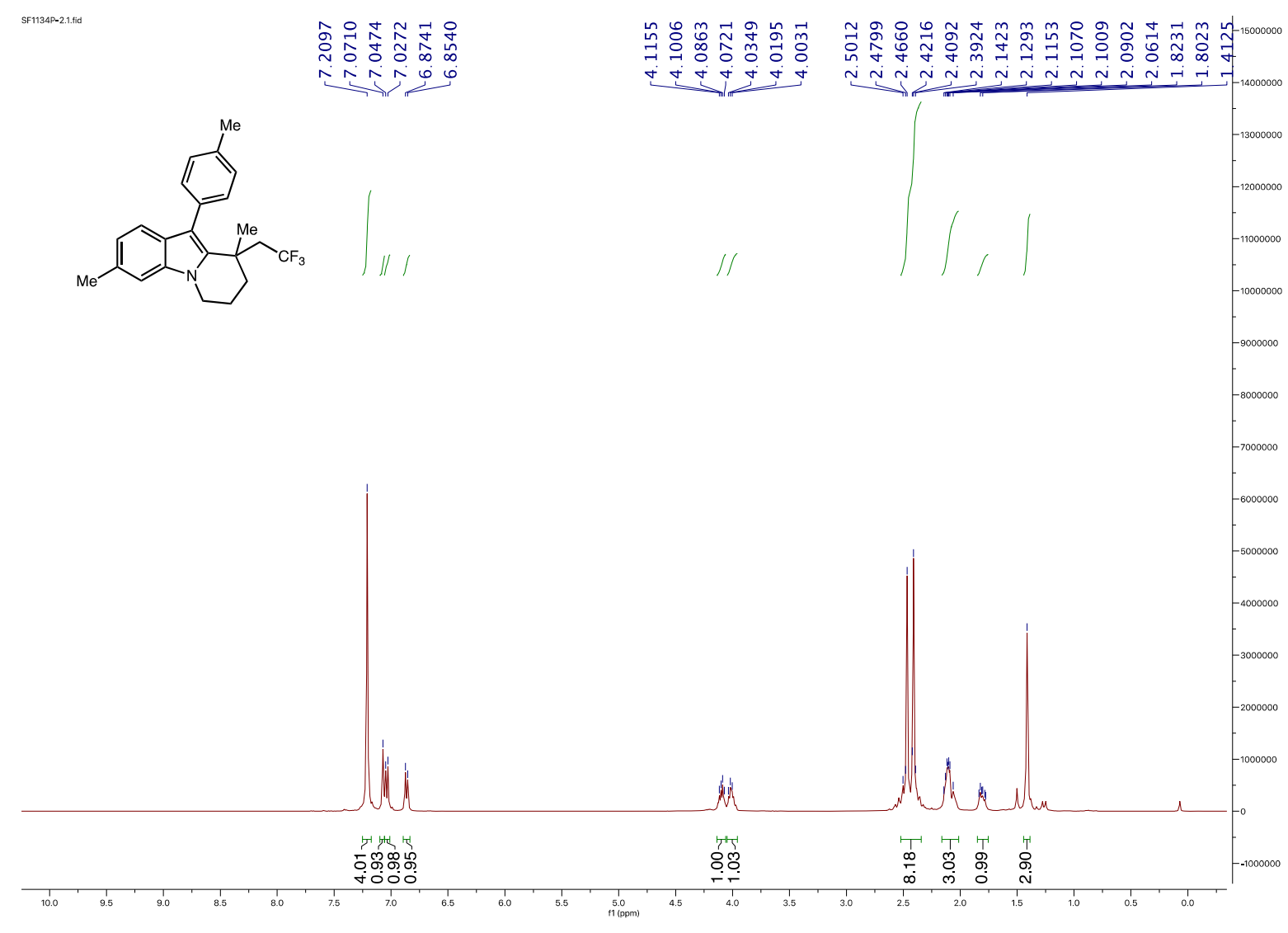

7a ${ }^{1} \mathrm{H}$ NMR $\left(400 \mathrm{MHz}, \mathrm{CDCl}_{3}\right)$

SF1134P-1.13 fid
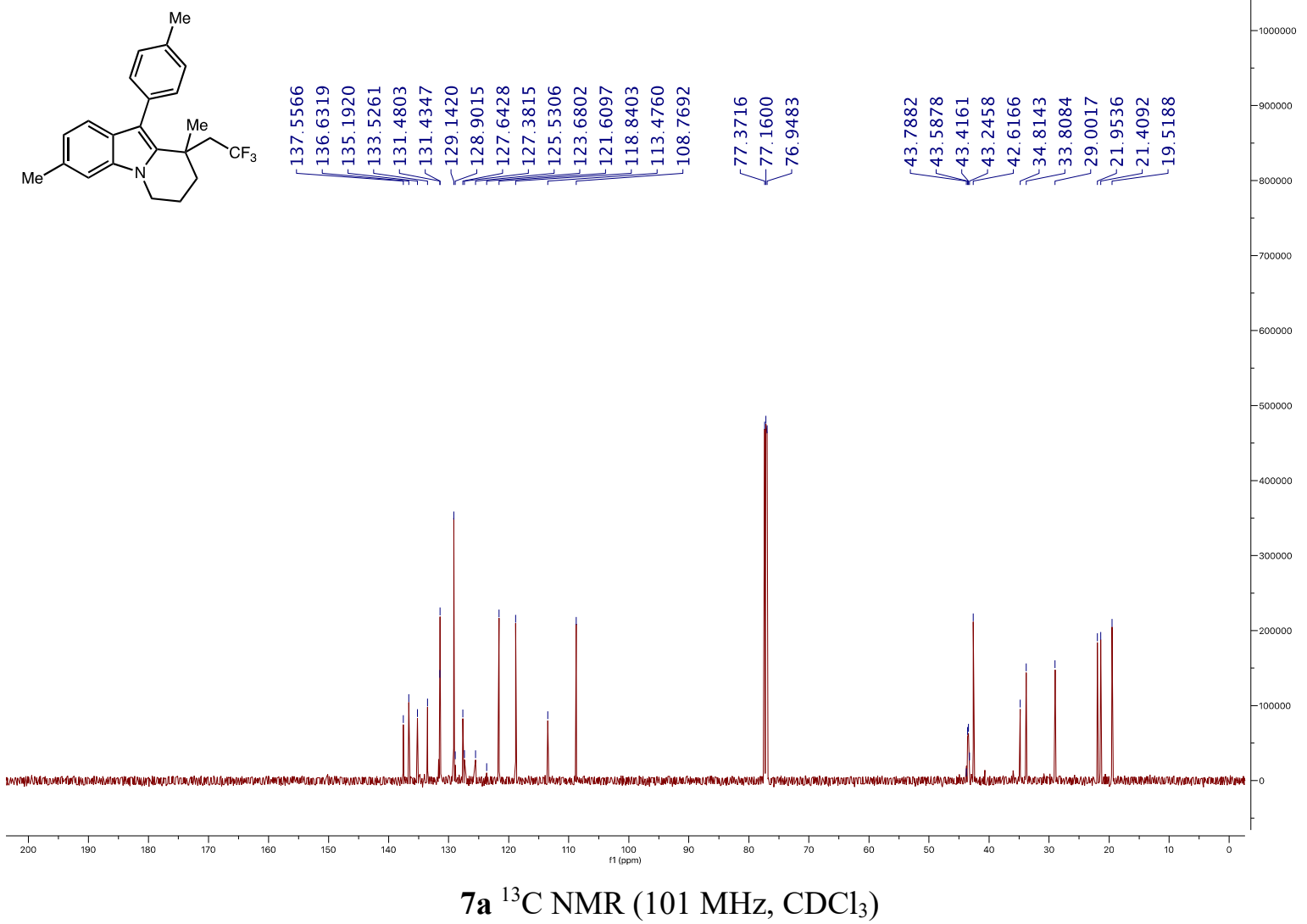

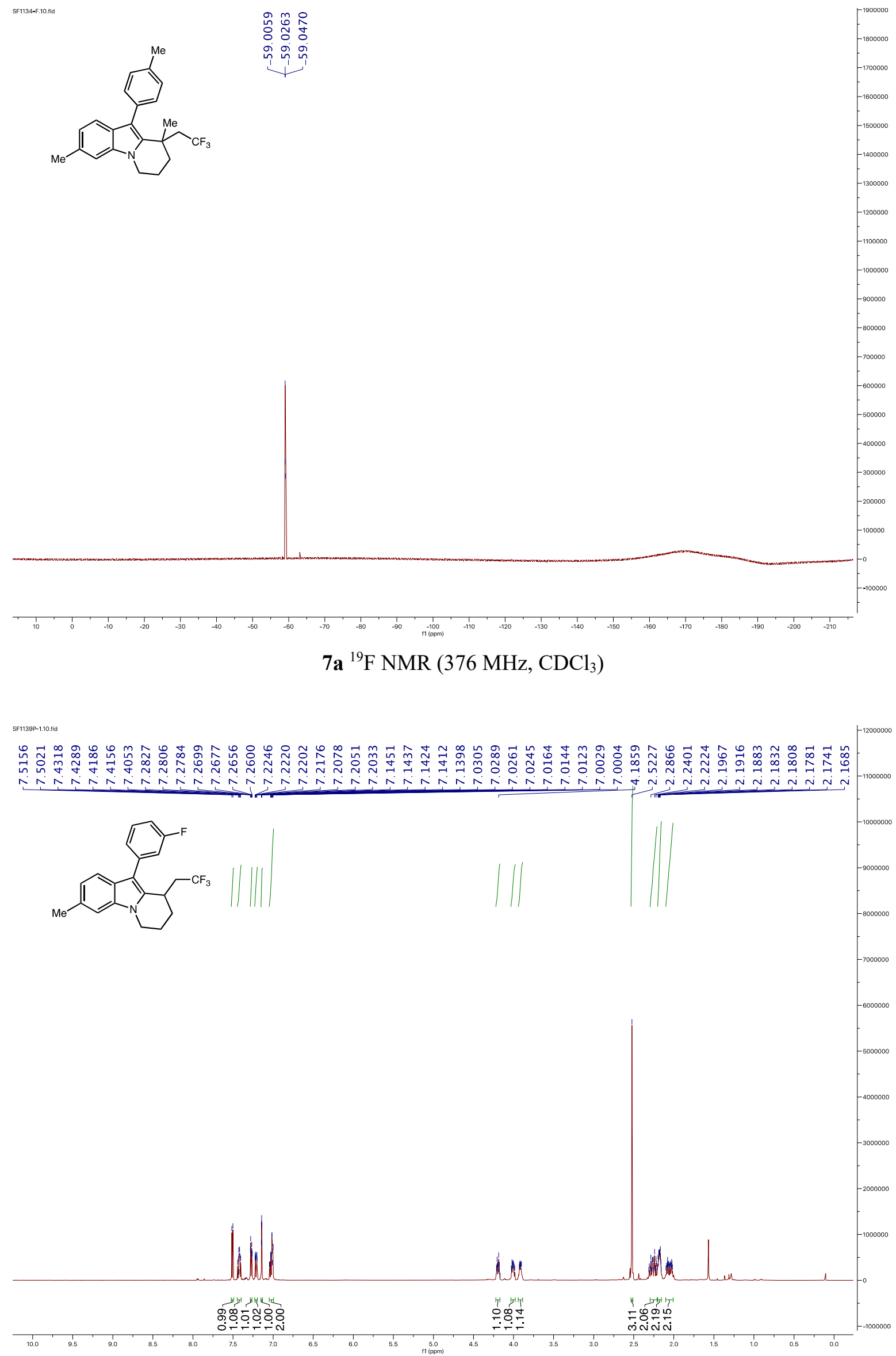

7b ${ }^{1} \mathrm{H}$ NMR (400 MHz, $\mathrm{CDCl}_{3}$ ) 


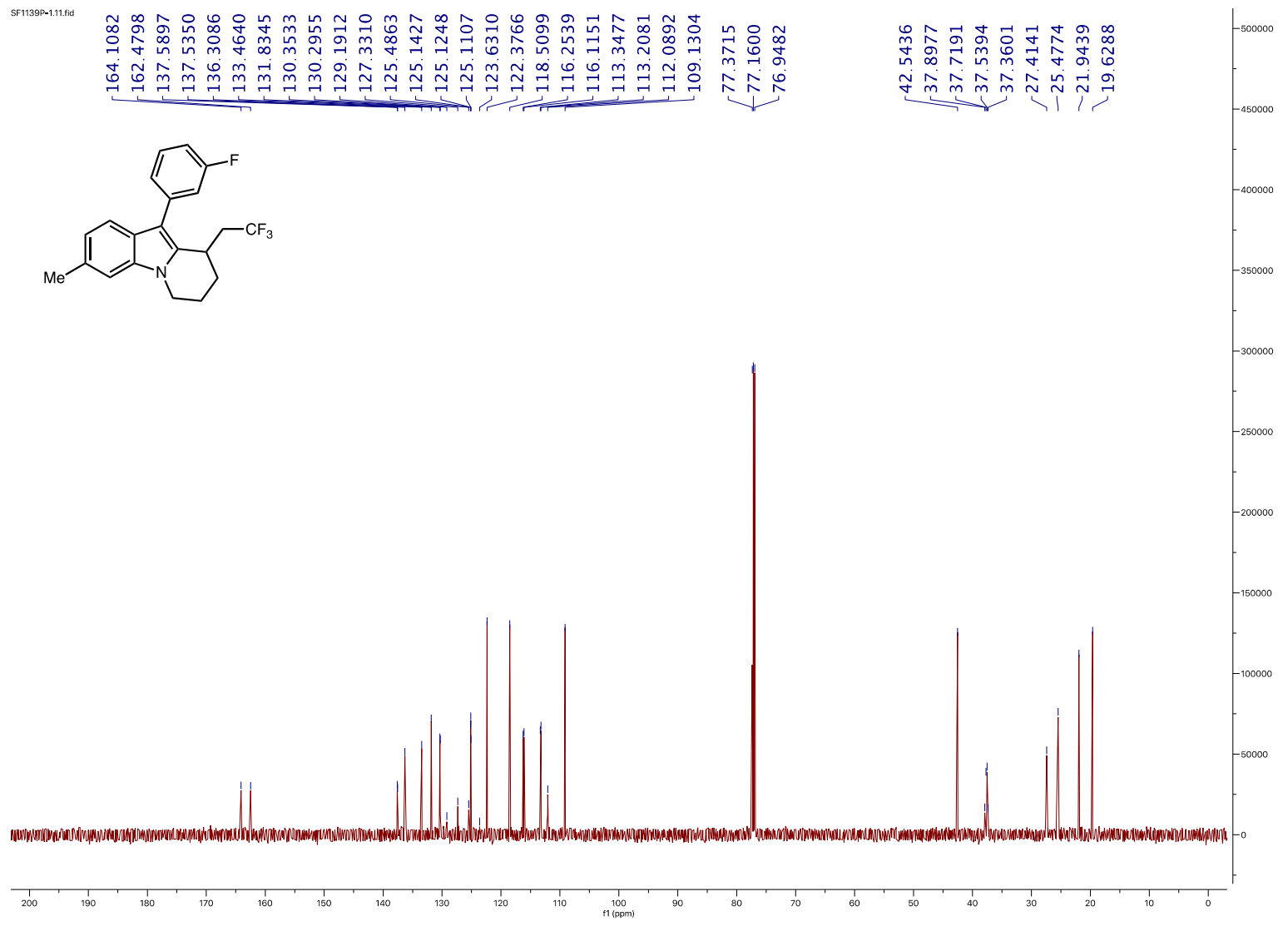

7b ${ }^{13} \mathrm{C}$ NMR (101 MHz, $\left.\mathrm{CDCl}_{3}\right)$

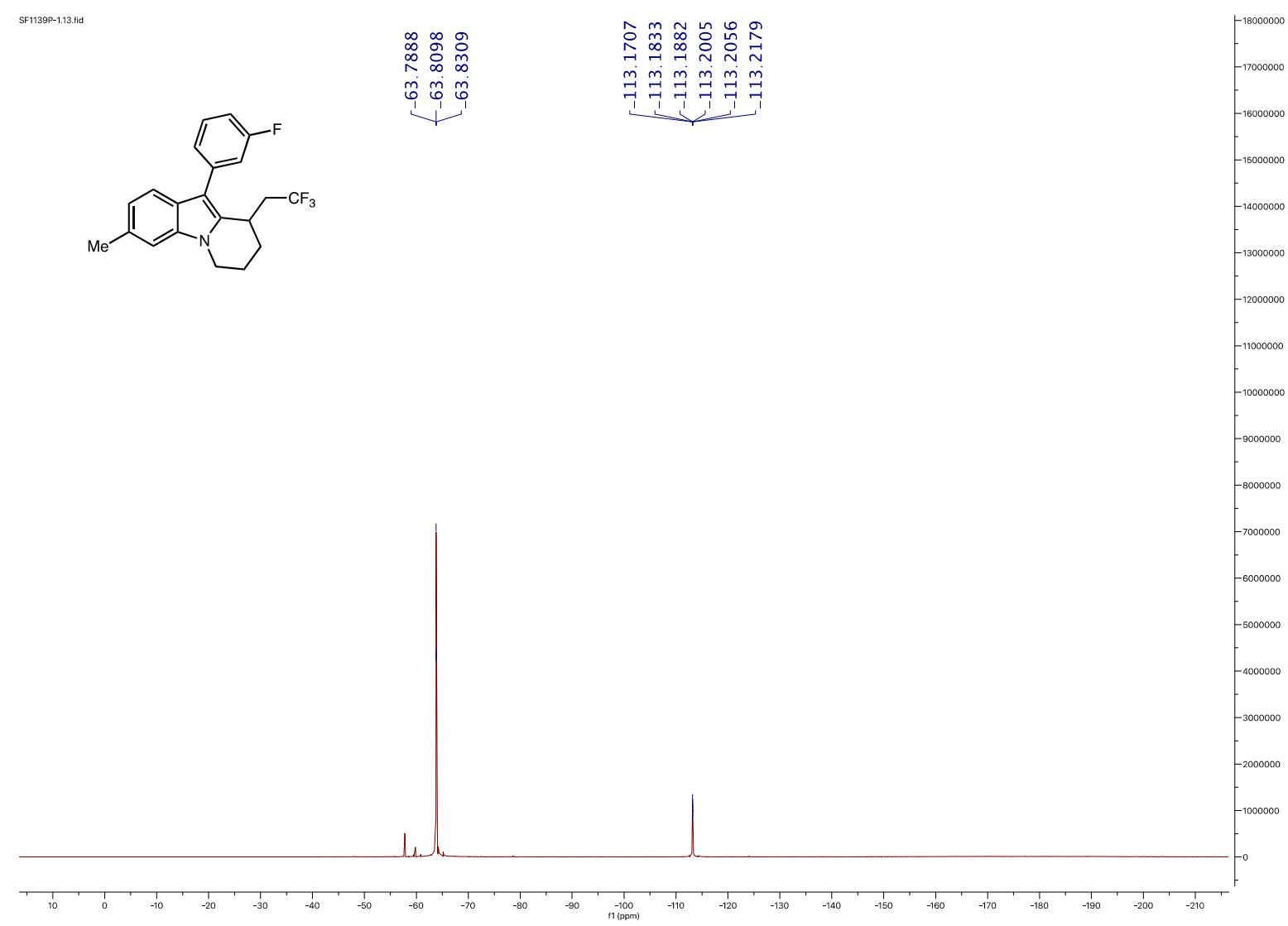

7b ${ }^{19} \mathrm{~F}$ NMR (376 MHz, $\mathrm{CDCl}_{3}$ ) 


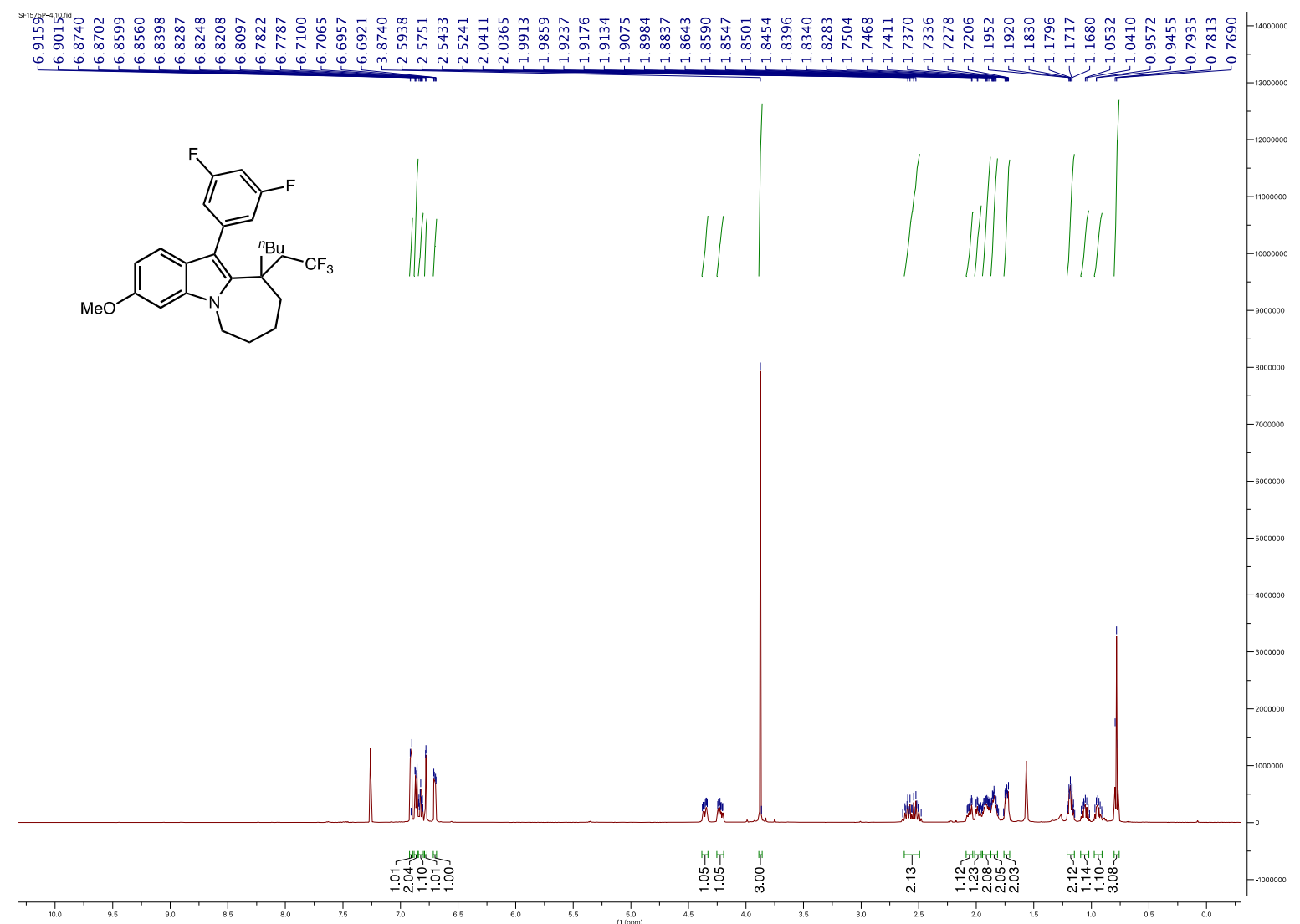

7c ${ }^{1} \mathrm{H}$ NMR $\left(600 \mathrm{MHz}, \mathrm{CDCl}_{3}\right)$

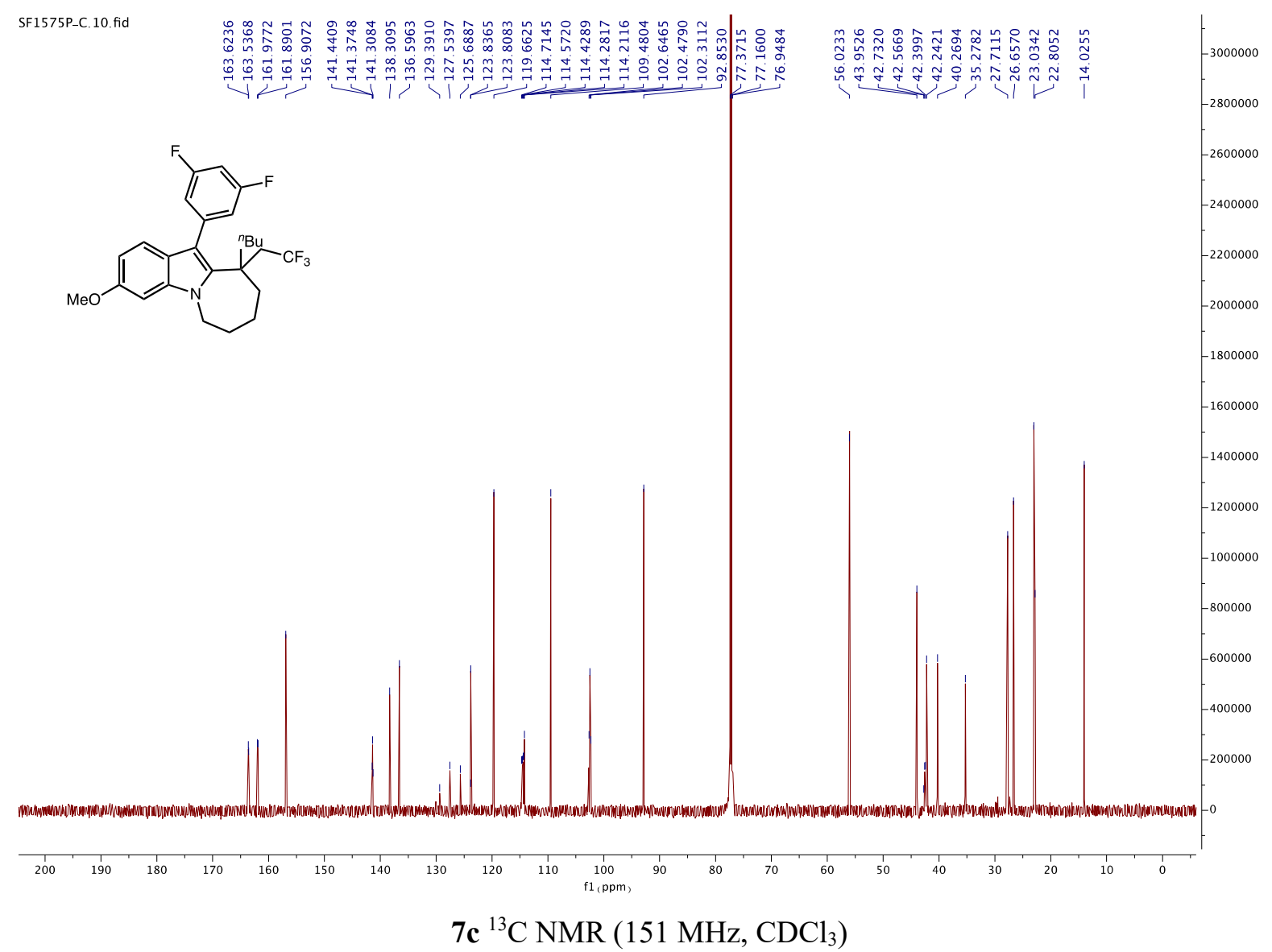



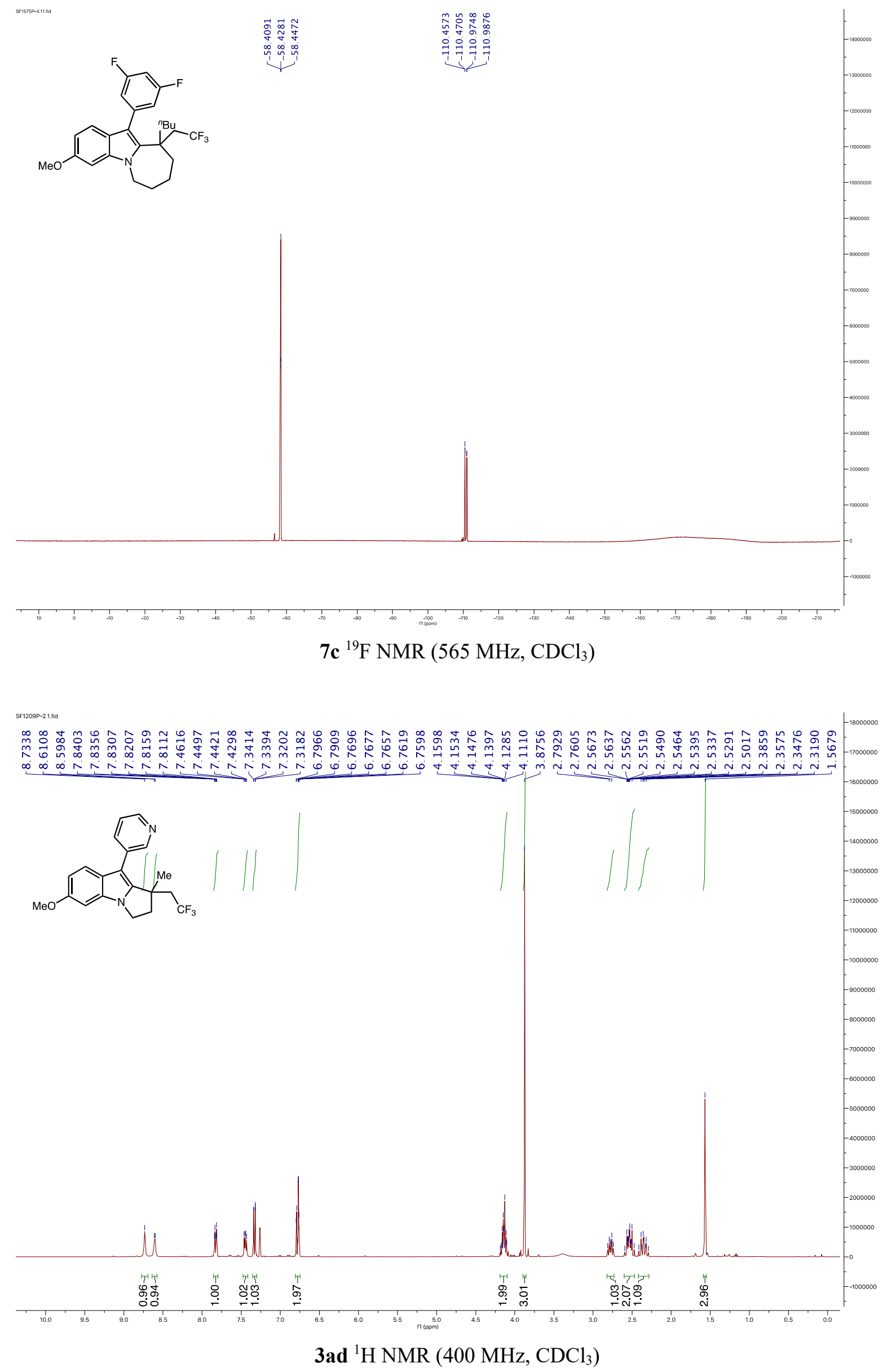

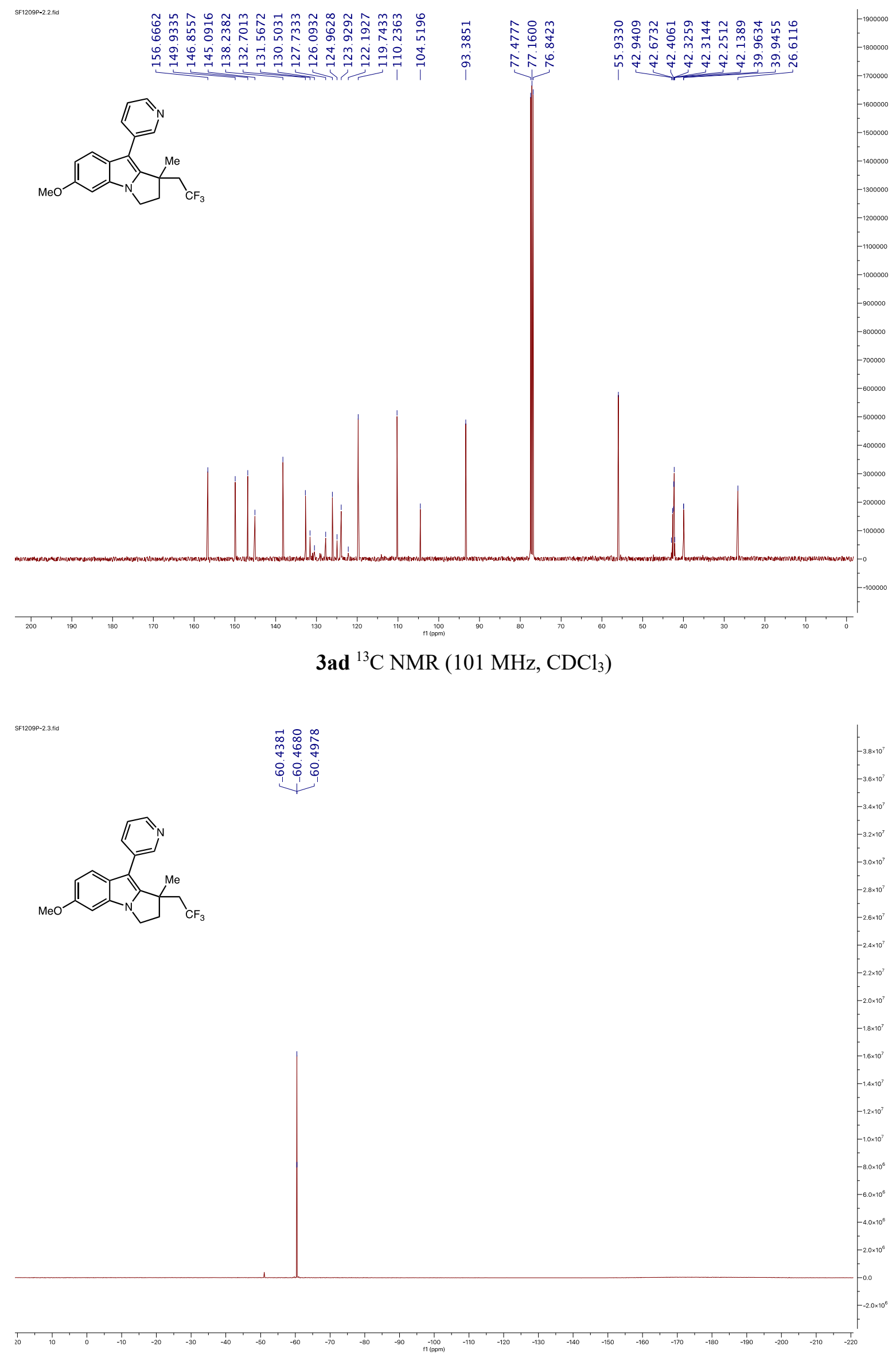

3ad ${ }^{19} \mathrm{~F}$ NMR $\left(376 \mathrm{MHz}, \mathrm{CDCl}_{3}\right)$ 


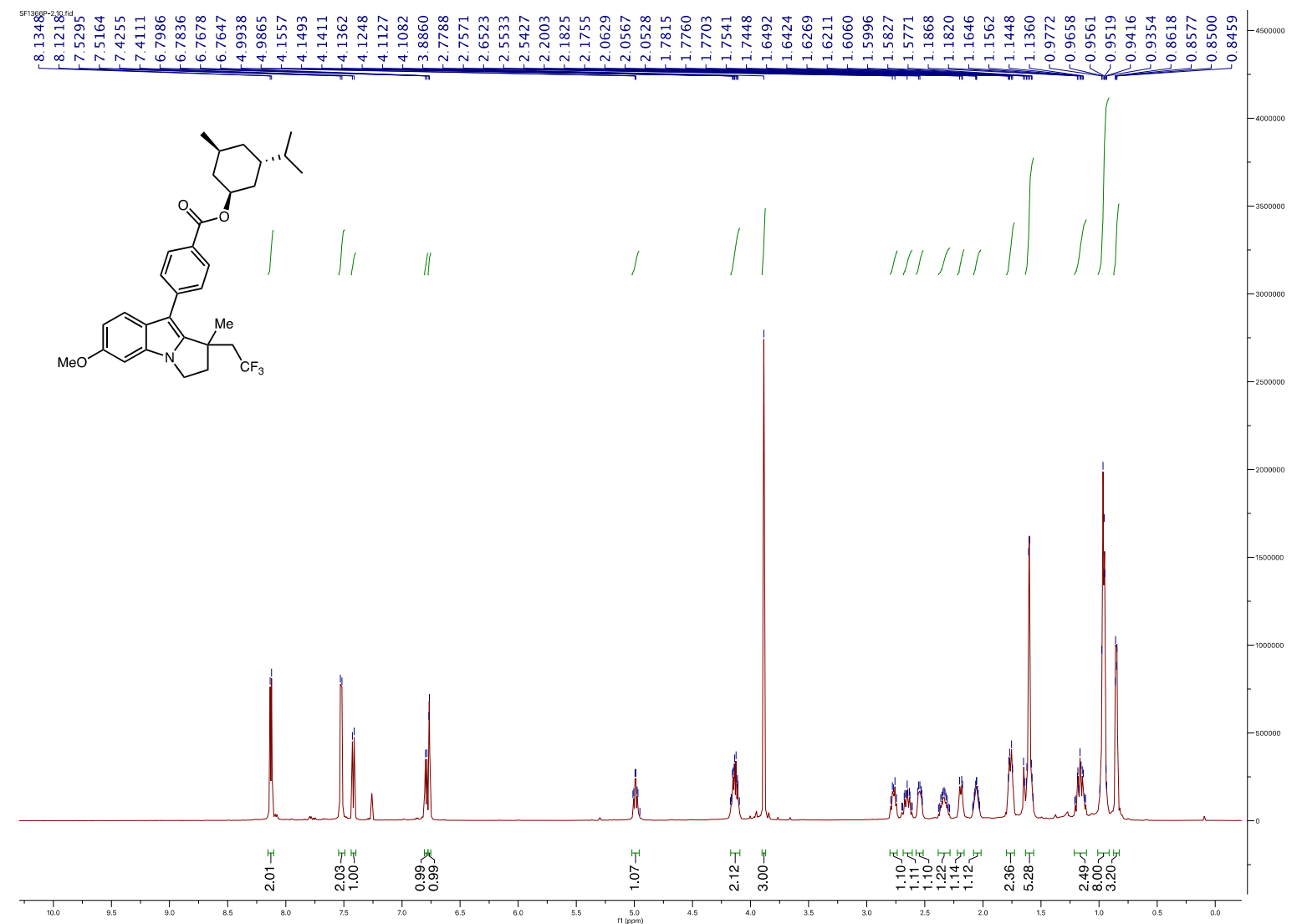

$3 \mathbf{r}^{1} \mathrm{H}$ NMR $\left(600 \mathrm{MHz}, \mathrm{CDCl}_{3}\right)$

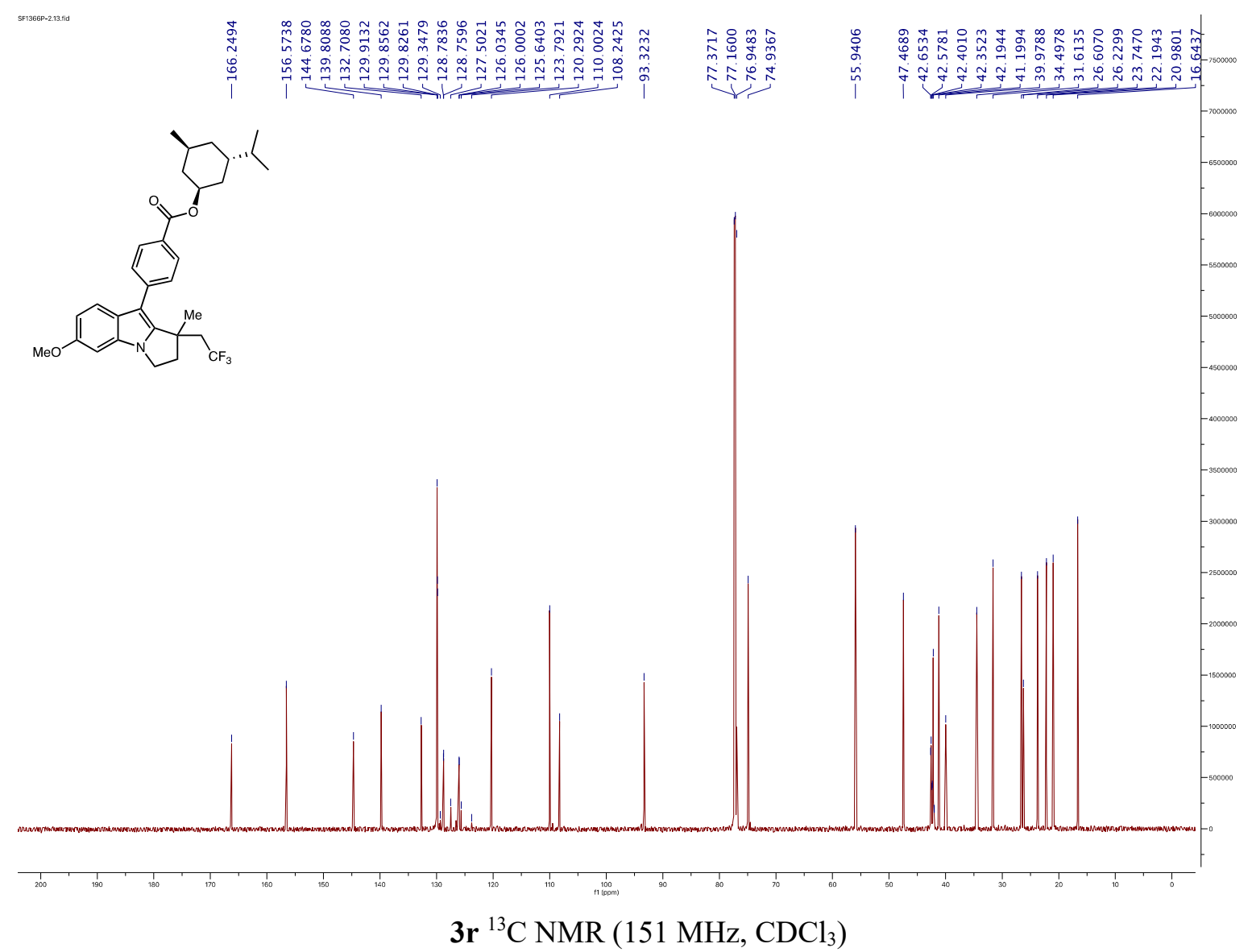



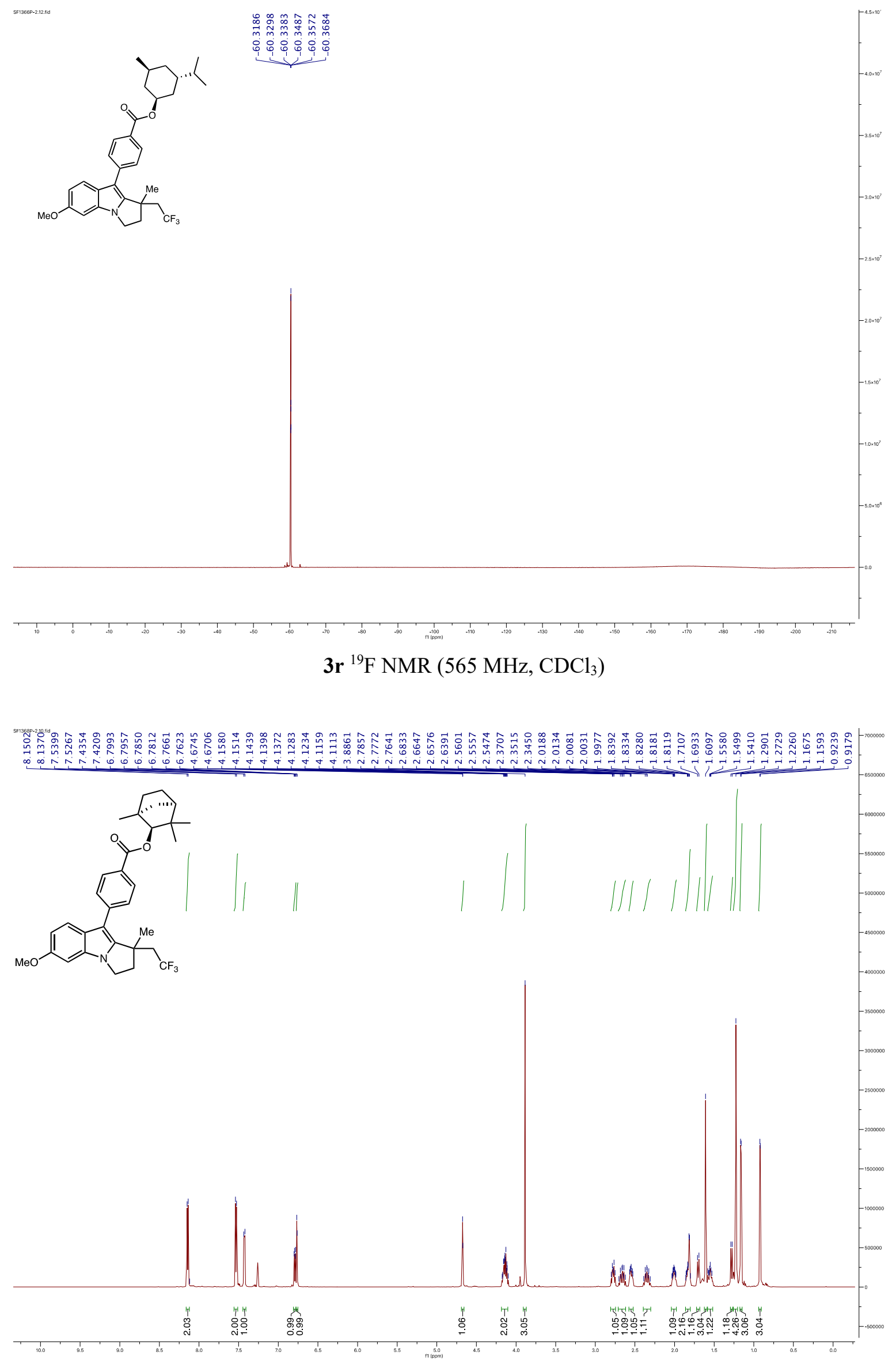

3s ${ }^{1} \mathrm{H}$ NMR $\left(600 \mathrm{MHz}, \mathrm{CDCl}_{3}\right)$ 


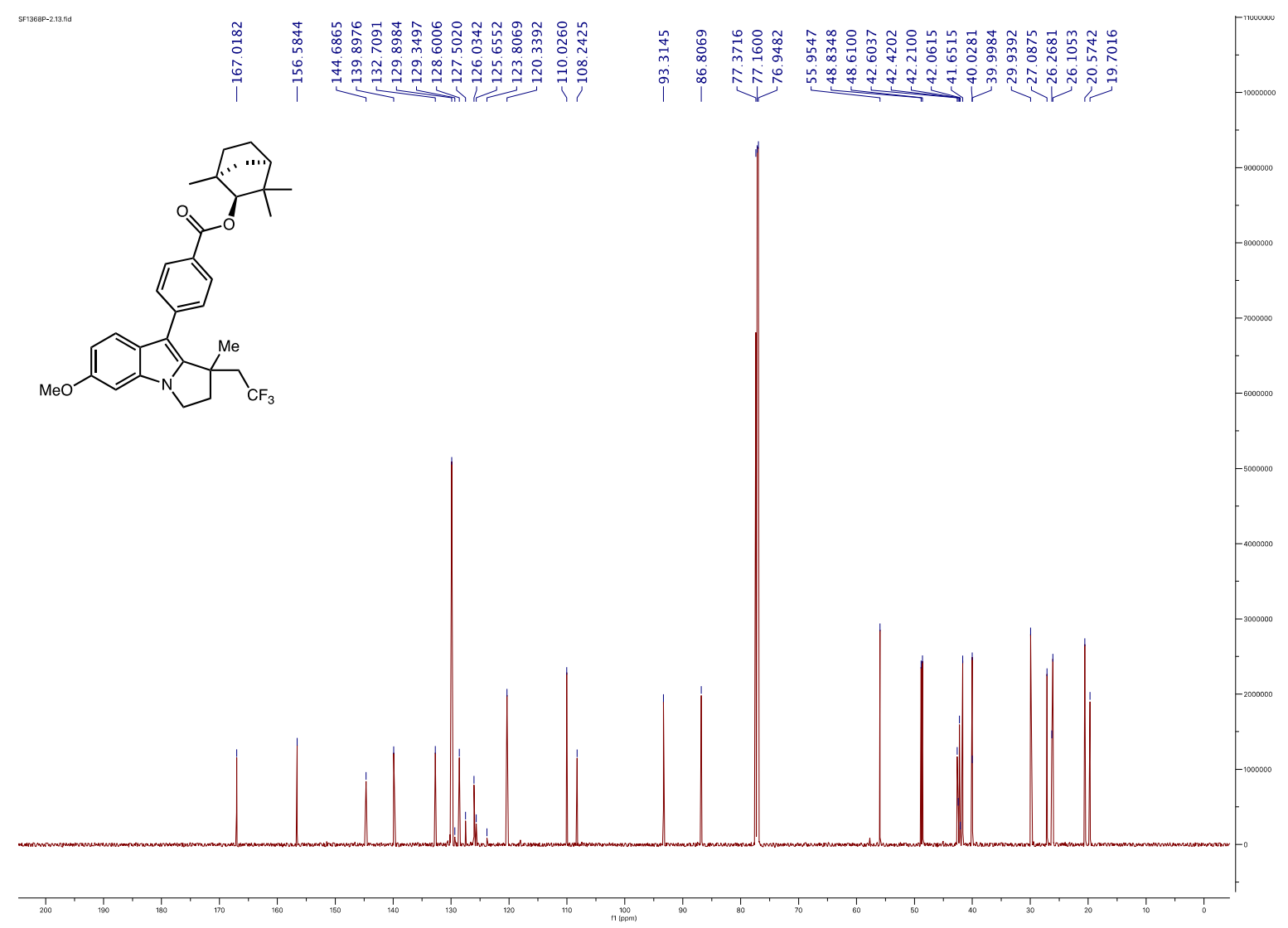

3s ${ }^{13} \mathrm{C}$ NMR (151 MHz, $\left.\mathrm{CDCl}_{3}\right)$

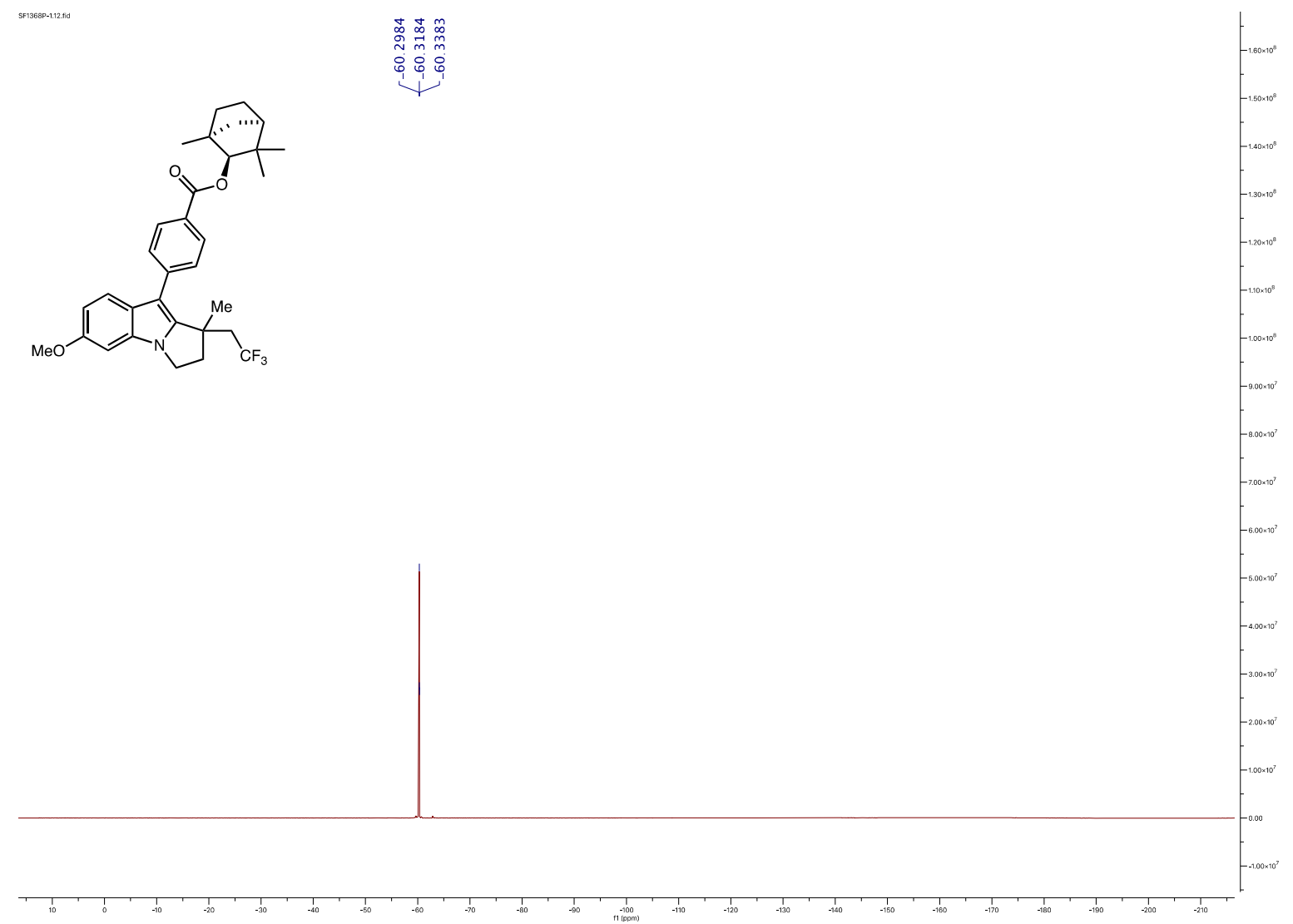

3s ${ }^{19} \mathrm{~F}$ NMR $\left(565 \mathrm{MHz}, \mathrm{CDCl}_{3}\right)$ 


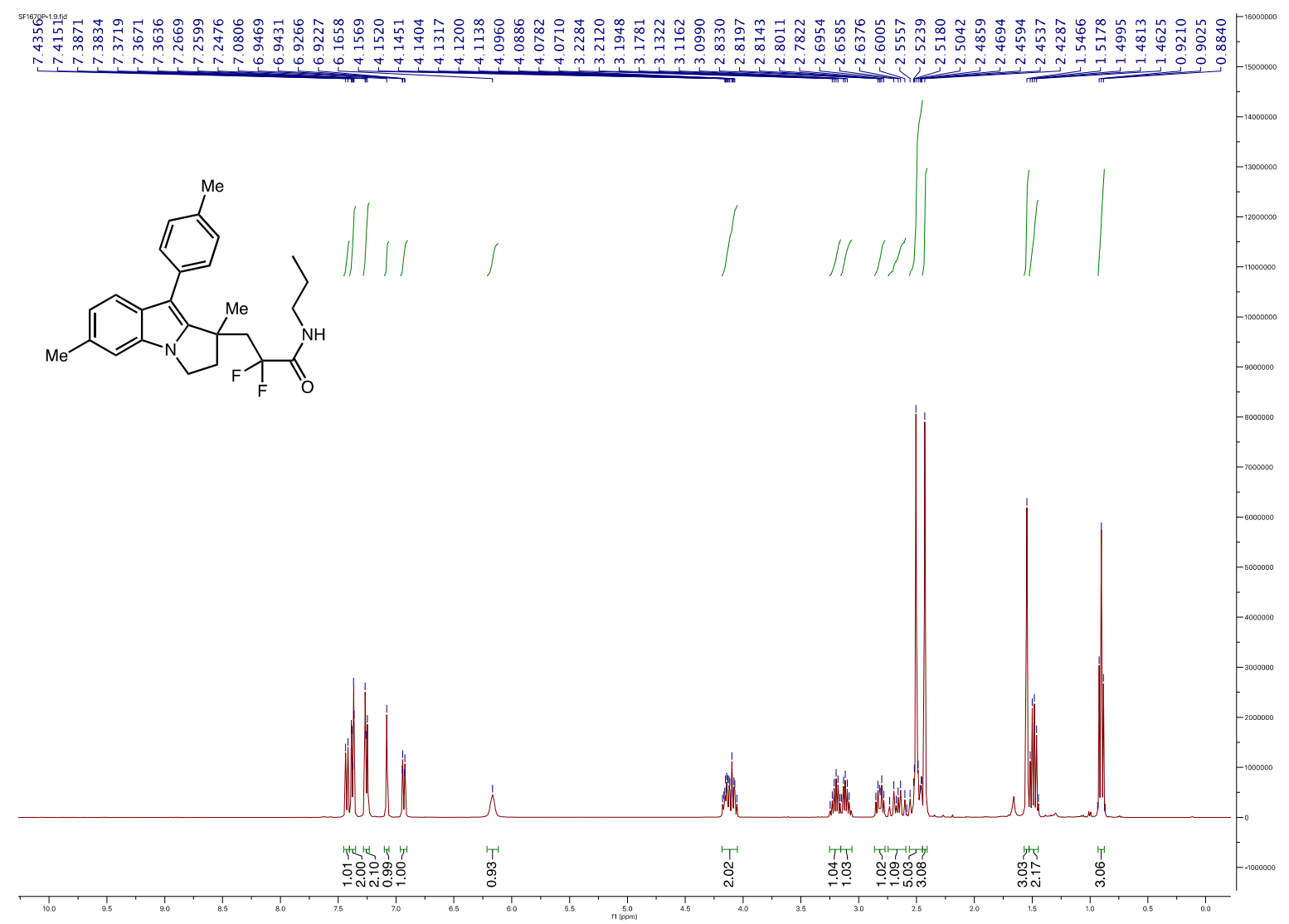

8a ${ }^{1} \mathrm{H}$ NMR (400 MHz, $\mathrm{CDCl}_{3}$ )

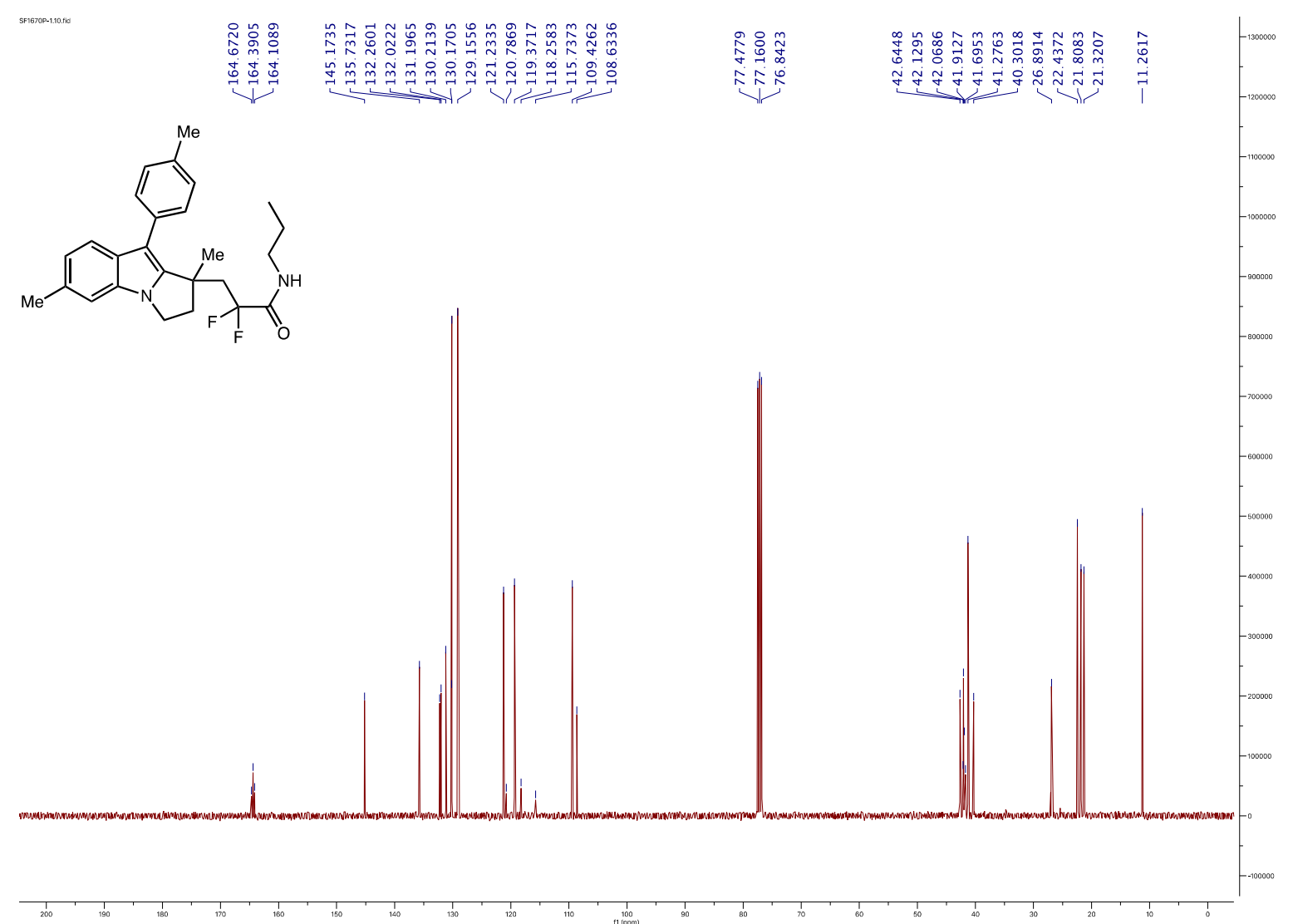

8a ${ }^{13} \mathrm{C}$ NMR (101 MHz, $\left.\mathrm{CDCl}_{3}\right)$ 


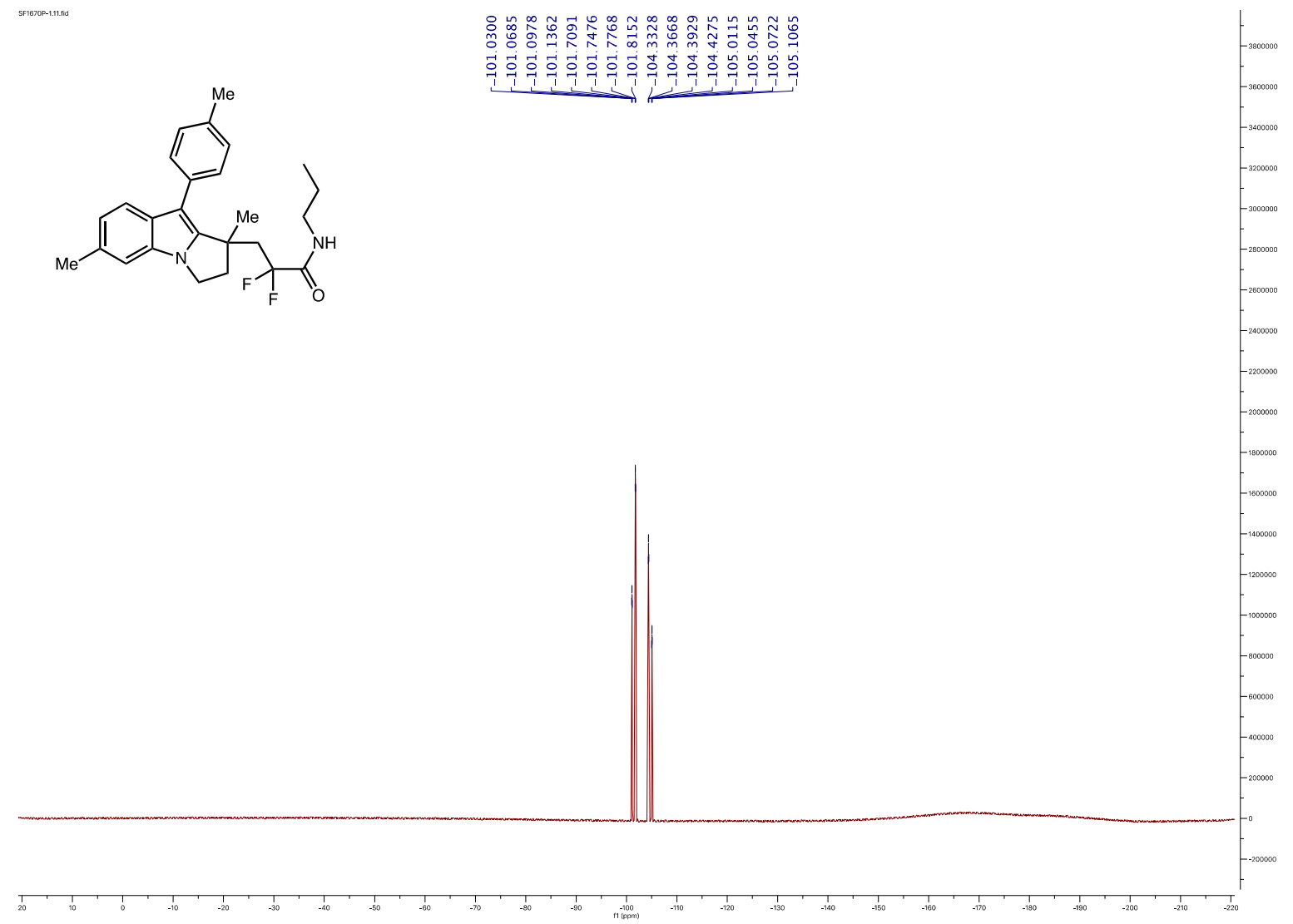

8a ${ }^{19} \mathrm{~F}$ NMR (376 MHz, $\left.\mathrm{CDCl}_{3}\right)$

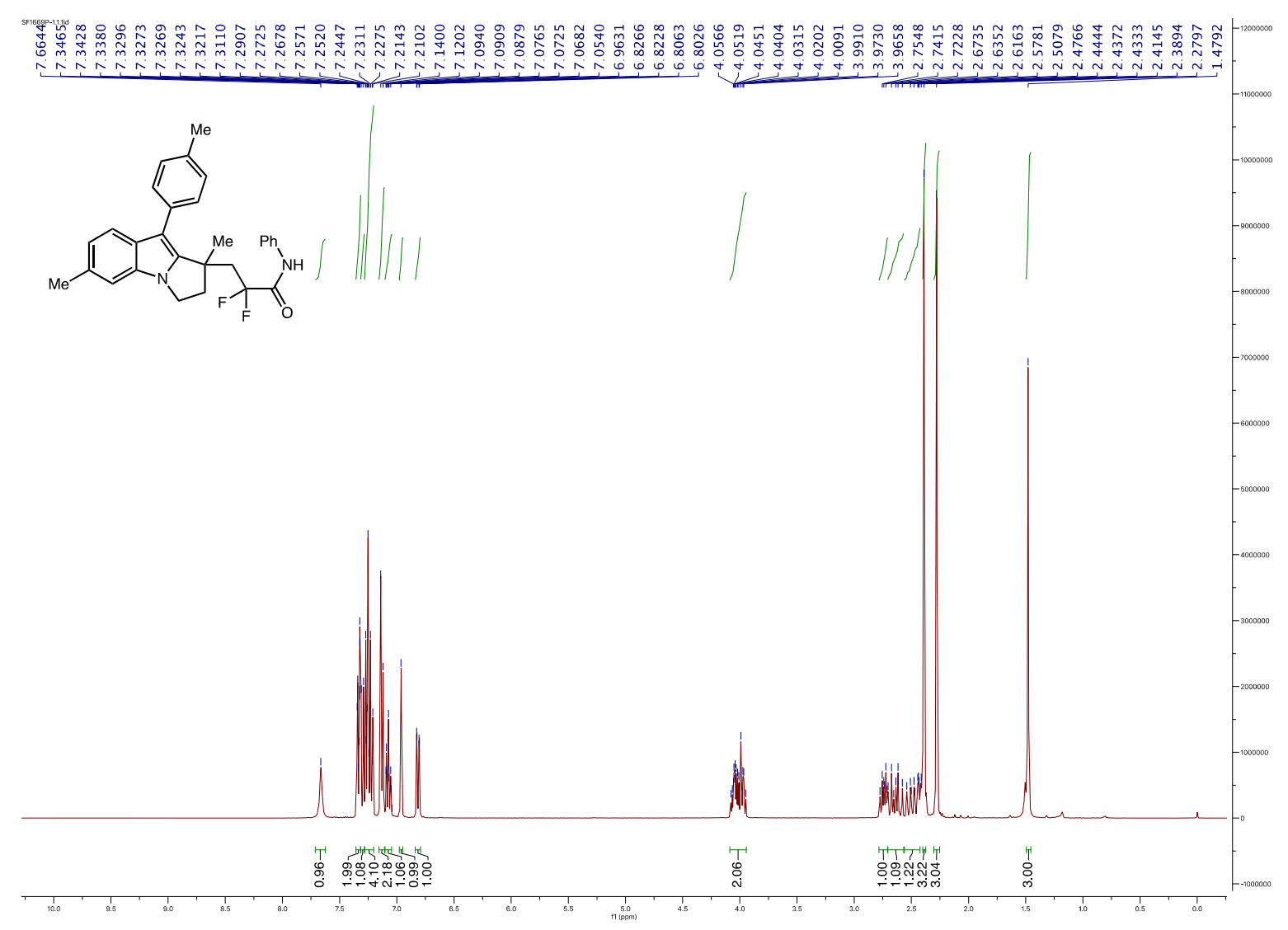

8b ${ }^{1} \mathrm{H}$ NMR (400 MHz, $\mathrm{CDCl}_{3}$ ) 


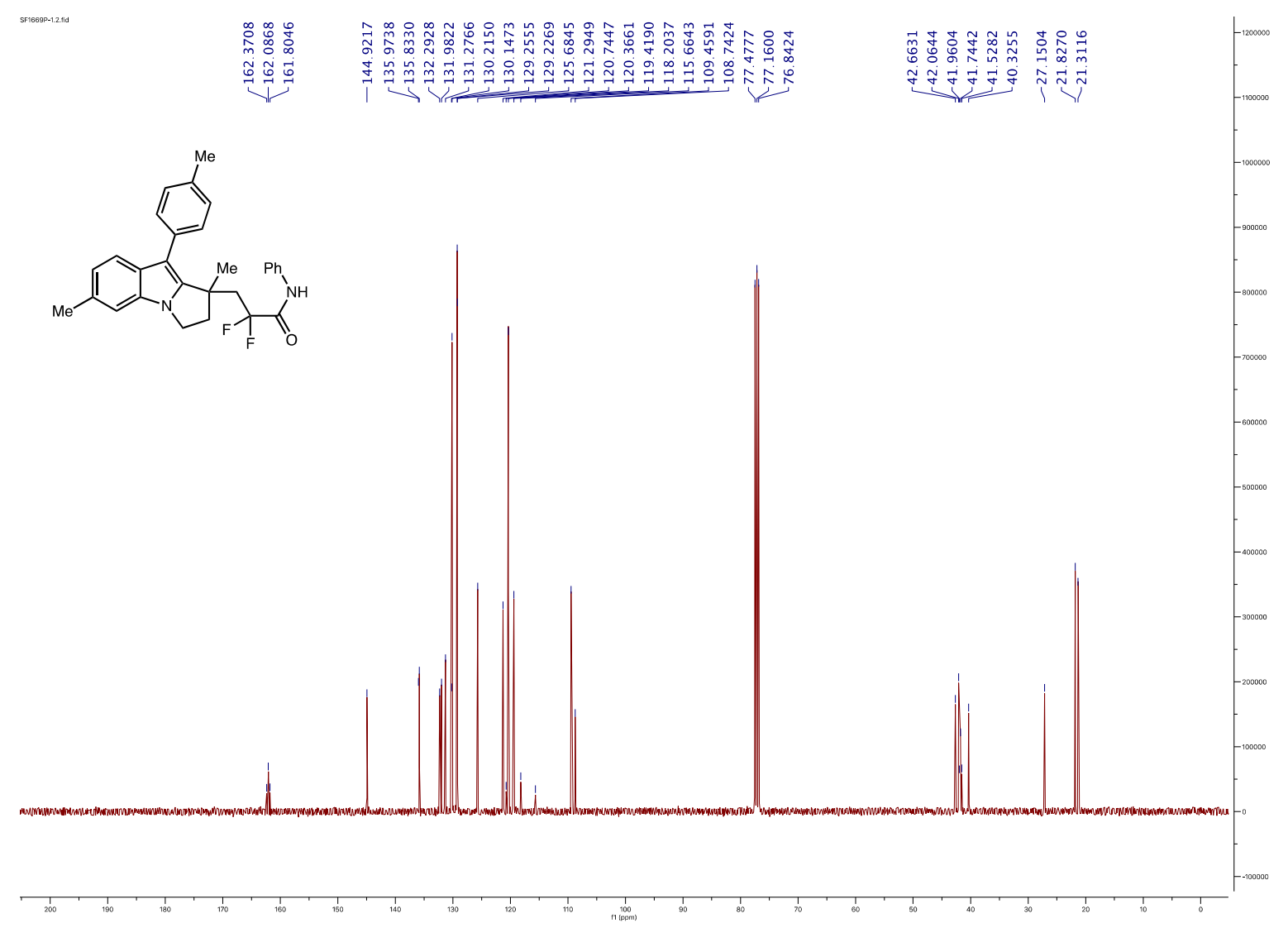

8b ${ }^{13} \mathrm{C}$ NMR (101 MHz, $\left.\mathrm{CDCl}_{3}\right)$

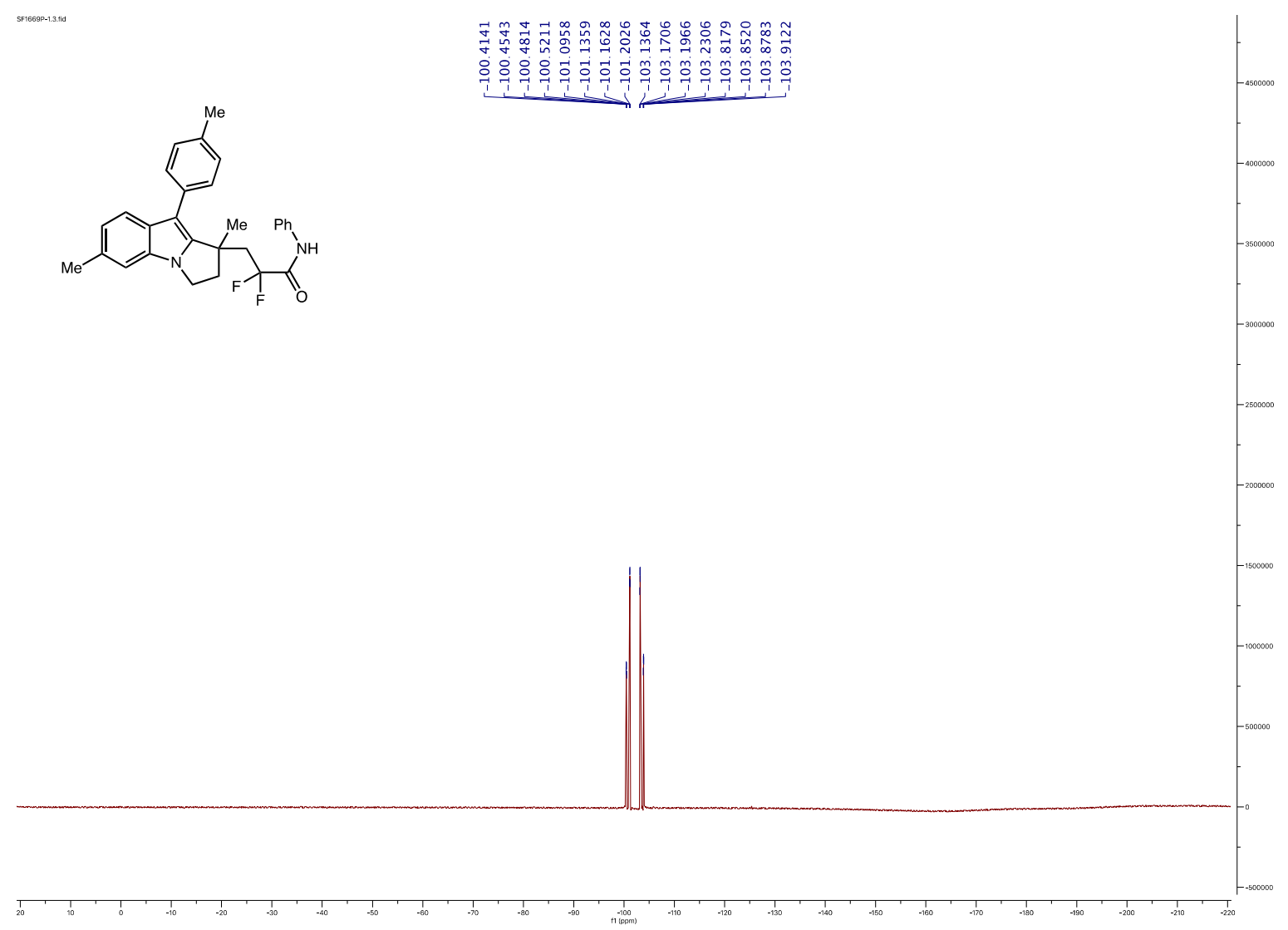

8b ${ }^{19} \mathrm{~F}$ NMR $\left(376 \mathrm{MHz}, \mathrm{CDCl}_{3}\right)$ 


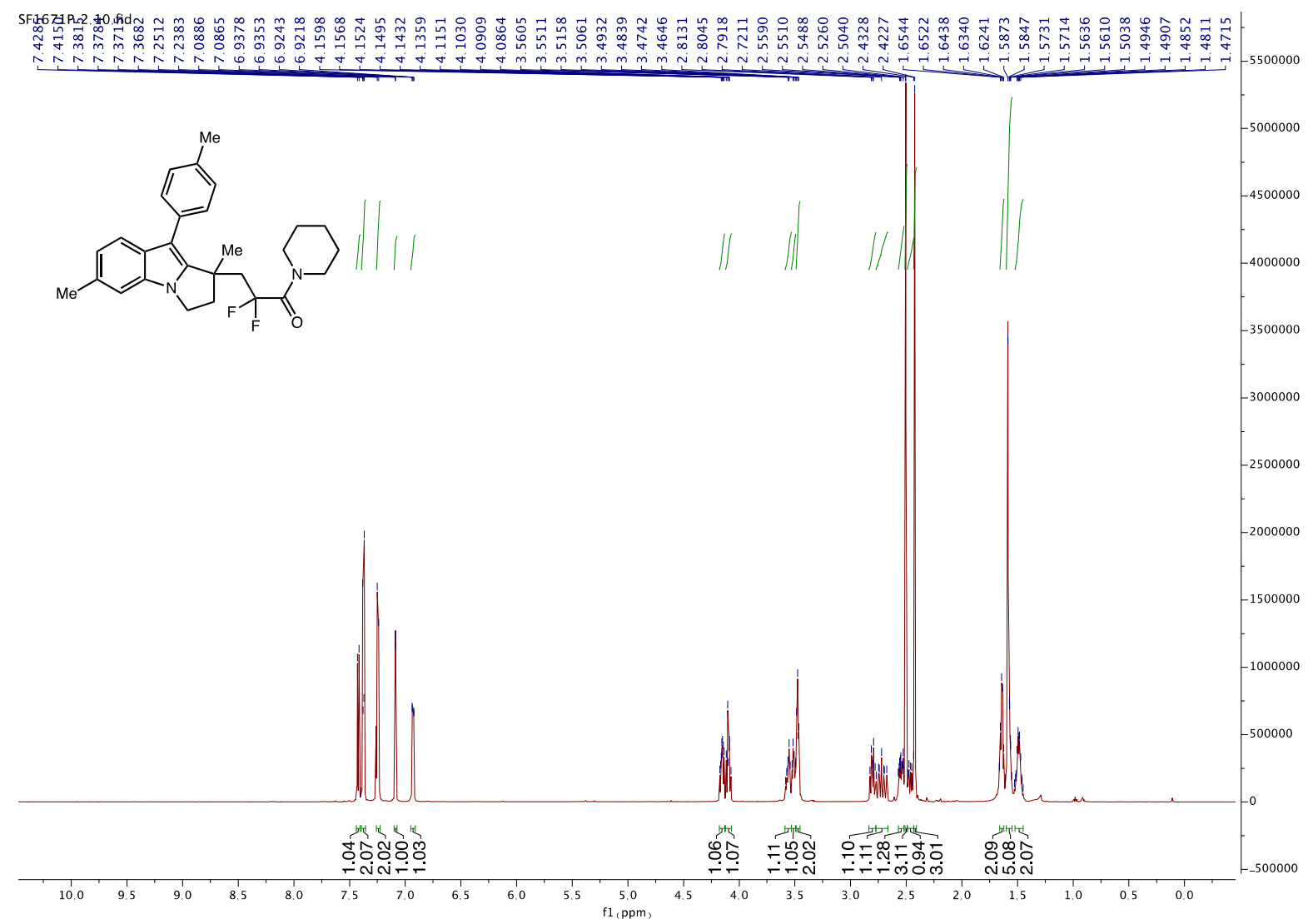

8c ${ }^{1} \mathrm{H}$ NMR $\left(600 \mathrm{MHz}, \mathrm{CDCl}_{3}\right)$

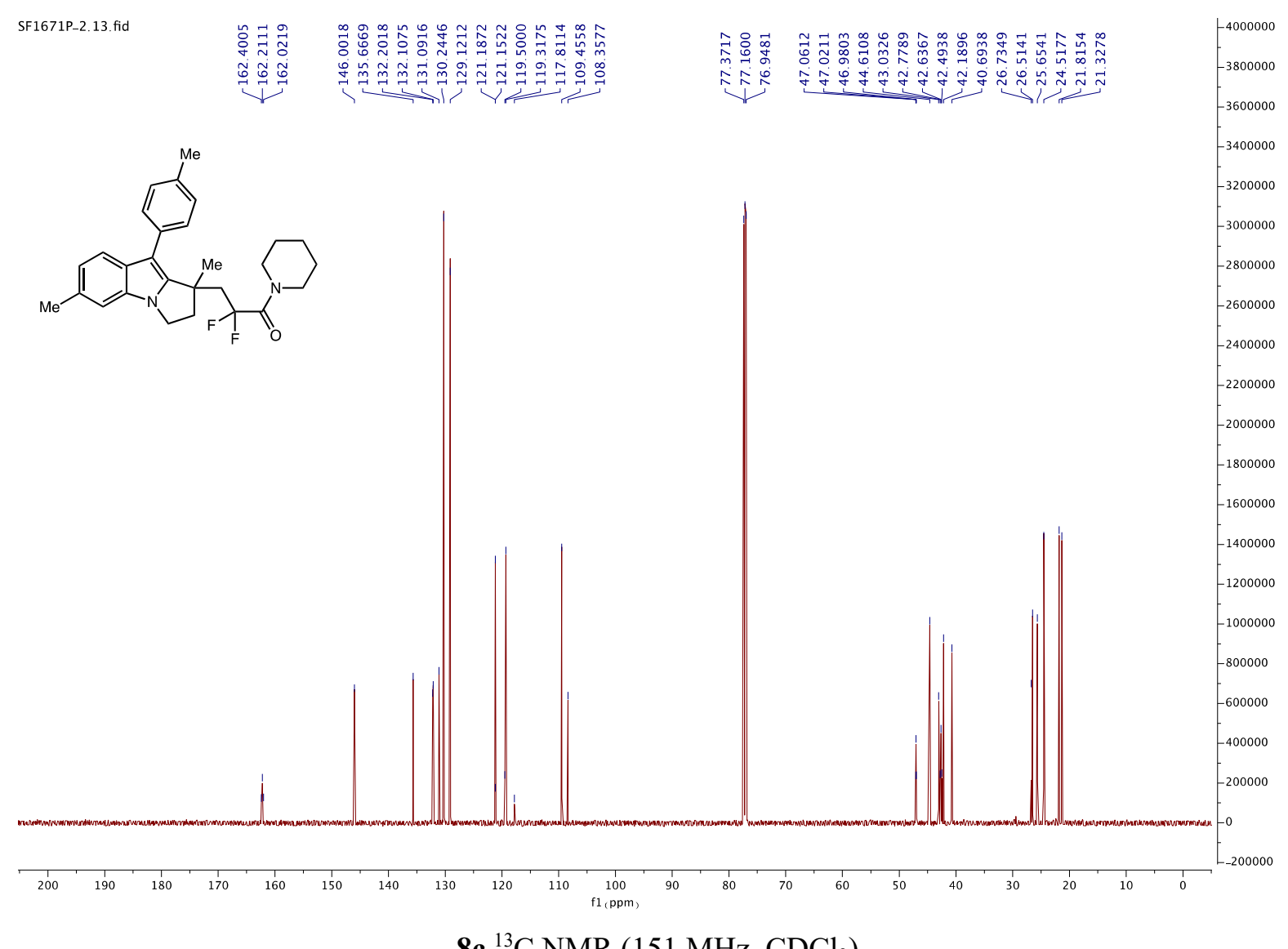

8c ${ }^{13} \mathrm{C}$ NMR $\left(151 \mathrm{MHz}, \mathrm{CDCl}_{3}\right)$ 

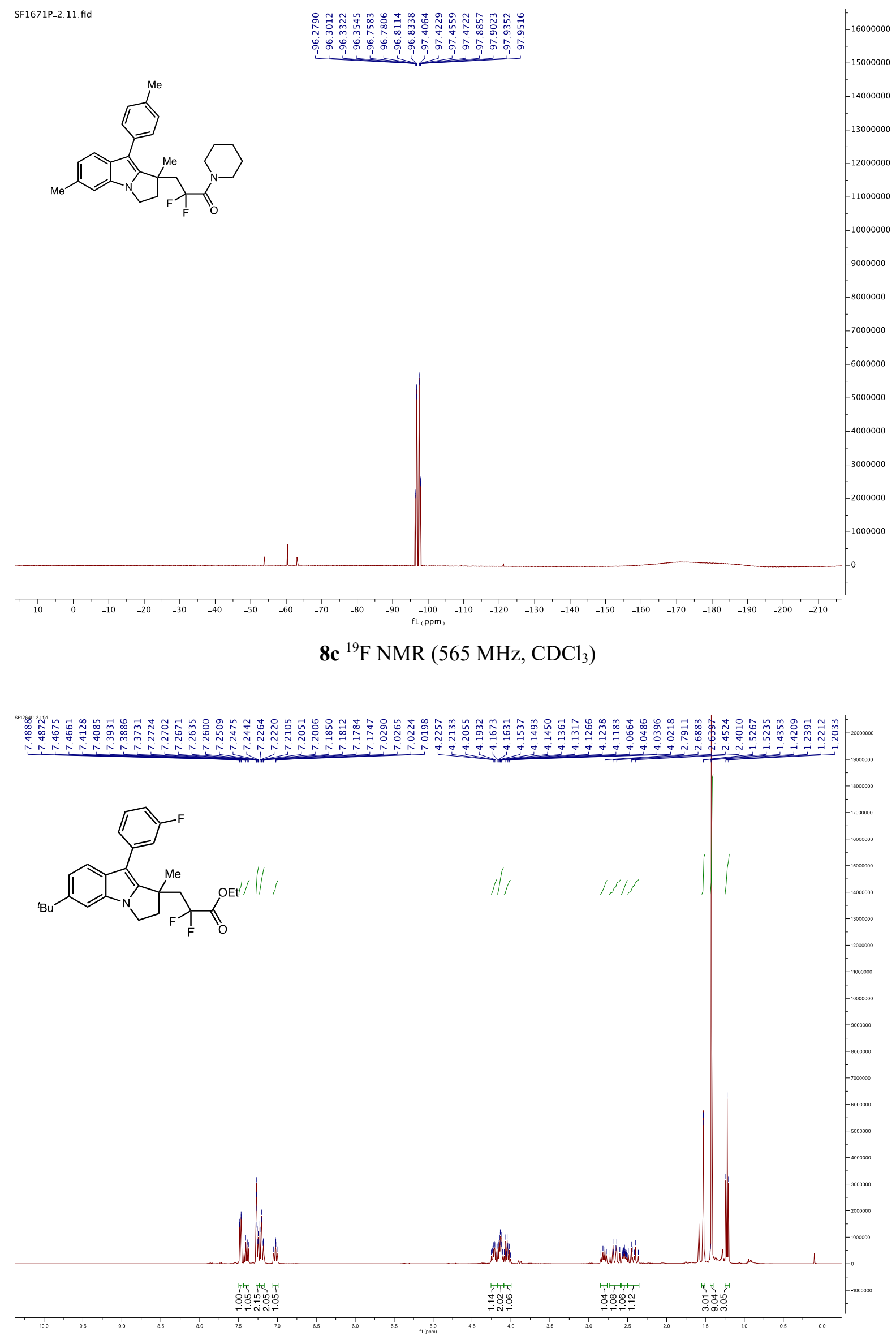

8e ${ }^{1} \mathrm{H}$ NMR (400 MHz, $\mathrm{CDCl}_{3}$ ) 


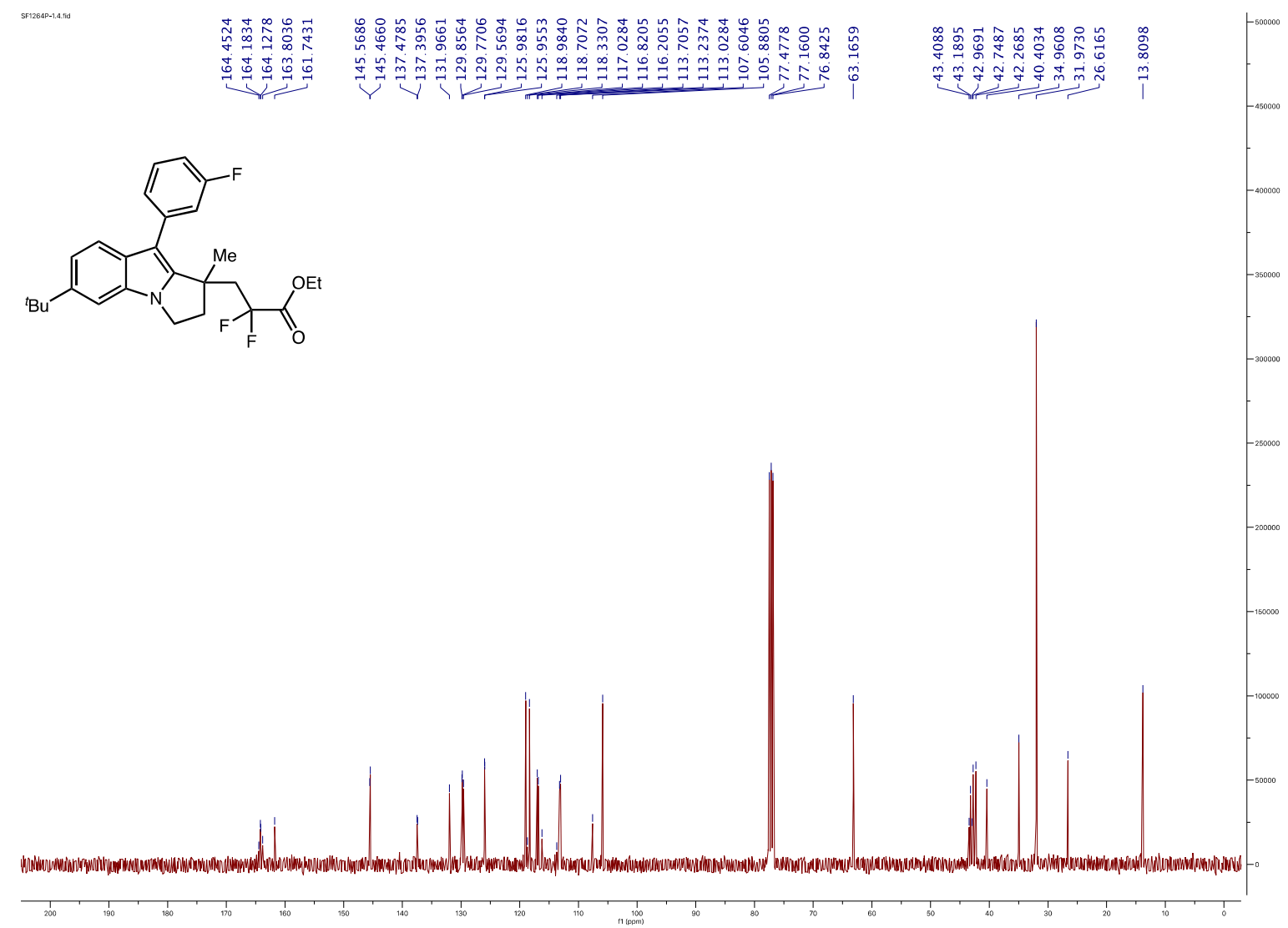

8e ${ }^{13} \mathrm{C}$ NMR (101 MHz, $\left.\mathrm{CDCl}_{3}\right)$

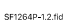

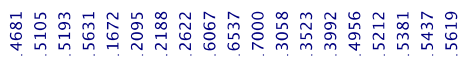

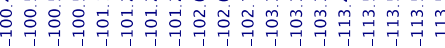
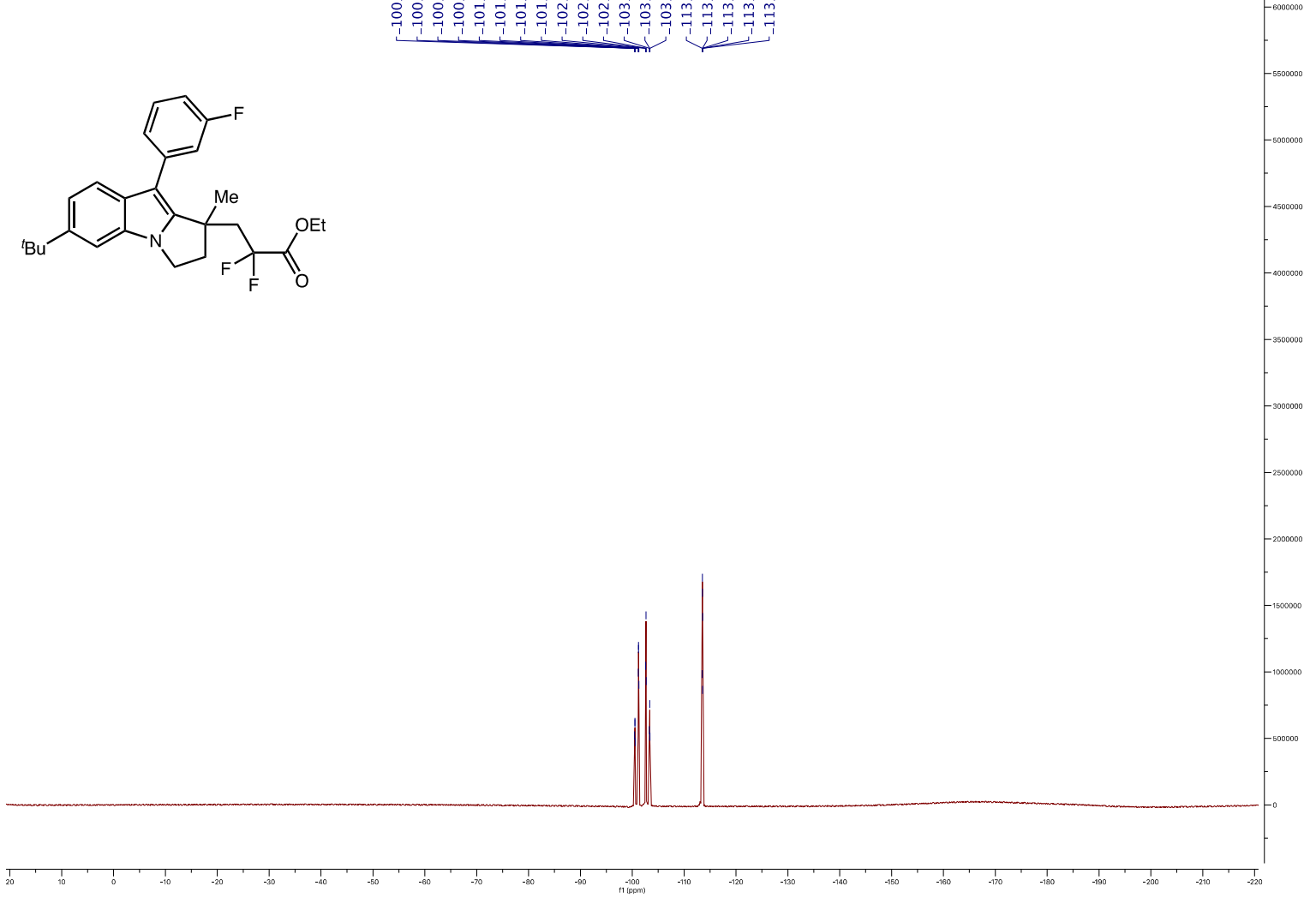

8e ${ }^{19} \mathrm{~F}$ NMR (376 MHz, $\left.\mathrm{CDCl}_{3}\right)$ 


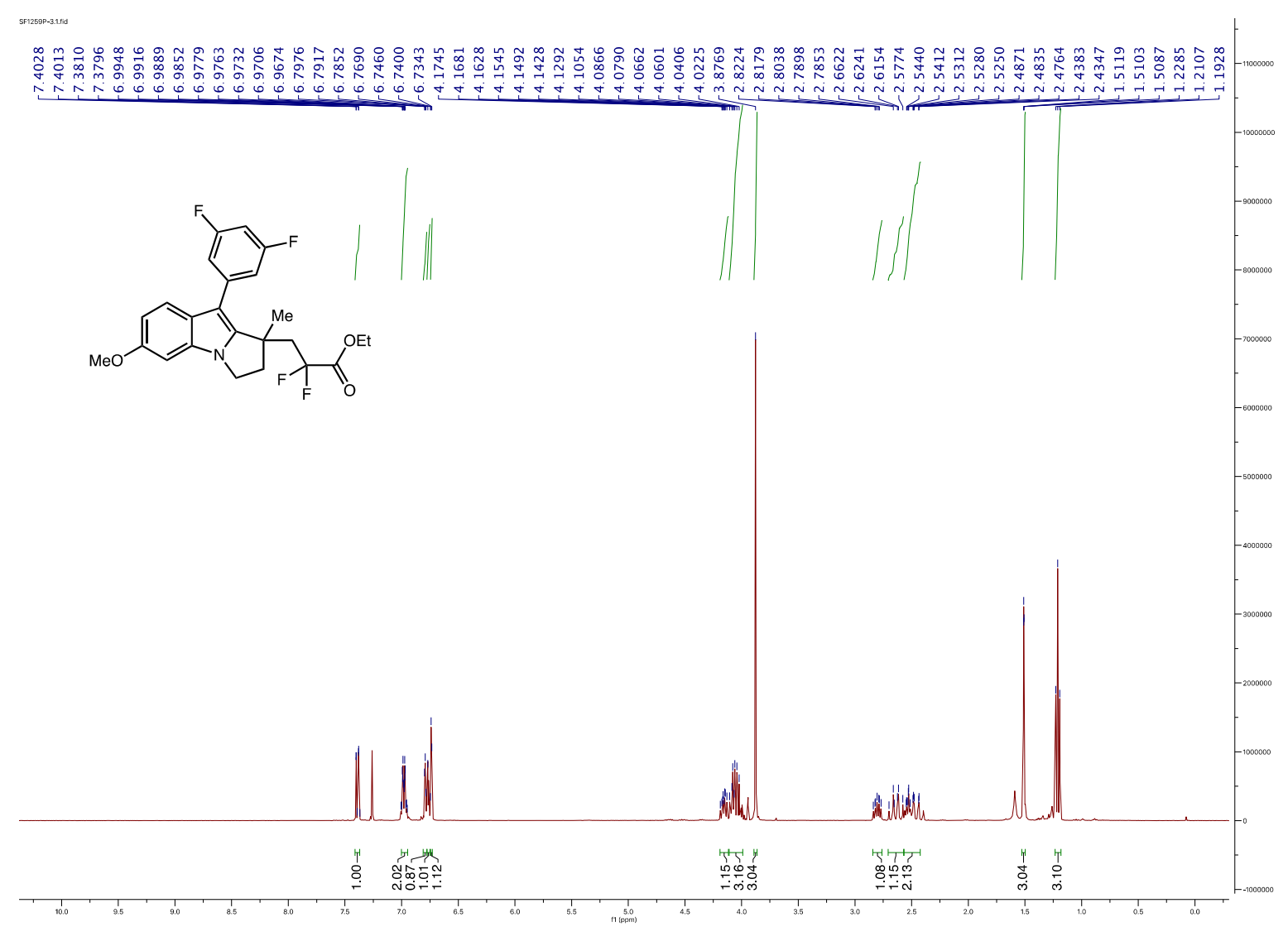

8d ${ }^{1} \mathrm{H}$ NMR (400 MHz, $\left.\mathrm{CDCl}_{3}\right)$

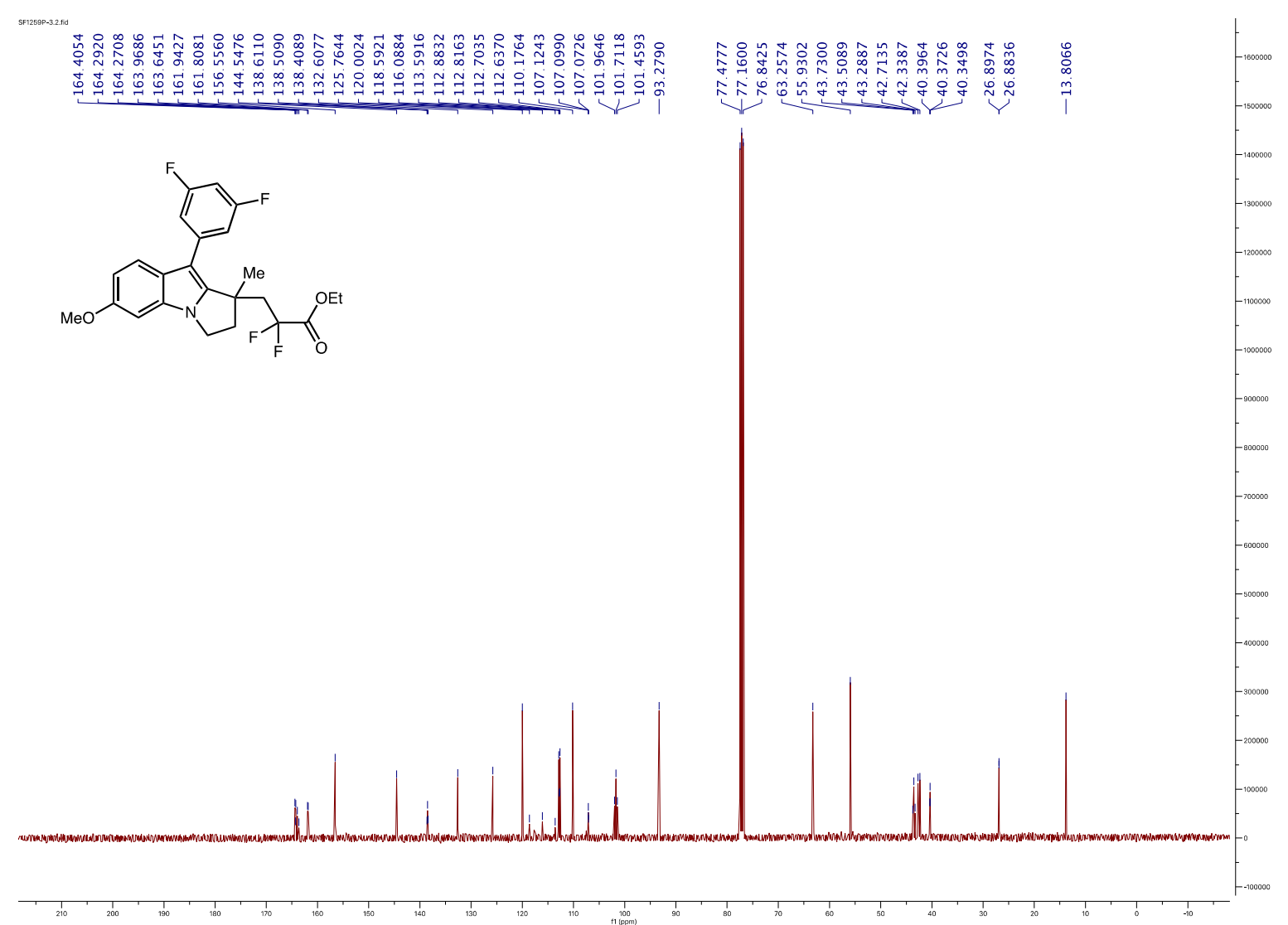

8d ${ }^{13} \mathrm{C}$ NMR $\left(101 \mathrm{MHz}, \mathrm{CDCl}_{3}\right)$ 


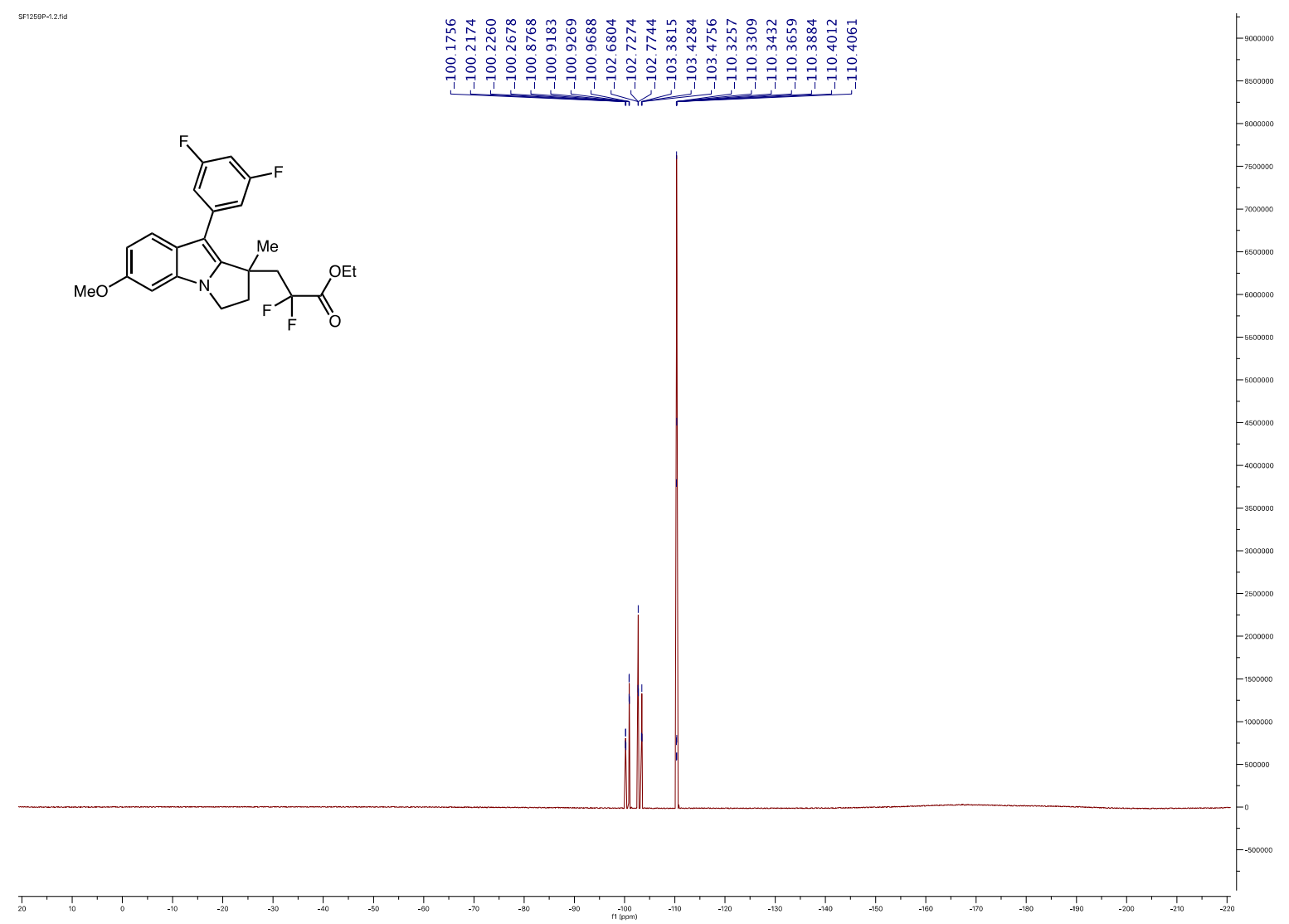

8d ${ }^{19} \mathrm{~F}$ NMR (376 MHz, $\left.\mathrm{CDCl}_{3}\right)$

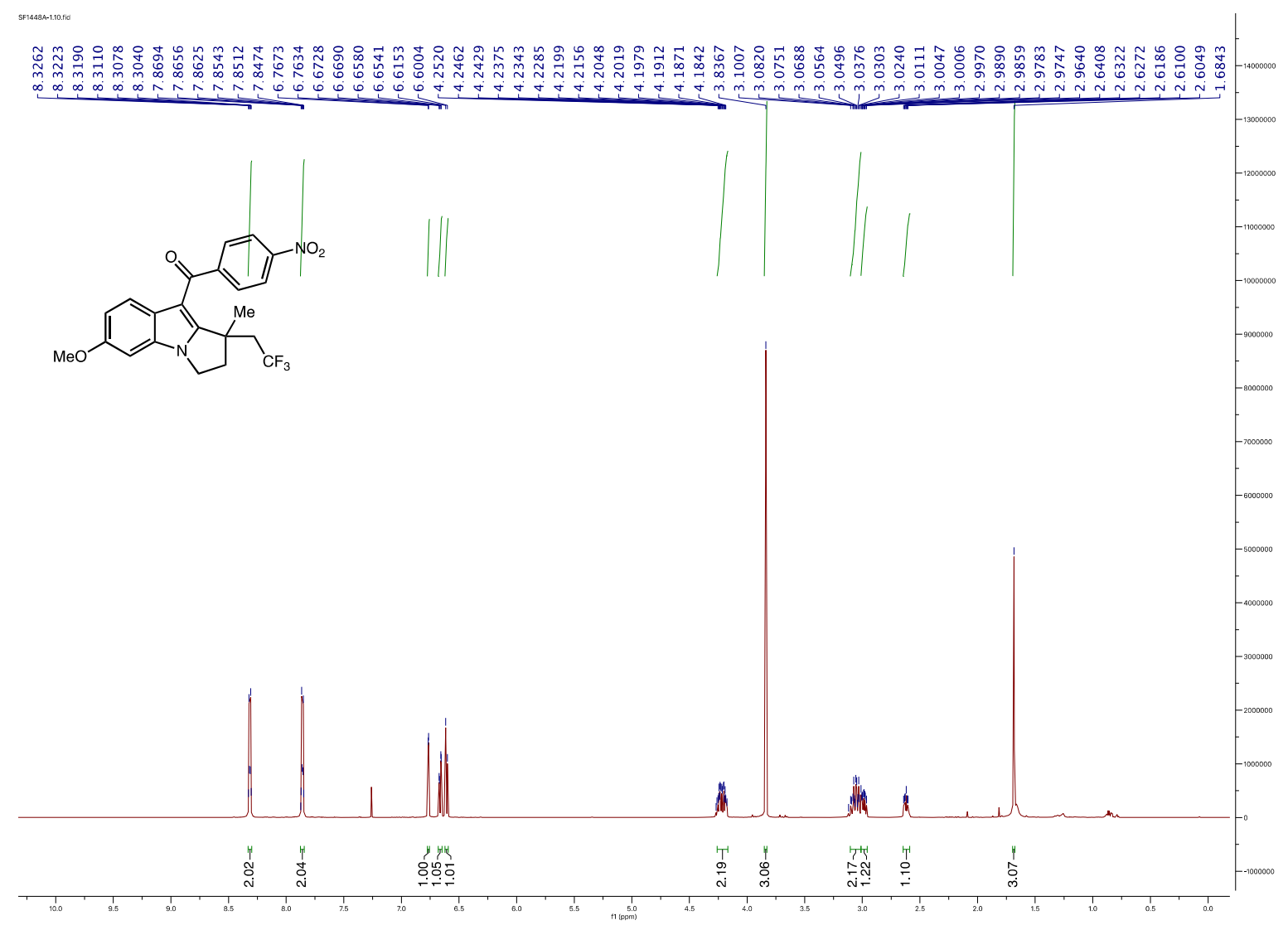

10a ${ }^{1} \mathrm{H}$ NMR $\left(600 \mathrm{MHz}, \mathrm{CDCl}_{3}\right)$ 


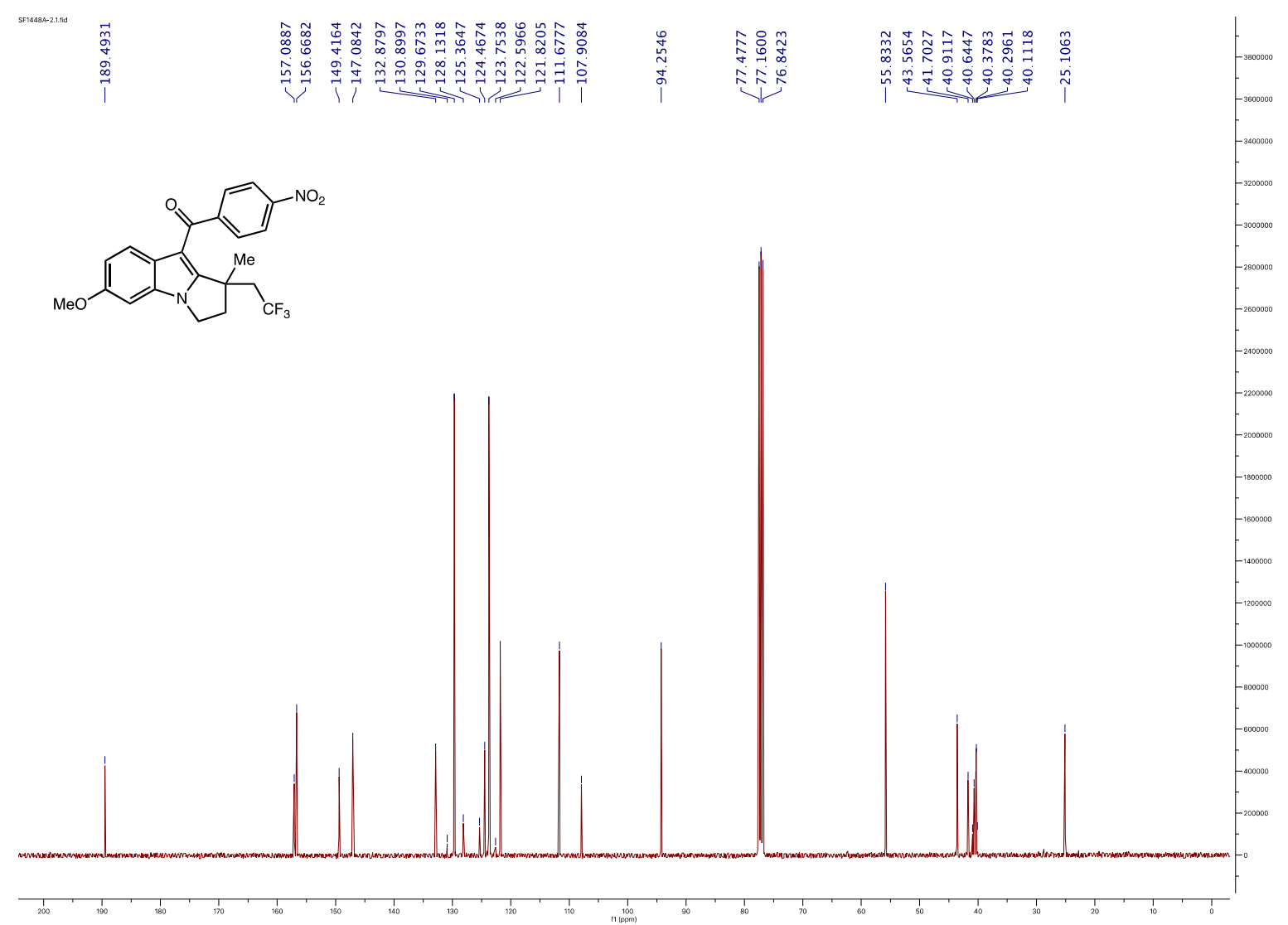

10a ${ }^{13} \mathrm{C}$ NMR $\left(151 \mathrm{MHz}, \mathrm{CDCl}_{3}\right)$

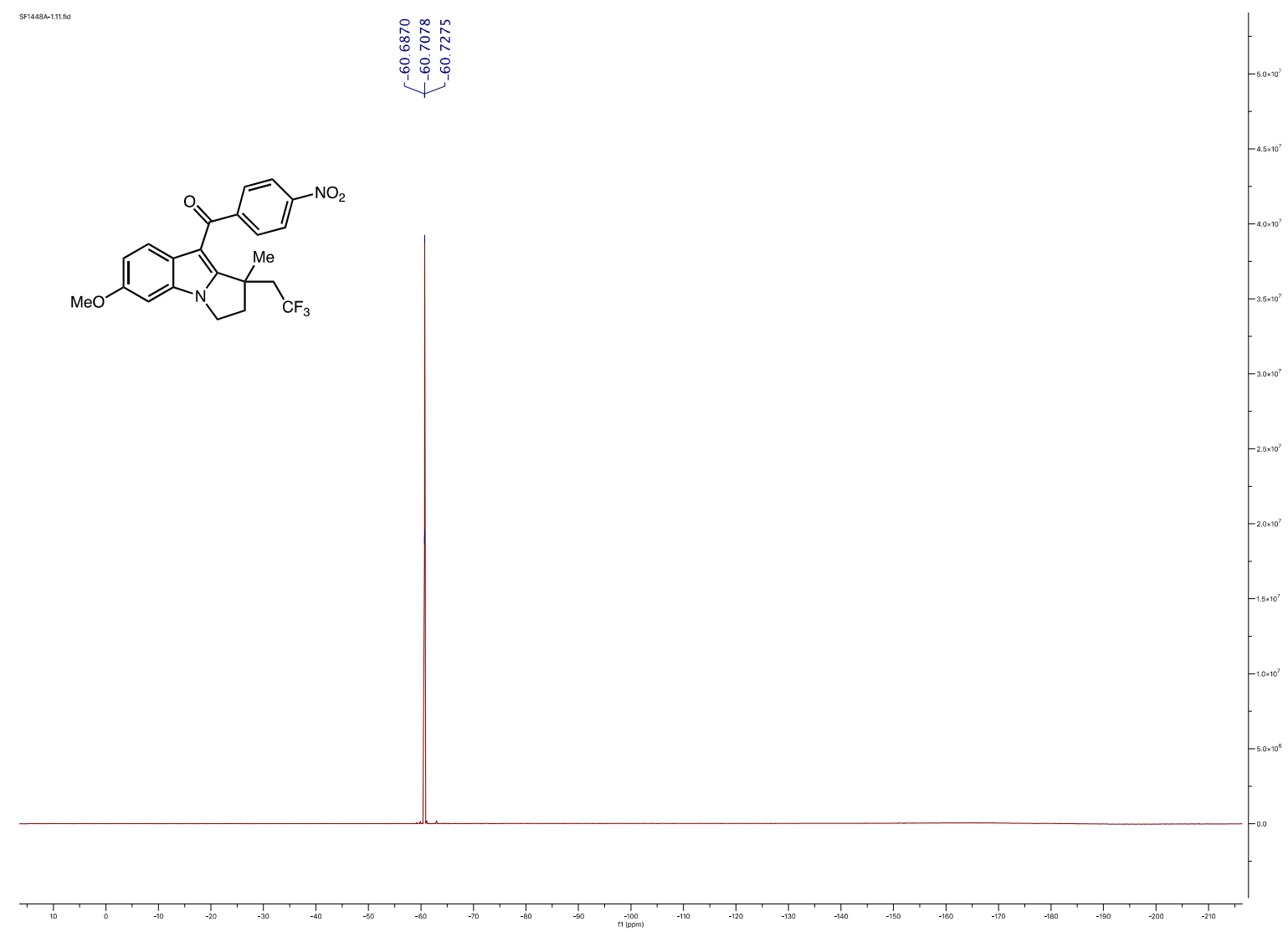

10a ${ }^{19} \mathrm{~F}$ NMR (565 MHz, $\mathrm{CDCl}_{3}$ ) 